\title{
$\sin _{4}$
}

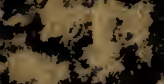
motion.

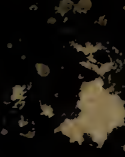

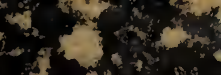

$2+5,2 y+2$

int

婙

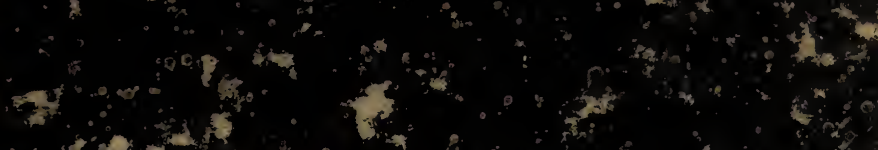

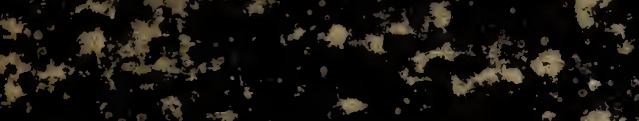

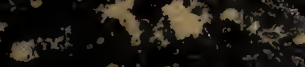

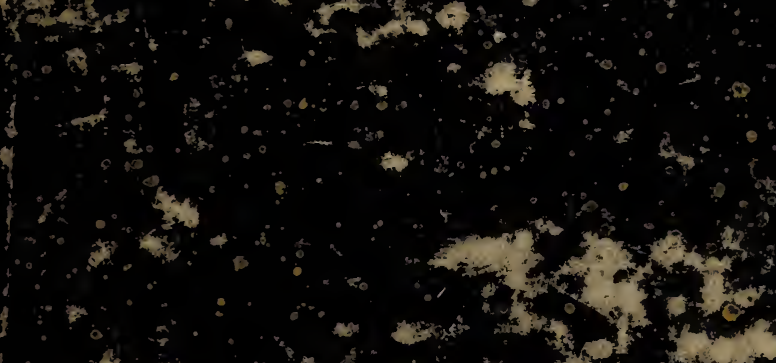

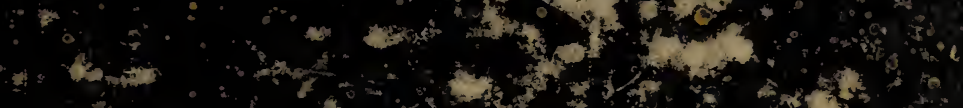

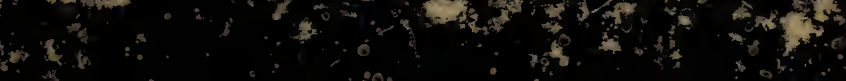

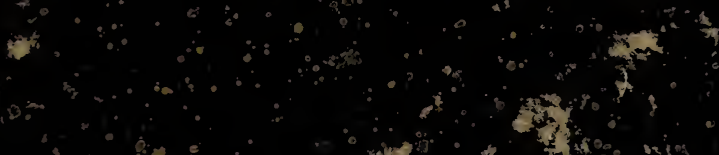

2. $c^{4} x^{2}-x^{2}+x^{2}$

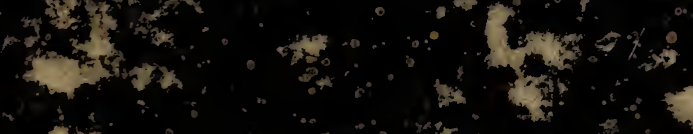

\section{ate 40 \\ .}

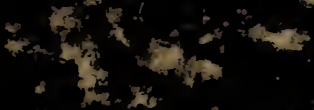

$$
\text { W" }
$$

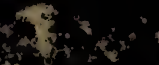

sy

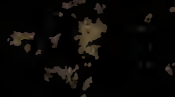

$$
\begin{aligned}
& -\frac{1}{3}
\end{aligned}
$$$$
3
$$

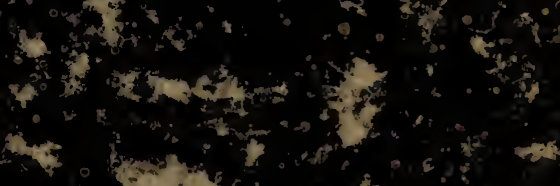

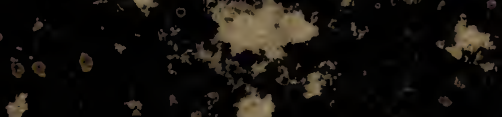$$
-c^{2}-2
$$

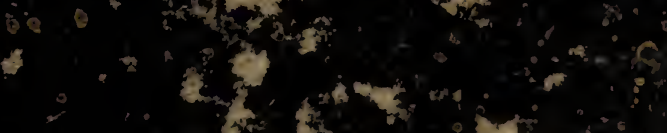

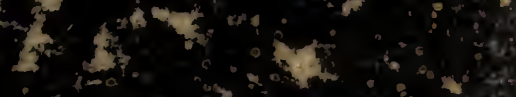




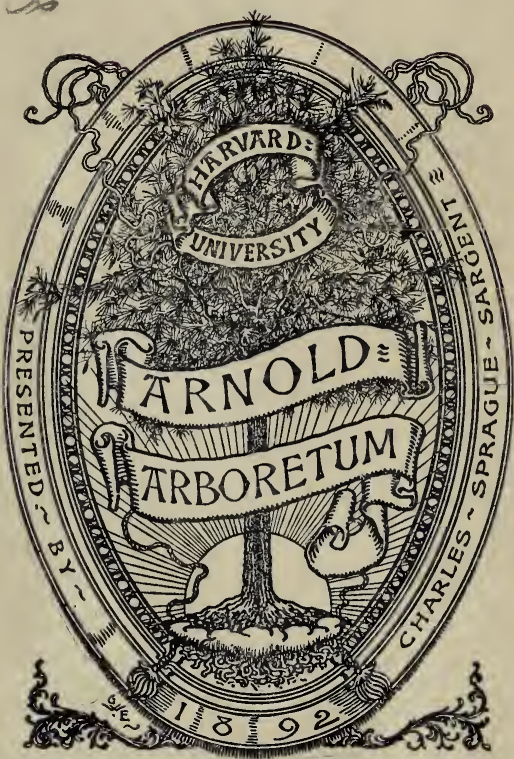


$x^{7}$

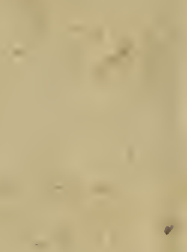

$<$

, .

-,

$\checkmark$

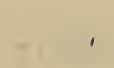

( 



\section{G E N E R A}

\section{P L A N T A R U M}

SECUNDUM

\section{CHARACTEPES DIFFERENTIALES}

A D

IMIRBELII EDITIONEI

REVISA ET AUCTA

EDENDA CURAVIT

\section{ROMANUS ADOLPH HEDWIG}

PROFESSOR BOTANICES IIPSIENSIS, SOCIETATUM BOTANRCA. RUM VARIARUM SOCIUS。

\section{I P S I A I 806}

A P U D I. H. R E C L A M. 


$$
\begin{aligned}
& \text { Mul1968 } \\
& 17847
\end{aligned}
$$

$x^{2}=1+19$
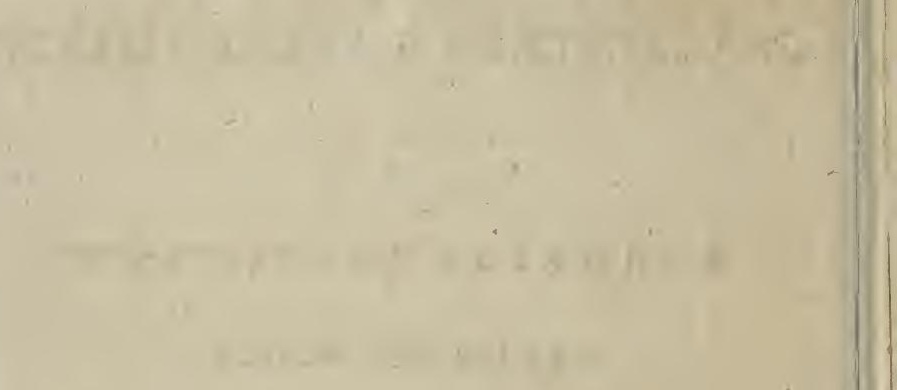

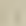

0,1897 in he

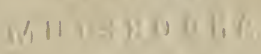

Whashali?

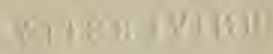




\section{PRAEMONENDA.}

Species plantarum qui ediderunt gravissimi bota: nici atque extare iusserunt earum Synopsin, non dubitarunt quidem generum characteres essentiales addere, eo ut videtur consilio, quod vegetabilium disquisitores ad manus haberent succinctum et brevem differentiarum conspectum. Angustioribus autem iusto limitibus esse conscriptas, ita, ut neque ubique perspicuitatem absolutam referant, neque, qui illis uti cupiunt, perfectam et iustam notam semper supedi ent ad genera disquirenda et dignoscenda. et alii botanci saepe sensere et tirones conquesivere. Neque enim, quam curavit venerandus de Schreber, generum editio omnibus ad manus versatur et si esset, nunc non cuncta vel nuperrime detecta vel disquisitione fideliori vegetabilium enata genera comprehendit. Nam cotidie botanicorum sagacissimorum et strennissimorum disquisitione et perseverantia nova indagantur, numerusque dirimitur et augetúr et cumulatur. Hinc generum editionem qunad characteres essen tiales veras et genuinas ordinare gravem esse laborem facile intelligitur, inprimis quum non unius sit, omnia vidisse et perlustrasse, vel omnia et infinita disciplinae volumina perlegisse et invicem contulisse: scilicet quod anctores vegetabilia variis ten poribus observarint et descripserint, unde dis- 
crepantia et incerta oriri debet notitia, aut non partes omnes essentiales inflorescentia, instante et absoluta, vel inde a germinandi tempore notarint; tandem partium aut rerum novarum studio flexila: quia induxerint. Inter ea non dubitavi hanc generum elitionem curare, necessitatis obsequio, disciplinae studio fideli, imo amicorum cohortatione ductus, inprimis quum ad hunc gravem laborem et Lamarkii et Mirbelii opus anno 1803 . Parisis edi. tum, *) animum meum non excitaret sed incitaret, quod potius augere non emendare volui.

Jam etsi in adornanda hac editione Linnei sequendum erat systema sexuale, rationali constru. ctione et harmonia amabili facile primurn et praestantissimum, tamen non dubitavi gravissimorum botanicormm emendationes suscipere et in usum referre quasdam classium natura ipsa iubente remotiones. Diu enim iam incerta erat polyadelphia, et polygamia potius respuebat veran veri systematis normam, ita, ut ante me summi extiterint viri qui easiem supprimerent. Arbitror etiam quemque experientia esse edoctum quantis fallaciis variae systematis Linnei quotque erroribus superstructae fuerint classes, classium ordinumque genera: quat cum accuratius perlustratis vivis in locis natalibus cerptis, exemplaribus perspicerent fidelissimi nostrae aetatis botanici plura refutata sunt, reliqua dies revelabit, ubi probis viris cordi erit veritatis studio naturam incedere arctissimo invicem amicitiae et hospitii vinculo; tunc enim, spes laetis. sima affulget, Linnei systema genuinum erit et purum extiturum.

Sed quum huius systematis classes quaedam naturales quasdam teneant familias sociali hospitia

con:

") Histoire zaaturelle des Vegetaux, tomus III, 
connexas: non vituperandus esse arbitror, qui in hac generum editione obsequutus sum praeter sexuale et systema naturale. Nam quae a Laurenbergio, Scopolio, aliis, hanc in rem suscepta suspiciuntur, a Jussieuis fere perfecta, certe fini pro. prius ducta admirantur. In quo systemate summi viri ordines, natura ipsa iubente, sibi invicem succedere, et familias tribusque aftabili modo ita vestigia sua legere voluerunt, ut mitissimo incessu classis classem, ordo ordinem, genera genus suscipiat, atque adeo ubi transitus facillimus non esse videatur, arbitrandum sit, coniunctionis membra adhuc ignota latitare. Quum autem in eo labores inpendam, hoc systema proxime disquisitionibus variis illustrare et in usum communem juvenibus disciplina nostrae studiosis aptum commendare, et vel vindicare contra Sprengelii aliorumque obiurgia vana et inepta; hoc unice addam omnia quae extant systemata sive artificialia sint sive naturalia ea propter perfecta et absoluta non esse posse, quod vel propriam naturam atque indolem vegetabilium omnium botanici nondum accurate satis investigarint, vel in tanto et fere indefinito detectorum numero flexiloquantur, vel in terminologia dissentiant, vel partium stndio suique ip. sius amore novarumque rerum indulgentia dncantur; ita, ut Jussieui systemati potius laudes quam vituperia inducat, quod aut plurima genera his illisque familiis affinia proponat vel statuat adhuc alia multa sedis incertae.

Equidem patris desideratissimi mei vestigia Jegens non dubitavi quosdam terminos varios, licet non omnes, ne plane inusitata vel nova indu. cam, adhibere, inprimis quibus uti debere ipsius naturae iussu vel accuratiori partium et fideli disquisitione arbitratus sum. Sic fasciculum ductu. lerum nominavit partem, quam vulgo nervum ap. pellant: truncum (vel corpus cl. Humboldti) su. perficialem, subterraneum etc. quae pars auctori- 
bus audit radix; sporas, quibus nomen est semina, et cet.; atque saepius, inprimis in addendis lussieuum sequutus sum, qui, secundum reram definitionem calycis et corollae, contra consuetum morem hos terminos adbibuit e. g. in irideis liliaceis etc. vel pro ortu harnm partium. Denique Gaertneri auctoritatem quoad albumen sporarum retinui, ubi Jussieuus vace perisporii usus est.

Caeterum iam laboribus prelio subditis ad manus venit Jaume St. Hilaire ,expositoon des familles nuturelles et de la germination des plicules" " Parisiis 1805. Aureum sane et praestantissimum opus, cui plurima, et neglecta in adidendis, debeo..

Tandem varia genera suspecta quidem esse arbitratus sum; sed praeterire nolui; huc pertinent Aubletii et Loureïri quaedam, aliaque ab obscrvatoribus inperfecte descripta anthesi vel absoluta vel instante, quo pertinent Ruizi et Pavonis varia: sed et horum gravissimorum virorum observata dies revelabit curiosiori peregrinationi et dilegentiori in veritatem perseverantia, imo arctiori botanicorum vinculo.

Equidem si boni quid in communem utilitatem his laboribus retulerim iudices probi sedeant botanici dilectissimi; quos rogatos esse volo, ut ad continuandam prospere opellam observata sua benevoli communicent. Dabam Lipsiac ante Id. Maii MDCCCVI.

R. A. Hedwig. 


\section{GLASSIS PRIMA.}

\section{O N A N D R I A.}

\section{III $O N O G Y N I A$.}

\section{Fructu infero, mono-triloculari.}

I. CA N N A, (BASILIER) IINN. LAMARK。 tab. I. (Scitaminea *) Calyx coloratus, brevis, trifidus; (triphyllus pers.) corolla hexapetata, (sexpartita PERs.) petalis quinque rectis, sexto extrorsum revoluto; stylus lanceolatus corollae adnatus; capsula muricato scabra, trilocularis, triangularis **) Nectarolyma bipartitum, revolutum (petalum sextum?)

2. HELLENIA, (HELENIE) RETZ. WILLD, HERETIERA retz.; LANGUAS Kölc. (Scitaminea).

Calyx

*) SCITAIMINEAE Juss, genera: sunit plantae canle herbacco, petiolis vaginantibus tecto, foliis nervosis; junioribus convolutis; floribus spathaceis, filanentis ad styli basin insertis; stamina dno in Globba $P$.

* ) Irnmortalis PROF. VAHt in pracfat, ad Vol. I. cnumerationis plantarum, Havnize 1805. pag. 10. jussit hanc aliaque Scitaminearum familiae genera ad Classem Gynandriam recedete, quum, teste ipso Limnaco Philos. but. 68. et fide Richardi, Desfoniainii, aliorum, vel stylus minus plusve cum filamento cohacreat, atcine tyronum ranli Canzam aliasque lunjus familiae plantas inter Gynandriam ipsam investigarent, cf. et RERSOON syuopg. plautt, 1805. P.I. 
Calux spathiformis, campanulatus, bifidus, fissuris inaequalibus; corolla limbo duplici, exteriori subtrifido, Nectarolyma diphyllum aut bifidum; capsula inflatr, coriacea, trilocularis, subglobosa.

3. C A T I M I I M , ( CATIMBAN) JUss. GLOBBA *) IINN. RENEALMIA IINN, et PERs. (Scitaminea); Calyx monophyllus tubulosus, bi-tridentatus, dentibus inaequalibus, trifidusque, Corolla tubulosa, trifida, superioribus fissuris duabus truncatis infra nectarilymate oblongo munita, basi bi-dentata, supra triloba, at medium ántherifera; bacca umbilicata, carnosa.

4. AMOMUM (AMOME) IINN, LAM, tab 2. (Scitaminea) Calyx trifidus, inaequalis, cylindricus; Covolla tripartita, patens, inaequalis; Nectarolyma bilabiatum, erectiusculum: capsula baccata, tri-locularis.

5. HOR NSTEDTIA, (HORNSTEDTIE) RETZ. willd. AMOMUM, rönı. (Scitaminea). Calyx bifidus; Corolla tubulosa, tubus longus, filiformis; limbus duplex, exteriori tripartito, trifidoque; Nectarolyma tubulosum; capsula trilocularis, oblonga.

6. HEDYCHIUM, (GANDASULE) KOENIC. ram. (Scitaminea). Calyx monophyllus, tubulosus, membranaceus, duplex, quinquepartitus; Corolla tubulosa, tubo longissimo, limbo duplici, trifido; Nectarolyma diphyllum; stylus longissimns; Fructus....?

7. COST,US, (COSTUS) LINN. AMOMUM LAN. (Scitaminer) Calyx trifidus, gibbus; Corolla bilabiata tripartita, ringens; Nectarslyma bilabiatum, labio inferiori latissimo, maximo, trilobo; Capsula (bacca? pens.) trilocularis, angulosa.

8. ALPINIA, (ALPINIA) IINN. AMOMUM LAR. (Scitaminea) Calyx tubulosus, tridentatus, aequalis, brevis; Corolla tubulosa, basi ventricosa, sexfida; fis-

*) Globoja perrooon Synopsis, No, $\mathbf{x}_{\text {. }}$ 
suris interioribus brevioribus; (tripartita aeqialis PEPs.) Nertaroluma bilabiatum, labio inforiori patente; Capsula trilocularis, polyspora.

9. MYROS M A , (MYROSME) IINN. (Scitaminea) Calyx duplex; exterior triphyllus, intetior tripartitus; - Corolla irregularis quinguépartita; Capsula trigona, tri- locularis, polyspora.

10. INARA NTA, (NARANTA). GALANGA IIN.

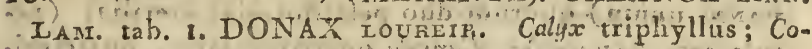
volla trifida; fissuris tribus exterioribus maloribus; Nectarolyma tripartitum, lacinia tertia superiori latero antherifera; drupa nuce mono-dispora (Capsula 1. locularis, I. spora. Pipisoon. Synops. p. 3.)

I1. CU RCUMA, (cUrcyna) zrN (Scitaminea) Spatha minima; Calux bifdus; Corolla tubulosa, quatriloba, Inbo altero interiori, et maiori filamenta quatuor sterilia, Attherae basi bicalgaratas, Capsula, trilocularis, polyspora.

I2. IR A E M FERIA, (ZEDOAIRE) IINN, IARg, tab. 1. (Scitaminea) Calyx monophyllus, ad summune obsoletus; Corolla tubulosa sexpartita, laciniis tribus alternis inaioribus lanceplatis patentibus otalibus; una inforiori bifida, maiori; Stigma bilamellatum, "Capa sula irigona, trilocularis, polyspora.

13. THALIA, (THALIA) scHREB. WILID. (Sci- taminea). Calyz triphyllus: "Corolla pentapetala, peta. lis duobus interioribus minoribus; Nectarolyma petaliforme, lanceolatum, coucavam; drupa oblonga, con$>$ tinens uucem urildculatom.

I4. PHPY N I U M, (RHRYNE) wrLLD. PHYLLODES TOUREIR. PONTEDERIA SWARZ (Scitaminea) Calux triphyllus; Corpllae petala tria, aequalia, tubo necrarolymatis adnata: ... nectarolyma monoplyilum tubulosum, tubo filiformi, limbo quatripartito; Capsula trilocularis; mices tres *):

*) IIó loco locum tenere videtur novum IVEXDLANDI genus, quod condutit in Sexti. Hannoverani Tol• I. 


\section{Fructu infero, 4. loculari.}

15. L O P EZ I A, (LOPEZIA) cavañ. (Epilobiacea) Calyx coloratus, caducus, tetraphyllus; Corollae inaequalis petala tria (secundum ventenAt; sed sec. CAvan. et vahr quinque) duo lateralia, plana, obtusa; uno inferiori reflexo, ad summum emarginato, breviori; filamenta tria (sec. Venten; sed sec. c.. VANN, unum) quorum duo petaliformia, sterilia, (petula sec. CAvaN.); Capsula quatrilocularis, quatrival. vis, globosa, polyspora.

\section{Fructu superiori.}

16. PHYLIDRUM, ( PHYLIDRE) BANCKS, GAERTNER; GARCINIA LoUREIR. Spatha uniflora, monophylla ; Calyx nullus; Corolla tetrapetala, irregularis, incompleta, petalis duobus exterioribus maioribus; Capsula trilocularis, polyspora.

17. VOCHISIA, (VOCHY) JUSSIEU, LAMARIE ill. t. 35. VOCHY aUbL. CUCULLARIA schreb. vahy. (PERs. p. 4.) (planta ex ordine indeterminato) Calyx minimus inaequalis, quatrilobus; Corolla tetrapetala, irregularis, petalis calyci insertis, quorum alterum superius, majus, basi calcaratum, inferius alterum magnum, non calcaratum; duo interiora mini. ma; breviora linearia; flamentum petaliforme, mombranaceum, artheris duobus leculis discretis; Fructus....

18. Q U A L EA, (QUALIER) IINN. I.AM, tab. 4. (ex ordine indeterminato) - Calyx coriaceus, inaequalis, quatripartitus; (tetraphyllus VAHL. foliolo unico majori basi calcarato); Corolla petalis duobus in aequalibus, superiori ad basin calcarato; Fructus globosus, monolocularis, polysporos.

fasciculo IVto. ZERUMLET (Scitaminea), nos supe: sus, spathaceus; Corolla tubulosa, papillionacea, tetrapetala; filamentnm unum; bacca trilocularis, polyspora. 
19. USTERIA, (USTERIE) SCHREB. MONODYNAMIS cMelin, - Calyx quatridentatus, dente altero laciniae ad instar reliquis multo majore; Corolla infundibuliformis, quatridentata ; Capsula compressa, mo* nolocularis, dispora; Sporae aristatao.

\section{Fructu monosporo.}

20. BOERHAAVIA, (BOERHAAVE, TASSO. LE) IINN. IAM. tab. 4. (Nyctaginea) (PFRSOON. Sy. nopsis p. 36. ad Class. III. 1, retulit.) Calux nullus, (Cal. margo tubuloso obconicus, integerrimus PERs.) Corolla campanulata, monopetala, plicata, infera, ovario inserta, quinquefida; fissuris obtusis; (Fructus tuberculatus); Spora unica basi corollae texta. (Stamina 1. 2. 3. 4. et 10. PERs.) (VAHL ad hanc classem nonnullas Boerhaviae species referri jussit. Caeterum Boerhavias ad classis diandriae monogyniae plantas flore completo infero monopetalo retulit; PERsOoN in enchir. ad classem tertiam.)

21. SALMONEA, (SALMONEE) VAHL. IOU. REIR. - Calyx inferus, compressus, quinquefidus, la. ciniis subulatis; Corolla formata o lamella oblonga, in tubum cylindricum trifida, fissuris longitudinaliter conniventibus; limbi laciniae rotundatae media lon. gior, cucullata; Ovarium subrotundum, compressum; stylus inflexus, medio ventricosus; Stigma crassiusculum; Cupsula (Silicula LoupeIR) compressa, bilocularis, scabra, monospora.

22. P O L L I CH I A, (POLLICHE, Pollique) AIT. IAM. NECKERIA gMELIN. - Calyx monophyllus, quinquedentatus; Corolla nulla; (pentapetala VAHX) Spora una, basi squamato calycis inclusa, et affixa medio squamae baccatae, receptaculo inservienti; (Receptaculum squamae baccatae, aggregatae, fructum olovan(eS VAIIL).

23. SA L I COR NIA . (SALICORNE) IINN. LAM. tab. 4. (Chenopodiaceae) Colyx ventricosus, tetragonus; 
trancatus, integer; Curolla nulla; Stigma bifidum; Spo: ra unica in fundo calycis inflati.

24. "HIPPURIS (PESSE) IINN. IANI. tab. 5. (Fluviatilis) - Calyx (sec. LrNN. nullus) obsoletus, integer; (Calycis margo bilobus vaHL); Corolla nulla; Stigma simplex; nux. una, unilocularis, monospora, infera.

25 LACISTE M A (LACISTEME) SW. WILLD. PIPER, REPa. NAEVIATOSPERMUM RICHAPD - Amentum tectum squamis imbricatis, concavis, unifloris; squamulis duabus linearibus, lateraliter cuiquesquamae adpositis; Curolla quatrifida; filanentum bifidum ad medium nectarolymatis positum; (Capsula baccata pedicellata, monolocularis, monospord, Iatere dehiscons; Spora evalvis filipendula pedicellata - VAHL ad monandriae trigynias retulit.)

\section{$D I G Y I I A$.}

26. CORISPERMUM, (CORISPERME) IINN. IAM: tab. 5: (Chenopodiacea) Calyx nullus; Corolla diphylla: (al. Calyx diphyllus, cor. nulla, varru). Spora unica, elliptico ovalis, compressa, (convexo plana YAHL) Innda.

27. CALLITRI CHE, (CALLITRIC) LINN. LAM. tab 5. - Calyx inferus, diphyllus; Corolla sulla; Capsula membranacea, marginata, tetragona, biloeularis tetraspora; (Flores nomulitis nonoici 1'Bns).

28. B LI TUM, (BLITE) IINN. IAM. tab. 5. (Chenopodiacea). Calyx trifidus; Corolla nulla; Spora una, calyce succulento et baccato inclusa.

29. C A N N , (CINNE) IINN. (e familia graminum.) - Gluma calycis uniflora, bivalvis, inaequalis; corollae gluma bivalvis, valvula exteriori ad summitatem mucronata; spora unica, cylindrica.

30. JARAVA, (JARAVA) rourz et pAvon. (c familia graminum) Caiyx gluma uniflora, bivalvis, val- 
vulis inaequalibus; Corollae gluma univalvis, minor, papposo aristata, ad basin pilis copiosissimis cincta; spora una, oblonga. (Alopecuri genus rediro videtur ${ }^{*}$ ).

\title{
CLASSIS SEGUNDA.
}

\author{
D I A N D R I A \\ $I M O N O G Y I N A$.
}

I. Flore infero, monopetalo, regulari.

3I. NYCTANTHES, (NYCTANTHE) IIN⿴囗 IAN. tab. 6. (e fam. pll. Jasmini) Calyx integer; Corolla hypocrateriformis; laciniis quinque oblique trunc2tis enarginatis; (quinque-octofida); capsula compressa, mprginata, cordiformis, bilocularis, bipartibilis, loculis monosporis.

32. MOGORIUM, (MOGORI) JUSSIEU, IAM. tab. 6. NYCTANTHES IIAN.; JASMINUM WIILD. (e famil. pll. Jasmini) - Calyx octofidus; Corolla

*) Sub hac Monandriar, ord. Monogyniae, Classe Hippolit. FUIZI et Jos. PAVUNIs, (auctorum praestantissimoruso forac peruvianac et Chilensis prodromi ed. 2 da auctior. et cmend, ; Romae 1797.) apud rensoontura in enchir. desidero genus AciOSTA, cujus charact, generis differentialem, hanc contituerum: Calyx subbilabiatus. contortus; recturolyma conico carinatum, antheriferum: pomum quincueloculase: anthere ut in cucusbitaceio, amdialata. 
hypocrateriformis, octofida; bacca regulariter didyma, vel dicocca, trilocularis, dispora (distinctum genus ob constantem octenarium laciniarum numerum).

33. JAS M I N UM , (JASMIN) IINN. LAM. tab. 7•; (e familin pl. Jasmini) - Calyx quinquedentatus, aut quinquefidus; Corolla hypocrateriformis, quinqueocto-fida; bacca bilocularis, dispora, dicocca; Sporae solitariae, arillatae,

34. SYR I NGA ; (LILAC.) LILAS. Juss. IAM. tab. 7. (e famil. plantt. Jasmini). - Calyx quatridentatus; Corolla infundibuliformis, quatrifida ; Capsula ovalis, oblonga, bilocularis, bivalvis, dispora; Sporae membranaceo alatae. (margine membranaceo cincta vaHL.)

35. FOR SYTHIA. (FORSITHIE) VAHL. SYRINGA THUNB. Japon. RENGIO TRAEMPF. DECUMARIA LINN, WALTER - Calyx quatripartitus, laciniis lanceolatis; Corolla campanulata, quatrifida, fisşurae ad medium; fructus - - ? (Genus jam willdenov1o a Syringa distinguendum.)

36. LIGUSTRUM, (TROENE) XINN. LAM. tab. \%. (e familia plantt. Jasmini) - Calyx brevissimus, minimus, quatridentatus; Corolla quatrifida ; bacca globosa, monolocularis, (bilocularis, loculis disporis vAIIL) tetraspora.

37. PHILLYREA, (FILARIA) LINN, LAM. tab, 8. (e familia plantt. JASMINI) Calyx minimus, quatridentatus; Corolla quatrifida, tubo brevissimo; Drupa baccata, globosa, bilocularis; loculis monosporis; una saepe abortiva; putamine chartaceo; Sporae solitariae.

38. OLEA, (OLIVIER) IINN. IAM.tab. 8. (e fa, milia plantt. Jasmini) Calyx quatridentatus, Corolla quatrifida, laciniis subovatis; drupa ovalis, putamine os$\mathrm{seo}$, nuce uni-biloculari, mono-di-trispora, altera saepe abortante. 
39. OR NUS, (ORNE) MILI. Calyx quatripartitus; Corolla quatripartita, petalis longis ligulatis, filamenta longa; nux alata.

40. CHION A N T HUS, (CHIONANTHE) IINN. IAM. tab. 9. (e familia plant. Jasmini) Calyx quatridentatus; Corolla quatrifida, laciniis longissimis linearibus; drupa ovoidea, putamine osseo striato, nucleum continens uniloculsrem, monosporum, striatofibrillosum.

4I. FONTANESIA, (FONTAINESE) IABIL. IARD. LAMARK tab. 23. (e fam. plantt. Jasmini) Calyx inferus, quatripartitus; Corolla quatripartita, laciniis duabus profundius incisis (petata duo, unoquoque in duas lacinias diviso IAB1LL.); Capsula compressa, membranacea, bilocularis, non dehiscens; loculis monosporis. (VAHL retulit ad plantas flore completo, infero, polypetalo.

42. P I M E L E , (PIMELEE) sMiтн. ІАM. tab. 9.

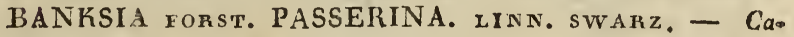
lyx nullus; Corolla infundibuliformis, unbulosa, quatrifida; stamina inserta corollae fauci; $n u x$ superior, corticata, unilocularis, monospora. (VAHr retulit ad plantas flore incompleto, ante Margaricarpum.)

43. ERA N T H E M U M, (ERANTHEME) IINR. IAM tab. 7. (pyrenacéa) - Calyx quinquefidus; $C_{0}$ volla quinquefida fere regularis, tubo filiformi, medio curvato; antherae extra tubum corollae; Stigma simplex; Fructus capsula polyspora.

44. MAYTENUS, (MAYTENUS) FEVILI. Chil. mol Ir, hist. n. Chil. Juss, varre. - Calyx quinque. lobus, monophyllus, minimus; Curolla campanulata, in. tegra ; stigma simplex; Capsula parva, compressa, ovata, bi. valvis bilocularis, dispora, marginibus dehiscens; Sporae ovato oblongae, imo loculo affixa. keviriex et JusSrev. 
45. HOPPEA, (IIOPPEE). wirld *). Calyx qua. tripartitus, aequalis; Corolla cyathiformis, quatrifida, laciniis revolutis; Capsula unilocularis, bivalvis, polyspora $\left.{ }^{* *}\right)$.

46. CARANGA, ( GAPANGE) vaHL. JUss. - Ca - lyx bivalvis, valvula altera minori; Corolla calyci bre- vior, monopetala, lobo inferiori latiori; stamina duo oodem lobo inserta: ovarium superum; stylus unicus; Capsula calyce tecta, bivalvis, valvulis dissepimento orali paralielis, bilocularis, polyspora. JUSSiEU.

47. G A L I ' EA, (GALIPIER) AUBLET, (o familia plantt. ordinis indeterminati) Calyx tubulesus, tetrapentagonus, quatri-quirque dentatus"; Corolla hypocrateriformis, profunde quatri-quinquepartita, subinaequalis ; stamina quatuor; dnobus brevioribus, sterilibus; cvarium subpentagonum; stigma quatrisulcuin; Fructus... ( $P$ AHL retulit ad sequentem et secundum ordinem, post micrainthemum).

43. ROETTLERA, (ROETTLERE) ROETTLR in epistolis ad VArru. - Calyx monophyllus, pilosus, co. rolla brevior, coloratus, laciniis laneoolatis persistentibus; Corolla monopetala campanulata; tubus nullus; faux inflata, incurvata; limbus quinquefidus, laciniis subaequalibus; stamina quatuor, filamentis e fundo corullae oris, brevibus, incurvatis, quorum duo superiora sterilia: antherae didymae; ovarium ovatum; stylus filiformis, staminibus longior; stipma simplex; capsula orata, acuminata, bivalvis, polyspora.

II. Flore infero, monopetalo, irregulari; fructu capsula aut bacca.

49. PAE DEROTA. (IEDEROTE) LINN. IAM. tab. 1j. MULIENIA Jacq. vaIIx. (Personata

*). Nora acta Socicrat, Nat. scrizt. Berol. Tom. III. igor, et -. Heyale Term, bot, tab, 30. f. 3 .

**) VAHT in auren sito opere monandriac monogyilae insernit, et ad ordincm flore compicto infero militare jussit. 
nata *), Calyx quinquepartitus laciniis linearibus; Sorolla ringens, tubulosa, bilabiata: labium superius integrum, aut emarginatum; inferius trifidum; (quatrifida. Pers.): fauce nuda; capsula bilocularis, quatrivalvis, polyspora. (VAIIL post Justitiam sistere jussit).

50. VERONICA, (VRPÓNTQUE) LiNN, LÁN. tab. 13. (e fam plantt, rhinanthoideum) Calyx quatri-quinquepartitus; Corollae limbo quatripartito, lacinia imfima angustiori; Capsula ad summum emarginata, bilocularis.

5I. GRATIOLA, (GPATIOLE) sINN. IAM. tab. 16. (personata) Calux heptaphyllus; foliola duo exteriora patula; Corolla irregularis, resupinata, quatriloba, lobo superiori emarginato; staminum filamenta duo sterilia; stigma bilabiatum; capsula bilocularis.

52. C OLU MELLIA, (COLUMELIEE) RUı. et PAvon. Calyx quinquepartitus; Corolla rotata; artherae reniformes, plicatae; Capsula didyma, bivalvis, valvulis duplicatis bilocularis. (vAHI ad plantarum flore incompleto supero, ubi Circaea, ordinem amandavit).

53. SARMIEN TA, (SAPMIENTE) rurz. flor. peruv. URCEOLARIA fEVILI. - variL. - Calyx inferus quinquepartitus, laciniis quatuor aequalibus, quinta latiori emarginata; Corolla urceolata, tubulosa, tubo superne ventricoso, basi angustissimo, fauce cóarctato, limbo quinquefido laciniis aequalibns; filamexta quinquo; tria sterilia inclusa; duo fertilia exserta; Capsula polyspora, nnilocularis, circumscissa **),

54. SANCHIEZIA, (SANCIEZIE) rUIz.' pAv. flor. peruv. prodr. vafr. - Calyx quizquepartitus laci-

*) Ab hoc genere differre videtux WULFENIA carinthicica $J_{\Lambda C Q}$. Miscc, et Icon. rar. et Paederota IVulfenia I_am. encycl. tâl. 13. f. 2. generis hac ingulari discrejantia: ,Corolla ringens, labio superiori brevi integ:o, inferjori ,tripartito, fauce lobata, capsula biloculari."

**) Hoc genus staminum indole proximum est STVENiLAE. 
laciniis obtusis; Corolla tubulosa, quinquefida; laciniis revolutis, duabus superioribus brevioribus; stamina quatuor, duobus sterilibus; antherae calcaratae? aristatae, basi bifidae. Capsula oblonga, bivalvis, bilocularis (Flores involucrati).

55. CYRT A D RA, (CYRTANDRE) FORST. gen. WAHL. BESLERIA TORST prodr. JUSTICIA LAM. - Calyx quinquefidus, subbilabiatus; Corolla irregularis quinquefida, tubata; stamina quatuor, quinque, quorum duo vel tria sterilia; bacca bilocularis.

56. SCHWEN Í I A, (SCHWENKE) LINN. (personata) Calyx tıbulosus, striatus, quinquedentatus; Corolla tubulosa, fere regularis, fauce inflata, decemdentata, dentibus alternis glandulosis maioribus; stamina tria sterilia; Capsula bilocularis, bivalvis, poly. spora.

57. GLOBIFFRA, (GLOBIFERE) GMEx. MI. CRANTHEMUM MICHAUX VAHL. - Calyx quatriparritus, laciniis duabus inferioribus majoribus: Corolla subcampanulata, quatripartita, subbilabiata, lacinia, superiori minore: filamenta incurva, basi appendiculata; Capsula subglobosa, polyspora, bivalvis, unilo, cularis.

58. JUST I CIA , (CARMANTINE) LAM. tab. 12. DIANTHERA IINN. (acanthoidea) Colyx quinquepartitus, saepe tribus bracteis munitus, vel subrogularis; hinc vel simplex, vel duplex; Corolla irregularis tubulosa. bilabiata; labio superiori emarginato, inferiori trifido; hinc subaequalis aut ringens; filamenta aut mono-aut diantherifera; Capsula bilocularis, bivalvis elastico ungue dissiliens; dissepimentum valvis contrarium adnatum, retinaculis seminum uncinulatis.

59. ELYTRARIA, (ELYTRARIE) michaUX. TUBIFLORA oMeL. JUSTICIA LiNN. - Calyx subaequalis, coriaceus, quatri-quinque partitus; lacinia antica fissa; Corolla quinquefida; laciniis subaequalibus; filamenta quatuor, duo castrata sterilia: stigmata ligula.

ta: 
ta; Capsula oblonga, bilocularis, bivalvis, valvulis semiseptiferis, (Scapus radicalis bracteatus) Sporae dissepimento valvis contrario inferne annexae. MICHAUX.

60. CATALPA, (CATALPA) JUSSIEU: BIGNO. NIA LINN. (Bignonaicea) Calyx bipartitus; Corolla campanulata, lobis quatuor inaequalibus, undulatis; filamenta tria sterilia; capsula cylindrica, siliculosa, bilocularis, polyspora: (VAHL hoc genus non sus: copit).

61. P I NGUICULA, (GRASSETE) IINN. LAM. tab. 14. (Primulacea). Calyx campanulatus, bilabiatus, quinquefidus, irregularis; Corolla bilabiata, ringens, calcarats, labium superum trifidum, inferum bifidum; Capsula ovalis, monolocularia, polyspora.

62. UTRICULARIA, (UTRICULAIRE) IINN, LAM. tab. 14. (primulacea). Calyx caducus, aequalis, regularis, diphyllus; Corolla bilabiata, ringens, calca. rata: labium superius integrum, inferius maximum; Capsula globosa, unilocularis, polyspora.

63. SCHIZANTHUS, (SCHIZANTHE) PAVON. et nUiz. varu. Calyx quinquepartitus; Corolla irre gularis, bilabiata, resmpinata, labio superiori quinquepartito, inferiori tripartito; stamina quatuor, rudimenta duorum filamentorum sterilia; Capsula bilocularis, bivalvis.

64. CAL CEOLARIA, (CALCEOLAIRE) IINR. I.AM. tab. :5. VAHL. (personata) - Calyx quatripartitus, aegualis; Corolla bilabiata, labium superius minimum, inferius maximum, infatum, calceiforme; $C_{\text {s.p. }}$ sula ovata, bilocularis, semibivalvis, valvulis bifidis, polyspora.

65. BAEA , (BE'OLE) JU5S. IAM. tab. 15. VAHL (personata) Calyx persistens quatri - quinque partitus (subtus in medio floris), Inciniis lanceolatis; aequalis ; Curolla subringens, cymbiformis, bilabiata; tubus brevissimus; labiis planis non inflatis, superiori lato, 
rotundato, tridontato, inferiori,emarginato, fere bifido reflexo; stamina incurva, breviora corollae; Capsulu.calyce longior, corniculata, torta, bilocularis, quatrivalvis, polyspora *).

J O. V.EL LANA, (JOVE'LLANE) gAvon et aviliz. cayan: (porsonain) Culyx tetraphyllus; Co6. rolla cymbiformis, lobis duobus fere aequalibus conca. - vis Capula ovato-coniea, bisulca, biloçularis, apico bivalvis, valvulis bifidis, polyspora - varil spocies hujus generis ad genus Culceolaviae referri dobere auctor est pag. 173. no. 1, pag. 177 . no. 15, pag. 1sit no. 26.

66. PA MONEA, (TAMONE) AUBLIT. IAMARCK 4.tab. 542... GHINIA SCHHEB. VEPßENA LINN. (pyrenacea) Culyo quinquefidus; Corolla quatriloba, lobis inaequalibus; stamina duo brevissima, storilia; filamenta medio cincta squama ; bacca sicca, calyci inclusa, nucem continens quatrilocularem, tetrasporam.

67. RAPUTIA (PAPUTIER) AUBLET, IAMAFiq. t. 10. SCIURIS SCHREB. FITS $P$, 30. enchir.;. VAHL. (planta ordinis indeterminati) Calyx brevis, quinque. fidus; Corolia inaequalis; irregularis, tubulosa, curva, quinquefida, subbilabiata;' lobis inaequalsbus', 'superiori trifido, inferiori bifido, breviori; stamina quinq̨e, quorum tria castrata, sterilia, villosa; stigna.subtrilobum; Capsulae quinque, conlitae, uniloculares, interne bivalves, monosporae,

III. Flore compleio infero, monopctalo, irregulari; fructu oymosporo.

68. VERBENA, (VERVEINE) IINN. IANI. tab. I\%. (pyrenacea). - Caly $x$ quinquedentatus, dente quinto quasi truncato; Corolla infundibulitormis, irregularis, curva, quinquefida; stamina quatuor; sporae quinque. 69. S T A-

*) sussizu gen, plantt. Capsula longe comiculata, contonta, bilacularis, quatrivalvis. 
69. 'STACHYTARPHETA, (STACIYTAR. PHETA) vaHL. VEREENA XINN. JACQ, - Culy $x$ tubulosus, quatridentatus; Córolla lyypocrateriformis, inhaqualis, quinquefida, curva; staminum quatuor duo stcrilia; spoo rae duo.

70. ZAPANIA (ZAPANE) IAM. tab. 17. VERBENA IINR. (pyrénacea). Calyx tri-quatrifidus; cos volla tubulosa, irregularis, quinqueloba; 'stamina duo aut quatuor; sporae duae. An praecedentis generis famula?

72. LYCOPUS, (LYCOPE) LINN. LAN. tab. 18. vayt (labiatá) Calyx parumper tubuloso-campanulatus, quinquefidus; Corolla irregularis, quatrifida, tubulosa, lacinia superiori latiori, emarginata; stamina distantia; sporce quatuor, retusac.

73. A MET'HYSTEA, (AME'THYSTE'E) LINN. IArr. tab. 18. VAMI. (labiata) Calyx subcampanulatus, quinquefidus :- Corolla tubulosa, subaequalis, subbilabiats, quinquefida, lacinia infima patentiori et longiore, concava; starina approximata; sporde quatuor, gibbae.

74. HOSL,UNDIA, (HOSLUNDIE) TXONING. - vanL. - Calyx tubulosus, quinquedentatus, mono: phyllus, striatus; Corolla ringens, calyce fore duplo 2. longior, limbi labio superiori concavo, erecto, ovato, gibbo, inferiori hiante, trifido, recurvo; stamina qua- tuor intra calycem baccatum tubo adnata, duo brevis. - sima, vix longindine faucis, antheris minntissimis - sterilibus; dus corolla longiora, antheris reniformibns fuscis; hinc dno fertilia; ovarim superum quatripartitum; stylus filiformis longicudine staminum fertiliun: stigma bifidum. Racca spuria, e calyce orta, magnizua dine Ribeos, subrotunda, decemangulata, dentibus calycis umbilicata, pubescens, intus cava; Spoiae quatuor ovatae intra calycem baccatum. THON INic.

75. ZIZIJHORA, (ZIZTPIORE) xINN. IAK. tab. 18. CLINOPODIUM AMMAN. THYMUS bUXe. (labia. 
(labiata) Calyx tubulosus, striatus, ad faucom barbatus, quinquedentatus; Corolla ringens, bilabiata; labio superiori integro, retuso s. reflexo, inforiori patente, trilobo: sporae quatuor.

76. MONARDA, (MONARDE) LINN, IAM. tab. 19. (labiata) Calyx tubulosus, cylindricus, quinquedontatus, striatus : Corolla ringens, bilabiata; labio superiori lineari, filamenta simplicia involvente; inferiori trilobo; sporae quatuor.

77. ROSMARINUS, (ROMARIN) LINN. LAM. tab. 19. (labiata) Calyx ad supremum compressus, bilabiatus; labio superiori integro; Corolla ringens, bilabiata, labio superiori bipartito, inferiori trifido; filamenta longa, curva, simplicia, dente laterali distincta; sporae quatuor.

78. SAL VIA, (SAUGE) LINN. IAM. tab. 20. SCLAREA MILL. HORMINUM MILL. (labiata) Calyx subcampanulatus; bilabiatus, labio superiori tridentato; Corolla ringens; fauce dilatata, bilabiata, labio superiori curvo emarginato, inferiori trifido, filamenta transverse pedicello adfixa; sporae quatuor.

79. CUNILA, (CUNILE) LINN. LAM. tab. 19. vaHL. ZIZIPHORA LAM. CALAMINTHA PLUK. MOris. HEDYPNOIS Mitehel. MELISSA THYMUS IINN. (labiata) Calyx striatus, cylindricus, quinque dentatus; Corolla ringens, bilabiata; labio superiori erecto, plano, emarginato, inferiori trilobo, lobo medio emarginato; stamina quatuor, filamenta duo castrata, sterilia; sporae quatuor.

80. COLINSO N I A, (COLLINSONE) LIN I. LAM. tab. 21. VAнt. (labiata) Calyx tubulosus, bilabiatus; labio superiori tridentato; Corolla tubulosa, quinqueloba, inaequalis, labio inferiori margine multifido, capillari, truncato, longissimo; spora e quatuor una perfecta fertilis, tria abortiva. 


\section{- IV. Flore coinpleto infero, polypetalo.}

(Hunc in locum vaHriUs FONTANESIAM retulit.) 8I. L I T HOPH IL A, (LITHOPHILE) SWAFz. VAHI. Calyx inferus, triphylius; Corolla tripetals, petalis conniventibus; nectarolyma diphyllum, petaloideum; stigma emarginatum; perica:pium biloculare?

82. LIN O C IER A, (LINOCIERA) SCHRER. WILLEMT. WILLD. spec. VAHI. THOUINIA SWARz. CHIONANTHUS IAM. PERs. enchirid. GARIETHA. GAS herm. Zeyl. (jasminacea ) Calyx inferus, brevissimus, quatridentatus; Corolla tetrapetala, petalis linearibus, concavis; antherae duae sessiles, lineares, petala duo opposita connectentes, et longitudine aequantes; bacca exsucca, bilocularis; loculis monosporis SWARz.

83. ACAENA, (ACAENE) Flor. Peruv. IINN. VAHL. AGRIMONIA LINN. ANCISTRUM IINN, FORST. PROQUIN FEVILL. - Caly $x$ monophyllus, (4. 5. phyllus superus PERs, ench,) aristis glochidatis armatus. Corolla tetrapetala, apici calycis inserta (nulla PERs.) stamina duo, quatuor, quinque; stigma penicellatum; Spora una calyce corticatnm (Nrupa angulata, obovata, glochidibus echinata P'ERs. qui in Class. IV. ut alii redire jussit *).

84. $\mathrm{CO}$.

*) Concessum sit hoc loco verbotenus referre quod immortalis v "florae peruvianae Ancistris congeneres sint, patet tam ", ex earum habitu, quam ex fructificatione. Auctores "praedicti operis inde verosimile seducti fueruut, "quod acaena juxta descriptionem LINNEl patris in ", mantissa habcat calycem superum absque corolla; $A n$. ", cistrum vero sccundum fopstenum in nov. gen. ", et LINNEUM filium in supplem, gaudeat calyce infe. "ro, corollaque tetrapetala; hinc genere differre cre. ", diderunt. Character generis aucistri hortikevensis con"venit cum charactere acaenae LINNEI patris. Ex ob. , servatione vero LAMATIL, VENTENATII et GAERT"NERI, flores completi sunt; sic character aucistri ", horum virorum non a FonSTERI et LINAEI lilii "recedit, nisi co, quod sporae calyx aduascitur, 
84. CODARIUM, (CODARIE) VAHI. pag. 38?. Banks. - Calyx pentaphyllus, foliolis aequalibus ses. silibus, ovato oblongis, patentibus, deciduis; Corollae petalum unicum, lineari lanceolatum, calyci brevius, caducum, annulo nectarolymatis adfixum; Nectarolyma in centro floris receptaculiforme orbiculatum, margine distincto, plano concavum, porsistens; Stamina duo longitudine calycis, utrinque margine receptaculi inserta crassiuscula, patentia; antherae oblongo ovatae, erectao; ovarii pedicellus declinatus pubescens; Stylus subulatus, incurvus, stamina longitudine exaequans; Stigma subacutum; pericarpium legumen magnitudine fabae, oblongiusculum, subrotundum, tomentosum, pedicellatum, evalve, uniloculare, farinaceo subsicca pulpa repletum, subtrisporum: $S_{p o-}$ ra compressa, dura, variegata, longitudinaliter posita. thonning. - Char. differ.; Calyx pentaphyllus; Corollae petalum unicum lineare lanceolatum, annulo nectarolymatis insertum; legumen pedicellatum, farina farc-

tum

" quod quoque naturae consentaneum expropriis obser", Vatiouibus didici. Ex hisce omnibus concludere licet, "quod alii auctores petala superieri part is calycis insertas ", lacinias calycis nominarint, et tubum calycis sporae aeta. "te accretum germen inferum; alii dutem, qui flores $j u$ ", nioves viderunt ot calycem a germine distinctum, has "lacinias petala ori calycis inserta dixerunt et germen "distinctum superum. Hinc differentia omnis auctorum ", in eo consistit, quod ratione a etatis partes fruc"tificationis alio modo consideratas alio nomine consigna, rint. Et rel adeo ex his sequi luce clarius videtur, , unius ejusdemque generis esse Acaena et Ancistrum, "nam si character Acaenae LINNEI patris assumitur, ", onınia Ancistra ad Acaenam veniant, necesse cst; si "vero Acaenae considerantur secundum characterem an" Cistri FORStent, aliorumque, qui petala dixerumt cas ", partes, grae LINNEo patri laciniae calycis audiunt, om. " ucs Acaenac, et vel A. clongata LINN, specics anci"stri fore, natura ipsa jubere videtur. Ancistra igi", tur et acaenas genere coniunxi, et nomen Acaenae, "antiquitate sancitum, rctinui. Numerus staninum in ", diversis spccicbus diversus; frequentius tamen duo ", adsunt, ideoque in classe diandric locum dedi. Si"tus partium floris fere idem ac in Boexhayia". 
tum, subtrisporum, ovalve. VAHL. (Distinctum sano a Dialio genus ipso soLANDro auctore).

85. D I A L I U M, (DIALI) LINN. VAHL. (ex ordine indeterminato) - Caly $x$, nullus; Corolla pentapetala ; petalis ellipticis, caducis; stamina ad latus superius receptaculi posita; fructus?....*) (VAHL ad plantas flore incompleto rotulit).

\section{Flore supero, completo.}

86. MORIN A, (MORINE) LINN. LAM. tab. 2x. VAHL. (dipsacea) Calyx duplex; exterior fructus su. bulatus, monophyllus, denticulatus, inferus; interior floris bifidus, superus: Corolla inaequalis, bilabiata, tubol: longissimo; spora unica, floris calyce coronatus.

87. CIRCAEA, (CIRCE'E) IINN. IAM. tab. I6. varru. (epilobiacea). Calyx superus, tubulosus, brevis, diphyllus; Corolla dipetala; stigma emargina. tum 3 Capsula pilis hamatis hispida, bilocularis, non dehiscens, loculis monosporis.

88. GLOBBA. (GLOBBE'E) LINN. (amomea) Calyx brevis trifidus; corolla tubulosa trifida; cap. sutu infera, coronata, trilocularis, trivalvis, polyspora.

V1. Flore

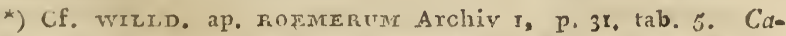
lyx mullus. Corolla pentapetala coriacea, petalis aequa. Jibus externe pubescentibus, petalo supremo latiori oblongo emarginuto, reliquis oblongis acutis. Staminum filamenta duo crassa, subulata, incurva, sub petalo supremo; antherae duse in quolibet filamento connatae, olulungae;. Pistilli germen superunı filamentis uppositune stylus subulatus, incurvus; Stigma subulatum. Pericarpium capsula oblonga, magnitudine cerasi, unilocularis, evalvis, monospora, denso tomeuto atro tecta, cortice fragili, Spora nuica magnitudinc pisi, oubxu. tuida, compressa, fuscit, diitzd.s. 


\section{Flore incompleto.}

\section{(Huc vahisus Pimeleam retulit).}

89. MARGYROCARPÙS, (MARGYRO.

CARPE) PAVoN et RUIz. VAHL. ANCISTRUM IAr. EMPETRUM LAM. Calyx quatri, quinque partitus; Corolla nulla; stigma poltatum; drupa nucleo monospora *).

90. A NCISTRUM, (ANCISTRE) IINN. LAN. tab. 22. (rosacea). Calyx quatridentatus, tetraphyllus, terminatus aristis quatuor hamatis, crucfiormiter locatis; Corolla tetrapetala, (nulla Pers.) stigma multifidum; drupa exsucca, hispida, monolocularis; (spora una calyce tectus?). Conferri possunt, quao ad acuenue genus vahLius dixit, et concedi, hoc genus ancistrum esse delendum $\left.{ }^{* *}\right)$.

\section{Flore apetalo.}

9I. ARUNA. (ARUNIER) IAN. tab. Io. AROUNA AUbLet. (leguminosa) Cuity $x$ brevis, quinquepartitus, laciniis reflexis; Corolla nulla; pericarpium bacca ovalis, interné pulposa, ex uno latere sulcata, monolucularis, mono-dispora. (VAHLIUS maluit paz. 303. cum genere Diulium conferri.

VIII.Flore monopetalo infero, fructu capsulari.

92. SUFFRENA, (SUFFRENE) BELEARD buller. de la soc. philom. 1805. (salicaria) - Calyx nullus; corolla monopetala, quadrifida; foliolis ovatis acutis aequalibus; stamina duo corolla breviora, eidem basi oppo-

*) Genus Acaeuae valde affine, conferri potest et wiLder. in nov. actt, nlat, curios. Bcrol, vol, 2, LAMARK ill, gen. pag. 77.

**) vaHruvs ctiam Fraxini genus sccundum ea, quae in pracfatione operis sui dicta proposuit, sul, hac classe et ordine locum tucri jussit. 
opposite insidentia; ovarium superum, subrotundum; stylus simplex longitudine corollao; stigma capitatnm; capsula oblonga unilocularis; sporae plures, subrotundae, receptaculo insidentes ${ }^{*}$ ).

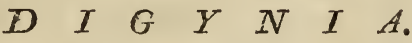

93. ANTHOXANTHUM, (FLOUVE) LINN. 2AxI, tab. 23. VAIIL. (e graminum familia). Calyx gluma bivalvis, uniflora; Corollae gluma bivalvis, acuminata, aristata: spora una.

94. CRYPSIS, (CRIPSIDE) AIT. LAM. tab. 42. ANTHOXANTHUM I.IN., JACQ.; PLLEU.M IINN. SCHOENUS LINN. AGROSTIS scop. (e familia graminum) Calycis gluma bivalvis unifora; Corollae gluma bivalvis longior inaequalis, mutica; stamina duo, tria; Spica foliorum vagina involucrata, seu floribus in capitulum foliatum congestis. Spora una. (PERsoor enchir. p. 79. ad Triandriae digynias juxta Phalarides amandavit.)

G U N N E R A, (GUNNERE) IAN. tab. 80\%. f. I, VAHI. MISANDRA JUSS. Amentum thyrsoideum; calijx superus, bidentatus; Corolla nulla; Stylus bipartitus; drupa monospora, coronata calyce accreto, subcarnosa.

95. M N IARUM . (MNIAR) FORST. LINN. IAM. tab. 6. (ex ord. plantt. indeterme) VAHL, DITOCA

GAERT.

*) Staminum numero tantum a generc glaux remota; - Corolla est secundum Jussieu in hoc genere calyx; qui, eodem auctore, non foliolis multis constat, sed est monophyllus quatridentatus, dentibus punctatis. Caeterum, hace generis planta apud Lовец p. 227. tab. 416. oclorrit, atque est P'olygala repens auctorum. 
CAERTN, - Involucrum subuniflorum, tetraphyllum; calyx quadrifidus superus, persistens; Corolla nulla; Sfora una oblonga, calyce incrassato corticatum. GAERTN. *)

96. PIPER, (POIVRE) LINN. SWARz. LAM. tab. 23. LOMPIA, SIRIUM RUMPH. SAURURUS PIUM. (urticata). - Spadix cylindrica, amentiformis, plena tectaque floribus numerosissimis, et serratis. Calyx nullus; Corolla nulla; Stylus brevissimus; Stigmata tria manifesta; aut (ut in sequenti genere) punctum vel maculam unam - duas sistentia; Bacca globosa, monospora. (stamina $2,4,5$ et 6 .

97. PEPEROMIA, (PEPEROMIE) PAVON ot RUIz. (CONGONA ?) Calyx squama orbicularis peltata, depressa; Corolla nulla; Stigma punctum unum vel duo in apice germinis. ${ }^{* *}$ )

\section{CLASSIS TERTIA.}

TRIANDRIA.

$\begin{array}{lllllllll}I M & O & N & O & G & Y & N & I & A\end{array}$

I. Flore supero.

98. VALERIA N A, (VALÉRIANE) IINN, IAM. tab. 24. fig. 1. 2. (dipsacea). Calyx minimus, quasi margo,

*) ,Ex observatione SOLANDPit stamina utplurimum duo ,ideoque potius ad Diandriam retuli.“ vA HL.

**) Diferre augurantur PAvon et nurz. a genere PIPER: Spathis ovatis minimis; squama minima, orbiculari (ovata) 
margo, aut nullus; maturitate enascens in ariftam sessilem; Corolla monopetala, tubulosa, tubo longo, subinfundibuliformis, quinquefida, saepe calcarata, aut basi gibba; Spora unica, calyco tecta. (Stamina 1. 2. 3. et 4. exserta. )

99. P HYL L A C T IS, (PHYLLACTIS) pERs.pag. 39. VALERIANA rutz. PAV. - Flores iuvolucrati: involucrum monophyllum, vaginans; calycis margo minimus; corolla trifida; spora una: Genitulia exserta.

100. FEDIA, (MACHE) AdANSon. Venten. VA. LERIANA IINN. LAMI tab. 24. f. 3.4 (dipsacea) Calyx minimus, tri - sexdentatus; corollu tubulosa, tubo brevi, quinquefida; Capsula calyce coronata, trilocularis; loculi monosporae. (duo fere semper steriles) $=$ Sporae nudae, aut dentibus coronatae.

1OI. MELOTHRIA, (MÉLOTRIE) IINN. LAM. tab. 28. (cucurbitacea). Calyx campanulatus quinque fidus ; Corolla campanulata, quinqueloba; bacca parva, exigua, trilocularis, polyspora.

102. CROCUS, (SAFRAN) IINN. IAM. tab. 30. (iridea) Spatha radicalis, monophylla; corolla tubuloso iufundibuliformis; tubo longissimo gracilescente; sexpartita, laciniis aequalibus; stygmata tria, profunde incisa, denticulatim cristata, (crocea); Capsula ovalis, trigona, trilocularss, polyspora.

103. GALAXIA. (GALAXIE) тнUмr. ran. tab. 568. IXIA LINN. (iridea). Spatha subradicalis univalvis, uniflora; calyx nullus; Corolla infundibuliformis, sexlciba, lobis aequalibus, tubulosa, tubo filiformi, Iongo; filamenta connuta? Stigmata tria multifida, 'Inbato fimbriata; Stylus unus; Capsula infera, trilocularis, tri-

(ovata) peltatr, depressa sub) singulis fosculis; staminibus porro ovario longioribus; stigmate denique puncto unico (vel duo) minimo, vel macula, vid. prodr. flor, peruv, p. \&. Utrumque gemus rant, cone junxit in genus Piper. 
trivalvis polyspora. (Plantulae subacaules; flores plerumque lutei.) *)

304. WATSONIA, (WATSONIE) MILI. JACC. GLADIOLUS LINN. THUNB. ANTHOLIZA THUNB. IINN. fil. - Corolla recurvata, tubuloso cylindrica, aut plerumque tubuloso dilatata, infundibuliformis; laciniis omnibus aequalibus strictis (Spica disticha saepius, foliolis sparsis obtecta, genitalia exserta.)

J05. ANTHOLYZA, (ANTHOLYZE) LINN. MILL. WILID. - Corolla stipitata, longe tubulosa; tubo recurvo subcylindrico; laciniae ovato-lanceoatae, suprema fornicata longiori; Spatha bivalvis, ovata; Genitalia exserta; antherae saepius caeruleze; stigmata tria - sex, )

I06 GLADIOLUS, (GLAYEUL) IINN. IAM. tab. 32. ANTHOLIZA IINN. (iridea). Spatha bitrivalvis, valvulae utplurimum lanceolatae, magnae; Corolla irregularis, infundibuliformi tubolosa, varia, erectiuscula; limbo bilabiato, sexpartito; laciniae ovato lanceolatae, undulatae; Genitalia adscendentia, pistillum pone stamina versus laciniam superiorem. Stigmata tria, trifida; capsula ovalis, trigona, trilocularis, polyspora. Sporae in multis alatae. (Corolla ringen's; laciniae inaequales; tubus incrassatus subincurvus.)

107. HEBEA, (HEREE). Corolla tubo brevi, lla. ciniis divaricatis, suprema lanceolata fornicata; laterales obovatae; inferiores angustae, pendulae, subunguiculatae.

a. LE MONIA, (LEMONIE) POURRET act. Tolos. vol. 3. tab. 13. Corolla campanulata, tubo sub-

*) Hoc et quaedam sequentia genera e Classi Monadelphia retulerunt nuperrime auctores gravissimi in hanc hujus ordines classem: nescio an uaturae jussu, suo, si quod est, systemati et normae faventi, an propter differentiam genitalium masculinorum vix vel nunquam comatorum. 
subbrevi, parum incurvo, laciniis profundis, subaequalibus, ovatis,

b. PEYROUSIA, (PEYROUSIE) POURRET. Corolla tubo gracili subfiliformi, laciniis patulis, subregularibus.

c. MICRANTHUS, (MICRANTHE) Spica disticha, spathis imbricatis, flores parvi sessiles; stigmata ....?

c. A G L A EA , (AGLE'E) PHALANGIUM muna. - Corolla subcampanulato bilabiata, sessilis seu tubo vix ullo; Capsula triloba, retusa, Sporae subrotundae, mucronatae; Spatha duplex, bivalvis sub corolla, bi - trivalvis ad basin pedunculi.

IOg. MO NTBRETIA : (MONTBRETIE) DEcandorce. Spatha diphylla, scariosa; Corolla monopetala, supera, infundibuliformis, sexfida; auriculao tres, callosae, sessiles, perpendiculares, in laciniarum trium inferiorum pagina supera solitariae; Stamina tria libera, imo tubo inserta; stylus unicus; stigmata tria, gracilescentia; capsula trilocularis, $=$ (Gladiolus securiger AIT. et flavus EJUSD.)

109. IRIS, (IRIS) LINN. LAM. tab. 33. (iridea) Spatha bivalvis; Corolla sexpetala, laciniis alternatim rectis et reflexis; stylus nullus; stigmata tria, petaliformia; stamina incumbentia; capsula ovalis, trigona, trilocularis, polyspora.)

IIO. IXIA, (IXIA) xINN, IAM. tab. 3r. (iridea) Spatha bi - trivalvis, ovata, brevis; corolla tubulosa, tubo subgracilescente, longo aut brevi, cum genitalibus recto; sexpartita, laciniis aequalibus, hypocrateriformibus, subellipticis, planis; stigmata trifida, subfiliformia; - (filamenta stylum includentia, in nonnullis connata) -; capsula ovata, trigona, trilocularis, polyspora. 
a. ROMULEA ( ROMULEE) Corolla campanulata; folia linearia.

b. EURIDICE, filamentis connatis.

c. BELEMCANDA, (BELEMCANDE) Corolla tubo subbrevi, apice dilatato, limbo inciso subcampanulato; laciniae ovatae; stamina in nonnullis subincurva, in tubo medio.

III. DIASIA, (DIASIE) DECANDote. (iridea) Spatha diphylla, valvulis subfoliaceis oppositis, Corolla monopetala, supera, rotsta, sexpartita, post florescentiam scissa et caduca; tubo nullo; laciniis acuminatis; stamina tria, libera, imo corollae inserta; stylus unicus; stigmata tria, gracilia; capsula trilocularis, depressa, trigona, angulis divaricatis, superne dehiscentibus *).

II2. GEN O SIRIS, (GENOSIRE) LABILIARD. **) (iridea) Corolla supera, tubulosa, triloba, petalis aequalibus; stamina tria; stylus unus; stigmata fere recta ${ }^{* * *}$ ).

1I3. ARISTEA, (ARISTE'E) Art. SChreg. IXIA rinN. MORAEA тнилв. (iridea) Spatha bivalvis; corolla (caerulea) hexapetala, tubo breviusculo; stylus de. clinatus; stigma simplex, infundibuliforme, obtuso subconcavum; capsula infera, poiyspora; sparae compressae scabrosae.

II4. MORAEA, (MOREE) LINN. LAM. tab. 3 r. (iridea) Spatha bivalvis; corolla hexapetala, petalis tri-

*) Ab IXIA differt corolla sejuncta in profundas laminas, quae post inflorescentiam sese disjungit ab ovario; nec non capsula depressa, divergente angulis tribus lo. culisque supra dehiscontibus.

**) Nov. Hollandiae plantarum Specimen, Paris 1804.

***) Differt ab IXIA numero loborum corollae, receptaculo spurarum libero et centrali. 
tribus alternis rectis, patentissima, tubo subnullo; stigmata tria, sex; trifida, filiformia; capsula oblonga, trigona, trilocularis, polyspora. (Flores, marcescendo in spiram saepius convoluti. Filamenta in nonnullis connata, et petala alterna minora.)

I 5. S ISYR I N C H I U M, ( BERNUDIENNE) LiNN. LAM. tab. 569. MORAEA THür. JACl. (iridea). - Spatha diphylla, multiflora; Calyx nullus; Corolla Hexapetala, laciniis planis, saepe acuminatis, nunc aristatis; stylus unus; stamina utpluimum connata (Monadelpha), trifida; ovarium subrotundo-triquetrum, pedicellatum, extra spatham; hinc capsula infera trilo. cularis trivalvis polyspora.

II6. TIGRIDIA, (TIGRINE) JUSs. FERRARIA IINN. LAM. tab. 569. MCRAEA тHUNв. (iridea) Spatha bivalvis vel diphylla, uniflora; calyx nullus; corolla laciniis sex, tribus exterioribus ovalibus, interioribus tribus ninoribus, hastatis, margine nectariferis; stylus unus; stigmata tria bifida; capsula infera oblonga, angulata, trilocularis, trivalvis, polyspora. (Mona. delpha.)

FERRARIA, (FERRARE, Feraire) IRN, JUss. MORAEA JACQ. (iridea). - Spatha diphylla (no. nophylla?) uniflora; calyx nullus; corollae laciniao sex fere aequales, margine indulato - crispae; stylus unus; stigmata tria, bifida, laciniis penicellatis, undulato cucullata; filamenta monadelpha; capsula infera, trilocularis, trivalvis, trispora.

117. D ILATRIS, (DILATRIS ) IIN. Suppl. IAMARIR tab. 24. WACHENDORFIA LIN mantiss. (iridea). Calyx nullus: corolla hexapetala, supora, liirsuta, extrorsum Foluta; filamentum tertium reliquis brevius; stigma simplex; capsula infera, globosa, coronata, trilocularis, trispora.

\section{Flore infero.}

I18. WI T SENIA, WITSENE) THUNB. LAM. tab. 30. (iridea). Calyx (spatha) nullus; corolla tubu- 
losa, tubo utplurimum longo, sexpartita, laciniis iribus exterioribus extrorsum volutis, rectis inter folia disticha; stigma emarginatum, aut leviter subtrififidum; capsula supera.

119. C IPURA, (CIPURE) AUBLET. IArr. tab. 3०, MARICA scriree. PERs. enchir. pag. 54. Spatha bivalvis corollam involvens; corolla tubulosa, supera, hexapetala, laciniis tribus interioribus minoribus, alternatim cum exterioribus positis; stigma petaliforme, trifidum, laciniis indivisis acutis; stylus crassus; capsula angulosa, trilocularis, polyspora.

320. WACHENDORFIA, (TVACHENDOR. FEE) цINN. тнимв. LAм. tab. 34. (iridea) Spatha bivalvis: Corolla hexapetala, petalis inaequalibus, tribus superioribus repressis, tribus inferioribus planis; infera; Capsula supera, triquetra, trilocularis, trispora.

32I. H A M A D R U M, (IIAEMADORE) SMITH. transact. Vol. IV. (iridea) Petala sex; tria interiora supra medium staminifera; stigma obtusum; capsula infera, trilocularis, Juss. gen. I. 59. ${ }^{*}$ )

122. XIPHIDIUM, (GLAIVANE) AUBLEt. wiLLD. IAMr. tab. 36. (iridea) Calyx (Spatha) nullus; Corolla hexapetala, aequalis; stamina recta; stigma trigonum; Capsuta supera, trilocularis, trivalvis, poly. spora.

123. LEPTA N T HUS, (LEPTANTHE) MICHAUX PERs. p. 56. - Spatha uniflora; hinc dehiscens; corolla tubo longo gracili, limbo sexpartito; stamina laciniis inserta; antherae lineares uniformes, triangulares, flamenta aequalia; Capsula intra spatham, coronata, trilocularis, polyspora, angulis dehiscens.

124. H E-

*) A WAChENDorfia differt germine infero, petalis apicem rersus staminiferis, staminibus abortivis nullis. 
124. HETERA N T H RA, ( HETERANTHERE) PAVON et RUIz. WILID. (iridea) Corolla monopetala, limbo sexfido inaequali; antherae duae difformes, renuformes, una sagittata; filamenta longitudino inaequalia; capsula supera, trilocularis.

125. COM MELINA, (COMMELLINE) LINN. xAM. tab. 35. (e familia juncoideum) Calyx triphyllus, foliolis concavis; corolla tripetala, petalis unguiculatis; filamenta tria sterilia, cincta ad summum glandulis crno ciformibus: (nectarolymata tria, cruciata, filamentis propriis inserta); capsula fere globosa, bi-trilocularis.

I26. OXYBAPHUS, (OXYBAPHE) L' HERET. MIRABILIS cav. CALYXHYMENIA oRteg. VITMANNIA. TURh. CALYMENIA pers, enchirid. pag. 36. (nyctaginea) Calyx campanulatus, quinquefidus; Corolla parva, infundibuliformis, plicata, quinquefida; nux pentagona, striato sulcata, monospora, fundo calycis persistentis et ampliati membranacei inclusa et circumdata.

127. MACROLOBIU M, ( MACROLOBEE) SCHREB. WILLD. VOUABA AUBLET. LAM. tab. 26. (1eguminosa) Calyx duplex; exterior diphyllus, interior turbinatus oblique quatrifidus (basi cinctus duabus bracteis); petolum unicum unguiculatum; legrmess coriaceum, laxum, compressum, monosporum.

328. OUTEA, (OUTEY, Joutey) AUBL. IAM. tab. 28. MACROLOBIUM SCHREB. WiIID. (leguminosa) Calyx quinquedentatus, turbinatus, basi bibracteatus; Corollae petala quinque, superiori maximo; filamentum sterile petalo superiori annexo recondente, stamina lon. gissima; legumen pedicellatum.

129. H IPPOCRATEA, (HIPPOCRATE, Bejuco) IINN. LAx. tab. 28. (acerosa *) Calyx minimus quinquepartitus; Corollae petala quinque apice unobifoveara, concava; Capsulue tres, compressac, bivalves, valvis carinatis; uniloculares, bi-pentasporae; sporae ex uno latere alatae.

130. $S O N$.

*) Certe accrosis et Mulpighis affinis. 
130. TONSELLA, (TONSELLE) SCHREB. TONTELLA AUBLET. LAM. tab. 26. (ex ordine plantarum indeterminato) Culyx urceolatus, quinquefidus, (5. partitus?) Corollae petala quinque, inserta super nectarolyma discoideum, urceolatum; ovarium disco velatum; bacca parva, monolocularis, polyspora.

13I. LOEF L I N G A, (LE'FLINGE) LINN. IATr. tab. 29. Calyx pentaphyllus, foliolo quoque insignito dentibus duabus ad basin; Corollae petala quinque, conniventia, minima; capsula subrotunda, supera, uni-bilocularis, bi-tri-valvis, polyspora.

132, WI L L I CH I A, (WILLICHE, Villique) IINN. (ex ordine plantarum indeterminato.) Calyx quatrifidus; corolla rotata, quatriloba aut quatrifida; capsula fere rotundata, compressa, bilocularis, bivalvis, polyspora.

C A L L IS I A, (CALLISE) IINN. HAPALANTHUS JACQ. Americ. - IAM. tab. 35. (juncacea) Calyx triphyllus; Corollae petala tria; antherae geminatae; stigmata ponicilliformia; capsula ovalis, compressa, bilocularis, dispora; nectarolyma nullum.

*) 133. SYE NA, (SYENE) schred. wilid. MAYACA, Mayaque, aublet. LAm. tab.36. Calyx triphyllus; Corollae petala tria; Antherae oblongae; Capsula unilocularis, supera, globosa, trivalvis, hexaspora.

134. TAPURA, (TAPU'RE) AUBr. - Calyx campanulatus sexpartitus, basi tribracteatus; Corolla pentapetala, bilabiata; stigmata tria revoluta.

135. C A L Y S O', (CALYPSE) AUBERT. DE PET. тнovars, (rhamnea).. - Flores hermaphroditi, com. pleti, pentapetali, perigyni, fasciculatim dispositi axilares. Calyx persistens, quinquelobus, discus centralis,

a ANTHODON. par. et RUIZ, ante SYENAM, 
lis, staminifer et pistillifer; corolla pentapetala ; stamina tria; antherce adnatae; ovarium sub stamirubus latens; fructus bacca polyspora. Sporae perisporio donatae; embryo parrus; cotyledones planae, (Genus simile Hippocrateae madagascuricae IAM.; Ptelidio disco affine; sed neutiquam e falinia rhamnearum.)

I36. RUMHHIA, (RUMPHE) IINN. IAMT. tab. 25. (terebinhinacea) Calux trifidus: Corollae petala tria, oblonga; drupa trilocularis, coriacea, turbinata, et notata tribus striis vel sulcis; nux trilocularis, trispora.

137. F I S S IL I A , (FISSILIER) JUSS. LAM, tab. 28, (Hesperidea) Calyx urcoolatus, integer; Corolla tubulosa, regularis petalis tribus, binis bifidis; stamina octo, quinque sterilia; $n u x$ glandisormis; monospora, involuta particulatim calyce.

133. CNEORUM, (CAMELE'E) LINN. LAM. tab. 27. (terebinthinacea) Culy $\approx$ minimus, tri-quatridentatus; Corollae petala tria, quatuor, aequalia; stigma trifidum; stamina tria, quatuor; bacca sicca (drupa), tritetra - cocca, cocco singulo monospora.

339. COM O CLADIA, LINN. LAM. tab. 27. (terebintaceae) Culyx tripartitus; Curolla tripetala (tripartita?) ; drupa oblonga, arcuata, ad summum notata punctis tribus; nux membranacea, biloba, monospora.

140. XY R IS, (XYRIS) LINN. LAM. tab. 36. (juncacea) Calysis glumae bi - trivalves, in capitulum congeitae; Corollae petala tria, parum crenata, basi staminifera, aequa. lia; Capsula rotundata, supera, trilocularis sec. LIN di. Juss. (unilocularis LAM. PERs.) trivalvis polyspoza.

I4I. TRIPTERELLA, (TRTPTERELLE) MI. ciraux (an VOGELIA gnex.? yers.) Calyx sexficus, aiato angulosus, supra basin solidam tubulosus; $C_{0}$. rulla nulla; stigmata tria; capsula triquetra, trilocularis, polyspora ; (stumina inclusa.)

I42. $O L \Lambda X$, 
142. OLAX, (OLAX) urne. (hilospora) Calyx brevissimus, integer; corolla infundibuliformis, trifida, fissuris tribus fere aequalibus; ad faucem corollao ne. ctarolyma tetraphyllum; Fructus.... ?

CONDALIA , (CONDALIE) rulz. fl. peruv. Corolla infundibuliformis; calyx quatridentatus; bacca bilocularis,cava, spongiosa; sporae lentiformes, liberae.

143. ROTALA, (ROTALE) IINN. Mantiss. (caryophyllata) Calyx tubulosus, tridentatus; corolla nulla; capsula calyci inclusa, trilocularis, trivalvis, polyspora.

I44. OR T E G I A, (ORTE'GIE) IINN. LAR. tab.29. (caryophyllata) Calyx pentaphyllus; corolla nulla; capsula apice trivalvis, monolocularis, polyspora; stigma unum, et tria.

145. POL Y C NEMUM , (TOLYCNFME) IINN. rAm. tab. 39. (chenopodiacea) Calyx tri-pentaphyllus; Corolla (nulla?) petala quinque, calyciformia; capsula monospora, evalvis, stylo persistente, acuminata; spora una, subnuda; (

\section{Flores glumacei **).}

146. D ICH P O M A, (D:CHROME) MICHAUx. Spica ovata seu capitata, involucrata; Squamae paleaceae, membranaceae, subcongestim imbricatae; stylus setaceus; stigmata duo; spora nuda; rugellosa, apice tuberculo lanao

**) Cf. PEFs. ENCHIRID. p. 56. Seirpoidei caules cylindrici aut triquetri, farcti, enodes, rarius articulati. Flores spicati, sqnamis seu paleis imbricati, monogyni; stigmata duo, tria; filamenta numero et situ variant, ut plurimum tria; spora unica unda, basi involucella setulae, lanae, paleae - arilliformi ant nullo cinctum. Huc et Carices pertinercnt, nisi ob staminum situm ad classem uronocciam a LINNAEO et uatura relata esscut. 
lanae instar cincto; (folia floralia sive involucrum basi discolora, ut plurimum albicantia *).

147. SCHOENUS, (CHOIN) LINN, JUSS, LAir. tab. 38. (Cyperi adans. Cyperoidea juss.) Spiculae subconvolutae, acutae; squamae fasciculatim connivendo congestae, exteriores steriles, nitidae; calycis glumae univalves, paleaceae; Corolla nulla; spora una, subrotun$\mathrm{da}$ in forma nucis nuda, aut setis inter glumas cincta. Flores inferi saepe abortientes, squamas steriles relinquunt.

148. CYPERUS, (SOUCHET) IINN. LAM. tab. 38. (cyperoidea juss.) Calycis glumae paleaceae, disticho imbricatae, duobus loculis oppositis in'mucronem compressae; corolla nulla; spora una, nuda; spicula compressa.

149. DULICHIUM, (DULICHIE) PLEURANTHUS RICHARD CYPERUS WILLD. SCIIOENUS LINN. SCIRPUS мгсн. - Spicae subracemosae, ex axillis foliorum; spiculae lineari - lanceolatae, subcompressae; squamae subdistichae, amplexanti adpressae. Stylus longissimus, bifidus; ovarium setulae retrorsum asperae.

I50. SCIRPUS, (SCIRPE) TOURN. IINN. IAIT. tah. 39. (cyperoidea) Calycis glumae paleaceae sivo squamae undique imbricatae, conniventes ovoideas; corolla nulla; spora una, nuda aut villis setulisve brevioribus cincta.

I5 I. TRICHOPHORUM, (TRICHOPHORE) SCIRPUS MIGHAUX. ERIOPHORUM LINN. WILLD. Spiculae subovatae, squamis undique imbricatis; sporae setulae capilliformes (nec lanam densam referentes) demum longe exsertae numero definito sex. = (Genus distinctum et scirpum inter et Eriophorum locum tenens. )

152. ERIO.

*) Forte nonnulli schoeni auctorum, et Scirpus radiatus тнилв., et Cyperus leucocephalus RETz, huc pertinent. PERBOON. 
152. ER I OPHOR U M, (LINAIGRETTE) IINN. JUss. I.AM. tab. 39. (cyporoidea) calycis glumae paleaceae, undique imbricatae, rectae, ovales; corolla nulla; spora una: ad basin lana densa longissima cincta.

I53. M A PA N A , (MAPANE) AUBLET. IAM. tab. 37. (cyperoidea). Flores cíncti involucro triphyllo, capitati: calycis glumu sexvalvis, imbricata, dentata; corolla nulla; spora una, involucello (perisporio) sexpaleaceo involuta IICHARD.

154. L I M N T IS, (LIMNETIS) RICHARD. DACTYLIS SMITH. SPARTINA rоTH, TRACHYNOTIA Mrichaux. - Spica lateriflora, flosculis subbifariam imbricatis; calyx bivalvis, valvula una minori; corolla bivalvis, mutica, compressa, carinata; stylus longus; nectarolyma nullum. (Flores paniculati, stricti) PEFS. enchir. pag. 72. qui et nomen e loco notali, oras maris, depromtum, et alias in graminum familiam observationes ex amico PICHARDO gratus suscepit.

155. NARDUS, (NARD) LiNN. IAM. tab. 39, (graminea). Calyx uniflorus, univalvis; corollae gluma bivalvis, inaequalis, inclusa; stigma unum; spora una cincta gluma corollae. (Spica floribus alternis sessilibus.) (Monogynia?)

156. REMIREA, (REMIRE) AuBL. IAM. tab. 37 . MIEGIA schres. (graminea). Spiculae in capitulum congestae; calycis glurna bivalvis, unifora; corollae gluma bivalvis, calyco minor, palea intima (s, nectarolymate scнREL.) sporam non dimittente; stigmata tria; filamenta longissima; spora una glumae inclusa.

I57. I Y L L I N A , (KYLLINGE) - IINN. IANI. tab. 39. (cyperoidea). Spica (Amentum LINN.) subrotunda, aut oblonga sessilis, aut umbellata constans e spiculis subimbricatis; calycis gluma bivalvis, valvula inaequali, uniflora; corollae gluma bivalvis, calyce major: spora una triquetra. (Stamina unum ad tria: stig* mata duo, tria.) 
158. LYGEUM, (SPARDE, Alvardo) IINM. LAM. tab. 39. (graminea*). Calycis gluma univalvis, introrsum voluta, spathiformis, aristata, biflora; floros intra spatham convolutam gemini; gluma basi in tubum bilocularem villosum concrescens, bivalvis. (Monogynia?)

159. POMMEREULLA, (POMMEREULLE) IINN. IAM. tab. 37. (graminea). Gluma turbinata, bivalvis tri-quatriflora; valvulis quatrifidis, dorso aristatis; corollae gluma bivalvis, aristata, spora una. ( $\mathrm{Ca}$ lyx bivalvis, subdistans; quatriflorus; (involucrum $\mathrm{x} \mathbf{\mathrm { NN }}$.); corolla bivalvis; valvula altera major, quinquedentata, dente intermedio aristato; styli duo; spica unilateralis? = ex roxnoungr Plantt. Coromand. vol. II. fascic. 2. tab, 131. $\left.{ }^{* *}\right)$.

\section{SACCHARUM, (CANNAMELLE) IINN.} IAM. tab. 4I. (graminea). - Flores ad unum omnes liermaphroditi; calycis gluma bivalvis, lanugine longa involucrata; uniflora?; corollae gluma uni- bivalvis, mutica aut aristata minor; stamina unum, tria, monospora.

\section{a. I M P RATA, crnInI. Spicis cylindricis, floribus triandris.}

*) Gratmina, Flores glumacei, triandri, Radix fibrosa; culmi plerumque fistulosi, articulati, aut basi ramosi; Folia linearia, vaginantia, vagina latere fissili, apice membrana alba distincta, quac liguli aut stipula nunca. patur. Flores spicati, racemosi, plerumque puniculati, rarissime folis involucrati; nonnulli saepius abortivi; alii pulygami; panci monoici; Stumina plurimis tria, (quac in hac șeric solummodo enumcrantur), in nonnullis sex, (Class. Hexandria) aut plura; styli. communiter duo, in quibusdam uinus; plerumque tamen lifidus. In unico tautummodo gramine stigmate tria observata sunt. Glumae interiores in mult is sporam maturam obducunt, involvunt, quasi incrustant, et cum ea is. durantur.

**) PEnsoon enchir, ad digyniam triandriae amandavit. lag. 106. a. 
b. ERIANTHUS. Calycis gluma bivalvis, mutica; valvulis lineari oblongis, canaliculatis; basi villis densis involucrata; corollae gluma bivalvis, valvula interiori longe aristata, monandra, MICHAUX.

I6I. VAGINARIA, ( VAGINAIRE) PERS. Enchir. pag. 70. FUIRENA RICHARD. - Spica ovata, squamis undique imbricatis : sigmata tria; perisporium (sporae involucellum) alternatim tripaleaceum trise. tumque.

162. F UIREN A, (FUIRENE) IINN. LAM. tab.39. (cyperoidea). Squamae mucronatae in spiculas, undique aristatae; gluma nulla; corollae gluma trivalvis, valvulis aequalibus, cordatis, aristatis; spora una, involucellum latum, paleaeformis, trigona, saepius aristata.

163. HYP OLYTRUM, (IIYPOLYTRE) RICHARD - Spicula squamis undique imbricata; perisporium (sporae involucellum) glumam tri - quatrivalvem mentiens; stamina duo, tria; stigma unum, duo.

164. D IP L A S I A , (DIPLASIE) RICHARD. - Spica squamis undique imbricata; perisporium involucellum glumam quatrivalvem mentiens; stamina septem; stigmata duo.

165. LEPIRONIA, (LEPIRONIE) RICHARD. Spica ovato oblonga; spiculae squamis orbiculatis cartilagineis notatae; perisporium sedecim paleaceum; stamina quatuor - sex.

166. CHRYSITRIX, (CHRYSITRIX, Chrisite) LINN. fil LAM, tab. 842, RIHARD. (cyperoidea). - Flores hermaphroditi: spica spathacea, monophylla, gluma squamis multis, oblongis, coriaceis, fere aequalibus imbricatis parvis, in capitulum conglomeratis; covollae gluma numerosae squamae filiformes, fasciculatae; stamina multa, singula singulis squamis in- 
tersita; ovaria duo centrali disco insita, unoquoque stylo brevi et tribus stigmatibus supposito: fructus....? - Flores masculi, spica spathacea; glumae calycis et corollae nec non stamina ut in hermaphroditis.

167. ORYZOPSIS, (ORYZOPSE) MICHAUX. IICHARD. PERS. - Calyx uniflorus bivalvis laxus obovalis; corolla coriacea, subtereti ovata, bivalvis, valvula exteriori apico aristata; nectarolyma bipaleaceum lineare. FICHABD.

\section{$D I G Y N I A$.}

I. Flores glumacei; glumae uniflorae.

168. COR N U C O P I A E, (CORNUCOPIE, Co. queluchide) IINN. LAM. tab. 40. (graminea). Involucrum monophyllum, cyathiforme (infundibuliforme), multiflorum. Calux monophyllus, bipartitus; corolla univalvis, superne fissa; stylus brevis; stigmata duo, (raro unum) longissima; (

169. PAN ICUM , (PANIC.) IINN. IAM. tab. 43. (graminea). Inflorescentia varia ${ }^{* *}$ ); Calycis gluma trivalvis, valvula exteriori tertia dorsali minima; $\mathrm{CO}_{0}$ rollae gluma bivalvis; spora una.

I70. DIG I TARIA, (DIGITAIRE) JUss. PANICUM LINN. PASPALUM LAM. (graminea). Flores unilaterales, geminati, altero subsessili; calyx bi-trivalvis, concavus; valvula extima minutissima aut nulla; secunda variabili; intirna longitudine corollae; corolla bivalvis, oblongo ovata, teres, mutica; styli

lon-

*) Retulit anctoritas rensovir enchir. pag. 7I, 2, ad txi. andriae monogyniam.

**) Spicata ; spica composita; paniculata. 
longissimi; nectarolyma fissum; Spicae digitatae, lin6ares; ( FICHARD.).

I7I. CERES I A, (CERESIE) pers. enchir. pag. 85. PASPALUM ram.tab. 43. f.2. Flosculi laterales bifarii, sub rachi tenui lata, membranacea, subinyalina cymbiformi ; calyx bivalvis, uniflorus, lanatus; antherae bifurcatae.

172. CYNODON, (CYNODON) RICHARD. PANICUM LINN. WITHEr. - Caly $x$ bivalvis, patens, lanceolatus; corolla major, bivalvis, valvula exteriori maxima, ovoidea: nectarolyma truncatum; spica digitata, floribus unica serie imbricatis, solitariis.

I73. TRACHYS, (TRACHYS) PICH. CENCHRUS IINN. PANICUM RETz. WrLID. - Spica digitata, flosculi in rachi membranacea unilaterales, pedicellis pinnatis, bracteae sive involucrum ovatum cartilagineum; calyx uniflorus, bivalvis; corolla bivalvis.

174. PASPALU M, (PASPALE) IINN. LAM. tab. 43. (graminea :- Flores unilaterales, gemini; calycis gluma bivalvis, aequalis, orbiculata; corollae gluma ejusdem magnitudinis, bivalvis; stigmata penicillifor* mia; spora una.

I75. A R IS T IDA, (ARISTIDE) LINN, IAM, tab. 41. (graminea). Calycis gluma bivalvis, biflora; corollae gluma univalvis, aristis tribus terminalibus; spora una.

I76. A LOPECURUS, (VULPIN) IINN, IAM. 1ab. 42. (graminea). Spicata. Calycis gluma bivalvis, fere sessilis; corollae gluma univalvis, basi aristata aut mutica; spora una.

377. POLYPOGON, (POLYPOGON) DESFONT. WILID. ALOPECURUS IINN. PHLEUM SCHREB. PAZJAS. SMITH. CHAETURUS IrNx. (gramina). Calycis gluma biralvis aristata uniflora; corollae bivalvis, val. vula exteriori aristata, calyce minor.

I78. PHLE. 
178. PHLEUM, (FLEOLE) IINN, LAM. tab, 42. (graminea). Calycis gluma (indurata) bivaivis; valvulis linearibus, sessilibus, truncatis, apice retusis seu carina prominente, utrinque bicuspidata; corollae gluma inclusa, bivalvis, monospora.

179. PHALARIS, (ALPISTE) IINN, LAM. tab, 42. (graminea). Calycis gluma uniflora, bivalvis; ralvu. lis subovatis, dorso membranaceo carinatis aut lanceolatis, aequalibus, nervosis; corollae gluma bivalvis, inclusa, hinc multo calyce minor, basi (saepius) pilosa. Spora una.

I80. M IL I U M, (MIL) IINN. AGROSTIS Iam. (graminea). Calycis gluma bivalvis, uniflora, ventrico8a, fere aequalis; corollae gluma bivalvis, brevior, imo brevissima; stigmata penicellata, villosa; spora una.

18I. A GROSTIS, (AGTOOSTIS) LINN. LAM. tab. 4I. (graminea). Calycis gluma bivalvis, valvulis acutis uniflora; gluma corollae bivalvis, major calyce; stigmata longitudinaliter hispida, sive plumosa; spora una. (Flosculi patentes sive sint aristati, sive mutici.)

I82. TRICHODIUM, ( TRICHODIUM ) RICHARD. MICHAUX. CORNUCOPIAE WALTEP AGRO. STIS wrILd. - Calyx bivalvis, valvulis subaequalibus, acutis; carina spinulosa; corolla brevior, unilocularis, mutica; stigmata subsessilia, hispidula, (Flores paniculati. J RICHARD.

183. S T UR M I A, (STURMIE) PERSOON. HOPPE. AGROSTIS IJNN. WILLD. - Calyx bivalvis; valvulis aequalibus truncatis; corolla minor, bivalvis, ovata, lanuginosa, mutica; - (Spica filiformis, floribus alternis muticis.) - PERs. Enchir, pag. 76.

184. TRICHOON, (TRICHOON) готн. Саtal. II. (graminea). - Calyx bivalvis, nudus, uniflorus; 
rus; corolla bivalvis, calyce duplo major, nuda; ova. vium cinctum lana longitudine corollae ${ }^{*}$ ).

185. S TIPA, (STIPE) LINN. LAM. tab. 4I. (graminea). Calycis gluma bivalvis, uniflora; corollae gluma brevior, bivalvis, valvulis involutis truncatis; arista terminalis, basi articulata et contorta, decidua, longissima; spora una.

186. LAGURUS, (LAGURE) LINN. LAM. tab. 4 I. (graminea). Calycis gluma bivalvis, uniflora, aristis villosis elongata; gluma corollae bivalvis, exterior munita aristis duabus terminalibus rectis; tertia dorsali retorta; monospora.

387. MA TRELLA, (MATRELLE) PERS. AGRO. STIS LINN. WILID. LAII. - Calyx nullus; corolla car* tilaginea sive indurata compressa, mutica, bivalvis; valvulae demum connatae?

188. MÜHLEN B ER G I A, (MÜHLENBERGE) schreb. Rотн. DILEPYRUM mínaux. (graminea). Gluma calycis uni-bi-valvis, (uniflora?), minutissima; corollae gluma bivalvis, basi villosa, valvula exteriori apice aristata; interiori breviori, mucronata; dua squamae internae. Spora una corollae inclusa.

189. STEG O S I A, (STE'GOSIE) LOUREIRo. (graminea). Calycis gluma bivalvis, uniflora; corollae bre. vior, trivalvis, valvula interiori latiori longiorique; spora una, corolla tecta.

190. PEN N I SET U M, (PENNISETE) PER. CEN. CHRUS auctorum; PANICUM IAM. (graminea). Involucrum multisetum; calyx bivalvis, biflorus, floro altero hemaphrodito, altero masculo, (rarius neutro)

utro.

*) Arundo Karka netz.; character enim essentialis Arundinis consistit in lana corollam cingente; (in saccharo laua ambit corollam) ejusdem fere longitudinis; sed in Trichoon lana germen cingit, corolla extus nudä. 
utroque sessili; - flores spicati, polygami; in plerisque speciebus involucrum heterochaeton, setisque nonnullis inferne plumosis. - CENCHRUM subordine tertio videas.)

191. PEROTIS, (PEROTIS) AIton. SACCHARUM LINN. LAM. tab. 40. f. 3. (graminea). Calycis gluma nulla; gluma corollae bivalvis; valvulae aequales, aristatae, exteriori villo vel lanugino involucrata; spora una.

192. SP I N I F EX, (SPINIFEX) IINN. IAM. tab. 840. (graminea). Flores polygami. Gluma bivalvis, valvulis parallelis rachis axi; biflora, flos unus hermaphroditus, duo masculi; flores hermaphroditi corolla mutica bivalvi, squamae nectarolymatis duae internae; stamina duo; styli duo; spora una. Floris masculi calyx et corolla ut in hermaphwditis, nectarolyma nullum, stamina tria.

193. Z OYDIA, (ZOYDIA, ZOYSIE) WILLD. nova actt. Berol. Calycis gluma univalvis, carinata; corollae gluma univalvis, membranacea.

194. LEERSIA, (LE'ERSE) SWARz. MICHAUX. ASPERELLA SCHREB. IAMARCK. PHALARIS IINN. EHRHARDTA wrocers (graminea). Calycis gluma nulla; corollae bivalvis, claussa; valvulis compresso navicularibus, muticis, ciliatis; spora, una.

195. M A N IS UR IS, (MANISURIS) IINN. IAM. tab. 83. (graminea). Flores polygami : flores masculi et neutri, calycis gluma bivalvis, valvula regulares, variae; uniflora, corollae bivalvis, inclusa; flores feminei, calycis gluma univalvis nnifora; valvulae cartilagineae, inclusae, fissae, et parte anteriori persistentes; corollae gluma minor calyce, bivalvis; styli duo; sporae laeves. gluma iuvolutae.

II. Flores glumacei, glumis bi-trifloris. 1g6. AIRA, (CANCHE) EINN. LAM. tab. 44. (graminea). Gluma calycis nitens, bivalvis, biflota; Corollae gluma 
gluma bivalvis, mutica, aut basi aristata; spora una.

197. MEL I C A , (MELIQUE) I INN. IAM. tab. 44. (graminea). Calycis gluma colorata, bivalvis, biflora; flore medio abortivo, sterili; glumae laxae, obtusae, membranaceae, inaequales: Corollae gluma bivalvis, minor, ventricosa; rudimentum floris; spora una.

198. COLL A D A , (COLLADOA) cAv. ISCHAEMUM LINN. *) (graminea). Flores spicati, rachisf flexuosa. Involucrum bipartitum, triflorum; flores hermaphroditi duo, unus masculus; florum hermaphroditorum calycis gluma bivalvis; corollae bivalvis interne basi aristata, arista torta, versus medium geniculata? stamina tria, styli duo, spora una ovato-compressa. Masculi flores: calycis gluma bivalvis, corollae nulla (calycis nulla, corollae bivalvis CAv.) mutica; 'stamina tria.

199. A E G ILOR S, (AEGILOPE) LINN. LAM. tab. 839. (graminea). Polygama. Flores hermaphroditi spicati. Gluma bivalvis coriacea, latissima, multi aristata, aristis rigidis divergentibus, triflora; flore uno masculo inter duos hermaphroditos collocato; flores hermaphroditi corollae gluma bivalvis, valvula exteriori bi-triaristata, aristis terminalibus, ovata; nectarolymatis squamae duae internae; stamina tria; styli duo; sporre una; - flores masculi; corollae gluma, nectarolymate, staminibus ut in hermaphroditis; pistillum saepe abortiens.

200. HOLCUS, (HOUQUE, SORGO) IINN. IAM. tab. 838. (graminea). Flores polygami paniculati; ca$2 y x$ biflorus, bivalvis, opacus, nervosus; corolla minor, valvula altera aristata; nectarolyma lineare, bipartitum; stigmata subsessilia; - (huc pertinent $\mathrm{H}$, mollis et lanatus L. SCHREB. - PERs. opinatur et has fere ad Airas

esso

*) Ccrte Ischaemum aristatum $\boldsymbol{L}$, non ab hoc genere distin. ctum. 
esse removendas species. SORGIUM peculiare genus infra locatum.) -

201. THUARIA, (THUARE'E) AUBLET. PETS. pag. 110. (graminea). Flores laterales polygami; inferiores hermaphroditi; rachis membranacea, demum involuta, decidua; culyx biflorus, bivalvis; corolla bivalvis, ovata, muica; - peculiare arenosarum Madagascariae ab AUBERTIO PERsooxio communicatum gramen, cujus spicula, ut in Arachi hypogaea obser. vatum scimus, terram intrat.) -

202. ECHINARIA , (ECHINARIE) DESFONT. atlant. 2. pag. 385. CENCHRUS IINN. (graminea.) FloIes capituliformes; calyx brevis, membranaceus, aristatus, bivalvis, bi-triflorus; corolla bivalvis, valvula exteriori inaequali, quatri-quinquefida; interiori minima, bi-trifida; spora una. ${ }^{*}$ )

203. TRISE T UM, (TRISETUM) PERs. pag. 97. AVENA IAM. LEERs. DESF. IINN. - Calyx bi - triflosus, acuminatus, carinatus; corolla aristis duabur, terminalibus, subdentiformibus, (glumis apice setoso bifidis ;) una dorsali, recta nec contorta; flosculis ut plurimum glabris: (spiculae compressao, pallescentes.

204. SORG HUM, (HOUQUE, SORGO) M IAM. tab. 838. HOLCUS IINN. MIEG. SCHREN. (graminea). - Flores polygami, panicnlati; flosculi gemini, altero hermaphrodito, sessili, altero postico pedicollato masculo neutrove. Flores hermaphroditi: Calux gluma bivalvis, uni-interdum billora; corolla bi-trivalvis, calice minor, valvula exteriori secunda ad supremun aristala, tertia squamas nectariferas tres villosas connectendo includens; stamina tria ; styli duo; spora una majuscula ovalis; flores masculi minores; calyx bivalvis, corolla nulla, (aut secundum scirREBErum, clarissimum virum, bivalvis, ) mutica; squamae tres interio-

res

*) Iens. Enchir, sub CENCHRO No. 2; militari jussit. 
res minores; stamina tria; ovarium abortiens, instar, rudimenti; styli duo.

205. SEHIM A, (SE'HIME'E) Forsk. (graminea). Polygama. Glumae quatuor approximatae, duao dua. bis coalitae, unaquaque bivalvis; biflora; altera geminata, gerens flores masculos et pedicellatos; altera sessilis gerens hermaphroditum, et masculum; tllius co. rolla bivalvis; stamina tria, styli duo; spora una; hujus corolla bivalvis, stamina tria.

206. ISCHAEMUM, (ISCHAEMON, Ischeme) IXNN. LAM. tab. 839, (graminea). - Flores polygami. llermaphroditorum calyx biflorus, bivalvis, valvulis subaequalibus, cartilagineis; exteriori subovata, gibba, apice bifida; corolla minor bivalvis, valvula exteriori ventricosa, mutica, aut aristata, bifida; nectarolyma foliola duo, spathulata, truncato emarginata; stamina tria; styli duo; spora una; flores masculi idem: sed mutici, flores spicati geminati. SCHREB.

207. TETR $\triangle$ POG O N, (TETRAPOGON) DESront. - Calycis gluma bivalvis, mutica, inaequalis, triflora; flores duo laterales sessiles; corollae gluma bivalvis, valvula exteriori truncata, et aristata; flore uno centrali pedicellato, minori, sterili; corollae valvulae duae truncatre, aristatae.

III. Flores glumacei, peniculati; glumae multiflorae.

208. D A C T YLIS, (DACTYLE) LINN. LAM. tab. 44. (graminea). Calycis gluma bivaIvis, compressa; altera valvula majori, carinata; inaequalis, multiflora; corollae gluma bivalvis; spora una; (spiculae aggregatae, et capitatae.)

209. UNIOLA, (UNIOLE) LINN. BRIZA IAM. tab. 45. fig. 3. (graminea). Spiculaemultiflorae, planao anci- . 
ancipites, ovatao; calyx multiglumis; valvulis imbricatis duplici serie; corolla bivalvis, mutica; valvula interiori minore, intra exteriorem recumbente; carinatis squamis compressis; stamina unum, tria; appendices duae, subbicornes ad basin ovarii; stigmata longa, muricata.

210. BRIZ A, (BRIZE) LINN. IAM. tab. 45. fig. 1. 2. (graminea). Calycis gluma bivalvis, multiflora; glıma corollae bivalvis, valvulis ventricosis parumper cor. datis, obtusis; valvulis interioribus minimis; spicula disticha.

2II. POA, (PATURIN) IINN, IAM. tab. 45. (graminea). Calycis gluma bivalvis, multiflora; gluma corollae bivalvis, valvulis margine scariosis, et paululum punctatis (discoloribus); spicula ovata; mutica; monospora.

212. FEST UCA, (FESTUQUE) IINN. IAM. tab. 46. (graminea). Calyx bivalvis, multiflorus; corolloe gluma bivalvis, valvulis acuminatis et punctatis; spicula oblonga, teretiuscula, aut divergens, mutica aut arista terminali instructa; spora una.

213. KOELERIA, (KOELERE) PERs. tab. 96. POA LAM. KROK. LINN. PHALARIS TOIRET. AIRA. VAHL. SMITH. AVENA LATr.? - Calyx multillorus. bivalvis, compresso carinatus; corolla bivalvis, brevi aristata, glumis nervosis; - spica composita ex spiculis compressis, saepius pubescentibus, subsessilibus. PERS.

214, BROMUS, (BROME, DROUE) LINN. IAM. tab. 46. (graminea). Calycis gluma bivalvis, multiflora; corollae bivalvis, valvulis sub apice, (apice saepo bifida) aristatis; spicula oblonga disticha; - (gluma interior pectinato ciliata, secundum smitr.) spora una.

215. AVENA, (AVOINE) IINN. LAM. tab. 47. (graminea). Calyx bivalvis multiflorus; corollae gluma bi- 
valvis, valvula exteriori arista dorsali contorta munita; glumae membranaceae, subfolliculosae; spora una.

2I6. ARUNDO, (ROSEAU) IINN. LAN. tab. 46. (graminea). Calyx bivalvis nudus, uni-multiflorus, glaber; corolla lana longa ad basin cincta, bivalvis; spora una.

217. MIEG IA, (MIEGIE) pERs. ARUNDINARIA MIcIlAUx. - Stigmata tria; fores polygami, panicu. lati; calyx multiforus, bivalvis, brevis, inaequalis; corolla bivalvis, mutica; appendices bini lato lanceolati, acuti, plani in utroque flore; spora nuda maxima. = (Ab omnibus hucusque cognitis graminibus tum staminibus tribus tum spora grandi sane distinctum genus. PETSOON pag. 102.)

218. APLUDA, (APLUDE) IINN. IAIr. tab. 841. (graminea). Flores polygami vel monoici. Involucrum diphyllum, foliolo uno majori, parum distante; caly $x$ quatriflorus, cartilagineus, bilamellatus, sive bivalvis; valvula anteriori truncata; flosculi inferiores gemini: altero hermaṕlhrodito, aliero femineo; hermaphroditi corolla univalvis, navicularis, striata, tridentata, dento intermedto aristata; stigmata crassa hirsuta; flosculi superiores masculi valvulae truncatae insistenses. (Flores duo masculi pedunculati, unus femireus sessilis; florum masculinorum calyx bivalvis; flor. femin. corolla bivalvis; styli duo; spora ual. (An subordinis quarti?)

219. PAPPOPHORUM, (PAPPOPIORE) scFR. varis. Symbol. (graminea). Calyx bivalvis, bi-quatriflorus, flore uno hermaphrodito, inferiori, sessili, basi barbato: unus - tres flores masculi superiores, pedicellati, glabri, non barbati; flores hermaphroditi: corollae gluma quaeque bivalvis, calyci brevior; valuula exteriori inflato ventricosa, munita tribus - quatuor-decem etlongissimis aristis; valvula interiori angustiori, longiori, punctata; duae squamae interiores; stamina tria; styii duo; spora una. Flores masculi: valvulae et squamao ut in hermaphroditis; stamina tria. 
220. LAPPAGO, (LAPPAGINE) RETZ. SCHREB. WILID. TRAGUS DESFONT. CENCHRUS LINN. ('graminea). Duo, tres, quatuor flores mutici; terminali uno sterili, approximati forma aristae; caly $x$ subtrivalvis, muricatus, echinatus pilis rigidis; corolla bivalvis, membranacea, inaequalis, aristao duae interiores; spora una.

22I. CENCHRUS, (RACLE) LIN. MrChAUX. DESF. WIILD. (graminea). Involucrum monophyllum, laciniatum, echinatum, aut formatum multis pilis mollibus, bi - tri-quatriflorum, muticum; corolla bivalvis, mutica, longior; masculi uni-biflori; stylus bifidus. (MONOGYNIA. - PERs.)

IV. Flores glumacei, dispositi in aristam serratamin.

222. ROTTBOELLA, (ROTTBOELLE) IINN, IAM. tab. 48. AEHILOPS IINR. MORIs. (graminea). Flores polygami, spicati. Rachis articulata, teretius. cula, in pluribus linearis, velfiliformis; calyx gluma cartilaginea, ovato lanceolatus, planus, uni-bivalvis, uni-biflorus; corolla bivalvis, minor calyce; stamina tria; styli duo; splora una; flosculi alterni: unus mascrllus, alter hermaphroditus, partibus in utroque eodern modo formatis; sessiles, in sinu rachis flexuosae; spora una.

223. SECALE, (SEIGLE) LINN. tant. tab. 49. TRITICUM LINN, ROTII. AGPOPOGON GAERTAEH.

- (graminea). Calyx bivalvis, bi aut multiflorus, solitarius in quoque axeos dente; valvulae oppositae, flore minores; glumis lineari lanceolatis, laevibus, aut utrinque 'canaliculatis; spora una.

224. TRITICUM, (FROMMET) IINN. DESF. коти. WILLD. LAM. tab. 49. ELYMUS LINN. WIILT. 
Monis. (graminea). Calyx bivalvis, solitarius in quoque axeos dente, multiflorus; flos obtusiusculus, glumis muticis, aut interrupte aristatis; corolla bivalvis; spiculae breviusculae, a latere plano rachi approximatae; spora una,

225. HORDEUM, (ORGE) IINN, ROTH. wIIID. LAM. tab. 49. ELYMUS schreB. (graminea). Flores spicati; calyx uni-biflorus, hexaphyllus, involucriformis: foliolis per paria digestis, setaceis; flosculi terni, intermedio sessili, lateralibus stipitatis (saepius sterilibus); corolla stipitata, bivalvis, acuta; exteriori valvula aristata; spora una.

226. E L Y M U S , (ELYME) LINN. SCHREB. WILLD: IAM. tab. 49. ASPERELLA HUMBоtid. (graminea). Flores spicati; calyx bivalvis, involucriformis, tetraphyllus, lateralis, aggregato - ternus, saepe multiflorus, floribus duobus - tribus in quoque axeos dente conjunctis; corolla bivalvis; spora una.

227. LOLI U M, (JURAIE) LINN. LAM. tab. 48. (graminea). Calyx univalvis, vel monophyllus, fixus, multiflorus; spicula simplex, approximato - sustentata versus rachin latere acuto; spora una.

228. CYNOSURUS, (CRETELLE) LINN. VAHL. DESF. IAM. tab. 47, (graminea). Flores sustentati receptaculo unilaterali; calyx bivalvis, multiflorus, bractestus; vel receptaculum proprium unilaterale foliaceum spiculis bracteis subjectis ; corolla bivalvis, calyce longior; spora una.

22\%. ELEUSINE, (ELEUSINE) GAERTNER, I,AMARK, tab. 48. CYNOSURUS LINN. - Flores omnes hermaphroditi, in receptaculo unilaterali, secundi, mutici; calycis gluma bivalvis, carinata, compressa, biquinque - multiflora; corollae gluma bivalvis, inaequalis, mutica; spora una, subglobosa, tunica membranacea obtecta. 
230. CHLORIS, (CHLORIS) swarz. WILID. TETRAPOGON DESFONT. CYNOSURUS VAHL. ANDKOPOGON LINN. - Calycis gluma bivalvis inae. qualis bi-sexflora; flore uno hermaphrodito sessili, altero masculo, pedicellato, hermaphrodito minori; flores hermaphroditi: corolla valvulis duabus exteriori majori aristata; interne squamae duae minutae; stamina tria; styli duo; spora una. Masculi flóres: corolla unibivalvis: valvula exteriori aristata; stamina tria. - Polygana. Spica ut plurimum digitata unilateralis. Val. vulae corollarum carinatae muticse, aut aristatae.

\section{I. CHRYSURUS, (CHRYSURE) PERS. CYNO-} SURUS XINN, WILLD, TKOELER, - Florés spicato racemosi; spiculae biformes; steriles pendulae, muticae, multivalves, ad basin fertilium involucrum mentientes; fertilium calyx bivalvis, bi - triflorus; corolla bivalvis, valvula exteriori longe aristata.

232. SESLERIA, (SESLERIE) ADANS. JUSS. CYNO. SURUS LINN. HOFFR. WILLD. APD. WULT. POA WUIF. (graminea 7.). Gluma bi-quinqueflora, bivalvis, valvulis antrorsum versus vel apice dentata dorso barbata, bi - triflora; calyx bivalvis, valvula exteriori triaristata, interiori bicorni aut furcata; stigmata subglandulosa; flores spicati, saepius cuerulei, brsi involucrati; spora una. (TRIANDR. MONOGYNIA PERS.)

233. A NDROPGON, (ANDROPOGON, Barbon, squénante) IIIN N. LArr. tab. 840. (graminea 3.). Flores polygami, geminati; altero hermaphrodito sessili; altero masculo sive rarius neutro pedicellato masculo. P. Flores hermaphroditi; calycis gluma bivalvis, uniflora; corollae calycis gluma brevior sessilis, trivalvis; exteriori et secunda majori, basi arista longa, fere spirali, munita; tertia intima minuta; (duae parvae squamae interiores); stamina unum, tria; styli duo; spora una oblonga; receptaculum s. rachis plerumquo villosa; involucrum villi fasciculus ad basin spicularum, in nonnullis folium cymbiforme. - Flores masculi: ca. lycis gluma bivalvis uniflora; corollae bivalvis, pedun- 
culata : valvula exteriori majore, nuda; squamae duae internae parvae; stamina tria.

234. A N T H ISTIRIA, (ANTHISTE'RIE) IINN. suppl. LAM. tab. 47.841 ANDROPOGON LiNN. TIIUNB. STIPA porf. (graminea 4.). Flores polygami fasciculati in panicula laxa: flores hermaphroditicentralos solitarii sessiles et basi aristati; calycis gluma bivalris, corollae gluma vix ulla, aut nulla; stamina tria, styli duo, arista tortilis e basi pistilli vel e fundo corollac; spora una oblonga; flores masculi peduriculati, non aristati numero sex, quorum quatuor sessiles, verticillati; duo pedicellati mutici; calycis gluma, uno-bivalvis; uniflora; corollae bivalvis; stamina tria.

\section{$\begin{array}{llllllll}T & R & I & G & Y & N & I & A\end{array}$}

\section{Flore infero.}

235. HOLOSTEUM . (HOLOSTEE) IINN. swafiz. IAm. tab. 51. (caryophyllata). Calyx penta. phyllus, sujerus; corollae petala quinque bifida; capsula monolocularis, subcylindrica, polyspora, apice dehiscens. (Folia opposita; flores corymbosi, axiliares ot terminales.)

236. POLYCARPON, (POLYCARPE) xINN. THUNB. SWARz. Lam. tab. 5l. S'TIPULICITA MichaUX. (caryophyllea), Calyx pentaphyllus; corollae petala quin. qque, muinima, emarginata, ovata, persistentia; styli tres; capsula ovata, unilocularis, trivalvis, polyspora. (Folia stipulacea, verticillata quatuor foliis; flores corymbo dichotomo terminali dispositi).

237. LE C HEA, (LE'CHE'E, Léquée) IINN. MICIIAUX. WILLD. IAM. tab. 5\%. MENANDRA criquov. (caryophyllata 7.). Calyx triphyllue persistens; corollae petala tria, linearia; stigmata tria plumosa; capsula trilocularis, trivalvis, valvulis totidem interioribus pla. 
placentiformibus, trispora; spora una in loculo; embryo leviter arcuatus.

239. CENTROSEPIS, (CENTROSEPIS) $x_{A}$ BILI. notae Hollandiae specimen. Paris. 1504 . (spathacea). Spatha multiflora; callyx nullus; corolla nulla; glumae centrales simplices; stamen unicum, basi ovarii insertum; ovarium subditum stylo trifido; capsula trilocularis, monospora ${ }^{*}$ ).

139. ERIOCA ULON, (JONCINELLE) IINN. PíU1. Mopis. IAMr. enc. 3. tab. 50. (juncacéa I.). Ca. lyx communis quatrifidus; capitulum imbricatum, hemisphaericum, multiflorum; corollae petala tria-sex, aequalia; stamina supra ovarium; capsula parum globosa, bi-trilocularis, bi - trispora; tricocca, monoica. Flores masculi quatuor stamina; feminei stylus bifidus.

240. MONTIA, (MONTIE) LINN. IAM. tab. 50. (portulacacea 1.). Calyx diphyllus; persistens; corolla irregularis, monopetala, petalo quinquefido, fissuris tribus alternis, minoribus, staminiferis; stamina tria, rarius quinque; capsula turbinata, monolocularis, trivalvis, trispora, calyce tecta, sporue iu fundo capsulae funiculo umbilicali affixae.

24I. MOLLUGO, (MOLLUGINE) I:NN. IAM, tab. 52. (caryophyllata 1.). Calyx tri- vel pentaphyllus, interne coloratus; corolla nulla; capsula ovalis, trilo. cularis, trivalvis, polyspora. (Folia opposita, aut verticillata; flores axillares, aut terminales.)

242, MINUARTIA : (MINUARTE) LINN, LöFLING. LAM. tab. 52. (caryophyllata 1.) Calyx pentaphyllus; corolla nulla; capsula monolocularis, minima, oblonga, triangularis, trivalvis, polyspora. (Folia opposita; flores coaliti.)

243. QUE-

*) Notabile genus absentia calycis et corollae, in familia juncincarum. 
243. QUERIA , (QUE'RIE) IINN. THUNB. WILID. IAMr. tab. 52. (caryophyllata I.). Calyx pentaphyllus; corolla nulla; capsula rotundata, unilocularis, trivalvis, monospora.

244. TO E N I G I A (KE'NIGE) IINN. IAM. tab. 5 I. (polygonacea) *). Calyx triphyllus; corolla nulla, stigmata tria velata pilis s spora una, ovata, nuda.

\section{Flore supero.}

245. DONATIA, (DONATIE) FORSt. IAM. tab. 51. POLYCARPON IINN. (caryophyllata 1.). Calyx triphyllus; corolla polypetala, octo-decem petala integra, linearia, oblonga; styli tres; capsula trilocularis, infera.

246. PROSERPIN A CA, (PROSERPINAQUE, Trixide) LIMN. LAM, tab. 50. TRIXIS michaUx. (onagraria). Calyx liberus, tripartitus, superus, persistens; corolla nulla; stamina tria sub calycis laciniis affixa; ovarium adhaerens; stigmata pubescentia; nux ossea, triquetra, trilocularis, calyce coronata; spora loculi summo affixa; perisporium striatum; embryo bilobus, altero lobo breviori; radicula horizontalis.

*) Facie polycarpi. 


\section{CLASSIS QUARTA.}

\section{TETRANDRIA.}

\section{$M I \quad O \quad N \quad N \quad O \quad G \quad G \quad Y \quad N \quad I \cdot A$.}

I. Flores aggregati, monopetali, inferi; spora una, umbrata.

247. GLOBULARIA, (GLOBULAIRE) IINN. MIORIS. WILLD. LAMT, tab. 56. (primulacea 3.). Flores aggregati : involucrum commune polyphyllum, imbricatum; seceptaculum paleaccum; calyx proprius tubulosus, inferus, quinquefidus, persistens; corolla inaequalis, quin quefida; labio superiori bipartito, partitionibus minoribus; inferiori tripartito; stamina tubo inserta tria; spora una, calyce connivente tecta.

II. Flore aggregato, monopetalo, supero; spora una, velata (uubrata).

248. DIPSACUS, (CARDERE) IINN. JACQ. WILID. LAM. tab. 5\% (dipsacea r.). CARDUUS LobEd. Ilores compositi, aggregati; involucrum commune polyphyllum: culyx proprius duplex : superus adhaerens, tere integer, exterior liber; curolla quatrifida; tubnlosa, tubo brevi; spora una calyce circumdata; receptuculum c.0- 
nicum paleaceum, floribus longius, paleis rigidis amictum.

249. SCABIOSA, ( SCABIEUSE) LINN, IAM. tab. 57. (dipsacea 1.). Flores compositi, aggregati; calyx involucrum commine polyphyllum, muliflorum, proprius superus, duplex, exterior brevis, plicatus, interior saeyissime quinque aristatus, rarius plumosus; corolla tubnlosa, saepe inaequa!is, quatri-quinquefida; spora una, calyce coronata; receptaculum convexum, nudum aut paleaceum.

250. IK N A U T I A, (KNAUTIE) IINN. TILII. WILID. I.AM. tab. 58. (dipsacea I.). Flores aggregati terminales; calyx: involucrum commune cylindricum, rel oblongum, simplex aut multipartitum, quinqueflorum: proprius duplex: exterior denticulatus, aut fere integer ovarium cingens; interior urceolatus, ciliatus, aut plumosus, superus; corolla tubulosa, inaequalis, quatrifida; spora una, basi tuberculata, calyce exteriori tecta, interiori coronatal; receptaculum nudum aut pilosum.

251. A L L I N I A, (ALLIONE) LINN. L'HFRet. PUIZ. WILLD. LAMr. tab. 53. (nyctaginea 1.). WEDELIA LOEFL. Flores aggregati. Calyx: involucrum commune oblongum, simplex vel triphyllum, triflorum; proprius radiatus, superus, obsoletus; corollulae irregulares, quaeque fere quatrifida; spora una, tecta; receptaculum nudum,

1II. Flore monopetalo, infero; duae aut quatuor sporae nudae.

252. PERAMA, (PE'RAME) AUBLET. guian. $Y$. IAsI. tab. 68. MATTUSCHKAEA SCHREB, PERS. (py. renacea). Calyx quatripartitus; corolla infundibuliformis, tubulosa, limbo quatrifido, inferior; sporae duo ant quatuor nudae; stamina alterna cum lobis, iisque aequalia. 
IV. Flore monopetalo, infero; sporis pericarpio inclusis.

253. PYROSTRIA, (PYROSTRE) JUSS. LAM. tab.68. (rubiacea 8.) Calyx minimus, superus, fere intcger vel subquatridentatus; corolla campanulata, semiquinquefida patens, fauce tomentosa: drupa pyriformis, infera, octo striis notata, includens nuces octo, monosporas.

254 MYONIMA, (MYONIME) JUSS. WILLD. IANT. tab. 68. (rubiacea). Calyx superus, minimus, subin. teger; corolla quatripartita, tubu brevi; drupa globosa, nuce quinqueloculari, tetraspora.

255. PETI TIA, (PETITIE) JACR. (verbenacea). Calyx inferus, quatridentatus; corolla tubulosa, qiratrifida, vel quatripartita; drupa nuce biloculari, dispora.

256. A QUARTIA , (AQUART) LINN. JAGQ. WILLD. LAM tab. 82. (solanacea). Calyx campantlatus, quatrilobus, lobis duobus majoribus: corollu ro" tata, quatrifida, laciniis linearibus oblongis; bacca globosa, unilocularis, polyspora; antherae longae apice biperforatae.

257. ROUSSEA, (ROUSSE'E, ROUSSEAU) SMITIX. IAM. tab. 75. Calyx tetraphyllus, ligulatus, reflezus; corolla campanulata, infer, quatrifida, laciniis extrorsum volutis; filamenta dilatata; bacca quatrangularis (tetragona), pyramidalis, polyspora; antherae sagitta. tae; ovarium pyramidatum, glabrum.

258. CALLICARPA, (CALliCARPE) PLUr. IINN. THUNB. IAM. tab. 69. (verbanacea). Calyx quatrifidus, campanulatis ; corolla monopetala, quatrifida; bacca globosa, unilocularis, tetraspora; sporae osseao.

259. H I GGINSI A, (HIGGINSIE) ruiz. Flor. peruv. rens. Calyx quatridentatus; corolla infundibuli- 
formis, limbo quatripartito; stigma bilabiatum prominens ; bacca bilocularis, bisulca, umbilicata, polyspora.

260. IVALLENIA, (WALLENE) SWArz. PETISOIDES JACQ. BAYONIA sLOAN. - Calyx inferus, quatrifidus, laciniis obtusis; corolla tubulosa, hypocrateriformis, quatrifida, laciniis erectis : bacca unilocularis, monospora.

26r. WI T HER I N G I A, (WITHE'RINGE) L'HEhET, LAM, tab. 82. (solanacea). Caly $x$ minimus, urceolatus, quatridentatus; corolla fere campanulata, quatrifida, tubo urceolato quatrigibbo; antherue conniventes; bacca supera, bilocularis; nectarolyma fossae quatuor melliferae.

262. A E G IPHIL A, (AEGIPHILE) IINN. VAIIL. SWARz. IAM. tab. 7O. MANABEA AUBLET. JUSs. (verberacea). Calyx campanulatus, quatridentatus; corolla longa tubulosa, quatrifida; stylus filiformis, semibifidus; stigmata bifida; bacca inferne cincta calyce, bilocularis, loculis disporis in osseis nucibus.

263. N UXIA, (NUXIER) LAMARK. tab. 7r. AEGIPHILA swarz. WILLD. MANABEA Juss. (vorbenacea). Calyx turbinatus, quatridentatus vel quatrifidus; corolla monopetala, subinfundibuliformis, quatrifida; tubo brevi; stamina iu fauce; sligma truncatum; capsula carnosa, dispora, ovalis.

264. CEPHALA N THUS, (CEPHALANTHE) pLUK. LINN. LAMr. tab. 59. (rubiacea). Flores aggregati, capitati, insidentes receptaculo globoso, piloso; calyx: involucrum commune nullum; proprius superus, infundibuliformis, quatridentatus; corolla quatrifida; capsula bi-quatripartibilis, totidemque locularis; loculis monosporis; sporae solitariae, oblongae.

265. ROUHAMON, (ROUHAMON) AUBLET. LASIOSTOMA SCHREB. WIILD. STYCHNOS JUSS. (apocinacea). Calyx quatrifidus (quinquefidus SCHP.EB.) basi munitus duabus squamis; corolla infnndibuliformis, 
mis, quatrifida, fauco villosa; capsula unilocularis, dispora, turbinata, compressa, ot uno laterale striata.

*) 266. SCOPA il IA, (SCOPAIRE) IINv. JAcQ. vahi. LANi, tab, 85. (personata). Calyx quatripartitus tubo brevi; corollu quatripartita, rotata, fauce pilosa; capsula uni-bilocularis, bivalvis, polyspora, sphaerica.

267. BARTONIA, (BARTONIE) wiLin, Calyx tetraphyllus; corolla campanulata, quatrifida, persistens; capsula unilocularis, bivalvis, (monospora, vel polyspora.

268. CENT U N C ULUS, (CENTENILLE) LINN. VAll. LAM. tab. 83. (primulacea 1.). Calyx quatrifidus; Corolla rotata, patente limbo quatrifida; capsula monolocularis, globosa, circumscissa, polyspora. (Interdum stamina quinque, tunc congener anagallide.

269. FRASERA, (FRASERE) MighaUx. GMEL. pens. - Caly $x$ quatrifidus; corolla quatrifida, patens; petala medio glandula barbata; capsila compressa submarginata, bilocularis; sperce paucae, imbricatae.

270. PLANTAGO, (PLANTAIN) IINN. IAM. tab. 85. (plantaginea). Caly $x$ quatrifidns; corolla tubulosa, quatuor limbis reflexis; stamina longissima; capsula bilocularis, polyspora, circumscissa, Flores spica serrata, bracteata.

271. PSYL L I UM , (PSYLLE, Pulicaire) JUSSIEU. PLANTAGO LINN. pers. (plantaginea). Tlores capitati terminales, bracteati; calyx quatrifidus; corolla tubulosa, quatuor limbis reflexis; capsula bilocularis, dispora, circumscissim se apperiens.

272. $\mathrm{P} \mathrm{O}$

*) SPIELJMANIA africana IAMARK. tab. 85. LANTANA LINN. JASIIINUM MEDICUS. (verbenacea). Caly $x$ quinquefidus; corolla fauce barbata, limbo quinquclobo, subaequali; stigma uncinnatum; drupa nuce viloculari dispora. 
272. POLYPREMUM, (POLYPREME) LINN. MICHAUX. WILLD. LAM. tab. 7r. (personata 3.). Calyx tetraphyllus; corolla rotata, quatrifida, lobis rotundatis, vel cordatis; capsula compressa, emarginata, bilocularis, bivalvis, polyspora.

273. B U D L EIA, (BUDLE'IE) LINN. LAM. tab.69. IANTANA IINN. BUDLEA AIT. (personata I.). Calyx quatrifidus; corolla tubulosa, quatrifida; stamina tubi incisuris inserta; capsula sphaerica, bivalvis, bilocularis, polyspora, apice dehiscens.

274. CODONIUM, (CODONIE) roHr gen. plantt. (caprifolia?). - Calyx duplex; inferus monophyllus, inaequaliter bifidus superus turbinatus subinteger; corolla campanulata; drupa obovata; stigma capitalum. (An prope LINNEAM collocandum genus.)

275. EXACUM, (GENTIANELLE) IINN. SMITH. I.AM. tab. 80 GENTIANA AIT. LINN. (gentianea 1.). Calyx tetraphyllus: corolla tubulosa, quatrifida, tubo globoso; capsula bisulca, bilocularis, loculis disporis, apice dehiscens.

276. CENT A UR I U M, (CENTAURELLE, CEN. TAURELlA) michaux. (gentianea). - Calyx quatripartitus adp̧ressus; corolla subcampanulata quatripartita; stigma crassum glandulosum subbifidum; capsula (calyce et corolla persistentibus involucrata) unilocularis, bivalvis, polyspora.

277. C OUTOUBEA, (COUTOUBEE) AUBLET. IAst. tab. 79. EXACUM vAHi. (gentianea 1.). - Calyx quatrifidus bracteae tres; corolla monopetala, quatrifida, calice longior, tubo brevi; squamae quatuor cucullatae; stigma bilamellatum; capsula bivalvis, polyspora.

278. TACHIA, (TACHI) AUBIET. IAM. tab. 80. NiYrimeCIA schreb. peris. (gentianea 2.). Calyx tubulosus, campanulatus quinquedentatus; corolla tubulosa, quinquefida, fauce dilatata, vel inflata; glandulae quin- 
que cingunt ovarii (germinis) basin disco quatrangulari; capsula bilocularis, bivalvis, polyspora.

279. POUTERIA . (POUTERIA) AUBLET. LAM. tab 72. LABATIA swariz. WILLd. (ebenacea). Calyx inferus, persistens, tetraphyllns; corolla subcampanulata, ovalis, quatrifida: laciniis ruabus minimis, quarum diuisuras vel sinus tenent obsitas filamenta; stigma quatrifidum; capsula ovalis striato sulcata, tecta pilis rigidis, quatrivalvis, tetraspora. Sporae solitariae, arillatae.

28c. PENAEA, ( PENE'E, Sarcocollier ) puUk. IINN. THUNB. IAM. tab. 78. (planta ordinis indeterminati). Calyx diphyllus bracteaeformis, caducus; corolla campanulata, aut infundibuliformis longior, quatrifida; stigma quatrilobum; capsula tetragona, quatrilocularis, quatrivalvis, valvulis septiferis, loculis di-octosporis, axi nulla.

28I. BLAERIA, (BLARRE) IINN. BERG. IAII. tab. 78. ERICA tiNN. тнUNB. (ericae). Calyx quatripartitus; corolla campanulata quatrifida; stamina receptaculo inserta; capsula quatrangularis, quatrilocularis, ad angulos delsiscens, polyspora.

$\boldsymbol{V}$. Flore monopetalo, supero; sporis pericarpio inclusis.

282. CHOMELIA, (CHOME'LE) JACQ. (rubia. cea 6.). Caly $x$ superus, tubulosus, quatripartitus; corolla liypocrateriformis, tubo cylindrico, limbo patente quatripartita; stigmata duo, crassiuscula; drupa infera coronata; nux bilocularis, dispora.

283. MA LANEA, (MALANI) AUBLET. WIILd. IAM. tab. 66. ANTIRHEA GMEL. CUNNINGHAMIA scirrer. (rubiacea). Calyx superus, minimus, quatridentatus; corolla rotata, infundibuliformis, quatrifida; stylus 
stylus bifidus; drupa ovalis, coronata; nux bilocula. ris, तispora.

294. COLOSANTHUS; ( SCOLOSANTIIE)

VAIL. WILLD, CATESBAEA LAM, tab, 6 \% (rubiacea 3.). Calyx superus, parvas, quatrifidus; corolla tubulosa, quatrifida, limbo extro1sum revoluto; stamina basi fere coalita; drupa succulenta, globosa; $n u x$ uniloculaxis, monospora.

285. PAVETTA, (PAVETTE) LiNN. VAHLIUS. wrLld. IXORA Forsi. CRINITA moutr. (rubiacea 6.). Calux superus, minimus, quatridentatus; corolla infundibuliformis, quatripartita; tubus angustatus; stigma incrassatum, curvum; bacca pisiformis, unilocularis, secuud IInN. mono-dispora; sporae uno latere planae, altero convexae.

=86. IX OR A, (IXORE) IINN. VAHL. SWARZ. IAIV. tab. 66. (rubiacea 6.). - Calyx superus, minimus, quatridentatus; corolla infundibuliformis, longa, monopetala, quatripartita; tubus longus; stamina supra faucem tubi inserta; bacca bilocularis, loculis di-tetrasporis.

287. PETESIA, (PETESIE) LINN. LYGISTUM 1Rown. LAM. tab. 67. f. 2. (sub Fernelia.) (rubiacea 3.). - Calyx superus, quatridentatus; corolla infundibuliformis, quatriloba; stigma bifidum; bacca globosa, coronata, bilocularis, polyspora.

=88. C A T E SB A A, (CATESBE'E) IINN. LAM. tab. 67. fig. x. (rubiacea). - Calyx superus, minimus, quatridentatus; corolla infundibuliformis, quatriloba; tubus longissimus, dilatatus; stamina tubi basi inserta; bacca globosa, coronata, bilocularis, polyspora.

289. F ROELICHIA, (FROELICHE) WILLD. LILLARDIERIA vaHL. ${ }^{*}$ ) (rubiacea 6.). - Calyx su-

perus,

*) Symbol. I. qui ipse ait : ,impresso fasciculo ad manus venit - of the botany of new Holland - ubi smrin jam novum genus BILLARDIERIAM constituit, " hinc FROELICHIAII numcupendam suadet, Cf. Spec, in Iracfatione. 
perus, quatridentatus; corolla tubulosa, quatrifida: drupa sicca (bacca exsucca VAHL.) monospora; spora arillata.

290. HOFF MANNI fl. ind. I. pag. 242. (rubiacea 3.). - Calyx superus, quatridentatus; corolla hypocrateriformis, quatripartits, tubo brevissimo; antherae sessiles conniventes; filanenŁum nullum; bacca (capsula pers.) pulposa, bilocularis, bivalvis, polyspora, infera *).

29I. 'ER N O E A, (ERNQDE'E) sWARZ. IAMART. KNOXIA BROWN. (incertae sedis) **). Calyx superus, quatri-quinque-partitus; corolla hypocrateriformis, monopetala, quatripartita, limbi laciniis linearibus: stamina longitudine corollae; stigmata duo extrorsum revoluta; bacca pisiformis, coronata, bisulca, bilocula. ris, polyspora; sporae solitariae.

292. SIDERODENDRUM. ( SIDERODEN. DRON) YAHL. Eclog. I. (rubiacea 6.). SIDEROXYLOIDES JACQ. PLUx. - Calyx subquatridentatus; corolla tubulosa, quatrifida; stigmata lanceolata, duo extrorsum recoluta; bacca dicocca, bilocularis, loculis monosporis; sporae solitariae.

293. FER N E L I A , (FERNEL) COMMERS. JUSSIEU. IAM. tab. 6\% f 1. COCCOCYPSILUM swARz. LAM, tab. 64. WILLD. CONDALIA ruiz. et PAv. (rubiacea 3). Calyx superus, quatridentatus; corolla infundibuliformis,

*) Huic generi novum genus RUrzi et PAv, O-HIGGIN. SIA maxime congencr, cujus fructus etiam baccatus, licet valvulis dehiscat. Cacterum valde affinis CATES. BEAE.

**) persoor in Enchiridio suspicatnr, Ernodeae species et Siderodendri potins ad Ixoras aut Pavettas pertinere, quum multa rubiacearum genera iutcr se nimis affinia sint; atque olsservatores strennos rogat, velint in viyis accuratius denuo examinare, quo firmiores gcnerum characteres crucutur. 
mis, parva, quatrifida, (quatriloba); stylus semibifidus; bacca coronata, inflata, bilocularis, polyspora; sporce lentiformes, liberae; receptaculum sporiferum, centrale, loco dissepimonti versus medium ovaniti. *).

294. GONZALEA, (GONZALEE) PERs. ench. pag. 132. b. GONZALAGUNIA RUIz. BUENA cAv. Calyx campanulatus, minimus, quatridentatus; corolla infundibuliformis; pericarpiurn bacca drupacea, obsolete tetragona, cocculos quatuor polysporos includons.

295. PALTORIA , (PALTORIE) RUIz, - Calyx minimus, quatrifidus; corolla rotata; stigma magnum; bacca quatrilocularis; sporde solitariae. (Spora una, duae, ad maturitatem perveniunt. reliquae abortiunt ${ }^{* *}$ ).

296. M I T C H E L L A , (MITCHELLE) PLUน.LINN. IAM. tab. 62, CHAMAEDAPHNE $2 \mathrm{ITCH}$. gen. 27. (rubiacea 10.). Duo flores gemini; calyx quatridentatus Juss. (quinquefidus IAM.); corolla monopetala, infundibuliformis, interne villosa, quatrifida; stigma quatrifidum; bacca globosa, bifida, didyma, tetraspora.

297. E VEA, (EVEE) IAM. tab.59. Calyx communis polyphyllus, foliolis quatuor exterioribus latioribus, proprius turbinatus, quatridentatus; corolla infundibuliformis quatripartita; pericarpium .... (Flores octo, decem, in recoptaculo paleaceo, aggregati.)

298. HEDYOTIS, (HE'DIOTE) IINN. IAM. tab. 62. (rubiacea 3.). - Caly $x$ superus, quatridentatus; corolla infundibuliformis, monopetala, quatrificia; capsula

*) Coccocypsiluir вrows, I 1 M. tab. 64. (rubiacea 6.) - Calyx quatripartitus, laciniis linearibus; corolla infundibuliformis . quatriloba; capsula calyce icorouata, bilocularis, polyspora; sporae dissepimento affixac.

**) Gravis Ol. swarzi veritatis investigatio hoc genus ad genus Ryginda rccedere jussit apud schrADEnun in Diario IV., 
globosa, didyma, coronata sub apice, bilocularis, po. lyspora, infera, transversim dehiscens; receptaculo dis. sepimento undique adnata. GAERTN.

299. OLDENLANDIA, (OLDENLANDE) LINN. WILLD. SCHREB. HEDYOTIS LAM. tab. 6:. (sMb OLDENLANDIA) HEUCHERA MUPR. (rubiacez). Calyx superus, quatripartitus; corolla vix tubulosa, quatrifida, (tetrapetala PERs.) ; capsula minima, coronata, infera, bilocularis, polyspora, ad apicem linea transversali dehiscens; receptacula nuda. (Styli unus, duo.) GAERTN. ").

\section{HY D R O H L A X, (HYDROPHYLAX)}

IINN. suppl. IAM. tab. 75 . SARISSUS OAERTN. (rubibiacea). - Calyx quatripartitus; corolla quatrifida, monopetala, infnndibuliformis, fauce villosa; bacca (capsula ?) exsucca, angulosa, bilocularis, dispora, dissepimento transversali; sporae in loculo solitariae.

301. N A CIBE A, (NACIBE) AUbLet. I 1 Ir. tab. 64. MANETTIA IINN. PAV. et RUIz. PETFSIA IINN. Brown. (rubiacea 3.). Calyx superus, quatri-, quinque-octopartitus; corolla infundibuliformis, quatriquinquepartita, fauce barbata; stamina quatuor, quinque; capsula calyce coronata, ovalis, compressa, oblonga, bilocularis, bivalvis, valvis duplicatis, polyspora; sporae orbiculares, imbricatae, alatae, numerosae. (Plantae volubiles aut scandentes.)

302. CAR P HA L E A, (CARPHALE, JUss. writ. LAN. tab.59. (rubiacea 3.). Calyx superus, quatrifidus; laciniis spathulalis, scariosis ; corolla infundibuliformis, monopetala, intus hirta, quatrifida; capsula coronata, bilocularis, bivalvis, polyspora, dissepimenta duabus valvulis opposita, semiseptifera.

303. TON.

**) Plures ut videtur Oldenlandiae specics cl. a Cavanilles Vol. sexti Ic. plantt. hisp. sul, genere Hedyotis descriptae, in mentem Lamafixi, schueberix, et forte non immerito, dua hacc gencra haud diversa putanda esse, autumat IEISOON Enchir, pag. 146. 
303. TON TANEA, (TONTANE) AUBx. IAM. tab. 64. BELLARDIA wiLLD, scineb. (rubiacea 3.' Calyx superus, quatrifidus; corolla infundibuliformis, quatrifida; bacca (capsula?) coronata, bilocularis, bipartibilis, minima, ovalis, polyspora; sporae omarginatae, dissepimento affixae. Nectarolymu margo quatrilobus stylum cingens.

304. SANG UISORBA, (SANGUISORBE) IINN. . MORxs. WILID. LAM. tab, 85. (rosacea 3.). Calyx 811perus (inferus Xers.) quatrifidus (diphyllus PERs.), basi duabus squamis cinctus; corolla nulla (supera PEPS.); (ovaria duo inter calycem et corollam PERS.); capsula e calyce formata uni-bilocularis, dispora.

\section{Flore monopetalo, infero; fructu dicocco.}

$30 \tilde{5}$, H O U S T O NI A, (HOUSTONE) MORIS. LINN. MICHAUX. ANDREW. LAII, tab. 79. (rubiacea). KNOXIA? LAMT. MichaUX. Calyx quatridentatus; corolla infundibuliformis, quatrifida; capsula supera, didyma, bilocularis, bivalvis, dispora, transversim dehiscens.

306. A N THOSPER M U M, (ANTHOSPERME) LINN, JUss. (rubiacea 1.). - Calyx minimus, quatridentatus; corolla tubulosa, qnatripartita; fructus ovalis vel oblongus, in duas sese disjungens sporas.

307. RUBIA, (GARANCE) LINN. IAM. tab. 60. (rubiacea 1.). Calyx superus, quatridentatus; corolla campanulata, quatri-quinquefida; baccae duae, rotundatae, monosporae; sporae scilicet membrana tectao molli. (Stamina quatuor, quinque.)

308. G A L L I U M, ( GAILLET, CAILLE - LAIT) LINN. LAM. tah. 60. (rubiacea 1.). Calyx superus, fero integer, aut quatridentatus; corolla monopetala, plana, rotata, quatrifida; sporae duae, subrotundae, unitae, 
aut glabrae, aut scabrae, aut hispidae: Interdum corollas laciniae tres, tunc et stamina tria.

309. ASPERULA, (ASPE'RULE) IINN. LAN. tab. 61. (rubiacea I.). - Calux superus, quatridentatus; corolla infundibuliformis, quatrifida; sporae duae, globosae, unitae, non coronatae.

3IO. PUTORIA, (PUTORIE) PERs. - Callyx quatridentatus, persistens, demum baccans; corolla tubuloso infundibuliformis; limbo quatrifido; stylus bifidus, acutus; bacca subcompressa; sporce duae, oblongae. (Asperula calabrica IINN. SHERARDIA crr. IAMT. )

3II. SHER A R D I A, (SHE'RARDE) IINN, JUSS. IAM. tab.61. (rubiacea I)). - Caly $x$ superus, quatridentatus; 'corolla infundibuliformis, quatrifida; sporae duae oblongae, coalitae, dentibus calycis coronatae.

312. SPERMACOCE, (SPERMACOCE) IINN. Juss.tam. tab.62. (rubiacea 2.). Calyx superus, quatridentatus; corolla monopetala infundibuliformis, quatrifida; capsulae duae connatae, oblongae, coronatae, biloculares, disporae; sporae duae, bidentatae.

313. K N OXIA, (KNOXIE) LINN. GAERTN. WILID. rAM. tab. 59. (rnbiacea 2.). Calyx superus, quatrifidus folium unum majus; corolla infundibuliformis, filiformis, quatrifida; sporae duae, rotundato acuminatae, uno latere planae, trisulcatae altero latere, coronatae, supra axin filiformem insitae.

3I4. DIODIA , (DIODE) IINN. vaHL. SWARz. IAM. tab. 63. (rubiacca 2.). Calyx superus, bifidus; corolla infundibuliformis, interne villosa, quatrifida; capsula bilocularis, ovalis, quatrangula, coronata, bivalvis, bilocularis, dispora.

315. CRUCIA N ELA, (CRUCIANELLE) IINN LAM. tab. 61. (rubiacea 1.). Calyx di - triphyllus; corolla infundibuliformis, quatri - quinque[s] fida, 
fida, tubo filiformi, limbo unguiculato; sporas duaè, oblongae, unitae. (Folia saepe linearia; flores bra: cteati, in spica attenuata et terminali.)

\section{Flore monopetalo, infero; fructu tetracocco.}

316. SIPHONANTHUS, (SIPHONANTHE) IINN, AVIMAN. WILLD. IAM. tab. 79. (borraginea 5.). Calyx magnus, quinquepartitus; corolla infundibuliformis, quatrifida, infera; tubo filiformi patente, longissimo; baccaequatuor, rotundatae, superiores, monosporae.

\section{Flore infero, tetrapetalo.}

317. EPI M D I UM, (EPIME'DE) IINN. IAM. tab. 83. (berberidea 1.). Calyx tetraphyllus, caducus; potala quatuor; nectarolymata quatuor, irregularia, cyathiformia, petalis inter incumbentia et staminibus; corolla patens duobus foliis ad basin bractea munitis ; silicula oblonga, unilocularis, bivalvis, polyspora.

318. P T E L E A, (PTELEA, PTELE'E) IINN. LANI! tab. 84. (térébintacéa 4.). Caly $x$ inferus, quatripartitus; corollae petala quatuor patentia, calyci majora; stamina petalis alterna; stigmata duo; styli breves; capsula rotundata, orbicularis, compressa, membrana alata, ad medium inflata, bilocularis, dispora.

319. PTELIDIUM, (PTELIDIE) AUBERT DI PEтіт thoUARs. Plantt, aff. austr. tab. 4. (rhamnea). - Flores hermaphroditi, completi, tetrapetali, perigyni. isostemones, monogyni; paniculao axillares. Discus centralis, staminifer, et pistillifer; calyx quatrifidus persistens corolla tetrapetala, calyci major; stamina qua. tuor petalis alterna, disco centrali inserta; stigmata brevissima; stylıs vix ullus ; samara inaperta, cycloptera, comprossa, 
pressa, alata, bilocularis, loculis disporis, una abortiente; spora recta; perisporium carnosum, embryo rectus; cotyledones planae, virides.

320. BLAKBURNIA, (BLAKBURNE) FORST. PTELEA LINN. (terebintacea). Calyx inferus quatridentatus; corollae petala quatuor; stigma simplex; bocca? monospora.

32I. S I I M M I A, (SKIMME) THUNe. JUss. (ex ordine plantarum indeterminato) - Calyx inferus, minimus, quatri-rarius quinquepartitus; corella petala quatuor; stamina quatuor brevissima; ovarium libe. rum; bacca ovalis, umbilicata, quatrisulca, fere quatrivalvis continens substantiam farinoso pulposam, tetraspora; sporae ex uno latere angulosae; ex altera convexae.

322. A Z I M A, (AZIME) I,AM. tab. 80\%. 1. p. 339; MONETIA L'HEPET. WILID. SCHREB. ( $x$ ordine plantt. indeterminato). - Calyx tri-quatrifidus, urceolatus; corollae petala quatuor; bacca bilocularis; lo, culis disporis, una spora saepe abortiva.

323. SAM A RA, (SAMARA) LJNN. JUSS. IAM. tab. 74. MEMECYLON LINN. BURM. PAPANEA AUBLET. (rhamnea). - Calyx minimus quatripartitus; corollae petala quatuor, basi excavata; stamina quatuor petalorum cavitati inserta; antherae fere cordatae; stig. ma infundibuliforme; drupa rotundata, monospora.

324. HART T G I A, (IIARTOGE) THUNB. wILID, IAM. tab. 76. SCHREBERA IINN. JUSS. (rhamnea). - Calyx quatrifidus; corollae petala quatuor plana pa. tentia; drupa supera, ovata; nux dispora.

325. C URTISIA, (CURTISE) АाтоN. THUNe. WILLD. (Planta sedis incertac). Calyx quatripartitus; corollae petala quatuor; drupa succulenta, subrotunda, supera, continens nucem qnatri-quinque locularem. 
325. F A G A R A, (FAGARIER) IINR. LAMr. tab. 84. (térébintacea). - Calyx quatri - quiuquefidus; corollae petala quatuor, quinque, patentia; stigma bilobum; capsula simplex, aut multiplex, globosa, calyce cincta bivalvis, bilocularis, monospora; spora globosa. (Stamina quatuor, quinque, octo.)

327. Z IE R I A, (ZIERIE) sMITH. transact. IV. (rutacea Juss. gen. 297.) - Calyx quatripartitus; corollae petala quatuor; stamina glabra, glandulis insidentia; stylus simplex; stigma quatrilobum; capsulae quatuor, coalitae; sporae arillatae.

3:8. OTHERA, (OTHERE) тичкв. Juss. (berberidea). Caly $x$ quatripartitus persistens; corollae petala quatuor, lancolata; stamina quatuor petalorum basi iuserta, brevissima; stigmata sessilia; capsula?

529. O R I X A, (ORIXE) тнuгi. juss. '(ex ordine plantt. indeterm.). Calyx brevis quatripartitus; corollae petala qnatuor, lanceolata; stamina quatuor, brevia; stigma capitatum; capsula ?....*)

330

A M M A N N I A, (AMMANNE) HOUST. SLOAN. IINN. WILID. LAM. tab. 77. (lythraria 2.) - Calyx monophyllus, campanulatus, striatus plicatusve, octodentatus, coloratus, inferus; corollae petala quatuor, (interdum corolla nulla) calyci inserta; stamina quatuor didyma; capsula quatrilocularis, supera, calyce tecta, (passim bilocularis et stamina octo) polyspora. (Planta herbacea, aquatica; folia opposita; flores axil. lares plurimi, sessiles, minimi, oppositi, quasi verticillati.)

1X. Flore

*) PERsoon enchir. p. 145. b. ORIXAM cum OTHERAE genere conjunxit, incertus num OTH, japonica, et Orixa japonica sint diversi aut ejusdem generis, quum utriusque fructus numc usque ignoretur! 


\section{Flore supero, tetrapetalo.}

33I. TRAPA, (MACRE) IIINN. WITLD. GAERTN. LAM. tab. 75. (onagraria 2.) - Calyx superus, quatripartitus, persistens; corollae petala quatuor; antherae declinatae; ovarium biloculare; stigma emarginatum: $n u x$ coriacea, duabus aut quatuor cincta spinis, for. matae foliorum calycis laciniis; spora magna, carnosa, - (trunci elongatio simplex, aquae inmersa.)

332. CISSUS, (CISSUS, Achit.) IINN. PLUK. VAHL. LAM, tab, 84. VITIS TOURN. SAELANTHUS rorsx. (vinifera). Calyx vel subinteger, vel quatridentatus, inferus, minimus; corollae quatuor petala libera, reflexo petula, decidua; stamina quatuor disco, ovarium cingente, inserta; stylus unus; stigma acutum; nectarolyma discus orarium cingens; baccagl.bosa, bilocularis, mono-di-tetraspora, basi calyce cincta. (Stamina passim quínque); (Folium unum, interdum ternata, aut digitata; flures disposti in corymbum, aut umbellanı cinctam involucro polyphyllo.)

333. VOTOMITA, (VOTONIT) AUBLET. JUSS. GLOSSOMA SCHREB, PERs. WIJLD. ( rhamnea 6.) Calyx superus, turbinatus, quatridentatus; corollae petala quatuor; stcmina quatuor; antherae oblongae mem. brana in cylindrum coalitae, fere sessiles, termmatae ad apicem mucrone saepe laterali; ovarium adhaerens; stylus tectus ostaminum cylindro, stigmata quatuor; drupu coronata calycis dontibus, unilocularis; nux sulcata, monospora.

334. CORNUS, (CORNOUILLIER) TOURN.IINN. L'HETER, JUSS. LAM. tab. 74. (caprifoliacea 4.). Flores saepe munitae involucro tetraphyllo; caly $x$ superus, quatridentatus; corollae quatuor petala parva, basi dilatata; stumina quatuor cum petalis alternantia; antherce declinatie; drupa ovoidea, aut globosa non coronata; $n u x$ bilocularis, dispora. (Arbuscula aut arbores mediae altitudinis; folia opposita, basi nuda, in una tantum specie alterna.)

3,5. L U D. 
335. LUDWIGIA, (LUDWIGIE) IINN. MICIr. JUss. LAm. tab. 77. (onagrea 2.). Calyx superus, truncatus, limbo Iongo, quatripartito, laciniis longis persistentibus; corolla aut nulla, aut tetrapetala; staminum antherae oblongae, rectae; stigma quatrangulum; capsala tetragona, quatrilocularis, infera, calyce persistente coronata, ad apicem foramine circulari perforata polyspora: (Elongatio trunci herbacea, aut suffrutescens; folia alterna, aut oppcsita, simplicia; flores solitarii, 'axillares).

\section{SAN TALUM, (SANTAL, SANTALIN)}

IINN. Mantiss. JUss, noxb. ram. tab. 74. (onagrea 4.) Calyx nrceolatus, margine quatridentatus; corollae qua. tuor petala, calyci innata; glandilae quatuor cum petalis alternantes; bacca infera, monospora.

\section{Flore incompleto, infero.}

337. STRUTHIOLA, (STRUTHIOLE) LIRN. tinunb. Juss. retz. LANi. tab. 73. (daphnacea). Calyx tubulosus, longus, filiformis, limbo quatrifido, munitus duabus bracteis ad basin oppositis (perianthium diphyllum PERs.); corolla tubulosa, ore glandulis quatuor - octo munita; stylus filiformis; stigma capitatum; bacca exsucca, monospora; - (Folia opposita; flores solitarii, axillares.)

\section{OPE ILCULARIA, ( OPERCULAIRE )} GAertw. Willd. Juss. LAM. tab. 58. (planta sedis incertae). - Flores aggregati; caly $x$ involucrum commune monophyllum, campanulatum, sex - novem inaequaliter dentatum, clausum receptaculo operculiformi, tri vel sexfloro; proprius nullus; corolla infundibuliformes, epigyna, monopetala, tri-quatri-quinqueparpartita, aequalis; unum do quinque staminibus basi corollae insertum; filamenta et antherae distinctae; ovavium adhaerens; stigma bipartitum; spora una calyce persistente tecta, receptaculo inferne infixa uno latere striato sulcata, altero convexa; embryo cylindricus, 
ciss, locatus ad medium perisporii carnosi; (trunci elongatio herbacea; intereum basi lignosa; folia opposita, simplicia, formant per basin sulcum; flores capitati aut umbellati.)

339. CRYP T O SPER M U M , CCRYPTOSPERME) YoUnc. transactt, of IINN. Soc. Vol. III. ROEMER. II, I. SCHRADER. Diar. 1799. II. OPERCULARIA young. (rubiacea). - Calyx communis hexaphyllus; foliolis patentibus, inaequalibus; proprius? e paleis receptaculi triphyllus; corollulae (subviginti) monope. talae, quatrifidae, primo ovatae; receptaculum globosum, paleaceum, subtus sporiferum; capsulae uniloculares, in receptaculum subglobosum coalitae, medio longitudinaliter dehiscentes ${ }^{*}$ ), young.

340. PROTEA, (PROTEA, Protée) IINN. WiLtid. JUss. LAMI. tab. 53. GLOBULARIA ToUrn. (proteacea 1.). Flores aggregati; calux proprius nnllus, communis imbricatus; corolla quatrifida, aut tetrapetala, petalis apice conniventibus, interno intra summitatem excavatis sulcatisque; lacinia suprema multoties altiusque fissa; stamina quatuor inserta pesalorum cavitate sub apice; antherae lineares, oblongae, et juxta laciniarum sulcos insertae; stigma rarius bifidum; oblongum articulatum saepe super stylum; $n u x$ aut capsula supera, non dohiscens, calyce tecta, unilocularis, monospora; - (folia alterna; flores interdum distincti, in spicam dispositi et monoici; saepissime hermaphroditi, et coaliti in receptaculo communi interdum nuco, interdum vero obsito pilis aut palois, aut imbricati in conum; florum capituli terminales, rarius axillares).

34I. CONOSPERMUM, ( CONOSPERME) smith. JUss, gen. 78. (proteacea). Calyx nullus; corol-

*) In ordine naturali decimo octavo L. P. B, aggregatis locandum; in systemate JiNNEANo inter Allionam ct Knautiam, interque Crinitam et Eveam editionis Gmelini; pertinet ad Jussieui classem undecimam rubiaceas, ordiuem decimum inter Pataheam ct liveam. 
la monopetala, ringens, staminifera; labium superius fornicatum, inferius trifidum; stigma obtusum; spora unica, nuda, pappo coronata *).

\section{A D E A N THOS, (ADENANTHOS) IA-} bILIARD. **) (proteacea). Corolla quatrifida, laciniis basi squamis cincta; antherae quatuor lineares, insertae lobis corollae sub apice; spora unica supera, corolla tecta, quae exsiccat, et desilit basi, quatuorque glandulis squamas format, corollae basi approximatis***).

343. ROUPALA; (ROUPALE) AUBLET. JUSS. LA. MARr. tab. 55. RUPULA WILLD. (proteacea 1.) Calyx nullus; corollae petala quatuor, spathulaeformia, excavata superiori parte, basi cohaerentia; stamina medio petaloı um cavitati insera; antherae quatuor, fere sessiles, post corollae expansionem rectae; pericarpium uniloculare, monosporum?

344. L A MBERT I A, (LAMBERTE) sMITH. transactt. IV. JUss, 79. (proteacea). Calyx perianthium commune polyphyllum, imbricatum, squamis interioribus longioribus, uni-septemflorum; corolla quatrifida, (aut petala quatuor) laciniis revolutis, stamini. feris; stigma subulatum, sulcatum; capsula tricornis, unilocularis, dispora; sporae marginatae.

345. B A N KSIA, (BANKSIE) LINN. suppl. JUss. cavan, willd. LAM. tab. 54. (proteacea I.). Flores aggregati; inflorescentia: conus strobiliformis squamosus; solitaria; receptaculum commune elongatum, squamosum; calyx nullus (quatrifidus GAERTN.); corolla quatrifida vel tetrapetala, laciniis longis lineari lanceolatis, excavatis interne sub apice, tnbo brevi; antherae sessiles in cavitate limbi; stigma filiforme;

cras-

*) sмrth act. soc. LINi. IV, P. 213.

**) Novae Hollandiac plantarum specimen, Paris, 1804.

***) Quocum gencre protea racemosa potest conjungi. 
crassum ; capsula lignosa, bivalvis, mono-dispora, dissepimento semibifido sporis interjecto; sporae ala. tae; - (Folia sparsa aut conferta, saepe obliqua; coni squamae coriaceae, duplici ordine dispositae, alterius magnis bifloris, alterius minoribus florem nullum continentibus).

346. VALA IN T I A, (VALANCE, Croisette) LINN. JUSS. IAM. tab. 843. CRUCIATA et APARINE TOURN. GALIUM scop, GAERTN. (rubiaceà r.). Saepe Polygarna, per aportum partis allterae sexus; flores hermaphroditi; calyx nullus (vel vix ullus subinteger Juss.); corolla plana, vix tubulosa, tri - quatrifida; stamina quatuor; stylus bifidus, spora una globosa; altera saepe abortiva; - Flores masculi: calyce nullo, vel vix ullo conspicuo; corolla tri - quatrifida; stamina tria, quatuor: pistiltum abortivum. (Folia post anthesin reflectuntur, fructumque obtegunt; - elongatio trunci herbacea, folia quatuor saepenumero in verticillo; flores axillares.)

347. CONCHIUIM . (CONCHIE) sMITH. trans. act. IV. HAKEA sert. Hannover t. 17. BANKSIA wrTHERING. GAERTN. - (Juss. gen. 79.) (proteacea). - Flores distincti; calyx nullus; petala corollae quatuor, staminifera, stigma turbinatum, mucronatum; cupsula unilocularis, dispora; sporae alatae *).

343. B R A B E J U M, ( RRABEI) IINN. JUSs. LAM. tab. 847. BRABYLA INN. mant. ("proteacea 1.)."Flores hermaphroditi: amentum parvum cylindricum pubescens, tectum squamis ovatis, obtusis, imbricatis, trifloris; calyx nullus; corolla infundibuliformis, quatripartita, deinceps connivens, laciniis tandem revolutis; stamina quatuor; stylus unus; stigmata duo; drupa sicca subrotunda, villosa, monospora; flores masculi amento et calyce ut in hermaphroditis; corolla quatriquịn-

*) A Xylomelo differt stigmate mucronato; a Banksia fru. ctu milurulari, ab utrisyue floxims calyce destitutis nec anculaceis. 
quinquefida; laciniis deorsum rerolutis; stamina qua. tuor, quinque, stylus unus abortiens. (Folia fere verticillata; flores in spicam axillarem.)

349. II A ILE A, (HAYEE) WENDL. I, fasc. 3. (proteacea 1.). Fiores distincti : caly $x$ communis imbricatus, polyphyllus, squamis deciduis (gemma?): corollae quatuor petala linearia, apice concava, ibique staminifera ; ovarium pedicellatum, basi glandulosum; capsula lignosa, unilocularis, bivalvis, dispora, latere interno rima longitudinali dehiscens; sporae duae, alatae *).

350. XYLOMELUM, ( XYLOMELE) SMIY. transact. IV. JUss. fam. 79. (proteacea 1.) - Amenti squama simplex; corollae quatuor petala, staminifera; stigma clavatum, obtusum; capsula unilocularis, dispora; sporae alatae ${ }^{* *}$ ).

35I. EM BOTHRIU M, (EMBOTHRION)FORST. IINN, ruIz, JUSs. IAM, tab. 55. (proteacea). Calyx nullus; margo semicircularis $R_{U} \mathrm{Iz}_{\text {. ; }}$ corolla inferne tubulosa, ***) inflata, apice clausa, interdum apice aperta, quatrifida; laciniis dilatatis, ad summum excavatis;' (petalis spathulatis revolutis, apice-concavis RUrz.;) laciniae stigma tegunt; antherae saepe foro sessiles cavitati limbi petalorum insertae; ovarium podicellatum; stylus, vix ullus; stigma dilatatum; folliculus fere oblongus, cylindricus, longitudinaliter deliscens, polysporus; sporae ex unú latere alatae, imbri-

*) Perss, in enchir, p. 117. 2, haec:, genus vix a Banksia divcrsum; forum forma, et foliis integerrimis ab ca distinctum, eique nomen Conchium impositum rst a ccl. $\sin 11.1$. 1. sed mihi inter Conchium et Hakean discrepantia gravis existere videtur inprimis calyce, si verum existit genus, "s

* j Habitus Brabeii ; gaudet vcro capsula lignea, apice hinc dehiscente, sporis binis alatis.

***) ILrs, ench, p. ri7, ,Tetrapetala,“ 
bricata**). - (Folia alterna; flores spica aut fere corymbus, terminalis aut axillaris; pedunculi binati.) -

352. PERSOONIA, (PERSOONIE) вMITH. LINKIA cAv. (proteacea?). Calyx nullus; corollae petala quatnor, primo conica, laciniis revolutis, basin versus staminiferis; glandulae quatuor ad basin ovarii; stamina quatuor; flamenta nulla; antherae rectae, medio laciniarum corollae insertas; ovarium stylo recurvato, filiformi superatum; stigma obtusum; drupa ovalis monospora ${ }^{* *}$ ).

353. GUEVINA, (GUEVINIE) IIOLINA. ***). PEPs.' QUA D R I A PAV. et RUIz. (proteacea). Cat lyx nullus; corolla tetrapetala, petalis apice concavis, tribus revolutis, quarto erecto; drupa monospora.

354. POTHOS, (POTIIOS) AUBI. IINN. PLUM. JACQ. Juss. LArr. tab. 738. (aroïdea 1.). Spatha globosa; spadix simplex, brevis, spicatus, floribus obtectus; calyx nuilus; petala corollae quatuor; stamina quatuor; ovarium truncatum; stylus nullus; bacca monospora Juss. (dispora LrN.)

\section{IRA-}

*) Gchus, et a Forstero et LINNEO aliisque editum, sancitum a smriro, ,in botany of neiv Holland." In corollis anomalias praebet: Sic $E$. dentatum et obliquum sub ovario et corollae petalis offert glandulas tres; $E$. pinnatum et monosporum glandulas quatuor; E. lanceolatum glandulam unam, corollae petalis usque ad medium conmatis; E. emarginatum glandulan nullam halet, corollae petalis connatis vel monopetala corolla; folliculus in E. emarg. dentato, obliquo, et lanceolato est polysporus; in E. pinnato follic. disporus; E. monosporo spora est zula.

* Caeterum jam ventendt auspicatus est pefisooviant suan cum LINIrA constituere debere zuum genus, etsi in pensoonra filamenta sint lincaria, compressa, longitudinaliter dehiscant. A Lorantho discrepat numero partium, et calyce nullo.

***) Hist. Chil. Comp. p. 198. anni 1788 . cdito; cditio Lulognae 1782 . inscripta ,Saggio della Storia etc, “ 
355. IR A MERIA, (KRAME'RE) LOEFL. LINN. CAV. PAV. et PUIz. (ex ordine plantarum sedis incertae). - Calyx tetraphyllus (nullus PERs.); corolla tetrapetala, fere aequalis, vel difformis, (nectarolymata duo diformia superum trifidum, lineare, mombranaceum unguicilatum; inferum diphyllum, foliolis sessilibus, brevioribus; stamina quatuor sub necta. rio superiori inserta; antherae parvae biperforatae ad apicem, foraminulis, minimis; bacca (drupa PEPs.) Jappacea, sicca, globosa, tecta, echinata pilis rigidis, unilocularis, non dehiscens, monospora; spora dura, glabra.

356. RIVINA, (RIVINE) LINN. MILL. IAM. tab. 8. SOLANOIDES TOUP.N. (atriplicea i.). Culyx persistens, quatripartitus; corolla nulla *). bacca monospora; sporae lentiformes, scabrae. (Stamina quatuor, octo, duodecim); trunci elongatio suffrutescens; flores spica axillaris; folia colorata.

357. CHLORANTHUS, (CHLORANTIE) SWARz. L'Heret. JUSS. NIGRINA thUNB. LAM. tab. 71. (planta sedis incertae). Calyx semi adhaerens (h. e. ovarium cum calyce corpus constituens) extrorsum uno dente munitus, parum exsertus, ad basin bractea munitus; corollae unum parvum petalnm, forma squamae concavae, trilobum, affixum lateri medio ovarii paulisper sub calycis dente; antherae sessiles insident interne medio lobo petali bivaives; ovirium semiadhaerens; stylus nullus; stigma capitatum, fero bilobum; bacca minima, ovalis, exsucca, supra insignita vestigiis calycis et petali, adhaerens, monospcra. - (Stipulis hoc genus analugium habere videtur cum rubiaceis, )

359. SAL VADORA, (SALVADORE) IINN. poxb. JUSs. LAM. tab. 81. (atriplicea 1.). Calyx quatrifidus laciniis extrorsum revolutis; corvila nulla (qua-

tri-

*) Ap. Pers. ench. P. 549. a. res vera perverse eutumcratur. 
trifida LAM. PEKS.); bacca monospora; sporae arillatae. - (Frutex; folia opposita; flores in racemos terminales.)

359. EMPLEURUM, (EMPLEURE) SOLANDR. AIT, LANI, JUSs. DIOSIMA IIN. suppl. (rutacea 3.). - Calyx turbinatus, quatrilobus, inferus, punctis glandulosis insignitus; corolla nulla; stamina quatuor exserta; antherae magnae, apico glandulosae, utrinque dehiscentes, filamentis adnatae; summitas ovarii uno latere ala terminata, aut stylo laterali inserta; capsula oblonga, compressa, forma leguminis, apice alata, unilocularis, apice dehiscens, monospora; spora arillo coriaceo cincta.

360. CA M PHOROS A A, (CAMPHRE'E) BUXB: IINN. suppl. LAM, tab. 86. CAMPHORATA TOURN. (atriplicea 2.). Calyx urceolatus, quatridentatns, den. tibus duobus alternis majoribus, ternisquo minimis; corolla nulla ; stamina exserta; stylus bifidus : capsula unilocularis, monospora, calyce tecta. - (Trunci elongatio ramosa; flores axillares,)

36r. P T ERAN THUS, (PTERANTHE) FonsR. DESF. VENT. IAM. tab. 764. LOUICHEA L'IIERET. CAMPHOROSMA IIN . (urticata 2.). - Calyx quatripartitus laciniis oblongis concavis, mucrone recurvo terminatis, inaequalibus; duabus oppositis majoribus utroque margine versus summum ala membranacea munitis, cristatis; corolla nulla; filamenta basi connata, subulata; stigma bifidum; capsula membranacea, monospora, calyce tecta.

362. A L C H E I L L A, (ALCHEMILLE) TOURN. I.INN. JUSS. LAM. tab. 86. APHANES LINN. JUSS. IAM. tab. 87. (rosacea). Calyx tubulasus, limbo aperto, octofido, laciniis alternatim positis minimis; corolla nulla; stamina quatuor minima; stylus ad basin ovarii; spora supera, una, calyce vestita. (Trunci elongatio herbacea; folia palmata, aut fere digitata; inflorescentia corymbus axillaris aut terminalis.)

363. D R O. 
363. D ORSTENIA, (DORSTENE, Contrayerva PLUM. LINN, juss. LAMr. tab. 83. (urticata) *). Rieceptaculum commune concavum squamosum, tectum floribus sessilibus, interdum monoicis; calyx quatrangulus; corolla nulla; sporae solitariae, pulpae recoptaculi immersae. - (Receptaculum commune carnosum, nunc angulosum, nunc rotundatum, antrorsum elevatum vel ventricosum, deorsum planum, circum. circa septum squamis minimis, acutiusculis; interna receptaculi superficies repleta flosculis, quorum discoidei sunt feminei, radii mascula organa habent. Flores masculi in proprio calyce tetraphyllo, receptaculo quasi insculpto; corolla his propria, filamenta brevissima, antherifera; flores feminei codem modo constituti; pistillis duo, solitaria in ovario; stigmata rotundata: haes PAIVA. Cf. WENDLAND apad nOEMERUM Arch. I, p.5r. tab. PAIVA nec non sprengelius e classe quarta in vigesimam tertiam hoc genus referri jubent.) (Trunci elongatio herbacea: folia radicalia petiolata; involucra solitaria ad trunci partem foliis denudatam.

364. COMETES, (COMETE) BURrM. LINN. JUss. IAM. tab. 76 . (ex ordine plantt. indeterminato). Involucrum tetraphyllum, foliolis aequalibus ciliato villosis, trifloram; calyx tetraphyllus foliolis aequalibus; corolla nulla; stigmata tria; capsula tricocca, trispora.

365. CA N S IERA, ( CANSIEPE) roxB, - Calyx globoso urceolatus; quatridentatus, inferus; nectarai $y$ mata dentata, ovarium cingentia; bacca monospora, JUSBIEU.

\section{$X I$, Flore incompleto, supero.}

366. GO NOCARPUS, (GONOCARPE) THUNB, JUSS. LAM. tab.73. GONATOCARPUS SCHREB. WILLD,

(ex

*) Vid. Mannel Jong, Henri de Paiva, med, pract, Lisboar 1790. "Memorias de historia natural。“ 
(ex ord. plantt. sedis incertae). Caly $x$ superus, libeJus, quatrifidus, persistens; corolla nulla; stamina quatuor calyci inserta; ovarium adhaerens; drupa calyce coronata, minima, globosa, octogona, monospora.

367. FUSANUS, (FUSANE) LAN. tab. 77. COLPOON BERG. THESIUM LINN. suppl. (eleagni). Calyx superus, turbinatus, quatrifidus; corolla nulla; stylus subnullus; stigmata quatuor, brevia, opposita calycis laciniis; antherae didymao; uovarium inferum, apice glandulosum; stylus nullus aut brevissimus; stigmata quatuor; drupa? turbinata, monospora.

368. IS N R R.D I , (ISNARDE) IINN. Fотн. RUIz. IAMT. tab. 7\%. LUDWIGIA auctorum *). (onagrea 2.). Calyx tubulosus, campanulatus, adhaerens, quatrifidus; corolla nulla; stamina ad summum calycis, circa stylum simplicem stigmate terminatum; capsula tetragona, quatrilocularis, laciniis calycinis cincta. - (Planta herbacea, fere semper marcescens.)

369. E L A E A G N US, (FLEAGNE, chalef) TOURN. IINT. PALI. THUXB. JUSS. IAM. tab. 73. (elaeagni I.). - Calyx superus, campanulatus, externe rugosus, interne coloratus, quatrifidus; corolla nulla; stamina qquatuor alternantia cum laciniis calycinis; antherae fere sessiles; drupa nuce monospora; radicula infera. - (Frutices floribus axillaribus, quibusdam tantum masculis).

370. GYROCARPUS, (GYROCARPE) JACQ: roxв. - Flores polygami. Calyx quatripartitus, inaequalis ; corolla nulla ; nectarolymata quatuor; stigma sessile; capsula unilocularis, monospora, quinquealata. (An genus juxta Skimmiam.)

372. DRA.

*) Cf, PERs, enchir. P, 146 . 
37I. DRAPETES, (DRAPETES) LAM. tab. Io. hist. nat. diar. - Calyx nullus ; corolla infundibuliformis, limbo quatrifido; receptacula pedicellata, barbata; spora una, tecta. (Flores aggregato fasciculati; habitu genus Passerinae, et generi Dais affine.)

\section{$D I G Y N I A$.}

372. B U,F F O I A, (BUFFONE) PLUK. LIN . JUss. I.AM. tab. 8\%. (caryophyllea 2.). Calyx tetraphyllus; corollae petala quatuor, calyci breviora; capsula ovalis, unilocularis, bivalvis, dispora.

373. HYPECOUM, (HYPECOON) AMMAN. IINN. JACQ. LAII. tab. 8\%. MNEMOSILLA FORST. (papaveracea 2.). Calyx minimus, diphyllus, corollae petala quatuor, triloba, duobus exterioribus majoribus ot latioribus, interioribus minoribus conniventibus; siliqua leviter articulata, articulis monosporis, longa. (Folia multifida, alata, aut pinnatifida; flores terminales, umbella corymbiformis; flavi.)

374. PAGAMEA, (PAGAMEE) AUBL. JUSs. IAM. tab. 83. (rubiacea Ir.). - Calyx quatridentatus, turbinatus, persistens; corolla limbo quatrifida, urceolata, tubo brevi, intus villosa; antherae quatuor sessiles non exsertae; ovcrium semiadhaerens; stylius basi bifidus; drupa (bacca?) supera bilocularis, calyce tecta, loculo quoque nuculis duabus, disporis, altera abortiente.

375. HA MA MELIS. (IIAMAMELIE) AUBIET. I INN. DUHAMET. JUSS. LAM. tab. 88. (berberidea i.). - Involucrum triphyllum; culyx proprius quatrifidus, (tetraphyllus PERs.), munitus externe squamis duabus; corollae petala quatuor longa, cum calycis laciniis alternantia, ad unguem introrsum squama munita; stamina cum petalis alternantia; antherae biloculares, rotundatae in filamento brevissimo; munito utroque latere valvula operculiformi; linearia, capsula (nux T'EP.s.) 
bicornis, coriacea, bilocularis, ad medium usque basi calycis persistentis cincta, duabus valvulis apice bifi. dis, dispora; sporae oblongae, apice umbilico notatae, tectae arillo coriaceo clastice dehiscente in duas valvulas; perisporium crassum; embryo rectus; cotyledones planae; radicula umbilico opposita. - (Frutex foliis alternis, stipulatis; floribus sessilibus, saepo diclinis.)

376. DICORYPHA, (DICORYPIIA) AUBसA DE PEtit thouArs. plantt. aff. austr. tab. \%. (rhamnea). *). - Flores hermaphroditi, completi, polypetali, isostemones epigyni, tetrandri, fasciculati, terminales. Calyx tubulosus, quatridentatus, basi ovario adhaerens, persistens; corolla tetrapetala, petalis alternantibus cum calycinis laciniis; staminum filamenta fertilia qua. tuor, quatuor sterilia fertilibus alterna, omnia cum potalis alternantia; stylus bifidus; ovaria duo in unum coalita, basi calycis inmersa; fructus dicoccus, coccis calycis basi persistente capsularis cinctis, apico disjunctis bicornibus, maturis elastice dehiscentibus; sporae duao inversae; perisporium corneum; embryo foliaceus marginibus convolutis; radicula cylindrica; cotyledonibus foliaceis.

377. CUSCUTA, (CUSCUTE) TOURN. IIN⿴囗. JUss. LAm. tab. 88. (convolvulacea 3.) Calyx turbinatus, quatri-quinquepartitus; corolla limbo quatriquinquefida, globosa, attenuata, interne ad basin limbi munita squamis quinque bifidis; stamina inserta co. rollae orificio et cum ejus laciniis alternantia, squa-

$\mathrm{mis}$

*) Magna est hujus generis affinitas cum to Hamamelis; sed distinctum calyce profunde diviso in quatuor lacinias; ovario levitcr fundo calycis adhacrente, antheris denique, quibus duo sunt loculi in ipsa filamentorum substautia sulcati, singulis clausis valvula extrorsum dehiscente, ut in familia berbcridum et lanrinorum, a quibus familiis naturalibus tamen scilicet berberid is et $\mathrm{H}_{\mathbf{2}}$ mamelis staminibus perigynis, sporarum structura dis. crepat, et, natura ipsa jubente, ad xhamncas pertinct. 
mis opposita; capsula bilocularis, sphaeroïdea aut conica, transverse circumscissa basi dehiscens, basi calyce tecta; sporae in loculo binae, rotundatae; (stamina passim quatuor; sáepe quinque ${ }^{*}$ ); Embryo spiralis, G.; nectarolyma squamae in corollao basi quibusdam. (Plantae parasiticae.)

378. G O M OZ I A, мUт. IINN. suppl. JUss. IAM. tab. 87. NERTERIA SMITH. ẂILLD. PEPS. (rubiacea 3.). Caly $x$ integer, planus; non prominens suss. (nullus IIN N. ; curolla supera, infundibuliformis, limbo campanulato, quatrifido: tubo brevi, filiformi; bacca bilocularis, polyspora Juss. (dispora GAERTN, PERs. ${ }^{* *}$ ).

379. G A L O P I N A, (GALOPINA) THUNB. JUss. ANTHOSPERMUM thunb. (rubiacoa 2.). Calyx integer, planus (non prosiliendo exsertus) JUss. (nullus тHUNв.); corolla supera, quatrifida, laciniis reflexis; antherue oblongae, rectae; fructus minimus, se dividens in sporas duas, umbratas, muricatas, parum globosas.

380. APHANES, (APHANE) IINN. ruIz. ALCHEMILLA willd. PiUiz. et PAT. (rosacea). Calyx nctofidus: laciniis alternis, minimis; corolla nulla; sporae duae, calycevestitae; una interdum abortiva. (Staminu unum, duo, quatuor.) ${ }^{* *}$ ).

38I. CRUZI T A, (CRUZTTE) LINN. IAM. tab. 548. (atriplicea). Calyx quatripartitus (tctraphyllus pris.) basi bracteis tribus vestitus (exterior triphyllus PERs.); corolla nulla; spora una, calyce tecta.

*) Hinc a pers. ench, p. 289. ad pertandriam digyniam xelatum est genus CUSCU'TAE.

**) Genus hoc nimis affine Psychotriae, etsi Gomozia numero quaternario in flore gaudet, quinario in Psychotria, nec non sporae Gomoziae laeves sint, sulcatae in Psychotria.

***) Stamina alii observarunt quatuor, quorum tria abortiunt; alii ıumm. 


\section{$\begin{array}{llllllll}T & R & I & G & Y & N & I & A\end{array}$}

382. BOSCIA, (BOSCIE) THUNB. - Caly.x quatridentatus; corollae petala quatuor; capsula quatrilocularis. (Diversum genus a POSC1A LAIr, illustr, tab. 395.)

\section{$T E T R A G Y N I A$.}

383. I LEX, (HOUX) IINN. THUNB. JAcQ. AIT. IAM. tab. 89. AQUIFOLIUM TOURN. (rhamnea 2.). Caly $x$ minimus, quatridentatus; corolla rotata. quatripartita, aut petala quatuor ungue dilatato coalita; stamina quatuor; styli nulli; stigmata quatuor; bacca parva rotundata, continens quatuor nuces monosporas. (Fru. tices, aut arbores mediae altitudinis; folia alterna, interdum persistentia; pedunculi axillares, multiflori.)

384. C OLDEN I A, (COLDENE) PLUK. LINN. JUSs. LANr. tab. 89. (borraginea 3.). Calyx quatripartitus (tetraphyllus pens.); corolla infundibuliformis, lim. bo patente; (quatriloba secundum ADANs.); ovarium quatrilobum; fructus: capsulao quatuor, mucronatae, tactu asperae, approximatae, monosporae. - (Plantae trunci elongatione pendula, flores axillares, fere sessiles.)

385. SAGINA, (SAGINE) IINN. EMITH. CURT. IAM. tab. 90. ALSINE tourn. (cariophyliea 1.). Ca* lyx tetraphyllus; corollae petala quaruor, rarius nulla; capsula quatrilocularis, quatrivalvis, polyspora. (Plantae exiguae; flores fere solitarii, terminales, et axillares, sustenti longis pedicellis.)

380. MOENCHIA, (MOENCHIE) EHRH. SAGI. NA T.INN. ALSINELI,A MoEncir. (caryophyllea). Calyx tetraphyllus, connivens; laciniis lanceolatis, acutis; corolla subbrovior, petalis integris; capsula evalvis 
unilocularis, apice quinquefariam dehiscens; sporae renitormes, scabrae.

387. R A D I L A , (RADIOLE) SMITH. ROTH. GMEL. LINUM LINN. MILLEGPANA RAJJ. CHAMAELINUM vaILI. (caryophyllea 2.). Calyx quatripartitus, laciniis trifidus (multifidus pers.); corollae petala quatuor; capsula globosa, supera, octovalvis, octolocularis, octospora.

388. TIL L AEA, (TILLEE) MICH. IINN. wILLn. Juss. LAm. tab. 90. CPAASSULA тнUnв. (crassulea; sempervira juss. seda ADAns.). - Calyx tri-, quatri-quinquepartitus; corollae petala tria, vel quatuor aequalia; stamina tria, quatuor, totidemque ovaria, et vel capsulae tres, quatuor, uniloculares, polysporae. (Plantae exiguae foliis oppositis; flores axillares, minimi.)

389. BUILLARDA, (BUILLARDE) DECAND. plantes grasses; L'HERET. Calyx quinquepartitus; $c 0$ rallar petala quinque, aequalia: ovaria ${ }^{*}$ ) quinque, basi squamis quatuor munita; capsulae quinque, monoloculares, polysporae ${ }^{* *}$ ).

390. MY Y ( I N D A , (MYGINDE) SWARz, JAC2. juss. HiH $\beta_{\wedge}$ COOMA LINN. (xhamnea 2.) - Calyx minimus, quatripartitus, corollae petala quatuor, aperta; stamina quatuor, filamentis brevibus, antheris rotundatis; styli quatuor breves; stigmata duo - quatuor; drupa globosa, pisiformis; $n u x$ monospora.

391. R I Q UER I A, (RIQUERIE) PERs, PAv. RUIZ. RIQUEUR.TA (planta sedis incertae) Caly $x$ triplex: duo interiores ex una parte constantes, bipartitao; interior diphyllus ;

cos

*) In Tillaea triä, quatuor.

**) Persoonius ipse enchir. pag. 153. concedit, ob calycem, et syuamas nectarolymatiformes integras, lineares fon hoc genus a Tillaea separandum esse. 
corolla tetrapetala, petalis rotundate concavis; staminos. quatuor sub ovario inserta; filamentis compressis; ovavium liberum; styli quatuor perbreves; totidem stigniau ta; capsula tetragona, stylis coronata, quatriloculario, quatrivalvis, polyspora.

392. POTA MOGETON, (POTAMOGETON, EPI - d'eau) TOURN. IINN. ROTH. SCHUMACHER JUSS。 IAx. tab. \$9. (Fluvialis; Najades fuss.). Calyx culom ratus, caducus, tetraphyllus foliolis obtusis; corolla nulla *); stylus nullus; ovaria quatuor stigmata quatuor: sporae quatuor, nudae; approximatae. - (Folia arso plexantia, elongationis caulina alterna, fioralia saepe opposita; flores in spica terminali aut axillari rami et spicae saepe ad basin duabus spathis muntae.)

393. RUPPI A, (RUPPIE) Michel. LiNN. JUSS. LAM. tab. 90. CORALliNa tourn. (fluvialis). Cím lyx caducus bivalvis (nullus PERs.); corolla nulla: ovavia quatuor subsessilia; styli nulli; stigmatu quatuor; nuces quatuor, ovales, acuminatae, monosporae; sporue pedicellatae pedunculo filitormi, **)

*) Áp. TERs, enchir. p. 152. vice versa calyx dicitur nullus, corollae petala quatuor.

* * Planta monoica videtur. - Peduncnli subspirales longissimi vel bréves, Valisneriae in modum, coutra:" lnuntur aut laxantur vasorum spiralium beneficio: vid. SMIII. Jrit, I. 199. PERS. 


\section{CLASSIS QUINTA.}

\section{PENTANDRIA.}

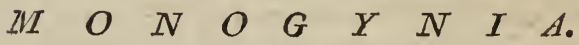

I. Flore monopetalo, infero; spora una.

394. NYCT A G O, (NYCTAGE, BELLE-de nuit) JUSs. JALAPA toURn. NYCTAGE royen. MIRABI. LIS IINN. BATSCH. IAM. tab. 105. ADMIRABILIS cLUS. (nyctaginea I.). Calyx involucrum inferum, quinquefidum, uniflorum; corolla (calyx petaliformis) infundibuliformis basi ventricosa, attenuata ad medium, vel inferne ad ovarium coarctata, supera, ad summum dilatata, Iimbo patente, fere integro vel obsoleto quinquefido; ovarium cinctum nectarolymate membranaceo, glanduloso, globoso, formato coalitione baseos quinque filamentorum, hinc staminifero; stigma globosum; spora (nux) solitaria, globosa, striata, infra corollam, tecta basi calycis incrassati et quasi capsulari. - (Truncus superficiali-subterraneus tuberosus; elongatio herbacea, cylindrica; folia opposita; flores terminales, fere corymbosi.) 
395. FABIA NA, (FABIANE) ruIz. pav. Corolla infundibuliformis, limbo plicato revoluto; tubus longissimus, inferne attenuatus: stamina inaequalia; stigma emarginatum; capsula bilocularis, bivalvis.

396. NIEREMBERGI A, (NIERENRERGIE) rulz. PAV. (incertae sedis). - Calyx quinquefidus; corolla subhypocrateriformis, tubo calyco dupio longiori, fauce coarctato flicata, limbo quinquelobo, lobis patentibus; stigma bilobum; stamina inaequalia, inserta fauci corollae, superrie versus stylum adpressa quatuor didynama, stamine quinto broviori, posito inter duo majora; ovarum liberum, rapsula ovoidea, bilocularis, bivalvis, calyce comitante; sporue numero plurimae.

397. XUARESIA, ( XUARESIE ) rulz. pav. CAPRARIA FEWILI. - Corolla rotata; stigma compressum; capsula bilocularis, bivalvis, valvulis bifidis *).

398. ABRONIA, (ABRONE) JUss. IAM. tab In5. TRICPATUS" L' heret. SCHReB. Willd. (nyctagynea 1.). Involucrum compositum quinque aut sex foliis minoribus squamaeformibus, duodecim aut quindecim flores includens; calyx (nullus, corolla) infera, hypocrateriformis, vel tubulosa, involucro longior, ad medium supra ovarium coarctata, ad summum in lacinias lobosve quinque emarginatos cordatos divisa; ovarium cinctum staminibus vaginantibus basi coalitis; spora una, gauinquangularis, tecta calycis (coro:lae) basi indurata, cujus superior pars caduca est. (Pedunculi solitarii, axillares, terminati floribus ag. gregatis.)

399. PLUM BAG O, (Plombagine, Dentelaire) TOURN. SLOAN, LYNN. JUSS. LAM. tab. IOS. (plumbaginea). Culyx oblongus, tubulosus, quinque. den-

*) Ccrec Caprariae biflorae habitu simillima: schnsd. 
dentatus, glanduloso scaber et viscosus; corclla monopetala, infundibuliformis, quinquefida laciniis colora. tis; stamina quinque hypogyna; filomenta dilatata, basi reunita, ovarium cingunt, squamis inserta; stigma quinquefidum; capsula monospora; spora oblonga, tunicata. - (Trunci elongatio herbacea, aut frutescens; folia semiamplexicaulia; flores spica terminalis.) ${ }^{*}$ ).

400. WEIGELA, (WEIGELE) KAEMPF. тHUNB. WILLD. JUSS. LAM. tab. 105. (ex ordine plantarum sedis indeterminatao). - Calyx pentaphyllus (quinquepartitus) laciniis (foliis) subulatis; corolla infundibuliformis; tubo interne villoso; limbo campanulato, subquatrilobo; stamina quinque tubo inserta; antherae basi bifidae; ovarium liberum, quatrangulum; stylus e basi ovarii lateralis; stigma peltatum; spora una.

40I. QUINCHAM ALIUM, (QUINCHAMALI) MOLIN. PAV, ot RUIZ. JUSS. LAM. tab. 142. QUINCHAMALA Willd. (eleagni adans. Juss.). - Culyx parvus, inferus, inaequalis, leviter quinquedentatus, et coriaceus, globosus; corolla supera, tubulosa, tubo longo quinquefido, (quinquepartito); stamina basi laciniarum corollae inserta: antherce fere sessiles; stigma truncatum; spora una, infera, minutulo calyce persi. stente tecta.

402. C ORY M B I U M, ( CORYMBIOLE) LINN. JUSS. IAM. tab. 723. ( cinarocephalea 3. Juss, compositae ADANs.) - Calyx diphyllns, longus, glumaceus, connivens, cylindricus, basi squamis quatuor, quinque vestitus; corolla infundibuliformis, quinquefida; antherae approximatae, unitae; stigma bifidum; spora una, oblonga, maxime villosa, coronata calyculo; receptaculum nudum.

I1. Flore

*) Corolla plumbaginearum, auctore $\operatorname{venteñTt,~considerari~}$ debet tanquam verus caly $x$ : est marcescens, saepe persistens, neque refert unquam tracheas aerophagas vel vasa pneumatochymifcra, quae conspicuntur in veris corollis. 


\section{Flore monopetalo, infero; fructu disporo.}

463. CERINTHE, (MÉLINET) TOURN. LINN. потн. Juss, LAM tab. 93. (borraginea 2.) - Calyx quinquepartitus; corolla tubuloso ventricosa, limbo quatridentato, aut 5. fido; fauce nuda, pervia; anthevue rectae, vix exsertae; capsuiae (nuces) duae osseae biloculares, disporae. - (Folia glabra, glandulosa; flores solitarii, axillares, aut terminales.)

604. M E S S E R S C H M I D I A, (MESSERSCHMIDE, ARGUSE) IıNN. suppl. Juss. LAar. tab. 95. ARGUZIA Amm. ruth. (borraginea 2.). - Culyx quinquepartitus; corolla infundibuliformis, aut hypocrateriformis, tubo globoso, fauce nuda, limbo quinquefido, plicato aut plano; stamina in tubo; antherae rectae, subulatıe ; stigma capitatum ; capsula (bacca? ) muricata, suberosa, cylindrica, bipartibilis: singula parte hemisphaerica dispora: vel haemisphaeria quaeque continet nucem trilocularem, loculis lateralibus monosporis, ad medium sterilibus; spora ossea. (Herbae aut frutices; pedunculi axillares, et terminales, multiflori.)

III. Floremonopetalo, infero; sporae quatuor nudae.

405. ECHIU M, (VIPÉRINE) TOURN. LINN. IAN. tab. 94. (borraginea 3.). Culyx quinquepartitus; corolla tubulosa, tubo brevi, limbo lato campanulata, utplurimum labis quinque inaequalibus oblique fissa irregularis: fauce nuda, ampla; stigma bifidum; spovae quatuor. - (Inflorescentia spica simplex, aut panicula unilateralis; trunci elongatio interdum suffrutescens, )

406. ECHIOCHILON, (ECHIOLON) DESFONT. (borraginea 3.) - Calyx persistens quatripartitus, la. 
ciniis subulatis; corolla tubulosa: tubo gracili, arcua. to: limbo patente bilabiato: labio snperiori bilobo, inferiori trilobo, lobis rotundatis; hinc quinquedentata; superne ventricosa; staminum quinque filamenta brevissima, ad summum tubi, non exserta; stylus unus; stigmata duo; ovaria quatuor, supera; sporae totidem nndae. (Flores solitarii axillares; nomen a $\varepsilon \gamma$ เov et $\chi^{\varepsilon i \lambda o \%}$ Echium labiatum,) cf, schnad, Diar. 1799. pag 354.)

\section{HELIOTROPIU M, ( HÉLIOTROPE)}

TOURN. IINN. JUSs. IAM. tab.9r. (borraginea 2.). Calyx tnbulosus, quinquedentatus; corolla hypocrateriformis, quinquefida, interpositis aut dentibus aut plicis : fauce nuda; stigma emarginatum; nux interdum cortice tecta. (Inflorescentia spica recurvato involuta, unilateralis; quinque dentes minutulae inter limbi corollae lacinias positâe in Hel. supino et europaeo.)

408. PUL M N N I A , (PULMONAIRE) toun. IINN, JUSs. IAM. tab. 93 (borraginea 2.). Calyx prismatico - pentagonus, quinquefidus; corolla infundibuliformis, limbo semi vel quinquelobo, lobis patentibus, tubo cylindrico, fauce nuda, pervia; stigma emarginatum; sporae quatuor. - (Iuflorescentia corymbus terminalis; folia fere glabra in quibusdam speciebus.)

* 409. MERTENSIA, (MERTENSIE) Rотн, (borraginea). Calyces abbreviati, tubo corollae dimidio breviores.

410. TIQUILIA, (TIQUILIE) ruIz. Flor, Tperuv. LITHOSPERMUM LINN. - Culyx quinquepartitus; corolla infundibuliformis; laciniis emarginatis, fauce nuda; stamina exserta; stylus flliformis, longior; stigmata duo simplicia; sporae quatuor, unum saepius abortivum.

4I. L I THOSPERMUM, ( LITHOSPERME, Gremil) LINN. IAM. tab. 91. OSKAMPIA MoEx̣ç. *). AN. 
*). ANCIfUSA barret. BUglossum tourn, gaRID. ( borraginea 3.). Calyx quinquepartitns; corolla infundibuliformis, parva, quinqueloba: fauce pervia, nuda; stigma bifidum; sporae nuces qnatuor, interdum nitidae, osseae; genituliu utraque inclusa. - (Flores solitarii, axillares aut spica terminalis bracteis associata; folia floralia saepo geminata.)

412. BATSCHIA, (BATSCHE) VAHL. MrCH. (asperifolia). - Calyx subyuinqueficus; corolla hypocrateriformis, majuscula, tubo recto, calyce longiori; intus basi annulo barbato; tauce nuda; laciniis rotundatis: sporae nudae, nitidae.

4I3. ONOSMA, (ONOSNE, orcanette) LINN. PAIL, juss, xam. tab. 93. SYMTHYTUM toURNEF. (borraginea 3.). - Calyx quinquepartitus; corolla campanulata, infundibulitormis, limbo tubuloso-ventricoso, quinquedentato: fauce nuda, pervia; sporuequatuor. (Flores axillares et terminales.)

4I4. SYMPHYTUM, (CONSOUDE) TOUr. IINN. JUSs. IAM. tab. 93. (borraginea 4.). Calyx quinquepartitus; corolla infundibuliformis, campanulata, tubuloso - ventricosa, limbo recto, param attenuato, quinquedentato: fauce clausa squamis (radiis) oblongis subulatis, glanduloso fistulosis, conniventibus, conicis; stigma simplex. - (Inflorescentia fere disposita in corymbum terminalem et axillarens; folia floralia geminata, in quibusdam speciebus caulina decurrentia.)

415. O NOSMO DIUIM, (ONOSMODIE) MICH. LITHOSPERMUM LINN. (borraginea). - Calux profunde quinquepartitus; corolla oblonga, subcampanulata; fauce nuda; limbo ventricoso, semiquinquefido; laciniis subconniventibus, acutis; antherae sessiles, inclusae; stylus exsertus.

4I6. BOR.

*) Quae tamcr habitu a lithospcrmis recedit calyce subdeca. goulo, corolla flava, fauce ad marginem villosa, stig. mate capitato; sporis subpubescentibus. Oskampia trie chutoma Moensh. Micth, 1'. 420 . 
416. BORPAG O, (BOURRACHE) TOURN. PLUK. IINN. BUXB, JUss. LARx. tab. 94. (borraginea 4.) Calyx quinquepartitus; corolla rotata, saepe aperta, quinquefida: fauce squamis emarginatis, obtusis, radiatim clausa; sporae quatuor rigidiusculo - asperae, calyce connivente tectae. (Pedunculi axillares aut terminales, uni-multiflori.)

417. L Y C O P S I S, (LYCOPSIDE, Grippe) LINN. JUsS. I.AM. tab. 92. ANCHUSA LAM. ASPERUGO LIN N. WILLID. (borraginea 4.) Ealyx quinquefidus, inflatus aut ventricosus; corolla infundibuliformis: tubus incurvatus, calyce longior; limbo brevi, bi - vel subquinquelobo; fauce clausa squamis convexis, conniventibus; stigma bifidum; sporae quatuor. - (Flores oolitarii aut fere spicati, axillares aut terminales.)

418. ECHIOIDES, (ECHIOIDE) TOURN. DESFONT. LYCOPSIS IINN. NONEA MOENCH. (borraginea 4.) Calyx inflatus, angulosus, quinquefidus, persistens: corolla infundibuliformis, limbo quinquelobo; tubo erectiusculo: fauce squamis clausa; !stamina non exserta; sporae quatuor liberae *).

\section{ANCHUSA, (BUGLOSSE) pLUK. moris.} LINN. JUSs. LAM. tab. 92. (borraginea 4.) - Calyx quinquefidus; corolla infundibuliformis, quinqueloba, lobis rectis, patentibus : fauce squamis exsertis, fornicatis, convexo - conniventibus clausa; stigma emarginatum; sporue quatuor, basi insculptae, superficie utplurimum venosae gibbae. - (Flores axillares, aut terminales, in glomerulum approximati, aut dispositi in spicam simplicem, unilateralem, aut paniculatam **). 420. A S.

*) Natura enim ipsa jubente cl, DEsfontrarnes Lycopsin vesicariam ad hoc novum genus amandavit, (quo et $I$. aesyptiaca, aliae forte, pertinent,) quod jam MoENchrus Method. pag. 422, monuisse nobis videtur.

* *) BUGLOSSUM, RETz. squamulis faucis corollae pe. nicilliformibus. - (Quo et Cynoglossum fulvum, Iuv DOLPII ap. SCHRAD, referri potest.) - 
420. A SPERGO, (RAPETTE) TOURN. LINR. Juss. LAMr. tab. 94. (borraginea 4.). Calycis laciniae quinque, inaequales denticulatae, plano parallelae, sinuatae; corolla infundibuliformis, tubo brevi semi quinqueloba: faux clausa squamis conniventibus, convexis; sporae quatuor in fructum calyce duplicato et compresso formatae. - (Flores axillares; folia floralia geminata.)

\section{I. CYNOGLOSSUM, (GYNOGLOSSE)}

TOURN. LINN. JUSS. LAM. tab. 92. (borraginea 4.) Calyx quinquepartitus; corolla infundibuliformis, quinqueloba, lobis brevibus; fauce squamis quinque convexo - fornicatis conniventibus clausa; stigma emarginatum ; sporae quatuor, depressae, latere interiori stylo persistenti adfixae. - (Flores axillares aut terminales, dispositi in spicam, aut in glomerulum approximati; folia tomentosa aut glabra.)

422. MYOSOTIS, (MYOSOTE, SCORPIONE) IINN. JUss. IAM. tab. 92. (borraginea 4.) - Calyx quinquefidus; corolla hypocrateriformis, tubo brevi; limbo plano, quinquefido, lobis fere emarginatis: fauce clausa squamis connivendo fornicatis convexis; stigma simplex; sporae quatuor laeves, - aut *) echinatae. - (Flores saepe in spicam terminalem dispositi, unilaterales.)

IV. Flore monopetalo, infero; sporis pericarpio inclusis.

A00 IVOLA NA. (NOT,ANE) I.INN. JUSS. I,AM. tab. 97. Auctcres florae Peruviana **). (aftinis familiao

*) Ut in Lappulis.

**) Qui N'olanas dicere solent plantas succosas, inferne ramosissimas, altera unica specie excepta, prostratas, habiru solanaceas, fructu vero diversas, cujus mucmlae epidermide succulenta obtectae, yuadriloculares sumt, loculis uno-trilms sacpe abortientibus. 
liae borraginearum.). Calyx quinquangularis, quinquefidus, limbo patente, laciniis latis, inferne basi turbinatus; corolla campanulata, plicata, patens, obsolete quínqueloba; filumenta subulata, erecta, aequalia, corolla multo breviora; antherae erectae; ovaria quinque; stylus inter ovarium; stigma capitatum, subpentagonum; drupue quinque tri - quinquelosulares, in'sidentes disco in fundo calycis, receptaculo carnoso coali. tae, arillatae? loculus quisqne unisporus.

424. CORIS, (CORIS) TOURN. IINN. IAM. tab. 102. (primulacea $\Upsilon_{\text {. }}$ ). Calyx ventricosus, quinquedentatus, dentibus subspirosis, certe quinque spiniformes processus ad basin dentium, persistens; corolla monopetala, tubulosa, irregularis per laćinias quinque; cupsula supera, quinquevalvis, calyce cincta; sporaë plurimae. - (Trunci elongatio humilis, ramosissima, basi suffrutescens.)

\section{HYDROPHYL L UM, (HYDROPHYLLE)}

TOUHN. LINN. JUSs, LAM. tab. 97. (borraginea fructu capsulari.) - Calyx quinq̨uepartitus; corolla campanulata quinquefida, interne quinque striis melliferis et longitudinalibus munita, cingentibus staminum filamenta corolla multo longiora; antherae oblongae, versatiles; stigma bifidum; cupsula globosa, bifida, unilocularis, bivalvis, monospora; sporae numero quatuor, una fertilis. - (Trunci elongatio herbacea; folis palmatis aut pinnatifidis; floribus corymbosis pedunculatis et terminalibus.)

426. GALAX, (GALAX) IINN. JUss. VITICELLA MITCir. gen. 24. (ex ordir plant. sedis incertae.) Calyx decempartitus (10. phyllus Ptro.), laciniis quisy.-. internis alternantibus et longioribus, corolla monopetala, hypocrateriformis, tubo cylindrico, limbo quinquefido; stamina quinque in filumentis brevioribus; antheris ròtundatis, conniventibus, et corollae fauci insertis; ovarium liberum in stylo uno; stigmata duo; capsula unilocularis, di- vel polyspora, elastice in 5alvulas duas dissiliens. 
427. PORAQUË̈BA, (PORAQQUÉBE) AUELET. JUSS. tant, illustr. tab. 134. BARRERIA scireb. (ber.

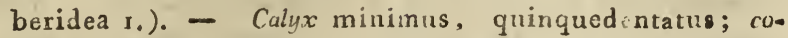
volla rotata, hypogyna, quinquepartita, laciniis quinque oblongis, concavis ad basin et ad summum, scrobiculos in superficio gerentibns, foveola superior ga:minata, inferior ternata; stamina quinque hypogyna, laciniis corollae opposita, et occulta in corollae foveolis; filmenta emarginata, dilatata; antherae longae, tetragonae, articulatae, marginatae, marginibus cohaerentibus; stigmata tria; frustus.... ?

428. CORTUSA, (CORTUSE) LINN. JACR, JUSS. LAM. tab. 99. (primulacea 2. Anagallides ADANS lysimachiao Juss.) - Culyx quinquefidus; corolla rotata, quinqueloba, fauce parumper inflata et annulo extrorsum elevata, limbi laciniis ovatss acutis; antherae ad. natae, billamellatae; cupsulu unilocularis, ovalis, apice bi - quinquevalvis.

429. A N A G A L L IS, (MOURON.) I.INN. тHUזв. JUss. LAM, tab. 101. (primulacea I). - Calyx quinquefidus; corolla rotata, quinqueloba; stamina interne velata, filamentis hirsutis; cupsula globosa, saepe circularis, unilocularis, circumserssa. -- (Trunci elongatio tetragona; fulia opposita; flores solitarii axillares. )

430. LYSIMACHIA, (IYSIMACHIE) тOURT. LiNN. JUSS, LAM. tab. 101. (primulacea 1.) - Calyx quinquefidus : corollu rotata, quinqueloba; capsula globosa, unilocularis, quinque - decemvalves, mucronata. Filamenta in quibusdam speciebus basi connata. (Folia opposita; fures axillares aut terminales, solitarii, spicati, aut corymbosi.)

43I. LUB IN I A ; (LUBINIE ) commers, VenteNAT. LYSIMACHIA LAM. WiLLD. (primulacea 1.). - Calyx quinquepartitus; corolla hypocrateriformis, fere rotata; tubo longitudine calycis; limbo plano, quinquepartito, aequali; filamenta medio tubi adnata, 
superne libera; antherae ovales, obtusae; stigma obtu. sum; capsula ovalis, unilocularis, polyspora, mucro. nata; (non sponte, neque in duas aut quatuor valvulas dehiscens, sed si digitis comprimitur bi - quatrivalvis; secundum veNTEN. quinquevalvis sec. LAM,) Folia alterna.

432. D D R A N A , (DORENE) тнUNв. japon. \$4. Juss. (planta ordine et sede incerta). Calyx quinquefidus; corolla monopetala, quinquefida; stamina quinque tubo corollae inserta; antherae fere sessiles, oblongae, non exsertae; ovarium liberum; stigma emarginatum; capsula supera, pisiformis, unilocularis, univalvis, polyspora.

433. CYCLAMEN, (CYCLAME, pain de pourceau ) tounN. IINN. LAM. tab. roc. (primulacea 2.) Calyx quinquefidus; corolla rotata, quinquefida, laciniis reflexis, tubo globoso, brevissimo, fauce prominente; stamina tubo inclusa; antherae conriventes; bacca globosa unilocularis, quinquevalvis, tecta capsula.

434. DODECATHION, ( DODÉCATHEON, Gyroselle) LINN, Juss. LAM. tab. 99. (primulacea). Calyx quinquefidus, laciniis reflexis; corolla rotata quinquefida; tubus brevis; laciniae longae reflexae; stamina extra tubum; antherae sagittatao, conniventes; capsula unilocularis, fere cylindrica, oblonga, apicein quinque partes dohiscens. - (Flores penduli.)

435. SOLDANELLA, (SOLDANELLE) TOURN. IINN. Juss. LAIr.' tab. 99. (primulacea 2.) - Calyx quinquepartitus; corolla campanulata, lacera, limbo tenerime multifido; antherae sagittatae, adnatae infra apicem bifidam filamentorum; stigma simplex; capsula unilocularis, apice multivalvis seu multidentata.

436. VOHIRIA, (TOYERE) sUSs. LAMT. tab. 109. VOYIRIA AUBLET. LITA SCHREB. WIILD. GENTIANA LAM. (gentianea 1.). - Calyx brevis quinquefidus, basi duabus, vel tribus squamis munitus; corolla calyce multo longior, tnbuloso hypocrateriformis, 
limbo patente quinquelobo (quinquefido), laciniis ovatis patentibus; tubo longissimo; et ad basin, et ad summum dilatato, inflato, tumido; antherue juxta faucem tubi insertae subsessiles; stigma simplex, capitatum; capsula unilocularis, bivalvis; sporae numerosissimae, scobiformes, - (Plantae fere subterraneae, foliis inferioribus carnosis, subterrestribus; flores terminales.)

437. PR I M ULA , (PRIMEVERE) IINN, JUSS. I.AM. tab. 98. PRIMULA veris, et AURICULA ursi, TourN. ( primulacea 2.). - Involucrum umbellulae; calyx tubulosus, quinquedentatus, (multo brevior corolla in auricula ursi T.); corollae tubus cylindricus, calyce longior, fauce libera patula, limbo quinquelobo emarginato; stigma globosum; capsula unilocularis, ore decemvalvulato vel decemfido. - (Numerus partium cultura variat, )

438. A N D OSACE, (ANDROSELLE) TOURN. LINN. JUSs. LAM. tab. 98. f. 1. 2. (primulacea 2.). Involucrum umbellulao; calyx quinquefidus, persistens; corolla hypocrateriformis, quinqueloba; tubo calyco breviori ovato, fauce attenuata, glandulosa; capsula ovato - globosa, unilocularis, quinquevalvis, vel quinquefariam dehiscens, polyspora.

439. A RETIA, (ARETIE) LINN. HALI. PRIMU. LA VILI. ANDROSACE VIIL. LAM. DIAPENSIA WILLD. ARETIA. - LAM. tab.98. - (primulacea) Calyx quinquefidus; corolla hypocrateriformis, quinquefida, tubo ovato, ad summum serrato; stigma dopresso capitatum; capsula unilocularis, globosa, quinquevalvis, subpentaspora (pentaspora JAcQ.). (Trunci elongationes floriferae foliis denudatao numerosissimae.)

440. ВАСOPA, (BACOPE) AUBLET, JUSs. LAir. tab. 102. (portulacea 1.) - Calyx quinquepartitus vel fissus, inaequalis, lacinia superiori majori ; corolla monopetala, perigyna, rotato hypocrateriformis, quinquefida; tubo brevi, staminiforo; limbo quinqueden- 
tato; stamina quinque sub denticulis corollae inserta; antherae rectae sayittatae; ovarium semiadhaerens, aut adhaerens basi sua calyci; stigma capita. tum ; capsula membranacea, unilocularis, polyspora; sporae minimae. - (Trunci elongatio herbacea: folia opposita, basi connata; pedunculi solitarii axillares, bibractonti.)

44I. HOTTONIA, (HOTTONE, Plumean) IXNN. JUSS. LAII. tab. 100. STRATIOTES V́aILL. (primulacea 1.) - Culyx quinquopartitus; corolla hypocrateriformis, limbo plano quinquelobo, tubo brevi; stamina tubo corcllae inserta; stigna globosum: capsula globosa acruninata, unilocularis. - (Plantae aquaticae, folis verticillatis, alatis aut denticulatis; floribus solitariis, axillaribus aut verticillatis, spicam terminalem formantibns; verticilli involucro polyphyllo muniti.)

* 442. SCHEFFIELDIA, (SCHEFFIELDE) FORST. gen. 18. SMITH. SAMOLUS IINN. suppl. (primulacea 1.) - Cuigx quinquefidus, persistens; corolla calyce longior campanulata, quinqueloba; stamina quinque fertilia, lobis corollae opposita; quinque filamenta sterilia altérna cum fortilibus; stigma capitatum; capsula unilocularis, quinquevalvis, polyspora ${ }^{*}$ ).

443 MEN I A T THES, (MENIANTHE) TOUR. LINN, juss. LAM, tab. 100, f. I. Mrchavx. VILLARSIA GMEL. veNT primulacea 3.). - Calyx quinquefidus; corolla infundibuliformis, tubo calyce longiore; limbus patens, quinquepartitus, laciniis ovalibus, interno barbato hirsutis; stigma capitato bifidum, striato sulcatum; capsula unilocularis, bivalvis, polyspora; sporae glabrae, longitudinaliter medio valvularum adnatae; radicula supera, - (In plantis emersis capsula biralvis, valvula media placentaeformi; in natantibus ruptilis MCHAUX.)

*444. NY M-

*) PErs. enchir, cum Samolo tinx, coniunctum hoc gc* nus proposuit, No. 388, pag. 17I. 
* Gi4. NY MPHO I DES, (NYMPIEAU) TOURN. VENT. WALDSCMIDIA HAYNE MENIANTHES LINN. IAM. tab. 100. f. 2. (primulacea 3.). - Caly $x$ quinquefidú; corolla rotata, laciniis quinque rotundatis, ciliatis; stigma bifidum; capsula unilocularis, bivalvis, polyspora; sporae membranacene, ciliatae, longitudinaliter suturis valvularum innatae.

445. A L L A M N D A, (ALLAMENDE, Orelio) IINN. Juss. IAM. tab, I 71 . ORELIA AUBLet. (apocinacea 3 , contorta). - Calyx quinquepartitus; corolla infundibuliformis, magna; tubo longo; fauce dilatata; limbo patente, magno, quinquefido; stamina fauci coroilae inserta; antherae fere sessiles sagittatae; stylus longus; stigma bilobum, laminis duabus rotundatis associatum; ovarium basi disco annulari cinctum; capsula lentiformis, ovalis, coriacea, echinata punctis longis, compressiuscula, unilocularis, bivalvis, polyspora; sporae rotundatae, marginibus membranaceae, duplici ordine disposita, margini valvularum insertae.

446. THEOPHRASTA, ( THEOPHRASTE, Coquemollier) LrNn. JUSS, LAN. tab. 119. ERESIA plum. (apocinea). Calyx quinquelobus, persistens; corolla campanulata, brevis, quinqueloba, lobis aequalibus obtusis; stamina quinquo brevia; capsula pomiformis, globosa, permagna, fragilis, unilocularis, polyspora, interne fere pulposa; sporae rotundatae, receptaculo centrali libero et cylindrico insertao; em. bryo in perisporio magno et coriaceo.

* 447. GENIOS T O M A, ( Geniosteme ) xопвт. tab. 12. JUSS. LAM. tab. r33. (ex ordine plantarum sede incerta). - Calyx turbinatus, inferus, quinquefidus, persistens; corolla monopetala tubulosa, limbo quinquepartito; fauce barbata; stamina in corollae orificio, alternantia cum lobis; ovarium liberum; stigma sulcatum; capsula oblonga, bilocularis, polyspora. 
448. SPIGELIA, (SPIGELE) IINM. IAM. tab. 107. ARAPABACA plum. (gentianea 3.) - Calyx quinquepartitus; corolla infundibuliformis, limbo patente, quinquefido, laciniis aequalibus; tubus calyce longior; ovarium didymum; stylus persistens; capsula didyma, bilocularis, quatrivalvis, polyspora. (Trunci elongatio herbacea ; folia opposita; flores terminales, bracteis minoribus muniti, spicati, secundi.j

449. PONGATIUM, (PONGATI) jUSS. SPHENOCLEA gaERTN. WILLD. GAERTNERA retz. (ex ord. plantt. sedis incertae). Calyx inferus, semiadhaerens, munitus ad basin bractea; quinquefidus; co. rolla (tubus interior petaloideus minimus, quinquefidus) quinquefida, calyce minor; starnina quinque ad basin interiorem tubi inserta; ovarium semiadhaerens; stylus nullus; stigma capitatum, persistens; capsula semiadhaerens, compressa, bilocularis, circumscissa; sporae minimae, numerosae, subteretes, adhaerentes duobus receptaculis fixis utroque latere dissepimenti.

450. OPHIOPHIZA, (OPHIORIZE) IIXN. sWARz. LAM. tab. 10\%. CYNOCTONUM GMEL. (gen. tianea 3.). - Calyx quinquedentatus; corolla infundibuliformis, limbo patente quinquefido; ovaium bifidum; styius unus; stigmata duo; capsula biloba, bilocularis, introrsum dehiscens, polyspora. - (Trunci elongatio herbacea, foliis oppositis, floribus bractentis, spica terminalis aut axillaris.)

45I. BENO MIA, (BENOMIE) AUBERT DE PE. TIT THOUARS BONAMIA JAUHE II. p. 349, (convolvulacea $)^{*}$ ). - Flores completi, monopotali, quinque-

*) Proximum genus est Cordiae. Sed auctor jubet ipsam Cordiam e familia borraginearum, cui nunc usque associata, excludere, et annumerari civibus Cordiae; bonamiam convolvulaceam esse forma calycis quinene foliolis partiti; dispositione et forma cmbryonis, - Forsair genus cum OPHIORRHIZA conjungendum, a qua Bonamia tantum sporarum numero differe videtur: in Ophiorrhiza multac, in Bonamia duae, 
quefidi isostemones, brevi panicula dispositi. Calyx quinquefidus, laciniis profundis concavis, inferus; curolla monopetala, hypogyna, limbo quinquefido, campannlata; stamina medio corollae inserta, lacinis corollae alterua; antherae dorso affixae; ovarium oblongum -biloculare, tetrasporum; stylus ultra medium bipartitus; stigmata capitata; fructus capsula bilocularis, dispora; sporae duae, etiam tres fundo affixae, aut per abortum una; perisporium nullum; embryo replicatus; vadicula infera; cotyledones foliaceae.

452. GEN I O S T O M A, (GENIOSTOMA) FORST. tab. 12. Juss. (sedis incertae). - Calyx turbinatus, quinquefidus, persistens; corolla monopetala, tubulosa, fauce pilis munita; limbo quinquefido; stamina quinque ad faucem corollae, alternantia cum lobis; ovarium liberum; stigma striato-sulcatum; capsulu oblonga, bilocularis, polyspora.

453. RETZIA, (RETZIF) LINN. YHUNB. Juss. LAM. tab. 103. (convolvulacea 1.). - Calyx quinquepartitus, inaequalis; corolla tubulosa, cylindrica, quinquefida, extus intısque villosa; stigma bifidum; capsula oblonga, bilocularis, bivalvis, polyspora.

454. MARIPA, (MARIPOU) AUBL. Guy. tab. 9 I. juss. LAMr. ill. tab. 110. (convolvulea r.). Calyx quinquepartitus, laciniis obtusis; corolla tubulosa, basi dilatata; limbo quinquefido; stamina quinque apice tubi inserta; antherae longae, sagittatae; stylus unus; stigma peltatum; fructus ...., bilocularis, loculis disporis. (Elongatio trunci frutescens, scandens; folia integerrima; flores in paniculis terminalibus aut axillaribus; pedunculi bracteati.)

455. MURUCOA , (MOUROUCOU) A Tra, Guy. tab. 54. Jus8. LAM. ill. tab. r03. (convolvulea r.). Calyx quinquepartitus, connivens;' laciniae duae magnae, exterioros, reliquas obtegentes; corolla infundibuliformis, limbo patente, amplo, quinquelobo; stamina quinque inserta summitate tubi lobisque opposita; 
antherae declinatae; stigma bilamollatum; capsula ad medium calyce cincta, coriacea, fibrosa, trilocularis, trispora. (Frutex scandens; folia integra, apice plicata; podunculi axillares multiflori.)

456. CONVOLVULUS, (LISERON) TOURr. Iinn. Juss. LAn. t. 104. (convolvulacea 3.) Culyx quinquepartitus; corolla campanulata, aut rarius infundibuli. formis; limbus quinque plicis fere semper integris no. tatus angulosus, angulis argutis aut dentatis; obsolete quinqueloba; stamina inaequalia ; filamenta approximata ; ovarium ad basin glandula cinctum; stigma bifidum; capsula saepe trilocularis, rarius bi - vel quatrilocula. sis; loculis mono - disporis. - (Plantae herbaceae, aut rarissime suffrutescentes, lactescentes, regulariter volubiles; pedunculi axillares, uni-multiflori, duabus bracteis muniti.)

457. IPOMAEA, (IPOMÉ, Qnamochit) IINN. JUss LArr. tab. 104. (convolvulacea 3.) - Caly.x quin. quepartitus vel fissus; corolla (rarius infundibuliformis) campanulata, longa, limbus quinque plicis fere semper integris notatus, angulosus, angulis argutis ot dentatis; quinquefida; stamina inaequalia; filamenta approximata; ovarium ad basin cinctum glandula; stigma capitato globosum, papillosum; capsula bi - fere semper trilocularis, polyspora*). - (Plantae herbaceae, volubiles, lactescentes; pedunculi axillares, terminales, uni-multiflori.)

458. MI A C ROSTO MA, (MACROSTOME) PERs. pag. 185. CALBOA cav. nov. gen, tab. 476. (convolvalacea). - Calyx inferus, persistens, semiquinquepartitus; corolla infundibuliformis, laciniis lanceolatis, fance ventricosa; starnina longissima, exserta, basi nııda; stygma simplex; capsula quatriloçularis, quatrivaslvis,

*) Ipomaea bracteata c $\Delta \mathrm{v}$. nov, gen, tab. 477. ob corollac limbur integerrimum, revolutum, flamenta glabra, stigma simpliciter oratum, distinctum genus constitucre monet gravissimus PERs. P. $\mathbf{1} 85$. 
vis, dissepimento valvulis parallelo cruciata; sporae solitariae.

459. L ISIA NTHUS, ( LISIANTIE ) Brown. IINN. JUSs. IAM. tab. 107. GENTIANA LINN. (gentianea 2.) Culyx campanulatus, quinquepartitus, laciniis margine membranaceis, carınatis; corollu infundibuliformis calyce multo longior, limbo quinquefido, laciniis recurvatis, tubo parum ventricoso, basi attenuato, ore patente; stigma capitatum, bilobum; capsula oblonga, bilocularis, bivalvis, margine valvularum introrsum, voluta; sporue numerosae, subimbricatae, margine minimo membranaceò cinctae. - (Flores uno, duo, terminales.)

460. DATURA, (DATURE, Stramoine) LINN. IANT. tab. II3. STRAMIONIUM toupn. (solanea I.) Caly $x$ magnus, tubulosus, ventricosus, quinquangulus. apice quinquefidus, caducus, basi orbiculari tantum persistente, et sese revertente; corolla magna infundibuliformis, tnbo elongato, limbo quinquangulo, quinque plicato, obsolete quinguedentato, dentibus acuminatis; stigma obtusum bilamellatum; capsula ovalis, bilocularis, loculis in duo aut plures parietes per dissepimentum divisis, quatrivalvis, quatrilocularis, laevis vel spinosa, polyspora; sporae reniformes. - (Plantae herbaceae, rarius frutices, interdum scandentes; folia geminata in quibusdam speciebus.)

46r. B 1 U G M A N I A, (BRUGMANSIE) pers. pag. 216. ruiz. DATURA ruiz. (solanea). - Calyx latere ruptilis, persistens; corolla infundibuliformis, plicata; antierae conglutinatae; stigma utrinque lineatim decurrons; capsula bilocularis inermis. (Forte et huc MOENCHII Brugmansia pertinet.)

462. HYOSCYAMIUS, (JUSQUIAME) TOURN. LINN. JLss. LAM. tab. II7. (solanea 1.). Calyx tubulosus, quinquefidus; corolla infundibuliformis, obtusa, irregularis, limbo patcnte, oblique in quinque lobos iuaequales fisso; stigma capitatum; capsula oblonga, ob- 
tusa, leviter compressa et sulsata in utroque latere stria una, operculata, bilocularis, bivalvis, circumscissim dehiscens, polyspora. - (Plantae herbaceae; folia floralia saepe geminata; flores solitarii, axillares.)

463. N ICO TIANA, (NICOTIANE, Tabac) TOUPN, LINN. IAM. tab. 113. (solanea 1.) - Calyx urceolatus, quinquefidus; corolla calyce multo major et longior, infundibuliformis, regularis, quinquefida, limbo plicato; stigma emarginatum; capsula bivalvis, bilocularis, polyspora. - (Plantao herbaceae: flores terminales, dispositi in spicam vel paniculam.)

464. PETUNIA, (PETUNIE) juss. (solanea?). Calyx profunde quinquefidus, laciniis subspathulatis; corolla tubulosa, limbo dilatato, subquinquelobo; stigma capitatum, bilobum; capsula bivalvis, biloculauis, polyspora.

465. I A A A KEA , (LAMARKÉE) RICHARD. Carlyx oblongus, pentagonus, quinquefidus; corolla hypocrateriformis, quinquepartita, limbo subaequalii obtuso ; capsula teres, bilocularis, polyspora.

466. VERBASCUM, (MOLÉNE) TOURN. LINN. Juss. LAM. tab. 117. (solanea I.) - Calyx quinquepartitus; corolla rotata, patens, lobata, lobis quinque inaequalibus; stamina quinque inaequalia; filamenta inclinata, versus basin barbata; stigma simplex; capsula ovalis aut globosa, bilocularis, bivalvis, valvulis inflexis, polyspora. - (Plantae herbaceae, saepenumero tomentosae; folia interdum decurrentia; petiolus interdum appendiculatus.)

467. RAMONDA, (RAMONDE) RIснARD. PERs. pag. 216. (personata 3.). VERBASCUM LINN. EHRH. Scapi radicales; caly $x$ campanulatus, fissus usque ad medium in partes quinque cibtusas; corolla rotata, quinqueloba, lobis inaequalibus; tubo brevissimo; stamina quinque alterna cum laciniis corollae, et inserta basi corollae; antherae approximatae, circum ovarium stylo munitum positae, apice perfora- 
tae; stigma rotundatum; capsula unilocularis; placentae duae laterales, formatae margnibus, valvulas, quae sese cingunt $a b$ interiori parte circumcirca, mentientibus; receptacula duo parietalia; polyspora; sporae oblongae, numero plurimae *).

468. CHIRONIA, (CHIRONE) LINN. JUSS. LATr. tab. 108. ROESLINIA MOENCH. CHLORA LINN. WIILD. (gentianea 2.) - Calyx quinquefidus, fere quinquepartitus, subcampanulatus; corolla infundibuliformis, (subrotata pers.) tubo calycis magnitudine, limbo magno quinquepartito; stylus unus, striatus, antrorsum inclinatus; stamina tubo corollae insidentia; antherae foecundatione absoluta spiraliter tortae; stigma capitatum, bilobum; pericarpium : capsula bilocularis aut bacca unilocularis.

469. ERYTHRAEA, (ERYTHRAEE) (gentianea). Calyx adpressus, subpentagonus, quinquedentatus; corolla infundibuliformis, tubo longo; antherae post anthesin spiraliter tortae; stigmata duo approximata, rarius simplex; capsula sublinearis, unilocularis; receptacula duo extantia. ( Richapo Catal. hort. mod. parisini. pers.)

470. PORANA. (PORANE) IINN. BURM. IAM. tab. 186. (ex ord. plantt. indeterm.) - Calyx quinquepartitus, in fructu major; corolla monopetala, campanulata, calyco multo longior, quinquefiáa, laciniis aequalibus; stamina quinque intersita corollac laciniis; stylus semibifidus, longior, persistens; stigmata duo capitato globosa; pericarpium bivalve. - (Proximum hoc genus est familiae Borraginearum.)

47 I. COBAEA, ( СOBAÉE) (bignoniacea). cAv. tab. 16. 17. et 500. VENT, - Calyx inferus, persistens, penta-

*) Hoc genus valde convenit cum Beslevia secundum ri. CHARDI observata; nihiloninus margines valvularum intrantes et sese convolventes ad̉ formanda receptacula genus Ramondia approximant gentianis. 
pontagonus, angulis basi compresso alatis, quinquepartıtus, laciniis patentibus; corolla infundibuliformis; tubo longissimo, vix dilatato, limbo campanulato, laciniis quinque patentibus, crenulatis; stumina declinata; antherae oblongae versatiles; filumentu declinata; ovarium basi cinctum corpore glanduloso vel disco q̧uinquangulo; stigmata quatuor aut quingune subulata; capsula obovata, punctata, leviter angulosa trilocularis receptaculo trigono distincta; sporae imbricatae, marginatae.

472. DIA PENSIA, (DIAPENZE) IINN. JUSS. LAM. tab. 102. ANDROSACE TOUR.N. (convolvulacea 2.). - Calyx quinquepartitus ant pentaphyllus, imbricatus externe foliolis squamaeformibus tribus; corclla hypocrateriformis, limbo quinquetido plano; stamina quinque tubo inserta, et alternantia cum laciniis corollae; stylus unus; capsula trivalvis, trilocularis, polyspora.

473. PHLOX, (PHLOX) LINN. JUSs, IAM. tab. 108. (polemonacea). - Calyx prismaticus, profunde quinquefidus aut partitus, connivens, persistens; corolla hypocrateriformis aut infundibuliformis, tubulosa, tubo longo, limbo plano, quinquepartito; stamina super tubum inserta in magna lataque dimensione; an- therae sagittatae; filamenta starninum inaequalia, tubo inclusa; stigma trifidum; capsula trilocularis, loculis monosporis - (Trunci elongatio herbacea; folia opposita, simplicia; foralia interdum alterna.)

474. GILIA, (GILIE) RUIZ. pAv. IPOMOPSIS MIchaUx. WIIID. IPOMOEA IINN. (convolvulea). - Corolla infundibuliformis; filamanta aequalia, incisuris corollae inserta, prominula; stigmata tria; capsula trilocularis, trigona, polyspora; sporae nudae, non emarginatae.

475. BONDLANDIA, (BONPLANDIE) CAVAN: nov. gen. tab. 532. - Calyx liber, tubulosus, quinquedentatus; corolla subbilabiata, quinquepartita, laciniis emarginatis; stamina declinata, infra faucem tubi 
inserta; stigma trifidum; capsula ovato trigona, trilocularis, trivalvis, trispora; sporae ellipticae.

476. NAVARETTIA, (NAVARETTIE) Ruİ。 pAv. (incertae sedis). - Caly $x$ campanulatus, quinquefidus, quaeque laciniarum munita tribus dentibus acutis; corolla infundibuliformis, tubo quinguangulo, limbo plano quinquangulo, angulis acqualibus; stami'na quinque tubo inserta: ovarium liberuen; stigma bifdum ; capsula membranacea, bilocularis, bivalvis, calyce tecta; sporae hand multae.

477. POLEMONIUM, (POLÉMOINE) TOUR. IINA. JUSs. LAMr. tab. 106. (polemonacea). - Calyxa urceolatus, quinquefidus; corolla rotata, limbo quinquepartito, (quinquelobo), fundo clauso valvulis sta. miniferis, tubo brevi; staminum fllamenta dilatata ad loborum basin; antherae versatiles; stigma trifidnm; capsula trilocularis, trispora, supera; sporae angulatae. (Folia alterna.)

478. CANTUA, (CANTU) JUSs. IAM. tab. ro6. PERIPIIRAGMOS ruiz. flor. Peruv. (polemonacea). Calyx monophyllus, urceolatus, tri-quinquefidus; corolla infundibuliformis, (hypocrateritormis nutz.); limbo patente, dilatato, quinquelobo vel partito, laciniis obcordatis sive emarginatis; tubo cylindrico, calyce longiori; stamina magnitudine aequalia, interdum exserta; ovarium corpori glanduloso insidens; stigma tripartitum; antherae sagittatae; capsula subtrigona, trilocularis, trivalvis, polyspora; sporue imbricatae, margine membranaceo alatae.

479. HOITZIA, (HOITZIT) CAVAN. JUSS. CAN. TUA wilid. (polemonacea). Calyx duplex; interior monophyllus, tubulosus, quinquefidus; exterior polyphyllus (an bracteae?), foliolis quatuor - octo polymorphis, dentato serratis, conniventibus; corolla infundibuliformis, longa; limbo quinquelobo, lobis fero aequalibus, ovatis, paulisper patente; staminum filanien. ta aequalia, longa, extra tubum; ovarium triangulum; 
capsula ovalis, interdum leviter striata. (Frutices foliis alternis, simplicibus; flores axillares, solitarii.)

480. BROS SAEA, (BROSSE) IINN. PLUM. JUSs. zAMr, tab. 111. (ericae I.). - Calyx carnosus, superus, quinquefidus, laciniis oblongis; corolla conoïdea, magnitudine calycis, truncata, ad summum recurva, limbo crenulato aut integro; capsula quinquesulca, quinquelocularis, calyce persistente tecta, quinque fissuris pervia, polyspora.

48 I. A Z A L E A , (AZALEE) IINN. JUSs. IAM. tab. I10. CHAMOEPHODODENDROS TOURN. (rhodoracea 1,). - Calyx quinquepartitus; corolla campanulata aut infundibuliformis, limbo plano, subobliquo, laciniis quinque inaequalibus; stamina quinque receptaculo sub pistillo inserta; stigma obtusum; capsula quinquelocularis, polyspora. - (Flores axillares aut terminales, fere solitarii aut in racomis.)

482. CRYPTA N D R , (CRYPTANDRE) sMrTH. transact. IV. (rhododendracea). Juss. gen. 158. - Calyx pentaphyllus; corolla tubulosa, limbo quinquefido, squamis quinque cucullatis inter segmenta; stamina fauci inserta sub singulis squamis; stigma trifidum; capsula supera, trivalvis, trilocularis e valvulis inflexis; sporae solitariae compressae.

483. EPACRIS, (EPACRIS) TORST. LINN. JUSS. zAM, tab. 367. (ericae 1.). - Calyx simplex aut duplex, exterior imbricatus, quinquepartitus; corolla in. fundibuliformis, velata pube, tubo paulisper magnificato versus limbum quinquefidum; stamina quinque brevia, tubo inserta; antherae ovales aut oblongae; stigma capitatum; ovarium basi cinctum quinque squamis brevibus; capsula pentagona, quinquelocularis, quinquevalvis, polyspora. - (Frutices foliis imbricatis aut remotis, foribus in racemis aut fere solitariis.)

484. TR I CYCLA, (TRICYCLE) cAv. tab. 598. juss. (nyctaginea 2.). - Calyx (involucrurn) tripbyl- 
phyllus, foliolis magnis ovalibus, subroduntis, venosis, unifloris, persistens; corolla (calyx) infundibuliformis, calyce (involucro) brevior, tubulosa, tubo leviter ad medium inflexo, limbo (quinquelobo) quinquefido, (lobis) laciniis tricenatis ; filamenta infra ova* rium inserta; samara ovata, corollas tubo ampliato cincta; sporae basi (calycis) corollae tectae, limbo coronatae, et cinctae (involucro) calyce exsiccato, persistente. (Stamina interdum septem.) - Trunci elongatio spinis alternis obsita, fasciculatis; flores solitarii, e medio fasciculi foliorum egredientes.

485. VEN T ENATIA, (VENTENATIA) Auctorum flor. peruv. - Calyx inferus, duplex; externus polyphyllus, internus pentaphyllus; corolla monopeta. la, infundibuliformis; stigma globosum; nux quinque. locularis, loculis monosporis.

486. STYPHELIA, (STYPIELÉE) SмITH. WILLD. ARDISIA GAERTN. EPACRIS IINN. VENTENATIA cAv. (ericae 2.). - Calyx imbricatus; corolla tubulosa, quinquefida, laciniis saepe revolutis, hirsutis aut glabris; nectarolyma membranaceum, lacerum, ovarium cingens; stigma capitato quinquefidun; stamina quinque fauci corollae inserta; drupa quinquelocularis, loculis disporis; sporae geminatac.

487. PEROA, (PEROA) cAVAN, ic, tab. 349. PEROIV a auctt. flor. peruv. - Calyx duplex; externus triphyllus, internus pentaphvllus; corolla hypocrateriformis, laciniis acutis tomentosis; ovarium ovatum, nudum ; stigma simplex; capsnla ovata, unilocularis, monospora; spora oblonga.

\section{PYXID A N T IIE R A, (PYXIDANTHERE)} McнаUх. - Ealyx quinquepartitus, laciniis incumbentibus, paleacco membranaceis; corolla campanulata, quinquepartita; filamenta lamellata; antherae loculis subglobosis circumscissis; sty/us crassus; stigmata tria; capsula....? (An hujus loci ?) 
489. NERIUM, (NERION, Laurouse) Toun. LINN. JUSs. LAM. tab. 174. (apocinea 2.). - Calyx quinquepartitus, parvus, persistens; corolla infundibuliformis, oblonga, limbi laciniis quinque obtusis obli. quis, saepe ad basin internam appendiculis multifdis lacero, non exsertis extra tubum, coronatis; stigma truncatum, membrana annulaxi insistens; folliculi duo cylindracei, recti, conniventes, longe acuminati ; sporae coma instructae. - (Arbores, aut frutices folia opposita, aut tria tribus opposita; flores dispositi in corymbum.)

490. ECHITES, (ECHITE) JACQ. LINN. JUSS. IAr. tab. 174. APOCYNUM ritr. (apocinea 2.). Calyx minor, quinquepartitus; corolla infundibuliformis, fauce nuda; limbo plano, quinquefido; antherae conniventes convergentesve in conum, acuminatae; receptaculum ovarii quinque glandulis munitum; stylus simplex; stigma capitatum, bilobum; folliculi duo, longissimi, recti, senıpor fere angusti, et quasi articulati; sporue coma instructae. - (Frutices: trunci elongatio saepe volubilis; folia opposita; pedunculi axillares aut terminales, uni-multillori; corollae forma varia.)

49I. STOPHA N THUS, (STROPIANTHE) DECAND, bullet, philom. no. 64. tab, 8. f. 1. ECHITES WIIID. NERIUM IAM. (apocinea 2.). Calyx quinquepartitus; corolla campanulata, infundibuliformis: inferne curvato attenuata, quinque appendices acutao simplices; quincqueloba, terminataque cauda longissima duorum rostrorum; antherae sagittatae; ovarium duplex; coronulae faucis dentibus decem simplicibus; folliculi duo cylindrici, recti; sporae comatae. (Arbores aut frutices, elongatione trunci cylindrico, saepe scandente; foliis oppositis, integris; floribus pedicello brevi saepe bifurco.)

492. PLUMIERIA , (PLUMIERE, frangipanier) PLUM, tourn, IINN. JUSS, taM, tab. 173. Auctt, florae 
peruv. (apocinea 1.). Calyx quinquepartitus, laciniis obtusis, minimus, deciduus; corolla infundibuliformis, limbo quinquefido, laciniis obtusis patentibus Pl. (rectis in pudicu); tubo longo, subincurvo, vix dilatato; antherae connirentes; stylus fore nullus; stigma bifidum; folliculi duo ventricosi, longi, deorsum flexi 3 sporae oblongae, basi ala membranacea crenato denta. ta adauctae. - (Arbores aut frutices gratissinat, et formosissimae; folia alterna, integra, magna; flores corymbus terminalis, multi saepiuls abortivi.)

493. CAMERARIA, (CAMERIER) pLUM. IIXx. suss. LAMr. tab. 173. (apocinea 1.). - Calyx minimus, quinquefidus, acutas; corolla infundibuliformis, cylindracea, limbo plano quinquefido, laciniis quinque obliquis, tubo utrinque et ad basin et ad summurn ventricoso; staminum filamenta ad basin appendiculata; antherae conniventes, filis longis duobus terminatae; stylus brevis; stigma capitatum,' bifidum: folliculi duo, basi utrirque bi-trilobi; lobis obtusis, fere hastatis; lobi latorales, breves, medio longiore; horizontales, compressae; sporae compressae, imbricatae, membrana alari ad summum notatae. - (Arbores aut frutices ramis dichotomis; folia opposita; flores axillares aut terminales.)

494. TABERNAEMONTANA, (TABERNEMONTANE, "Taberne) PIUM. IINN. Juss. IAMr. tab. 170. (apocinea 1.). - Calyx quinquefidus aut quinquepartitus, caducus; corolla longa, infundibuliformis, obliqua, limbo plano quinquelobo; tubus an. gulato striatus, basi subglobosus; antherae acuminatae, conniventes; ovarium basi munitum glandulis quinque bifidis; stigma capitatum; folliculi duo disjuncti, horizontales, acuminati, ventricosi, roflexi, uniloculares, univalves, interne pulposi; sporae rugosae, pulpa immersae. - (Arbores aut frutices; folia opposita, rarius alterna; pedunculi multifloti, axillares aut terminales.) 
495. A M SONIA, (AMSONIE) miciraux. (apocinea). - Corolla infundibuliformis, fauce clausa; folliculi duo erecti; sporue teretes, nudae, oblique truncatae. (Stigma margo annullaris: folia alterna.)

496. URCE O LA, (URCEOLE) VANDELI. ROXB. SPRENGEL. ap. SCIRAD. 1801. p. 236. (apocinea 3.). Calyx hexaphyllus; corolla urceolata; tubo longo; limbo quatrilobo patente et laciniis extrorsum flexis; stamina exserta; ovarium liberum; nectarolyma cylindricum integrum, ovarium cingens; folliculi duo, uniloculares, bivalves, - (Nescio an URCEOLA VANDELl, delineata in flora Brasiliensi tab. 1. f. 4. ab URCEOLA SPRENC, diversa sit.):

497. CERBERA, (CERBERE, Ahouai) IrNe. LAMr. tab. 170. AHOUAI toUris. (apocinea 3. s. contorta). - Calyx patens, quinquepartitus; corolla infundibuliformis; limbo magno, obliquo, quinquefido: tubo clavato, fauce quinquangula, et quinquedentata; antherae conniventes; stigma bilobum; drupa magna, striata vel bipunctata ex altero latere, monospora; continens nucem osseam quatrivalvern, bilocularem, disporam. (Arbores foliis alternis; floribus saepe terminalibus, fore corymbosis.)

498. I G NATIA, (IGNATIE, vomique) juss. STRYCHNOS IINN. suppl. (apocinea 1.). - Culyx quinquedentatus; corolla longissima, infundibuliformis; drupa unilqcularis, polyspora; sporae irregulares, angulatae. (Distinctum esso genus a tò STRYCHNOS JUSSiev auctor est. Cf, PErs. Enchir. p. 26. ) (Folia ovata, spithamea; flores in panicula axillari; fructus ovatus magnitlidine pyri.)

499. A N A S ER, (ANASSERE) IAM. JUSS. (apocinea 3.). - Calyx parvus, quinquefidus; corolla longa, urceolata, quinqueloba, intus villosa; antherae cum laciniis corollae alterna; stigma didymum; capsula oblonga, bilocularis, bivalvis, valvulis marginibus intrantibus, et loculamentum formantibus; receptacula 
duo contralia, sporifera, approximata; sporae nume. rosae.

500. VIN C A, (PERVENCHE) IINN, LAM. tab. 172. PERVINCA tourn. (apocynea 1.). - Calyx quinquepartitus, persistens; corolla hypocrateriformis, tubo longo, limbo plano, quinquelobo, laciniis obtusis, obliquis; fauce pentagóna; antherae membranaceae; stigna infra urceolatum concavum, interne plano tuberculari cinctum et orbiculari; folliculi duo cylindrici, acuminati, conniventes, teretes, recti; sporae oblon. gae, nudae. - (Suffrutices "trunci elongationibus rectis aut decumbentibns, sarmentosis; foliis oppositis; floribus axillaribus.)

50I. GELSEMIUM, (GELSEMIN) JÜs. MIcirAux. BIGNONIA linN. (apocynea). - Calyx parvus, quinquedentatus, corolla infundibuliformis, laciniis quinque fere aequalibus, limbo patente; capsula ovalis, compresso - plana, sulcata ad modium, bipartibilis, bilocularis, bivalvis, polyspora; sporae planae, valvularum marginibus adnexae. JUssiev.

502. EN DRACHIU M, (ENDRACH) JUSS. LAM. illustr. tab. 103. THOUINIA sMith. HUMBERTIA commess. (convolvulea I.). - Calyx coriaceus, quin. quepartitus (pentaphyllus); corolla campanulata sive urceolata, ventricosa, extus pilis hirsuta, limbo quinque plicis fere integris anguloso, angulis argutis sive denticulatis; subquirqueloba; stamina inaequalia, exserta; filamenta approximata; ovarium cinctum ad basin glandula una; stigma bifidum; capsula stipitata, baccaeformis, lignosa, tri - rarius bi - aut quatrilocularis; loculis mono - disporis. - (Plantao herbaceae, rarissime suffrutescentes, lactescentes, ut plurimum volubiles; pedunculi axillares aut terminales, uni-multiflori, duabus bracteis muniti.

503. TEC T O N A, (TECTONE, Tec.) IINN. suppl. roxB. WILID. THEKA. RHEED, JUSS, LAM. tab. 136. (verbenacea u. . - Calyx campanulatus, quinque[8] 
sexlobus; corolla tubulosa, tubo brevi; limbo patente, quinque - sexlobo, laciniis crenulatis; stamina quinque vel sex; stigma dentatum, bi - trifidnm; drupa magna ex́succa, spongiosa, calyce magnificato, et inflato vel vosiculoso reddito inclusa, continens nucems rri-quatrilocularem, tri-tetrasporam.

504. A R DISIA, (ARDISE) swARz. WIILD. ICA* COREA AUBL LAMr. tab. 136. fig. ?. TINUS rURM. ANGUILARIA GAERTN. (planta ordinis et sedis incertae.) Calyx monophyllus, quinquepartitus; corolla monopetala, hypocrateriformis, limbo quinquelobo laciniis aequalibus reflexis ovario approximatis; stamina quinque infra lobis corollae approximatis; antherae magnae, erectae; ovarium liberum; stigma simplex; drupa baccaeformis, carnosa, supera, globosa, tegens nucèni monosporam; spora arillo pulposo striatoque tecta; embryo elongatus, cylindricus, in medio perisporio carnoso collocatus. - (Hoc genus nullam familiam cognitam attingens, novam constituit, Sapotis vicinam, distinctam staminum puncto insertionis, sporae numori unitate, nec non dispositione et forma embryonis.) *).

505. ACHRAS, (ACHRAS, Sapotillior) IINN. jUss. LAM, tab. 255. Puiz. PAv. SAPOTA rLUM. (Sapotea). - Calyx quinque - sexpartitus, laciniis hr. dino duplici dispositis, ovatis, concavis, incurnbentibus; coroila campanulata, ovata, limbo quinquesexfido, laciniis rotundatis; squamis totidem emarginatis, alternis ad faucem; POVIUM mammosum, uniduodecimloculare; sporae solitariae (duodecim) hilo umbilicati marginali munitae, apiceque unguiculatae; stigma obtusum, striatum; stamina quatuor - sex.

506. SIDEROXYLON, (SIDEROXYLON, Ar. gan.) IINN. JUSS. ACHRAS LINN. IAAM. tab. 120. (sapotea).

*) Huic generi valde affinis EMBBELIA вUnM, juss. cui: Caly $x$ ninimus quincuepartitus; corolla pentayetala, Arbor zeyaniae BaDULA Jess. 
potea). - Calyx pentaphyllus vel quinquepartitus; corolla rotata, quinquefida, subrotunda; lacinia quaeque ad basin appendiculata duabus parvis, alternis squarvis, serratis; stamina quinque, (interdum decom, ubi appendices sunt antheriferae); ovarium cinctum quinque nectarolymatibus foliaceis; drupa ovata.

507. ROEMERIA, (ROEMERIE) THUNP. nOV. gen. pars IX. et ap. roemer II, 1. - Calyx perianthium pentaphyllum, obsolete pentagonum, foliola ovata acuta, concava, extus hirsuta, persistentia; corolla pentapetala; petalis ovatis, obtusis, concavis, ezectis; stamina filamentis quinque brevibus: antheris ovatis; pistillum: ovarium superum, ovatum; vertice do. presso; stylus crassus, erectus, longitudine staminum; stigmata tria subrotunda. Pericarpium: nux majuscula, rotundata, basi dilatata, unilocularis, rugoso striata, in embryone trispora; spora una ad maturitatem perve. niens oblonga, latere pedicello adfixa, rubra, (differt a Sideroxylo calyce pentaphyllo; corolla pentapetala ; stigmatibus tribus.)

508. OLINI $\Lambda$, (OLINIE) тHUNB, nov. gen. apud Roemer II, I. Char. generis: corolla pentapetala; nectarolymatis squamae quinque; stigma pentagonum; fructus locularis, polyspora. = Calyx perianthium monophyllum, tubuloso campanulatum, glabrum, lineam lon. gum, -quinquedentatum, dentes rotundati, erecti, brevissimi; curolla pentapetala, petala margini calycis inter dentes interne inserta, lineari lanceolata, obtusa, alba, intus supra basin subvillosa, crecto patentia, persistentia, lineam dimilean longa; nectarolymatis squamae quinque, raro sex, basi petalorum insertae, antheris superimpositae, subtus concavae, supra convexiusculae, pallide flavescentes. 'Stamina: filamenta quinque, raro sex, brevissima, fere nulla, calyci sub nectarolymatibus inserta; antherae parvae, globosae, didymae, inflexao, flavae; pistillum: ovarium suporum, concavum, glabrum; stylus brovissimus; stigma incrassatum, obtusum, subbifidum, pentagonum; pericarpium: fructus in fundo calycis eoque persistento 
coronatus, oblongus, obsolete pentagonus, pentasporus, quinquelocularis? - (Obs.: corolla cum calyce videtur formare corllam monopetalam, quinquepartitam: odor florum gratus. - Sideroxylon cymosum IINN, hujus generis est.)

509. B U M EL I A, (BUMELIE) swArz. (sapotea). Caly $x$ minimus, quinquepartitus; corolla quinquefida; nectarolyma quinque appendices internae pentaphyllum; stylus unus; stigma simplex aut nullum; drupa (bacca) ovalis aut globosa, unilocularis, monospora. - (MASTICHODENURON JACQ. CORNUS CAtesb.) (Arbores aut frutices foliis alternis; flores verticillati et fere sessiles.)

510. MA N GLIL LA, (MANGLILLE) JUss. mss. CHRYSOPHYLLUM LAM. SIDEROXYLON JACQ. BUMELIA WiLLd. - Calyx minimus, quinquepartitus, laciniis profundis; corollu rotata, quinquepartita; nectarolymatis squamulae nullae; staminum antherae sessiles; stylus nullus; stigma incrassatum, sessile; drupa globosa, unilocularis, monospora ; JUss. gen. pl. ; an ROEMERIA THUNB. idem genus? CABALLERIA PAV. et ruiz.; Certe Gueitardae congener. 8CHRAD.

511. GYNOPOGON, (GYNOPOGON) FOHST. Juss LAM. tab. 118. (apocynea 3.). - Calyx minimus, quinquefidus, persistens; corolla tubulosa, interne pilosa, attenuata versus faucem, parum inferius dilatata, limbo obliquo, quinquepartito; antherae sagittatae, fere sessiles; stigma didymum s. bilabiatum, apico villosum; drupa (bacca) pedicellata, pisiformis, coriacea, continens nucem semi-bilocularem, cartilagineam, disporam.

512. GUETTARDA, (GUETTARDE) IINN. JUSS. LAM. tab 154. LAUGEKIA swarz, RUIz. (rubiacea 8.) - Caly $x$ oblongus, fere integer, inaequalis, obsolete dentatus aut crenatus; corolla tubulosa, tubo longo, fere intundibuliformis, limbo patente quinquenovemfido, laciniis obtusis; staminum antherae òblon. gae sessiles, non exsertae: stigma capitato clavatum; 
drupa exsucca, rotundata, spice retusa, umbilicata, continens nucem sulcatam, sinuosam, quinquepassim bi-sexlocularem. (Stamina quatuor, quinque, sox, septem, et novem.) - Arbores; pedunculi longi axillares, solitarii, multiflori ad summum ; flores dispositi in cymam bifidam, bracteatam, quibusdam sterilibus.)

513. LAUGERIA, (LAUGERE) JACQ. IINN. swarz. Juss. (rubiacea 8.). Calyx minimus, limbo fere integro; corolla tubulosa, limbo plano, quinquelobo, tabo oblongo, tenui; antherae quinque fere sessiles, non exsertae; stigma capitatum; drupa pisiformis, non corónata, subrotunda, dispora, continers rucem quinquelocularem quinquesulcatsm, pentasporam. (Frutices non spinosae mites; flores dispositı in racemos axillares.

514. SCHRADERA, (SCHRADERE) VAHL. eclog. I. 35. URCEOLARIA GMEL. FUCHSIA SWARz. Involucrum universale multiflorum; calyx margo superus, integerrimus, urcoolatus; corolla quinque - sexfida, campanulats, fauce hirsuta; stigmata duo; bacca unilocularis, polyspora.

515. VARRONIA, (VARRONE, Majoli) IINN. LAMr. tab. 95. TOURNEFORTIA LINN. (borraginea I.). Calyx quinquedentatus, tubuloso campanulatus; corolla tubulosa, calyce paulo longior, limbo quinquepartito, ore patente crenato plicato; antherae cernuae; stylus dichotomus : stigmata quatuor; drupa ovata, continens nucem unilocularem (quatrilocularem LINN.); tetrasporam, calyci inclusa; RUIz. - (Frutices foliis saepo asperis, interdum fere oppositis, petiolis interdurn persistentibus; pedunculis axillaribus aut torminalibus, floribus in glomerulis aut spicis.)

516. ALDEA. (ALDEA) ruiz-pAv. HELIOTROPIUM LINN: (borraginea? sode incerta JAUME.) Calyx quinquefidus: corolla campanulata, laciniao aequales calycinis; stamina quinque longe exserta, basi co- 
rollae affixa; filamenta villosa; antherae oblongae; ova a vium liberum; stylus furcatus; stigmata duo; capsula unilocularis, bivalvis, calyce quinquefido obtecta, dispora; sporarum una maturoscens, altera abortiens.

517. PHACELIA . (PHACELIE) JUss. IIICHAUX. f. am. bor. tab. 16. (borraginea 2.) - Calyx quinquepartitus; corolla fere campanulata, quinquefida, basi interna striata, striis margine membranaceis, basin filamentorum cingentibus; stamina corolla multo longiora; stylus brevis; stigmata duo, longa; capsula bilocularis, loculis tetrasporis, bivalvis, valvula quaque divisa receptaculo sporifero. - (Planta herbacea pubesccns; folia alterna, pinnata; flores unilaterales, spicati.)

5IS. CORDIA, (CORDIE, Sebestier) PLUM. IINN. JUss. willd. IAM. tab. 96. (borraginea 1.) POTAGONULA IINN. Ditu. hort. elth. - Calyx tubulosus, apice quinquedentatus, persistens et fructifer maximus; corollainfundibiliformis, rotata, tubo aequali aut longiori, limbo quinquepartito (raro quatuor ad octo lacinias habet); antherae oblongae; stylus apice dichoto. mus, vel duplicato bifidus; stigmata quatuor; drupa continet nucem quatrilocularem, tetrasporam, duobus loculis a ut tribus abortientibus; sporae .... ? ( Arbores aut frutices; folia aspera taciu; flores in panicula aut corymbo tcrminali vel axillari.)

519. EHRETIA, (EHRÉTEE, Cabrillet) IINT, JUss. IAMr. täb. 96. BEUPRERIA JACQ. (borraginea 1.) Calyx quinquefidus; corolla tabulosa limbo quinquelobo; stumina quinque corollae tubi ad medium inserta, exserta; stigma bilobum, omarginatum; bacca (drupa) dissiliens in duas haemisphaerias; quaque haemisphasria biloculari, dispora; nuces solitariae; (sporae duac?). (Arbores aut frutices: folia glabra aut tactu aspera; flores in panicula terminali et axillari.)

520. H IPPOTIS, (Hippote) ruIz. et PAV. Corolla infundibuliformis; stigma bifidum; nectaralyma 
ovarium ambiens; capsula baccata, bilocularis, calyce magno auriculaetormi coronata.

52I. A IBELANIA, (AMBELANIER) AUBEET: sUss. IAM. tab. 169. TVILLUGHBEIA SGHFEB. (apocinea 3.) - Calyx brevis, quinquepartitus; corolla tubulosa, hypocrateriformis, cylindrica, fauce attenuata, limbi laciniis quinque obliquis, undulatis; stamina brevia; antherae triangulares; stigma ovale, bicuspidatum, in disco rotundato positum; capsula magna, ovalis, carnosa, bilocularis, polyspora; sporae affixae receptaculo gracili, compressae, exasperatae.

522. OPHIOXYLON, (OPHIORYLON, Ophiose). IINN. Juss. LAM. tab. 842 QCHROSIA Juss. (apocinea 3.). Flores hermaphroditi : calyx minimus quinquedentatus; corolla limbo quinquepartita, tubulosa, tubo filiformi, medio incrassato; stamina quinque brevia; stylus unus; bacca didyma, bilocularis, dispora. Flores masculi: calyx ut in hermaphroditis, (bifidus LINN.); corolla infundibuliformis, quinquefida; fauce appéndice interno cylindrico cincta; stamina duo;.flores masculi hermaphrpditis mixti ${ }^{*}$ ).

523. CARISSA, (CARISSE, Calac) IINN. JUss. IAlr. tab. 118. ARDUINA IINN. ANTURA FORsk. (apocinea 3.) - Calyx quinquefidus aut quinquepartitus, breris; corolla calyce multo longior, tabulosa, juxta faucem leviter inflata, limbo quinquefido; stamina inclusa; ovarium simplex; stigma simplex aut fere bifidum; bacca parva, sphaerica, bilocularis, loculis mono-di-aut polyzporis; sporis compressis, receptaculo affixis. - (Frutices ramis dichotomis; spinac oppositae, interdum floriferae; folia opposita, in-

tegra;

*) o chrosia, (ociroosie) juss. (apocinca r.) Calyx minimus. quinquedentatus; corolla tubulosa, infundibuliformis, limbo quinquepartito, patente; stylus unus; stigma incrassatum; folliculi disjuncti, ovales, drupaeformes, continentes nucem bilucularcm; sporae duae vel tres in quoque loculo, inacquales, plamac, maxginc membranaccac. 
tegra; pedunculi uni-bi-multiflori, flores axillares aut torminales.).

524. VOGELIA, (VOGELEE) IAM, tab. 149. Calyx pentaphyllus; foliolis complicatis, magnis, transversim undulato sulcatis; corolla tubulosa, plicata; stigna quinquefidum. - (Spica scrobiculiformis, bra* cteae plicatae, folia obcordata cum mucrone.)

525. J ACQUINIA, (JACQUINIER) LIN J JUss. IAM, tab. 121, MEDEOLA LINN. DILr. (sapotea). Calyx quinquepartitus; corolla campanulata, ventricosa, limbo decemfido, laciniis quinque alternantibus brevioribus et introrsum positis; stamina receptaculo inserta; drupa baccaeformis, monospora; spora rotundata, cartilaginea. - (Arbores aut frutices, foliis verticillatis aut fere oppositis.)

526. SICII N GIA, (SICKINGIE) wIIID, nov. gen. - Calyx quinquedentatus; corolla campanulata; capsula lignosa, bilocularis, bivalvis; sporae alatae.

527. MYRSINE, (MYRSINÉ) IINN. JUsS. IAM. tab. 122. ( sapotis affinis). - Calyx quinquepartitus, parvus, persistens; corolla semi vel quinquefida, connivens; stamina brevia, epipetala, corollae laciniis opposita; ovarium globosum, corollam interne replens; stigma magnum, striatum, lanigerum, exsertum; drupa monospora, nuce quinqueloculari; (bacca immatura pentaspora, matura monospora; GAERTNER.)

528. BLA D I A, (BLADHIE) тнUNB, I,AM. tab. 133. (ex ordine plantt. sedis incertae). - Calyx brevis, quinquepartitus; corolla rotata, quinquefida; drupa baccaeformis, monospora; spora arillata.

529. PAEDERIA, (PÉDERE, Danaïde) IINN. IUSS. JAM. tab. 166. fig. 1. DANAIS PERs. (rubiacea 7.) - Calyx parvus, quinquedentatus ; corolla infundibuliformis, puinquelcba, interne fauce villosa vel hirsuta; antherae oblongae, fero sessiles, inclusae; stylus apico 
bipartitus; bacca umbilicata, coronata, nitida, ovalis, fragilis, apice bivalvis, dispora.

530. DANAIS, (DANAÏ̈E) commers. (rubiacea 4.). - Calyx quinquedentatus, brevissimus; corolla monopetala, infundibuliformis; tubo longo, limbo quinquelobo, lobis oblongis, erectis; filamenta corolla multo longius exserta; stylus simpliciter bifidus, corollae tubum longitudine exaequans; capsula bilocularis, polyspora, ad medium dehiscens in duas partes; sporae margine membranaceae ${ }^{*}$ ).

53I. DISODEA, (DISODEE) (LYGODISODEA) nurz. PAv. - Calyx quinquepartitus; corolla infundi. buliformis; capsula compressa, unilocularis, basi bivalvis, dispora; receptaculum filiforme; sporae orbiculatac, membrana cinctae.

532. RA U IV OLFIA, (RAUW'OLFE) PLUM. IINN. JUss. LAM. tab. 172. (apocinea 3.) - Calyx minimus, quirquedentatus, persistens; corolla tubulosa, infundibuliformis, limbo quinquepartito; basi glandulosa; stamina brevissina; stylus brevis; stigma capitatum; drupa baccata, subgloboso rotundata, succulenta, ex uno latere striata; nuce biloculari, dispora; sporae solitariae. - (Frutices erectae, foliis verticillatis quatuor; flores saepe terminales, corynbosi.)

533. VALLESIA, (VALLESIE) FuIz. PAV. (apocinea). RAUWWOLFIA cav, WIIID. - Calyx minimus; corolla hypocrateriformis, utrinque globosa, faux inflata; stamina fauci inserta; drupae duae, divergen. tes; nux striato reticulata, unilocularis.

534. CESTRUM, (CEstre, Cestreau) IINN. JUSs. IAM. tab.II2. JASMINOIDES TOURN. (solanea 2.)

Calyx

*) Hoc genus, ab auctoribus quibusdam cum PAEDERIA conjunctum, plurimis characteribus differt; secundum cl. VENTEATI observationes sejunctum proposuit JAUME st. HILAIRE si vel ipse ordo rubiacearum, quo locumteset, non juberet. 
Caly $x$ brevis, urceolatus, quinquedentatus; corolia calyce multo longior, tubulosa, tubo tenui, vix dilatato ; infundibuliformis, limbo plicato, quinquefido, $\mathrm{l}_{2}$ ciniis acutis marginatis; stamina glabra; filumenta inser. ta medio tubi saepe aut dente brevi in medio munita, aut nuda edentula; non exserta; antherae tetragonae; stigma obtusum, interdum bilobum: bacca ovalis, bilocularis, (unilocularis GAERTN.) polyspora; sporae angulato rotundatas. - (Frutices; folia interdum persistentia; pedunculi axillares, multiflori.)

535. FAGRAEA, (FAGRÉ) THUNB. JUSS. IAM. tab. 16\%. (apocineis affinis). - Calyx campanulatus, quinquepartitus, laciniis margine rnembranaceis; corolla infundibuliformis, limbo qrinquepartito, retorto, tubo longissimo, apice dilato; stamina quinque tubo inserta; ovarium liberum; stigma peltatum; bacca oralis, contoita carnosa, bilocularis, epidermite tecta, poly: вpora; sporae globosae rotundatae.

539. TOURNEFORTIA, ( TOURNEFORTE, Pittone), LINN. JUss. lam, tab. 95. PITTONIA plum. 227. fig. 2. (borraginea I.). - Calyx brevis, quinquepartitus; corolla infundibnliformis, limbo patente, quinquefido, tubo basi globoso; stamina versus summum tubi inclusa; antherae mon exsertas; stigma inte. grum; bacsa minima, apice duobus poris perforata, supora, continens nuces duas vol quatuor biloculares disporas. - (Frutices; folia saepo tactu aspera; flores in cyma vel spica laterali.)

540. STRYCHNOS, (STRYCHNOS, Vomique) IINN. LAIr. tab. 119. IGNATIA LINN. suppl. WILLD. TENTANCOTTA rETz. RoXB. *) (apocyneis valde affinis). Culyx quinquepartitus aut quinquefidus, cadu. cus; corolla tubulosa, limbo quinquefido aut quinquepartito, patente; stigma incrassatum; bacca globosa, magnथ,

*) Quae sub hoc genere a roxburgro deseripta est species, fere verum novum genus constituit, 
magna, cortice saepissime fere lignosa, fragilis, unilocularis, interne pulposa, polyspora (rarius monotrispora); sporae insertae recéptaculo contrali; embryo planus, tenuis et ad summum perisporii magni et cornei positus. - (Arbores aut frutices non lactescentes; rami cylindrici; folia opposita, integra; flores in corymbo axillari aut terminali.)

54r. CAPSICUM, (CAPSIQUF, Piment) tours. IINN. JUss. IArr. tab. 116. (solanea 2.). Calyx quinquefidus; coroila rotata, limbo magno patente, plicato, quinquefido, tubo brevi; antherae oblongae conniventes, longitudinaliter dehircentes; stigma obtusum; bacca exsucca, interdum basi trilocularis, corticata, polyspora, - (Trunci elongacio herbacea aut suffrutescens; folia geminata; flores extraaxillares, solitarii; sporae paucas.)

542. SOLA N UM, (SOLANUM, Morelle) IINN. IAM. tab. 115. (solanca 2.). Calyx quinquefidus, persistens; corolla totata aut campanulata, limbo magno, patente, plicato, quinquefido vel lobato, tubo brevi; antherae subcoalitae, couniventes, oblongae, apice poro gemino dehiscentes, (passim liberae, inprimis in corolla basi hyalina); stigma obtusum; bacca saepissime rotundata, succulenta, apice punctata, bilocularis cum placenta adnata dissepimento aut multilocularis; loculi semidivisi in LYCOPERSICON, polyspora. (Elongatio trunci herbacea aut frutescens, inermis, rarius spinosa, munita aculeis, interdum scandens; folia simplicia. saepissime geminata, interdum pinnata cum impari; pedunculi axillares, numorosi, unimultifori, extraaxillares, sejuncti aut terminalesq)

543: ULLOA, (ULLOE) - JOUANULLOA RUIz. PAv. - Calyx ovatus, inflatus, quinquepartitus; co: rolla tubulosa, fauce globosa; stigma oblongum; bacca bilocularis, calyce magno obvoluta; sporae renifornes.

544. JABOROSA, (JABOROSE) JUSs. IAM, tab. 114. (solanoa 2.), - Calyx brevis, quinquefidus; 
corolla multo calyce longior tubulosa, limbo quinquefida; filamenta plana, ad summum tubi inserła; stigma capitatum; fructus bacca? ...

545. A T ROPA, (ATROPE, BELLADONE) LINN. juss. IAM. illustr. tab. I14. BELLADONA TOURN. (solanea 2.). - Calyx quinquefidus, vesiculosus; corolla campanulata, duplo calyce longior, quinquefida; staminum filamenta filiformia, distantia; antherae breves; bacca fere globosa, calyce persistente vestita, bilocularis, polyspora; placentae adhaerens dissepimento mediante lamina membranacea. - (Frutices aut herbaecaulescentes; folia saepe geminata; flores extraaxillares, fere solitarii aut dispositi in parvos glomerulos.)

546. NICA N DRA, (NICANDRE) ADANS. JUSS. GAERTN. ATROPA LINN. CALYDERMOS flor, peruv. (solanea 2.). - Calyx pentagonus, angulis compressis, quinquepartirus, laciniis sagittatis, maturitate conniventibus fructum tegentibus; corolla magna, campanulata, quinquefida, lobis parum distinctis; filamenta filiformia, incurva, basi dilatata, superiora versus conniventia, ovarium tegunt; antherae ovales; bacca sphaerica, carnosa (exsucca rutz.), tri - quinquelocularis per placentae suturas, tota per calycem magnum angulis compressis membranaceum tecta, polyspora. (Calyx ergsus RU1z.)

547. SARACHA, (SARACHE) antt. flor, poruv. (olanea). - Bacca unilocularis per aborturn, fero ad medium calyci involuta; calyx quinquangulatus; $c 0$. rolla rotato campanulata; sporae in cellulis nidulantes; receptaculum carnosum *),

548. M A N.

*) Differt hoe certe proprium genus a Physsalide et Nicandra: corolla rotata, staminibus erectis, bacca, uniloculari, calyce vix semicincta; ab Atropa LINN. calyce distinctum quinquefido, corolla rotato campanulata, laciniis aequalibus reflexis; staminibus ercctis, infcrue dilatatis, basi corollae insertis; stylo crecto, stigmate 
548. MA NDRAGORA. ( MANDRAGORE)

TOURN. JUSS. GAERTN. ATROPA IINN. IAM. (solanea 2.). - Calyx turbinatus, quinquefidus; corolla campanulata, fere calyce duplo longior, quinquefida: filamenta staminuın basi dilatata et conniventia, apice fliformia et incurva, emarginata; ovarium ad basin duabus glandulis munitum; stigma capitatum, striatum; bacca globosa, solida, receptaculis introrsum dehiscentibus polyspora. - (Herbao radiculis maximis; folia radicalia; elongationes nudae aphyllae multae, uniflorae, internascentes foliis.)

549. L I N IK I A. (LINKIE) PERs. ench. p. 219. DESFONTAINIA auctt: flor. peruv. (solanea). - Calyx quinquepartitus, laciniis lineari lanceolatis, erectis, corolla campanulata, tubo pentagono; antherae sagittatao ${ }^{*}$ ).

550. PHYSALIS, (PHYSALIS, Coqueret) IINN. juss. LAm. tab 116 . ALKEKENGI tounN. (solanea 2.) - Calyx quinquefidus, inflatus, maturitate vesiculosus fructum includens; corolla magna campanulato - rotata, quinquefida; staminum antherae oblongae, conniventes; stigma obtusum; bacca inter calycem inflatum globosa, bilocularis, polyspora; sporae reniformes. - (Elongatio trunci herbacea aut frutescens; folia quarundam specierum geminata; flores extraaxillares, solitarii, aut plurimi coaliti.)

55 I. E L.

truncato; bacca uniloculari, sporis compressis nidulan. tibus in totidem cellulis. (Scopolia carniolica huic generi valde adfinis.) - Praeterea cavanilles hoc genus ad ATHOPAs refert et cum eO PERSOON ellch. p. 219. nuIz sane non temerarius contrarium probare videtur.

*) Aliud genus sub nominc LINKIA edidit CAvANILLIg icon. tab. 187. e nuva Hollandia. - Calyce quatrifido, ad basin tubiformi; stamina quatuor, filamenta nulla; antherae rectae, ad medium laciniarum pusitae; ovarium stylo recurvo, fliformi superatum; legumen ovale, polysporum. 
55 I. ELLYSIA, (ELLISE) IINN. JUSS. IAM. tab. 197. (borraginea 2.). - Calyx profunde quin: quefidus, patens; corolla calyce multo-minor, infundibu= liformis, angustata, recta, quinquefida; stamina non ${ }^{\circ}$ exserta; antherue rotundatas; stigma bifidum; capsula baccaeformis, sicca, coriacea, bilocularis, bivalvis, valvulis calyce in forma stellae adhaerentibus, loculis disporis; spora altera super alteram. - (Herba humifusa; folia pinnatifida; pedunculi uniflori.)

552. SESSEA, (SESSEE) auctores fl. Peruv. Corolla infundibuliformis; stigma bilobum, lobo altero breviori; capsula teres, curvata, unilocularis, bivalvis, valvulis bifidis.

553. LYCIUM, (LICIET, Jasminoïdes) LINN. JUss. IAM. tab. 112. JASMINOIDES TOUnN. (solanea 2.). - Calyx brevis, urceolatus, tri- aut quinque. fidus, aut quinquedentatus; corolla calyce multo lon. gior tubulosa, tubo cylindrico, limbo plano, quatriquinquefido, aut quinquelobo, fauce clausa; staminum filamenta inflata, basi barbata, medio corollae inserta, interdum exserta; stigma bifium, aut striatum; bacca rotundata, bilocularis, polyspora, - (Frutices fere semper spinosae; ramis terminatis in mucrones; folia interdum fasciculata; flores axillares, solitarii aut geminati.)

554. POGONIA, (POGONIE) ANDreit. (campanulata). - Calyx pentaphyllus, persistens; corolla in. fundibuliformis, ore villis ciauso, limbo quinquefido; stylus sursum subcurvatus; stigma concavum; drupa compressa, quatrilocularis.

555. MOU TABIA, (MOUTABIE) AUBL. JUss. CRYPTOSTOMUM Schreg. WiLID. (ex ord. plantt. incertao sedis). - Calyx tubulosus, quinquefidus; corolla, monopetala, infundibuliformis, laciniis quinque inaequalibus, ad summum calyci inserta; nectirolyma (quasi antherae uno filamento lato, ad apicem curvo, unitae) monophyllum, quinquedentatum, anthexa sub 
singulo dente faucem corollae claudens; 'ovarium liberum ; stigma capitatum; bacca pruniformis, exsucca, trilocularis, loculis monosporis; sporue arillatae, basi umbilico munitae, nidulantes in pulpa; - (umbilico hoc genus proximum famliae sapotearum.)

556. ROPOUREA, (ROPOURIER) AURT. IAM. tab. 121. CAMAX schres. (ex ordine plantt, incertae sedis). - Calyx quinquepartitus vel quinquefidus; Corolla hypogyna, monopetala, rotata, limbo quinquelobo, tubo brevissimo; stamina quinque inserta super coroliam, alternantia cum limbi lobis; ovarium liberum; stigma tri-quatrifidum ; bacca magua, ovalis, villosa, quatrilocularis, loculis introrsum pulposis et polys sporis.

557. TRİUERA. (TRIGUÉRE) cAv. WIIID. Juss. LAM. tab. 114. ( solanea 2.). - Calyx quinquefidus; corolla campanulata, irregularis, ad faucem dilatata, ad limbum inaequalem leviter quinquefido, plicata; nectarolyma breve, ovarium cingens, quinquedentatum, membranaceum; filamenta scilicet hrevissima, dilatata, ad basin coalita videntur et vel inserta nectarolymati; antherce oblongae, sub ovario in conume coalitae; stigmu capitatum; drupa parva, globoso, quatrilocularis; leculis disporis, ad medium calyce tecta. - (Elongatio trunci herbacea; pedunculi extraaxillares, biflori.)

558. SOLAN.DRA, (SOLANDRE) 8WARZ. WILID. IAM. tab. 114. (solanea). - Catyx quinquefidus, sim. plex, longus, fructu maturo rumpens iu duo triave segmenta; corollu clavato - infundibuliformis, maxima, laciniis quinque undulatis; bacca ovalis, acuminata, quatrilocularis, polyspora.

559. MENAIS, (MENAIS) IINN. JUss. (borraginea 1.). - Calux triphyllus vel tripartitus, persistens ; corolla by pocrateriformis, limbo plano, quinquepartito, tubo longo; untherue subulatae, fere sessiles juxta corollae faucem; stigmata duo oblonga in stylo 
uno; bacca globosa, quatrilocularis, tetraspora; sporae solitariae.

560. LEEA, (LÉE) rpYEN. IINN. JUSS. STAPHYLEA BURM. AQUILICIA CAVAN. (sapotearum familiae vicina). - Calyx campanulatus, quinquefidus; corolla monopetala, tubo brevi, limbo quinquefido, laciiis inaequalibus; nectarolyma monophyllum, tubulosum, certe tubo corollae inpositum, quinquelobum, apice bifidum, erectum; (squamae?): flores masculi: stamina quinque basi corollae inserta, et inter squamas nectarolymatis internas alternantes posita;' stylus unus; stigma unum; ovarium abortiens; flores feminei: squamae ut in floribus masculis; internao minores; staminum rudimenta; ovarium liberum, stylus unus et stigma unum; bacca infera, globosa, pentaspora. (Nam est Monoica secundum JUSSIEU.)

56r. AQUILI C I A, (AQUILICIE) IINN. JUSs. cav. diss. 7. p. 372. tab. 218 . (meliacea 2.). - Calyx turbinatus, quinquedentatus; corollae petala quinque ovalia; staminum quinque filamenta coalita in tubum urceolatum quinquelobum; cintherae interne et inter singulum lobum affixao; stigma obtusum; capsula carnosa, sphaeroïdea, quinquesulca, quinquelocularis, loculis monosporis. - (Frutex habitu sambuci; folia bipinnata; flores in corymbis. ) *).

562. CHRYSOPHYLLUM, (CHRYSOPHYLLE, Caïmitier) IINN. JUss. IANr. tab.120. CAiNITO PLUm. (sapotea). - Calyx quinquepartitus; corolla campanulata, quinquepartita vel quinqueloba, laciniis vel lobis alternis, patulis; stigma fere quinquefidum;; bacca magna, globosa, decemlocularis, decaspora, (etiam mono-dispora); sporce compressae, umblico notatae.

563. B A S.

*) Pers. chlí. I. p. 233. congenerem Aquiliciam cum $\tau \omega$ Leea habet, secundum juss, gen, p. 176 . 
563. R A S OVIA, (BASSOVE) Auret, Guy. t. 85. Juss. LAMr. tab. 102. (planta sedis incertae). Calyx quinquepartitus vel fissus: corolla monopetela, rotata, hypogyna, limbo quinquepartito, laciniis punctatis; stamina quinque brevia, basi laciniarum corollae inserta; ovarium liberum, in disco positum; stylus brevis; stigma obtusum; bacca ovalis, nodulnsa, superficie inaequali, polyspora; sporae reniformes, membrana emarginatae, nidulantes in pulpa.

564. A RION A, (ARIONE) C.AvAN. ic. t. 383. (daphhnacea). Calyx inferus infundibuliformis, basi munitus duabus bracteis; corolla infundibuliformis, tubo longo cylindrico, limbo quinquefido; autherae inclusae; ovarium squamis munitum; stigmata bilamellata; bacca globosa, bilocularis. *)

565. D I GERA, (DIGERE) Forsk. IUss. (amaranthacea 1.). - Cdlyx quinquefidus, externe bibracteatus; laciniae tres interiores in cylindrum conniventes; filamenta staminum quinque distincta; stylus simplex; stigmata duo; capsula monospora. - (Elongatio trunci pendula, striata; folia alterna, glabra; pedunculi axillares, recti.)

566. PACURIA, (PACOURIER) AUBi. Guy. t. 105. Juss. WILLUGHBEIA scireb.' (apocinea 3.) Calyx quinquepartitus, persistens; corollae tubus brevis, limbus undulatus, quinquepartitus, oblique versus; stamina quinque brevia; antherce cuspidatae; stylus simplex; stigma ovalo super discum rotundatum, colloratum, duobus mucronibus terminatum; bacca pyriformis, magna, intus pulposa, polyspora; sporae angulosae, magnae, durae, in pulpa nidulantes. - (Frutex lactescens, rami nodosi, scandentes; folia opposita ad ramorum internodios, integerrima; flores in race mis axillaribus,) Locum post Ambelaniam tenet.

$$
\text { 56? } \mathrm{R} A \text {. }
$$

*) Habitu vix a QUINCHA ruAlo diversa; sed partitus fruclificationis quilusdam sane distincta est ARIONA. 
567. RAPANEA, (RAPANEE) AuBlet. Guy. tab. 46. suss. (berberideis affinis). - Culyx parvus, quinque vel sexfidus; corolla hypogyna, quinque - sexpartita; stamina quinque aut sex, basi laciniarum calycis opposita; stylus brevissimus; stigma simplex; bacca pisiformis, penta-hoxaspora, spora unica saepe perfecta. - (Frutex ramosa; folia alterna coriacea; flores subsessiles super ramos,)

568. AID IA, (AIDIE) IOUREIR. Juss. mss. (caprifoliacea 2.) - Calyx tubulosus, quinquedentatus; corolla hypocrateriformis, tubulosa, terminata limbo plano, fauce tubi lanata; anthere quinque sessiles, lineares, corollae incisuris insertae; bacca monospora, parva, ovalis, umbilicata.

569. HELIXANTHEPA, (HELIXANTHERE) IoUREIR, conch. I. 176. JUss. mss. (caprifolicea 2.). - Calyx truncatus, basi bractea una carnosa muni. tus; corolla tubulosa, margine inversa profundo quinquefida, interne ornata appendiculo urcenlato quinquangulo, quinquefido, et cingente stylum; stamina quinq $l i 3$ inserta fauci corollae; antherae spiraliter tortae; stylus unus; stigma simplex; bacca oblonga; spora una. (Frutex parasitica; folia lanceolata, integerrima; flores in spicis axillaribus.)

57\%. BAEOBOTRYS, (BÉOBOTRYS) тоRST.

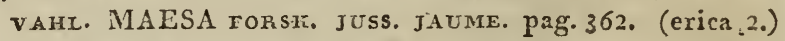
- Calyx semiadhaerens duplex, superus: exterior diphyllus vel squamaeformis, squarnis duabus basin cingentibus, persistentibus; interior campanulatus, quinquedentatus ${ }^{*}$ ); corolla tubuloso campanulata, quinquefida; stamina quinque brevia; antherae ovales; ovarium somiadhaerens; bacca globosa, unilocularis, cincta ad medium calyce cujus laciniae conniventes supra versus tegendo coronant; loculo polysporo; sporae insertae receptaculo centrali.

* 57. $\mathrm{ROH}$.

* Calyx squamula duplici infra persistente cinctus jussifr. 
57r. ROHRIA, (ROHRIE) schreb. tabura AUBIET. Juss. (plantt. incertae sedis). - Calyx sexpartitus, munitus bracteis tribus; corolla hypogyna, monopetala, bilabiata, labio superiori concavo, recto, longo; inferiore breviori, latiori et bilobo; stamina quinque epipetala, quorum quatuor didyma, inserta super labium superius, qrintum longissimum et ad basin labii inferioris; ovarium liberum, trigonum; stylus longus; stigma tuilobum; fructus ....? * *). Genus Bignonearum familiae affinis.

572. PYRGUS, (PYRGE) LOUR. JAUME. II. 352. (sedis incertae). Calyx quinquedentatus, dentibus brevibus; corolla rotata, quinqueloba; stamina brevia, súper corollam aftixa, super medium approximata; antherae magnae, in conum una versus alteram inclinatae; ovarium liberuin; stylus brevis; bacca globosa, calyco tecta; spora una parva, non arillata. - (Frutex fo. liis alternis; floribus racemosis.) Genus ante Pongatium inserendum.

573. CAMPYLUS, (CAMPYLE) LOUR. JAUME. II. p. $35 \%$ ( sedis incertae). - Calyx tubulosus, quinquefidus, laciniis inaequelibus, tuberculosis; corolla tubulosa, bilabiata, labio superiori recto, arguto, acuminato; inferiori ovali; síamina quinque inaequalia, fundo tubi inserta; ovarium liberum; stigma quinquelobum in stylo uno; capsula quinquelocularis; sporae numerosae. (Frutex repens, foliis cordatis, acuminatis, integerrimis; flores racemosi terminales.) - Ante Nievembergiam isserendum genus **).

574. STA-

*) Diversa sane a Tapura tertiae elassi3.

**) TApUpait atelzt. juss. (sedis incertae). Huic classi inseruit JAUME II. p. 348. Calyx sexpartitus, trous bracteis munitus; corolla liypogyna, monopetala, bilabiata; Labio superiori concavo, recto; inferiori breviori, latiori, biłobo; stamina quinque epitetala; quatuor didynama, labio superiori inserta, quintum longissimun et collocatum ad basin lalii inforioris; ovarium libcrum, uriangulum; stylus longus; stigma trilobum; frus stus ....; geuts Bignoncis affine. 
574. STAPELIA, (STAPEIIE) LINN. JUSS. LAM. tab. 178. ASCLEPIAS TOUn, (apocinea 2.). C'alyx parvus, quinquefidus, persistens; corolla rotata; magna, plana, quinquopartita, laciniis ad basin, inferno dilatatis, acuminatis, versus apicom angustatis; necturolyma operculata stellula duplex, una altera quinquepartita, sexus organa tegens; filumenta plana; antherae filamontis adnatao; styli nulli; stipmata duo parum apparentes; folliculi duo oblongi, subulati; sporae plumosáe. - (Plantae crassae; truncus aphyllus, tuberculis obsitus; pedunculi uniflori.) ${ }^{*}$ ).

575 CYNANCHUM, ( CYNANQUE ). IIN. JUss. IAM. tab. 277. APOCYNUM et PERIPLOCA TOURN. (spocinea 2.). Calyx minimus, quinquedentatus, persistens; corolla rotata, tubo brevissimo, limbo plano, quinquepartito, laciniis lougis linoaribus; fauco coronata nectarolymate urceolato, cylindrico, oblongo, quinquefido vel quinquedentato, laciniato, simplici; organa sexus ut in Asclepiade; stylus fero nullus; stigmata duo; folliculi duo oblongi acuminati; sporae plumosae. - (Frutices aut suffrutices, saepe volubiles: folia opposita; flores axillares aut terminales, in spicam dispositi, aut in corymbum, aut in umbellam.)

576. GDNOLOBIUM, (GONOLOBE) MIcIIAX. amer. bor. I, IIg. VINCETOXICUM WALT. ASCLEPIAS cAv. (apocinea 2.). - Calyx quinquefidus; Corolla rotata, quinquopartita, laciniis profundis; appendix (nectarolyma) brevissima, inclusa; corpus. cula pollinis fere transverse locata propter styli brevita. tem; stylus discoïdeo pentagonus; folliculi ut plurimum costati vel angulati; reliqua ut in Asclepias et Cynanchum. - (Planta truuco scandente; incola America septentrionalis.)

\section{* VIN.}

*) Persoon enchir. p. 277. 380. ob differentias specificas, MASSONI eX autopsia redditas, WILIDENOVIANIS praeferre roluit, et hinc e digynia in monogynian cum aiiis generibus retulit, quem sequuti sumus. 
* VINCETOXICUIM, ( VINCETOXICE) MOENCH. ASCLEPIAS IINN. - Corolle erocta; nectrvium squamulas 8. callas quinque, carnosas, retusas sistens. - ( $\mathrm{Ob}$ appendices uniformes solidas Cynachis proximum hoc peculiare genus, sed distinctum locum tenot.

57\%. PERIPLOCA. (PERIPLOQUE) IINN. JUSs, IAMt. tab. 17\%. APOCYNUM TOURN. (apocinea 2.). Calyx minimus, quinquefidus, persisteus; corolla rotata, flana, quinquepartita; face circumdata corona urceolata (nectarolyma), quinquefida, selis quinǫue exsertis, stigma capitatum, pontagonum, glandulis quinque stipitatis munitum; staminum filamenta conniventia, villosa; folliculi duo oblongi, ventricosi, peritagoni; sporce planae, plumosae. - (Frutices saepe scandentes; folia opposita ; fores fere dispositi in corymbum axillarem aut terminalení)

578. MATELEA, (MATELÉE) AUzLET. IUss. LAM. tab. 179. HOSTEA WrLID. (apocinea 1.). - Ca. lyx quinquepartitus; corolla rotata, tubo brevi, limbo quinquepartito patente; antherae fere sessiles, in capitulum pentagonum conjunctae; styli dun; duo stigmata dilatata et cernua; folliculus unus (altaro saepe abortivo) longus, verrucis scaber, pentagonus, angulis acutis; sporce planae, margine crenuiatao.

577. APOCYNUM, (APOCIN), TOURN, LINN. Juss. LAM. tab. 176. (apocinea 2.). - Calyx minimus, quinquefidus; corolla; campanulata, ad medium usque quinquefida; lacinis ad summum reflexis aut deorsum revolutis; filamenta quinque, cum laciniis alterna; antherae couniventes basi bificae; 'styins subnullus; stigme latum, bilobum; avarium gininģue corpusculis glandnlosis cinctum; folliculi duo longi, acuminati, lineares; sporcie plumosae, arista longa munitae. - Friatices ant herbac; folia opposita; flores in corymbo aut panicula, axillari aut terminali.)

580. PE R- 
580. PER, ULARIA, (PERGULAIRE) LINN. JUss. IA Arr. tab. 176. (apocinea 2.). - Calyx persistens, quinquefidus; corolla contorta, hypocrateriformis, tubo calyce longiori cylinárico; limbo plano quin. quepartito, laciniis obtusis; nectarolyma quinque squamae parvae, semisagittatae, apice mucronatae, basi dentatae; antherae sessiles; stylus nullus; stigma magnum, truncatum; folliculi duo, recti, saepius asperi, versus apicem attenuati; sporae plumosae. - (Frutices elongatione trunci volubili; folia opposita; flores axillares, fero corymbosi.) ${ }^{*}$ ).

581. A S L EPIAS, (ASCLEPIAS, Asclepiade) TOURN. IINN. LAM. tab. 175. APOCYNUM TOUBN. (apocynea 2.). - Calyx parvus quinquefidus, laciniis minimis, persistens; corolla rotata, quinquepartita, laciniis aut planis aut reflexis; nectarolyma squamae aut peculiaria corpuscula cum lacinis corollae alterna, callosa, ovata, concava, cucullata, in corniculum velata; tukum staminum plane obtegentia, applicata contra partes fructificationis, exeunte ex ipsorum fundo saepe filo inclinato versus floris medium. Circum haec corpuscula quinquangula posita sunt autherae quinque aut fila pendula applanata, unoquoque diviso in loculos duos; ad summum horum ipsorum corpusculorum, in peripheria sui platea inveniuntur foveae quinque parvae, cum flamentis alternantos, quarum quaeque continet corpusculum nigrum, quod elongatur in filameuta duo plus minusve longa, (antherae variorum auctórum) interdum truncata apice dilatata, ophatulaeformia; haec filamenta sese insinuunt in loculos propinquos antherarum, ita, ut quaeque anthera respondeat huic coalitioni duorum corpusculorum, quorum

*) ANDREw hoc genus ad GYNANDRIAE decandriam retulit, propter nectarolyma, ut definire voluit, duplex genitalia involvons; squamulis exterioribus quinque cuspidatis, interioribus quinque - (vera filamenta!) membranaceis, singulis stamina occultantibus (antheras), duobus alternis (quae tumc non pistillo, ut est in Gymandris, sunt adnexae.) 
rum quodque continet duas antheras; stamina in tubum approximata; antherae corneas, longitudinaliter dehiscentes; ovarium duplex; styli duo brevissimi; stigma unum, simplex, pentagonum; sporae plumosae. (Herbae aut frutices; pedunculi solitarii, axillares, multiflori; flores dispositi in umbellam, cincti involucro polyphyllo.) VENTENAT.

582. CEROPEGIA, (CĹROPEGE) IrNN: JUss: IAM. tab. 179. APOCYNUM TOURx. (apocynea 2.). - Calyx minimus, quinquedentatus, persistens; corolla ad summum tubulosa, basi ventricosa, limbo quinquefido aut quinquedentato, fere connivente; antherae hastatae; stylus vix ullus; stigmata duo; folliculi duo cylindracei, recti, longissimi; sporae plumosia - - (Plantae volubiles; folia opposita; pedunculi axillares aut terminales; bi-tri-multifori; flores in umbellam dispositi.)

583. MELODINUS, (MELODIN) Forst. JUss. IAM. tab. 179. (apocynea 3.). - Calyx quinquepartitus persistens; corolla hypocrateriformis, tubulosa, limbi laciniis quinque obliquis, fauce munita et clausa coronula appendiculata quinquefida, lacera, ut in $\mathrm{Ne}$ rio; stigmata duo; bacca carnosa, globosa, magnna, bilocularis, pulpa farcta, polyspora; dissepimentum carnosum; sporae compressae.

\section{Flore inonopetalo, supero.}

584. SAMOLUS, (SAMOLE) TOURN. IINN. JUSs. LAM, illustr. tab. 101. (primulacea 3.). - Calyx semisuperus, quinquefidus, persistens; corolla hypocrateriformis, quinquefida (loba), squamae quinqu fue fliformes o sinu corollae pronatae; stamina quinque basi corollae inserta, ejusque laciniis opposita, tubo inclusa; ovarium basi adhaerens; capsula ad basin calyce cincta, unilocularis, sporifera, dehiscens apice quinque valvularum hinc quinquedentata, polyspora; pla- 
conta centralis libera, pedicellata; perisporium carno. sum; embryo parumper cylindricus, leviter curvatus; radicula infera.)

585. VIRECTA, (VIRECTE) LINN. suppl, VAHL. Juss. RONDELETEA potтв. (rubiacoa 4.). - Calyx quinquefidus dentibus interpositis; corolla infundibuliformis, limbo quinquepartito; antherae non exsertao, conniventes; stigma bipartitum; capsula infera, angulosa, coronata, villosa, unilocularis, polyspora; recepta* culum internum carnosum, tectum uno sporarum ordine.

586. SIPA NEA, (SIPANEE) AUBLET. LAM. tab. 15I. VIRECTA vahl. (rubiacea). - Calyx quinquepartitus; corolla infundibuliformis, quinqueloba; capsula coronata, bilocularis, bipartibilis, polyspora.

587. BELLONIA, (BELLON) PLUM. IIN. sWAP.Z. Juss. LAM. tab. I49. (rubiacea 4.). - Calyx quinquefidus, laciniis lanceolatis; corolla rotata, limbo plano, quinquepartito, laciniis obtusis; tubo brevi; antherae conniventes, praulisper exsertae; capsula infera, turbinata, laciniis calycinis conniventibus rostro terminata, unilocularis, polyspora.

588 MACROCNEMUM, (MACPOCNEME) BROWN. IINN. SWARZ, VAYL. (auct. flor. peruv. genus PERUVIA certe parum ab hoc genere diversum) JUss. (rubiacea 4.). Culyx turbinatus, campanulato cyathiformis, quinquedentatus; corolla campanulata aut infundibulifornis, quinqueloba; stamina inserta calycis tubo; antherae inclusae; capsula infera, turbinata, bilocularis, polyspora; sporae imbricatae. (Frutices.)

589. VANGUIERA, (VANGUIER) vahL. JUss. LAM. tab. 159. VAVANGA VAHL. (rubiacea 8.). Caly $x$ minimus, quincquedentatus, patens; corolla parva, campanulato globosa, quinquepartita, fauce villosa; antherae oblongas, vix exsertae; stigma bilamellatum; bacca infera, pomiformis, umbilicata, quatri-quinquelocularis, non coronata, tetra - pertaspora; sporae amyg- 
amygdalinae. Juss, (Arbor humilis; fores axillares; pedunculati: folia magna, ovalia, laxa, punctata.) ·

590. DENTELLA , (DENTELLINE) Forst. JUSS. LAM.. tab. IIS. OLDENLANDIA IAM. RUMPH. (rubiacea 4.). - Caly $x$ quinquepartitus sive fissus, sub limbo attenuatus, superus; corolla infundibulifor. mis, limbo recto quinquefido, laciniis rectis, tridentatis, dente medio longiori; filamenta brevia; antherae oblongae, non exsertae; capsula pisiformis, coronata, bilocularis, loculis monosporis; receptacula sporifera in loculis proeminenţ.

59I. CHIMARRHIS, (CHIMARRHIS, bois de riviere) JACQ. Juss. (rubiacea 7.). - Calyx integer; corolla infundibuliformis, tubo brevi; limbo plano, quinquefido, laciniis interne villosis; stamina inserta tubi summo; filamenta basi villosa; stigma bipartitum; capsula infera ovö̈lea, coronata, bilocularis,- loculis monosporis, bivalvis, valvulis apice dehiscentibus; sporae duae.

592. RONDELETIA, (RONDELÈTE) PLUM. IINN. JUs8. LAM. tab. 162. ('rubiacea 4.) *). - Calyx quinquefidus; corolla infundibuliformis, limbo obtuso, quinquepartito, laciniis subrotundis, planiusculis, tubo apice parumper ventricoso; antherae inclusae; bacca capsularis exsucca, rotundata, coronata, infera, quatri. locularis, apice in duas partes dehiscens, polyspora.(Fratices aut arbores mediae aititudinis; flores in corymbo terminali.)

593. CINCH ONA, (QUINQUINA) IINN. JUSS. LAM. tab. 154 . (rubiacoa 4.). - Caly $x$ campanulatus, turbinatus, quinquedontatus; corolla tubulosa, infundibuliformis, limbo quinquefido, ant quinquepartito, recto, glabro, ut plurimum interne hirto, laciniis pla. nis aut raflexis; stamina inserta versus medium tubi, brevia

*) Non omnes hujus geueris species videntur vere ipsi esse triwlendale. 
brevia, in corolla villosa, fere exserta in corclia glabra; stigma clavatum, fere bifidum; capsula oblonga, teres, coronata, bilocularis, matura bipartibilis, aut qua. si in duas capsulas introrsum dehiscentes disceders, dissepimento parallelo; sporae numerosae, compressae, margine membranaceo lato cinctae, affixae in lquoquo loculo placentae centrali. - (Arbor mediae altitudinis; flores in panicula terminali.)

*) 595. COS MIBUENA, (COSMiBUENE) áuctorum flor. peruv. (rubiacea). - Calyx deciduns, quinquedentatus; corollae tubus longissimus, limbo reflexo obliquo glabro; stamina sub fauce inserta; capsula apice bivalvis, valvulis involutis, subbilocularis; receptacu1a lamellata, hinc valvulis adpressa, inde sporifera; sporae avales, cinctae ala lineari et reticulata.

596. PINKNEA, (PINKNÉE) Juchaux. (rubiacea). - Calycis laciniae inaequales, una altera bractezeformes, magnae; corolla longe tubulosa : filamenta ad basin tubi inserta; capsula superne bivalvis, valvulis medio septiferis; sporae alatae transverse invicem superpositae. $=$ (PINKNEA et COSMIBUENA Cina chonae generi maxime affines)

597. PORTLANDI A, (PORTLANDE) вRоWN. IINN. JUss. IAM. tab. 162. (rubiacea 4.). - Calyx magnus, "quinquefidus s. pentaphyllus; corolla clavato infundibuliformis, limbo quinquefido, laciniis patentereflexis, tubo vix dilatato; stamina ad basin tubi inserta; antherae longae, erectae, fere exsertae; capsu'a ovali- globosa, fere pentagona, retusa, coronata calycis limbo, simillima capsulae acanthearum; bilocularis, bi= valvis, valvulis duplicatis bifidis, polyspora.

598. ROELLA, (ROELLE) LINN. JUSS. IAN. tab. 123. (campanulea 1.). - Calyx turbinatus, quinque-

*) ExAstonta. Staminibus erectis, quum in Cinchona sint inclusa; auctores florae peruviuna ceusent EXASTOAIAE species ad Portlandias esse referendàs. 
quefidus, laciniis magnis, dentatis; corolla infundibu. liformis, limbo quinquepartito, fundo clauso valvulis staminiferis; filamenta basi dilstata; stigma hifidum; capsula infera, cylindrica, bilocularis, coronata laciniis calycinis; (capsula unilocularis apice pervia GAERTN.). - Folia ciliata, conferta circum flores forma bractearum; flores axillares aut terminales, solitarii.

599. GOODENIA, (GOODÉNE) SIIITH. CAV. venter. (campanulea 1.) - Calyx monophyllus, apice quinquepartitus, adbaerens; corolla labiaka, monopetala, irregularis, labiata; tubo brevissimo, ad basin usque longitudinaliter fisso, genitalia exserens, labio superiori recto bifido, labio inferiori reflexo, trifido; quinquefida, undulata, limbo laterali; staminum quinque filamenta exserta in spatio, quodest inter duas lacinias labii superioris; stigma urceolatum, ciliatum; capsula linearis, bivalvis, uni-bilocularis, polyspora, dissepimento parallelo; sporae compressae, imbricatae, nuarginata.

600. PHYTEUMA, (PHYTEUME, raponcule) LINN. JUSS. LAM. tab. 124. RAPUNCULUS TOURN. (campanulea 4.). - Caly $x$ quinquefidus; corolla rotara, primo tereti conica, dein quinquepartita, laciniis linearibus, laxis, tubo brevissimo; staminum filamenta parum ad basin dilatata; antherae oblongae, rectae; stigina bi - vel trifidum; capsula infera, bi - aut trilocularis, dehiscens foraminulo laterali, polyspora. (Flores bracteati, interdum remoti, saepissime terminales, capitati aut spicati.)

60\%. PETROMARULA, (PETROMARULE) VENT. RAPUNCULUS baUh. (campannlea). - Calyx quinquefidus; corolla primum ovata, deinceps quinquepartita, laciniis refexis; filamenta basi dilatata; stigna capitatum, simplex; ovarium globosum; capsula... ? *)

602. FOR.

*) Ilabitu et filumentis cum Canpanulae genere, - corolla rotata, quamvis facie aliena, cum Pliytcumate convenit; set. ab ntroque stylo crasso, et stigmate simplici capitato differt, hinc vere verum cst groprium genus. 
602. FORGESIA, (FORGESIE) commers. JUSS. gen. DEFFORGIA rAm. ill. tab. I25. ESCALLONIA sirtit? (certe huic valde affinis?) (campanulea 1.) Culyx turbinatus, quinquefidus; corolla quinquepartita aut petala quinque basi dilatata et coalita, apice rostrata; antherae oblongae filamentis adnatae; stigma bilobum; capsula sporifera, semiadhaerens, stylo bipartito acuminata, bilocularis, apice in duas valvulas dehiscens, poJjspora.

603. TRACHELIUM, (TRACHELIE) TOURA. IINN. JUss. IArr. tab. 126. (campanulea I.). - Calyx quinquefiaus; corclla infundibuliformis, tubo longo, limbo quinquelobo; stumina quinque filamentis basi non dilatatis; stylus longus; stigma globosum; capsula infera, trilo. cularis, polyspora. - (Flores in corymbum termina. lem dispositi, bracteis parvis muniti.).

60. CAMPANULA, (CAMPANULE) TOURN. IINN. JUSS. LAMI. tab. r23. PRISMATOCARPOS L'HE. Fет. (campańulea 1.). - Calyx quinque aut decemfidus; lacinis quinçue reflexis; corolla campanulata, quinquefida, fundo clauso valvulis staminiferis; fila. menta basi dilatata: antherce oblongae; stigma tri-quinquefidum; capsula infera, tri-rarius quinquelocularis; variat enim forma vel naturas vel arţis beneficio lo. culis; polyspara, poris lateraliuas fere semper, dehiscens. - (Plantae herbaceae, interdum suffrutescontes; flores bracteati, saepe axillares, solitarii aut fasciculati, interdum terminales dispositi in spicam aut paniculam, rarissime solitarii.)

605. LEGOUSIA, (LEGOUSÉE) DURANDE. PRISMATOCARPUS I'IEPT. POLEMO panulea). - Corolla sibhypocrateriformis;' stigma bitrifidum; capsula longissima, prismatico - cylindrioa, bi-trilocularis, polyspora.

6̊5. L O BELIA, (LOBÉLIE) LIX. JUSS. IAM. †ab. 724. IRAPUNTIUM et TRACHELIUM TOUPN. (campanulea 10.) - Calyx quinqnedentatus, persi- 
stens; corollu monopelata, tubulosa, irregularis, longitudinaliter ad infmurn fissa; limbo bilabiato, labio superiori bipartito, labio inferiori maiori trifido; stamina sex; flamenta longitudine tubi; antherae in cylindrum coalitae; stigma simplex, rarius bifidum, villosum; capsula infera, bi - trilocularis, apice dehiscens, polyspora. - (Elongatio tranci erecta aut decumbens, interdum suffrutescens; flores duabus bracteis muniti, solitarii aut axillares; vel tribùs bracteis muniti et in spicam laxam et terminalem dis. positi).

609. SCAE V OLA, '(SCAEVOLE) IINN. JUSS. LAM. tab. 124. GOODENIA cURTIS. LOBELIA pLUM. (campanulea 1.) - Calyx quinquefidus; corolla monopetala, irregularis, tubo longitudinaliter usque ad basin fisso; limbo quinquefido, laciniis inaqualibus unilateralibus; styli e corollae tubi fissuris exeunt; stigma urcealatum, ciliatum, planum; drupa infera, rotundata, nuda aut calycis laciniis coronata, striata, monospora, nuce bilocuları mono - dispora.

610. SELLIEPA, (SELLIERE) nov. gen. cavan. (campanulea). - Calyx fuperus, quinquepartitus; corolla irregularis, tubo longitudinaliter fiffo; stigma globosum; bacca unilocularis, polyspora; sporae quadrifarium imbricatae, marginatae, hoc est sacculis totidem membranaceis contentae *).

6II. VELLEIA, (VELLEIA) sMith transact. IIIr. (campanulea Juss, ord. 165.) - Calyx triphyllus, inferus; corolla tubulosa, supra hians, limbo quinquefido: capsula quadrivalvis, unilocularis, polyspora, Sporae imbricatae **)。

6I2. SCHOE.

*) Ob sporas imliricatas sane a COODENEIS removendum, etsí auctor huins foneris ob pericarpii naturam achuc incex: tus hacrere nuisis videatur.

**) GOODENIAE proximum; diversum calyce naguo, iris pisyllo, inforo; capsula quadrivalri: corolla non ad basin 
* 6iz. SCHOEPFIA (SCHOEPFIE) SCHPEB. CO. DONIUMI VAHL, - Calyx duplex, exterior bifidus, inferus, interior integer, superus; stigma capitatum; drupa monospora.

6r3. MA T THIOLA, MAT'THIOLE) PLUM. IINN. Juss. (rubiacea s.) - Calyx superus, oblongus, marginibus integer; corolla tubulosa, orificio dilatato, infundibuliformis, tubo corollae tenui, elongato, limbo integro, undulato; stamina inclusa; stigma paulisper incrassatum; drupa cerasiformis, depressa, coronata, nuce sexloculari, hexaspora.

6I4. MORINDA, (MORINDE, ROYOC) VAILI. IINN. IUSS. LAM. tab, 153. ROYOC PIUM. BADCUDUS RUMPH. (rubiacea) Flores aggregati et collecti in capitulum superpositum receptaculo sphaerico sloboso; $c a$ lyx proprius, obsolete quinque dentatus; corolla tubulosa, subinfundibuliformis, supera, quinquefida, patens; antherae lineares, fero sessiles, non exsertae; fructus formatus o multis baccis approximato aggregatis, ob mutuim compressionem angulatis, apice retusae, quatrilocularibus, di-tetrasporis; sporae une latere pla* nae, altero convexae. - (Arbores aut frutices; flores capitati aut axillares aut oppositi foliis).

6I 5. PSYCHOTRI A, (PSYCHOTRE) IINN. JUSs. LAMr. tab. 16i. PSYCIOTROPHUM DROWN (rubiacea \%.) - Culyx parvus, quinquedentatus, persistens; corolla tubulosa, infundibuliformis. quinquefida, stamina summo tubi inserta, fere sessilia, inclusa; bacca coronata calyce, matura striata, bilocularis, rotundato globosa aut ovalis, coriacea, dispora; sporae duae, altero latere planae, altero convexae, sulcatae, osseae; (Frutices aut therbae repentes, flores terminales approximato conferti aut in corymbum dispositi).

6I6. NO NA.

usque fissa; stigmate caeterum GOODENJAE gaudet, habitu herbacco acauli! - genus certe prope SCALVo. LAlH collocandum. 
6I6. NONATELIA, (NONATELIER, AZIER) AUBL. JUSs. IAM. tab. 155. ORIBASIA 5CHREB. PSYCHOTRIA willd. (rubiacea 7.) - Caly $x$ parvus, quirquedentatus; corolla tubulosa, limbo quinquefido, laciniis parum reflexis; stamina exserta; bacca parva, sphaerica, coroplata, striata, quinquelocularis, pentaspora. - (Trunci elongatio herbacea aut frutescens; flores in panicula terminali).

617. COFFE A, (CAFFEYER) IINN. LAM, tab. 160. COFFE raj. (rubiacea $\eta^{\circ}$ ) - Calyx minimus, quatriquinquedentatus, dentibus deciduis; corolla tubulosa, oblonga, fere infundibuli - vel hippocrateriformis, limbo plano quinquepartito; stamina exserta supra tubum; antherae sagittatae; bacca infera, rotundata vel ovoïdea, rarius oblonga, umbilicata, idispora; sponae arillatae, uno latero planae, altero convexae. - (Frutices: flores axillares, fere sessiles).

* Gr8. PO TIMA, - corollae Iaciniae acuminatae, deflexae; bacca monospora; porisporangio in quatuor lobos diviso, et embryone in eius substantia immerso, (convolutae in Coffea arabica); spora una; WILtD. I ACQ.

619. CHIOCOCCA, (CMOCOQUE) BROWN. LINN. JUSS. LAM. tab, I60. (rubiacea 7.) - Calyx quin. quedentatus; corolla infundibuliformis, limbo quinquefido, aequali, laciniis argutis, reflexis; stamina non exserta, medio tubi inserta, fere sessilia; antherae lineares; bucca infera, coronata, rotundata, compressa, dilyma, dispora; sporce oblongae, compressae. (Arbores atit frutices; flores in panicula terminali aut in racemo axillaxi.)

620. SERISSA, (SERISSE) COMMERS. JUSS. IAM. illustr, tab. 151. JUUCHOZIA L'HeRET. DYSODA LOU. REIT. LYCIUMT IINN. (rubiacea 11.) - $\varepsilon_{a l y x}$ quadriquinguefidus; corolla breris, quadri-quinquefida, fance barbata; antherue quatuor, quinque, sessiles; ovarium adhacrens; stylus bifidus; fructus: bacca infera, poly. spora. 
spora *). - (Frutex a Commersonio detecta : foliis oppositis, ad basin per expansionem membranaceam (folii pars inferior, qua cingit aut ambit plus minus trunci elongationem) ciliatam quasi in spinam persistentem coadunatis; odoro post frictionem inamoeno; flo. res saepe solitarii in apice ramorum).

62I. TAPOGOMEA, (TAPOGOME) AUBг. t. 60. JU6s. IAM. tab. 152. CALICOCCA SCHPEB. CEPHAËLlS sw'́Anz. (rubiacea I0.) - Flores capitati in receptaculo paleaceo, et cincto squamis aut calyce communi polyphyllo involucrati, involucro bipartito, mono-penta-pulyphyllo, saepe magtio; caly $x$ proptius minimus quinquedentatus; corolla parra tubulosa, fere infundibuliformis, quinqueloba; antherae quinque fere sessiles, inclusae; stigma bipartitum; bacca infera, ovalis, minima, ad summum glandulosa, continens duas nuces monosporas, altero latere planas, altero convexas. - (Frutices aut herbae fere lignosae; flores capitati saepe terminales).

622. POSOQUERIA; (POSOQUERIER) AUBL. JUSS, LAI. tab. 163. CYRTANTHUS SCMREB. SOLENA

WIILD.

*) SERISSAE characterem genericum THUNBEfG, in nov. generibus suis parte nond, his retulit verbis: calyx ,perianthium monophyllum inferum, glalorum, mini„, inum, quinquedentatum; corolla tubulosa, glabra, ", virescens, quinquepartita; laciniae ovatae, reflexae; ", tubus fauce barbata clausus. Stamina filamenta quinque, ,brevissima, ori tubi inter lacinias inserta; antherae , ovatae, didymae, favo nigrae; pistillum : ovarium su"perum, convexum, glabrum; stylus filiformis, exser"tus, tubo paulo longior; stigma globoso capitatum: "pericarpium: capsula didyma compressa, emargin.ta, ", bilocularis." (Lycium barbatum et iaponicum huc refert; alteram speciem iam je'ssrev eodem proposuit nomine in gen. plantt, et vel L'HER. et IOtREIRo utrasque species a Lycii genere disiumxerunt. Sed I.ycium barbatum a Serissa iaponica adeo discrepat, quoì utra. que minime sub uno genere milutari posse videantur. Practerea THUNEng. iu hac generis definitione magis respexisse videtur ad partes fructificatiunis; Serissae ca= pensis, quam iaponicae. 
wirzo. (rubiacea 4.) - Calyx superus, turbinatus, quinquefido - dentatus; corolla tubulosa, tubo longis simo, limbo patente, quinquepartito, laciniis longis, rectis; fauce pilosa, parum dilatata; antherae exsertae; filamenta inserta fauci corollae; stylus filiformis; stigma fere trifidum; bacca oviformis, magna, coronata, suc. culenta, unilocularis, polyspora.

623. GARDENIA, (GARDENE) ELI, LINN. JUss. IAM. tab. 158. THUNBERGIA MONT. act. holm. (rubiacea 4.) - Calyx quatridentatus aut quatrifidus, laciniis verticalibus, interdum obliquis; corolla primum contorta, infundibuliformis, limbo plano, quinque novemfido, tubo saepius longo; staminum antherae sessiles, insertac ad tubi introitum, parum vel non oxsertae; stylus elevatus; stigma bilobum; bacca exsucca, infera, bi-quatrilocularis, polyspora; sporae numeresae, duplici serie in quoque loculo dispositae. - (Arbores aut frutices; flores fere solitarii, terminales aut axillares; folia verticillata, aut numero tria in una specie).

624. GENIPA, (GENIPAYER) TOURT. IINN. JUSS. IAM. t. 158. f. 2, RUIZ, PAV. GARDENIA swARz. wiIID. (rubiacea 40) - Calyx tubulosus aut turbinatus, marginibus subintegris; corolla cylindrica, hypocrateriformis, calyce duplo longior, limbo magno, patenve, quinquepartito; antherue in fauce tubi sessiles exsertae; stigma clavatum, integrum sive simplex; bacca magna, ovalis, carnosa, apico attenuato truncato, bilocularis, polyspora.

625. STYLOCORYNA', (STYLOCORYNE) cAV: tab. 436. - Calyx superıs, quinquedentatus; corolla infundibuliformis; stylus clavatus; stigma simplex; bacca coronata, bilocularis; sporae numerosae, nidulantes $=($ an praecedenti genori subiungendum? $)=$

626. RANDIA, (RANDIE) HOUST. LINN. JUS8. IAM. t. 156. RUIZ-PAV. GARDENIA WILLD. - Calyx quinquepartitus; laciniis lineari lanceolatis contortis; [10] curolla 
corolla hypocrateriformis, tubulosa, tubo calyce $\nabla$ ix longiori, cylindrico, limbo plano quinquefido; antherae fere sessiles, inclusae; stigma bilobum, lobis oblongis, inaequalibus; bacca exsucca, dissépimento incompleto, semibilocularis, cerasiformis, coriacea, vix calyce tubuloso coronata; sporae plurimae, per quatuor series alternatim superimpositae. - (Frutices spinosae; quatuor spinas verticillatae, aut plurimum duae oppositas in crucem cum foliis; flores axillares, fere solitarii aut approximati et terminales).

627. C A NTHIUM, (CANTHIE) IAM. JUSS. GARDENIA THUNB. IINN. SUPpl. WEBERA WILLD. RONDELETIA IINN. (rubiacea 7.) - Calyx quinquefidus, corolla tubulosa, brevis, quinquefida, patens; stamina non exserta; stylus elevatus; stigma capitatum; bacca ovalis, coronata aut rotundata, nuda, corticosa, bilocularis, dispora; sporae uno latere convexae, altero planae; sulco longitudinali munitae. *) - (Frutices spinosae; hores fere sessiles, axillares et terminales).

628. TOCOYENA, (TCCOYENNE) AUBL. t. 50, juss. IAM. tab. 163. VCRIANA WIILD. (rubiacea 4.) Calyx superus, quinquedentatus, turbinatus; corolla hypocrateriformis, tubulosa, tubo longissimo, superiora versus dilatato; limbo quinquepartito dilatato; fauce ventricosa, nuda; antherae fere sessiles, vix exsertae exira tubum; stylus pilosus parum clavato inflatus super stigma bilamellatum; capsula baccaeformis, ovalis, coronata, bilocularis, polyspora; sporue pulpa cinctae.

629. CANEPHORA, (CANEPHORE) JURS. LAM. tab. 151. (rubiacea 10.) - Tres ad sex flores positi ad sumroum calycis commuris tubulosi, quinque parvis laciniis insigniti, disiuncti invicom squamis; Calyx proprius quinque vel sexfidus, longitudine corollae; corolla parva, campanulata, subaequalis, quinque - sex loba;

*) CUPIA convenit, si genere distinctum a CANTHIO, in caeteris, nisi quod stigma clavatum; frutices inermes. 
Joha; antherae quinque, sex, oblongae, sessiles; stigma bifidum; fructus capsula? coronata, pisiformis, bilocularis, mono - dispora.

630. BERTIERA, (BERTIERE) AUBL. tab. 69. vaHi. Juss. IAM. tab. 165. (rubiacea 4.) - Calyx tur. binatus, quinquedentatus; corolla tubulosa, limbo quinquefido, fauce villosa; antherae fere sessiles, vix exsertae; stigma bilamellatum; bacca infera, pisiformis, coronata, bilocularis, polyspora; dissepimeatum sporiferum.

63I. LONICERA, (LONICERE Chevrefeville) IINN. LAM. tab. 150. SYMPIIORICARPOS, DIERVILLA, XYLOSTEUM, CAPRIFOLIUN JUSs. (caprifolia 1.) - Calyx superus, quinquedentatus, basi brac̀teatus; corolla irregularis, laciniıs fere aequalibus, tubulosa, quinquefida; stumina quinque longitudine corollae; stigmia globosum; bacca infera, umbilicata, bi tri-quatrilocularis, polyspora. - (Frutices elongatione trunci volubili ;i folia basi connata in quibus. dam speciebus; flores sessiles, approximati in capitulum et terminales, aut verticillati et axillares).

\section{* Caprifoliun suss. periclyvenum} Juss. floribus in pedicello solitariis, laciniis fere irregularibus et bilabiatis; tubo ut plurimum longo, bacca distincta triloculari.

632. XYLOSTEUMI TOURN. JUSS. LAM, tab; 150. CHAMAECERASUS TOURN. LONICERA LiNN. (CHAMí́lisier) (caprifolia §.). Fedunculi hifori, inserti pedicellorum summo; caly $x$ quinquedentatus, basi munitus bracteis approximatis invicem aut adnatis; corolla infundibuliformis ant campanulata, quinqueloba aut quinquefića, laciniis fere aequalibus; stumina exserta; stigma incrassatum; fructus: baccae duae; basi connatao, biloculares, polysporae; aut in unicam, apice duobus umbilicis notatam, coadunatae. - (Frutices elongatione trunci recta; pedunculi biflori, axillares, solitarii). 
633. SYMPHORICARPOS, Dir... JUss. IAM. t. 15\%. (SYMPHORIA) (caprifolia 1.) - Caly $x$ parrus quarridentatus, basi bracteis minoribus munitus; corolla tubulosa, brevis, limbo quinquefido, laciniis fere aequalibus; stamina quinque vix exserta; stigma globosum; bacca ovali globosa, coronata, quatrilocularis, loculis interdum duobus abortivis, tetraspora.

634. DIERVILLA, (DIERVILLE) TourN. Juss IANI. tab. 150. LONICERA IINN. (caprifolia 1.) Calyx oblongus, quinquefidus, basi bracteatus; corolla duplo longior, infundibuliformis, quinquefida, laciniis patentibus; stamina quinque exserta; stigma capitatum capsula oblonga, acuta, non cbronata, quatrilocularis, polyspora; sporce parvac.

635. TRIOSTEUM, (TRIOSTE) IIN⿴. JUss. IAM. tab. 150. (caprifolia I.) - Calyx superus, quinquefidus, laciniis lanceolatis, persistentibus, basi bracteatus, longitudine corollae; corolla tubulosa, vix calyce longior, quinqueloba, lobis inaequalibus; stamina quinque inclusa; stigma incrassatum; bacca infera, coronata, ovali-globosa. trilocularis, trispora.- (Plan. tae herbaceae, erectae; poidoli basi coaliti ; flores nume. rosi, axillares, sessiles).

636. PLACOMA, (PLACOME, plocome) AIT: GMEI. WILID. - Calyx superus, quinquedentatus; corolla campanulata, quinquefida; bacca trilocularis, 10culis monosporis.

637. MUSSAEN D A, (MUSSENDE) HERPM. LN JAcQ. JUss. IArr. tab. 157. (rubiacea 4,) - Calyx quin: quefidus, laciniis linearibus; corolla infundibuliformis, tubo longo ad faucem dilatato, limbo plano, quinquepartito; antherae oblongae, fere sessiles, inclusao; stigmata dıo, crassiuscula; capsula ovalis, oblonga, mem. branacea, ad summum nuda aut coronata, bilocularis, loculis placenta disiunctis ; polyspora ; receptaculum exsertum, sporiferum et dissepimenta efformans; spoxis quadrifario dispositis; stamina tubo interno inserta. - 
(Frutices saevissime villosae; flores sacpe in corymbo).

638. SABICEA, (SABICE) AUBL. t. 75. JUS8.LAM. tab. 165. SCHVVENKFELDIA SCMPEE. WILLD. (rubiacea 9.) - Involusrum di - iptraphyllum; calyx oblongus, quinquefidus; corolla infundibuli - vel hypocrateriformis, tubo longo, gracili, limbo quinguepartito, laciniis mucronatis, argutis; antherae fere sessiles, parum aut non exsertae; stigma quinquepartitum; bacca coronata, pisiformis, quinquelocularis, polyspora. (Numorus partium interdum quaternarius.) - Frutices trunci elongatione volubili; flores axillares, fere sessiles.

639. DUHA MELI A , (DUHAVELE) LINN. JACQ. Juss.' LAM. tab. 155. (rubiacea 9.) - Calyx parvus, quinquefidus ; corolla tubulosa, oblonga, pentagona, limbo quinquefido; stamina quinque inserta versus tubi medium; antherue oblongae; stigma obtusum; bacca infera, ovalis, apice coronata, striata, retusa, quinquelocularis, polyspora. - (Frutices : racerai partiti; folia verticillata; flores in cyma terminali):

640. ERITHALIS, (ERITHAL) вROWN. IINN. Juss. IAM. tab. 159. (rubiacea 8.) - Cialyx minimus, urceolatus, quinquedentatus; corolla quinquepartía, laciniis patentibus aut recurvis, tubo brevi; stamina quinque exserta; stigma punctatum; bacca infera, pisiformis, coronata, dęcemstriata, decemlocularis, decaspora. (Frutex: flores dispositi in corymbum axitlarem ot torminalem).

\section{Flore tripetato.}

6.fT. TRIPHASIA, (TRIPIIASIE) LOUP. conch. p. 189. (hosperidea 1.) - Calyx brevis, tridentatus; corollae petala tria, basi in tubum approxinata, ad summum reversa; stamina quinque receptaculo inseria ; ova- 
rium simplex; stylus incrassatus ; stigma trigonum; bacca ovalis, monospora. - (Frutex foliis ternatis: flores solitarii; spinae axillares).

\section{Flore tetrapetalo.}

642. CADABA, (CADABE) Forsi. Juss. STROE. MIA vAHL. WILLD. CIEOME IINN. (capparidea 1.) Calyx parvus, tetraphyllus, caducus; corolla tetrapetala, petala unguiculata, interdum nulla; stamina quinque inserta ad summum pedicelli qui ovarium sustinet; filamenta filiformia; antherae rectae; ovarium pedicellatum, ad pedicelli basin nectarolymate vel appendice ligulato munitum; stylus nullus; fructus: siliqua pedicellata, cylindracea, corticosa, unilocularis, bivalyis, interne pulposa, polyspora, sporis triplici serie dispo, sitis, pulpa cinctis. - (Arbores aut frutex foliis simplicibus, parvis, glandulis munitis; flores in spica terminali).

\section{Flore infero, pentapetalo.}

643. HIPTELLA, (HRTELLE) IINN. JUSS. IAM, tab. 138. (rosacea 6.) - Caly $x$ quinquepartitus, laciniis reflexis; corollae potala quinque; stamina tria, quinque, sex, onmino ómnia eodem calycis latere inserta; filamenta longissima, spiraliter torta, persistentia; stylus longus, ad basin ovarii lateralis; drupa baccata, exsucca, ovalis, compressa, obsoleto apice dilatata, fero trigona, uuce "uniloculäri, monospora; spora magna ; stylo persistente terminata. - (Frutices aut arbores modiae altitudinis; foliis stipulaceis; floribus in spica aut racemo terminali).

644. WIBELIA , (TVIBELEE) PERs, enchir. pag. 210. PAYROLA juss. LAM tab. 126. PAYPAYROLA AULI, t. 90. cMer. (incerize sedis). - Calyx inferub, quin- 
qquinquepartitus; corollae petala quinque, super discum inserta; uuguibus rectis, in tubum conniventia, apico reflexa; stamina inserta ut corolla; antherae approximatae; ovarium liberum, biloculare; stigma bilobum, pericarpium biloculare fructus - ?

645. RHAMNUS, (NERPRUN) TOURN, LINN. SUSS. IAM. tab, 128. FRANGULA et ALATERNUS ToURN. (rhamnea 3.) - Calyx urceolato campanulatus, quatri quinquefidus; corollue petala minima quatuor, quinque, (rarissimo nulia); squamaeforaia qua includuxt stamina quatuor, quinque, calyci inserta; stigma bi - tri quadrifidum; bacca bi--quatrilocularis, tri-tetraspora. (Frutices aut' arbores mediae altitudinis, ramis interdum terminatis in mucronem; foliis alternis, interdum fere oppositis, floribus axillaribus, passim polygamis, interdum dioicis perabortum.

646. COND ALIA, (CONDALIE) cAv. ZIZIPHUS ortega (rhamnea). - Calyx ureeclatus, quadri-quinquedentatus (fissus?), porsistens; coroila infundibuli. formis: nulla. „Exs. Discus glandulosus ; stylus unus; drupa baccata, bilocularis, ceva, spoligiosa; sporae lentiformes, liberae (drupa ovata, nuce monospora PERs.) - Rhanino vel Zizipho omnino affinis: cf. Pers. pag. 241. Arbitror fere pertinere ad no. VIII.

* 647. OENOPLEA, (OENOPLÉ) michaux. ZIZIPHOS Willd. - Calyx patens, quinquefidus; petula quinque; discus carnosus; drupa succosa, bilocularis, altero loculo saepe abortiente, monospora. (Frutex flore Rhąmni, fructu Ziziphi. - Umbellao axillares. WIILD. Racemi subterminales. MrCHAUX. flores hermaphroditi AITON, dioici $\mathrm{MICH}$. -).

648. CEANOTHUS, (CÉANOTHE) IIN. JUSS. I.AMr. tab. 129. (rhamnea 4.) - Calyx turbinatus, quinquefidus; corollae petala quinque, squamaeformia, saccata; concava aut sulcuta in ligulam ad apicem; basi unguiculata; stamina quinquo petalis inclusa; ovarium triangulum; stylus trifidus; stignzata tria; bacca 
eapsulaeformis, exsucca, sustentata basi calycis persistente, continense tres, quatuor coccos, trilocularis, cocculis monosporis, introrsum dehisientibus. - (Frutices foliis-alternis; flores dispositi in racemos axillares aut terruinales).

649. CELASTRUS, (CELASTRE) IINN. LHE. RET. JUSS. HAENKEA? ąuct. f. peruv. CASSINA IAx.? (rhamñea 1.) - Calyx minimus, quinquelobus, coroilae petala quinque unguiculata, patentia; filamenta longa ; ovarium parvum, in discum latum decemstriatum depressum; stylus brevis; stigmata tria ; capsula trigona, trilncularis, trivalvis; sporce insertae margini centrali dissep.mentorum, glabrae, semiarillatae, arillo quatrifido, calyptratae. - (Frutices aut arbores mediae altitudinis; tolia alterna; pedunculi axillares, dichotorni, multiflori).

* CATHA, (CATHE) Forsk. juss. (sedis incertae). - Culyx quinquedentatus, enervius; petala corollae quinque patentia; stamina brevia; ovarium liberum, basi disco urceoli forma cinctum undulato, inter stamina exserto; stylus brevis; capsula oblonga, bi - trilocularis, di - irispora. - (Arbores ramis alternis, foliis oppositis aut alternis, interdum spinis in axillis ramorum; pedunculi axillares, solitarii; - genus rhamneis analogum).

650. MALESHERBIA, (MALESHERBIE) DoMLEY. RUIz-PAv. (sedis incertae). - Calyx tubulosus, coloratus, limbo quinquefido; corollae petala quinque lanceolata, breviora laciniis calycinis; squamae multao tubi fauce insertae; stamina filiformia; antherae rectae, sagittatae; ovarium stipitatum; styli tres insertae fere ad basin ovarii; stigma obtusum; capsula triangularis, unilocularis, trivalvis; sporae numerosae.

651. HAENKEA, (IIENKÉE) auctorum florao peruv. tab. 6. (rhamnea? sedis incertae). - Calyx parvus, diphyllus, altero biiobo, quinquedentatus; corolla (urceolata) petala quinque sursum convexa, deoraum 
concava; stamina quinque petalis opposita circumposita disco; ovarium neutiquam calyci adhaerens, sed semiinfusum disco; stigma sessile, obtusum, trigonum; drupa (capsula) ovalis, unilocularis, bivalvis (nux trilocularis); sporae duae (rarius una, tres, quatuor) ob. longae, osseae, arillo pulposo vestitae.

652. SENA CIA, (SENAKĖ) - CELASTRUS LAM. (rhamnea). - Calyx minimus, quinquedentatus; çcrolla pentapetala; capsula sphaeçica, pedunculata, bịvalvis, tetraspora; sporae angulatae, nudae.

653. EVONYINUS, ( EVONYME, FUSAIN ) TOURN. xINN. JUSs. LArr, tab. r3r. (rhamnea r.) Calyx patens, quatri - quinquepartitus vel fissus, laciniis planis, basi particulatim tectus disco peltato; corollae petala quatuor aut quinque inserta exteriori margine disci; staminum quodque glandula exserta sub disco insertum ; capsula tetra - pentagona ; tri - quinque locularis, tri-Iquinquevalvis; sporae arillato calyptratae aut cinctae tunica propria et colorata, atque pulposa, insertae angulo centrali loculorum. - (Arbores plus minus elatae, ramis tetragonis; foliis oppositis; stipulis vix notabilibus; pedunculis axillaribus, solitariis, oppositis, unifloris aut multifloris; floribus umbellatis).

654. BURSARIA, (BURSARIE) cavan. JUss. mss. (rhamea I.) - Caly $x$ inferus, quinquefidus; corollae petala quinque linearia, erecta, applicata disco in fundo calycis locato; stigma simplex; ovarium super discur collocatum; 'capsula cordata, compressa, matura bipartibilis in duos coccos compressos et dehiscens in duas tresve valvulas; in loculo quoque tres aut quatuor sporae reniformes, resinae inclusac.

655. STAAVIA, (STAAVIE) DAHL. THUNB. prodr. VENT. BRUNIA TLUK, MORIS, LEVISANUS SCHREB. (rhamnea 2.) - Flores in receptaculo paleaceo aggregati barbato villoso; involucrum: calyx communis polyphyllus, foliolis coloratis patentibus; calyx proprius ad. haerons quinquepartitus; corollue petala quinque unguir culą⿰氵 
culata; stamina calyci insorta ; styli duo, coaliti; capsula (bacca) ovoïdea, coronata laciniis calycinis, corticosa, unilocularis, trivalvis, monospora. - (Suffrutices; foliz linearia, remota, saepe punctata).

6j6. OPELIA, (OPELIE) roxB. - culyx quinquedentatus; corolla pentapetala; nectsioly-mata quinque, staminibus a!ternantia; baccu monospora.

657. IMBRICARIA, (BARDOTTIER) SMIT. JUNGIA GAERTN. PIIILADELPIUUS gMeI. - Curollae petala quinque; stigma capitatum; capsula calyce tecta bilocularis, polyspora. sMITH - (Capsulu unilocularis evalvis, apice ampla foramine dehiscens. GAERTN.) *)

658. EUPAPEA, (EUPARE) BANKS. GAERTA, IAMr, tab. I33. - Calyx pentaphyllus; corolla pentadodecapetala; bacca exsucca supera, globasa, unilocularis, polyspora.

639. CORTESIA, (CORTESIE) cAvan. tab. 177. (borraginea vel sebestana sec. cavax.) - Culyx inferus, decemdentatus; coroila quinquepartita; siylus bipartitus; stigma globoso peltatum; bäcca dispora. (ROCHEFOR. TIAE generi, a swarzio condito, valdo affinis secundum auctoritatem CAVANILIES).

660. BILLARDIERA, (BILLARDIÉRE) SMITIY. (solanea 2.) - Calyx quinquefidus; corollae petala quinque tubulosa, cum foliolis calycinis alternantia; nectavolyma nullum; filamenta antheris parum versatilibus terminata; ovarium adhaerens, cylindricum; stylus brevis; stigma bilobum; bacca supera, ellyptica, tillosa, stylo terminata, polyspora; sporae rotundatae, paulisper cmarginatae; embryo minimus.

66I. RUY.

*) Ab hoc genere IMBRICARIA commer,s. Juss, ita distind cta: Calyx octo laciniis coriaccis, duplici serie dispasitis; corolla rotata, laciniis octo multifilis; appendices octo filiformes, curvatae; stamina octo ; fructus: pomum octoloculare, octosporum; loculi plures, pluzesque sporae abortiut; sporae furma irregulari. 
66r. RUYSCHIA, (RUISCHE) JACQ. JUSs, xAM, tab. 135. fig. 1. inf. SOUROUBEA AUBI, JUSs. IAM. tab. 135. f. 2. inf. (sub PUYSCHIA), LOGANIA scop. omex. (ex ordine plantt. sedis incertae). - Calyx parvus, penta - hexaphyllus, involucratus, foliolis im. bricatis, persistens; corollae petala quinque magna, reflexa; filamenta plana; ovarium liberum; stigma sessile, divisuris fere cruciatis angulosis quatuor, quinque; stylus nullus; bacca subquinquelocularis, polyspora. (Hoc genus affine Commersoniae, distinctum a LAMAriro).

662. SURUBEA, (SOUROUBEA) AUBL. JUSs. xAM. ill. t. 135. f. 2. (incertae sedis). - Calyx penta - hexa= phyllus, coriaceus; corollae petala quinque, hypogyna, carnosa; stamina quinque hypogyna, petalis opposita, cernua; filamenta basi dilatata; ovarium liberum, quinquestriatum; stylus fere nullus; stigma planum, quinqueradiatum; fructus quinquelocularis. ...

663. VITIS, (VIGNE) TOURN. IINN. JUSS. IAN. tab. 145. (vinifera). - Calyx minimus, quinquedentae tus; corolla pentapetala, petalis fere semper apice calyptratim cohaerontibus, deciduis non separabilibus, emarcitis; stylus nullus; stigma capitatum; bacca supera, glo. bosa, rarius ovoïdea, unilocularis (quinquelocularis ante maturitatem) pentaspora. - (Folia simplicia aut ternata, aut digitata, aut bipinnata; flores dispositi in racemum, interdum sexpotalis formati, et muniti sex sta. minibus).

664. LEONIA . (LEONIE) PAV. et FuIz. - Cals'x minimus, quinquepartitus; curollae petala quinque, obovata, concava; nectarolyma cyathiforme, quinquecres natum, antheriferum; bucca corticosa, maxima, unilocularis; sporae obovatae, nidulantes.

665. ESCALLONIA, (ESCALLONE) INN, suppl. sMrTI. STEPEOXYLON ruUIz fl. peruv. (erica. 1.) - Calyx quinquedentatus, persistens; corollue petala quinque lingulata; ovarium adhaerens; stigma capitatum, depressum; fructus: bucca rotundata, sporifera, bilocu- 
laris, calyce coronata, polyspora; sporae multae. (Capsula bilocularis, calyce coronata, basi rimis duabus, quatuor dehiscens; receptucula duo in loculo singulo; dissepimentum interrupturn. sYsT. veg. florae peruvianse p. 65: sub STEREOXYLO). Nam secundium recentes JUssieur observationes (annales de Museum no. 7.) hoc genus, antea sub onagrariis locatum, locum tenet post vaccinium, (hinc ericas familiam habet), quocum plurimum convonit.

"666. IMA N G IFERA, (MANGUIEP) REEL. RUMPH. xINN. JAcQ. JUSS. IAM. tab. 138, (terebintacea I.) Calyx quinquefidus; corolla pentapetala, petalis calyco longioribus; antherae fore cordatae; drupa oblonga, subreniformis, includens nucem oblongam compressam, extroreum filamentosam, monosporam *). - (Arbores foliis simplicibus et floribus parvis, albis, in panicula terminali bracteatis; interdum per ovarii abortnm masculis).

*667. SCHREBERA, (SCHRERERA) RETz. IINN. WILID. Juss. CELASTRUS vaHL. MANGIFERA rotrb. (rhamnea 2.) - Calyx parvus, quinquepartitus; jcorolla pentapetala, infundibuliformis; stamina inserta fauci corollà; filamenta interne squama ciliata munita; antherae rotundatae; styli duo; stigmata dủo; ovarium liberum Juss: (ovarium disco orbiculari cinctum); fructus bilocularis, disporus (drupa sicca, ovali oblonga, nuçe semibiloculari). ${ }^{*}$ )

668. ELAE O DE N D U M, (OLIVETIER) JAeQ. LAMT. tab. 132. RHAMNUS LINN. SIDEROXYLON IINN. (rhamnea). - Calyx penta - decaphyllus, foliolis quinque squamosis subrotundis, concavis; corolla quinqquepartita (pentapetala), laciniis o çavis ;

*) Jam vero immortalis varr post QUALEAE genus aliud IMANGIFEPAE genus condidit in nuperrima specierum. aurea editione sua.

*) PERs, enchir. pag. 7. aliam ROXEUígIJ plantam sub SCHPEBERA exhibuit; an igitur sequenti generivinculo fanniliari coniuncta haec SCHREBEHA? 
cavis; nectarolymata lineari subulata, petaliformia; drupa exsucca, ovalis, nuce bi-triloculari, (loculis duobus abortientibus), mono-dispora; putamen crassum, durum, bi-trisulcatum.

669. WALKERA, (WALYÈPE) SCHREB. WILID. MEESIA GAERTN. IANi. tab. 143. (menispermea). Caiyx inferus, quinquepartitus; corolla pentapetala; drupae quinque, monosporae, uniloculares; nuces reniformes.

670. CONORIA, (CONORIE) JUSS. CONOHORIA Aubiet. Guy. tab. 95. (berberidea). Calyx quin. quepartitus; corollae petala quinque conniventia, et introrsum rounita peialo minori, ad summúm patente; stamina quinquo ungue petalorum interiorum inserta; antherae subsessiles; siylus unus; stigma simplex; frucius....? (Frutex ramosus, foliis oppositis stipula= tis; floribus in spicis.)

67r. R I A N A, (RTANE) AUBLET. Guy. tab.94. JUss. (berberideis affinis). - Calyx quinquepartitus; $\mathrm{CO}^{*}$ rollae petala quinque basi conniventia, limbo revolu. to; squamae quinque minores, hypogynae, interne es alternando cum petalis positae; stamina quinque super squamarum unguem inserta; antherae subsessiles; ovavium quinquestriatum; stylus incrassatus; stigma clavatưm; capsula oblonga, unilocularis, trivalvis, valvulis compressis, ad medium sporis tribus onustis. (Frutex ramosa, foliis oppositis, floribus in Bpicis terminalibus.)

672. C AL ISPE R M UM, (CALISPERME) IOUR. conch. I, 194. (berberideis affinis). - Calyx quinquefidus, laciniis minimis; persistens: corolla pentapetala; stamina quinque basi petalorum inserta; stigma in stylo simplici incrassatun; bacca unilocularis; sporae numerosae, in pulpa baccae locatae. (Frutex scandens foliis alternis; foribus in racemis oblongis, simplicibus, subterminalibus.) 
673. COR,Y NOCARPUS, (CORYNOCARPE) EORST. LINN. suppl. JUSS. LAM. tab. 143. (berberidea). - Calyx inferus, pentaphyllus; copolla pentapetala; nectarolyma squamae quinque petaliformes, cum petalis alternantes, minores, interne munitae ad basin glandula; stamina quinque petalorum unguibus inserta; antherae oblongae; nux turbinato-clavata, monospora.

674. HUERTEA, (HUERTEE) rUIz. PAv. tab. 6. et 227. (aralioïdea? sedis incertae; sec. auctores $\mathrm{Al}$.pes ruv. rhamnea). - Calyx campanulatus, quinquedenta* tus; corollue petala quinque ovata, sessilia, essicantia, jmo calyce deinceps ita adpressa, ut primo intuitu denticuli ipsius calycis esse decem appareant; stamina quinque ovario apposita, cum petalis alterna; antherae ova. les; ovarium liberum; stylus brevis; stigma bifidum, acutum; drupa? parva, ovalis, calyce associata, conti。 nens nucem? unilocularein (pericarpium enim auctores immaturum offenderunt; ovarium autem illud monosporum indicabat. RUIz. PAV.) una spora repletam.

675. HUMB OLDTIA, (HUMBOLDTE) VAIIx. BATSCHIA vaHt. (leguminosa 2.). - Calyx monoplyyllus, quatripartitus, laciniis oblongis; corolla pentapetala, petalis oblongis, fere aequalibus, vix unguiculatis; stamina corolla multo longiora; antherae oblongae, versatiles; ovarium pedicellatum; sigma obtusum; legunen, oblongum, compressum.

676. CERDANA, (CERDANE) rurz. - pAv. t. 6. (sedis incertae). - Caly $x$ tabilosus, decemstriatus, quinquedentatus; corollae petala quinque unguiculata; antherae oblongne; ovarium liberum, oblongum; stylus tenais; stigmata bifida; drupa oblonga, calyce comitata; mux unilocularis, monospora; necturoiyma cyathiforme, ovarium ambiens; (auctores pericarpium inmaturum of fenderunt, hinc an drupa esset nec ne certificare noluerunt) CORDIAE forsan congener SCHRAD.

677. PILOCARPUS, (PILOCARPE) vaHL eclog. fasc, I. prunr.' (sedis incertae). - Calyx pentaphyllus: 
corolla pentapotala; stamina sub ôvario inserta; capsulae (cocculi duo ad quinque rant.) quinque, (duae - tres abortientes) basi coalitae, elastice dehiscentes, monosporae; sporae arillatae. - (Evonymo valde affine genus.)

678. CEDRELA, (CEDPELE) BROWN. LINH. Jús, LAMr, tab. 137, CEDRUS MiLI, (meliacea 3.) Calyx minimus, quinquedentatus, marcescens; corollu infundibuliformis, pentapetala, petalis obtusis, 'basi dilatata approximatis; vel ipsa basi trifario receptaculo adnatis; fíamenta brevia, distincta; antherae oblongas; ovarium elevatum super discum clavatum incrassatum, cujus basi corolla et summo stamina sunt inserta; stigmts capitatum ; capsula ovoïdea minor, parum lignosa, quinquelocularis, longitudinaliter quinque valvulis dehiscens, polyspora, receptaculum placenta pentagona; tota tecia sporis multis alatis basi ala membranaceis, planis, deorsum imbricatis. - (Arbor; quoad fructum Swieteniae affinis; folia pinnata sine impari; Iores, in panicula laxa.)

\section{CALODENDRUM, (CALODENDRON)} THUNB. diss. JUSS. LAM. journ. d'hist nat. No, 2. t. 3, DICTANINUS LINN. PALLASIA noUTTUYN. (rutacea ? sedis incertae). - Calyx quinquepartitus, parvus, persistens, externe villosus ; corolla pentapetala, petalis canaliculatis, lanceolatis, nnguiculatis, undulatis, barbatis; necturolyma squamae quinque supra locatae petaliformes, apice glandulosae, petalis interpositae, receptaculo inserıa; filumenta staminum quinque, quatuor fertilibus, antheriferis, uno plerumque sterili; ovarium pedicellatum, liberum; stylus lateralis; capsula striis quinque rotata, quinquangularis, quinquelocularis, loculis disporis, calyce associata, pedicellata, quinquevalvis; spurue pisiformes, duae in quaque valvula per abortum.

680. VILL ARESIA, (VILLARESIA) nUIZ-TAY. yuss. (rutacois affinis). - Calyx parvus, quinquefidus; corolla pentapetala; stamina quinque; antherae bi- 
fidae; ovarium superatum stigmate sessili, applanato; capsula oblonga, putamine corticata, in mucronem attenuata, unilocularis, bivalvis; spora und, tetragona, in villo carnoso contenta. (Frutex.)

68I. TODDALIA, ( TODDALI) JUSS. IAM. t. ỉj9. VEPRIS TOURN, PAULLINIA IINN. JACQ. SCOPO. LIA smitif, ined. WILID, CRANZIA schrib. (terebintacea 2.). - Calyx minimus, quinquedentatus; (quinquefidus?); corolla pentapetala; stylus brevis aut nullus; stigma truncato - capitatum; capsula baccata, (exsucca $\mathrm{LAMI}_{\mathrm{A}}$ ), pisiformis, punctata, quatri-quinquelocularis, loculis quibusdam abortientibus, monosporis. - CFrutices spinosae Asiae; folia terma punctata; flores in panicula axillari; fructus obtecta punctis glandulosis.)

\section{POLYCARDIA, (POLYCARDE) JUss.}

xAMr. tab. 132. (rhamnea I.). - Calyx minimus; quinquelobus; corollae petala quinque, rotundata; filamenta brevia; antherae rotundatae; ovarium dilatatum; stigma lobatum; capsula coriacea, tri-quinquelocularis, valvalis tribus vel quinque semiseptiferis; dissepimeutis sporiferis unoquoque latere ad valvularum basin; sporae paucae, oblongae, ad hilum semiarillo laciniato munitae. (Flores tres, quatuor, ex apice foliorum nonnullorum, (petaliformium?) provenientes !)

683. PITTOSPORUM, (PITTOSPORE) BANTS caertin. venten. LAMr. tab. 144. (rhamnea r.). - Ca. lyx inferus, quinquefidus laciniis profundis, hine fero pentaphyllus, statim caducus; corollae petala quinque ad summnm plann, ad basin in conum approximata, unguiculata, fere in tubum urceolatum conniventia; ovarism liberum; capsula fere globosa, parum angulosa, bi - trilocularis, dehiscens in duas vel tres valvulás; loculo quoque tri-tetrasporo, sporis resina cinctis; sporcie pulposae.

684. BYTTNERIA, (BYTTNÉRE) IIN. JUSs. BUTTNERIA wrldd, LAM. tab. 140. (malvacea 6.) Calyx simplex quinquefidus, patens; corolltae petala quinque, 
quinque, concava et ad basin in conum retracta, et intus curvata, ad partem superiorem dilatata divisam in tres lobos, lobis lateralibus brevissimis, medio filiformi, longissimo; nectarolyma pentaphyllum; filamenta staminum connata in cupulam brevem, limbo nectarolymatis quinquedentato, dentibus quinque sterilibus, quinque fertilubus, duabus antheris onustis basi concava petalorum obtectis; ovarium sessile, cinctusn cupula ; stigma quinquangulum; fructus: capsula globosa, muricata pentacocca, formata quinque capsulis fore adbaerentibus, introrsum dehiscentibus, coccis monosporis, quinquevalvis. - (Frutex spinis armata; rami plurimum angu. losi, interdum sarmentosi; media pagina foliorum glandulosa aut basi inflata; flores axillares).

685. AYENIA, (D'AYÉN) IINN. JUSS. LAMI. t. 732. AYENIA MEDIC. monad. p. 125. (malvacea 7.) *) Calux simplex, quinquepartitus, inferus; corollae potala quinque, inserta basi stipite unguibus arcuatis, recurvis, capil-

*) DAYENLA men. monad. p. 122. - Calyx basi monophyl lus, profunde quiriquęf kns; stamina duplicia: monadelpha et disiuncte libera: illa primum tubum formant, dein campanulato dilitata, e tubi margine incisurac orimutur quinque introrsum curvac, et quinque extus curvata filamenta antheris declinatis; haec orimutur in filamentis quiuque semicirculariter curvatis, aî apiccm cordata dilatatis ct incisis, hisque incisuris intrant filancnta montdelpha curvata; incisuris cordatis insident fllamenta imperfecia quinque; ovarium intra tubum filunentorum pedicellatum vel intra campanam tubi insidens; sty?us sime plex; stigma infundinuliforme; fructus: capsulde quinque; singula exirorsum convexa, altero et opposito latere arguto, margine rima parva notato quam intrant receptacuii alae, quibus ctpsulae monosporae comectuntur: L. g. Ayenia pusilla liss. (Dayenia inermis MIEN.) obs, bot. 1782. p. I14. f. 4-7. - AYLNIA зАCQ. autem : calyx Dayeniac; stamina duplicia: Monadelpha, et disiuncto - libera; illa tubo destituta canspauformiter dilatata terminautur filamentis quinque imperfectis; haec in receptaculo orta adscendunt circulariter curvata, apice dilatata incumbunt campanae filamentorum monadelphorum; autherae snbtus declinatae; ovarium iintra filimenta monadelpha; stylus brevis, filiformis; stigma capitatum, quiuquesulcatum. 
capillaribus, longis, in ceronae formam munita, superins dilatata et in siellulam planam conniventia, ad medium glandula pedicellata superdta: cupula staminum tecta stellula a petalis formata, ad limbum nunita dentibus decem, quinque alternis obtusis staminiferis, erectis, quinque filiformibus, monandris; nectarolyma cyathiforme, quinquefidum; stamina sub petalis circa crenam inserta, iisque recondita: ovarium pedicellatum; stigma foro quinquelobum; fructus: capsula globosa, par$\nabla a$, muricata, depressa, quinquesulcata, pentacocca, formata quinque capsulis conniventibus bivalvibus, monosporis, hine fructus quinquelocularis et quinqueral. vis; valvulis bifidis (hinc videtur decemvalvis), elastico dissilientibus; spora solitaria (auctores flora poruv. 3. pag. 11.). Suffrutex humilis, floribus axillaribus, minimis; pedunculi uniflori. ${ }^{*}$ )

686. GLUTA, (GLUTE) IINN. JUss. WILID. (ex ord. plantt. sedis incestao). - Calyx nembranaceus, campanulatus, caducus; corollue petala quinque, calyce multa longiora, apice patentia, medio infero pedicello ovarii adposita et adglutinata, ad summum diffusa; stamina quinque aequaliter inserta ad pedern membranao ovarii; antherae versatiles; ovarium pedicello oblongo columnaeformi insidens; filamenta apice stipitis inserta; fructus...?

687. DIOSMA, (DIOSMA) IINN. LAM. tab. 12\%. (rutacea). - Calyx subquinquepartitus, persistens; covolla pentapetala; nectarolymo discus quinquefidus, ovarium includens; capsulae tres, quinque, coalitae; sporae calyptrato arillatae.

* 689. BURSARIA, (BURSARIE) cav. pav. ruiz. Juss. mss. (rhamnea 1.). - Calyx inferus quinquefido dentatus ; corollae petala quinque erecta, linearia, approximata disco in fundo calycis locato; filamenta rece-

ptacu-

") Hoc et antecedens genus potius ad eorum familiam classis Monalelphiae vel potius malvaceám translocanda, PERsoon. ench. pag. 263. 
ptaculo inserta; ovarium super discum positum; stigina eimplex; capsula cordiformis, compressa, bipartibilis in duas tresve valvulas, uniloculares, loculo quoque tri - tetrasporo, sporis resina cinctis.

689. POIRETIA, (POIRETIE) CAVAN. SPREN. GELIA smitr. (incertae sedis). - Calyx persistens, quinquepartirus, imbricatus; corolla pentapetala; stamina receptaculo inserta; antherae connatae; stigma truncatum; capsula quinquangularis, quinquevalvis, dissepimentis medio valvularum adpositis, polyspora. (Calyse duplex: exterior imbricatus, interior pentaphyllus $\mathrm{CA}_{\mathrm{A}}$ VAN.: ante Stipheliam).

690. HOVEN I A, (HOVENE) THUNB. JUSS, IAM. tab. 13I. (rhamnea 4.). - Calyx quinquefidus, vel quinquepartitus; corolla pentapetala, petalis introrsum revolutis; stumina quinque petala cingunt, (stigma trifidum) stigmata tria; capsula pisiformis, crenata tribus striis, inferne per basin calycis persistentis cincta, trilocularis, trivalvis, loculis monosporis.

691. NAUCLEA, (NAUCLEE) IINN. JUSS. LANI. tab. 154. CEPHALANTHUS IINN. IAM. OUROUPARIA AUBL. UNCARIA WILLD. ap. USTERT. (rubiacea 10.) Flores in globum capitatum collecti, sustentati receptaculo communi sphacrico, globoso, piloso; calyx proprius angulosus fere integer, aut obsolete quinquefidus, corolla infundibuliformis, gracilis, tubo quinquefido; antherce fere sessilis, parum exsertae; stigma incrassatum; capsula infera, subtrigona, angulis vicinis compressa, bilocularis, dispora.

692. IMPA TIENS, (BALSAMINE) IINN. LATr. tab. 725. BALSAMINA TOURN, t. 235. Juss. MEDIC. de Monadelphis pag. 70. (geranieis affuis). - Culyx minimus diphyllus; corollae petala quatuor, (quinque) irregularia, laypogyna, inaequalia, inferiori brevi, basi calcarcato, superiori fornicato; lateralia duo maiora basi appendiculata, aut interdum profunde bifida; stumina quinque hypogyna; filamenta brevia, prisum monadelpha galeasa, deinde maturitate distincta; antherae in tis. 
bum coalitae; ovarium simplex; stylus nullus; stigma arcuatum, acutum; capsula supera, oblonga, quinquelocularis, (oralis inflata MED.) polyspora, dehiscens elastice et dissiliens in valvulas quinque, quae introrsum spiraliter involvantur; dissepimenta membranacea, uno latero adhaerentia placentae centrali; altero affixa modio valvularum, maturitate seso contrahentes; embryo rectus; albumen nullum. - (Plantae herbaceae, foliis alternis, rarius oppositis, semper stipulis privatis; pedunculi axillares uni-- multiflori). ") In Balsamina lacca sty. lus conicus, stigmata quinque radiata.

693. VIOLA, (VIOLETTE) TOURN, IINN. JUSS. LAN. tab. 725. (cisteis affinis). - Calyx quinquefidus (pentaplyyllus?) laciniis corniculatis, prolongatis sursum e basi; corollue irregularis petala quinque inaequalia, postice cornuta, hoc est: superiori maximo, basi calca. rato cornuto; filamenta distincta, basi duobus appendiculis notata, suspensa in calcare petali superioris; antherae apice membranula in tubum quasi cohaerentes unitae aut distinctae exsertae; stigma arcuatum aut urceolatum; capsula supera, triangula, unilocularis, polyspora, dehiscens elastice in valvulas tres vel quinque; sporae longitudinaliter medio valvularum affixae per frenula parva umbilicalia; albumen crassum; embryo rectus; lobi orbiculares; radicula infera.

694. JONID I UM, (JONIDIE) VENTEN. jardin. do Malmaison tab. 27. JON. MEDIC. monad. p. 102. et obs. bot. anni 1786. pag. 186. fig. $41-44$. (cisteis affnis). Calyx pentaphyllus, foliolis basi in pedunculum et re. copta-

*) Alias hoc genus ad familiam Papaveracearum relatum erat a cl Jussrze, et vere quidem proximum adsistit calyce diphyllo, corolia tetrapetala, styli absentia; sed remotum tamcin distat antheris numero constante et coalitis; fructu multiloculari ct multivalvi : placenta centrali : foliis denique interdum oppositis. Ipsis Geranieis propriis Africue affine est hoc genus, sed dissidet suo calcare ommino libero ct diffcrenti structura staminum, fuuctus, sporartum, JUSS. VENI. 
eptaculum productis; corolla hypogyna, irregularis, pentapetala, fere bilabiata, labio superiori horizontali duobus petalis formato, inferiori e tribus figurato ; petalum medium maximum, non calcaratum; stamira quinque opposita laciniis calycinis; antherae plerisque distinctae; tres filamentis destitutae corollae insertae, dwae in filamentis liberis apice in tubum coalitae; stylus filiformis; ovarium superum; stigrna simplex capitatum; capsula calyco cincta, unilocularis, trivalvis, valvulis medio sporiferis, et apice dehiscentibus, oligosporis. - (JONIA PERs. ench. pag. 256. a. SOLEA SPRENGEI ap. SCIPAD. in diar. 2. 1800. p. 190. tab. 6.) Trunci elongatio herbacea aut suffrutescens; folda stipulis munita, saepissime alterna; pedunculi solitarii, axillares, uniftori.

695: CLAYTONIA, (CLAYTONE) GRON. IIN. JUSs. IAMr. tab. 144. (portulacea 2.). - Calyx bivalvis; corollae petala quinque leviter unguiculata; stumina quinque inserta unguibus petalorum; antherae cernuae; stigmata tria, (stigma trifidum PERs.); capsula trilocularis, elastice dissiliens in valvulas tres, trispora. (Elongatio trunci herbacea, folia radicalia).

\section{RORIDULA, (RORIDULE) LINN. JUSS. LAM.} tab. 14I. IRION BURM. DROSERA thUNB. (planta ex ordine sedis incertae). - Calyx quinquepartitus seu phyllus, persistens; corollae petala quinque, bypogyna, aequalia magnitudine, alternantia cum calyce; stumina quinque hypogyna, cum petalis alternantia; antherae oblongae, rectae, basi scrotiformes vel tuberculosae, apice duobus parvis poris deliscentes; ovarium superum, stylo unico terminatum; stigma peltatum et fere trilobum; capsula oblonga, acuminata, trilocularis, trivalvis, valvulae medio septiferae, loculis monosporis, hinc trispora; receptaculum centrale tenue, triangulum, angulis singulis respondentibus dissepimento singulo capsulae; sporae oblongae, rigido - asperae.

697. I TEA, (ITEA) x.INN. IUES. LAM. tab. 147. DI. CRONANGIA MITCI. CYRILLA IINN, Juss. (rhodo- 
racea 2.). *) - Calyx minimus, quinquefidus, campanulatus; corollae petala (quatuor) quinque elongata, li-nearia, reflexo patentia, calyci inserta; antherae nutantes; stylus persistens; stigmata duo obtusa, capitata, (bilobum); capsula mucronata, bilocularis, bivalvis, valvulis margine introflexo sporiferis michaUX. - (Flores in spica terminali, bracteati).

698. LASIOPETAL UM, ( LASIOPETALE) siriti. transact. IIII. p. 21\%. Vent. (erica. gen. 160. JUss, vel sedis incertae). - Perianthium calyx monophy• lus, rotatus, tri-quatripartitus, tomentosus, persistens; corolla minima (rotata) quatuor, quinque petala squamaeformia, inserta basi calycis; hispida (lanuginosa ANDP.) quinquefida, laciniis ovatis incurvatis; stamina quatuor vol quinque, basi calycis inserta, petalis opposita; antherne postice bilobae, apice poris duobus dehiscentes; ovarium superum; stylus brevis; capsula supera, calyce tecta, trilocularis, trivalvis, dissepimentis e medio valvularum sмiтr. Sporae vix numerosae, insertae angulo loculorum centrali; (rhamnearum familiae affinis).

699. AEGICERAS, (AEGICERE) caErtN. RHIZOPHORA IINN. MANGIUM RUMPH. - Calyx quin. quefidus; corollae petala quinque; capsula arcuata, unilocularis, univalvis, monospora.

700. SAUVAGESIA, (SAUVAGÉSE) LINN. JUSS. IAx. tab. 140. (sedis incertae aut cistea?) **) - Calyx pentaphyllus (quinquepartitus), persistens, rectus; corollae petala quinque, hypogyna, fimbriata, cum calycis foliolis alterna, caduca; nectarolyma pentaphyllum, squamatum, squamis cinctís externe ciliis glanduliferis, staminibus similibus, petalis duplo minoribus et cum iis

*) Etiam ordini naturali, quo Saxifrageae a Cyrillae genere, quocum ab aliis anctoribus coniunctum fuit, differunt dissidere Richard apud Michauxium in flora 1. pag. 15\%. asserrere voluit.

**) Nam cum viola familiam naturalem effccere videtur. 
iis alternantibus; hypogynis, neque tam cito ut petala caducis, insertis super ovarium; stamina quinque hypogyna, cum squamis alterna; antherae longae, fere sessiles, quadrangulares, apice dehiscentes; ovarium superum ; capsula oblonga, trilocularis juss. (unilocularis L.) trisulca, trivalvis, valvularum marginibus introflexis et sporiferis, polyspora; receptaculum contrale nullum aut minimum; (stipulac pennato ciliatae).

7OI. VENT ILA GO, (VENTILAGINE), GAERTN. - Caly $x$ tubulosus, integer, decemstriatus ; corollae petala squamaeformia calyci inserta, muniunt stamina quinque; capsula sphaerica, supera, ala longissima membranacea terminata; spora una solitaria.

702. BRUNIA, (BRUNIE) LINN. JUSE. LAM. tab. 126. LEVISANUS PIUK. PLUM. (rhamnea 5.). - Flores terminales, aggregati in capitulum super receptaculum commune paleaceum, villosum; involucrum calyx communis polyphyllus; calyx proprius quinquepartitus, superus; corollae petala quinque longa, erecta, unguiculata;. staminum filamenta quinque unguibus patalorum inserta; ovarium superum; stylus simplex aut duplex, aut bifidus; stigmata duo; drupa exsucca, ovoïdea, continens nucem fere osseam et bilocularem, includentem numero paucas sporas. - (Frutex ericis et proteis similis).

703. KUHNIA, (KUHNIE) vENT. hort. cels. t. 9r. LIXN, fil JUSS. CRITONIA GAERTN. EUPATORIUMT onteg. (corymbifera 1.) - Flores compositi flosculosi; calyx imbricatus, oblongus, cylindricus, includens circumcirca duodecim vel quindecim flores; antherae quinque liberae distinctae, cylinúricae; stigna longum; spora solitaria, pappo plumoso; receptaculum nudum.

\section{L O PHA N THUS, (LOPHANTHE) FORST.}

gen. t. 14. Juss. JAUnie II. p. 353. (sedis incertae). Calyx t:ibulosus, laciniis quinque a equalibus, persistens; corollete petala quinque unguiculata ungue tenui, hypogyna; stamina quinque hypogyna; ovarium superum, vil- 
losum; stylus brevis; stigma leviter emarginatum; fructus monosporus, calyce tectus. - (IValtheriae hoc genus proximum).

705. RUBENTIA, '(RUBENTIE) commers. JUSS. (rhamnea 2.). - Calyx minimus, quinquepartitus; corollue petala quinque patentia; ungue dilatato; filamenta staminum quinque brevia; antherae rotundatae; stylus brevissimus; stigma simplex; drupa olivaeformis, continens nucem bilocularem, disporam. - (Frutices ramis oppositis, gongylosis; tolia opposita, longa, in ramis junioribus angusta et in antiquis ovalia; flores nxillares ).

706. T'RALLI A A, (TRALLIANE) Lour. conch, 1. p. 195. Suss. mss. (rhamnea 2.) - Calyx quinquefidus, laciniis profundis rotundatis; corolla pentapetala; stamina quinque, collocata in disco magno recto decemcrenulato; stylus unus ; stigma ....; bacca bilocularis, dispora. - (Elongatio frutescens, scandens, inermis; folia cordata, glabra; flores dichotomi).

\section{Flore supero, pentapetalo.}

707. RIBES, (GROSEILLER) IINN. JUSS. IAM. tab. 146. GROSSULARIA TOURN, (nopalea 1, cacti juss.) - Caly $x$ ventricosus, quinquefidus laciniis coloratis; corollae petala quinque, summo calycis inserta et cum laciniis calycinis alternantia; stylus bifidus; stigmate duo; stamina quinque, opposita, calycina; antherae cernuae: bacca infera, globosa, umbilicata, unilocularis, polyspora; sporae affixae filis parvis; placentae duae oblongae, oppositae et locatae super parietes baccae.

* RIBESIA, - inermis; floribus racemosis.

** GROSSULARIA, - aculeata; pedunculis plerisque paucifloris, fructubus maiusculis.

708. HE D E. 
708. HEDERA , (LIERRE) TOURN. IINN. 'Juss: IAM. tab. 145. (caprifoliez 4.). - Calyx superus, quinquedentatus, deniibus ad basin et persistentibus; $c \theta$ rollae petala quinque basi dilatata, oblonga; stamina quinquo alterna; antherae cerruae, basi bifidae; ovarium ad medium calyci adhaørens; bacca globosa, quinque. locularis, loculis monosporis, limbo calycis coronata, dissepimenta angusta, maturitatis statu disparentes.

709. PLECTRONIA, ( PLECTRONIE) IIN, JUSS. LAN: tab. 146. (rhamnea 6.). - Calyx turbinatus, quinquedentatus, persistens, clausus ad faucem squamis quinque pilosis; corollae petala quinque calycis fauci inserta; filamenta brevissima; antherae didymae; ovarium inferum; stigma capitatum; bacca infera, bilocularis, loculis monosporis, hinc dispora. - (Arbores ramis tetragonis; foliis oppositis, integerrimis; foribus in corymbos dispositis).

710. GOUANIA, (GOUANE) POLYCAMA MIONOECIA. JACQ. IINN. JUSS. IAM. tab. 845. (rhamnea). Tlores hermaphroditi: calyx superus, turbinatus, quinquefidus, interne munitus disco membranaceo, pro. longato in quinquo partes oppositasi calycis laciniis; corollae petala quinque, squamaeformia (corollu nulla IINN.); stamina quinquo petalis opposita et involuta; ovarium inforum; stylus unus; stigma trifidum (stigmata tria) ; fructus triqueter, formatus tribus cocculis sive quinque capsulis trigonis dorso fero semper bialatis, monosporis evalvibus, hinc non dehiscentibus, tripartibilis juss.; Flores masculi: calyx, corolta, stamina ut in flore hermaphrodito; pistillum abortiens. - (Fruticex sarmen. tosae et scandentes; foliis alternis, stipulaceis; floribus dispositis in racemos terminales, interdum simpliciter per abortum pistilli masculis).

7II. STRUMIFIA, (STRUMPFIE) IINN. JACQ. juss. (planta sedis incertao). - Calyx superus, minimus, quinquedentatus, persistens; corollae petala quinque; stamina quinque; antherae sessiles, in corpus ovale coalitae, striis quinque cinctae, basi quinqueden. 
tatae; ovarium adhaerens; bacca coronata, unilocularis, monospora.

7I2. PHYLICA, (PHYLIQUE) IINN. JUSS. IAN. tab. 127. ERICA ses. BECKEA BURM. (rhamnea 4.) Calyx perianthium superum, quinquepartitum, turbinatum, villosum; coroliae petala nulla, vel quinque minima squamaeformia stumina quinque munientia; stig. ma simplex; capsula (interdum fere baccaeformis) tricocca, infera, calyce coronata, cocculis monosporis altero latere convexis, altero angulosis. - (Frutices ha. bitu ericarum; folia alterna aut in verticillos conge. sta, plerumque stipulis privata; flores fere semper terminales).

713. CARPODETUS, ( CARPODET) TORT. gen. t. 17. IINN. WIILD. LAM. tab. 153. (Thamnea 6.) Calyx turbinatus, quinquedentatus, dentibus caducis, ovario adnatus; corollae petala quinque, perigyna, inserta margini calycis; ovarium seminferum; stigma capitatum; bacca exsucca, sphaerico globosa, semiinfera, ad medium cincta annulo, quinquelocularis.

7I4. GRONDVIA, (GRONOVE) HOUST. IIN⿴囗 juss. IAir. tab. 144. (cucurbilacea 1.). - Hermaphro. dita: Calyx campanulatus, quinquefidus, coloratus (mu. nitus interne quinque squamis parvis; - petala IINN.); corollae petala quinque squamaeformia, calyce aeque ac stamina, quae cum petalis alternant, inserta; bacca infera, exsucca, parva, monospora.

7I5. IASIONE, (JASIONE) IINN. JUSS. IAx. tab. 724. IAPUNCULUS ToURx (campanulea 2.) - Flores aggregati intra calycem communem, polyphyllum (involucrum), insiti receptaculo nudo; culyx quinquefidus; corolla rotata, tubo brevissimo; laciniis quinque longis linearibus, subtortis; gntherae in tubum coalitao; stigma bifidum; capsula infera, quinquangula, bilocularis, calyce coronata, polyspora.

7I6. CYPH I A, (CYPHIE) EERG. JUSS. LOBELIA IIxN. (campanulea 1.). - Calyx turbinatus, limbo quin. que- 
quepartito ; corollae petala qninque basi dilatata, conniventia, ad summum emarginata, linearia, supera; staminum filamenta barbata, basi coalita; antherae liberao oblongae; stigma truncatum gibbosum, cernuum, uno latere pubescens, Fructus ....?

7I7. A R G OPHYLL UM, (ARGOPIYYLLE)FOPST. JUSs, IAN. tab. III. (erica 2.). - Calyx semisuperus (semiinferus) quinquefidus; corolla quinquepartita vel pentapetala; tubus interne pentagonus pyramidalis, ad summum reflexus truncatusque (nectarolyma); stamina quinque non exserta, brevissima, calyci inclusa; ovarium semiinferum; capsula cumi calyce coalita, sporifera, trilocularis, trifariam dehiscens, polyspora.

718. L I GHTFOOTIA, (IIGHTFOOTE) I'HEret. LOBELIA IINN. (campanulea) *), - Calyx pentaphyllus; corolla pentapetala, fundo clausa valvulis staminiferis ; stamina inserta squamis in fundo corollae con. niventibus; stigma tri-quinquefidum; capsula semisupera, tri-quinquelocularis, tri-quinquevalvis.

719. LAGOECIA, (LAGOECIE) IINN. IAM. tab. 142. CUMINOIDES Tours, (umbellifera 4.). Involucrum universale octophyllum, partiale (involucella) quatriflorum, capillaceo pennatum; calyx superus, quinquefidus; laciniis calycinis capillaribus multifidis; petaIa quinque breviora, bicornia s. bifida; spora una coro. nata laciniis calycinis. (CUMINUM ваUн.).

720. SCHRADERA, (SCHRADERE) VAHr. WILLD. FUCHSIA 8 WARZ. URCEOLARIA GMEI. Involucrum universale multiflorum; calyx superas, urceolatus; corolla quinque - sexfida, campanulata, fauce hirsuta; bacca polyspora, (an hujus loci? vel juxta GRONOVIAM?)

721. $\mathrm{CO}$

*) Scilicet hoc genus nec non ROELLA valvulis staminiferis, charactere familiac campanulatac aeque essentiali, ac necsasolyma Ranumculis, campanulcarum est cives, 
721. CONOCARPUS, (CONOCARPE) LINY. Juss. LAM. tab. 126. (éléagni). - Flores aggregati in capitulum; calyx superus, parvus, quinguepartitus, laciniis subulatis; corolla pentapetala aut nulla; capsulae minimae, numerosae, applanatae, monosporae, marginibus membranaceae, non dehiscentes, axi comuni approximatae, et in coni rotundati formam squamaeforrnes imbricatae; (spora infera solitaria, nuda. PERS. pag. 211.)

\section{- X. Flóre incompleto, infero.}

722. A CHYRANTHES, (ACHYRANTHE, Cadelari) IINN. Juss. IAM. tab. 168 . (amarantliacea r.). Calyx quinquefidus, externe munitus squamis tribus; calyciformîbus; corolla nuila; stamina quinque basi caalita in tubum intogrum aut fissum; ovarium cinctum quinque squamulis apice imbricatis, cum staminibus alternantibus; stigma simplex; capsula monospora, non dehiscens ; spora solitaria, calycinis foliolis conniventibus obtectum. - (Stipulae nullae; trunci elongatio frutescens, aut herbacea, ramosa; spica terminalis; flores sessiles.)

723. AER UA, (ERUA) FоRsi. JUSS. ILLECEBRUM IINN. (amaranthacea I.). - Calyx quinquefidus, externe duabus vel tribus squamis munitus; stamina quinque basi coalita in tubum dentatum, ot quasi interposito cuique staminum filum sterile abortivum; stylus unus; duo, tria stigmata; capsula monospora *).

724. CHENOLEA, (CIFNOLE) THUNB. nov. gen. F. 10. (sedis incertae?). - Calyx quinquepartitus; corolta nulla; styliss filiformis; stigrnata duo reflexa; capsula umbilicata, unilocularis, monospora **).

\section{CE.}

*) Hoc genus cum antecedenti conjunctum esse voluit PERsoov ellchir. p. 258 . 8 .

**) PERs, in enchir. pag. 295. cum genere SALSOI,AE conjunxit, 
725. CEL OSIA , (CÉLOSIE, PASSE-VELOUR) LINN. MED. monad. p. 92. JUSS. LAM. tab. 16\%. AMARANTIIUS toUnN. (amaranthacea 1.). - Caly $x$ corollae pentapotalae facie, externe duabus, tribusve ntplurimum, squamis munitus; corolla nulla; stumina basi conjuncta in cupulam plica:am vel tubum brevem cam. panulatum, e cujus margine filamenta quinque exeunt, vel intersita habent fila quinque alterna (nectarolyma) styius simplex, ant bi - vel trifidus: capsula circumscisse vel transversim dehiscens in valvulas duas, altera inferne posita, altera supra insidens, utraque polyspora. - (Folia opposita; flores approximati in spicam aut paniculam, interdum compressi in capitulum.) *).

726. ILLECEBRUM, (ILLÉCĖBRE) IINN. JUSs. x'HeRET. stirp. tab. 37. med. monad. p. 93. (amaranthacoa 2.). - Calyx quinquofidus, laciniis apico fornicatis, externe squamis tribus munitus; corolla nulla; stamina basi in tubum urceolatum coalita; stylus brevis. simus; stigma simplex aut bifidum planum; capsula quinquevalvis, monospora. - (Flores glomerulo dispositi axillari aut torminali; trunci elongationes diffusa..)

727. A M A NOA , (AMANOA) AUBL, Guy. tab. 10 . zuss. (sedis incertae). - Calyx minimus, quinquepartitus, laciniis magnitudine aequali; corolla nulla; stamina quinque hypogyna, alterna curn laciniis calycinis; antherae latao, tere sessiles; ovarium superum, triangulum; stylus nullus; stigma concavum triangulum; fructus ....?

\section{A NY-}

*) * C.ANDELAPI sIE.D. monad, p. 91. Involucrum $\mathrm{ccm}$. mune quincue squamis extcrioribus in fasciculos setosos divisis formatum, triflorum; caly $x$ pentaphyllus, foliola ovalia; corolla rulla; stamina quinque patcliaeformiter basi coalita; filamenta quinque e margine tubi ad basin cordato dildtata; ovarium intra staminum tubum; stljlus fliformis ; stigma roumdum; capsula monospora; Ex gr. Achiyranthes. (Celosia. MED. obs, tot, 1782. P, 60. t. 45.) huppacea LINA, 


\section{CLASS. V. PENTANDRIA.}

728. A NYCHIA, (ANYCHIE) мICHAUX.amer. bor. I. p. 113. (amaranthacea ?). - Calyx connivens, quinquefidus, laciniis oblongis, ad summum munitis mucrone parvo vel apice subsaccatis; corolla nulla; filamenta libera, distincta, setulis nullis interpositis; stigmata duo oblonga; capsula utricularis, non dehiscens, calyce cincta, continens sporam unam, subreniformem. - (Folia opposita, stipulacea; stamina interdum tria, trunci elongatio herbacen, ramosa, prostrata; fores fasciculati et bracteis disjuncti.) *).

729. PARONICHIA, (PARONIQUE, panarine) TOURN. JUSS. GAERTN. IAN. tab. I80 ILLECEBRUMI IIN. (amaranthacea 3.). - Calyx quinquepartitus, laciniis sub apice acuminatis, fornicatis? parum infra summum interne coloratis, squamis quinque linearibus parvis, curn staminibus alternantibus; stylus bifidus; stigmata duo; capsuia quinquevalvis, monospora, calyce connivente tecta ${ }^{* *}$ ).

730. HAGAEA, (HAGAEE) VENTEN. **) POLYCARPAEA ram. (caryophyllea 3.). - Calyx pentaphyllus vel quinquepartitus; corcliae petala quinque emarginata, calyce multo breviora: stylussimplex; capsula trigona, unilocularis, trivalvis, folyspora; sporae fundo capsulao insertae per fila parva umbilicalia.

73I. GYM NOCARPON, (GYMNOCAPE) Fonsr: Jess. PERs, p. 26:. TRIANTHEMA VAHL (portulacea I) Caigr

*) Ad hoc genus, illecebris valde aftine, Mrснаеx etian QUERIAW canadensem IINN. referri jussit, quan sylvestrem plantam semper pentandram, cultam semper diandram observasse profitetur.

**) Conferri potest inprimis GAERTNer de Scm. et feuctt. tom. 2. pag. 213.

***) Ad nominum Polycarpea cum Polycarpo confusionem evitantam, veNTENAT ipse loo commutavit in hortulani Lahaye memoriam nomen genericum. JAtsIE II. F. 112 . 
Calyx quinquepartitus, laciniis coloratis interne, persistens; corolla nulla; stamina decem, duo alternantia fertilia et sterilia; stigma simplex; spora una, calyceindurato tecta. - (Frutices diffusae).

732. GLA UX, (GLAUX, Glauce) TOURN. IINN. JUSS. LAM. tab. 141. (lythraria 2.). - Calyx monophyllus, campanulatus, coloratus, quinquelobus, lobis extrorsum revolutis; corolla nulla; antherae rotundatae; capsula globosa, calyce cincta, unilocularis, quinquevalvis, pentaspora; placenta globosa, sporis sulcata.

733 COLLETIA, ( COLLETIER ) commers. JUss. LAM. tab. 129. (rhamnea 4.). - Calyx urceolatus, limbo quatri - sexfido, patente aut reflexo, basi intus villosus; quatuor vel sex squamae petaliformes margini calycis inter lacinias insertae conduplicatae; corolla nulla; quatuor, sex stamina petalis inclusa; stigma trilobum; fructus: cupsula parva, baccata, sicca, cincta per basin calycis persistentis, tricocca, tripartibilis, trispora; cocculis monosporis. - (Frutices spinosissimae; rami oppositi, fere aphylli; flores solitarii aut per parvos glomerulos congesti in axillis spinarum.)

734. CERVA NTESIA, (CERVANTESIE) rUIz. PAV. CAV. tab. 475 ? - Caly $x$ minimus, crescens; corolla nulia; squamae nectariferae quinque, ovales, crenatae, infra lacinias calycis insidentes; stamina plana, basi calycis inserta; $n u x$ calyce carnoso pentagono colorato magno, inferne nuci accreto, involuta, unilocularis.

735. ALZATEA, (ALZATEE) hUIz. PAv. - Ca. lyx quinquefidus, pentagonus; corolla nulla; stamina receptaculo inserta: capsula obcordata, bilocularis, bivalvis, valvulis medio septateris; dissepimentum contrarium; sporce superinpositae, membrana cincrae.

736. MYOSCHILOS, (MYOSCHILOS) rux. PAv. (eicagni). - Calyx pentaphigllus, coloratus, persistens; curolla mulla; stamina lacinis calycinis oppo- 
sita, receptaculo inserta; antherae rotundatae; ovarium oblongurn; stigma et stylus bi - trifidus triangulus; drupa oblonga monospora, calyce persistente coronata; nux unilocularis rotundata; squamae tres sub singulo ovario concavae.

737. LIGA I A , (LICANI, Caligni) Auzr. Juss. IAM. tab. 122. HEDYCREA \&CHREB. WILLD. (rosacea 7.) Calyx inferus, limbo quinquedentato sive quinquefido, turbinatus, basi bracteis duabus munitns; corolla nulla schreb. (aut petala sex fauci calycis inserta); stamina fauci calycis inserta et laciniis ejus opposita; stylus recurvus; drupa carnosa, olivaeformis, continens rucem ovatam, fibris tectam, unilocularem, monosporam.

\section{Flore incompleto, supero.}

738. THESIUM, (THÉSION) LINN. JUSS. IAM. tab. 142. ALCHIMILLA roupin. (eleagni). - Calyx superus, monophyllus, quatri-quinquefidus; corolla nulla; stamina calycis basi inserta et ejus laciniis opposita; capsula infera, monospora, calyce persistente coronata, non dehiscens; radicula supera: - (Plantao herbaceae aut suffrutescentes; flores dispositi forma diversa, basi munita duabus vel tribus bracteis.)

739. HEL I C ONIA, (HELICONE, Bihai) IINN. JUSs. LAM. tab. 148. BIHÁ PLUM, MUSA AUBL LINN. (musacea). - Spadix universalis recta; spatha partialis disticha, concava, aut cymbiformis ircludens thyxsum florum, dispositorum in spicam confertam sive glomerulum; calyx nullus; corolla (calyxjuss.) supera, bilabiata, vel laciniis duabus profundis; inferiori lacinia simplici, canuliculata; superiori triloba, lobis duobus erectis lateralibus . et positis ad dorsum lobi medii latioris et canaliculati; (nectarolyma diphyllum, corollae potala tria); stamina sex, uno abortiente filamento sterili brevi spathaeformi; stylus filiformis longitudino sta- 
minum; stigma simplex, oblongum, prismaticum, ad summum recurvatum; capsula oblonga, triangula, trilocularis, loculis monosporis, hinc trispora.

740. STRELITZIA, (STRELITZ) AJT. BANKS. VENT. WILLD. LAM. tab. 148. HELICONIA IIN. (mu. sacea). - Spatha universalis et partialis monophylla terminalis, multifora; flores muniti spatha partiali. Calyx nullus; corolla (calyx vent.) sexfida, laciniis coloratis; tres exteriores magnae, duabus rectis et acuminatis formantibus labium superius, tertia emargisata et canaliculata; laciniae tres interiores cum exterioribus alternantes: una fingens cucullam liquorem molliferum continens, reliquae duae interstitiis duorum labiorum interpositae, basi attenuatao membranaceam expansionem fingunt sexus organa involventem; stamina quinque, duo tecta margine alterius laciniae, reliqua tria alterius laciniae margine tecta; antherae lineares, bngissimae; (nectarolyma triphyllum, genitalia involvens); ovirium oblongum; stylus filiformis; stigmata tria agglutinata et exserta; capsula coriacea, oblonga, leviter trilocularis, trivalvis, loculis polysporis; sporae numero plures, duplici serie dispositae, insertae placentao centrali.

741. VILLARESIA, (VILLARESIE) PIIZ-PAV. juss. mss. (rutacea 3.). - Calyx parvus, quinquefidus: corolla pentapetala; stamina quinque; antherae bifidae; ovurium stigmate sessili, applanato superpositum ; cap. sula oblonga, calyce associata, cortice tecta, et attenuata in mucronem, unilocularis, bivalvis; spora una tetragona, in arillo crasso conterta. *)

$D I G Y$

*) Rxrz et PAv. in fora peruviana duo haec genera adhuc in pentandriae primo ordine commemorarunt: 1.) NYCTE. RISTON: pericarfio quinqueloculari, pentasporo; sporis solitariis. - 2.) IJESFONTAINA: bacca quinquelo. criliri; corolla subcampanaformi, tubo pentagono; an theris sagittatis. - (Inscii iam duo suis noc DESFONT. ioraine gencra extate). 


\section{$\begin{array}{llllllll}D & I & G & Y & N & I & A\end{array}$}

\section{Flore monopetalo supero.}

742. GENTIANA, (GENTIANE) TOURN. LINN. JUSs. LAMr. tab. 109. CENTAURIUM moURN. (gentianea 1.). - Calyx fere quinquepartitus; corolla basi tubulosa, campanulara in Gentiana totsin., aut infundibuliformis in Centaurium tounN, rarius rotata, limbo quinquefido, rarius quatuor - novemfido, varia forma, laciniis obtusis aut acutis, ciliatis aut integris, patentibus aut rectis; stamina quatnor aut quinque; styius bipartitus; stigmata duo; capsula biralvîs, unilocularis, polyspora, receptaculis duobus longitudinalibus. ") -

*) Wulisaid smidt. apud roemervi nova genera e Gen. tiana consistens, liunc char. genericum dedit: , Calyx g,spatha membranacea, bicornis; corolia rotata quinque,partita; ficumente intra corollae lacinias innata; anthegrae oblongae, sagittatae, circa stylum coalitae; cap,sulu ovata, unilocularis, livalvis; et haec exinde genera ,reliqua constituit. 1.) PNEUMUNANTHE: Calyx mo,nophyllus persistens; corolla campanacformis; antherae ,in tubum connatae; stigna didymum, sessile; capsula ,oblonga milocularis, bivalvis, apice dehiscens, bifida. ,2.) HIPPION : Calyx monophylius; persistens; corolla ,tubulata, plicata, limbo quadri - vel quinquefido; , antherae simplices liberac; stigmata sessilia; cupsula „fusiformis in stylum attemuata, unilocularis bivalvis ,apice dehiscens, bifida. - 3.) CHIRONIA: Calyx mo= ,nophyllus persisteus; corollu tubulata, linbo quinquespartito; fiamenta ex apice tubi cnata; antherae deflora,tae spiraiiter contortae; capsula bilocularis, bivalvis, ,longitudinaliter dehiscens. - 4.) GENTIANA. ,5.) SWERTIA. - 6.) CHLORA: Calyx octophyllus; "corvlia tubulata, octo - duodecimfida; stigmuta in. „crassata, stylo longitudine tubi, simplici vel semibifido ginsidentia; capsula miliocularis, Livalvis."

Caeterum eodem apud RoEMERTM loco GENTIANAE IINNEI species, ila BORTHAUSEN in genera dispescuit:

I.) GENTIANA: Calyx quadri - quinquefidus; corolia infundibuliformis ant hypocrateritormis, quadri aut quinquefila, absque peripetalis; stamina numero lisciniarum corollae; stigma simplex bilohum, vifidimcile. (et huc refert G, filiformam L., tetragonam noth., 4uil- 
(Flores axillares aut terminales, solitarii aut fasciculati aut verticillati).

743. VI L-

quefidam $I_{10}$, maritimam $L_{.,}$auream I., macrophyllam PALL., dichotomam PALL.)

2.) GENTIANELLA : Calyx quadrifidus; corolla hypocraterifotmis, quadrifida, absque peripetalis, fundo nectarifero poris quatuor; stamina quatuur, antheris supra stigma comiventibus; cvarium subpedicellatum, olsolete tetragonum; stigmate latiusculo revoluto; capsulu angulis olstusis dehiscens; sporis quadrifariam dis. positis. - (GENT. luter L., serrata BonKH.)

3.) CHLORA $\triangle$ DANs, famille des plantes 503 RENEALM. sp. So. tab. 76. - Calux octn - duodecimfidus; corolla cicto - duodecimficta, infundibuliformis; stamina numero laciniarum corollae; stigma bilobum bifidumque. (Genf, perfoliata L., quadrifolia L., dodecandra.L.)

4.) CENTAUREUMr: Calyx tubulosus, subincurvus, quadrilentatus; corolla tubulosa, irregularis, limbo bipartito, laciniis biparitis, lacinulis duabus superioribus erectis, duabu inferioribus deflexis, antheram nıaiorem inciudelitibus, extcrise patentibus; stamina quatıor inaequalia, tria intra tubim, quartum fauci insertum antheram maiorem gcreus; stulus filiformis, nexuosus; stigmata bilabiato interne villoso, labiis reflexis; capsula elastice dissilièns. - (Gent, heteroclita LINN.)

5.) EPYTHRAEA RENEAL, 51. 7\%. tab. 76. CENTAUREUM пьоелсн. plantt. Marb. pag. 449. CENTAUREULI minus toun. tab.48. - Calux pentagonns, quinquefidus; corolla infundibuliformis, fauce denticulata; stamina quinque aequalia; antherae post pollinis dimissionem spiraliter toriae; stigmata duo sessilia incrassata. (Gent, centaureum L.)

a) AsTERTAS. Calyx spathaceus; corolla rotata, quin: que - octofida, absque siectarolymatibus et parapetalis ; stigma hifidum, laciniis reflexis, - (Gent. 1utea.)

b) CoIlANTIA : Calux spathaceus, truncatus; corolla campanulata, quinquefida; stamina antheris $\mathrm{li}$ bera, RENEALAr. sp. 65. - (Gent. purpurea L.)

c) DASYSTEPHANA IENEALM, sp. 67. tab. 68. Calux ousolete auguiosus, dentatus, tot angulis et dentibus, quot corollae segmenta; corolla campanulata, yuiuque - septemfidal stamina antheris liberis; stigma bifidum. - (Gent, punctata L., panuonica L., glfuca I,. r rifora PALI., adscendens paLL., algida $P \perp I, L_{0}$, auriculata $\left.P \perp L L_{0}\right)$ 
743. VILLARSIA, (VIELARSIE) Bosc. (gentianea). - Calyx monophyllis, quinquepartitus, laciniis ovato acutis, lanceolatis, exectis, persistentibus, minutis, atropurpureo punctatis; corolla monopetala, alba, campanulata, profundo quinquefida, laciniis ovato lanceolatis, ad margines superiores appendiculatis, appendice tenuiori plicato, linc corollae figura ovato subcordiformis; stamina quinque; filamenta fusiformia, longitudinaliter corollae adhaerentia; antherae erectao, acutae, luteae; nectarolymata decem, quinque corollae insidentia, quinque receptaculo; illa cum corollae laciniis alternantia, sphaerica, stipitata, villosa, staminibus hreviora; haec ad basin ovarii sessilia, ovata, subreniformia, glabra, lutea; pistillum: ovarium ovato oblongum; stylus nullus; stigmáta duo, tria, membranacea, approximata, submembranacea, ovarium coronant; fructus capsula subtetragona, glabra, unilocularis, bival-

d) CIMINALIS AD ANs, familles des pl, pag.504. CYANA RENEALM, sp. 69, tab, 69. THYLACITIS RENEALMI: sp. 7o. tab. 68. - Calyx obsolete quinquegonus, quinquedentatus; corolla campanulata, quinquefida stamina antheris in tubum connatis; stigma simplex. (Gent. pneumonanthe $L_{\bullet}$, acaulis $L_{0}$ vel longillora MOENCH.).

e) ERICOILA RENEALMr, sp. 75. tab. 68. TPETOR. RHIŻA REN, sp. 74. tab. 73. GENTIANAE IINN. specics. - Calyx tri - quadri - quinqtzefidns; corolla hypocrateri - att infundibuliformis, quadri quinque - sexfida; totidem parapetalis non barbatis inter lacinias ancta; stamina nutumero laciniarum corollae; antherae liberac; stigma unicum simplex aut hilobum bifidumve. - (Gent. verna, bavarica, nivalis, prostrata, aquatica, utriculosa, verticillata, pumila, pyrenaïca, altaica LINN.)

f) ERYTHALIA RENEALM. \$P. 72. OPSANTHA REN. sp. 7I. GENTIANELLA MOENCH. p. 482. - Calyx quadri - quinque - sex - septemfidus: corolla tubuloso campanulata, aut hypocrateriformis, tot laciniis quot calycis segmcnta, laciniis parapetalis barbatis interstiuctis; stamina numero laciniarum corollae; stigrna bilobum, bifinumve. - (Gent.

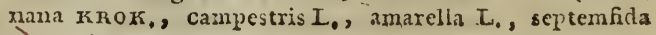
PALL.). 
bivalvis; sporae númerosae, globosae, compressae, fuscae, margini valvularum affixae.

744. SWERTIA, (SWERCE) IINN. JUSS. IAM. tab. 109. GENTIANA Tourn. (gentianea I.) - Calyx fero quinquepartitus; corolla rotata, tubo brevissimo, limbo plano, laciniis quinque lanceolatis, quarum quaeque ad basin interne munita est glandulis duabus ciliatis, (pori nectáriferi PERs.); stamina quinque corolla multo breviora; ovarium terminatum stylo brevi et duobus stigmatibus; capsula unilocularis, bivalvis, poly• spora. ${ }^{*}$ ) - (Flores axillares et terminales; pedunculi saepe multiflori).

745. CERA T IA, (CERATIE) NeIchaux. - Corolla subcampanulata, basi cornicula exserens. (HALENIA BonkH. ap. Roemer. I. qui haec , corolla rotata, quadri-quinquefida, laciniis parapetalis subulatis interstinctis." SWVERTIA corniculata IINN.)

746. F A L K I A , (FALKIE) IINN. fil. supl. JUss. (borragineis affinis) ANDRew. - Calyx subinflatus, quinquepartitus, quinquąngularis; corolla campanulata, limbo amplo emarginato crenato, (crenato - decempartita ANDr.); stomina sex? magnitudine inaequalia; ovariu quatuor; styli duo divaricati; stigmata duo subglobosa, lanata; sporae quatuor globosae, nudae, avillatae, in fundo calycis calyce tectae ANDREW. ${ }^{*}$ )

747. CRESSA, (CRESSE) IINN. JUSS. IAM. t. 183. QUAMOCLIT tourn. (convolvulacca 2.). - Calyx (pentaphyllus) quinquepartitus, ntunitus duabus parvis bracteis; corolla hypocrateriformis, vix calyce longior, tubulo-

*) scharrir ap. noem. haec dedit: Calyx quadri - quinque. iartitus, patens; corolla rotata, quadri- quinquepartita, nectariferi pori ad basin laciniarum; stigmata sessilia; copsula unilocularis, bivalvis.

*) Qui osulatisimus hums lutuic gencri locum sancivit-ex hexandria senoto; - convolvulum pracicrea exacte refert, sed fructu a convolvulaccis ad borragincas amandandum. 
- bulosa quinquefida; filamenta tubo insidentia; antheris rotundatis terminata; capsula supera, quadrilocularis, quadrivalvis, mono-vel tetraspora, basi dehiscens.

749. NAMA, (NAME) Brown. LINN. JUSS. IAM. tab. 184. (convoIvulacea 2.). - Calux pentaphyllug vel quinquepartitus, laciniis linearibus; corolia tubulosa, limbo amplo, quinqquefido rel partito, vel niarsine quinquecrenulato; styli duo; stigmata dun; capsula oblonga, bilocularis, bip̧alvis, polyspora; sporue dissepimento affixae.

749. HYDROLEA, (HYDROLE, Coutarde) IINN. JUss. IAm. tab. 184. (convolvulacea 2.). - Calyx quina quepartitus vel pentaphyllus: corolla rotata aut campanulata, tubo brevi, limbo patente quinquepartito obtuso; filamentu ad basin tubi inserta; antherae cordatae versatiles; styli duo longi divergentes; stigmata duo ca, pitato peltata; capsula bilocularis, bivalvis, polyspora; sporae in dissepimento centrali. - (Elongatio herbar cea aut suffrutescens; flores bracteati, terminales aut axillares).

750. ROCHEFORTIA, ( ROCHEFORTE) - SWARz. - Calyx quinquepartitus; corolla infundibuli. formis, fauce aperta, quinqueparsita; fructus bilocularis, polyspora.

75I. EVOLVULUS, (EVOLVULE, Liseralle) IINx. JUss. LAM. tab. 216 . (convolvulacea 2.). - Calyx quinquepartitus; corolla rotata, campanulata, limbo quinquefido, lobis emarginatis; styli duo, profunde bifidi, ${ }^{*}$ ) laciniis capillaribus divergentibus; stigmata simplicia; capsuta bilocularis, quadrivalvis, tetraspora; sporae binae (semen solitarium LINN.); cf. RUIZ et PAV.

752. DICHONDRA, (DICHONDRE) FORST, SMIth. IAM. tab.183. DIMIDOFÍs omel. (convolvula cea 2.). - Calyx quinquepartitus, laciniis subspachulatis:

\#) Hinc ex ordine tetragyniae ad hunc locum referendum. 
latis; corolla rotata, fere brevicampanulata, limbo quinquepartito, tubo brevi; stamina quinque subulata; astherae rotundatae; styli duo; duo stigmata peltato capitata; capsula partito - didyma, (capsulae duae globulosae) subcompressa, bilocularis, loculis monosporis; sporae globosae.

753. S I M I RA, (SIMIRE) AUr. Gry. t. 65. (rubiacea 7.). - Calyx minimus, quinquedentatus; corolla parva, tubulosa, quinqueloba; stamina summo tubi inserta; bacca parva, ovalis, coronata, bilocularis, dispora. - (Arbor; folia integerrima, petioli perbreves; flores minimi in racemis terminalibus).

\section{Flore infero, pentapetalo.}

754. CARMONEA, (CARMONÉE) cAv. tab. 438. (borraginea $\mathrm{r}_{\text {) }}$ - Calyx persistens, quinquepartitus; corolla rotata, tubulosa, tubo brevissimo, limbo quinquepartito; antherue ovales, oblongae, didymao; ovarium globosum, stylis superpositum; styli duo capillares; drupa globosa, includeas nucem sexlocularem; sporae solitariae.

755. ZIZI PHUS, (JUJUBIER) TOURN. JUSs. IAM. tab. 185. RHAMNUS LIN : (rhamnea 3.). - Calyx patens, quinquefidus, urcoolatus; corollae petala quinque, squamaeformia, disco orbiculari carnoso glanduloso pistillum ambiente inserta; stamina quinque disco inserta; ovariun disco cinctum; styli duo; stigmata duo; drupa ovoïlea, continens nucom bilocularem, mono - dispo. ram. - (Arbores mediae altitudinis; folia alterna stipulis saepe spinescentibus; flores axillares). pers. enchir. p. 240. sub RIIAMNO.

756. VELEZIA, (VELEZE) xINN. JUSS. IAM. tab. 386. (caryophyllea 2.). - Calyx tubulosus, filifurmis qrinquedentasus; corollae petala quinque unguioulata, brevissima; stamina quinque, sex; styli duo; capsula cylin- 
cylindrica, unilocularis, apice quadrivalvis, polyspora"; sporae plurimae receptaculo filiformi, centrali affixae.

757. LINCONIA, (LINCONA) IINN. JUSS. LAM. tab. 52\%. (ex ord. plantt, sedis incertae). - Calyx basi urcoolatus, apice quinquepartitus, laciniis scariosis, canaliculatis, persistentibus; externe quatuor bracteis per paria approximatis duplici serie cinctis, exterioribus minoribus; corollae (caly $\mathrm{x}$ juss.) laciniis paleaeformibus, canaliculatis, basi foveatis; stamina quinque cum laciniis (icalycis J.) alternantibus; antherae sagittatae; ovarium semiadhaerens; styli duo filiformes; stigmata duo; capsula semiinfera, bilocularis, bipartibilis, loculis monosporis, nitida.

758. B UMALDA, (BUMALDE) THUNB. diss. et Jap. 114. Juss. (rhamnea 5.). - Culyx quinquepartitus, corolla pentapetala, hypogyna; stumina quinque inserta petalorum unguibus; ovarium superum, villosum; styli duo villosi; capsula bilocularis, birostris.

759. HEUCHERA, (HEUCHER̀E) IINN. JUSS. IAM. tab. 184. CORTUSA MrchaUX. (saxifragea I.). Calyx semisuperus, quinquedentatus ; corolla pentapetala; stamina quinque exserta; ovarium inferum; capsula birostris, bilocularis, polyspora.

\section{Flore incompleto.}

760. GUMILLEA, (GUMILLEE) RUIz flor. per. - Calyx campanulatus, quinquefidus; corolla nulla; ovarium subbifidum; capsula bilocularis, polyspora, birostris.

76I. A NABA SII !', (ANABASE) IINN. JUSS, IAM. tab. 182. SALSOLA CAV. BUXE. (atriplicea 3.) - Calyx quinquepartibus, basi bracteis tribus munitus; corolla nulla; - (calyx triphyllus; corolla pentapetala pers. p. 237.) - styli duo; bacca monospora, tecta calyce cincerte, basi baccato, infra attenuato, limbo patonte; sport spiraliter voluta. 
762. CAROXYLUM, (CAPOXYLON) TIUNB. Juss. (atriplicea 3.). - Calyx quinquepartitus, adauctus externe squamis duabus, interne quinque appendiculis; stumina quinque, ad latus ovarii inserta; ovariun liberum; stylus simplex; stigmata unum aut duo; spora una tunicata, spiralis, appendicibus calycis couniventibus obtecta; (folia imbricata, sessilia).

763. S S L SOLA, (SOUDE) IINN, JUSS. IAM, tab. 18r. KALI TOURN. WILLEMETIA MAERKLIN; CHENOLEN wIILD. BASSIA ALIION. CHENOPOD, IIN - Calyx quinquepartitus (pentaphyllus?) basi capsuląris; corolla nulla; styli duo, tres (bi - trifidi); stigmata 'duo, tria; capsula monosporo; spora cochleata, vol spiraliter intorta; calyce recurvato, limbo coarctato, tecta, calyco in quibusdam margine mernbransceo. ${ }^{*}$ ) (Elongatio trunci frutescens aut herbacea; folia oppo. sita aut alterna, cylindrica aut plana; floresterminalos aut axillares).

764. TOCHIA, (KOCIIA) rotr. (chenopodea).Calyx inonophyllus, camparulatus, quinquefidus, in fructu in marginem foliaceum pontapetaliformem excrescens; fauce clausa dentibus quinque triangularibus; corolla nulla; stylus unus, brevis ; stigmata, duo, tria, longa; capsula unilocularis, mono-dispora; spora incurva.

765. A TRIPLEX, (ARROCHE) TOUR, IINIV.'JUSS. xAM. tab. 853. (atriplicea 3 ). - Polygama: - Floves hermaphroditi: calyx quinquefidus vol pentaphyllus; coralla nulla; stumina quinque; stylus bifidus vel bipartitus; spora una, calyce tecta, angulosa, dentata, depresșa; flores feminei: calyx diphyllus, compressus, intermixtus floribus hermaphroditis; corolla nulla; stylus bipartitus; spora una, compressa ut in flore hermaphrodito. (Qua spora nota a Chenopodiis differt, cum quo genere rụN-

BER-

*) Quilunsdam specieluus generi Chenopodio affnc, hinc solertius examinandac et seimgendae; quare oculatissinus notrs quasdam sub IrOCHIA comprehendere voluit, 
BERGTUS hoc atriplicis combinavit). Flores glomerati, paniculati; folia interdum fere opposita ; elongatio trunci rarius suffrutescens.

765. CHENOPODIUM, (CHÉNOPODE, Ansé. rine) TOURN. LINN. JUSS. LAM. tab. 181: OSYRIS Dosox. (atriplicea 3.). - Calyx quinquepartitus, (penta. plyyllus) maturus pentagonus connirens totus obtegens sporam unam, superam, lentiformem, angulosam; corolla nulla; stylus bi - trifidus. - (Flores approximati per fasciculos dispositi in paniculam).

767. HETA, (BETTE, Poirée) TOUR. IINN. IUSS. I.AM. tab. 182. (atriplicea). - Calyx quinquepartitus, (pentaphyllus), laciniis carinatis; corolla nulla; ovarium parum adhaerens vel semiinferum; styli duo; spora una, reniformis, intra substantiam baseos calycis infusa, capsulae locum tenentem. - (Elongatio trunci striato sulcata; duo - tres - quatuor flores conferti in fasciculos sessiles et axillares.

768. HERNIARIA, ( herniaire, Herniole) - TOURN. LINN. JUss. LAM. tab. 180 . (amaranthacea 3.). Calyx quinquepartitus, interne coloratus; corolla nulla; squamae quinque parvae, filiformes, cum staminibus quinque altornant. (vel filamenta quinque sterilia cum staminibus alterna pers.); capsula monospora, calyce tecta, lıon dehiscens. - (Eiongatio trunci ramosa, re. pens; flores glomerati, axillares).

* 769. GOMPHRENA, (GOMPHRÉNE, Amaran. thine) IINN. JUSS. IAM. tab. ISO. MEJ. monad. p. 93. AMARANTHOÏDES toUR. (amaznnthacea 2.). - Calyx fere pentaphyllus; externe munitus squamis duabus magnis coloratis, acuminatis, conniventibus (coloratus exterior triphyllus, foliolis duobus conniventibus carinatis PERs.); corolla nulla; ( petala quinque rudia villosa rẹs.) staminum filamenta per totum ipsorum longitudinem coalitae in tubum longum quinque - decem dentatum, (nectarolyma pens.), filanientis vix u!lis, ad ori- ficium munitum quinque antheris; capsula mowospora, circum- 
circumscissa; ut in Celosia; stylus semibifidus *). (Flores capitati vel axillares vel terminales).

770. BOSEA, (BOSE) IINN. JUSS. LAM. tab. 182. (atriplicea 1.) - calyx quinquepartitus (pentaphyllus P.); corolla nulla; stylus nullus aut punctiformis; bacca globosa, monospora. - (Frutex elongatione parum torta, foliis acuminatis; floribus in racemis axillaribus).

77r. PLA NERA, (PLANERE) MrCHAUX. (rham. noïdea?) - Polygama: calyx membranaceus, subcam. - panulatus, quadri-quinquefidus; corolla nulla; stigmata duo oblonga, glandulosa, recurva, divergentia? capsula (nux?) subglobosa, membranaces, unilocularis, non dehiscons, aut laevis, aut squamulosa, (non alata), monospora; masculi flores intermixti staminibus quatuorsex. (Frondescentia ulmi, cui valde affinis.)

272. ULMUS, (ORME) TOURN. IINN. JUSS. LAMT. tab. 185. (amentacea 1.) - Culyx campanulatus, qua dri-quinquedentatus (vel 5 . fifus P.) coloratus, persistens; corolla nulla; stamina tria, sex, pluriniam quatuor, quinque, filarnenta calyce multo longiora; ovirium compressum; styli numero duo aut nulli; (cupsula) samara GAERTN. fere orbicularis, planc-compressa, membranacea, versus medium inflata, alata, monospora, sporae lenticulares. - (Arb res, rarius frutices; folia uno baseos latere longiora; llores saepe sessiles, interdum pedunculati, conglomerati per parvos fasciculos squamaeformes, saepè axillares).

773. CELTIS, (MICOCUIIER) TOURN. IINN. SWARz, JUSS. LAM. tab. 844. RIIA HNUS IINN. (amentacea 1.) - Fiores polygami, hermaphroditi. Ca.jx quinquepartitus; corolla nulla; stumita quinque brevia, antheris fere sossilibus; ovarium ovoideum; styli duo divaricati, crassiusculi; drupu ğlóbosá, monospora; co-

*) PEns. enchir, p, 257. sub monogynia. 
tyledones sinuati et plicati; fores masculi inferiores: calyx sexpartitus, stamina sex; corolla nulla. (filamenta elastica.) - Arbores; flores axillares, fore solicarii aut - in racemis, interdum tantum masculi, mixti herma. phroditis aut distincti, positi in axillis inferioribus.)

774. MICROTEA. (MICROTEE) SWAFz. IAM. tab. 182. SCHOLLERA ROHR. (atriplicea 1.).- Calyx pentaphyllus vol quinguefidus laciniis profundis, patens; corolla uulla; ovarium duobus stylis terminatum; drupa sicca, coriacea, echinata; nux monospora.

775. ANDREDERA (ANDREDERE) JUSS CMEL. BASELIA vesicaria LAM. (atriplicea?) - Culyx bipartitus, dorso carinatus; corolla nulia, stylus bifidus; spora una, calyce compresso, membranacen bialato tecta. (locum inter ANABASIN et BOBEAMI dedit PERs. p. 297.)

IV. Flore supero, pentapetalo; fructu capsulari, aut dicocco.

776. VAHLIA, (VAHLE) TIIUNB. JUSs. LAN. tab. 183. RUSSELIA IINN. suppl. *) (onagraria 1.). - Calyx superus, pentaphyllus, (vel quinquefidus); corollae potala quinque duplo minora calyce; stamina petalis alterna; ovarium inferum; capsula infera, coronata calyciniș laciniis, unilocularis, bivalvis, polyspora.

777. PHYLLIS, (PHYLLIS) LINN. JUSs. LAM. tab. 186. (rubiacea 2.) - Calyx minimus, diphyllus vel bifidus; corolla qninquepartita, vel pentapetala; styli duo; stigmata duo pubescendo hispida; sporue duas, conniventes, uno latere planae, convexae altero, oblongae, supra dilatatae et axi centrali filiformi annexae. - (Frutices foliis verticillatis, lancelatis; flores in corymba terminali aut axillari).

778.

*) a RUSSLLIA JACQ. VAHL, LAMT, t. 539, didynamia distincta. 
778. COPROSMA, (COPROSME) FORST. EINN. supl. Juss. LAM. tab. 854. (rubiacea 7.) - flores hermaphroditi: calyx minirous, quinquefido dentatus (inter dum sex -septemfidus); corolla infundibuliformis; guinquefido dentata, (vel sex-septemfidus): símina quinque (interdum sexvel septem); antherae oblongae: styli duo alte fissi in duas partes basi cohaerentes; bacca infera, rotundata, parum ovata, globosa, pulposa, dispora; sporae uno latere planae, convexae altero; flores masculi: calyx integer, caeterum idem ac corolla et stamina ut in flore hermaphrodito; - (Frutex foliis oppositis stipulaceis; pedunculi axillares, uni-muliiflori).

779. PANA X, (PANAX, Gin-seng) IIN⿴. JU8s. LAM: tab. 810. ARALIASTRUM vaill. (araliacea) Polygama; flores hermaphroditi involucro polyphyllo, brevi ; caly $x$ quinquedentatus; corolla pentapetala; stamina quinques styli duo; stigmata simplicia; bacca infera, fere cordata, umbilicata, bilocularis, dispora. Fiores masculi: involucrum polyphyllum; calyx integer; petala quinque; stamina qninque. - (Elongatio trunci herbacea, simplex, ad medium munita-tribus foliis in verticilfo; ad summum umbellifera; folia digitatas umbella simplex aut ramosa, munita involucro brevi.).

780. CUSSONIA, (CUSSONE) IINN. JUSS. IAM. tab. 187. (araliacea $\left.x_{*}^{*}\right)$ ). - Calyx superus, monophyl. lus, truncatus, quinquedentatus, margine dilatatus, crenatus; corollae petala quinque, oblonga, trigona, acuta; fructus didymus, calyce stylisque coronatus, angulosus, compressus, bivalvis, bilocularis, loculis monosporis, (dicoccss?) - (Frutices foliis digitatis; floribus in spica aut in racemis umbelliferis),

*) THUNBErig. diss. x. pag. 12. inter umbellatas hoc genus retulit; Jussict, natua ipsa monente, ad familiam Ara. liacearum. 


\section{Flore supero, pentapetalo; sporis nudis; uinbellatae.}

\section{A. Umbellae involucro (peripodio EHRH.) munitae; umbellulac involucratae.}

78I. ERYNGIUM, (PANICAUT) TOURN, IINN. JUSs. LAxi tab. 187. (umbella anomala 4.) - Flores aggregato capitati; congesti in receptaculo conico, disiuncti paleis lanceslatis asperisque; involucrum polyphyllum; culy $x$ proprius pentaphyllus, superus, persistens; corollae petala quinque oblonga, curva; fructus calyco coronatus, ovali oblongus, bipartibilis, glaber aut muricatus: (umbellulae distichae aut formantes umbellam involucratam, ramosam, saepe irregularem; folia simplicia aut composita, saspe spinosa. Folia Cardui vel Bromeliae, in his integerrima, margine ciliato spiatulosa )

782. ARCTOPUS, (ARCTOPE, oursine) IINN. JUSs. LAII. tab. 855 (umbella anomala 4.) Polygama dicica. Flores masculi: umbella composita; involucrum et involucella pentaphylla; calyx minimas, quinquepattitus: corolla pentapetala : stamina quinque; ovarium nullum; styli duo; fores hermaphroditi: umbella simplex; involucrum magnum, monophyllizm, patens, quinquepartítum, laciniis spinulasis; flores masculi multi umbellae centrum vel discum occupant; fiores feminei quatuor circum circa radiatim positi; flores mascuili in disco, calyce minino, quinquepartito; petalis quinque, integris, oblongis; stamina quinque: flores feminei quadem in peripheria calyce et corolla ut in mas. culis; styii uno; fructus quatuor hispidi, biloculares, dispori, 1nvolucro persistente et connivento reconditi. (flures masculi in distincta planta) Juss. - Habitus Eryngiii folia coalita, sinuata, ciliata; flores terminales, ad medium foliorum positi).

783. HY D R O C O TYLE, (HYDROCOTYLE, Hydrocote) gOURN. LENN. JUSS, LAM, tab. 18S. SOLANDRA 
IIN. (umbella anomala 4.) - Umbella simplex; involucrum tetraphyllum; calyx integer, obsoletus; corollae petala quinque integra, plana; fructus compressus, on bicularis, didymus; sporae semiorbiculatae, munitae quibusdam elevationibus filamentosis; - (Plantao ut plurimum reptantes.: Folia simplicia, integra, interdum peltata, rarius lobata; umbella pedicellata, saepe simplex, terminalis aut axiliaris, interdum ramosa).

784 A Z O ELLA, (AZORELLE) IAM. tab. 189. - HYDROCOTYIE (tridentata, triloba) THUNB.? (umbellifera) - Umbella simplex; involucrum tri-quinqueocto-phyllum; calyx quinquedentatus! corollae petaia quinque ovata. oblonga, integia, obtusa; fructus fero globosus, striatus, calyce persistente coronatus, monosporus.

785. FRAGROSA, (FRAGROSE) ruIz et pAv. Flores radiati fertiles. Involucrum octophyllum; corollae petala inaequalia; fructus ovatus: sporae striatac. (PERSOON certe pag. 303. enchir. Sub HYDROCOTILE Militare iussit)

786. SPANANTHE. (SPANANTHE) IACQ. Icon. 3. et Collect. 3. HYJROCOTYLE wiLLD. (umbellifera 4., - Umbella simplex; involucrum subheptaphyllum; corollue aequales; petala lanceolata, pateutu; fructus ovalis, in dorso tristriatus.

787. CHA MI TIS, (CHAMITIS) GAERTN. t. 22. f. 4. (umbella anomala) - Umbella nulla aut simplexs involucrum nullum aut fere octophyllum; calyx quin. quedentatus, persistens; corollac petala quinque ovalioblonga, obtusa, integerrima, alba; styli duo filifor: mes; stigmata incrassata; fructus parum globosus; striatus, seso dividens in duos. (Folia integra, versus elongationem serrata).

788. $13 \mathrm{OWLESIA,} \mathrm{(BOWLESIE)} \mathrm{RuIz} \mathrm{et} \mathrm{pav.}$ (umbellifera) - U'mbella simplex, triflora; flosculi terciles, paralleii; petalu aequalia; frictus terragonus, subpyramidalis; sporce echimatae, exius concavae. 
789. SAN I C ULA, (SANICULE) TOURN, LIN⿴囗十 JUss. LAM. tab. 191. ARTEI)IA PALIAS. (umbellifera 3.) - Umbellae in capitulum coarctatae et confertae, involucella parva, poly phylla; calyx subinteger; corollae petala quinque integra, apice curva ; flores centri masculi,

- disci abortientes; fructus ovoïdeus, fere globosus, scaber punctis duris vel maricato aculeatus; (Flores fere sessiles, contri masculi; folia palmata aut digitata).

790. ASTRANTIA, (ASTRANCE) TOURN. LIN N。 JUss. LAM. Itab. 191. (umbellifera 3:) - Umbella triquadriradiata; involucella partialia polyphylla, foliolis lanceolatis latiusculis, coloratis, patentibus, aequalibus, petalis longioribus, plantae totius foliis similibus; umbellulae multiflorae; $\operatorname{calyx}$ quinquedentatus persistens: corollae petala quinqne, curva et bifida; Hores plurimi steriles abortientes; fructus ovoüdeus, calyce. superante coronatus; sporaeduae, uno latere planae, ǵlabrae, altero convexae et quinque angulis elevato transverse rigidae aut asperae; - (folia saepe palmata).

79I. BUPLEURUM, (BUPLEURE) TOUR. IINN. JUSS, LAM, tab. 189. (umbollifera 3.) - Involucrum polyphyllum, brove, rafíts tri-quinquefidum 3 folia interdum basi coalita; involucella pentaphylla, umbellulae maiora; calyx integer; curcliae petala quinque integra, brevia, iutrorsum semicirculariter involuta, aequalia; fructus ovalis vel subrotundus, gibbus, coronatus, striatus, leviter compressus. - (Vilia inte. gerrima, interduru basi coalıta; flores flavi; umbellae et umbellulae compositae utplurimuin parvo radiorum numero.)

792. HERMAS, (HER.MAS) IINN. JUSS. LAM. tab. 85r. BUPLEURUM I/AN. (umbellifera 3 ). Polýgatna; umbelia hemisphaericá radii floribus masculis deciduis) truncata, centri hermaphrodita; umbollae larerales masculae, invoiucrum polyphyllum, (3-12.); involucella di-triphylla, tunilateralia; flores hermaphruditi: calyx: integer, minimus, quinquedentatus, petala corollae quinque integra, aequaiia; staminu quinque sterilia, 
styli dıo; fructus rotundatus, parum äpplanatus, formatus sporis duabus ellypticis, suborbiculatis, compressis, stria lon ritudinali elevatis. Tlores masculi: calyx et corolla ut in hermaphroditis; stamina quinque fortilia.

793. HERACLEU M, (IERACLEE) IINN. JUSS. IAM. tab. 200. SPONDILIUM TOURN. (umbellitera 3.) Involucrum polsphyllum, caducum; involucella tri-heptaphyl!a, foliolis longis, linearibus, inaequalia ; - calyx fere integer; corollue potala emarginata, apice inflexo curvata; petala circumferentiae inaequalia, difformia, externa bifida, centri aequalia; fructus ellipticus, levirer striatus, compressus, ad summum emarginatus; sporae duae masgine membranaceae. - (Umbellae et umbelIulae formatao magno radiorum numero; flores centrales abortu subiecti.

794. OEHANTHE, (ENANTE ) TourN. Morrs: LAN JUSS. xAM. tab. 203. ( umbellifera.) - Involucrum interuirn nullum, rarins mono-diphyllum; sae. pissime polvphyllum; involuceila polyphylla; - caigx quinquedentatus; curollae petala quinque, disci coráta, curvata, subaequalia; circumferentiae raiora, bifida, inaequalia; flosruli difformes, in disco sessiles, circum. ferentiae steriles; fructus oblongus ant fere ovoüdeus, retusus, suberoso-corticatus, calyce ct pistillo coronatus; sporae duaé striatae. - (Hlantae aquaticne; $1 \mathrm{~m}$. bellae efformatae e parvo radiorum numero; umbellulae globulosae).

79). ECHINOPHORA, (ECHINORIORE) IINN. LA I. tab. 190. (umbellifera 3.) - Involucrum tri-tetrapliyllum; involucelía turbinata, quinque-sexfida; calyx quinquedentatus; corollue petala guinque inaegnalia; flores laterales masculi; centrales hermaphroditi, calyce nullo; spora uma, tecta involucello inmersa, spinosa.

796. CA UCALIS, (CAUCAIIDE) TourN, uINN. JUSs. IAM. tab. 1u2. (umbellifera 3.) - Involucrum polyphylium, simplex, rarius nullum, aut monodiphyl. lum, foliolis integris; invoiucella polyphylla simplicia; [I3] calyx 
calyx quiaquedentatus; comilie petala inflexo emarginata, rccurvato cordata, disci aequalia, circumferen. tiae, inaequalia; exteriori maiori, bifido; corolla radiata ; flores disci masculi; fructus ovalis oblongus, exasperatus, vel hispidus setis mox dissitis, mnx ad latus sporarun positis; sporae duae striatae, asperae. -

- (umbella minori radiorum numoro composita).

797. ARTEDIA, (ARTEDIE) IINx. JUSS. IAM. tab. 193. TAPSIA Totra. (umbellifera 3.) - Iuvoluc: um polyphyllum, pinnatifidum foliolis saepe super elungationem reversis, basi membranaceis, superiori parte fere pectinatis; fores disci fere abortientes; caly $x$ quinquedentatus: corcllae radiatae petala quinque recurrato - cordata, disci aequalia, circumferentiae inaequalia, exteriora bifida; fiores disci masculi; fructus orbicularis, compressus; sporce duae, planae in lo. bos decem, undecim, margine membranaceo, ro. tundatae, extrorsum notatae quinque lineis parum exsertis, scariosae; - (Folia multifida, linearia; elongatio cylindrica).

DAUCUS, (CAROTTE) TOURN. IINN. JUSS. IAn. tab. 192. (umbellifera 3.) - Involucrum polyphyllum, pirnatufidum; culyx integer; corollae radiatae petala quinque, curoata, cordata, exterinra maxima: flo. res circumferentiae ab rtiunt; fructus ovoïdeus, pilis rigidis hispidus aut muricatus; sporce planae, interne striatae, cenvexae, et parvis lateribus membranaceis externe elevatae; (Umbellae planae per florescentiae sta. tum sese coutrahentes, et concavae redditac tempore quo fructuls maturitati accedit.)

799 TORDYLIU Ḿ, (TORDYLIER) TOURN. Ixx. Juss. LAM tab. 193. (umbellifera 3.) - Invulucrum polyphyllum, foliolis integris: involucella subtriphylla dimidiata; (involucrum longum, indivisum P.) - calux quinquedentatus; curollae radiatae petala recurva, cordata; - disci aequalia, circumterentiae inaequalia, exteriora maiora; omnes flosculs teriles aut hermaphroditi; fructus conpressus, suborbscularis; 
sporae duae leviter planae, margine inflato-callosae et crenatae.

800. L A S ERPITIUM. (LASER) TOURN. IINN. JUss. LA MI. tab. 199. ANGELICA et LIGUSTICUM ToURn. (umbellifera 3.) - Involucrum et involucella po. lyphylla foliolis inaequalibus et membranaceis; calyx fere integer; coroliae petala apice curva vel inflexa, omárginata, patentia; fructus ovalis aut oblongus; sporae duae alis duabus membranaceis saepe erosis angulatum fructum roddunt: - (Umbellae et umbellulao multis radiis efformatae )

801. PEUCEDAN UM, (PEUCEDAN) TOURN: IINN. JUSS. ANGELICA et OREOSELINUM TOUR. (umbellifera 3.) - Involucrum polyphyllum (rarius mono-pentaphyllum) reflexum; involucella polyphylla, reflexa; utraque, et involucrum, et involucella, brevissima; calyx parvus, quinquedentatus; corollae petala quinque, oblonga, inflexa apice, aequalia: fructus ovatus, loviter compressus: sporae duae striatae, marginibus attenuatae et fere ala cinctae; - (Elongatio praealta; folia multifido linearia. Centrales umbellae ut plurimum breviores quam radii; flores flave cunt.)

802 A M MI, (AMMI) TOURN. IINN. JUSS. LAM, tab. 193. (umbellifera 3.). - Involucrum polyphyllum, pirnatifidum; involucella polyphylla, simplicia; umbella laxa; calyx integer: corcllue radiatae petala recurvato cordata; disci aequalia, circumferentiao inaequalia; fores omnes hermaphroditi; fructus parvus, rot ndatus; spo. rae duae striatae, laeves. (VISNAGA cAERTN.)

\section{HASSELQUIS T IA,} ( HASSELQUIST)

IIN N. JACQ, Juss. (umbellifera 3.) - Involucrum quiliquefidum; involucella dimidiata, triphylla: calyx quinquedentatus; corollae radiata $\theta$ petala recurvata, cordata, bifida, disci aoqualia, inaequalia circumferentiae : flores disci masculi et abortivi; fructus circumforentiae vel umbellae exterioris sporis duabus ovali-orbiculatis margino crenulatis, disci vel interioris umbellae for- 
matus ex una spora interne concava, haemisphaerica, urceoiata: spora altera abortiens, et sese repraesentans forma squamae exsiccatae; - (Umbellula centra. lis abortiens.

8c4. C I CUTA, (CIGUE) Touriv. juss. IAM. tab. I95. CONIUM IINN. (umbellifera 3.) - Involucrum tri-pentaphyllum; involucellu triphylla, foliolis reflexis; (Involucrum nullum 3 involucrum partiale iri-pentaphyllum rers. $p$ 318.) dimidiata; calyx integer; corollae petala cordata, recurvata, inaequalia; fructus ovato leviter globosus; sporae duae, quinguangulae, angulis elevatis rel sulcatis, hinc lateribus quinquo crenulatae aut tuberculosae; angulis tribus dorsalibus, duobus lateralibus; - (Folia pleruniqualato-pinnata.)

805. EXOACAN THA, (EXOACANTHE) IABILI. syr. tab, 2. LAM. tab. 190. ViLID. (umbellitera anomala). - Involucrum polyphyllum, foliolis integris, canaliculatis, spinosis: invelucellis dimidiatis, compositis e radiis magnitudine inaequali; caly $x$ integer; corollac petala magnitudine aequalia, cordata et recurva; flures omues hermaplroditi; fructus ovalís; sporae duae ovales, striatae, convexae, uno latere planae.

80. BUNIUM. (BUNIE, Suron, Terre-noix) IIN . Juss. IAM. tab. 197. (umbelliera 3.) - Involucrum et involucella polyphyila; umbelia conferta calyx integer; corvilue unif rmis petala quinque curva, cordata, aequalia: fructus ovato-oblongus, striatus: sporue duae striatae, inter st iarurn interstitia tubesculosae; - ('Mruncus subcerraneus (radix) tuberosus, lere spbaoricus).

807 A T 1 A MA N THA . (ATHAMANTHE) IINN. JUSS. IAM tab. 194. CHAEROPHYLLUM et OREUSE. LIVUM TUURN (umbellitera j) - Inveincrum polyphyllum ant rarius fore triphyllum; invoincela poigphylla caly $x$ integer: coroliae petala emarginata, apice incurva, rere aequalia: fructus ovalis ant obiongus, striatus; spurue diade stratae. fere glabrae ant pubescontes, extrorsum elevatae expansionibus quinque memsranaceis, 
ceis, aut sulcatae striis quinquo interdum vix intellegibilibus. - (Umbellae multos radios habent).

808. S I U M, (BERLE) TOURN. IINN. JUSS LAM. tab. 197. SISARUM et $A M M I$ TOURN. (umbellifera 3.) Involucrum polyphyllum, reflexum; involucella polyphylla, brevia: calyx integer; corollae petala cordata, recurva, a equalia; fructus subovatus vel ovoïdeus, oblongus, compressps, striatus; sporae duae striatae; (Folia saepo alato-pinnata.)

309 SISO N, (SISON) IINN. JUSS. SIUM IAM. SIUM et FOENICULU.M et CARVI TOURN. (umbellifera 3.). - Involucrum tri-tetraphyllum, inaequale; rarius nullum; involucella fere tetraphylla; calyx integer; corollae petala lanceolata, apice recurva: fructus ovalis, striatus; sporae duae striatae; - (Umbellae paucis radiis constitutae.)

810. OLIVERIA, (OLIVIĖRE) VENTENAT (Umbellifera 2.) - Char generis: Umbella aequslis, tri-quadriradiata: umbellulaetres, quatuor, simplices. aeguales, multiflorae, frosculis omnibus fertilibus; involucrum tritetraphyllum, foliolis numero radiorum, umbella brevioribus, profunde trilobis, lobis trifidis; invoincella polyphylla, foliolis cuneiformibus, trifidis, umbellulis vix longioribus; calyx quinquedentatus; curollae petala quinque, bipartita, lacinis ad basin margine involutis, latere excavatis, superne expansis, undulatis, reflexis; stamina quinque; filamenta filiformia, primu h inflexa et petalorum cavitate recondita; mox.libera, et exserta; untherae subroiundae; ovarium subovatum; styli duo, teretes, erecti; stigmata capitata; fructus subovatus, hirsutus, bipartibilis; sporue duae, ovatae, hinc convexac, et quinquejugatae, inde planse et unisulcatae. - Char: essentialis: Involucrum et involucella polyphylla, foliolis trifidis; umbeila paucis radis cousttuta; umbellulae simplices: flosculi onnes fertiles; ca$i y x$ quinquedentatus; petala bipartita, aequalia, laciniis profundis; fructus subovato-oblongus, hirsutus; 
sporae duae dorso quinquejugatae, commissura monosulcatae.

8I1. SELINUMT, (SELIN.) IINN. JUSS. LAM, tab. 200. ATHAMIANTA IINN. THYSSELINUM TOURN. CARVIFOLIA VAJLL. (umbellifera 3.) - Involucrum polyphyllum, reflexum; involucella polyphylla; - calyx integer; corollae petala cordata, aequalia; fructus corrpresso planus, ovali oblongus aut rotundatus; sporae duae, quinquenerviae, processibus membranacois duabus lateralibus exsertis striatae.

812. MULINUM, (MUIINE) TERS. SELINUMI cAVAN. (umbellifera.) - Umbella simplex; involucrum polyphyllum; calyx denticulatus; corollae petala quin. que lutescentia; fructus turgidus, ovatus, profunde sulcatus, angulis rotundis. TErs. ench. pag. 309. *)

8I3. GIN GIDIU M, (GINGIDIE) Fors1. aust. $t$. 2:.Juss. (umbellifera 3.) Calyx quinquedentatus; corollae petala lanceolata, cordato recurva; fructus ovalis, calyco coronatus; sporaequadristriatae. (Umbellae inaequali magnitudine; umbellula pauciflorae; involucrum hexaphyllum ).

814. CUMINUM, (CUMIN) IINN. JUSS. IAM. tab. 199. CAVAN. tab, 360. FOENICULUM TOURN. (umbellifera 3.) - Involucrum et involucella regulariter quadrifida, vel tetraphylla, foliolis interdum trifidis et simplicibus; umbellulae quatuor; caly $x$ integer; corollae petala emarginata, apice introrsum curva, fere aequalia; fructus ovalis, calyce coronatus, parvus, striatus; sporae duae, striatae.

8I5, FERULA, (FERULE) TOURN. IINN. JUSS. I'AM. tab. 205. (unbellifera 3.) - Involucrum cadu. cum ; involucella polyphylla, brevia; calyx integer; corollae petala oblonga, apice incurva, subaequalia; fructus ovalis, compressus, trinervatus processibus membranaceis parum exsertis, marginibus lateraliter exsertis

*) Plantas huius generis nmnino habitu a Selinis differre con. teadit generis cunditor gravissimus. 
tis et erectis; sporae duae ellipticao, trifervatae:(Umbellae et umbellulae globulosas, formatae plurimis - radiis; flores flavi.)

8I6. CRITH MUM, (CRITHME, Eacille) ToUrN. IINN. JUSS. IAMr, tab. 197. (umbellifera 3.) - Inrolucrum polyphyllum reflexum; involncella polyphylla; calyx integer; corollae petala quinque integra, apice in. curva, fere aequalia ; fructus ovoideus, compressus, striatus; sporae duae striatae; - (Flosculi aequales, albi; umbellae et umbellulae homisphaericae contormatae magno radiorum nuinero.)

817. BUBON, (BUBON) rINN. IANt. tab. 194. APIUM et FEPULA tounN. (umbellifera 3.) - Irvolucrum quinquefidum, saepissime pentaphyllum; involucella polyphylla; calyx fere quuinquedentatus; corollae petala quinque lancenlata, apice reflexa; fructus ova. lis, striatus, in quibusdam speciebus villesus; sporae duae, striatae, glabrae, saepissime villosae.

Sis CACHRYS. (CACIRYTF, Armarinte) tourn. xINN. JUSs. LAM. tab 20.5. (umbellifera 3.) - Invclucrum et involucella polyphylla, interdum ramosa; calyx minimus integer, aut quinquedentatus; coroliae petala quinque obinnga, lanceolata, apice recurra, aequalia; fructus magnus, (subovatus), ovalis, pasum compressus, cylindricus, ut plurimum angulosus aut lacvis; sporae duac convexae, tectae testa fungosa et suberosa, incrassatae: - (Umbellae ct umbellulae efformatae mag. no radiorum numero; flores ftavi).

819 L I GUS T ICU M, (LIVÊCHE) TOURN.IINN. JUss. LAMr. tab. 198. APIUM BAUH. GINGIDIUM Forits. CICUTARIA rounN. (umbellifera 3.) - Involucrnm saepe lieptaphyllum; involucella fere tetraphylla, faliolis membranaceis ut in involucro; calyx subinteger vel quinquedentatus; corollae petala integra, introrsum voluta; fructus oblongus; sporae duae, glabrae, altero latcre planse, altero convexao, quinque costis incras. satis et exsertis elevatae, hinc quinquesulcatae; (Umbellise et umbellulas magno radiorum nusiero conformetae). 
830. A N GELIĆA, (ANGÉLIQUE) TOURN. LIN. JUSs. IAM. tab. 193. IMPERATORIA TOUnN. (umbellifera 3.) - Involucrum tri-pentaphyllum; rarius mo* nophyllum; involucellu penta-octophyila, rarius nulla; calyx leviter notatus quinque dentibus; corollae petala lanceolata, apice reflexa, aequalia; styli horizontales aut reflexi; frictus fere subrotundatus aut ovalis, angulosus, solidus; sporae duae costis quinque elerato profunde sulcatae, trialatae, glabrae; - (Umbella radiis multis constructa).

821. HUANACA, (HUANACE) cAvan. tab. 528. (umbellifera). - Involucrum diphyllum, foliolis tripartitis longis; involucella polyphylla; calyx persistens, denticulatus, denticulis minimis; corollae potala quinquelanceolata, patentia; fructus ovatus, acutus; sporne planae, unisulcatae, externe convexae, tribus lineis elevatis striatae.

B. Umbellae fere semper nudae; umbellulae involucellatae.

822. AETHUSA, (AETHUSE) IINN. JUSS. IAM. tab. 19\%. (umbellifera 2.) - Involucrum nullum, aut mono-diphyllum; involucella dimidiata, tri-pentaphyl1a, pendula; caly $x$ integer; corollae petala recurva, cordata, inaequalia; fructus ovö̈deus aut oblongus, striatus ant sulcatus; sporae duae striatae vel sulcatae. (Umbellulae centri breviores.)

823. MEUM, ( MEUM) JACQ. 'sNITH. LIGUSTI. CUM CPANZ. AETHUSA IIN. SESELI GAERTN. (UM. bellifera). - Involucrum monophyllum; involucella pancifolia, sublateralia; calyx obsoletus; corollae petala quinque integra, inflexa; fructus elliptico oblongus; sporae duae tricostatae.

824. CORIANDRUM, (CORIANDRE) TouRN. LINN. JUSS. LAM. tab. 196. (umbellifera 2.) - Involucrum monophyllum, rarius nullum; involucella dimidiata, 
dista, triphylla, aut nulla; calyx quinquedentatus; corollae radiatae petala quinquo emarginata, curva sive inflexa, cordata, disci aequalia, circumferentia inaequalia; flores centri abortientes; fractus sphaericus aut didymus; sporae duae striatae.

\$25. CHAEROPHYLLUM, (CERFVIL) TOURN. GAERTN. IINN. JUSS. MYRRHIS TOURN. SCAN1) IX LINN. Juss. (umbellifera $2_{3}$ ) - Ivvoincrum nullum (reflexum concavum pers.); involucclia ut pluximum pentaphylla; calyx integer; corollae petala cordata aut emarginata, inflexa, inaequalia; fructus oblongus, cylindricus, laevis, subulatus; sporae duae, stria. tao; -. (fores centri abortientes).

*) CEREFOLIUM, sporis laevibus; involu. crum passim mono-diphyllum.

826. MYRRHIS, (MYRRHIS) GAERTN. SCANDIX IINN. JUSS. CHAEROPHYLLUM IAM. ODORATA PENT. (umbellifera) - Involucrum nullum; involucella regulariter pentaphylla, ereçta; calyx integer; corollae potala quinque cordata aut emarginata, inaequalia; umbella composita; fructus oblongus, attenuatus a pice in rostrum brevem; sporae duae striatae, imo pro. funde sulcatae, angulis membranaceis, glabrae aut hispidae.

827. SCA N D IX, (SCANDIX, myrrhide) TOURN.

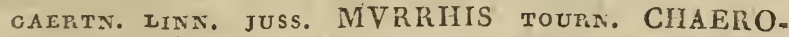
PHYLLUM TOURN. I.AM. tab. 201. f. 6 . (umbellifera 2.) - Involucrum saepissime nullum, rarius polyphyllum, pinnato incisum aut emarginatum; involucella pentaphylla; umbella simplex; flores radiaii; calyx integer; corollue petala guinque emarginata, apice recurva inaequalia, exteriora maiora: fructus oblongus, rostro longissimo terminatus, tenerrime striatus; sporae duae glabrae aut hispidae; - (Flores centri abortivi)。

828. A N THRISCUS, (ANTHRISCUS) PERS. p. 320. SCANDIX IINN. CAUCALIS Roth. CHAEfio. 
PIYT LUM IAM. (umbellifera) umbella compositas iuvolucella lanceolata; fructus ovatus, pilis scaber, rostro glabro.

\section{8:9 PHELLANDRIUM, ( PHELLANDRIE)} TOURN. LINN. JUSS. OENANTHE raM. (umbellifera 2.). - Involucrum nullum; involucella hepraphylla; catyx quinquedentatus corollae petala infexo curvata, cordata, inaequalia; fores omnes fertiles, disci sive centri minores; fructus ovatus, striatus aut sulcatus, dentibus calycis et pistillo coronatus; sporae duae laeves, elonEatae.

830. IMPERATOR IA, (IMPÉRATOIRE) TOUAN. zIRn. JUss, LAM. tab 199. (umbellifera 2 ). - Involucrum nullum; involucella mono - diphylla, brevissima; calyx integer: corollae petala emarginata, inflexa, fere aequalia ; fructus subrotundus, ellipticus, compressus; sporae interne planae et notarae duabus lineis fuscis, arcuatis ; dorso tribus sostis elevatae, medio gibbae, margine alato membranacoae. - (Folia terna; floralia fere opposita.

\$3r. SESELI, (SESELI) LINN. JUSS. IAM. tab. 202. FOENICULUM toURN. (umbellifera 2.). - Involucrum nullum aut monophyllum; involucella mono - triphylla, aut polyphylla; calyx integer: corollae petala quinquo recurva, cordata, asqualia; fructus parvus, ovoïdeus, striatus; spora una striata. - (Umbellulae breves, globosae).

832. CICUTARIA, (CICUTAIRE) LAM. tab.195. jUss. CICUTA IINN. ANGELICA TOURN. (umbellifera 2.). - Involucrum ut plurimum nullum, rarius foliolum unum lineare; involucella tri-pentaphylla, foliolis maxime erectis, umbellulam saepe comitantibus, brevia; calyx integer; corollae petala quinque integra, ovalia, apice curva, fere aequalia; fructus ovoïdeus, sulcatus; sporae duae striatao.

C. $U m$ * 
C. Umzella et umbellulis regulariter nudis.

833. SMYR NIUM . (MACERON) TOURN. IINM. JUSS. LAM, tab. 204. LIGUSTIC UM vILLARs. (umbellifera r.). - Involucro et involucellis nullis; caly $x$ integer; corollae petala quinque acuminata, carinata, apice leviter reflexa, subaequalia; flores flavi, centrales steriles vel abortientes; fructus fere ovalis, globosus, gibbosus, subcompressus, striatus; sporae duae maxime convexae, hinc lunulatae, nervis tribus striatis elevatae. - (Folia elongationis simplicia aut teruata).

834- CARUM, (CARVI) IINN. JUSS. SESELI LAM. CARVI TOUPN. (umbellifera 1.). - Involucrum submonophyllum; involucella nulla; calyx integer; corollae petala qunque sursum reflectendo carinata, inaegaalia, emarginata; flores albi, centrales abortu subiecti; fructus ovato - oblongus; sporae duae, altero latere convexì, altero planae, quinque nervis striatae.

835. THAPSIA, (THAPSIE) TOURN, IINN. JUSs. IAM. tab. 206 (umbellifera 1.). - Involucrum et involucella nulla; calyx integer; corollae petala quinque lanceolata, apice recurva, flavescentia; fructus oblongus, compressus, duobus extremis emarginatus, alis membranaceus; sporae duae striatae, marginatae uno latere membrana alata, supra infraque emarginatae.

83\%. PAS T I N A CA, (PANAJS) TOURN. IINN. JUSS. LAM. tab. 206. (umbellifera 1.). - Involucrum et involucella ut plurimum nulla; calyx integer; corollue petala quinque integra, introrsumflexa vel involuta, fere ae. qualia; fructus ellipticus, compresso planus; sporae duae, leviter apice emarginatae, tribus nervis prominentibus munitae, fore ad margines ciliatae, - (Flores flavi; folia alato pinnata).

337. A NE'THUM, (ANETH, Fenouil) TOURN. IxNN: JUss. xAM. tab. 204. FOENICULUM TOURA (umbellifora 1.). - Involucrum et involucella nulla ; calyx integer; corollae petala quinque integra, introrsum involuta in semi- 
semicirculum, brevissima, fere aequalia; fructus lenticularis, striatus, compressus; sporae duae striatae, membrana marginatae aut nudae. - (Flores flavi; falia multifida).

\section{\$38. AEGOPODIUM, (AEGOPODE, Podagraire)} IINN. ANGELICA tourn. PIMPINELLA lam. (um. bellifera 1.). - Involucrim et involucella nulla: calyx integer; corollae petala quinque, ovalia, apice incurvo, subaequalia; fructus ovato ablongus, striatus, costatus, leviter convexus; sporae duae sriatae.

839 A P I UM, ( ACHE, Fersil.) TOURN, IrNn. JÜs. r.AM. tab. 196. (umbellifera 1.) - Involucrum monotri - tetraphyllum; involucella involucris consentarea vel utraque nulla; calyx integer; curollae petala quinque rotundata, apice curvata, aequalıa; fructus ovoïdeus, globosus; sporae duae striatae, notatae duabus expansionibu\& membranacess (nervis) parum exsertis. - (Flores flavescentes).

840. PI M INELLA, (BOUCAGE) IINN. JUSS. LAM. tab. 203. TRAGOSELINUM tounn. (umbellifera I.). - Involucrum et involucella nulla; umbellulue subglobosae, primum nutantes; calyx integer corollue petala quinque apice curvato cordata, fere aequalia; stig mata subglobosa; fructus ovali - oblongus; sporae dua leviter striatae.

\section{$\begin{array}{llllllll}T & R & I & G & Y & N & I & A\end{array}$.}

\section{Flore supero.}

84T. VI B.UR N UM, (VIORNE) TOURN. IINN. JUS8. xAM, tab. 211. TINUS et OPULUS tourn. (caprifolia 3.). - Culux superus, parvus, quinquepartitus; (quinquefidus) bracteis basi adauctus; curollu campanulata, quinquefida; stamina quinque, cum laciniis corollae alterpantia; stylus nullus; drupa aut bacca infera, nuce 
monospora ${ }^{*}$ ) coronata in Tinus TourN., nuda in reliquis speciebus. - (Trutices foliis oppositis; flores dispositi in umbellam corymbiformem et terminalem).

842. SA MBUCUS, (SUREAU) TOURN. LINN. JU8S. LAM tab. 211. (caprifolia 3.). - Calyx parvus, quinquefidus, (quinquepartitus) \& corolla rotata, quinqueloba, (quinquefida); stumina quinque alterna cum corolla lobis; stylus nullus; bacca rotundata, unilocularis, tritetraspora, vix coronata. - (Arbores aut frutices: fo. lia opposita, pinnata cum impari aut bipinnata, in paus cis speciebus stipulacea aut in plurimis duabus glandus. lis stipulaceis munita; flores dispositi in umbellam corymbiformem et terminalern).

\section{Flore infero.}

843. RHUS, (SUMAC) TOURN. IINN. suppl. WILID. JUss. LAir. tab. 2 ?. TUXICODENDRON ot COT\&. NUS TOURN. ANACARDIUM LINN. supl. IAM. tab. 268. (terebinthacea 5. - - Culyx inferus, quinquefidus; curolla pentapetala; stamina brevia; antherae minimae; styli nulli aut tres brerissini; bacca parva reniformis, compressa, inserta receptaculo in pedunculo dilatato, magno, carnoso, turbinata aut cupulitornis, continens

nucem

*) BonkifuUsen ap. Roem. I. 2. p. 19. ita characterem generis differentialem constituit: "Calux persistens, minimus, "quinquepartitus; corolla campanulata, quinquepartita; ,sturnina quinque aequalia, laciniis corollae alicrnantia; ,sti, ma $m 11 \mathrm{ml}$, tria, sessilia; drupa globusa, monospo„ra;“ asque idem ancsor seinngit l'iburni genus in tria alia genera. I ) TrNUS: culuce persistente, minimo, quinquepartito; corolla campanulata, quinquemartisa; staminibus quinque; stylo mullo: stigmate unico scssiii; fructu autro corucoso, uniloculari, monosporo 2.) UPULUS: caluce persistente, mmimo, quincuedentate: coro'la et stammobus ut in TrNo; stipmatious tribus ses-ilibus; fructu antro pomo monosporo. 3.) IIBUrifun: culyce, corollu, stamtabus uit in TIVO: stigmatibus tribus scsoilibus; fructs drupa monospora. 
nucem monosporam. - (Frutices aut suffrutices foliis simplicibus, ternatis aut pinnatis cum impari; floribus in panicula aut in racemis confertis, interdum mere masculis )

* 844. SUMACRUS, (SUMACRE) IINN. IAM. tab. 20\%. (terebinthacea). - Calyx inférus, quinquepartitus; corolla pentapetald; drupu baccaeformis, nuce monospora.

845. SPATHELIA, (SPATHELIE) IINN. IAM. tab. 209. (terebinthacea). - Calyx pentapliyllus? (quinquepartitus); corolla pentapetala; stamina basi dilatata et barbata; capsula trigona, trilocularis, trispora.

846. STACKHOUSIA, (STACKIOUSIE) smith transact. IIII. (terebinthacea juss. gen. $369_{.}$). - Calyx quinquepartitus; corolla pentapetala, petalis unguibus coalitis; capsula tricocca, loculis monosporis.

847. TRICEROS, (TRICEROS) rour. conch. p. 230. (terebintacea 2.). - Calyx profunde quinquefidus; corollae petala quinque calyce longiora; stamina quinque; styli tres, uno persistente; stigmata tria; bacca coriacea, calyce cincta, terminata stylis, trilocularis, loculis disporis. - (Irutex: folia impari pinnata; flores in racomis laxis, subterminalibus).

848. OCHROXYLUM, ( OCHROXYLON) schrer. (terebinthaceis affnis). - Calyx parvus quinquefidus; corollae petala quinquo, parum incrassata, versus summum cavitate munita; stamina quinque brevia; ovaria tria locata super discum annularem, trilobum, lobis subdistinctis; styli tres breves; stigmata tria; capst:iae? tres, approximatae, interno latere dehiscentes; loculi dispori. - (Arbores spinosae aut inermes; folia simplića, alterna, obsita muricationibus pellucidis; flores in panicula terminali).

849. SAGONEA, (SAGONE) AUBI. IUss. IAM. tab. 2:2. REICIELIA scinEz. TERS. (convolvulea 2.). Culyx maguus, quinguepartitus ; corolla campanulata, quis- 
quinquefila, calyce minor; stamira tubo inserta; antierae oblongae, versatiles, ad extremum bifidae; stigrnutu capitata; capsula trilocularis, polyspora, circumscissa; sporae parvae, receptaculo centrali maximo adhaerentes, triangulares.

850. PALIUTUS, (PALIURE, Argalou) ToUrn. IINN. JUSs. LAMI. tab. 210. pens. sub Rhamno p. 24r. RHAMNUS IINN. (rhamnea 3). - Calyx patens, quinquefidus; coroliae peta!a quinque, squamaeformia, disco glanduloso ad basin calycis locato inserta; stamina disco inserta; ovarium disco cinctum; styli tres; drupa exsuc. ca, ala membranacea lata orbiculari et striata cincta, heinisphaerica, truncata, continens nucem bi - trilocis. larem, di - trisporam. - (Flores axillares Ziziphi).

8รI. CASSINE, (CASSINE) LINN. JUSs. LAM. tab. 130. EVONYMIUS IINN. mantiss. (rhamnea 2.). - Cam lyx minimus, quinquepartitus; corollae petala quinque patentia, basi dilatata et fere coalita; styli nulli; bacca trilocularis, trispora, stigmate persistente umbilicata. (Frutices aut arbores mediae altitudinis; folia opposita aut alterna; pedup̣culi axillares).

852. STAPHYLEA. (STAPHYLIN, NTez-coupé) IINN. JUSS. LAM. tab. 130. STAPHYLODENDRON TounN. (rhamnea 1.). - Calyx profunde quinquefius, concavus, coloratus, basi interna disco urceolato mu. nitus ; corollae petala quinque inserta disci margine, călyce concolora; stamina disco inserta; ovarium bi - tripartitum, styli et stigmata duo vel tria; fructus: capsulae duae, tres, membranaceae, versus medium coalitac seur connatae, vesiciloso inflatae, dehiscentes interne ad summum, mono-disporae: sporue nuces duae osseae, basi umbilicatae et truncatae, fere globosae cum cicatrice. - (Arbores nediae altitudinis; folia opposita, longis stipulis munita, ternata aut pinnata cum impari; flores dispositi in racemum terminalem, bracteatum).

853 TAMARIX, ( TAMARILUE, TAMARIS ) IINN. JUSS, LAM, tab. 213. TAMARIOCUS TOUR MEN. mo:a- 
monadelph. p. 113. Ej. obs. bot.1782. p,162. ubi genera TAMARIX et TAMARISCUS disiunxit. (portulacea). Calyx campanulatus, quinquepartitus, persistens; corol. lae petala quinque calyco maiora; stamina quinque - decom; antherae rotundatas; siylus nullus; stigmata oblonga, pubescentia; capsula oblonga, triquetra, milocularis, trivalvis, polyspora; sporcue coma instructao. (Frutices Cupresso et Junipero simillimae).

854 DRYPIS, (DHYPIS) MICH. IINN. JUSS. IAM. tab. 214. (caryophyllea 6.). - Calyx tubulosus, quinquedentatus; corollae petala qainque unguiculata, ad faucem apice bipartitam bidentata; capsula unilocularis, monospora, circumscisse dehiscens; spora reniformis.

855. MALESHERBIA, (MALESHERBE) DOMBEY. PUIZ-PAV. GYNOPLEUKA cAv. ic. tab. 375. (sedis incertae). - Calyx inferus, tubulosus, coloratus, limbo quinquefido, persistens; corollae petala quinque, lanceolata, breviora, Jaciniis calycinis et faci calycis infra lacinias insidentia; nectarolyma coronula decem vel plurimis squamis vol sex, membranaceis, duabus, quatuor, crenatis, petalis alternis constans, fauci corollse insertum; stamina filiformia; antherae incumberstes basi excavatae nectarolymatis, pedicellatae, erectae, sagittatae; ovariurn stipitatum; styli tres ad basin (infra apicem cAv.) ovarii orbiculatim adfixi ; stigmata obtuso capitata; capsulu triangularis, unilncularis apice, trival. vis; sporae plurimae affixae receptaculis tribus linearibus, per capsulae parietem ciecurrentibus.

856. TURNERA, (TURNERE) PIUN. IINN. JUSS: I Ax. tab. 252. PIRIQUFTA AUßL. BUPC RDIÁ sснREB. (portuacea r.). - Calyx tubulosus, infundibuliformis, limbo guinquefido; exterior diphylius; corollae petala quinque unguiculata, calyci inserta; stamina perigyna; antherae rectao; styli tres; stignata multifida; capsula unilocularis, trivalyis, polyspora, sporas in medio gerens. (Elongatio trunci herbacea aut sufirutescens; folia alterna non cernosa; flores axillares, solitarii aut super foliorum periolos inserti). 
857. TACHIBOTA, (TACHIBOTE) AUBг, Guy. t. 112. JUSS. LAM. tab. 208. SALMASIA SCMrE. PERS. (cisteis affinis). - Calyx quinquepartitus, persistens; corollae petala quinque, hypogyna, cum calyce alterna; stamina hypogyna; antherae rotundatae: stylus nullus; capsula calyce cincta, triangula, trilocularis, trivalvis, valvulis septiferis et versus medium sporiferis, polyspora.

858. SAROTHRA, (SAROTHRE) IINN. JUSS IAM. tab. 215. HYPERICUM MichaUX (caryophyllea 6. ). Culyx profunde quinquefidus (partitus), connivens; corollae petala quinque linearia; capsula oblonga, arcuata, unilocularis, trivalvis, polyspora, colorata.

859. A L SI NE, (MORGELINE) TOUR. IINN. JUSS. IAM. tab. 214. (caryophyllea 3 ) - Culyx quinquepartitus, (pentaphyllus P.); corollae petala quinque, integra aut bifida, aequalia; capsula supera, unilocularis, trivalvis, polyspora; receptaculum centrale superum. (Flores axillares ant terminales).

* g6o. SE GE T E L L A , (SEgeteLLE) Hoffm. Petala integra; stamina passim tria, quatuor; folia stipulacea. *)

86I. TELEPHIUM, (TÉLEPHE) TOURN. LINN。 Juss. LAm. tab. 2 İ3. (portulacea 1) - Calyx pentaphyllus (quinquepartitus) persistens; corollae petala quinque, receptaculo inserta, calycis longitudino; filamenta subulata; antherae deflexae; stigma simplex; capsula triquetra, unilocularis, trivalvis, polyspora. - (Elongatio trunci herbacea, cernua; folia alterna, stipulacea; fores in corymbo terminali).

862. COR-

*) APENARLA for dan. ALSINE vilt. - ALSINE genus enim dividendum aut potius supprimendum, et cius species pro petalorum forma vel stellariis, vel arenariis insereuclae, a quibus numcro staminum inconstante tantum differre videntur. PEns. ench, P. 33r. 
862. CORRIGIOLA, (CORRIGIOLE) IINT. JUss. LArr. tab. 2:3. POLYGONIFOLIA varlx. (portulacea 1.). - Calyx quinquepartitus, (pentaphyllus P.) marginibus membranaceis; corollae petala quinque longitudino calycis; mux una, calyce tecta, triangula, monospora; spora trigona vel triquetra, rotundata, ad summum nucis affixa per funiculum umbilicalem, enatum 0 fundo capsulae.

863. PORTULA CARIA, (PORTULACAIRE) JACQ! SGHRED. CLAYTONIA LINN. (portulacea). Calyx diphyllus, coloratus; corollue petala quinque ; spora una, ovato oblonga, alata, triquetra, basi'per calycem et corollam inclusa.

864. PHA R A CE U M, (PHARNACE) IINN. JUSS. IAN. tab. 2I4. CER VIANA MINUAFT. TRICHLIS HAIL. ALSINE PIUx. MOLLUGO IAM. (caryophyllea). Caly $x$ interne coloratus, quinquepartitus (pentaphyllus P.); corolla nulla; capsula trilocularis, trivalvis, polyspora, - (Folia saepe verticillata; flores axillares aut terminales).

* 865. XYL OPHYLLA, (XYLOPHYLLE) LINN. GENESIPHYLLA I'HERET. pag. 29. tab 39. PHYLLANTHUS LINN. (euphorbiea 1.). - Hermaphrodita: Calyx coloratus, quinquepartitus; corolla nulla; stamina quinque aut sex; stigmata lacerata; capsula bi - trilocularis, liexaspora. - (Frutices foliis simplicibus alternis, dentatis, stipulatis; flures dentibus foliorum inserti, interdum per abortum ovarii steriles).

866. BASELLA, (BASELLE) IINN. JUSS. IAM tab. 215. (atriplicea 3.). - Calyx urceolatus, septemfidus, laciniis inaequalibus, duabus oppositis latioribus, corolla nuila; stigmata stylis adnata; spora una, calyce in bac. cam enato tecta, hinc baccseformis. (PERs. ench. p. 331. vice versa refert: calyx nullus: corolla septemfida; laciniis duabus latioribus oppositis, tanciem baccata; spora una). Elongatio trunci recta volubilis; flores in spica axillari. 
867. ARUBA, (ARUBE) AUBd. Guy. t. 115. JUss. (ru* taceis affinis). - Calyx quinque - sexpartitus; corol. lae petala quinque aut sex disco hypogyno inserta; sta. mina ut plurimum numero quinque, rarius sex vel octo, eodem ac corolla modo inserta; filamenta ad basin mu* nita squama villosa; antherae quadrangulae; ovarium trivel sexstriaturn; styli tres in unum approximati; stigmata tria, sex; fructus formatus e tribus vel sex capsulis monosporis. - (Frutex ramosa; folia alterna, simplicia aut tornata; flores fere in corymbis axillaribus et terminalibus). I

\section{$T E T R \cdot A G Y N I A$.}

868. PARNASSIA, (PARNASSE) TOURN. IIKH. Juss. LAx. tab 216. (capparidea), - Calyx quinquepartitus, persistens; corollae petala quinque hypogyna, calyce alternantia; nectarolyma squamae quinque interno positae, coroliae angulis insertae, apico glandulis ciliatis munitae, ciliis globosis; stamina hypogyna, cum petalis alternantia; antherue declinatae; stylus nullus; stig mata persistentia; capsula ovalis, quadrangula, globulosa, apice quadrivalvis, polyspora; receptacula pariesalia; sporcie membranaceo marginatae. GAEZTN.

\section{P $E N T$ T $A$ G $Y$ I I I A.}

\section{Flore supero.}

869. ARALIA, (ARALIE) TOURN. LINN. JUSS. tAAT, tab. 21\%. (araliacea). - Jnvolucrum umbellulae; involita cella brevia; calyx superus, quinquedentatus; corolla pentapetala; bacca globosa, coronata stylis persistentibus, quinquelocularis, pentaspora. - (Arbores aut frutices ant herbae, folia rarissime simplicia, saepius uno bi - triplicato pinnata; umbella simplex aut ramosa).

870. GOU. 
870. GOUPIA, (GOUPIE) AUBI. Guy. tab. 116. JUss. IAM. tab. 21\%. GI OSSOPETALUM schreb. (rhamnea 2.). - Calyx minimus, quinquedentatus; corollae petala quinque, lanceolata, externe disco calycinali, interne apice ligula membranaeformi pendula, munita; stamina super discum collocata; filamenta brevia; antherae quadrangulae; stylus nullus; bacca pisiformis, quinquestriata, unilocularis, tri-pentaspora, basi calycis persistentis adnata.

\section{Flore infero.}

871. CRASSULA, (CRASSULE) DILL.LIN. JUSS. LAM. tab. 220. (crassulea). - Calyx quinquepartitus; corolla pentapetala; necturolyma squamao quinque ad ba. sin ovarii; capsulae quinque, uniloculares, polyspora (in quibusdam speciebus altera pars additur aut demitur yers.). Elongatio trunci herbacea aut frutescens; folia alterna ant opposita, interdum connata.

872. LAROCHEA, (IAROCHEE) DECANDOLL. redoute. ROCHEA dec. (crassulea). - Calyx monophyllus, quinquefidus; corolla nonopetala, intundibuliformis, quinquefida; squamae quinque ad basin ovarii ; capsulae quinque. (CRASSULA MERr.).

873 GISEKIA, (GISEQUE) IINN. JUSS. IAM. tab. 221. (portulacea 2.). - Calyx quinquepartitus (pentaphyllus P.); coroila nulla; staminum filamenta ad inferiorem partem dilatata; avarium quinquepartitum; fructus: cupsulae quinque invicem approximatae, rotundatae, calyce tectae, monosporae, evalves.

874. L I N U M, (LIN) TOURN, 176. IINN. JUSS. LAM. tab. 219. MEDic. obs. botan. 1783. et monad. p. 90. (caryophylleis atfinis). - Calyx quinquepartitus (pentaphyllus PERs.), persistens; coroliae petala quinque unguiculata; filamenta quinque basi coalita, campanulatim dilatata; antherae sagittatao; squamae quinque cum staminibus alternae; capsula globosa, acuminata, quin. 
que - multivalvis, valvulis geminatis, quarum quaeque membranis intrantibus loculúm efformat; decemlocularis, loculis monosporis; sporae solitariae insertao angulo loculorum centrali, ovoideae, compressae, nitidae; albumen nullum; cotyledones planae, rectae; radicula supera. - (Plantae herbacese aut suffrutescentes; foliis saepe alternis, rarius oppositis).

875. ALDKOVANDA, (ALDROVANDE) IINN. JUSS. LAM. tab. 220. (ex ordine plantarum sedis incentae). - Calyx quinquepartitus, persistens; corolla pentapetala, porsistens; ovarium superum; capsulu pentagona, quinquelocularis, quinquevalvis, decaspora; sporae affixae parietibus internis.

876 DROSERA, (DROSERE, ROSSOLIS) IINN. JUSs. LAM. tab. 220. ROSSOLIS TOURN. (capparideis affinis). - Calyx quinquepartitus (quinquefidus), persistens; corolla pentapetala hypogyna, petalis calyce altornis; stamina quinque alternantia cum petalis; antherae filamentis adnatae; ovarium superum; capsula turbinata aut rotundata, angulata, calyce cincta, unilocularis, polyspora, apice tribus, quinque valvulis (subquinquevalvis тн.) dehiscens. (styli etiam sex) *) - Elongatio lerbacea; folia radicalia, alterna, pilis ciliisve glandulosis obtecta; flores in spica super trunci elongationern aphyliam.

* 877. MAHER NIA, (MAHERNIE) IINN. JUss. IAm. tab. 218. (uliácea 1.). - Calyx campanulatus, quinquefidus; corollae petala quinque basi coulita, unguibus non spiraiter convolutis; staminum filamenta rocta, basi complanata, versus medium dilatato cordata, apice filiformia; antherae sagittaţae, conniventes; styli

quin-

*) Sequutus sum thrsergoro, viro summo, qui dedit dissertationem monograplam de Drosera; coumugri iussit Oroseram et Toridulim, neque temerarius, clim Dros. lusitanica sit ruonogyna et vel decandra; denique ojinatur, armirabile gemus DIONAEAE cum Droseris coniungi posse. 
qquinque approximati coadunati in unum; stigmata nequalia; fructus (capsula?) parvus, quinquangulus, quin. quelocularis, quinquevalvis, ralvulis medio septiferis, loculis polysporis. - (Frutices foliis alternis, simplicibus, stipulaceis; flores axillares et terminales).

878. COMMERSONIA, ( COMMERSONE) IORST JUSS. LAM. tab. 218 (ex ordine plantt. sodis in. cortae). - Calyx monophyllus, quinquepartitus, laciniis ovato - mucronatis, corollifer; corollae petala quinque calyci inserta, cum eius laciniis alterna, basi utrogue latere dilatata, et urrinque lobo introrsum flexo adaucta; stamina brevissima, inserta petalorum unguibus; antherae rectao, rotundatae, didymae; nectarolyma rorst. tubus sive annulus inter stamina et ova. rium positum decempartitum, laciniis quinque alteruis filiformibus, barbato villosis, lanseolatis; ovarium villo' sum, globosum, quinque lateribus elevatum; styli quinque recti, approximati; stigmáta globosa; capsuta rotundata, globssa, echinata filis longis et plumosis, quinquelocularis, loculis disporis; sporae ad umbilicum suum arillo membranaceo ad marginem subtilissime laciniato munitae; embryo rectus, albumine crasso circumdatum; cotyledones planae, fero foliaceao; radicula infera.

879. SI B B A L D I A. (SIBBALDE) LIN JUSS. IAM. tab. 22i. FRAGARIA tourn. (rosacea). - Calyx patonte limbo decemfidus, laciniis quinque alternis magis erectis; corollae petala quinque calyci inserta; stamina parva ; styli excedunt e latere ovariorum; sporae quinque calyco comivente tectae. - (Elongatio trunci herbacea foliis ternatis; foliolis simplicibus, divisis; flores axillares et terminales; iuterdum styli decem).

880. STATICE, (STATICE) TOURN. IINN. JUSS, IAM. tab. 219. LIMONIUM TOURN. (plumbaginea). Calyx mono- diphyllus, iuteger, limbo plicato, scarioso, persistens; corollae petala quinque (rarius monopetala, quinquefida) unguiculata, inferne coalita; stamina inserta basi petalorum; capsula ovalis, tota calyce tecta, 
tecta, monospora; spora supera; placenta filiformis ad basin capsulae nascens.

* ARMERIA: Scapo simplici, floribus capitatis, caule communi polyphyllo.

* L IMO N I U M : floribus sparsis in scapo paniculato, aut spicato, s. caule folioso.

88 I. PIRIQUETA* (PIRIQUETE) AUbI. Guy.

t, 117. Juss. (cisteis affinis vel novae familiae civis). Calyx pentaphyllus, caducus; corollae petala quinque aequalia, cum calyce alterna; stamina quinque hypogyna; antheraf ovales; styli quinque, sex; stigmaśa quinque, sex, dilatata, quinquestriata; capsula unílocularis, tri quadrivalvis, receptaculum versus medium sporis septem vel octo onustatum. - (Planta villosa, folia alterna, pedunculi axillares, solitarii et uniflori).

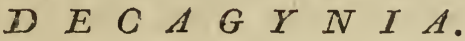

882. SCHEF F LERA, (SCHEFFLÉRE) rorst. JUSS. LAMr. tab. $22 \mathrm{r}$. (ex ordine plantarum sedis incertae). - Calyx minimus, superus, urceolatus, quinque: deutatus, persistens; corollae petala quinque calycis dentibus interposita; antheve rotundatae; ovarium superum; styli octo - decem se aperientes in radios, persistentes; stigmata octo, decem; capsula globoso depressa, octodecemlocularis, octo - decaspora, loculis monosporis, calyce tecta.

\section{$P \quad O \quad L \quad Y \quad G \quad Y \quad N \quad I \quad A$.}

- 883. MYOSURUS', (RANONCULE, Myosuro)

J. BAUH. IINN, JUSS. IAM. Eab. 22I, RANUNCULUS Tourn. (ranunculea 1.). - Culy $x$ coloratus, pentaphyllus, foliolis supra basin porrectis adhaerentibus, deciduus; ccrollae potala quinque brevia, unguibus filiformibus, 
mibus, tubulosis, (nectarolyma folia quinque ligulata, petaliformia P.); stamina quinque ad duodecim; capsulae numerosas, acuminatae, monosporae, receptaculo elongato caudato, tenui, et styliformi insertae.

884. CERAT OCEPHALUS . (CERATOCE. PIIALE) MOENCH. RANUNCULUS falcatus rINN. MORIS. JACQ. WILLD. (ranunculea I.). - Calyx pentaphyllus, persistens corollae petala quinque, poro ungue meliffero, squamula tecto: sporae plurimae, per paria dorso adnata, rostro acinaciformi; receptaculum demum clongaturn spicaeforme. ${ }^{*}$ )

885. ZANTHORHIZA, (ZANTHORHIZE)

I'HERE'T. JUsS. XANTHORHIZA MARSH. (ranunculea 5.). - Calux pentaphyllus aut quinquefidus (nullus P.); corollae petala quinque unguiculata, glanduliformia, subdidyma, minima, (corollae petala quinque, nectarolymata quinque, pedicellata PERs.); stamina quinque, decem; capsulae numerosae (quinque $P$ ), compressae, oblongae, membranaceae, apice semibivalves, monosporae.

*) Scapo antecedenti gẹnere affinis PERs. 


\section{$G L A S S I S S E X T A$.}

\section{HEXANDRIA.}

\section{$\begin{array}{lllllllll}I I I & O & N & O & G & Y & N & I & A\end{array}$}

I. Flore calyce et corolla instructo, spathaceo.

885. BROMELIA, (ANANAS) pLUNr. IINN. JUSS. LAM. tab. 223. KARATAS PLWM. ANANAS PL. TOURN. (bromeliacea 2). - Calyx superus, duplex; exterior tubulosus, trifidus, brevis; curolla (calyx interior) tripetala (laciniis cerie profundis tribus), petalis calyce longioribus, conniventibus, petalo quoque ad basin squama particulari munito nectarifera ; stamina summo calycis inserta, aut super glandulam calycinam tegentem ovarium (deinceps epigyna apparentia?); antherae rectae, sagittatae; stigma trifidum; hacca umbilicata, trilocularis, polyspora; spurae nidulantes ir cellulis propriis. (Plantae herbaceae, quibusdam parasiticis; folia radicalia canaliculata, saepe ad margines dentato - spinosae).

887. MUSA, (BANANIER) IINN. JUSS. IAM. tab. 836. 837. (musacea). - Polygama: spatha magna, supera, muliflora; calyx profunde bipartitus, lobo superiori et inferiori quinquedentato; flores hermaphroditi: calyx nullus; corolla (calyx juss.) dipetala, petalis oblongis, concavis, inaequalibus; scilicet altero externo, magno, erecto, quiriquedentato; altero interno, broviori et minori, integro, cordato, nectariforo; stamina sex, quorum 
nunc unum, nunc quinque perfecta (secundum LINN.); ovarium oblongum; stylus cylindricus; stigma capitatum, angulis sex parum exsertis munitum; bacca oblonga, forma Elateris, triquetra, attenuata duabus marginibus, trilocularis, loculis vix distinctis, polyspora; sporis fere semper abortiontibus. - Flores masculi: calyx et corolla at in hermaphroditis; stamina quinque sex, sterilia et imperfecta; pistillum abortiens. - (Elongatio praealta. herbacea, per omnem olongationem tecta expansionibus membranaceis foliorum; flures dispositi in axi solitaria cernua, superiores steriles, fertiles inferiores). ${ }^{*}$ )

888 PITCAIR NIA, (PITCAYRNE) I'HER, sert. angl. t. II. VENT. LAM. tab. 224. RENEALMIA FEUILL. (PUYA MOLIN, **)! HEPETIS SWARz, bromelliacea 2). Caly $x$ semisuperus, tubulosus, basi ventricosus, trıfidus laciniis conniventibus, brevioribus: potala'tria linearia, altero in alterum intus revoluto; squama nectarifera nulla; stumina inserta corollae; stigma trifidum, spiraliter tortum; stylus striis tribus sulcatum; cvarium semiinferum; capsula trigona, fere triccicca, polyspora, inirorsum dohiscens in partes tres, (hinc videntur capsulae tres); sporae alatae, aut utrirque in setam lorgam terminatae.

*PoURRETIA. ruz-pat. - Calye inferus, tripartitus; antherae sagiltatae incumbentes, lineaJes; capsulue valvis septiferis; corolla cum staminibus submarcescens spiralis. ${ }^{* *}$ )

889. PUYA, (PUYA) MoLIN. Juss. (bromeliacea 2.) Calyx fissus, laciniis interioribus tribus (corulla?) mul.

*) CASIMIR MEDICUS in praestantissimo libro "Pflanzenphysiologische Bemerkungen " 1803 , aliique oculatissimi viri coniecerunt, hoc genus ad pentandriam esse referenduin.

**) PErsoon in enchir. hoc ad genus rețulit.

***) Hoc geuus GAVANILLES HOH a RITCAIHNIAE genere distinctum putat; cui hurz obversatur, certe si quacdam intersit coniunctio, proxime cognatum esie pouendum genus. 
multo maioribus; stamina ad summum calycis inserta, sustentata filamentis basi squamosis; ovarium inferum; triangulare; stylus et stigma simplex; capsula trilocularis, polyspora, sporis parvis; - (Plantao trunci elongatiore incrassatae et suberosae; foliis alternis, elongationom plus minus cingentibus) num ante Xeropliyta ponenda?

850. GUZMA N NIA, (GUZMANNIE) RUIs. PAv. - Calyx inferus, tripartitus, laciniis revolutis; corollae petala tria, in tubum convoluta: antherce in cylindrum coalitae; capsula trilocularis, trivalvis, polyspora; sporae oblongae, nudae.

S9I. TILLANDS1A, (TILLANDSIE) PLUM. ZINN. LAM. tab. 224. RENEALMIA et CARAGUATA PLUM. (bromelliacea I.) - Calyx oblongus, trifidus, persistens, subconvolutus; corolla calyce maior trifida in RENEALMIA PLUM., aut tubulosa, campanulata in CARAGUATA PLUM, , colorata; stamina summo calycis inserta; capsula obtuse trigona; trilocularis Juss. (unilocularis LINN.), polyspora; sporae comosae; - (Folia saepe radicalia; flores super elongationem aphyllam spicati aut paniculati; plantae herbaceae, omnes fere parasiticae, habitu Aloës et Ananas.)

\$92. B UR M A N I A, (BURVANNE) IINN. JUSS. IArr. tab, 225. (bromelliacea 1.) - Caly $x$ prismaticus, tubulosus, angulis membranaceis, coloratus, limbo sexfido; laciniis tribus interioribus' minoribus, petaloideis (hinc corollae petala tria); stamina fere sessilia, ad calycis summum posita; ovurium oblongum; capsula trilocularis, polyspora, recta, trivalvis, calyce tecta; sporae minimae.

893. TRA DESCA N T IA, (TRADESCANTE, Ephémérine) LINN, JUss. xAM. tab. 226. EPHEME. RUM ToUnN. (juncinea 2.) - calyx triphyllus ; corolla tripetala; filamenta villosa pilis articulatis; stigma th. bulosum; cápsula trilocularis, trivalvis, oligospora; sporas parieti internae valvularurn affixae. 
* 894. Z A N O N I A, (ZANONIE) cramer enum. pl. p. 75. PEnar-valli RHEED. ?. - Calyx tripartitus; corolla tripetala, deinceps baccans; filumenta villosa; antherae geminao, uniformes; capsula trilocularis, corolla baccante inclusa; (COMMELINA IINN.)

895. STEPHA N IA, (STEPIANIE) wILID. CAPPARIS JACQ. - Calyx campanulatus, bilobus; corolla totrapetala; stamina bina, inferiora duo lóngiora; ovavium pedicellatum; stigma seosile, capitatum; capsula. ...?

895. FRA NIRENIA. (FRANQUENNE) IINN. JUSS. IAN. tab. 262. (caryophyllea 7.) - Calyx infundibuli, formis, fere cylindricus, quinquedentatus; corollae petala guinque unguiculata, unguibus interne canaliculatis; stigmata tria (stigma bi-tri-partitum P.); capsula uniincularis, trivalvis, polyspora; - (Plantae minimae, diffusae; foliis oppositis, brevissimis; floribus parvis terminalibus, per. glomerulos parvos approximatis, aut axillaribus et terminalibus; stamina interdum quinque ad decem; fructus trilocularis secundum ADANSON.)

397. C O S S I G N I A, (COSSIGNI) commers. Juss? CONIGNEA willd. COSSINIA LAm. tab. 256. (saponacea 2.) - Calyx interus, quinquepartitus, persistens; corollae petala quatuor, rarius quinque, ad basin unguiculata; ovarium leviter triangulum; stylus brevis; capsula ovalis, trigona, levicer plicata, trilocularis, loculis bi-trisporis, sexvalvis, apice dehiscens; 'sporae duae, tres, rotundatao, receptaculo centrali affixae; (Frutices; flores paniculato racemosi, axillares aut terminales).

898. CLEOME, (CLÉOME, mozambé) IrnN. JUss: x.AM. illustr. tab. 567. (capparidea) - Calyx tetraphyllus, parvus, patens, caducus; coroilae petala quatuor lateralia adecendentia et versus summum recurva: duo approximata minora: stumina saepissime sex; interdum tandum quatuor, aut decem, imo quatuordecim; nuno 
petalis approximata, nunc collocata super stipitem; filamenta declinata; ovarium podicello brevi aut elongato stipitaium, cinctum tribus glandulis positis sub foliolis calycinis superioribus; stylus nullus; stigma capitatum; siliqua pediceilata, aut fere sessilis, oblonga, cy. lindrica aut compressa, urilocularis, polyspora;(Frutices aut plantae herbaceae; folia basi duabus glandulis munita, rarius spinis kuabus; simplicia aut ternata aut digitata; flores in spicis terminalibus, fodicellis bracteatis). *)

899. L O RANTHUS, (LORANTHE) IINN, RUIZ. et pav. juss. LAM. tab. 259. LONICERA í prifoliea 2.) - caiy $x$ brevissimus, duplex; alter su. perus, fere integer, alter inferus eadem forma; (calyx nullus vel margo brevis concavus pers.); corolla sexfida (aut petala sex conniventia), laciniis reflexis, vel revolutis, lineari spathulatis, inferne adglutinata; sta. mina versus apicem laciniarum corollae inserta; iisque opposita; stigma obtusum; bacca infera, monospora, umbilicata, vel calyce coronata; embryo secundum Gaertner monocntyledoneus; (Frutices parasiticae; corolla etiam quinque, septemfida; stanina yuatunr aut quinque; folia incrassata, saepo opposita, rarius alterna; flores axillares, rarius alterni, corymbosi aut spicati).

900. HILLIA, (HILLE) JAcQ. IINN. JUSS IART. tab. 257. (rubiacea 5.) -- Culyx oblongus, duplex, inferus alter hexaphyllus, alter superus di-tetraphyl. lus, laciniis rectis; corolla tubuIosa, tubo cylindrico, longissimo, orificio parum inflato: limbo patente, mag no, quinque sexpartito, laciniis acuminatis; anthercie intra tubum corollae faucem obsidentes, subsessiles, nonexsertao; stigma capitatum ; cupsula infera, oblonga, compressa, bilocularis, polyspora; sporae comosae. **)

90I. RI-

*) Piecte cuim e decima quinta classe in hano relatum est ge. uUS a PERs, P. 391 .

**) DUROLA iam sub geNipa yerins locatum gcuus; ff. SCHRAD. 
90I. RICHARDIA, (RICHARDE) LINN. JUss. 2AAI, tab. 254. (rubiacea 2.) - Calyx sexpartitus, persistens, superus; corolla cylindracea, infundibuliformis, sexfida; stignata tria, capitata; fructus tripartibilis; sporae tres, conniventes, apice dilatatao, trunca. tae; (Capitula foliolis radiata; una pars floris interdum demitur; RUIz, P.Av.)

902. BARBACEN IA, (BARBACÈNE) VANDEL. II ap. Roem. LAM. tab. 252. - Calyx superus, sexdentatus, corolla hexapetala; filamenta petaliformia, denticulata; capsula glandulosa, trivalvis, polyspora.

903. BERBERIS, (VINNETIER, Epine-vinette) TOURN. IINN. JUSS. LAM. tab. 253. LYCIUM ALP. exot. (berboridea). - Calyx coloratus, hexaphyllus, exterioribus tribus minoribus (bracteis?); corollae petala sex unguiculata unguibus ad basiu biglandulosis, foliolis calycinis opposita; stylus nulius, stigma latum, umbilicatum, orbiculatum; bacca parva, ovoïdea, rarius sphaerica, unilocularis, bi-tri-tetraspora. (Filamenta primo glandulis petaiorum adhaerentia, deinceps eriguntur elastice momento foecundationis; frutices: folia alterna, saepe in fasciculiz cum trunci elon. gatione alternantibus; flores e medio fasciculorum; peduncili parva bractea muniti).

904. LE O N T ICE, (LÉONTICE) IINN. JUS I. IAM. ill. tab. 254. LEONTOPETALUM TOURN. (berberidea 1.) - Calyx hexaphyilus, caducus; corollae petala sex, opposita foliolis calycinis; squamae parvao nectorylomatis sex internae, unguibus petalorunı insertae, lim. bus patens; stylus brevis, oblique ovario insertus; stigma simplox; capsula vesiculosa, baccae formis, globosoacuminata, unilocularis, tri-tetraspora, sporis globosis'; - (Elongatio trunci herbacea, foliis alternis, pin. matis, uno-bi-triternata; flores dispositi in racemum terminalem, pedunculis basi bracteatis.)

905. CAULO PHYLLU M, (CAULOPHYLLUM) MICHAUX, LEONTICE wILID. - (berberidea). - 
Calyx bexaphyllus foliis planis, lanceolatis; corollac petala sex calycinis laciniis opposita et multo maiora; stamina ad basin ovarii inserta; filamenta brevissima; whtherae biloculares, loculis margine dehiscentibus; drupa stipitata, parum carnosa, includens nucem globo-1 sam, maxime incrassatam, monosporam.

906. DIPHYLLEIA, (DIPHYLLEIA) suchAUx. (berberidea). - Calyx triphyllus foliolis concavis deciduis; corollae petala sex, calyci opposita et maiora: stamina hypogyna; antherue membrana a basi ad apicem solubili dehiscentes, oblongae; ovarium ovale; stylus vix ullus; stigma capitatum; bacsa globosa, unilocularis; sporae duae, tres, subrotundae.

907. COUTAREA, (COUTAREA) AUEI. JUs. gen. IAMr. t. 25\%. PORTLANUIA JACQ. IINA. WIIID, (rubiacea 5.) - Calyx hexaphyllus foliolis subulatis caducis; corolla nagna, infundibuliformis, limbo sexfido, tubo incurvo ventricoso; filamenta basi tubi insorta ; antherae longae, lineares, exsertae, stigma sulcatum; capsula infera, ovali compressa, centro sulco uno notata, vir coronata, bilocularis bivalvis, valvulis carinatis, polyspora; dissepimentum formatum ex introitu valvularum sporiferum; sporae orbiculares, imbricatae, margine membranaceao; (Flores basi bibracteati, interdum heptandri A.) -

908. NA NDINA, (NANDINE) THUNB. JUSS. IAM. tab. 261. (ex ordine plantarum sedis incertae). - Calyx inferus, polyphyllus, foliolis caducis imbricatis serio sex et sex squamis in quaque serie; corollae petala sex calyce longiora; antherae oblongae, fere sessiles; ovarium superum; styli triarguli, brevissimi; stigma aequa. liter trigonum; baccu exsucca, pisitormis, dispora ; sporae henisphaericae, collocatae in receptaculo globoso. punctato.

909. PRINOS, (APALACHINE, Apalanche) I:INN. JUSs IAr. tab. 255. (rhamnea 2) - Calyx minimus, sexfidus; corolla monopotala, plana, rotata, sexpartita; 
filamenta subulata; antherae oblongae; stylus brevis; bacca rotundata, continens sex nuces monosporas; (Frutices aut arbores mediae altitudinis foliis altornis, interdum persistentibus; pedunculis axillaribus, saepo multifloris).

9Io. PSATHURA, (PSATHURE) comMERs. JUSS. WILID.-IAM. tab. 660. (rubiacea 8.) - Calyx minimus, superus, sexdentatus ; corolla campanalata, intus barbata, sexfida, tubo vix ullo; stigma tri-quadrilamellatum; drupa exsucca, striata, coronata, sexlocularis, sexspora, (Inculo quoque monosporo), sporis latero altero concavas, altero convexae.

9I I. ISERTIA (ISER'IE) vAHL. LAM. tab. 259. GUETTARDA AUBL. (rubiacea 8). - Cuy $x$ superus, quadri-sexdentacus; corolla infundibuliformis, limbo soxfido; stamina sex vel numerosa; filamenta brevissima; stigma sexfidum; fructus: pomum globosum, succulentuxn, sexloculare, polysporum. (Arbores foliisioppositis).

912. CANARINA, (CANARINE) IINN. IAM. tab. 259. CAMPANULA IINN. (campanulea). - Calyx he. xaphyllus vol sexpartitus laciniis lanceolatis, recurvatis; curolla campanulata, sexfida; stigmata sex; cap. sula infera, hexagona, sexlocularis, polyspora. ${ }^{*}$ )

913. STEVENSIA, (STEVENSIE) PоIтEAU annuar, mus. cah. 21. tab. 60. (rubiacea 4.) - Calyx bifidus, deciduus; corolla tubulosa, limbo plano, sexseptemfido; antheraesex, septem, in corollae fauce sessiles; capsula bilocularis, polyspora, apice in partes quatuor dehiscons, basi calycis approximante.

GI4. PAR-

*) A CHRAS, (SAPOTLliEr) IINN. IAM. tab. 255. (hilospora). - Calyx sexuaritus; corolla campanula. ta, scxfida, fauce munita sex squamis enarginatis, pomum globosum, decem-undecimloculare, deca dodeca spora; sporis compressis, umbilico laterali notatis; iam sub pentandria. 
914. PARSONSIA, (PARSONSE) ERown. JUSS. LYTHRUM LINN. (lythraria 2.) - Caly,x tubulosus, striatus, basi ventricosus, limbo sexdentato; corollae petala sex, unguiculata, inserta calyci; stamina sexnovem brevissima; capsula parva, merobraracea, calfce tecta, unilocularis, bi-sexspora; sforae infixae placentae centrali.

915. LDENIA, (ADENIE) FORsK. JUSS. (sedis incertae). - Calyx tubulosus, longissimus, limbo oblongo, sexfido, laciniis reflexis; corollae potala sex ad summum calycis inserta, breviora laciniis calycinis his. que inserta; stamina sex cum petalis alternantia, basi calycis inserta: filamenta extrorsum munita squama lineari, coalita parum, deinde tota libera; antherae rectae, lineari-lanceolatae; ovarium tubo calycis adnatum; stylus brevis; stigma emarginatum; fructus . . . .; (Frutex maxime vencnosa; folia alterna, peltata, palmata, petiolo glanduloso; flores in spica fasciculata).

9I6. APHYLLA NTHES, (APHYLLANTHE, Nonffuillee, Bragalou) tourn. IinN. Juss. i.Am, tab. 252. (juncinea 1.) - Calyx aequalis, persistens, formatus e glumis muitis univalvibus, imbricatis; corollae petala sex unguiculata, basi conniventia, limbo patente; stamina brevia; stigma trilobum; capsula supera, trilocularis, trivalvis polyspora.

91\%. PEPLIS, (PÉPLIDE) IINN. JUSS. LAM. tab. 262. GLAUX ToUriv. (lythraria 2.) - Calyx campanulatus ore dentibus duodecimalternis, recurvatis, minoribus; corollue petala sex (aut interdum nulla) calyci irserta, abortiva ; staminu brevissima; antherae rotundatae; capsulu calyce tecta', bilocularis, polyspora; placenta crassa duobus lateribus dissepirnento adnata.

919. FLOSCOJA , (FLOSCOPA) LOUREIR.JUS. vvilzd. (smilacea I) - Calyx exterior trifidus, infe. rus, pilosus; interior trifdus, laciniis alternantibus cum exterioribus; (corollae petala tria exteriora? P.; corolla tripetala ovata); antherae bilobae, lobis rotunda[I5] tis 
tis; capsula oblonga, biloba, bilocularis, monospora: sporae duae rotundatae, compressae, substantia cornea, notatae multis striis.

919. FLOERTEA, (FLOERKEA) wiLLD. - Calyx triphyllus; corolla tripetala; stylus bifidus; utriculus dicoccus.

*920. A I O VE A, (AIOVEe) aubl. Guy. t. 120. Juss. (laurinea 1.) - Calyx turbinatus, sexfidus; stumina sex; filamenta basi biglandulosa; stigma sexfidum ; bacca continens nucem fragilem, monosporam: (Arbor mediae altitudinis folia integra; flores in paniculis axillaribus et terminalibus).

II. Flore calyce tecto corollaceo, spathaceo.

921. CORYPHA, (CORYPHE) IINN. JUss. IAM. tab. 899. (palma 2.) - Hermaphrodita; Spatha polyphylla; calyx brevis, tripartitus vel triphyllus; corollae petala tria concava; bucca globosa, magna, drupacea, sphaerica, nucem globulosum continens monosporam; spora globosa, ossea, magna, rotundats.

922. LICUALA, (LICUALE) тHUNB. RUMP. JUSs. (palma 2.) - Hermaphrodita; calyx tripartitus, laciniis magnis extrorsum barbatis; corulis tripartita, laciniis alternantibus cum calycinis; stamina coalita, in (nectao rolyma) tubum brevem, apice truncatum, septiforme; drupa globosa, pisiformis, nucem continens monosporam; spora dura.

* 923. RAPATEA, AUBI. Juss. xam. tab 226. MNA. SIUM schreb. (juncinea 2.) - Spatha compressa, bivalvis, multiflora; calyx glumaceus, tripartitus; $c 0$. rolla monopetala, trifida, fissuris ovalibus convolutis; tubo brevissimo; circumcirca squarnae octo glumaceae, acuminatae, imbricatae, corollam (calycem 1.) cingentes; antheraesessiles, elongatae, appendice antheraeformi foliosovo terminatae; stigmata tria spiraliter torta; 
fructus. - ? (Capsula trilocularis, trivalpis, valvulis eeptiferis, trispora?)

\section{Flore calyce nullo, spatia munito.}

924. RAVENALA, (RAVENAL) SONNER. ADATS. JUSS. tAM. tab. 222. URANIA scHREB. WILID. (musacea). - Sputha unizersulis concava, multiflora; spa. tha partiales, bivalves; caly $x$ nullus; corolla supera, tripetala; nectarolyma bipartitum, diphyllum; foliolo uno bifido; (JAUME p. 152. „Calyx longissimus, quadrifidus, lacinia inferiori latiori basi ventricosa; spatha particularis bifida, infra posita); stamina longissima apice curvata; styli longissimi, subcylindrici; stigmata bidentata); capsulu infera, magna, coriacea, triargula, trilocularis, polyspora; sporae arillo (membrana caerulea) tectae, serie duplici dispositae, úmbilico laterali no. tatae.

925. TACCA, (TACCA) RUMPH. IINN. JUSS. LAH. tab. 232. IEONTICE IINN. (amaryllideis affinis). Involucrum polyphyllum, multiflorum; corolla (calyx) supera libera, sexfida; filamenta staminu:n (potala roRsT.) sex, basi dilatata, apice convexo-cucullaeformia, inserta medio corollae laciniarum lobis; - (calyx sexpartitus; corolla hexapetala, calyci inserta, antherifera pERs. pag. 382.); ovaviainfera ; stuli tres; stigma (stigmata tria) trilobatum, cordato-stellatum; bacca exsucca, ovalis, calyce coronata, striis sex nota. ta, hinc sexangulata, trilocularis, trivalvis, polyspora, infera.

926. HAEMAN THUS, (HÉMANTHE) TOURN. IINN. JUSS IAM. tab. 229. (amaryllidea 2.) - Involu. crum spathacenm polyphyllum, campanulatum, multiflorum; corollu (calyx) supera, tubo brevi, limbo sexpartito, laciniis aequalibus, linearibus; stamina tubo inserta, exserta i stigma simplex; bacca rotundata, trilocularis, trispora; - (Spatha sexpartita; floribus pe- 
dunculatis et coadunatis in umbollas; folia duo radicalia fere opposita.)

927. L E U C O I U M, (IEUCOIE) IINN, JUSS. LAM. tab. 230. NARCISSO-LEUCOIUM TOURA. NIVARIA MoEnch. method. (amaryllidea 2.) - Spatha monophylla, compressa, latere dehiscens, uni-multiflora; corolla (cal:-x) supera, tubo brevi; limbo campanulato, quinquepartito, laciniis aequalibus, apice parum incrassatis ; stamina glandulae, quae ovarium tegit, inserta ; antherae apice dehiscunt; stigma clavatum, simplex; capsula trilocularis, trivalvis, polyspora.

928. STRU MARIA, (STRUMAIRE) JACQ. WILID. LEUCOIUM THUNB. CRINUM LINN. (amaryllidea 2.) - Corolla (calyx petaliformis) supera, petala sex, patentia; stylus infra medium incrassatus, cum filamentis staminum cohaerens; stigma trifinm; capsula infera, fere rotundata, (certe infra) trilocularis.

929. GALANTHUS, (GALANTHE, Galantine, Perceneige) IIN N. JUss. LAM. tab. 230. NARCISSO-LEU. COIUM rourin. (amaryllidoa). - Spatha monopliylla, compressa, latere dehiscens, uniflora; corolla (calyx I.) supera, petala tria, (extériora) concava, tubo brevi; necturolyma (parapetala) foliola tria (exteriora petala) hreviora corollae petalis et margirata; stigma simplex, acutum; capsula ovato-obtusa, trilocularis, trivalvis, polyspora.

930. NARCISSUS, (NARCISSE) TOURN. IINN. JUSS. LAMr. tab. 229. (amaryllidea 2.) - Spatha monophyila, lateraliter sese aperiens, uni multiflora; corolla (calyx) supera, infundibuliformis; petalis sex, aequalibus; limbo patente et duplo; nectarolyma (corolla lim. bus internua) monophyllum, infundibuliformo, integrum aut divisum; stamina ad basin limbi interni inscrta, eoçue breviora; stigma trifidum; capsula triloculuris, trivalvis, polyspora. 
931. PA NCRATIUM, (PANCRAIS) DIXL. IINN. IAM, tab. 228. MEDrc. monad. p. 108. NARCISSUS TourN. (amaryllidea 2.) - Spatha monophylla, latero dehiscens, uni-multiflora; corolla (calyx) supera, infundibuliformis, tubo longo; limbo duplici: exteriori sexpartito, laciniis elongatis; interno (nectarolyma) breviori, duodecimfido; stamina obtusa, limbo corollae interno imposita, in membran a coalita intundibuliformi dilatata, apice filamentis sex terminata; filamentis interiacent sex incisurae; stylus longitudine filamentorum; stigma obtusum; capsula pyriformis, trilocularis, trivalvis, polyspora.

932. A M A RYLLIS, (AMARYLLIS) IINN. JUSS. IAM. tab. 22\%. LILIO-NARCISSUS tourN. (amaryllidea 2.) - Spatha monophylla, latere dehiscens, unimultiflora; corolla (calyx) supera, infundibuliformis, varia, ad faucem munita squamis sex parvis, limbo aoquali vel inaequali, laciniis quibusdam reflexis; filamenta declinata (aut recta, aut inaequali proportione vel directione), super tubi faucem inserta; stigma trifidum; capsula trilocularis, trivalvis, polyspora.

93.. CRINUM, (CRINOLE) IINN. JUSS. AMAPYYLIS LAM. (amaryllidea I. $_{\text {) }}$ - Spatha bipartita multiflora; corolla (calyx) supera, infunciibuliformis, limbo subsexfido, laciniis patulo reflexis, subulatis, canaliculatis, aequalibus; tubo filiformi; filamenta recta, libera vel discreta, ad faucom tubi inserta; stigma fere trifidum; capsula ovalis, trilocularis, trivalvis, polyspora; (sporae ad basin corollae viviparae ANDREW.)

934. CYRTANTHUS, (CYRTANTHE) AITON. TIMMIA GMEL. CRIN.UM IINN. AMARYLLIS L'HEPET . (amaryllidea) - Sputha multiflora; corolla supera, incurva, tubulosa, clavata, sexfida, laciniis ovato oblongis; filamenta apice conniventia, calycis tubo inserta; stigma trifidum; capsula trilocularis, trivalvis, polyspora. 
935. EUSTEPHIA, (EUSTÉPHIE) CAVAx, tab; 238. Vent. (amaryllidea 2.) - Spatha quadripartita, multiflora : corolla (calsx) supera, tubulosa, quinque. sexpartita, laciniis in cylindrum a pproximatis bifidis : tubo corollae interne foveis sex notato et undato; staminc calyce multo longiora, unoquoçue ingerto quaque fovea ad ipsius partitionem; filamenta tricuspidata versus apicem; laciniis duabus lateralibus brevioribus, media tertia triplo longiori et antherifera; discreta; stigma mucronatum, incrassatum; fructus....? (Flores colore purpureo in umbellas dispositi ut in plurimis Narcissis).

936. AGAPANTHUS, (AGAPANTHE) IHRRET. TENT. CPINUM IINN. IAM. tab, 234. MAUHLIA THUNB. (amaryllidea). - Spatha polyphylla, multiflora; coroila (calyx) infera, infundibuliformis, regularis, limbo sexpartito, patente; filamexta discreta, superiore tubi parte inserta, cernua; antherce peltatae; stigma fere capitatum; capsuli triangulata, trilocularis, trivalvis, poIyspors, sporis alatis; - (Flores in umbella).

937. PONTEDERIA, (PONTEDÉRE) LINA. JUS5. LAM. tab. 225. (amaryllideis!affinis). - Spatha oblonga, multiflora, latere dehiscens; corolla (calyx) in. fundibuliformis, monopetala, limbo saepo bilabiato, sex. partito, laciniis inaequalibus, (interdum sexpartito, Iaciniis fere aequalibus); stamina sex: tria tubo varia altitudine, tria altera ad basin limbi inserta; ovarium saepissime inferum; capsala carnosa, trilocularis, polyspora, lateraliter dehiscens; (Plantae aquaticae; radiculae fibrosae; folia radicalia et caulina elongationem amplexantia, saepo sagittata; flores spathacei, in spicam aut umbellam dispositi, terminales, aut exeuntes e sinu fisso foliorum amplexicaulium; - quaedarn haius generis species inter juncineas ponendae videntur. )*)

*) Geneta LeptANTHus, et hetenAxthena (class. III.) etsi triandra, vix loco naturali disiungi possunt a PONTEDE- 
939. B ULBOCODIUM, (BULBOCODE)RUDBECr. elys, IINN. JUss. IAM. tab. 230. (amaryllidea 1.) - Corolla (calyx) infera, infundibuliformis, hexapetala, petali's ungaiculatis, unguibus rectis linearibus, angustatis, elongatis, conniventibus; stamina inserta medio corollae (calycis); stigma trifidum; capsula triangularis trivalvis, supera, polyspora; - (Truncus subteraneus (radix L.) bulbosus; flores radicales solitarii; Croco similis).

939. GETHYLLIDIS, (GETHYLLIS, Gethilide) xIAN. THUNB, JUSS. HYPOXIS LINN. PAPIRIA thUNB. (amaryllidea i.) - Spatha simplex, persistens, capsulam tegens, croco similis, unillora; corolla (calyx) supera, tubulosa, tubo fliformi, longissimo; limbo bre-, vi, sexpartito, laciniis aequalibus; (calyx nullus nisi vagina oblique truncata THUNB.); stamina (secundum IINN. duodecim duodeviginti), coalita in sex fasciculos aequales: antherae spiraliter tortao; ovarium per calycis basin cinctum; (inferum?); stylus filiformis; stigma trifidum; capsula JUss. (bacca I INN. P.) radicalis, tecta, clayato ventricosa, unilocularis, polyspora: (Truncus subterraneus (radix IINN.) bulbosus; flores radicales, solitarii).

940. TULBAGIA, (TULBAGIE) IINN. JUss. Truve. IAM tab. 242. (amaryllidea 1.) - Spatha bivalvis, multiflora, similis Aliiis; corolla (calyx) infera, infundibuliformis, limbo sexpartito vel fisso, laciniis aequalibus, fauce cincta vel coronata squamis tribus (nectarolymate triphyllo foliolis -) parvis bifidis, magnirudine limbi; stamina tria ad introitum corollae posita, tria tubo inserta; stigma turbinatum; capsula supera, trianguia, anguiis vix distinctis, trilocularis, trivalvis.

Rra, cuins variac species eundem gchitalium numerux

habcre videutur, IAVME I. p. 140. 
941. SOWERBEA, (SOWERBEE) sMiTH. trang. act. IV. (asphodelea Juss.) - Corolla infera, hexapetala; filamenta tria, biantherifera, sterilibus tribus interstinctis. - Scapus nudus; umbella multiflora, bracteis scariosis; petala purpurea, persistentia; (Herba inodora, iuncea, radice fibrosa).

942. ALLIUM, (AIL) TOURN. LIINN. JUSS. IAM. illustr. tab. 242. CEPA et PORRUM TOURN. (asphodelea 5.) - Spatha membranacea bivalvis, multos flores in umbellam laxam vel capitulum reductos comprehendens; corolla (calyx) infera, sexpartita, laciniis profundis, patens; staminum filumenta interdum dilata. ta et tripunctata; capsula brevis, supera, trilocularis, trivalvis, polyspora: - (Truncus subterraneus bulbosus, bulbus cepae rovpiv. sphaericus, cylindricus in Porэum ToURN, compositus in Allium TOURN,; folia plana aut fistulosa):

943. CURCULIGO, (CUPCULIGE) roxB; cAERTN. ORCHIS RuMPH, (iuncinea 4.) - Spatha univalvis; corolla (calyx) infera, petala sex plana; (limbi laciniae sex planae I.) tubus gracilis et longissimus; stamina fauce tubi inserta, fere sessilią; ovarium triloculare, trisulcatum; stylus brevissimus; stigmato tria divergentia; fructus: capsula oblonga, triangularis, angulis rotundatis, spongiosa, rostriformis, rostro efformato stylis spiraliter tortis, uni-bilocularis per abortum, tetraspora; sporae appendiculatae.

944. MASSONIA, (MASȘONE) IINN. THUNB. JUss. IArr. tab. 233. (asphodelea 3.) - Corolla (calyx) intera, ad basin tubulosa, limbo sexpartito laciniis initio recurvis, deinceps applanatis; filamenta basi reunita, tubi fauce inserta; capsula triquetro alata, trilocularis, trivalvis, polyspora: - (Flores in elonga. tione aphylla spica brevi dispositi.)

945. HY POXIS, (HYPOXIS) LINN. JUSS. IAM. tab. 229. ORNITHOGALUM IINN. AMARYLLIS IINN. (amaryllideis affinis). - Spathabivalvis; corolla (calyx) 
8upera, parsistens, infundibuliformis, tubo brevi; limbo sexpartito, laciniis aequalibus; stamina brevia, (inserta super glandulam (calicinalem) corollae ovarium tegontem; ovarium inferum; capsula basi angustata, elon. gata, trilocularis, trivalvis, (calyce) corolla coronata, polyspora; sporae subrotundae, nudae; - (Radix fibro8a; folia gramineis simillima, radicalia aut alternantia; flores spathacei, terminales, solitarii aus fasciculati, aut corymbiferi).

\section{Flore calyce spathaque denudato.}

946. X ER OPHYTA, (XEROPHYTE) LINN. JUSS. IAM, tab. 225. (bromelliacea 2) - Corolla (calyx) sue pera, tubulosa, limbo sexpartito, laciniis aequalibus, tribus internis acuminatis angustioribus magisque erectis; stumina laciniis corollae inśerta; antherae longae, fere sessiles, limbo aequales; stigma clavatum oblongum, inflatum (simplex?); capsula scabra pilis rigidis, infera, corollae (calycis) laciniis tecta, trilocularis, polyspora.

947. A L S T ROEMERIA, (ALSTROEM亡̀RE) Pélegrine) LINN. Juss. LAMr. tab. 231. (amaryllideis affinis). - Corolla (calyx) supera, campanulata vel subbilabiata, limbo sexpartito, laciniis tribus exterioribus et superioribus cuneiformibus, interdum mucronatis; tribus interibribus et inferioribus alternis, lanceolatis, quorum duo basi tubulosae ant ad basin involutae; stumina inaequali magnitudine, declinata aut' erecta, basi corollae inserta; ovarium inforum, sexangulum; stigmata tria linearia; capsula infera, ad summum non tecta, subrozundo-ovalis, tri-hexagona, trilocularis, trivalvis, aut intus pulposa non dehiscens; loculis ipolysporis; sporae globosae, affixae placentao centrali, connatae cum dissepimentis; - (Radix fibrosa; fores terminales, fere solitarii aut umbellati; spathus nulla; folia saepius ob petiolos contortos resupinata;

olon: 
elongatio foliosa, recta àt scandens, foliis alternis sessilibus).

948. ARGOLASIA, (ARGOLASIE) suss. LANA. RIA AIt. HYACINTIIUS IINN. (irideis affinis). Corolla (caly.x) supera, tubulosa, externe alba et lanata, interne colorata, tubulosa, staminibus longior, limbo quinque - sexpartito, laciniis aequalibus patentibus, basi staminiferis; stamina sex fertilia; filamenta oblonga; antherae versatiles, basi bifidae; ovarium inferum; stigma leviter trifidun: capsula infera corolla coronata, lanata, trivalvis, trilocularis, loculis di - trisporis. (Planta capensis, maxime lanuginosa, radice fikrosa; folia elongationis sessilia, glabra; flores in paniculam attenuati ).

\section{HEMEROCALLIS, (HEMEROCALIE)}

IINN. JUŞS. IAM. tab. 234, LILIO-ASPHODELUS TounN. (amaryllidea r.). - Calyx (corolla) inferus, basi infundibuliformis, limbo campanulato, laciniis sex apice reflexis, tubo cylindrico; stumina declinata; stigma parvulum, simplex, triangúlum, villosulum; capsula calyce tecta, trigona, trilocularis, trivalvis, polyspora. - (Radix (truncus) bulbosa; flores radicales, solitarii; similis Croco).

950. A GAVE, (AGAVÉ) IINN. JUSS. IAMr. tab. 235. ALOE tourn. (bromelliacea 2.). - Calyx (corolla L.) supera, erecta, tubuloso infundibuliformis, limbo sexpartito, laciniis magnitudine aequalibus; stamina exserta ot apice corollae inserta; filamenta erecta, corollao longiora; stigna trifidum; capsula infera, duabus extremitatibus attenuata, obsolete trigona, trilocularis, trivalvis, polyspora. - Capsulae situ ab Aloës genero distinctum. - (Folia radicalia canaliculata, aspera apice et saepe margine spinosa; elongatio trunci aphylla florifera, floribus in panicula pyramidali).

25I. FUR CRAEA, (FOURCROYE) veNT. bull. philomat. AGAVE JACQ. (bromelliacea 2.). - Calyx (corollaL.) superus, campanulatus, sexpartitus laciniis magnitu- 
nitudine aequali; stamina inserta glandulae calycinae ovarium tegentis; filamenta non striata, infra applanatocompressa, incrassata; supra subulata, non exserta stylus' basi incrassatus; stigma obsolete trilobum; capsula infera, trilocularis, trivalvis, polyspora. *) - (Planta trunco bulboso et bulbifero; folia radicalia canaliculata, disposita multiplici serie, denticulato-spinosa ad margines; elongatio trunci aphylla, maxime elata, fere per totam longitudinem ramis alternis, saepius divisis, floriferis tecta, basi munita spatha fere foliis conforma. tione similis).

952. COINANTHERA, (CONANTHERF) rUIZPAV. BERMUDIANA TRE.W. ANTHERICUM WILED. ECHEANDIA onteg. - Calyx superus, folia sex reflexa; antherae in conum acutum coalitae (hinc Syngenesiae monogamiae L.); capsula oblonga, trilocularis, trivalvis; sporae paucae, subrotundae.

953. ALOE, (ALOËS) TOURN. IINN. JUSS. IAM. tab. 236. (asphodelea 1.).- Calyx (corolla L.) inferus, tubulosus, fere cylindricus, limbo subsexfido, laciniis plus minus profundis, rectis ad apicem extrorsum curvatis, nectarifero, patulo; staminum filamenta basi calycis ad receptaculum inserta; stigma obscure trilobum; capsula supera, oblonga, trigona, trilocularis, trivalvis, polyspora; sporae serie duplici dispositae, margine membra* naceae. - (Floris orificium nunc rectum nunc labiatum; radices fibrosae; truncil elongatio frutescens aut foliosa in quibusdam speciebus; flores dispositi in spicam axillarem aut terminalem; folia carnosa, sive succulenta crassa; sporae lobuis sessilis, et lateraliter affixus prima membranacea expansione inferioris partis germinationis).

954. ALETRIS, (ALETRIS) IINN. JUss. LAM. tab. 237. (asphodelea 1.). - Calyx (corolla L.) inferus, infundibu.

*) Ob calycem hexapetalum et filamenta basi obovata compressi, omninun crassissina, desinentia in superiorem partem subulatam, a praecedenti genere alisque notis sams distiuctum. 
dibuliformis, rugosus, sexpartitus vel fissus; staminum filamenta basi laciniarum inserta; stigna ir fidum; cap. sula semiinfera, superno calculo marcido vestita, trilocularis, trivalvis, polyspora.

955. VELT HEIMIA (VELTHEIMIE) GLDISTCH. WILLD. ALETRIS SEB. IINN. JUSS. (ąsphodelea (.). Calyx (corolla L.) inforus, tubulosus, margine sexdentatus, (pendulus); stuminu tubo inserta; capsula membranacea, alis tribus ad margines notata, trivalvis, trilocularis, loculis monosporis.

256. POLYANTHES, (TUBEREUSE) IINN. IUSS. IATr. tab. 243. HYACINTHUS TOURN. (amaryllideis affinis). - Calyx (corolla) inferus, infundibuliformis, limbo sexpartito, laciniis patentibus; tubo arcuatim curvato; staminum filamenta ad summum tubi vel faci inserta, terminata antheris elongatis; ovarium in fundo caJycis simpliciter tectum; stigma trifidum; capsula trilocularis, trivalvis, losulis polysporis, ad basin tubo calycis cincta; sporae planae, duplici serie dispositae, et angulo interno loculorum affixae. - (Truncus (radix) tuberoso bulbosus; foliis radicalibus longis, foliis elongationis squamaeformibus; flores dispositi in spicam spathacei, bini binis solitarii).

957. CQN VALLAR IA. (MUGUET) IINN. JUSS. LAM. tab. 246. LILIUM convallium, et POLYGONATUM, et SMILAX tourn. (smilacea 1.). - Calyx (curolla) inferus, varius: tubulosus in POLYGONATUM TOURN., sexfidus, laciniis patentibus in SMILAX Tourn., aut globosus, sexpartitus in LILIU.M convallium $x$; ; rarius sexfidus ; stamina tubo inserta; stigma triangulum; bacca supera globosa, trilocularis, ante maturitatem maculara loculis monosporis, (loculo uno abortivo). - Secundum calycem distinxeruut ita: 1. Corollis campanulatis, aut 2. POLYGONATUM corollis infundibuliformibus; aut 3. MAIANTHEMUM corollis rotatis. (Flores axillares, aut terminales, dispositi in spicam; folia alierna). 
958. LA P A GER IA, (LAPAGERIA) RUIZ • TAVON. (in honorem JOSEPHINAE IAPACERIAE, sanctissimao summi 'NAP, BonAPARTIS feminae). - Calyx hexapetalus, basi trigonus: calycis potala tria interiora laticra, subunguiculata ; untherae erectü $\theta$ stigma clavatum; basca supera, unilocularis, polyspora; sporae receptaculo triplici, per parietes decurrenti, adfixae.

959. L USURIAGA, (LUSURIAGA) rUIZ-PAVON. - Calyx (corolla) inferus, hexapiryllus, foliolis petaliformibus tribus exterioribus angustioribus; filanenta receptaculo inserta; antherae erectae, sngittatae; stigma triangularo; bacca trilocularis, dissepimentum membranacoum; sporae binae, altera passim abortiente.

960. SANSEVIERA, (SANSEVIÈRE) THUNB. SALMIA CAV. ALETRIS IINT. LIRIOPE LOUREIR. Calyx (corolla L.) inferus, monophyllus, persistens, hexagonus, tubuinsus, tubo filiformi, limbo sexpartito, laciniis lanceolatis, patentibus, erectis, deirde deorsum revolutis; staminum filamenta sex, subulata, basi laciniarum limbi inserta, longitudine corollae; antherae oblongae, erectae; ovarium ovatum; stylus subulatus, longitudine staminum; stigma trifidum; pericarpium bacca globosa, pulposa, mono-polyspora.

96r. HYACINT THS, (HYACINTIIE, Jacinthe) TOURN. LINN. DESFONT. LAM. tab. 238 (asphodolea 3). - Calyx (corolla) inferus, erectus, campanulatus, globoso ventricosis, aut tubulosus, semper fere suxfidus, passim sexpartitus, laciniis aequalibus; - ovarium no. tatum poris melliferis tribus, IINN.; - stamina receptaculo inserta, vel calycis laciniarum basi: capsula supera, trangularis, trivalvis, trilocularis, loculis saepissime disporis. (- ${ }^{*}$ ) Calyce sexpartito: SCILLOIDEA; **) calyce carnpanulato sexfido); Truncus (radix) bulbosus; flores in spica; germinatio ut in asphodelo.

962. MUSCARI, (MUSCARI) TOURN. DESF. (asphodelea j.) - (Corolla) calyx ovoïdeus, aut cylindricus, inflatus, limbo sexdentato; capsuía supera, triangularis, tri. 
trivalvis, polvspora. - (Truncus bulbosus; flores spicati in elongatione aphylla.

9ケ3. ZUCCAN GN I A, (ZUCCANGNIE) THUNB. nov. gen. plant, pars nona Ups. 1793. (asphodelea). Calyx (corolla) monophyllus, cylindricus, unguiculatus, sexpartitus vel fissus, laciniae limbi tres exteriores duplo longiores, lanceolato setacea $\theta$, saepe reflex̀ae; fila mentu sex; antherae ovatae; ovarium superum; pericarpium: capsula supera, ovata, trilocularis. (LACHENAIIA viridis THUNB. prodr. HYACINTHUS viridis IINN.).

964. D R I M I A, (DRIMIE) JAcQ. JUss: 2mss. (asphodelea 3.). - Calyx (corolla) inferus, campanulatus, limbo sexfido, laciniis extrorsum revolutis; stamina corollae inserta; stigma globoso - capitatum; capsula trilocularis, trivalvis, polyspora. ${ }^{*}$ ) - (Elongatio trunci herbacea).

965. MILLA, (MILLA) cAì. MILLEA wirxds (amaryllidea 3.). - Calyx (corolla) inferus, infundibuliformis, tubo longo, recto; limbo sexpartito, plano; antherae fero sessiles, ad tubi faucem insertae, rectao, oblongae, exsertae; stylus exsertus; stigmata tria, globosa, maxime villosa; ovarium pedicellatum; capsula supera, triangularis, trilocularis, trivalvis, polyspora.

966. ASPHODELUS, (ASPIIODĖLE) ToURN. IINN. IUSS. IANI. tab. 241. (asphodelea 2.) - Calyx (corolla) inferus, sexpartitus, laciniis profundis, patens $;$ filamenta basi dilatata, ovarium tegunt forma cupulao vaivulis sex; capsula globosa, trilocularis, trivalvis, polyspora; sporae angulosae. - (Truncus (radix I..) fibrosus aut fasciculatus; folia plana; flores in spica interdum ramosa; lobus sporae suspensus in germinatione,

*) PEnsooño enchir. pag. 374 . geints non satis ab IYYAIN. IHIS natura et arte distinctum videtur, inprimis quum insertio staminum, varia in varis Hyacinthi speciebus regcriatux, auctore WLLLDENOW. 
tione, ad summum declinatus ot attenuatus in modum fili primi folii).

967. BASILLAEA, (BASILÉE) JUSs. IAM. tab. 239. CORONA regalis DILI. FRITILLARIA IIN. EU. COMIS L'Hepet. (asphodelea 3.). - Calyx (corolla) inferus, campanulatus, persistens, sexpartitus (hexa. phyllus) laciniis oblongis, supra ungues cavitate nectarifora; staminum filamenta dilatata, basi conniventia, longitudine calycis; capsula trilocularis, trivalvis, polyspora; sporae planae, ovales. - (Flores colore viridi, dispositi in spicam; olongatio florifera aphylla, foliolum infra spicam).

968. PETILIUM, (PETILLE) KNORR. IINN. IM. PERIALIS JUSS. FRITILLARIA LINN. CORONA im. perialis rourx. (liliacea). - Scapus apice foliosus; ca lyx (corolla) campanulatus, laciniis rectis, ad basin foveola rotundata sulcatis; stamina stylo breviora; capsula angulis sex acutis maxime exsertis notata; sporae pla* nae. - (Trunci bulbus tuberosus, basi fibrosus).

969. ANTHERIC UM. (ANTÉRIC) IINN. JUss. IAM. tab. 240. ASPHODELUS TOURN. (asphodelea 2.): Calyx (corolla) inferus, hexaphyllus, patens aut connivens, persistens; staminum filamenia subulata, filifor. mia, villoso-barbata; capsula trilocularis, trivalvis, polyspora; sporae angulosae. (Truncus subterraneus fibrosus; folia succulenta, fistulosa, imbricata; flores in spica terminali aut axillari, saepe ramosa, Lutei ; Dulbins L.; lobus sporae in germinatione lateri primao expansionis mombranaceae folii affixus et suspensus filo).

970 PHA LA N G I U M, (PHALANGERE) TOURA. JUs3. LAMr. tab 240. LILIASTRUM et ASPHODELUS Tounn. ANTIIERICUM rinN. *) (asphodelea 2.). Calyx (corolla) inferus, hexaphyllus, foliis patentibus aut conniventibus, persistens; staminum filamenta subulata, filiformia, nuda vel glabra; capsula ovata, trilocula.

*) Omnia Anherica priorio sectionis Luss. 
cularis, trivalvis, polyspora ; sporae angulatae. (Truncus fibrosus in PHALANGIUM T., vel fasciculatus in LILIASTREM T.; folia plana; flores plezumquo albi aut purpurascentes, dispositi in spicam terminalem, interdum ramosam; germinatio ut in Asphodelo).

971. NARTHECIUI, (NARTECI) HUDS. SMITH engi. bot. tab. 535. Juss. LAN. t. 268. PHALANGIUM TOURN. ANTHERICUM IIN. WILLD. (iuncinea 4.). Calycis (corollae) folia aequalia cincta foliolis minoribus tribus, patentia, persistentia; staminum filamenta filiformia, hirsuta; ovaria sex aut plura; stigmata totidem; styli nulli; capsuiu supera, prismatica; sporue utrinque appendiculatae.

972. XAN THORRHAEA, (XANTHORRHOEE) sмITH transact. IIII. (asphodolea 2.). - Calyx (corolla) inferus, hexaphyllus, persistens; filamenta plana. linearia, nuda; capsula triquetra; sporae binae, compres- sae, marginatae. - (Scapus teres, longissimus, amento terminatus multifloro; floribus abortivis interstinctus squamoso $6 \mathrm{Mr}$. ?

973. CALLIXENE, (CALLIXENE) commers. JUSS. LAM. tab. 248. ENARGEA gAERTN. SCIMEB. (smilacea 1.). - Calyx (corolla) inferus, hexaphyllus, foliis aequalibus, tribus alternis basi glandulis duabus munitis; staminum filamenta basi dilatata latiora; antherae oblongae, versatiles; stigma trigonum; bacca supera. parva, trilocularis, loculis interne pulposis, saepe trispora, polyspora.

974. LACHENALIA, ( LACHENALE) JACQ. LAM. tab. 237. (asphodelea 3.). - Calyx (corolla) inferus, campanulato tubulosus, sexfidus; laciniis tubo approximatis; tribus interioribus conniventibus, obtusis, alternis, longioribus, tribas exterioribus brevioribus; stamina receptaculo inserta, erecta ; cupsula subovata, trilocularis, trialata, trivalyis, polyspora; sporue globosae. - (Elongatio trunci herbacea; flores in scapo aphyllo).

975. PHOR- 
975, PHOR MIUM, (PHORMIE) FORST. IINN. suppl. juss. (asphodelea 3.). - Calyx (corolla) inferus, campanulato tubulosus; laciniae sex in tubum coalitae, tribus interioribus longioribus, exterioribus brevioribus; stumina adscendentia, exserta; capsula oblonga, triquetra; sporal compressae, margine membranaceae. (Genere Hyacinthi simile).

976. ORNITHOGALUM, (ORNITHOGALE) ToUnN. IINN. JUSs. IAM. tab. 242. (asphodelea 4.) Calyx (corolla) inferus, basi connivens, persistens, erectus, sexpartitus (hexaphyllus) laciniis profundis, supra medium patentibus; filamenta staminum basi compressa, tria alterna ad basin magis dilatata, subulata: capsuia subrotunda, leviter trigona, trilocularis, trivalvis, polyspora; sporae subrotundae, nudae. - (Germina- tio ut in Asphodelo; truncus bulbosus).

977. ERIO SPE R N U M, (ERIOSPERME) JACQ. ORNITHOGaLUM LiNn. (asphodelea). - Calyx (corolla) inferus, persistens, hexaphyllus, campanulatus; filamenta basi dilatata; capsulu trilocularis, trivalvis, polyspora; sporue lana involutae.

978. S CILLA, (SCILLE) LINN. JUSS. LAM. t. 239. ORNITIOGALUM et LILIO- MIYACINTHUS TOURN. (asphodelea 4.). - Culyx (curolla) inferus, deciduus, sexpartitus, laciniis profundis (corolla hexapetala P.) interne patentibus; staminum filamenta filiformia, compressiuscuia, basi aequaliter dilatata petalorumque basi adnexa; capsula trilocuiaris, trivalvis, polyspora. (Truncus bulbosus; flores spicati; germinatio ut in Asphodelo).

979. C Y A NELLA, (CYANELLE) IINN. JUss. IAM. tab. 239. (asphodelea 4.) - Calyx (corolla) inferus, sexpartitus laciniis oblongis, planis, unguibus cohaerentibus, exterioribus tribus vel inferioribus propenàentibus; staminum filamenta quinque brevia, conniventia, sextum inferun reliquis longius et declinatum; stylus declinatus; capsula rotundata, trilocularis, [16] tri- 
trivalvis, polyspora; sporae oblongae; (stamina etiám tria deflexa pers. Scillae similis).

980. PHILESIA, (PHILESIE) commens. JUss. IAMr. tab. 248. (smilacea 1.). - Calyx (corvllu) inferus, campanulatus, sexpartitus laciniis magnis, regularibus, tribus interioribus obtusis duplo longioribus, tribus exterioribus acuminatis; staminum filamenta basi connata; antherce oblongae, versatiles; stigma trilobum; bacca leviter trigona, trilocularis? polyspora.

98I. OECHMEA, (OECHMEE) RUIZ-PAv. JUss. mss. (smilacea I.). - Calyx sexfidus, obtectus basi squamis tribus brevibus coriaceis, duabus rotundatis. una mucronata, laciniae interiores (corolla auctt.) cum reliquis alternantes, triplo maiores, latae, infundibuliformes, singula quaque duabus squamis parvis ad basin internam adaucta; marcescendo cum staminibus spiraliter convolutao; stamina affixa fundo corollae, et eiusdem longitudinis; (ovarium inforum?); stigmatatria; capsula ovoïdea, trilocularis, polyspora; sporae obovatae, in pulpa molli nidulantes. - (Planta herbacea).

982. LIN DERA, (LINDERE) THUNB. JUss. IAM. tab. 263. (ex ordine plantt sedis incerrae). $\rightarrow$ Caly $x$ (corolla) hexaphyllus, inferus; stamina inserta super ovarium superum; stigmata duo; capsula bilocularis.

983. DRACA E NA, (DRACONIER, Sang-dragon) IINN. JUSs. IAM. tab. 249 (smilacea 1.) - VANDIILr. (Corolla) calyx inferus, connivens, hexapetalus, petalis 1ectis, unguibus cohaerenibus; staminum flamentu versus medium incrassata aut simplicia: bacca trilocularis, 10culis monosporis, duobus abortientibus. - (Plantao similes Palmis; elongatio trunci trutescens; flores dispositi in paniculam terminalom).

984. HंERRERIA, (HERRERIE) rux etPAv.Juss. QUILA-SALSA EEUILL. to. 2. p.716. icon. VII. (smilacea i). - Culyx (curolla) inferus, sexpartitus; stumina in fundo corollae: antherae rotundatae; ovarium trianguJum; stigma trigonum; capsula triquetro alata, trilocu. Iaris, 
laris, trivalvis, valvulis septiferis, di - tetrasporis; sporae margine membranaceo cinctae, lenticulares.

985. DIA NELLA, (DIANELLE) JUSs. LAM. enc. t. 250. JAcQ. Schoenbr. t. 94. DIANA commers. DRACAENA IINN. WiLLd. (smilacea 1.). - Calyx (corolla) apertus, sexpartitus, laciniis aequalibus, tria alterna introrsum posita; filamenta apice tumida vel incrassata ; bucca oblonga, trilocularis, loculis tetra - pentasporis, amethystina; sporce ovales.

986. CORDYLINE, (CORDYLINE) COMMERS. petit-thouars. DIANELLA rar. (smilacea:.) - $C a$ lyx (corolla) externs pubescens; filamenta simplicia, basi corollae inserta ; stigma subincrassatum; bacca turbinata, unilocularis.

987. ASPARAGUS, (ASPERGE) TOURN. INNN. yuss. IAM. tab. 249. (smilacea 1.) - Calyx (coroila) inferus, campanaeformis, basi connivens, sexpartitus, laciniis tribus interioribus apice reflexis, erectis; stigma triangulum: bacca supera, trilocularis, loculis disporis. - CFolia ea epe setacea, fasciculata; elongatio trunci herbacea aut frutescens, ramosa; flores saepo axillares et terminales; spatha bivalvis; spina una interdum sub singulo ramo et sub' singulo foliorum fasciculo).

988. POLLIA, (POLLIE) тнUN3. JUss. (iuncinea 2.). - Calyx (corolla) inferus, sexpartitus, laciniis tribus interioribus rellexis, maxime erectis tribus; exterio. ribus maioribus et oralibus; bucca globosa, parva, trilocularis? prilyspora; sporae angulusae.

989. RIPOGONUM, (RIPOGONE) FORST. JUss. (smilacea 1.). - Culyx sexpartitus, laciniis aequalibus, tribus alternis interue positis, minianus; antherae elongatae, tetragonae, subsessiles; bacca globosa, bilocula. ris? dispora; sporue hemisphaericae.

990. METHONIC $\Lambda$, (MÉTHONYQUE) IRERM. JUSS, VENT, GLORIOEA IINN, LAM. tab. 247.(liliacea). Calyx 
Calyx (coroilla) inferus, patons, sexpartitus, laciniis lanceolatis, longissimis, undulatis, reflexis; stamina stylo breviora; stylus obliquus, apice trifidus; stigmata tria; capsulu ovalis, coriacea, turbinata, trilocularis, trivalvis, polyspora, dissepimenta formata per valvularum margines intrantes; sporae globosae. - (Genitalia mascula deflexa).

99I. ERYTHPONIUM, (ERYTRONE, Vioulte) IINN. JUss. IAM. tab. 244. DENS CANIS TOURN. (liliacea). - Calyx (corolla) inferus, campanulatus, sexpartitus, laciniis acuminatis, reflexis, quorum tria in. teriora basi interne munita sunt duobus callosis tuberculis, calycis foliorum alternantium basi adnexa (necta- rolyma); capsula globosa, basi attenuata, trilocularis, trivalvis, polyspora; sporae ovales..- (Folia radicalia, amplexicaulia; elongatio florifera aphylla, uniflosa, deflexa).

992. UVULARIA, (UVULAIRE) IINN. JUSS. IAM. tab. 247. (iiliacea). - Calyx (curolla) inferus, campanulatus, sexpartitus, laciniis rectis, basi fovea oblonga insignitis (nectarolyma L.); staminum filumenta brevissima; stigma reflexum; capsula ovalis, trigona, trilocala. ris, trivalvis, polyspora; sporae rotundatae et compres. sae. - (Flores solitarii in pedunculis biforis, axilla. ribus; folia sessilia aut amplexicaulia.

993. STREPTOPUS, (STREPTOPE) MCHAUX. UVULARIA IINN. (liliacea). - Calyx (corolla) campanulatus, profusde sexpartitus; stumina brevissima, stigmata brevissima; bacca subglobosa, laevigata, cartha. cea, trilocularis; sporae numerosae, aut numero paucae per abortum, ovö̈deae, hilo umbilicali nudo. мrснашx. *)

994. FRI TILLARIA, (FRITILLAIRE) TOURN. IINN. JUSS. LAM. tab. 245. IMPER'IALIS JUSS. (liliacea). - Calyx (corolla) inferus, campanulatus, laciniis sex, basi

*) Genera UVULARIA et STREPTOPUS multum analogiae cur smilaceis habent, et inprimis cum Convallariis. 
basi fovea rotunda aut oblonga munitis (nectarolyma); stigmata obtusa aut emarginata; capsula oblonga, leviter ıri - sexangula, trilocularis, trivalvis, polyspora; sporae planae.

995. L IL I U M . (LIS) TOURN, IINN. JUSS. IAM. tab. 246. (liliacea). - Calyx (corolla) inferus, campanulatus, laciniis sex rectis aut extrorsum revolutis, ad basin conniventibus, linea longitudinali nectarifera; fovea longitudinali nuda aut ciliata ad basin cuiusque petali (nectarolyma); stamina stylo breviora; capsula oblonga, striis sex sulcata, triangula, trilocularis, trivalvis, valvulis saepe connexis pilis cancellaeformibus, polyspora; sporae planae. - (Truncus subterraneus, tuberosus, imbricatus per basin foliorum; elongatio trunci adscendens simplex, foliosa; foliis alternis aut verticillatis; floribus spatha munitis, spicatis aut fere paniculatis, saepe declinatis vel cernuis).

996. TULIPA, (TULIPE) TOURN. IINN. JUSS. LANE. tab. 244. (liliacea). - Calyx (corolla) inferus, campanulatus, laciniis sax rectis; stylus nuilus; stigma sessile, triloburn; capsula oblonga, trigona, angulis obtusis, trilocularis, trivalvis, polyspora; sporae planae. - (Folia radicalia, amplexicaulia; elongatio florifera aphylia, uniflora, recta.

997. Y UC C A, (YUCCA) LINN. JUSS. LAM. tab. 243. (liliaced). - Calyx (corolla) inferus, campanulatos, sexpartitus, laciniis profundis non nectariforis; staminum filamenta ad apicem dilatata; cintherae minimae; stylus nullus; stigma tribus striis sulcatum, sessile; capsula oblonga, notata leviter angulis tribus obtusis, tri-sexlocularis, apice pèrtusa, trivalvis, polyspora; sporare planae. - (Elongatio trunci nulla aut suffrutescens; flores in spica aut panicula terminali).

998. $\triangle$ LBUCA, (ALBUCA) LINN, JUSS. LAM tab. 241. (asphodelea 4.). - Calyx (corolla) inferus, sexpartitus, laciniis tribus interioribus conniventibus, parum ad apicem incrassatis; tribus exterioribus patentibus, 
dorso saopius viridi coloratis; his laciniis exterioribus opposita sunt stamina sterilin tria, altera tria fertilia; vel ad unum omnia fertilia; stylus triqueter, inverse pyramidatus; stigma tribus mucronibus cinctum; capsula oblonga, trilocularis, trivalvis, polyspora; sporce planae. - (Truncus subterraneus bulbosus; flores spicati ; germinatio Asphodeli).

\section{Flore incompleto.}

999. OR O N T IUM, (ORONCE) IINN. LAM. tab. 251. (aroüdea 2.). - Spudix cylindricus, obtectus hoscúlis sessilibus; calyx (corolla).persistens, hexapetalus (sexpartitus), nudus; stamina cum corollae laciniis alterna; stylus nullus; stigma bifidúr ; folliculus unus minutus et subtilis, monosporus, corolla tectus, spadicis quasi inclusus substantiae.

1005 ACORUS, (ACORE) TOUnN. IINN. JUSS. I.1.r. tab. \$4. (aroïdea 2.). - Spadix cylindricus, floribus sessilibus altero contra alterum attenuatis obtectus; calyx (corolla) persistens, sexpartitus (hexapetalus - P.); ovarium oblongum; stigma stylo nullo sessile, parum exsertum; capsula trigona, pyramidalis, trilocularis, trispora JUss. (polyspora secundum LINN.) - Spadix enascens versus medium trunci elongationis foliis .c simillimus; - Bernh. d. Jussieu hoc genus stare iussit inter familiae iuncineartun cives, quibus maximopero analogum).

I00I. CALAMUS, (ROTANG) IINN. JUss. LAM. tab. 770. (palma). - Spadix tenellus, ramosus, sexpartitus, squamis imbricatis tectus; caly $x$ sexpartitus, (hexaphyllus P.), laciniis tribus exterioribus brevioribus; stylis trifidus; fructus: bacca turbinata aut globosa, squamis retrorsum imbricatis et nitidis tecta, primum pulposa, deinceps exarescens exsiccansque eveniens firma et coriacea; unilocularis, mono-rarius di-trispora. - Frtetices ramosae, arundine simillimae; folia pinnata, petiolo communi spinoso).

1002. THRI- 
1002. THRINAX, (THRINAX) STAEZ. JUSS. CORYPHA ERown. (palma 2.). - Hermaphrodita: Spatha universalis multipartita; spadix ramósus, cinctus spathis partialibus imbricatis; calyx minimus quinque - sexdentatus; antherae a summo ad basin bifidae; stylus brevis; stigma dilata to infundibuliforme, obliquum, unilabiatum; rarius bilabiatum; drupa baccata rotundata, pisiformis, exsucca, continens nucem osseam et cassantem; spora una.

1003. JUNCUS!, (JONC) TOURN. IINN. JUSS. IAM. tab. 250. (juncinea I.). - Calyx hexaphyllus, aequalis, persistens; stamina brevia; stigma trilobum, barbatum ; capsula calyce tecta, uni-trilocularis, trivalvis, tri - polyspora. - (Elongatio trunci simplex, nodis foliisque destituta, sive nodosa et unoquoque nodo foliis vaginando-amplexicaulibus cincto; flores terminales aut laterales, corymbosi aut paniculati, ad basin spathacei).

I004. CAPURA, (CAPURE) IINN. JUss. (ex ord. plantt. sedis incertae). - Calyx tubulosus, sexfidus, laciniis tribus alternis, exterioribus magis erectis et minoribus; stamina intra tubum inserta, non exserta: antherae fere sessiles, tres alternae elatiores; stylus brevissimus; stigma globosum; ovarium superum; bacca - . ? (an iuxta IINdersm hoc ponendum genus?) = Arbor foliis oppositis, integris, ovalibus; flores fasciculati, axillares.

\section{Flore glumaceo.}

1005. EHRHARDTA, (EMRIIARDTE) THUNB. LINN JUSS. LAMr. tab. 2G3. AIRA IINN. TROCHERA RICHARD. (graminea 9.) - Calycis gluma bivalvis, uniflora, abbreviata; corollue gluma duplex: utraque bivalvis ; exterior maior, compressiuscula, notata rugis sulcisque transversim striata, acinaciformis: interior laevis; nectarolyma squanas duao parvao; styli duo aut unus bifidus; spora una. - (Flores in spica paniculata).

1006. GAH. 
1005. GAH NIA, (GAHNIE) FORST. JUSS. IAM. tab. 263. (cyperacea 2.). - Calycis gluma uni - bivalvis, valvulis squamaeformibus, bi - quinqueflora; corollae gluma bivalvis brevior; staminum filamenta persistentia; stigmata duo aequaliter bifida; stylus dichotomus, persistens; spora una triangularis. - (Plantae Schoeno facio similes ipsique forsan congeneres, si observare velis et habere quemque florem quasi spicam compositam squamis, quarum inferiores steriles sunt, superiores fertiles uniflori).

1007. BAMBOS, (BAMBOU) WILL. BAMBUSA retz, sCHREB, BAMBUS gMEL. JUss. mss. ARUNDO IINN. (graminea 13.). - Calycis gluma quinqueflora, tribus valvulis navicularibus inaequalibus obtecta; dua-" bus oppositis, tertia exteriori; affixa super faciem planam spiculae. - (Squamae tres spiculas subquinquefloras tegentes; Calyx nullus; PErs. p. 393.); corollae gluma bivalvis, valvula exteriori ventricosa, interiori longiori et ciliata; nectarolyma squamae duae internae. parvae, apice barbato villosae; stylus superne bifidus; stigmata duo plumosa; spora una.

\section{D $\quad I \quad G \quad I \quad I N \quad I \quad A$.}

1008. NASTUS, (NASTE) JUSS. IAM. ill. t. 262. BAMBUSA schreb. (graminea 12.). - Gluma octodecenvalvis, uniflora; valvulae per paria imbricatae, glabrae, exteriori mivore; calyx bivalvis, lateraliter podicellatus, pedicello villoso; (flore altero abortiente?); squamae dua parvae ad interiorem calycis paginam; stamina sex; styli duo, aut unus et bifidus; stigma divisum; spora una, interdum abortiens. - (Flores paniculati, habitu arundinis arborescentis).

ICO). A T R A P:H A XIS, (ATRAPHAXE) IINN. JUSS. IAM. tab. 265. (polygonea). - Calyx quadripartitus; laciniis duabus regulariter oppositis minoribus, reflexis (calyx diphyllus PERs.); corolla nalla (corollae petala duo sinuata PERs.); stylus nullus; stigmata duo capitata; spora una, 
una, plana, calyce tecta; - (Elongatio trunci frutescens; flores axillares aut terminales).

Ioro. CABOMBA, (CABOMBE) AUBr. JUSs. IAM. tab, 265. NECTRIS schreb. WiLId. (juncinea 4.) Calyx coloratus, sexpartitus; lacini:s tribus interioribus petaloüdeis, parum minoribus, obtusis; corolla nulla; stamina inserta margini laciniarum calycinalium; ovaria duo; capsulae duae ovales, punctatae, subcarnosae, stylis coronatae, unîloculares, mono-di-polysporae; (Elongatio trunci lierbacea; folia non amplexicaulia digitata, foliolis linearibus; - planta habitu Ranunculi aquatici et Myriophylli aquatici).

IOI. ORYZA, (RIZ) TOURN, IINN. JUss. IAM. tab. 264. (graminea 9.) - Calycis gluma bivalvis, acuminata, uniflora; gluma corollae bivalvis, valvulis navicularibus, inaequalibus; exteriori striata et aristata; ovarium basi munitum squamis duabus oppositis; spora una, oblonga, striata, calyce tecta.

10I2. SUL A MEA , (SOULAMEA; Bouvati) IAM. Juss. (seäis incertae). - Calyx minimus, trifidus; corollae petala hypogyna, numero tria (aut mox guatuor), inserta super discum glandulosum; ovarium superum, compressum; stylus nullus; stigmata duo capitata; capsula compressa, fere plana, cordata, margine arguto, ad summum inter stigmata emarginata bilocularis, dispora; spurce oblongae; aibumen nullum; radicula recta; - (Frutex foliis alternis, confertis ad ramorum sum. mum; flores minimi, in racemos terminales aut axil. lares dispositi. Indiarum incola).

\section{$T R I G I I N I A$.}

1013. WUR MBEA, (WURMBE) TNUNB. diss. I. p. I8. t. I. f. 6. LAM. tab. 270. (juncinea 4.) - Calyx (corolla) inferus, tubulosus, tubo sexangulato, usque ad medium sexpartito; staminum filamenta fauci corollae insorta; styli subconnirentes; capsula oblonga, trique* 
tra, tristriata, trilocularis, polyspora; sporae rotundao; - (Elongatio trunci munita foliis amplexicaulibus, integerrimis, glabris; floribus spicatis sessiribus).

IOI 4 COLCHICUM, (COLCHIQUE) TourN. IINN. JUSS. IAM tab. 267. (juncinea 4.) - Spatha: ca. lyx (corolla) inferus, tubulosus, longus, limbo campanulato, (corollaeformi. Juss.)sexpartito ; tubo radicali, longissimo; stamina summo tubi inserta; antherae incumbentes; ovariurn trigonum; stigmata simplicia; capsulae (capsula triangularis sutura interna dehiscens) tres, inferne connexas, inflatae, uniloculares, polysporae; spo. rae muricatae, affixae medin marginis suturae internae; (cotchicumassimilis Croco, sed maiori affinitate accedit Veratro; autumno flores immediate e trunco subterraneo emittit (cf. IoH. HEDIvIc Aufsütze I.); folia vero insequenti prodeunt cum ipsis fructibus sessilibus ; tuberculum vel corpus antiqui. (praeeuntis anni) IIuncipersistit, et in sinu posito longitudinaliter ad latus anterius, novus truncus vel potius trunci elongatio circa basin enascit, sequaliter tuberculo munita; hoc tuberculum novum ex antiquo, quod functiones gerit albuminis aut cotyledonum, originem trahit. I. HEDWIG. JUS.S. vENT.)

1015. MEREN DERA, (MERENDÉRE) RAMOND Bull. soc, philom. no. 41. tab. 12. f. 2. REDOUTE Lil. t. 25. BULBOCODIUM DEsF. (juncinea 40? liliacea): Spatha calyx (corolla) inferus, infundibuliformis, campanulatus, sexpartitus $P$. (hexapetalus), laciniic exec. tis, unguiculatis, unguibus linearibus elongatis conniventibus, in tubum approximatis; antherae rectae, sagittatao; ovaria tria, baei coadunata; capsulae tres; basi connexae, uniloculares, univalres, polysporae, latere interiori dehiscentes; sporae suspensae duabus placentis linearibus.

1016. SABAL, (SABAL.) GUERNSENT bullet. Soc. philom. no. 67. tab. 25. Juss. CORYPHA JACQ. WALTHER. CHAMAEROPS MCHAUX; (palma) Polygama - flores hermaphroditi : spatha universalis nulla: spadix ramosus; spathae partialos membranaceae; caly $x$ sexpar- 
titus, lacinias tres extoriores minimae, persistentes; stamina sex, libera, flamentis basi incrassatis; ovaria tria, coadunata; stigmata tria, sossilia, subpubescentia; baccue tres; duae plerumque abortivae; subsphaericae, pisiformes, monosporae; caro pauca, subamarescens, sporis non adhaerens. Spora ossea, rufescens, punctis aspera, basi area umbilicali depressa notata; papilla exigua lateralis embryonein obtegens. Albumen cartilagineum, album; embryo parvus, conicus, horizontalis: hinc Character essentialis: Flores hermaphroditi: spathae partiales; stamina sex libera, filamentis basi incrassatis; ovaria tria, coadunata; baccae tres, monosporae, duae plerumque abortivae; spora ossea; embryo lateralis.

1017. CHA MAEROPS, (L'ÉVANTAIL) IINN. Juss. IAMI. tab. goo. (palma). - Polygama. Spatha monophylla, compressa, bifida; spadix ramosus; flores hermaphroditi: calyx sexpartitus, laciniis tribus exterioribus minoribus (calyx. P. corolla tripetala - internae laciniae-) - corolla nulla; filamenta connexa in cylindrum, monadelpha, et munita dentibus sex, singulo anthera ornatum; ovarie tria cincta cyatho; styli tres; drupae tres, parvae, globosae, monosporae; floresmasculi: calyx, curolla, stamina ut in hermaphroditis, dioici, (Ovarium triloculare, loculis monosporis, duobus abortientibus, mrcurux; - folia profunde palmata aut digitata, in petiolis utroque latere spinos

1018. TOFIE L D A , (TOFIELDE), HUDS. SMITH. ANTHERICUM IINN. NARTHECIUM JUSS, LAM. Lab. 268. HERITIERA Comes de SternBERG in diariis botanicis I. p. 83. IIELONIAS willd. (liliacea?) Caiyx trifidus; corolla persistens, infera, petala sex aequalia; capsulue tres, superae, basi connexae, polysporae.

1019. NOLINA, (NOLINEE) Mrchaux amer. bor. t. 241. (aspliodelea 2.) - Calyx sexpartitus, patens, brevior; ovarium triangulum; styius brevissimus; capsula trigona, membranacea tricocula- 
ris, loculis monosporis, duobus abortientibus, dissepimentis bipartibilibus, dehiscens; sporae solitariao hinc convexo incurvae, rectae, basi affixae, superficio inaequales.

1020. MELANTHIUM (MELLANTHE) LINN. Juss. Lam. tab, 269. (juncinea 4.) - Polygamum; ca$l y x$ (corolla) inferus, coloratus, rocatus, sexpartitus (hexapetalus) laciniis unguiculatis, basi biglandulosis; filamenta inserta unguibus elongatis calycis; ovaria tria coadunata; capsula ovalis, triquetra, tricocca? (capsulae coalitae), apice subtrifida, trilocularis, polyspora; sporae alato membranaceas; - (Folia elongata, debilia omnium huius generis specierum; flores paniculati, rarius spicati).

102I. VERATRU M, (VÉRATRE, Varaire) ToUR. IINN. JUSs. LAM tab. 843. (juncinea 4.) - Polygama; flores hermaphroditi: calyx (corolla) aequalis hexapetalus (sexpartitus), eglandulosus; stamina sex; ovaria tria distincta; in nonnullis floribus abortientia; styli tres breves; capsula triloba, (capsulae tres) dehiscens in unoquoque lobo per suturam internam; polyspora; sporae ala membranacea cinctae; Flores masculi: calyx, corolla, stainina ut in hermaphroditis, pistilli rudimentum; - (Flores in paniculam dispositi; folia nervis proeminentibus sulcata).

1022. ZIGADENUS, (ZIGADÈNE) MICHAUX am. bor.t. 22. - (juncinea 4.) - Calyx (corolla) maxime patens, sexpartitus laciniis profundis supra basin angustatam biglandulosís; stamina ad contactum ovarii inserta ; ovaric coalita; styli tres, contigui et ob. rusi; capsula calyce! brevior, rnembranacea, stylis persistentibue arcuata, trilocularis, polyspora; sporae numerosae, lineares oblongae, angulosae sive apterae. (Fructu a Veratro et Molanthio distinctum genus).

1023. MEDEOLA, (MEDÉOLE) LINN. JUSS. IAM. - tab. 265. (smilacea 1.) - Calyx (corolla) inferus, parens, sexpartitus, laciniis extrorsum revolutis, aequali- 
bus; bacca rotundata, trisulca, trilocularis, trispora; (Elongatio trunci recta aut scandens, simplex aut ra* mosa: ramis ad basin spatha munitis aeque ac folia; - flores solitarii, axillares).

1024. HELONIAS, (HELONIAS) IINI. IAM. tab. 268. (juncinea 4.) - Calyx nullus; corolla (calyx Juss.) infera, aequalis, colorata, hexapetala, peialis ob. longis; stamina corolla longiora; ovarium triangulum: styli rres, broves, distincti; capsulu rotundata, milocularis, tricornis, olygospora; - (Folia radicalia, lanceolata, super terram rosulam formantia).

1025 XEROPHYLLU M, (XEROPHYLLE) MI. cHAUX. HELONIAS pLUY. IINN. - Corolla rotata; filamenta basi contigua; stigmata tria, revoluta, basi subconnata; capsula subglobosa, apice triplici rima dehiscens, trilocularis, dispora.

1026. TR I L L I U M , (TRILI IE) IINN. JUSS. IAM. tab. 267.(smilacea 1.) - Culyx patens, sexfidus, laciniis exterioribus alternis, magis erectis; (corolla infera, petalis tribus calyce parum maioribus): bacca tere rotundata, trilocularis, loculis polysporis; (Elongatio trunci aphylla, apico uniflora, munitá tribus foliis versus medium).

1027. TPIGLOCHIN, (TRIGLOCHIN, TROS. CART) LINN.JUSs. IAM. tab 270. IUNCAGO TOURN. (juncinea 4) - Caly $x$ inferus, sexpartitus, laciniis profundis, rotundatis, superioribus cum inferioribusal. ternantibus; tribus interionibus petaloideïs; corolla nulla вотн.; (PER.s.: calyx triphyllus, petula tria calyciformia); starnina brevissima; cvuria tria aut sex comiventia; stigmata tria vel sex; styli mulli; copsuiae ovato oblongae, rectae, arcuatae, basi dehiscentes, tri-aus sexloculares, loculis monosporis.

1028. RUMEX, (OSEILLE, Patience) IINN. Jtiss. LAir. illustr. tab. 27r. ACETOSA et LAPATHUVI TOUnN. (polygonea). - Caly $x$ inferus, sexpartitus: laciniis

tri- 
tribus interioribus multo maioribus, approximatis; corolla nulla; (calyx triphyllus: corollae petala tria conniventia YERS.); stamina quinque ad novem; styli duo vel tres; stigmata multifida; spora una, triquetra, nuda aut calyce tecta. - *2 Floribus hermaphroditis; valvulis granulo notatis; $\left.{ }^{* *}\right)$ valvulis nudis granulovo destitutis; ***) LAPATHUM floribus diclinis; - (Flores paniculati axillares aut terminales.

1039. SCHEUCHZERIA , (SCHEUCHZÈRE) rinN. Juss. LAM. ill. t. 270 . (juncinea 4.) - Calyx inferus, aequalis, sexpartitus ; corolla nulla; (corolla sexpartita; calyx nullus rers.); staminum filamenta brevia; antherae lineares, oblungae; 'stigmata sossilia, lateralia ad externum ovarii; capsulae tres, compresso inflatae, distinctae, bivalves, mono-disporae.

3030. FLA GELLARIA, (FLAGELIAIRE) IINN. Juss. LAm. tab. 266. (smilacea 1.) - Calyx inferts, companulatus, sexpartitus laciniis aequalibus, patens; corolla nulla; stylus trifidus, persistens; bacca (drupa) rotundata, trispora aut per abortum monospora; - (Elongatio lierbacea; Solia arundinacea, amplexicaulia, cirrho spiraliter so involvente terminata; flores paniculati terminales).

* ro3i. HYDRO GE T O N, (IIYDROgeton) pers. OUVIRANDRA AUbe. Juss mss. minbel. (fluvialis). - Calyz coloratus, sexpartitus, laciniis profundis; corolla nulla; filamenta basi delatata; pistilla tria, simplicia; folliculi (capsulae rers.) tres, quisque nnilocularis, membranaceus, introrsum dehiscens, disf̧ori; sporae basi parietis capsulae adnatae; lobus embryonis plicatus; albumen nullum, (Scapus radicalis; corolla tripetala PERSOON. p. 400.)

1032. APONOGETON, (APONOGETON) IINN. supl. Juss. LAM, tab. 276 . (fluvialis). - Flores dispositi circum receptaculnm commune, lineare, singulo squama laterali tecto, amentucei; calyx formatus oquama una laterali simplici aut bipartita; corolia nul- 
la; stamina sex-duodecim secundum IAMARK; undecim-undeviginti sec. IINN., lateralia aut exteriora; ovaria tria, qnatuor; totidem styli et stigmata; capsulae tres, quatuor, trisporae, ad basin affixae; - (Tolia radicalia; flores in spica attonuata et terminali).

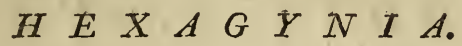

1033. WE NDLA NDIA, (WENDLANDIE) WILLd. ANDROPHYLAX wendr. hort. Herrenh. fasc. III. t. 16. - Calyx hexaphyllus; corollae petala sex succulenta; stylus reclinatus; capsular sex, uniloculares, monosporae.

1034. OT TELLIA, (OTTELIE) N. - STRATI OTES IINN. DAMASONIUM SCHREB. WILID. (hydrocharioïdea). - Spatha monophylla, quinquealata, unjflora; calyx superus, tripartitus; coroliae petala tria; bacca decemlocularis, polyspora. - (Scapus uniflorus).

1035. DAMASONIUM, (FLUTEAU) DAIECH. TOURN. JUSS. I.AM. ill. t. 272. ALISMA LINN, WILID. (juncinea 3.) - Calyx inferus, triphyllus, foliolis petaloideis? concavis; corolla tripetala; ovaria sex; styli sex; capsulae (nuces?) sex, acuminatae, evalves, stollatim positae, patentos, mono-di-trisporae.

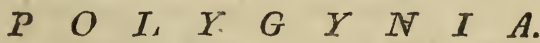

I035. A L I S M A, (ALISME, plantain 'd'ean) I 1 Nw. JUss. LAM. tab. 272. RANUNCULUS TOURN. (juncinea 3.) - Calyz inferus, triphyllus. foliolis concavis, petaloideis; corolla tripetala; stamina sex, interdum plura; ovaria numero plura, coadunata; totidem styli; capsulae numero plures, evalves, in capitulum parrum congestae, monosporae. 


\section{CLASSIS SEPTIMA}

\section{HEPTANDRIA.}

\section{$\begin{array}{llllllllll}I I I & O & N & N & O & G & Y & N & I & A\end{array}$}

I. Flore completo.

I037. TRIENTALIS, (TRIENTALE) TOURN. IINN. JUSs. IAMr. tab. 275. (primulacea I.) - C'alyx heptaphyllus (septempartitus?); corolla rotata, septemfida, laciniis aequalibus, plana; bacca sicca, globosa, evalvis, unilocularis, polyspora, angulis dehiscens. Stamina quinque, sex, septem.

1038. TOVARIA, (TOVARIE) RUIz, et PAV. (priznulacea?) - Calux heptaphyllus; corolla heptapetala; discus planus, heptagonus, stellaeformis, cui stamina insident; stigma peltatum; bacca unilocularis, pulposa; sporae compressas. - (Numerus partium interdum octonarius.) - *)

*) a TRIENTALI differt hoc geuus calucp cadnco; staminibus disco plano stellaeformi insertis incurvis, ovarium ambientibus; styio cohumuari; stigmute peltato, septem. fido; bucca; coronata; sporis reniformibus, nidulan. tibus. 
1039. D I SA N R A , (DISANDRE) LINN. JUS8. IAx. tab. 275. (rhinanthacea 1.) - Calyx quinque-septem-octopartitus vel fissus; corolla rotata, quinque-octoloba; laciniis lobisvo aequalibus; stamina quinqueocto; capsula ovalis, trilocularis, polyspora; - (Plan. ta herbacea, procumbens, repens; folia alterna, rotundata, crenulata et pubescentia; flores axillares).

I040. A E S C ULUS, (MARRONIER) LINN. HIP. POCASTANUM TOURN, VENT. LAM. tab. 273. (aceracea I.) - Calyx campanulatus, monophyllus, parvus, quinquedentatus, ventricosus; corollae petala quinque inaequalia, patentia, calyci inserta, pubescentia, limbo rotundato et undulato; filamenta subulata, declinata, inaequalia; antherae fero versatiles; stylus subulatus; stig: ma simplex; capsula rotundata, coriacea, processibus rigidis ochinata, trilocularis, trivalvis; dissepimentis medio valvularum adnatis; sporae duae in loculo quoque (una abortiens; tunc loculi monospori), fere globo. sae; embryo curvus; radicula declinato pendula super lobos maxime incrassatos, et adhaerentes maturitatis statu fructui; plumula megna, efformata duobus foliolis digitatis. (Arbores primae altitudinis; foliis digitais ; floribus pyramidalibus, terminalibus, interdum tantum masculis abortu pistilli rudimentum relin. quentis).

1041. PAVIA, (PAVIA) BOERH. VENT. LAM. tab. 273. AESCULUS LINN (aceracea 1.) - a praecedenti ge. nere differentia haec: calyx tubulosus, quinquedentatus; corollae petala quatuor, quinque, inaequalia, approximato-conniventia, calyci isserta, duo superiora magis erecta; sex ad octo stamina maxime exserta; filamenta capillaria, recta; cupsula pyriformis, laevis et inermis, trilocularis, trivalvis, loculis disporis; (Flores in spicam dispositi).

1042. PARINARIUM . (PARINARI) JUSs., PARINARI AUBLET. PETROCARYA SCHREB. WILLD. $\left(\right.$ rosacea $\left.7^{\circ}\right)=$ Calyx urceolatus, quinquefidus; corol[I7] 
i. lae petala quinque alternantia cum laciniis calycinis; stamina quatuordecim, quorum septem in eadem serie et fasciculo locata connexa, sterilia, septem opposita, fertilia; ovarium villosum; drupa ovalis, carnosa, differenti magnitudine, fibrosa; (cribrosa P.); continens nucem durissimam! sinuositatibus tectam, bilocularem, loculis monosporis; - (Arbores ramosi, ramis villosis; folia stipulacea.

1043. PANC OVIA, (PANCOVE) wrimd. (planta sedis incertae). - Calyx campanulatus, quadripartitus; coroilae petala quatuor, unguiculata, plicato-crispa, dentata, cucullaeformia; stamina adscendendo calyce multa longiora ; fructus.... ? - (Arbor Guineae, imperfecte cognita).

1044. IO N ESIA, (IONÉSIE) noxB. (leguminosa? sedis incertae). - Calyx diphyllus bifidusve; co. vollu infundibuliformis, limbo quadrifido tubn clauso, carnoso; recturolyma? annulus staminiferus insertus ad tubi faucem corollae; ovarium pedicellatum; legumen acinaciforme. tetra-octosporum; (Arbor; folia alterna, pinnata cum impari; flores in cyma terminali aut axillari).

1045. A NALECTIS, (ANALECTIS) JUss. mss. (pl. sedis incertae). - Caly $x$ urceolatus, qninquedentatus; corolla hypocrateritormis, calyce multo minor, vix exserta, tubulosa, modio attenuata, limbo septem. lobo stamina septem tauci tubi inserta, alterna cum lobis limbi: filamenta longa; antherae rotundatas; ovarilm superun; stylus tenuis; stigma furcatum; fructus calyce tectus. -

10.16. LA G UNEA, (LAGUNAEE) LOUR. JUss. mss. (aroìdea 1.) - Spatha squamis tri-quadrifloris : calyx companulatus, tubu brevi, limbo quinquefido: stamina septem: filamenta basi glanduloga; antherue ovales, de: clinatae; ovarium unuin; stulus furcatus: stigniata duo; spora una, pericarpio destituta; (Elongatio truncı herbacea; 
bacea ; rami prostrati ; folia integerrima, magna, utrin* que villosa; flores terminales.)

\section{Flore incompleto.}

10.47. P I S O IA, (PISONE) 'PIUN. LINN. JUSs. x AMr, tab. 86r. (nyctaginea 2.) - Calyx parvus, campanulatus aut infundibuliformis, limbo leviter quinquefido, aut integro, basi nudus, involucratus, involucrum compositum quibusdam squamis (duabus - quatuor) minimis, unum-plures flores comitantibus; corolla nulla; stamina sex, septem, octo, calycina; bacca leviter pentagona, unilocularis, monospora; spora elongata, basi angulosa calycis, cuius superior pars caduca est, tecta; pars calycis persistens evenit coriacea, et plerumque echinata super angulos pilis aut filis angulosis; - (Elongatio trunci in quibusdam speciebus spinis axillaribus restita; rami oppositi aut alterni aeque ac folia; pedunculi axillares, ramificati in paniculas aut corymbos, quorum quaeque divisio, basi squama caduca vestita, terminatur uno vel pluribus floribus).

1048. P E T I VER I A, (PETIVERE) PLUM, LINN: JUss. LAM. tab. 272. (atriplicea 2.) - Calyx tetraphyllus, foliolis linearibus, obtusis; corolla nulla; stylus lateralis vel styli quatuor; stigma penicilliforme; cap. sula monospora, compressa, calyce tecra, apice aristis quatuor reflexis munita, non dehiscens; (stamina sex, septem, octo.) - Folia acuminata; flores terminales, dispositi in spicam laxam.

\section{$\begin{array}{lllllllll}D & I & G & Y & N & I & A\end{array}$}

1049. L I M E U M, (LIMEOLE) IINN. JUSS. LAM. illustr. tab. 275. (portulacea 2.) - Calyx quinquepar-

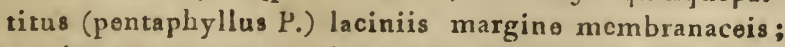
persistens; corollae petala quinque calyco breviora, ae- 
qualia; stamina septom, filamentis basi coaduratis; sty. lus bipartitus; capsula globosa, bilocularis, polyspora.

$$
T E T R A G Y N I A .
$$

1050. A S T R A N THUS, (ASTRANTHE) LOUREIR. wIILD. - Calyx nullus; corolla hypocrateriformis, limbo quatuordecimfido, laciniis linearibus, stellatim positis, septem alternis brevioribus; spora una, supera, tubo corolloe inclusa, parva:

105I. SAURURUS, (SAURURE) IINN. JUSS. IAM. tab. 276. (fluvialis). - Flores dispositi in amonto ; calyx persistens, amentum monophyllum (squama) laterale, coloratum, squama uniflora; corolla nulla; stamina sex aut septom, (hypogyna?); ovaria quatuòr ; stylus nullus; stigmata inserta interiori ovario; baccae quatuor, ovoüdeae, monosporae.

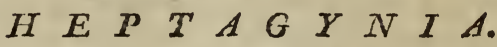

1052. SEPTAS, (SEPTAS) IINN. JUSS. IAM. tab. 276. CHASSULA тHU्NB. (crassulea). - Calyx septempartitus; corolla heptapetala; ovaria septem; capsulae septem, parallelae, univalves, polysporae; - (Herbas minutulae: folia radicalia, flores in umbella super elongationem apliyllam).

* 1053. GILIBEPTIA, ( GILIBERTA) RuIz ot PAv. - Calyx septemdentatus; corolla heptapetala; stigmata ovata, patentia; ovarium ovatum; capsulae loculamenta monospora, in stellae formam disposita; sporae oblongae. (Numorus partium interdum octo-novem, varius. Auctores fructus immaturos offenderunt.) *)

$$
105+\text { A c.' }
$$

-) Iam in speciebus Plantarum ed, wirID, II. p. 55x, et GMFI. systema I, pag, 682, existit aliud codem bub mo. - mine geuus. 
CLASS. VIII. OCTANDRIA: 26r

3054 ACTINOPHYLL UM, (ACTINOPHYLLE) RULz. PAYon. - Caly $x$ margo integer; corolla calyptraeformis, desiliens; ovarium truncatum; bacca septemangularis, septemlocularis; sporae solitariae, subosseae. (Flores congtomerati; 'styli quingue ad septem; stamina etiam quinque, sex, octo, novem). ${ }^{*}$ )

\section{CLASSIS OGTAVA}

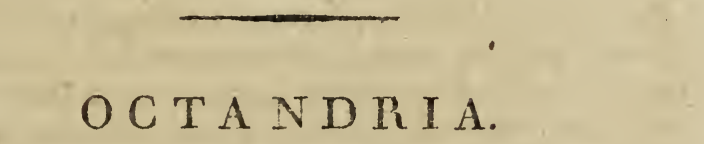

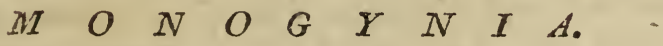

I. Flore completo.

1055. MIMUSOPS, (MTMUSOPE) IINN. JUSS. IANT. tab 300. BINECTARIA FoRsK. (sapotea), - Ca. lyx octoparticus laciniis dispositis in duos ordines quadrinarios, alternis mainribus; corolla octopartita, laciniis integris ant trifidis; nectarolyma appendices octosodecim squamaeformes, circumcirca ovarium ambien. tes; drupa acuminata, mono-dispora.

1056. HONCKENIA. (HONCKENIE) USTERT wiled. - Calyx pentaphyllus; corolla pentapetala;

- nec.

*) Conferri potest practer forac Peruvianac prodromum, D. 10s. PAVON dissert. liotan. sobre los generos TOVAMIA, ACTINOPIYLLUAI, ARANCAHIA, y SALIIA, Madrit. 1797. 
nectarolymata staminifera; capsula echinata, quinquelo. cularis, quinquevalvis, polys pora; sporae arillatae.

1057. SODADA, (SODADE) rORsK. NECTOUX. (capparidea). - Calyx tetraphyllus, foliolo superiori maiori et gibbo; corollae petala quatuor inaequali magnitudine, superiora duo ovalia et breviora; stamina octo inaequalis magnitudinis; antherae lanceolatae, recurvatae; ovarium sustentatum longo pedicello; stylus unus; stigma simplex; fructus ruber, paulo maior fructu coryli; (Frutex Aegypti, ramo singulp ad basin bispinoso; folia caduca; pedunculi uniflori; a Fors $\$$ primum observata; nuper ab hortulano expeditionis in Aegsptum allata hortis botanicis Parisiensibus).

10,58. C UPANIA, (CUPANI) PIUM. IINN. JUSS. SWARz. GUIOA CAV. MOLINAEA JUss. *) (saponacea 2.) - Calyx triphyllus vel quinquepartitus, persistens; flos masculus : corollae petala quinque, parva, apice cucullata, cum calyce alterna; stamina quinque; fila. menta basi villosa coalita; antherae rotundatae; flos $\mathrm{fe}$ mineus: coroilae petala tria; stylus minimus, trifidus; stigmata tria ; capsula turbinata, trilocularis, - (pedicellasa, coriacea,) triquetra, loculis mono-disporis, trivalvis: sporae oblongae, basi crassiusculo-arillatae. (Arbores : foliis magnis àlternis; floribus terminalibus paniculatis).

1059. EU-

*) Scilicet PERsoon in enchir. p. 413. confirmat, secuudura dilegentiores JUESIEU1, summi viri, observationes genus MOLINAEA ab co constitutum CUPANIIS inscti debcre; neC non PERSONIUM interrogantem legimus, , quibus etiam adicienda TOULICIA AUBI.ETII? - MOLINEAE comirers. Juss. LaM. to 305 enim char different. erat: calyx. quinquepartitus; petala corollae quinque parva, cum calyce alternantia, unguibus leviter villosis; stami. num filamenta basi villosa; ovarium super discnm positum et fere pedicellatim; stylus nullus; stigma sim. plex; capsula pedicellata, coriacea, triangula, trilociula. ris, trivalvis, trispord: valvulis ad medium septife. ris; - (Arbores: foliis pinnatis cum impari; Horibus in racemo axillari). 
1059. EUPHORIA, (IITSCHI) cOMMERS. JUSS. IAM. illustr, tab. 306. SCYTALIA GAERTN. DIMO. CARPUS WILLD. LITSCHI SONNER E LAM il.1.1. SAPINDUS AIT. (saponacea 2.). - Calyx monophyllus, parvus, quinquedentatus aut quinquefidus; corollae petala quinque, minima, interne ad mediam partem villosa, reflexa; stamina sex, saepius octo juss. (septem LAMr.); ovarium didyrnum; stylus apice bifidus; stigmata simplicia ; bacca didyma; altera parva abortions, altera globosa, coriacea, muricata tuberculis, unilocularis, monospora; sporae cinctao arillo molli..-- (Arbor; folia aequaliter pinnata; flores parvi, dispositi in paniculas terminales, bracteis brevibus muniti, quidam steriles).

I060. TROPAEOLUM, ( CAPUCINE) IINN. JUSS. LAM. tab. 277. CARDAMINDUM TOURN. (geraziea). - Calyx profunde quinquefidus, monophyllus, lacinia superiori ad basin calcarata, coloratus; corollae petala quinque inaequalia, calyci inserta, cum calycinis laciniis alternantia, superiora duo sessilia, tria inferiora ungue oblongo ciliato munita; stamina in disco collocata; filamenta distincta inaequalia, petalis breviora; antherae oblongae, erectae, biloculares ; ovarium disco cinctum, triangulare; stylus cylindricus, staminum longitudine; stigmata tria acuminata; fructus formatus baccis vel nucibus tribus, spongioso coriaceis, fere réniformibus, suberosis, sulcatis, connexis, subrotundis, monosporis affixis ad basin styli persistentis; embryo magnus sine albumine; cotyledones applanatao, apice bidentatao, maturae adhaerentes; radicula supera. - (Plantso herbaceae, exoticae; folia alterna, oxstipulacea, peltata aut rarius digitata; pedunsuli axillares, uniflori; hoc genus quodam modo Geraniis uffine, sive calcare superioris calycis laciniae, tubo superioris laciniae calycis Pelargoniis simile, sive sit situ staminum et petalorum; sive stylo unico et stigmate multiplici; sive pericarpiis aequaliter basi styli affixis; sive tandem albuminis absentia: sed a Geraniis differt defectu stipularum, staminibus distinctis, floribus foliis non oppositis, Lobis denique embryonis rectis. Juss, vent.)

10'1. B A E. 
I061. BAE CKEA, (BACKE) OsB. IINN. JUSS. IATh. tab. 285. (onagrea 4). - Calux turbinatus, quinquedentatus; corolla pentapetala; stamina octo; sex aequali magnitudine, duo breviora, solitaria; antherae ova. les; capsula globosa, coronata, tri-quadrivalvis, tri-quadrilocularis, polyspora, calyce tecta; sporae ńumero paucre, minores. - (Removeri ex hac naturali familia debet, quum oAERTAERUs ad sporas observavit albumen carnosum, quod neque in myrteis neque in onagreis existit).

1062. MA TAIBA, (MATAYBE) AUBL. JUSS. IAM. tab. 29S. EPHIELIS SCHREB. PERS. (saponaceis affinis). Calyx quinquepartitus; corollae petala quinque, interno ad unguiana basin squamis duabus appendiculata, inserta super discum calycinalem; stamina octo disco inserta; filomenta villosa; stylus nullus; capsula oblonga, utroque latere compressa, concava, unilocularis, bivalvis, altera valvularum inanis ot nuda, altera continens duas sporas reniformes et arillatas. - (Arbor foliis pinnatis sine impari, stipulaceis; flores in panicula axillari aut terminali).

I0ك3. HAGEN IA, (HAGÈNE, Cusso) IAM. tab. 3 II. - Calyx diphyllus; corollae petala quinque plana, appendices (nectarolymata) quinque petalis quadruplo minoribus vel brevioribus; capsula -?

1064. MEMECYLON, (MEMECYLON) IINN. JUss. LAM. tab. 294. (onagrea 4.). - Calyx turbinatus, suporus, margine integerrimus, fundo striatus; corollae petala quatuor (monopetala P.); filamenta dilatata et ad sumnum truncata; antherae his lateraliter insertae; bacca infera, calyce cylindrico coronata. - (Frutex foliis oppositis integerrimis; floribus axillaribus, fere capitatis).

I065. SCUTULA, (SCUTULE) IOUR. conch. I. p. 29 c. Juss. mss. (onagrea 2.) - Calyx carnosus, vasis destitutus, gemmaeformis, margine integer, interne octo poris munitus; corollae petala quatuor aut quinque, 
ad summum calycis affixa, in conum approximata; stamina octo; filamenta curva; antherae approximatae poris calycinis mediante filamentorum curvatura; stylus unus; stigma simplex; bacca concava, patollaeformis, octolo. cularis, octospora. - (Arbores aut frutices foliis op. positis).

7066. C O M BRE T U M, (COMBRET, CHigonnier) IOEFL. IINN. JUSS. IAMr. ill. tab. 282. (onagrea 3.). Calyx superus, campanulatus, limbo quadri - quinquedentato et caluco; corolla tetra - pentapetala, petalis calyci insertis; stamina octo - decem, corolla multa longiora; antherae oblongae; capsula oblonga, quadri quinquangularis, angulis quatuor vel quinque alatis, membranaceis, unilocularis, monospora; spora oblonga. (Flores quidam decandri, in spicá terminali aut axillari; folia opposita ; frutices sarmentosae).

1067. ROXBURGHIA, (ROXBURGHE) noXB. wrLl D. (pl. incertae sedis). - Calyx tetraphyllus; co* volla tetrapetala, quatuor appendices (nectarolymata) foliolis lanceolatis, conniventibus, medio petalorum insertis; antherae geminae e basi cuiusque folioli appendicis suspensae vel dependentes; capsula unilocularis, bivalvis, polyspora; sporae receptaculo spongioso affixae.

3068. EPILOBIUM, (ÉPILOBE) IINN. JUSS. IAM. tab, 278. CHAMOENERION TOURN. (onagrea 3.) - Calyx oblongus, tubuloso cylindricus, limbo qua. dripartito, caducus; corollae petala quatuor saepe emarginata; stamina octo, quatuor alterna breviora; dintherae ovales, declinatae; stigmata quadrifida; capsuia infera, longissima, cylindrica, angustata, quadrilocularis, quadrivalvis, polyspora; sporae multae comosae, placentae centrali affixae. - (Elongatio trunci plerumque herbacea, rarius frutescens; folia opposita aut alterna; flores solitarii axillares, aut dispositi in spicas termina. les, alternas super axin śpicae et ad basin bracteatas; filamenta staminum rectae aut declinatae).

1069. GA URA, (GAURE) XINN. JUSS. LAM. ill.tab. 28r. (onagrea 3.). - Calyx elongatus, tubuloso cylindxiv 
dricus, limbo quadrifido, caduco; corollae petala quatuor, adscendentia, solummodo ordinata in latus superius; antherae oblongae versatiles; stigma quadri-quinqielobum; nux (capsula) infera, ovalis, tetragona, striata, duobus marginibus punctata, unilocularis, (monospora LINN.), tetraspora sphenget., (polyspora J.), una spora tantum maturescit, reliquae abortiunt. - (Elongatio pubescens; folia alterna, lanceolata, parum splendentia, fere glabra).

1070. OENOTHERA, (ONAGRE) LINN. JUSS. IAm. ill. tab. 279. ONAGRA tourn. (onagrea 3.). Cályx elongatus, tubuloso cylindricus, limbo quadripartito, laciniis deflexis, caducis; corollae petala quatuor calyci inserta; antherae oblongae, declinatae; stigma qua. drifidum; capsula infora, cylindrica, elongata, quadrangula, quadriloquaris, quadrival ris, dissepimentum medio valvularum affixum; polyspora; sporae nudae, receptaculo centrali tetragono affixae. - (Elongatio herbacea).'

107I. VITMANNIA. (VITMANNE) VAHL. SA. MADERA GAERTN. SAMANDURA IINN. (onagrea? sedis incertae), - Calyx quadrifidus; corolla tetrapetala; flamenta ad basin squama parva nectarolymatiformi munita; antherae lineares; ovarium superum; stigma acutum; nux semilunulata, compressa, unilocularis, monospora. - (Arbores ramis cylindricis; fulia integerrima, alterna, petiolata; flores umbellati ad ramorum summum et in foliorum axillis ).

1072. RHEXIA, (RHEXIE) GRON. LINN. IUSS. IAN. illustr. tab. 233. MELASTOMA AURL. (mólastomea 2.). - Calyx tubuloso urceolatus, quadri-quinquefidus; corollae petala quatuor obliqua, calyci inserta; antherae arcuatim declinatae; capsula setosa, quadrilocularis, quadrivalvis, polyspora, inclusa ad basin calyce ventricoso; receftaculum sublunatum; sporae cochleatae. (Stamina etiam decem).

I073 OSBECIKIA, (OSBECKE) IINT. JUSS. IAM. ill. tab. 283. (melastomea 2.). - Calyx campanulatus, limbo 
limbó quadripartito, squamae" quatuor parvae ciliatao interstingunt fissuram quamlibet calycis; corollae petala quatuor, quinque; stamina octo vel decem; antherae terminatae filo longo, rostrato, filiformi, recurvo; ovarium coronatum filis rigidis; capsula echinato-villosa apice dehiscens in partes quinque, quadri-quinquelocularis, polyspora, limbo calycis truncato coronata; receptaculum compressum, semiovatum. GAERTN. (Stamina etiam octo, decemque). *) Elongatio trunci tetragona.

1074. BARBYLUS, (BARBYLLE) Brown. JUss. (sedis incertae). - Calyx campanulatus, limbo quadriquinquefido; corollae petala quatuor vel quinque margini interiori calycis inserta; stamina octo aut decem, ad basin calycis posita; filamenta compressa; antherce ovales; uvarium superum; capsula trilocularis, loculis disporis. - Genus Trichilice affinis.

1075. TETRATHE CA, (TETRATHECA) SMITH nov. Hollandiae plantt. - Caliyx inferus, quadrifidus; corolla tetrapetala; antherae quadriloculares; capsula bilocularis, bivalvis, mono - dispora, valvularum medio semisepto inserta; sporae subbinae.

1076. GRISLEA , (GRISLÉE) LOёrL. LINN. JUSs. (lythraria 1.). - Calyx tubuloso-campanulatus, coloratus, quadridenticulatus, persistens; corollae petala quatuor, minima, laciniis calycis innata; staminum filamenta longissims, adscendentia; antherae rotundstae; capsula globosa, fere pedicellata, supera, calyce bresior, unilocularis, polyspora, - (Flores corymbosi; folix fruticis disticha).

1077. KOELREUTEPIA, (FOELREUTÈRE) IAXMANN. L'HERET, VENT. IAM. Tab.308. SAPINDUS

IIN IN.

*) OSBECritAE a fIIEXIIS differre videntur ovario infero, - quum capsula RfIEXIAE calyce inclusa sec. wILLD. a MIELASTONIS non satis distingui, vel potius cum iis coniungi debere videantur LaAarckio ench. but. 4. pag. 647. 
IINN. (saponacea I.). - Calyx quinquefidus vel pente. phyllus; corollae irregularis petala quinque foliolis calycinis duplo longiora, unguiculeta, ad basin munita rectarolymatis squamis quatuor bifidis; stamina circum calycis longitudinem; filamenta villosa; antherae oblongae, rectae, villosae; ovarium pedicellatum; stylus triangulus; stigma trifidum; capsula fero ovoïdea, membranacea, inflato-resiculosa, trilocularis, loculis di-poris, spora altera abortionte. - (Frutex foliis pinnatis cum impari; flores in panicula terminali).

1078. CORREA', (CORREA) SMITH ANDREW.*) VENT. jard. de Malm. tab. 13. MAZENTOXYRON rABILL. (rutaceis affinis). - Calyx perianthium mono. phylium, inarginabus quadridentatum, campanulatum, erectum, porsistens; corollee petala quatuor, oblonga, concava, apice rellexa, marginibus crassis ; stamina octo, quatuor opposita petalis, quatuor alterna ; flamenta octo. erecta, filiformia, receptaculo inserta; ovarium superum, turbinatum, super discum hypogynum positum; stylus cylindricus, filiformis, longitudine staminum, porsistens ; stigma obtusum, quadridentatum ; fructus compositus quatuor cocculis maxime approximatis, ovalibus, compressis, truncatis, coloro brunneis, introrsum de. hiscentibus et ad summum : efformatis duabus membranis, altera interiori cartilaginea et elastica, includento duas tresve sporas, altera alteri invicem incumbente; (capsuia coriacea, lanata, valvulis quatuor dissiliens, loculis mono - disporis; ANEREW. - Frutex).

I079. SASSIA, (SASSIE) Morin. Juss. (planta sedis incertae). - Calyx patens, tetraphyllus foliolis patentibus; corolla tetrapetala; stamina brevia; ovarium superum; stulus unus; stigma unum; capsula ovalis, bilocularis, dispora. - (Plantac herbacea, foliis radicalibus; flores solitarii aut numero plures circa elongationem; observatao ad Ghili).

*) The botanists Repository T̃o. 1. tab. VI. 
1030. A N ICHORUS, (ANTICORÉTE, Antichore) IINN. JUSS. IAM. tab. 295. JUSSIEUA FOFsK. (liliacea 2.). - Calyx tetraphyllus, patens, caducus; corolla tetrapetala; antherae rotundatae; capsula supera, oblonga, siliquaeformis, subulata, quadrilocularis, quadrivalvis, polyspora. - (Elongationes declinato prorepentes; flures axillares; folia ovalia, denticulata).

108 . ALLOPHYLLUS, (ALI,OPHYLLE) IXN IV. (guttifera). - Calyx quadripartitus, laciniis rotundatis, duabus oppositis minoribus; corollae petala qua. tuor, calyci minora; ovarium didyıum; stigma quadri. fidum; fructus....?

1082. ORNITROPHE, (ORNITROPHF) cOMMERs. JUSS. LAM. tab. 509. fig. 1. RHUS IIN AN. ALLOPHYLLUS SWARz. SCHMIDELIA SWARz: (saponacea 2.). - Calyx quadripartitus; corollae petals quatuor barbata ad medium disci; ovarium didymum; stigma bifidum; drupa (alterum duorum ovariorum abortiens) baccata, parva, pisiformis, nuce monospora. *)

1083. GUIOA, (GUIOA) cAv. ic. 471. t. 373. (saponacea 2.). - Calyx pentaphyllus; corollae petala quinque calyce minora; stamina octo; ovarium sustentatum, disco staminiforo, subpedicellato; stylus brevissimus; stigma simplex; capsulae tres, affixae receptaculo communi centrali, compressae, uniloculares, biralves, monosporae. - (Frutex foliis alternis, aequaliter pinnatis; flores paniculati).

$1084^{\circ}$ T ALISIA, (TALISIE) AUBL. Guy. tab. 136. Juss. (saponacea 1.). - Calyx quinquepartitus; corollae petala quinque alterna cum laciniis calycinis, interno munita ad unguem squama simplici; stamina octo brevia; stylus brevissimus; stigma simplex; fructus.....

( Fru-

*) Cum hoc ORNITHOpHIS genere Pensoon in enchir. pag. 412. coniunxit pracedems ALLOPIIYLXI, uatura ut vittetur mosiente. 
(Frutex foliis alternis, impari pinnatis; flores pedun. culati axillares et terminales).

1085. APORETICA, (APORETICA) Forst.juss. (saponacea 1.). - Calyx tetraphyllus, foliolis duobus exterioribus; covollae petala quatuor totidem parvis squamis interioribus munita; stamina octo; antherae ro tundatae; ovarium didymum; styli duo basi coaliti; stig. mota duo disiuncta; fructus geminatus, singulo monosporo. - (Folia ternata; planta insularum australium maris).

Iogú. GIROA, (GIROA) cavan. - Calyx pentaphyllus, minimus; corolla pentapetala; capsulae tres coriaceae, recopiaculo colunirari adfixae, forma alarum, uniloculares, bivalves, monosporae.

1057. NIO TIA, (NIOTIE) POIRET. enc. bot. IAX. pag. 299. - Calyx quadrifidus; corollae petala quatuor, quinque; antherae sagittatae; stigma acutum; capsula lignosa, monospora.

I088. J A MBOLIFERA, (JAMBOLIER) IIN. JUSS. IAM. (onagrea 4.)." - Calyx quadridentatus; corolla totrapetala, infundibuliformis, petalis basi coali. tis; filamenta plana; antherae ovales; ovarium supra villo. sum; stigma simplex; fructus....? (Genus nondum satis cognitum; scilicet eius charactor diversimodo ab auctoribus describitur).

1089. XIMENIA, (XIMENIA) PLUM. IINN. JUSS. IAM. ill. tab. 297. AMYRIS BRown. (hesperidea 1.). Calyx minimus, quadrifidus, persistens; corollae petala quatuor, alterna cum laciniis calycinis, interns pilosa, basi approximata aut conniventia, versus apicem extrorsum revoluta; filamenta brevia; anthercie longae, rectae; fructus: drupa ovata, includens nucem monosporam. - (Frutices saepissime spinosae; foliaisimplicja, alterna; pedunculi axillares, uni - biflori).

1090. L A WS O N A, (LAUSONIE, Henné) IINN. Juss. LAM. tab. 296. CXPRUS, HENNA, ALCANNA 
RAUW. ALCANNA GAERTN ACRONYCHIA TORST. (lythraria 1.). - Calyx quadrifidus; curolla tetrapetala; stamina per paria petalis opposita; capsula infera sub calyce persistente et patente, globosa, stylo acuminata, quadrilocularis, polyspora; sporae angulatae, receptaculo centrali affixae.

109I. MELICOC CA , (MEIICOCCA, Knepier) IINN. JUSS. LAM, ill.'tab. 306. (saponacea 2.). - Calyx patens, tri-quadripartitus, persistens; corollae petala quatuor reflexa inter calycis lacinias; stamina brevia; stylus brevis; stigma umbilicatum, lateribus prolongatum, peltatum; drupa (bacca) corticosa, fore rotundata, laevis aut muricata, regulariter per abortum monospora, interdum di-trispora; sporae in pulpa nidulantes, cinctae arillo baccaeformi. - (Arbores; folia pinnata cum impari; flores dispositi in racemos terminales).

1092. MULLERA. (MULLERA) IINN, suppl. JusS. (leguminosa 4.). - Calyx campanulatus, inaequaliter quinquedentatus, persistens; corollae vexillum reflexum, alae oblongae, conniventes; carina brevior, dipetala, recta; legumen oblongum, moniliforme aut inflatum; articulo singulo monosporo, - (Arbor foliis impari pinnatis; flores in racemis axillaribus et terminalibus ;.

1093. AMYRIS, (AMYRIS, Balsamier) IINN. IUSS: xAm. illustr. tab. 303. (terubintacea 2,). - Calux quadridentatus, persistons; corollae petala quatuor oblonga, Patentia ; stylus incrassatus; stigma flobosum, fero capitalum; bacca drupacea, exsucca, fere rotunda, calyce cincta, continens nucem globosam, nitidam, per abor. tum monosporam. - (Arbores et frutices; folia ternata aut pinnata cum impari, quaedam adspersa punctis translucentibus; flores in paniculis axillaribus aut ter. ninalibus ).

1094. CLAUSENA, (CI,AUSENE) BURM. JUSS. (planta sedis incertae). - Caiyx parvise, quadridentatus, corollue potala quatuor sessilia; stuminum octo filamenta incrassata, dilatata et ad basin suicata, ovarum ciugentia; antherae versatiles; ovarium superum; styius 
brevis; fructus .....- (Frutex foliis alternis, pinnatis, foliolis integerrimis; floribus minimis in racemis paniculatis: Javae incola).

* 1095. ICICA, (ICICA) aubr. juss. lam. illustr. tab. 303. AMYRIS sinn." (terebintacea 2.). - Calyx quadri - quinquedentatus; corollae petala quatuor vol quinque oblonga, conniventia, affixa disco ovarium cingente, basi recta, limbo disposita; stamina octo, decem, disco inserta; stylus brovis; stigma capitatum, quatrisulcatum; dimpa (capsula?) coriacea, bi-quadrivalvis, di - tetraspora; sporae osseae, pulpa carnosa conditae. - (Arbor resinosa aut balsamifera; folia terrata aut pinnata cum impari, foliolis oppositis duplici rel triplici serie; flores saepe in panicula axillari aut terminali).

rog6. MELI C O E, (MÉLICOPE) Fórst. tab. 28. JUSS. LAM, illustr, tab.294. ENTOGANUMI OAERTN. t. 59. (ex ord. plantt. sedis incertae). - Calyx quadripartitus; corolla tetrapetala, basi urcoolata, tetragona ; ovaria quatuor, supera, cincto unoquoque (octo) duabus didymis glandulis vol crenulatis laciniis (nectarolymata); stylus brevis; capsulae quatuor, mono - tetrasporae.

1097. THOUINIA, (THOUINIE) POITEAU. (sapö nacea 2.). - Calyx quadripartitus, laciniis profundis ; corollae petala quatuor, interno ad medium amicta vil. lorum fasciculo; stamina octo libera; stylus unus; stigmata tria (st. trifidurn P.); capsulae tres monosporae, ad styli basin coalitao, supra in alam membranaceam terminatae. - Petala inserta basi exteriori super volvam glandulosam, extra staminum situm : haec volva omnibus saponaceis staminibus liberis propria viàetur).

1098. GNIDIA, (GNIDIENNE) IINN. JUSS. IAM. illustr. tab. 291. NECTANDRA BERc. PASSERINA IINN. (daplanacea). - Calyx infundibuliformis, elongatus, tenellus, limbo quadrifido (corolla infundibuliformis quadrifida P.); squamae quatuor petaliformes, alternae cum laciniis calycinis, fauci calycis insertae; 
stylus filiformis, lateralis; stigma capitatum, villosum; nux subdrupacea, calyce tecta, oralis; (spora subbaccata). - Folia rarius opposita, flores terminales.

1099. NECTANDRA, (NECTANDRE) BERG. juss. (daphnacea). - Caly $x$ infundibuliformis, limbo quadrifido; squamae octo, ad summum calycis insertae; stamina octo brevia; stylus filiformis ; stigma incrassatum; spora calyce tecta. - (Frutex foliis oppositis, alternis; floribus axillaribus et terminalibus).

1100. FUCHSIA, (FUCHSIA) pLUM. t. I33. f. r. IUss, IA VALLIA commers. (onagrea 4.). - Calyx infundibuliformis, coloratus, limbo quadrifido et caduco, superus; corollue petala quatuor (nectarolymata LiNN.), fauci calycis insidentia, et cum eius' laciniis alternantia; nectarolyma glandula octosulcata; stamina octo filamentis aeque calyci longa aut longiora; antherae oblongae, rectae; ovarium inferum, ovale-oblongum; stigma capitato tetragonum; bacca cblonga, obtuse tetragona, infera, quadrilocularis, polyspora; sporae numerosae obovatae, dissepimentis adhaerentes. Auctores flor. peruv. (Plantac herbaceae aut lignosae, folia opposita aut verticillata tria; flores axillares aut racemosi terminales, in pedunculis rectis pendulis; corolla calyci concolor, si excipis $F$. magellanicam IAM.).

IIOI. HED WIGIA, (HEDWIGIE, hois - cochon) SWARZ. SCHPEB. TETRAGASTIS? GAERTN. (terebintacea 2.). - Caly $x$ urceolntus, quadridentatus; corolla monopetala tubulosa, tubo brevi, limbo quadrifido, laciniis approximatis in conum; stamina basi corollae affixa, et inferne circa ovarium coalita; ovarium superum; stigna sessile; capsula coriaces, formata tribus lobis qui quasi totidem capsulae apparent, tricocca, trilocularis, nuce una solitaria in quoque loculo. ${ }^{*}$ ) - (Arbor;

folia

*) PERsoovir in enchiridio p. 474. haec dicta repetimus, "Spe, cies tantummodo generis BURSERAE videtur, a q(u) so„!.um.7:odo numero nartium fructificationis differt. Et nce [IS] 
folia pinnata, foliolis oppositis; flores in racemis brovibus terminalibus aut axillaribus).

IIO2. BURSERA, ( BURSÈrE, Gomart.) JACR. IINx. JUss. LAMr. ill. tab. 25\%. (térebintacea 2.). Polygama. Flores hermaphraditi: calyx plerumque triidus, rarius quadri-quinquefidus, parvas, caducus; corollae petala tria, interdum quatuor vel quinque, pa. tentia, ovata; stamina sex, quinque aut octo, aut decem sw., corolla breviora; stylus unus brevissimus; stigma capitatum, rarius trifidum; bacca capsulari carnosa, drupacea, coriacea, subtrigona, trilocularis, trivalvis, continens nucem solitariam unam aut quinque membrana carnosa cinctam. Fiores masculi: calyce corolla hermaphroditorum; stamina sex, interdum octo vel decem, corolla multo longiora; ovarium abortiens. *) (Arbores odoratissimae; folia alterna, ternata, saepissime pinnata cum impari, foliolis duplici vel triplici serio; flores in racemis axillaribus aut terminalibus).

IIO3. GOUAREA, (GOUARE) IrNN. JUSS. IAM. illustr. tab. 301. MELIA JACQ. (meliacea 2.). - Calyx parvus, quadridentatus : corollue petala quatuor; stamina octo, filamentis in tubum longum cylindricum integrum coalitis, versus summum facie interiori antheriferis; stigma capitatum; capsula subglobosa, quadrisul. ca, quadrilocularis, quadrivalvis; sporae quatuor arillo cinctae. - (Arbor; foliis oppositis, impari pinnatis; flores axillares, in racemis paniculatis).

II04. CARAPA, (CARAPA) AUBI. Guy. tab. 397. IAM. illustr. tab. 301. PERSUONIA wILID. - Calux quadripartitus; corolla tetrapetala; stamina in tubum cylindricum, octodentatun! coalita; sty-lus unus; capsula unilocularis, quadrivalvis, polyspora; nuces difformes, angulatae.

„,men HEDIVIGIA inter muscos conservandum, ob tiri ,immortalis merita in illustianda huc harum plantarum ",farnitia. "s

*) PEPSONio angurari licuit cum hoc genere genus antecedens esse connectendun, vid, enchir, add. P. 524 . 
xIO5. XYLOCARPUS, (XYLOCARPE) KOEN. schrer. - Calyx quadridentatus; corolla tetrapetala; stamina connata in tubum ovali - inflatum, octofidum (nectarolyma), antheriferum; drupa sCHREB. (bacca) globosa, magna, exsucca, quadri-' quinquestriata, includens nuces octo - decem angulosas. - (Arbor; folia opposita integerrima; flores in racemis).

1 106. MI I HAUXIA, (MICHAUXIE) L'HERET. VENT. LAM. illustr. tab. 295. MINDIUM ADANS. JUSs. CAMPANULA gron. (campanulea 1.). - Calyx octopartitus, laciniis deflexis, persistens; corolla campanulata, rotata, laciniis octo vel decem linearibus ad summum revolutis; nectarolyma octovalve, staminiferum; filamenta brevia, dilatata; antherae rectae, Iineares; stylus cylindricus; stigma octofidum, infundibuliforme; capsula turbinata, angulosa, octo - decemlocularis, poIJøpora, laciniis calycinis coronata.

I107. PORTESJA, (PORTESIA) JUss. (moliacea 2.). - Calyx parvus, quadridentatus; corollae petala quatuor conniventia; stuminum octo filamenta coalita in tubum, apice octofidum, laciniis interna pagina antheriferis; stigma capitatum; capsula villosa, coriacea, bilocularis, bivalvis; loculi dispori; spora una et loculo uno abortiente. - (Frutices foliis impari pinnatis; flores in racenis axillaribus). *).

I IOS. CHLO RA, (CHLORE) LXNN. JUSS. IAM. ill. tab. 296. GENTIANA IINN. HALL. CENTAURIUMI ToUnN. (gentianea $I_{0}$ ) - - Calyx octo - decempartitus ; corolla monopetala, hypocrateriformis, tubo brevi, limbo octofido; stamina brevissima fauci corollaeinserta ; stigma quadrifidum; capsula ovato-oblonga, unilocularis, bivalvis, polyspora. - (Flores terminales).

II09. OXYCOCCOS, (OXYCOCCUS) mICHAUX WILL円. SCHOLLERA TIOTH. VACCINIUM rINN. -

Calyx

-) Uoc genus coniungendum esse cum rü TRICHLLAE РДRsoun euch, p. 463. arbitratus est. 
Calux superus, quadrifidus; corolla quadripartita, laciniis sublinearibus, revolutis; filamenta conniventia; antherae tubulosae, bipartitae; bacca polyspora.

IIID. JEF FERSONIA, (JEFFERSONIE) BARTON. MICHAUX. PODOPHYLLUM IINN. (ranunculea vel incertae sedis). - Calyx pentaphyllus, rarius tri quadrifidus, coloratus, deciduus; corollae octo petala conniventia, campanulata, incurvo patentia; stamina ovarium ambientia; filamenta brevissima; stylus brevis; stigma peltatum: cupsula oborata, substipitata, unilocularis, infra apicem dehiscens; sporae plures, oblongae, ad basin arillatae.

III. M E N Z IE SIA, (MENZIÈSIE) sMITH. fasc. 3. t. 56. JUSS. LAM. tab. 285. ANDROMEDA et ERICA IINN. WILID. ( hodoracea 2.). - Calyx monophyllus, fasciculatus ; corolla monopetala, globosa, ovata, quadrifida ; stamina recoptaculo inserta ; siylus quadrangulus ; stigma obtusum, quadrilobum; capsula supera, recta, externe quadrangula, quadrisulca, quadrilocularis, dissepimenta e marginibus sulcato inflexis valvularum quatuor; sporcie numerosae oblongae.

III2. ERICA, ( BRUYERE) TOURN. IINN. JUSS. IAM. tab. 23\%. (erica 1.). - Calyx tetraphyllus, interdum duplex, persistens; corolla campanulata, persistens, saepe ventricosa, rarius ovalis aut cylindrica, quadrifida, marcescens; stumina exserta aut corollae inclusa; filumenta teceptaculo inserta; antherae simpliciter emarginatae aut bifidae; stigma tetragonum, interdum quadrifidum; capsula membranacea, quadri-octolocularis, quadrivalvis, calyce cincta: dissepimenta e valvalarum margine spurae iu singulis loculis plures. - (Suffrutices foliis minimis, oppositis aut verticillatis aut dissitis sparsisve; flores axillares aut terminales, ut plurimum in racemos dispositi).

III3. BORONIA, (BORONIA) sMith. ap. RоEM: III. ANDREW. VENT. hort. malm. t. 38. (rutacea 2.). Calyx persistens, aequaliter profunde quadripartitus, lacinis 
ciniis punctatis; corollae petala quatuor aequalia cum calyce alterna et longiora, sessilia, concava, persistentia; nectarolyma anuulus glandulosus, basin ovarii cingens; stamina octo, persistentia, corolla breviora, quatuor caeteris sublongiora; filamenta roceptaculo inserta. ovarium cingentia, complanata, decrescentia, ciliata, varie terminata; antherae biloculares, brevibus pedicellis incumbentes, infra apicem lateri inferiori filamentorum insertis, lateraliter dehiscentes. Pistillum: ovarium superum, nectarolymati impositum, conicum, ovali rotundatum. quadrisulcatum: styius rectus, brevissimus, ex apice ovarii, vel quatuor approximati; stigma capitato globosum, obtusum, glabrum, quadrisulcatum; capsulae quatuor, coalitcle initio, mox discedentes, compressae, unilaterales, bivalves, continentes arillum bivalvem, elasticum; sporae una aut duae in quavis capsula, compressae, gla. brae, nitidae.

* Ii 4. CORRAEA, (CORRAEE) suriti. transact. IIII. (rhodoracea). - Calyx monophyllus; corollue petala quatuor conniventia; "antherae incumbentes, biloculares, longitudinaliter dehiscentes; capsula supera, quadrivalvis, quadrilocularis e valvulis inflexis; stigma simplex acutum. *)

II 5. MAGALLANA, (MAGALLANE) CAVAN. tab. 374. - Calyx monophyllus, tripartitus, lacinia infima tripartita, postea calcarata; corollae petala quinque, irregulariter inaequalia; triá superiora pedicollata, basi cucullata; ovarium didymum; stigma bificum; fila. menta basi breviter coalita; fructus: samarae duae, trialatae, uniloculares, monosporae (per abortum P.); spora oblonga.

II 6. $\triangle \mathrm{CER}$, (ÉRABLE) TOURN. IINA JUSS. IAM. ill. tab. 844. (aceracea 2.). - Polygama. Flores hermaphroditi: calyx coloratus, saepe quinque-rarius quadrifidus,

7 LEIARIA (BEFARIA IINN, ) a CORRAEA differt partium aumero, pracipue antheris apice biporosis, stigmate in. crassato scptemfido. 
fidus, interdum novemfidus; coroliae petala quinque (interdum quatuor ad novem) alternantia cum laciniis calycis et ipsi saepe concolara; stamina octo (interdum quinque ad duodecim); stylus unus simplex aut interdum bipartitus; stigmata duo reflexa; samarae (CAERTN.) duae, tres, apice ala membranacea munitae, basi coalitae, uniloculares, mono-disporae; embryo curvus; vaticula pendula super lobos asperos in Acer tataricum vel plicatos in A. campestre, vel replicatos super sese invicem in A. rubro et pseudo - platano. Flores masculi: calyce, petalis, staninibus hermaphroditorum; ovarium etstylus nullus; stigma bifidum. = interdum flores dioici. (Arbores indigenae aut exoticae; folia saepe lobata, rarius pinnata cum impari aut ternata; petiolo dilatato basi et semiamplexicauli; flores axillares et terminales, dispositi in racemos aut fasciculos corymbiformes; pe. dunculi ad basin bracteati).

\section{Flore incompleto.}

II17. OPHIRA, (OPHIRE) BUKM. IINN. JUSS. IAM. illustr. tab. 293. (onagrea 4.). - Involucrum bivalve, triflorum persistens; corolla supera, tetrapetala, petalis conniventibus; antherae ovales; stigma emarginatum; bacca unilocularis, dispora. - (Frutex gongylosus: folia opposita, lineari lancoolata, parum acuminata; flores sessiles, laterales involucrati).

III8. GRUBBIA, (GRUBBIE) BERG. cap. tab. 2 . EMPETRUM JUSs. (erica 3.). - Involucrum (calyx) diphyllum foliolis ovalibus, bivalve, bi-triflorum; corolla infera petalis quatuor rotundatis, externe lanuginosis; filamenta subulata; antherae rotundatae; stigma simplex; bacca .... ? - (Frutex foliis linearibus extrorsum revolutis; flores axillares glomerati).

\section{IIID. BUGIN VILLEA, (BOUGAINVILLE)} commers. juss, IAMr. illustr. tab.294. (nyctaginea IC.). - Involucrum triphyllum foliis ovali rotundatis, triflo. rum; calyx involucro brevior, inferus, tubulosus, basi levi. 
leviter ventricosus, ad medium leviter coarctatus, limbo fere integro vel subquadridentato; stamina inserta disco squamarum ovarium cingentium, reseptaculo insidentia; fructus....? spora per calycis basin tecta, et cincta involucro persistente. - (Flos tubulosus, bracteae maiusculae, sive involucro insidens. Elongatio spinis axillaribus notata; folia alterna; pedunculi axillares, duobus vel tribus involucris onustati).

I $20 \mathrm{NECA}$, (NECA) RUIz-PAVON. JUss. (nyctagynea 2.). - Involucrum di - triphyllum foliolis parvis, squamaeformibus, uniflorum; culyx tubulosus, quinquedentatus; stamina octo, quatuor alternis longioribus; spora ovoïdea, tecta basi calycis inflati et carnosi, drupacei, forma et magnitudine olivae, limbo persistente coronata. - (Frutices foliis verticillatis aut oppositis).

II21. SCO POLIA, (SCOPOLIA) EINN. suppl. Juss. (sedis incertae). - Flores in involucro communi. diphyllo foliolis concavis; calyx proprius inferus. infundibuliformis, quinquefidus; antherae octo oblongae duas series constituentes, in quáque serie quatuor; coalitae aut conniventes in unum ordinem lineamve interruptam, applicatae super quatuor filamenta epigyna; ovarium superum, superatum pilis flamentis mixtis; stylus nullus; stigma unum; fructus ... .? - (Planta acaulis foliis coniugatis, pedunculis e trunco subterraneo crescentibus unifloris).

I I22. L A CH NAEA, (LACHNÉE) IINN. JUSS. LATr. tab. 292. GNIDIA LINN. (daphnacea). - Flores capitato glomerati: calyx (corolla L.) infundibuliformiș, lon gus, tenuis, limbo inaequali:er quadrifido, tubo basi inflato; stamina fere exserta; filamenta situ inaequalia; stylus filiformis, lateralis; stigma capitatum, villosum; nux subdrupacea pers. (spora una, fere baccaeformis $\mathrm{N}_{\text {. }}$ ) calyce tecta, - (Flores aggregati et terminales; frutex).

1123. DIR CA, (DIRCA) IINN. JUss. xAM. illustr. tab. 293. (daphnacea). - Calyx nullus; corolla (calyx Juss.) tubulosis, turbinatus, margine inaequali, co- 
loratus, limbo obsoleto; stamina tubo longiora, inaequalia; bacca drupacea, monospora. - (Frutices; flores axillares, excedentes o gemma terni).

I124. D A PHNE, (DAPHNÉ, Lauréole) IINN. JUss. IAM. illastr. tab. 290. THYMELAEA tourN. scop. (daphnacea). - Calyx nullus; corolla (calyx juss.) inferus, coloratus, infundibuliformis, marcescens, quadrifidus (corollaceus); stamina non exserta, sed inclusa; stylus brevis; stigma capitatum; drupa globosa, nucem monosporam continens. - (Flores axillares aut terminales, solitarii aut glomerati in fruticibus formae selectae. Gemmae in D. mezereuni tri-quadri-quinqueflorae).

II25. LA GET TA, (LAGET, Lagetto) JUSs. IAM. illustr. tab. 289. bois-dentelle incolis st. Domingo. (daphnacea). - Caly $x$ Juss. (corolla P.) superus, coriaceus, tubulosus, ad faucem attenuatus et munitus glandulis quatuor, caducus, limbo quadridentato vel quadrifido; glandulae quatuor petaliformes calyci insertae; stamina sessilia; drupa (nux) globosa, pilosa, pisiformis, monospora, non dehiscens, infera, calyce persistente ad basin sese horizontaliter aperiente tecta. IAM. encycl. pag. 376.*). - (Arbor cuius corticis interna pars finissi.* mum praebet reticulum; flores spicati terminales, spica geniculata, in geniculo quoque uniflora.

1I26. PASSERINA, (PASSERINE) IINN. JUSS: IArr. illustr. tab. 291. (daphnacea). - Calyx nullus; corolla (calyx juss.) tubulosa, medio ventricosa, limbo quadrifido, laciniis patentibus, nuda; stamina ad summum tubo imposita; stylus lateralis, filiformis, longus; stigma capitatum, villosum; nux una, calyce tecta. (Flores axillares aut terminales; folia opposita in quibusdam speciebus; frutices habitu Daphne similes).

II27. STELA

*) Genere et familia a Daphne distinctum; est enim e familia Elacagni. 
I12\% ' S TELLER A , (STELLÈrE). IINN. JUSS. IAM. illustr, tab. 293. ALSINE TOURN. (daphnacea). Calyx nullus; corolla (calyx juss.) infundibuliformis, elongata, filiformis, limbo quadri-quinquefido; medio ventricosa; stamina brevissima, quatuor inserta ad summum tubi, quatuor ad tubi medium; stylus brevis; stigma capitatum; nux una, rostro terminata, caljce tecta.

II29. DODONEA, (DODONEE) IINN. JUSS: IA îr. illustr. tab. 304. (terebintacea 5.) - Calyjx quadripartitus (tetraphyllus $P_{\text {. }}$ ) interdum tri-quinquefidus, caducus; corolla nulla; filamenta brevissima; antherae oblongae, fere sessiles; stigma leviter trifidum; capsula triquetra, trialata, trilocularis, loculis disporie, trivalvis, valvulis compressis, dorso ala membranacea munitis; sporae rotundatae, nigrescentes; (Frutices foliis simplicibus, alternis; flores dispositi in racemum terminalem et axillarem; interdum stamina septem).

I129. VALEN TINA, (VALENTINE) PIUK, PLUM. STARz. - Calyx inferus, coloratus, quinquepartitus, patens; corolla nulla; capsula baccata, interne pulposa, tri-quadrivalvis, tri-tetraspora.

1130. A N I B A, (ANIBE) AUBL. JUSS. 'IAMr. tab. 298: CEDROTA SCHREB, WILLD. (ex ordine plantarum sedis insertae). - Calyx sexpartitus laciniis profundis, obtusis, concavis; corolla nulla; stamina super ovarium posita; ovarium superum, stylo brevi superatum, glandula cinctum; fructus. ..... ? Secundum RICHARDI observata species est Lauri; quae si veritati et naturae respondent, fructus erit bacca spora una repleta; - (Arbor ligno citrino aromatico; foliis oppositis aut verticillatis; floribus minimis, axillaribus in paniculis maxime laxis, pedunculo longo tenui.) 


\section{$\begin{array}{llllllll}D & I & G & Y & N & I & A\end{array}$}

II3T. CODIA, (CODIE) FORST. IIN. suppI, JUsS. IAxr, illustr tab. 3 /4. (ex ord. plantt, sedis incertae) Flures is:capitalum globose congesti super receptaculum commune barbato villosuen, giobrisum, involucro tetra. phyllo cinctum; calyx tetraphyllus : corollae petala quatuor linearia, petalo queque ad basin munito duabus antheris; ovarium superuru, parvum; styii úuo; totidemque stigmata; fructus.....? (sporao quatuor?)

132. WE I N MI A N I A, (IVEINMANNE, Tan- rouge) IINN. JUSS. IAM. ill, tab. 313. WINDAIANNIA Brown. jam. tab 212. (saxifrageis affinis; - Calyx quadripartitus (tetraphyllus P.); corollae petala quatuor maiora; stamina brevia: ovarium superum, inferne ad ba. sin cinctum disco octo glandularum; capsula ovalis, birostris, rostris subulatis, bilocularis, hexa-octospora; valvularum membrana introitus dissepimentum efformat; - (Arbores aut frutices, foliis oppositis; flores in racemis axillaribus).

II33. MOEHRINGIA, (MERINGINE) IINN. 3Uss. IAM. illustr. tab. 314. ALSINE TOURN, HAIL. scHeucrz. (caryophyllea 3.) - Calyx tetraphyllus vel quadripartitus; corolla tetrapetala ; capsula unilocularis, quadrivalvis, polyspora; - (Flores fere solitarii axil. lares aut terminales, sustentati pedunculis longissimis).

II37 SCH M I E D E I A, (SCHMIEDÉLE) I IX A. JUSS. LAM tab. 312. USUBIS BURM. ALLOPHYLLUS SWARz RHUS SIOAx. - (saponacea 2.) - Calyx diphyllus, coloratus; corollie petala quatuor, calyce multo minora; antherae rotundatae; ovarium didymum, pedicellatum ; fructus duo, monospori ; .... (Frutex foliis ternatis) *)

*) ap. Pens, ench. p. 4r2, ad monogynias pentandriae relatim est hoc genus, et militat sub ORNITROPLE, mo, 4. 5. 6. 
I135. GALENIA, (GALIÈNE) IINN. JUSS. LAM: illustr. tab. 314. (atriplicea 2.) - Calyx minimus, quadrifidus; corolla nulla; capsula rotundata, bilocularis, dispora; - (Frutex ramis viscidis; folia linearia, recta; flores in panicula terminali). *')

\section{$T R I G Y N I A$.}

I136. SERIANA, (SERIANE) SCHUMACHER act. Hafniens. WILID. PAULLINIA LINN. JACQ. WILID. (saponacea r.) - Calyx tetra-pentaphyllus ; corolla tetrapetala: nectarolyma quatuor corollae appendices tetraphyllas et glandulae quatuor ad basin petalorum po. sitae; Samarae tres, globosae, longitudinaliter connatae, inferne in alam membranaceam dilatatae, monosporae.

I 37. PAULLINIA, (PAULLINE) IINN, JUSs. LAM. illustr. tab. 318. (saponacea I.) - Calyx tetra. pentaphyllus; corollae petala quatuor basi glandulosa; nectarolyma quatuor corollae appendices foliolis quatuor inaequalibus, et glandulae quatuor ad petalorum basin; capsula pisiformis, turbinata, interdum alis tribus exsertis trigona, trilocularis, loculis monosporis, trivalvis dissepimenta in valvularum suturis (seriania) aut opposita valvulis (cururu); receptaculum centrale; sporae fere ovales, ad dimidium arillatae; - (Fruticos sarmentosae; folia uno-bi-tri-ternata, aut pinnata cum impari, aut supradecornposita; pedunculi solitarii, axillàres, ad medium duobus filis cyrrhisve multifloris muniti; flores racemosi).

* I 3 38. SE M A R ILl a R I a . (semarillaria) RUiz. CURURU joum. gén. plant. p. 34. tab. 35. Calyx tetraphyllus; corolla tetrapetala; capsula unilocu- laris,

*) Ad hunc locum pertinere videtur genus TATAlBSIS ME. Dsc, vel Gypsophylla arburea. 
laris, trispora; sporce arillatae; receptaculum centrale, trigonum. *)

II39. CARDIOSPERMU M. (CARDIOSPERME ) IINN. IAM. tab 317. CORINDUM ToURN. **) (saponacea .). - Calyx tetraphyllus, foliolis duobus maioribus; corolla tetrapetala . nectarolyma appendices foliolis quatuor inaequalibus ad basin petalorum; capsulae tres (vel capsula una triloba, trilocularis et quasi triplex) connatae, inflatae, membransceae, monosporae; sporae globosae, ad umbilicum notatae cicatrice cordata, - (Elongatio trunci herbacea foliis biternatis; pedunculis solitariis axillaribus).

II40. TOUL I I A, (TOULICI) AUBL. JUUS. IAM. illustr. tab. 317. PONAEA schreB. Willd). (saponacea 2.) - Calyx quinquepartitus; corollae petala quatuor, ad sunumum cinctis tribus vel quatuor glandulis pilosis; filamenta alterna breviora; ovarium triangulum, super discum staminiferum positum; styli breves; capsula trialata; alis membranaceis bivalvibus; trilocularis, loculis monosparis; (Arbor foliis aequaliter pinnatis, floribus in spicis paniculatis axillaribus aut terminalibus),

I 14I. CORNIDIA, (CORNIDIE) RUIz. PAv, syst. flor. peruv. tab. 35. Juss. mss. (Saxifrageis affinis). Calyx campanulatus, cbtuse trigonus, semisuperus, ovario adhaerens, margine integer; corollae potala quatuor caly-

*) Differre a PAULLINIA IINN. fructus tantum partibus dicitur, caeteris cum illa convenire.

**) Disiunxit medic. monad. p. ro. genus CORINDUIM TounN. 246. calyce tetraphyllo, foliolis duobus maioribus, duobus minoribus; corollae petalis octo basi per paria connatis; exterioribus duobus dilatato patentibus, quatuor internis erectis, supra conniventibus, tubum fingentibus; staminibus basi in tubum brevem coalitis, tubi margine filamentis octo distinctis et ercctis, posterioribus longioribus notato; stylis tribus; capsula triloculari, inflata, triangula; loculis ronosporis; sporis cicatrice obcordata notatis. 
calycis margini affixa; ovarium trifidum; styli divergentes et persistentes; stigmata acuminata ; capsula tricorniculata, trifida, trilocularis, trivalvis, polyspora; interdum partium numerus uno augmentatus.

II42. SAPINDUS, (SAVONNIER) TOURN. IINN. JUSs. IAM. illustr. tab. $30 \%$ (Saponacea x.) - Caiyx coloratus, tetraphyllus, foliolis duobus exterioribus: corolla tetrapetala; nectarolyma appendices quatuor corollae et ad basin petalorum glandula quatuor; capsulce tres connatae, carnosae, globosae, ventricosae; duao abortientes, monosporae; sporae sphaericae; - (Arbores foliis pinnatis aut ternatis, floribus dispositis in paniculas terminales).

I 43. BRUNN ICHIA, (BRUNICHE) BANXS. POLYGONUM claviculatum GAERTN. - SCHREB. (́polygonea). - Calyx monophyllus, coriaceus, angulatus, apice angustato elongatus in pedunculum planum, arcuatum, basi attenuatum, ventricosus, quinquefidus, laciniis revolutis; corolla nulla; stamina octo vel docem; stigmata bifida; capsula supera, unilocularis, trigona, monospora; spora inversa, sexlobata.

1 144. C O C C O L O B A, (RAISINIER) IINN. 'Juss. IAM. illustr. tab. 316. (polygonea). - Calyx coloratus, quinquepartitus, demum baccatus; corolla nulla bacca (nux) calycina h. e. calyce succulento facto tecta, unilocularis: spora una; - (Frutex elongatione recta; foliis alternis, orbiculatis, parum cordatis, stipulis vaginantibus ad basin munitis; flores in racemis axillaribus.)

I145. POLYGON UM, (PERSICAIRE, Renouvée) TOURN. LINN. JUSS. LAM. illustr. tab. 315. FAGOPYRUM, BISTORTA, PERSICARIA toURn. (polygonea). - Calyx coloratus, quinquepartitus; corolla nullis. - (calyx nillus; corolla quinquepartita, calycina PERs.) - ; stamina quinque ad novem: styli duo vel tres; totidem stigmata; spora una, ovalis aut triangula. 
ris, certe angulata, calyce tecta. (Stamina et styli nu. mero incerto).

$$
T E T R A \text { G } Y N I A \text {. }
$$

I 46. КАLA NCHOE, ( DECAND. COTYLEDON LINN, VEREA WILLD. CRASSUVIA commers. LAM. (crassulea); Calyx quadripartitus; (tetraphyllus P.) corolla liypocrateriformis, quadripartita, laciniis scutis; ovaria quatuor, unumquodque basi squama nectarifera lineari cinctum; capsulae supcrae quatuor, uniloculares, interne quadrivalves, polysporae. *)

1147. A D OXA , (ADOXE, MOSCATELINE) IIN. JUss. LAM. illustr. tab. 320. MOSCATELLINA TOURN. (saxifragea 2.) - Calyx bi-trifidus, inferus, squamaeformis; corolla (calyx juss.) quadri-quinque-fida, supera, munita interne ad basin duabus vel quatuor squamis persistentibus; antherae rotundatae; ovarium inferum quatuor vel quinque stylis persistentibus terminatum; bacca globosa,quadri-quinque-locularis (matura unilocularis,) tetra-pentaspora, (loculis monosporis) calyce coalita; embryo rectus; radicula supera; (Flores laterales ennea-decandri.) **)

* Ir48. VEREA, (VEREA) ANDrews, wrird. Calyx tetraphyllus; corolla hypocrateriformis, quadrifida; tubus ventricosus; ovaria quatuor basi quatuor squamis munita; capsulae quatuor, superae, uniloculares, polyspörae.

II49. CERCODIA, (CERCODÉE) SOIAND juss. IAM. illustr. tab. 319. IIdLORAGIS rorst. tab. 3r. I'HERET, JACQ. PERS. 'TETKAGUNIA IINN. SUPl. SW.

(ona-

*) PER500xius ench. 445. ad octandriae tetragynias retulit.

**) PERs, ench. 1. 1, ad tetragynias retulit, 
(onagraria 1.) - Calyx urceolatus, tetragonus, quadridentatus dentibus rectis (vel tetraphyllus i, superus, persistens; corollae petala quatuor linearia, dentibus calycis interposita, caduca; antherae oblongae, striato-sulcatae, binae petalis oppositae; ovarium inferum; drupa sicca (capsula dura), tetragona angulis parum proeminentibus, limbo calycis coronata, continene nucem unam quadrilocularem, tetrasporam; - (Suffrutices floribus axillaribus.

1150. ELATINE, (ELATINE) IINN. IUSS. IAN. illustr. tab. 320. schкUHк. ALSINASTRUMI vaILL. (caryophyllea 3.) - Calyx tetraphyllus; corollce petala quatuor sessilia; ovarium orbiculare, depressum ; capsula orbicularis depressa, quadrilocularis, quadrivalvis, polyspora, dissepimenta suturis opposita; - (Plantae aquaticae, diffusae, minimae, foliis vérticellatis aut op. positis).

II I. PARIS, (PARISETTE) IINN. JUSS. IAII, ill, tab 319. HERBA PARIS TOURN. (smilacea 1.; Caly $x$ patens tetraphyllus; corollae petala quatuor alter. na, angustiora, magis erecta; antherue filamentorum me. dio adnexae; bacca globosa, quadrilucularis, loculis olygosporis; - (Elongatio ad modium quatuor folis verticillatis munita, ad summum uniflora; interdum pars quinta adest, interdum quarta pars deficit).

1552. GALVEZIA, (GALVĖZE) RU1Z. PAVON. Culyx quadriparitus (tetraphyllus?) Corollue petala quatu* or sessilia: stuminainaequalra, quatuoralternantia breviora; corpus glandulosum sub ovario; drupue quatuor. ${ }^{*}$ )

I153. POR.

4

*) $\mathrm{Ab}$ hoc genere difierre videtur GALvEZIA (GAlveze) DOSBEY. et J's: calyx quinquefilus parvus; curoila le. viter tubulosa, leviter al basin ventricosa, limbs bilabiato. labio superiori bilobo, inferiori tripartio ; stamina intra tusum (quatuor?); capsula tubulosa ; - (Frutcx ramosus, foliis alternis; floribus solituriis axilla. ribus). 
II53. PORLIERA, (PORLIERIE -, TURUCA. SA) RUiz. et PAvox. - Calyx tetraphyllus aequalis; corollae petala' quatuor obovata, conniventia, unguiculata; squamae octo nectariferae; stamina aequa-

2 lia, dorso nectarolymatis inserta; drupae quatuor connatae. ${ }^{*}$ )

II54. FRA NCOA, (FRANCOA) cavan. - Calyx quadripartitus, persistens; corolla tetrapetala; stylus nullus; stigmata plaza; capsulae quatuor, basi connatae, carinatao; sporae numerosae, suturis carinarum adfixao (a genere PANKE distinctum cf. PERs. encil. p.415.)

I155. POLYSCIAS, (POLYSCIAS) FOPST. austr. t. 32. Juss. (araliacea). - Calyx quinque ad octo den. tibus parvis munitus; corollae petala numero octo, rarius quinque ad septem, patentia; stamina octo, rarius quinque vel septem; antherae quadristriatae; stylus nul. lus; stigmata saepissime quatuor; rarius tria aut quinque; bacca globosa, calyce et stigmatibus coronata, saepe quadrilocularis; sporae triangulae. (Folia alata; umbella magna, verticillata).

*) GUALACO affine genus; ab anteccdente GALVEZIA ge. nere calyce tetraphyllo, nectarolymatis squami, octo, pe• talis obovatis unguiculatis, staminibus aequalibus, drupis conmexi, differre auctores gravissimi iubent. 


\section{$C L A S S I S, N O N A$}

\section{E N NEA NDRIA.}

\section{$\begin{array}{lllllllll}I M & O & N & O & G & Y & N & I & A_{0}\end{array}$}

II56. LAURUS, (LAURIER) TOURN. IINN. JUSS. IAM. illustr, tab 32t. (laurinea). - Calyx quadri-soxpartitus aut fissus, laciniis aequalibus ; caducus aut persistens: curolla nulla ; staminum filamenta duodecim, calyci multo breviora, duplici serie concentrica posita, sex exteriora fertilia, sex interiora, quorum tria fertilia, basi glandulis duabus bisetis, ovarium cingentibus sunt munita (nectarolyma), tria cum fertilibus alterna sterilia; stigma capitatum; drupa monospora, olivaeformis, supora ; nux unilocularis, monospora. 'Staminum numerus varius secundum IINN. tria, quinque, sex, septem, octo, quatuordecim; quo fieri potest quod species exotica accuratius observatae nova genera constituant. Folia alterna, ut plurimum integra; flores axillares interdum terminales, solitarii aut per fasciculos approximati, interdum dispositi in paniculam.)

* II57. PANKE, (PANKE) Morin. Willd. - Ca. lyx quadrifidus; corolla campanulata, quadrifida; capa sula bivalvis, monospora. ")

II $58 . \mathrm{ANA}$.

*) y $\mathrm{xsooN}$ enchir. pag. 30. in Diandriae digyniis sub GUNNERA militare iussit. ! conferri potest idem auctor libro laudato pag, 445, sub FRANCOA. 
II58. ANACARDIUM, ( ANACARDE) OIT. IINN. JACQ. CASSUVIUM (ACAIOU) IAM. tab. 322. SEMECARPUS IINN. sUppl. ACAIUBA GAERTN. (terebintacea). - Caly $x$ inferus, quinquepartitus ; corollae petala quinque, calyce maiora, reflexa; staminum filamenta decem, novem breviora antherifera, antheris rotundatis, docimo longiori, anthera caduca oblonga castrato; nux reniformis, monospora, inserta super receptaculum pyriforme, magnum, carnosum, sive pedunculum ampliatum incrassatum *); spora nuci conformis; - (Arbor resinosa, mediae altitudinis; folia alterna magna, simplicis, munita fasciculo ductulorum longitudinali transverse ramificante; flores parvi, albi, in panicula terminali, bracteati ; interdum masculi por ovarii abortum; fructus oleosus interne resinosus, affixus super pedunculum crassum, spongiosum, nominatur Pomme d'Acayou). "**)

I159 PLE GORHIZ A , (PLÉGORHIZE, Guä̈curu) Mox. Juss. Willd. (ex ordine plantt. sedis incertao). - Calyx nullus; corolla mox. (calyx juss.) monopetala, infera, limbo integro; staminum filamenta bre$\nabla$ ia; antherae oblongae: capsula oblonga, leviter com. pressa, unilocularis, monospora: sporae capsulae conformes : (Frutex foliis radicalibus, potiolatis, confertis ; olongationum alternis, sessilibus; flores parvi, fero um. bellati).

1160. CAS.

- Est planta polygama dioica; filamentum longius in apertis foribus sterile, in clausis autem antheram gerit magnam, torosam. Rоттв.-

**) Haec sul CASSUBIo juss. zarr. cum generis definitione, extant: sed ANACARDII characterem essentialem LAM. ill. t. 208. encycl. et Juss hune alias proposuerunt: calyx campanulatus, quinquefidus; corollae petala quinque magna; stamina quinque; antherae oblongae; styli tres; stigmata tria; $n u x$ compressa, cordiformis, basi multo latior, sustentata pedunculo incrassato, cupulaeformis aut turbinata; - (Arbores; folia simplicia, flores fructusque CASSUVII), IAUIME, II. Y. 849. 
1160. CASSY THA, (CASSYTHE, Cassuta) LINN. JUSS. LAM. illustr. tab. 323. CALODIUM LOUREIR. VOLUTELLA ronsK. (ex ordine plantt. sedis incertae.)Caly $x$ inferus, monophyllus, globosus, limbo attenuato ot sexfido, vol partito, laciniis conniventibus, tribus interioribus altecnis, petalö̈deis (petala LINN.) parum maioribus; ad basin bracteae tres parvae; corolla nulla; (calyx nullus : corolla calycina, sexpartita PERs.); staminum duodecim filamenta duplici ordine ad summum calycis disposita, quorum sex externe collocata antherifera laciniis calycinis opposita sunt; alia sex extra illa collocata habent tria fertilia antherifera, ad basin duabus glandulis minutis munita, tria reliqua sterilia, truncata, tuberculiformia (nectarolyma $L I N N$.); antherae filamentorum fertilium antrorsum affixae longitudine filamentorum; ovarium superum, cinctum (tribus tuberculis glandulosis truncatis) siaminibus sterilibus JUss.; stylus incrassatus ; stigma obtusum ; capsula drupacea, globosa, monospora, calyce grossificato tecta, baccae - vel drupaeformis.

I 6r. ERIOGONU M, (ERIOGONE) MrchaUX f. amer. bor. I. p. 246. tab. 24. PERs. (polygoneis affinis). - Calyx subcampanulatus, sexpartitus, laciniis tribus interioribus minoribus; corolla nulla; antherae ovalos; ovarium triangulum; stylus brevis; stigmata tria; spora una, triquetra, calyce tecta; - (Flores multi podicellati e communi involucro campanulato prodeuntes ; elongatio herbacea, dichotoma; folia ternata, verticillata, sessilia).

I162. O C O TEA, (OCOTEE). AUв. Guy. t. 3 ro. juss. (laurinea). - Calyx sexpartitus, laciniae tres interiores alternae et minores i staminum filamenta novem, membranacea, lata, ad summum truncata; sex exteriora laciniis calycinis opposita, tria interiora basi biglandulosa; antherae quatuor insertae interne singulo filamento, !o basi ad summum dehiscentes, duas inferiores, duae superiores; stigma concavum; drupa basi calyce pervistente et urceolato cincta, ovalis, monospora; (Ar- 
bor: folia alterna, integerrima; flores in paniculis axillaribus et terminalibus).

\section{$T R I G \dot{Y} N I A$.}

II 3. R HEUM, (RHUBARBE) IINN. JUSS. IAM. ill. tab. 324. RHABARBARUM TOURN. (polygonea). Calyx nullus; corolla (calyx juss.) sexfida, persistens; styius nullus; stigmata tria ; spora uria, triquetra, laevis, margine mombranaceo; (Flores paniculati terminales).

I164. PLAEA, (PLAÉE) мichAUX amer. bor. tab. 25. PEns. - Corolla (calyx juss.) sexpartita, patentissima; ovarium triangulum; stigmata sessilia; capsula subrotundo-trigona; (tres coniunctae?) calyco porsistento cincta trilocularis, sopto nullo manifosto; sporae numero plures, oblongae, cylindricae, margini valvularum adnexae. Spica in spathis unifloris*); (Plantae iunciformes, elongationibus foliisque glabris, maxime orectis, acuminatis; flores in spica terminali).

\section{$\begin{array}{lllllllll}H & E & X & A & G & Y & N & I & A\end{array}$}

1165. BUTOMUS, (BUTOME, Ionc-fieuri) TOURN. IINN. JUSS. LAM. illustr. tab. 324. (junciner 3.) - Calyx nullus; corollae (calyx Juss.) petala sex, fero aequalia, tritus interioribus minoribus; capsulae, ut ovaria, sex oblongae, univalves, polysporae; sporae ad parietes capsularum; - (Flores umbellati; folia ut in gramineis).

*) An genus potius cum TOFNIELDIA coniungendum, a qua staminum numero solummodo differre videtur. RXRs. 


\section{CLASSIS DECIIMA.}

\section{DE C A D R I A.}

\section{$\begin{array}{lllllllll}M I & O & N & O & G & Y & N & I & A .\end{array}$}

I. Flore polypetalo, irregulari.

II66. SOPHORA, (SOPHORA) XINN.JUSS. IAM. illustr. tab 325. (leguminosa 4.) - Calyx monophyllus limbo quinquedentato, campanulatus, superne gib. bus; corolla papilionacea; vexillo, versus medium vix dilatato, magno sed alis multo breviori; carina dipetala; legumen moniliforme, articulatum, articulis distinctis et reunitis invicem processibus plus minus. olongatis, in flatum ad sporarum insertiones; - (Arboxes et fruticos floribus ut plurimum favescents colare; folia pinnata cum impari; foliola plerumque nu. merosa).

II67. VIR G ILIA . (VIRGILIA) XAM. tab. 326. SOPHORA IINN. PODALYRIA WILLD. BROUSSENETIA orteg. decad. |V. (leguminosa 4.) - Calyx quinquedentatus, gibbus; curolla papilionacea; vexillum alis longius; carina dipotala ; legumen non articulatum, com. pressum aut subteres, polysporum (Folia pinnata).

1168. POD ALYRIA, (PODALYRIA) LAM. illustr. tab. 32\%. WILID. SOHHORA IINN. JUss, PÁLI. CRO. 
TALARIA IIN tus, quinquefidus; corolla papilionacea; vexillum et alas eadem invicem longitudine, carina dipetala; légumen non articulatum, lacve, breve, inflato ventricosum aut planum, polysporum; - (Arbores aut frutices foliis pinnatis aut ternatis, rarissime simplicibus; floribus axillaribus aut terminalibus).

II69. PUL T E N A E A, (PULTìNE) sMrtr. nov. holl. 1. p. 35. t. 12.) wrLld. (leguminosa 4.) - Calyx quinquedentatus, utrinque appendiculatus ; corolla papilionacea, alis vexillo brevioribus; legumen uniloculare, disporum; (Flores capitati terminales; folialinearia).

II70. G O M P H L Ó B I U M, (GOMIHOI, O. B IE ) sмitr. trunsact. IV. (leguminosa 4.) - Calyx campanulatus, simplex, quinquepartitus; corolla papillionacea; stigma simplex, acutum; legumen ventricosam, uniloculare, polysporum.

II7I. DAV IE S A . ( DAVIESIA)/SMITH. transact. IV. SOPHORA sert. Hannover. PULTENAEA wILLn. venten. (leguminosa 4.) - Calyx angulatus, simplex aut nudus, quinquefidus; corolla papilionacea; stigma simplex, acutum ; legumen compressum, monosporum. *)

1172. A NAGYRIS, (ANAGYRE) TOURN. IINN. JUss. IAM. illustr. tab. 328. (leguminosa 4.) - Calyx urceolatus, quinquedentatus, persistens; corolla papilionacea; vexillum obcordatum, brevissimum; alae paulo vexillo longiores; carina dipetala, longitudine vexil. lum et alas superans : stamina distincta; legumen elonga: tum, compressum, parum incurvum, fere gibbum, polysporum: sporae reniformes, maturao caerulescentes. (Frutex foliis ternatis, foliis bractea amplexicauli opposita munitis; flores spicati axillares).

I173. CE R-

*) Hoc et praecedens genus ex auctoritate gravissimi sMrTHII post sOPHOPAIM penenda sunt. 
II73. CER C IS , (GAINIER) IINN. JUSS, IAMI. illustr, tab. 328. SILIQUASTRUM TCUR, (leguminosa 4.) - Calyx urcoolatus, quinquedentatus, basi igibbus; corolla papilionacea; petala unguiculata; vexillum alis brovius, et sub alis positum; carinae petala duo approximata, aequalia; stumina distincta, inaequalia; ovavium substipitatum; legumen oblongum, polysporum, compressum, gibbum per sporarum affixarum proëminentiam, marginatum ala membranacoa: - (Arbores mediae altitudinis; folia simplicia; inflorescentia ante foliationem; flores thyrsoïdei vel fasciculati per longitudinem elongationum, purpurascentes, interdum albi).

1174. B A UH I NIA, (BAUHINE) PIUM. IINN. sUSB. LAM. illustr tab. 329. (leguminosa 3.) - Calyx irregularis, apice quinquefidus, dehiscens longitudinaliter latere inferiori, fissuris quinque ad basin, caducus ; corollas petala quinque, patula, oblonga, unguiculata, undulata, subaequalia, superiori magis distante, calyci inserta; stamina distincta inaequalia, novem corolla breviora et interdum sterilia, decimo libero, caeteris multo longiori semper fertili; ovarium pedicellatum; legumen pedicellatum, elongatum, sublanceolatum, compressum, polysporum; sporae planae, reniformes aut ellipticae; - (Arbores vel fruices intordum prostratae; folia simplicia, biloba; flores in racemos axillares aut terminales dispositi).

1175. PAULETIA , (PAULETIA) CAVAN. (leguminosa 3 ) - Calyx laciniis quinque longissimis revolutis; corollae potala quinque angusta, undulata; stamina decom basi connexa, in corpus crassum connata: stigma ovatum; legumen lineare, bivalve, polysporum. ")

1176. P A L O VEA, (PALOVÉ) JUSS. IAM, t. 323. - Paloue aubr. Guy t. 141. GiNANNIA schreb. (leguminosa 3.) - Calyx duplex: exterior urcoolatus, bilo.

*) PRRs, ench. P. 455. cum BAUHINIA hoc genus coniungi suasit. 
bilobus: interior infundibuliformis, corizcous, quadriquinquelobus lobo altero maiori; ccrollae petala tria (saepe quinque, duobus caducis). nlterna cum laciniis calycinis; filamenta longa, fertilia; antherae oblongae, versatiles: ovarium sustentatum pedunculo altero latero alato; stylus longissimus; legumen longum polysporum:...*) (Frutex floribus spicatis torminalibus; foliis simplicibus).

II7. HYMENAEA, (COURBARIL) IINN. JUss. IAM. illustr. tab. 330 f. r. COURBARIL prUm. (leguminosa 3.) - Calyx turbinatus, coriaceus, limbo quinquepartito; obtuso; caducus; corollae petala quinque, subaequalia; filamenta distincta, ad medium incurva; antherae magnae, versatiles; stylus intortus; legumen magnum, lignosum, ovali oblongum, parum compressum, interne pulpa farinosa repletum, dilatatum, polysporum ; sporae ovoïdeae, levitercompresaa, cinctae tela cellulosa fibrosa, pulpa infusae; (Arbores resinoszo; folia coniugata; flores in corymbum terminalem dispositi).

II78. A F Z ELIA, (AFZELIE) smirн.' transact. IV. (leguminosa ı.) - Calyx tubulosus, limbo quadrifido, deciduo; corollae petala quatuor unguiculata, snmmo maximo; stamina distincts decem, octo fertilia; filamenta duo suprema sterilia; ovarium stylo et stigmate superatum; legumen lignosum, multiloculare; sporae basi arillatae, arillo nigro, hinc nigrae **); (Arbores Africae, foliis alternis et pinnatis (sine impari; flores in racemis colore atrorubente).

II79. MYROSPERMUM, (MYROSPERME) JACQ. JUss, LAM. illustr. tab. 34I. MYROXYLON IIN N. (legu=

*) Secundum jussreus (gen. pl.) auctoritatem ex Enneandriae monoyyniis decimo flamento sterili ad hauc clas. sem revocandum genus; vid. PEIss, pag. 450, et JAUME II $p .210$,

**) AFZELIA Gmelini nimis incerta est, ut tanto condecoretur nomine. 
(leguminosa). - Calux campanulatus, limbo leviter quinquedentato, dentibus fore obsoletis; corollae petala quinque, papillionacea; alce et carina dipliylla foliis fere aequalibus unguiculatis, rectis; vexillum maximum, obcordatum, unguiculatum; stamina distincta, declirata, continuata in carinam; filamenta post petalorum ¿alapsum persistentia; antherae oblongae, versatiles; ovarium corolle longius, pedicellatum; legumen oblongum, alato membranaceurn, compressum ad basin, uniloculare, apice inflatum, mono-disporum, non dehis. cons. *)

II 8O. PARIKJ NSO N IA, (PARKINSONE, SIGALINE, Parkinset) pLUM. LINN. JUss. IAM. illustr. tab. 336. (leguminosa). - Calyx urceolatus, limbo quinquefido, laciniis reflexis caducis; corollae petala quin. que, ovata, unguiculata, fere aequalia ; inferiori látiori, superiori ovato subrotundo, ungue erecto longissimo: legumen elongatum, cylindricum, acuminatum, moniliforme, torulosum, tectum duabus tunicis bivalvibus, polysporum, ad sporarum insertionem inflatum. - (Arbor mediae altitudinis spinosa ; folia pinnata, foliolis numerosis; flores in racemis axillaribus aut terminalibus).

118I. HOFF M A NSE G GIA, (HOFFMANSEG. GIE) CAVAN. tab. 392. LARREA orteg. (leguminosa). - Calyx tubulosus, quinquepartitus, inferus, persistens; corollae petala quinque unguiculata, patentia, superiori latiore, basi glandulosa; filamenta duplici ordine calyci inserta, piloso glandulosa; stigma clavatum;

legu.

*) Ad hoc gellus SAUMERIUM JosePhI DE JUssiev referti debet, quod est LAMARKI Mlyros. pedicellatum; haec nova species a Myr. frutescente leguminibus super pedi. collum lougissimum distinguitur; nec non vera est Quina-quina incolarum de P'eru, Cinchona officinalis LINi., cui Europaei nomen Quinaquina ant Quinquina dare solent. Alque adeo hacc nominum confusio iude derivanda videtur, quod cortex Cascarae de Loxa, Cinchona officinalis L., fuerit substituta verae Quina-qui. nac perurianae, h. e. SAUMEFIo Jos. DE juss.; usus baumerii remedii loco comprobavit summam utilitalem, 
legumen linearo, compressum, bivalve, polysporum. (Petioli axillares in glandulis pedicellatis).

II 8:. CAESALPINIA， (BRESILLET, Sapan) PIUM IINN JUSS. IAM. illustr.tab. 335 (leguminosa 2.) Caly $x$ urcoolatus, quinquefidus rel partitus, lacinia infima longiori maiorique subfornicata; corollae petala quinque fere aequalia, inferiori amoenius colorato; filamenta distincta, arcuata, basi lanata, vix corolla longiora, omnia foecunda ; ovarium oblongum; legumen oblongum, compressum, bivalve, polysporum, ad summum interdum oblique acuminatum. - (Arbores aut frutices utiles ad tingendum, inermes aut saopissime munitae processibus spinosis ad elongationes petiolosque; folia bipinnata, foliolis oppositis; flores in racemos simplices aut paniculas axillares et terminalos dispositi).

II83. POMARIA, (POMARIE) CAVAN. tab. 402. (leguminosa). - Calyx turbinatus, quinquepartitus, caducus; corollae petala quinque, subunguiculata, superiori concavo, breviori; stamina declinata, filamentis inferne hirsutis; stigma capitatum; legumen unilocularo, disporum.

1184. TOLUIFERA, (BEAUMIER DE TOLUS) IINN. Juss. (terebintacea 2.). - Calyx campanulatus, quinquedentatus; corollae petala quinque, quatuor linearia, aequalia, quinto et infimo maximo, cordato; stamina decem brevissinu; stylus nullus; fructus.....? (pisiformis, quadrilocularis, tetrasporus secundum mrLIER.). - (Arbor quae suppeditat balsamum de Toulut nominatum; folia pinnata cum impari; flores in racemis axil. laribus).

II 55. CAS SIA, (CASSE) TOURN, IINN. JUSS. LAM. illustr. tab. 338. SENNA TOURN. (leguminosa 1.). Calyx coloraius, caducus, quinquepartitus (pentaphyllus ₹.); corollae petala quinque, aequalia, distantia, inferiori reliquis maiori; flamenta arcuata, tria superiora antheris sterilibus, inferiora tria longiora antheris arcuatis fortilibus onustata; lateralia quatuor brevia et 
2equaliter fertilia; ovarium pedicellatum; legumen olon. gatum, membranaceum, plus minusve compressum, polysporum, bivalve dissepimentis transversalibus, aut lignosum cylindricum, evalve ot saepe pulposum. - (Frutices aut suffrutices; folia pinnata, foliola opposita una vel serio multiplici; glandulae parvae ad basin potioli communis et inter foliola; flores axillares, dispositi in spicam, rarius fere solitarii).

I 86. CA THARTOCARPUS, (CATHARTO. CARPE) LAM. tab. 332. CASSIA IINN. VAHL. (leguminosa): - Calyx quinquepartitus, deciduus; corolla pentapetala, regularis; filamenta inferiora arcusta; legumen longum, teres, lignosum, multiloculare, loculis pulpe farctis.

1187. TACHYGALIA, (TACHIGALI) AUBL. JUSS. LAM. illustr. tab. 339. TACHIA PERS. AMASO. NIA IINN. suppl. CUBAEA schreb. (leguminosa). Calyx turbinatus, quinquelobus, lobis inaequalibus, persistens; corollae petala quinque, vix aequalia; staminum filamenta distincta, calyci inserta, villosa, exserta, omnia antherifera, tria superiora breviora; ovarium subpedicellatum ; stylus longus ; legumen coriaceum, longum, compressum, villosum, hexa - heptasporum, ad insertionem sporarum inflatum. - (Arbor ramis triangula. ribus; foliis pinnatis).

I 188. G Y M NO CLA D US, (CHICOT) JUSS. IAM. tab. 336. GUILANDINA LINN. WILLD. (leguminosa 1.). - Calyx monophyllus, infundibuli- vel hypocrateriformis, quinquefidus; corollae petala quinque, inserta calycis collo, brevia; stamina non oxserta, quibusdem. interdum sterilibus; legumen glabrum, oblongum, latum, leviter compressum, interne pulposum, fere semper multiloculare, rarius uniloculare, dissepimentis transversalibus; loculis monosporis; sporae osseae, fere globosae. - (Arbores mediae altitudinis, non spinosae; folia uni - bipinnata, foliolis alternis; flores dispositi in spicas paniculatas et torminalos).

II89. G UI. 
II89. G U I L A N D I N A , (BON1) UC, Queniquier) IINN. JUBS. IAM. illustr. tab. 336. BONDUC pIUM. HYPERANTHERA vaHL. WILE. (leguminosa 2.). Calyx urceolatus, quinquepartitus, laciniis profundis, fere aequalibus; corollue perala quinque, sessilia, subaequalia; filamenta non exserta, basi lanata; ovarium oblongum; stylus brevis; legunien breve, ovoïdoum, muricatum aut glabrum, ventricoso compressum, monotrisporum; sporae ossea, fero globosae. - Arbores aut trutices spinosio ad trunci elongationem et ad petiolos; folia bipinnata, foliolis oppositis; flores dispositi in racemos aut in paniculas axillares aut terminales).

I190. MORINGA, (BEN) IAM. tab. 337. JUSs. GUILANUINA IINN. HYPERANTHERA VAHL. WILLD. ANOMA IOUREIR. (loguminosa 2.). - Calyx quinquepartitus, laciniis profundis, caducus; corollae potale quinque sessilia, aequalia, altero superiori attenuato recurvato, quatuor inferiora; calyci inserta; stamina brevia, inaequalia, (quaedam sterilia aut antheris minimi。 donata?); legumen longissimum, capsulaeforme, subulatum, trigonum, dehiscens, in valvulas tres valvulis exsulcatis alternatim foveola, qua continentur sporae nunc alis tribus distinctas nunc non alatae. - (Arbo. res inermes).

119I. GO MPHIA, (GOMPHIE) vAHL, WIIID. OCHNa plum. Linn. (tulipifera). - Calyx ponta. phyllus; corolla pentapetala; antherae fere sessiles; drupae duas vel quinque, monosporae, disco carnoso superpositae; receptaculum subrotundum.

II92 DICTAMNUS, (FRAXINELLE) IIN. JUSS. LAM. illustr. tab. 344. FRAXINELLA TOURN. oAertN. reINEAim. (rutacoa 2.). - Calyx quinquepartitus (pontaphyllus p.), minimus, caducus; corollae petala quinque patula, lanceolata, inaequalia, unguiculata ; filamentu inaequalia, subulata, declinata, punctis glandulosis tecta; ovarium leviter pedicellatum; stylus declinatus, staminibus brevior; stigma simplex; capsu. las quinque, interno margine connexae, radiation dispositae, 
- itae, compressae, externa apice acuminatae, introrsum dehiscentes, continens quaeque capsularum arillum car̃tilagineum, bivalves, disporae - (Planta herbacea, glandulis atrorubentibus viscosis tecta; folia pinnata; foliolis serrulatis; flores in spica terminali).

II93. RHODORA, (RHODORE) IINN. I'HERET. tab. 68. Juss. IAM. illustr. tab. 364. (rhodoracea 2.). Calyx minimus, quinquedentatus; corolla tubulosa, tripartita aut bilabiata, disco calyci adnato inserta, tubo inflato, brevissimo; labio superiori oblongo-obtuso, bi - trifido in superiori sua parte, recto; inferiori bipartito laciniis oblongo-obtusis pendulis, longitudirte superiori aequalibus; stamina declinata; filamenta emarginata; capsula oblongo obtusa, stylo persistente superata, quinquesulcata, quinquelocularis, quinquevalvis, polyspora. - (Frutex foliis alternis, ellipticis, integerrimis; flores terminales fasciculati).

I194. TREMAN THUS, (TREMANTHE) PERs. FOVEOLARIA RUIZ et PAVON. STRIGILIA cav. TINUS juss. (meliaces). ${ }^{*}$ ) - Calyx campanulatus, inferus; corollae petala revoluta; necturolyma tubulosam; antherae post impraegnationem punctis setaceis stellatis asperae; drupa obovata. - (In drupa tria quasi apparent dissepimentorum rudimenta, qua fere exhibent tria loculamenta, ubi, ovariurn transverse dissecatur; quod curiose auctores, erroris evidandi causa, notarunt ). -

1195. STRIGILIA. (STRIGILIE) CAVAN. dis8.7० JUss. LAN. illustr. tab. 349. TINUS juss. (meliacea), Calyx quinquedentatus ; curolla urceolata, profunde quin. quefida; starninum decem ficumentu basi connata in tubum brevom, interna pagina antheriferum; antherae ex uno latere villosae echinorum in rrodum; ovarium superum: stigmata in stylo simplici tria; fructus ... . forsan trilocularis ot trisporus. - (Frutex foliis alternis, foribus in spica axillari).

$1196 \mathrm{QU} \mathrm{I}$.

*) Sane ad hoc genus STRIGILIA cavar, diss. 7. p. 358, tab, 20r, ipso furzxo auctore deferri debere videtur. 
II96. Q U I V I S I A, (QUIVI) commers. JUss. GILIBERTIA gMEL. (meliacea r.). - Caly $x$ urceolatus, quadri - vel quinquedentatus; corollae petala quatuor aus quinque, calyce breviora; staminum octo vel decem fila. menta connata in tubum brevem, octo-decemantheriferum; stigma capitatum; capsula coriacea, quadri quinquelocularis, apice dehiscendo quadri-quinquevalvis; dissepimenta affixa super medium valvularum; loculi dispori. - (Frutices; folia simplicia; pedunculi axillares et uniflori, aut multiflori spicati).

1197. TURRAEA, (TURRAEA) IINN. SMITH. JUSS. LAM. illustr tab.351. (meliacea I.). - Calyx quin. quefidus; corollae petala quinque, longissima, ligulata ; staminum decem filamenta connata in tubum longum, apice decempartitum, lacinia quaque anthera una gerento; stigma parum incrassatum; capsula pentacocca, coccis disporis. - (Frutices; folia simplicia; flores axillares).

II98. SANDORICUM, (SANTOL) RHUMPH. JUss. LAM. ill. tab. 350. WrLld. (meliacea 2.). - Calyx brevis, quinquefidus; corollae petala quinque linearias staminum decom filamenta connata in tubum, margine decempartitum, laciniarum apices antheriferae; stigmata in stylo simplici quinque bifidn; bacca pomiformis, interne pulposa, externe parum villosa, infra in duas valvulas dehiscens, tetra - pentaspora; sporae arillatae, arillo coriaceo, compresso. - (Arbor foliis ternatis, floribus in corymbo paniculato et axillari; fructus acidus).

I199. TR I CH I L I A, (TRICHILIE) LINN. JUSS. (meliacea 2.). - Calyx brevis tubulosus, quinquedentatus; corcllae petala quinque, lanceolata; staminum decem filamenta coalita in tubum terminatum quinque den. tibus facie interna antheriferis; stigma in stylo simplici tridéntatum; capsula rotundata, trilocularis, trivalvis, trispora; sporae arillatae et solitariae in loculo quoque. (Arbores foliis impari pinnatis aut ternatis; floribus in racemis aut paniculis saopo axillaribus). 
1200. E L C A IA , (ELCAIA) Forsk. Juss. (ineliacea 2.). - Calyx campanulatus, quinquepartirus, laciniis rotundatis; corollae petala quinque, calyce longiora, basi plana, linearia, villosa; staminum decem filamenta usque ad medium coalita in tubum prismaticum quatrangulum, prolongata in formam squamarum pagina interna antheriferarum: stigma capitatum; capsula ovoïdea, tomentosa, trilocularis, trivalvis, ivalvulis ad medium striatis; loculi dispori. - (Arbor foliis impari pinnatis, foliolis quadruplici serie; flores simillimi citri floribus, in corymbo axillares aut terminales). *)

1201. E K E BER IA, (EKÉBERGIE) sYARM. act. holm. 1779. t. 9. juss. (meliacea 2.). - Calyx quadrifidus; corolla tetrapetala ; staminum decem filamenta in tu. bum coalita brevem, integrum, interna facie antheriferum; stigma capitatum; bacca globosa, pentaspora; ...; (Arbor; folia impari pinnata aut sine impari; petioli communes compressi; flores in paniculis axillaribus).

1202. MELIA. (AZÉDARACH) IXNN. JUSs LABT. ill. tab. 352. AZEDARACH TOURN. MEDIC. monadelph. p.115. (meliacea 2.). - Calyx parvus, quinquefidus: corollae petala quinque oblonga; stuminum decem filamenta connata in tubum cylindricum, terminatum quinquo dentibus; anthera singula affixa facie interna denti singulo tubi ; stigma capitatum; drupa globosa; nux quinquelocularis, pentaspora. - (Frutex: folia impari pinnata, aut bipinnata; flores in paniculis axillaribus).

1203. S W IE TENIA, (MAHOGON) JACQ. LINN. JUss. (meliaceis affinis). - Calyx minor, quinquefidus, laciniis caducis; corolla pentapetala ; staminum decem filamenta coalita in tubum terminatum decem dentibus pagina interna antheriferis; stylus simplex; stigma capita. tum;

*) Plantam, quae hoc constituit genus, Forsk, auctore in Arabis detectam, b. vaHL aristratus est referrı debere ad Irichiliue genus, et nominari voluit Irichuia emetica: folia tenet pinuata, foliola ellyptica, inferiori pagina villosa, 
tum; capsula ovoïdea, lignosa, quinquelocularis, longitudinaliter inde a basi ad summum usque dehiscens in quinque valvulas affixas marginibus placentae pentagonae angulis, obtectae penitus sporis compressis, imbricatis, et ad apicem in alam prolongatis. - (Arbo. res; folia pari pinnata; flores in paniculis terminalibus ant axillaribus).

1204. GAERTNERA, (GAERTNERE) sCHRsB. MOLINA CAVAN. HIPTAGE GAERTN. SONNER VOY 2. t. 135. VENT. BANISTERIA IINN. IAN. (malpighiacea 2.). - Calyx quinquefidus, unica glandula munitus; corollae petala quinque, lacerato-ciliata, crenulata, ungue lineari; staminum decem filamenta subcoalita, uno cateris longiori, corolla breviora; stylus unus: ovarium simplex; samara quadrialata, alis aequalibus; monospora. - (Arbor humilis; folia opposita; flores in faniculis laxis).

\section{Flore polypetalo aequali.}

1205. CYNO MORA, (CYNOMÉTRE) IINエ. IAM. tab. 33I. IINN. WILID. LAM. tab. 331. (leguminosa). Calyx quadripartitus, laciniis reflexis; corollae petala quinque lanceolata, aequalia; antherae apice bifidao; legumen breve, lunatum, interne carnosum, externe tuberculosum, mono-dieporum. - (Folia coniugata).

I206. PROSOPIS, (PROSOPIS) BuRM. IIx.Juss. IArr. illustr, tab. 340. (leguminosa 2.). - Caiyx hemisphaericus, quadri-quinquedentatus ; corollae petala quinquo sessilia, aequalia, lineari lanceolata, apice recurva; stamina distincta, aequalia; filamenta basi petalorum adnata, capillacea; stigma simplex; legumen longum, inflatum, uniloculare, polysporum. - (Arbor foliis alternis, coniugatis; flores parvi in spicis termimalibus aut axillaribus).

1207. SCHOTIA, (SCHOTIE) JACQ. JUSS. IAM. 2ab. 331. GUAIACUMI LINX. THEODOKA MEDICUS, 
SCOTIA тнunв, nov. gen. IX. (leguminosa i.). Calyx coloratus, turbinatus, ad limbum quinquelobus, lobis caducis; corollae petala quinque, approximata, calyci inserta eoque longiớa, in tubum ventricosum conniventia, lateribus invicem incumbentibus clausa: filamenta subulata, recta, parum corolla longiora; antherue versatiles; ovarium pedicellatum; legumen compressum, mucronattum pedicellatum, longitudine digitis, glabrum; sporae oblongae, colore brunneae, munitae umbilico albente. - (Frutex foliis pinnatis, rigidis super petiolum communem striàtum; flores fasciculati aut spicati, rubri).

1208. CA D IA, (CADIE) FORSK, I'YERET. VENT. SPAENDONCEA DESFONT. PANCIATICA PICCIVOLI (leguminosa 2.). - Calyx campariulatus, quinquefidus 3 corollae petala quinque (rarius sex, septem) aequalia, disposita in eampanulam; obcordata, fere sessilia; stamiza decem, rarius duodecim vel quatuordecim, in circulum collocata, petalis approximatà; filumentu subulata, leviter arcuata, basi interne gibba, longitudine corollae. inter se aequalia; antherae oblongae, versatiles: ovavium pedicellatum; legumen lineare, compressum, membranaceum, polysporum; sporae octo, decem, ovali-oblongae, nitidae - (Frutex habitu simillima Tamarindo; folia pinnata cum impari; flores in racemis axillaribus aut terminalibus).

1209. A D EN A N THERA, (ADENANTHERE, Condori) IINN. JUss. LAM. illustr. tab 334. (leguminosa 2.). - Calyx minimus, quinquedentatus: corollae petala quinque vix aequalia; stamina distincta; antherae declinato-pendulae, apice exteriori glandula globosa adfixa munitae: lẹumen longum, compressum, membranacoum, polysporum: sporae remotae. - (Arbores non spinosae; folia bipinnata; flores in spicis laxis axillaxibus aut terminalibus).

1210. HAE MA TOXỲLUM, (HÉMATOXYLE, Campêche) LINN. JUss. IAM. illustr. tab. 340. (Íeguminosa 2. ). - Calyx turbinatus, quinquepartitus; corollae [20] pota- 


\section{CLASS.X. DECANDRIA.}

petala quinque aequalia, vix calyce longiora vel maiora; fllamenta distincta, basi interne barbata; stigma truncatum aut emarginatum; legumen capsulare, lanceolatum, parum compressnm, uniloculare, sese dividens ad mediam partem in duas vel tres valvulas, valvulis navicularibus, di - trisporis; sporae oblongae, appla. natae. - (Arbor spinosa; foliis oppositis triplici vel quatriplici serie, obcordatis; flores dispositi in racemos simplices et axillates).

1211. ZUCCAG N IA, (ZUCCAGNIE) cAVAN. (leguminosa 2). - Calyx inferus, quinquepartitus, persistens: corollae petala quinque, ovata, superiori latiore concavo: filamenta inferne pilosa; stylus curvus; stigma infundibuliforme; legumen subovatum compressum, uniloculare, bivalve; spora una, affixa apici valvularum.

* rzi H. H IPPO MA N I CA, ( hipponanica) molin. Juss. (ex ord. plantt. sedis incertae). - Calyx quinquepartitus; corollae petala quinque ovalia; stamina decem; ovarium unum; stylus unus; stigma unum; cap. sula quadrilocularis, polyspora; sporae reniformes. (Planta herbacea, elongationes trunci quatrangulares; folia opposita, simplicia, integra, parum incrassata; flores flavi, terminales; incola Chili).

1213. G UAJACUM, (GAYAC) PLUM. IINN. JUss. IAM. illustr tab. 342. (rutacea 1.). - Calyx quinquepartitus vel fissus; inaequalis; corollae petala quinque, inserta calyci; filamenta nuda; capsula fero pedicellata, turbinata, angulosa, angulis compressis, bi - triquinquelocularis, loculis monosporis; sporae ad apicem funiculi umbilicalis brevis, inserti angulo loculorum centrali. - (Arbores ligno duro; folia pinnata sine impari ; pedunculi axillares, uniflori).

I2I 4 RUTA, (RUE) TOURN. IINN. JUSS. IÁM. ill. tab. 345. (rutacea 2.). - Calyx quadri - quinquepartitus, parvus, persistens; corollae petala quatuor, quinque, unguiculata, concava; filamenta basi dilatata; receptaculum circa ovarium octo-decem poris melliferis

cin- 
cinctum ; stigma simplex; capsula quadri - quinqueloba, quadri - quinquelocularis, intra lobos dehiscens, polyspora; sporae renifnrmes. - (Stamina quibusdam spe. ciebus octo; elongatio trunci herbacea aut, suffrutescens, odore gravi; folia simplicia, saepius unum. bipinnata: flores terminales, dispositi in corymbos aut in cymam).

I215. ERIOSTEMON, (ERIOSTEMON) sMrty transact. IIIf. (rutacea). - Calyx quinquepartitus; co. rollae petala quinque, sessilia; staminum filarnenta plana, ciliata; antherae pedicellatae, terminales; stylus e basi ovarii; capsulae quinque coalitae, nectarolymati toruloso insidentes; sporae arillatae. ${ }^{*}$ )

12I6. CR O W EA, (CROWEA) sMrTH transact. IIII. ANDREW. tab. 79. VE.NT. jard. de Malm. tab. 7. (rutacea?). - Calyx quinquepartitus, laciniis unguiculatis ot in tubum approximatis; petala corollae quinque hypogyna, sessilia; staminum filamenta inserta super discum, plana, subulata, apice dilatata et dissita pilis ciliatis intertextis basi in tubum connexa; antherae longitudinaliter filamentis e parte interiori adnatae; ovarium rocundatum, quinquesulcatum, stipitatum, disco glanduloso cinctum; stylus e basi ovarii cylindricus stigma obtusum; capsulae (cocci) quinque coalitae vel conniventes, ovalos compressae, introrsum dehiscentes; sporae arillatae. ${ }^{* *}$ ) - (Frutex elongatione trunci triquetra; folia alterna, integerrima; flores solitarii, axillares).

1217. TRIBULUS, (TRIBULE, Herse) TOURN: IJNN, JUSS. LAM. illustr. tab. 346. (rutacea 1.). - Calyx quin-

*) $\Lambda$ genere DIOSIMA differt defectu squamarum cum petalis alternantium; staminibus non exsertis, ciliatis non glabris, defectu nectarolymatis ovarium coronantis;" staminum numcro Ad genus EHIOSTEMON pertinet autem Diosma uniflora LINN. cui stamina quinque abortiva. SMITH.

**) Antherarum praeprimis forma et situ ab antecedenti genero distinguitur. 
quinquepartitus; corollae petala quinque, bblonga, patentia ; stylus nullus; stigma fere quinquefidum; fructis formatus saepissime quinque capsulis aut des cem, appriximatis, hinc gibbis, extrorsum dilatatis et armatis spinis; sod contractis et coalitis rectissime introrsum ; capsula quaequo bi - tri - quadrilocularis, Inculis transversalibus, di- tetraspora, - iElongatio trunci herbacea, saepe disposita; folia opposita, parı pinnata, foliolo uno utplurimum minorí et abortiente; flores solitarii alterni, in axilla folii brevissimi collocati, flavi).

I2I \&. FA GONIA, (FAGONE) TOURN. IINN. JUss. LAM. illustr. tab. 346. (rutacea 1.). - Caly $x$ quínquepartitus, caducus; corollae petala quinque unguiculata; corlata; capsula rotundata, acuminata, pentagona aut quinqueloba, quinquelocularis, loculis bivalvibus, monosporis; sporae compressae, insertae párva corda umbilicali super placentarn pentagonam, subulatam, centralem. - (Planrae herbaceae, liguosae ad basin; folia opposita, simplicia aut ternata; flores solitarii, axilla. res; stipulae spinescentes in quibusdam speciebus).

1219. ZY GO PHYLL U M, (ZYGOPHYLLE, Fabagelle) IinN. Juss, iAM. illustr. tab. 345. FABAGO tourn. (rutacta 1.). - Calyx quinquepartitus, (pentapliylus P.): corolla pentapetala, petalis unguiculatis; filamenta ad basin internam munita squamulis conniventibus; capsula ovato oblonga, pentagona, quinquelccularis, quinquevalvis, dissepimenta longitudinaliter adnata medio valvularum; lnculi monospori; sporae affi. xae margini dissepimentorum centrali, duplici serie in singulo loculo dispositae. - (Frutices aut suffrutices; folia coniugata, rarissime simplicia; flores solitarii, axillares).

1220. LARREA, (LARREA) cAVAN, icon. 6. p. 40. t. 559. (rutacea 1.). - Calyx quinqueficius, laciniis concavis, inferus, deciduus; corollue petala quinque unguiculata; filamentorum quodque ad basin internam squama, ovarium cingente, munitum; antherae apice acumi- 
matao ad basin cordatae; stigma simplex: ovarium profunde quinquesulcatum; stylus subulatus, pentagonus; zuces quinque, ovali - oblongae, monosporae; albumen carnosum. *). - (Frutices ; folia simplicia aut pinnata; pedunculi saepissime solitarii).

322I. SI M A B A , (SIMABE) AUblet. JUSS. ZWINGERA schreb. wilid. (terebintacea 3.). - Calyx quadri - quinquepartitus; corollae petala quatuor, quinque, inserta super discum carnosum: filamenta inserta disco, basi dilatata et pilosa; ovaria quatuor aut quinque coalita; stylus unus (saepe quinque styli coaliti?); stigmata quatuor, quinque; capsulae quatuor, quinque (numero ovariorum) ovatae, coriaceae, disiunctae, monosporae, receptaculo carnoso insertae. - (Frutex; folia alterna, ternata aut pinnata cum impari, foliolis duplici serie; flores axillares, in corymbum dispositi; pedicelli ad basin squama una notati).

I222. CERATOPE T A LU IM, (CÉRATOPÉTALE) sMrtir WrLID. - Calyx persistens, quinquepartitus, staminiferus; corollae petalá quinque pinnatifida; stamina calyci inserta; antherce calcaratae; capsula in fundo calycis calyce tecta, bilocularis.

1223. THRYALLIS, (THRYALLE) IINN. JUSS. (aceracea 2.). - Calyx quinquepartitus, persistens; corolla pentapetala; antherae rotundatae; ovarium simplex; capsula triangularis, tricocca, trilocularis, in tres partes se dividens; loculis monosporis, angulo interiori dehiscentibus; sporae mucronato-incurvae, gla. berrimae. - (Frutex Brasiliae, ramis articulatis; folia opposita, stipulacea; flores in racemis terminalibus).

I224. G UIERA, (GUERA) JUss. IArr. tab. 360 . (onagraria 3.). - Calyx oblongus, fere cylindricus, gracilis, quadridentatus; corollae petala quinque; stamina exserta, corollạe alterna longiora; antherae globo-

sae;

*) Distinctum est hoc genus a genere LARfEA antegi qugd supra sub HOFFMINSEGGIA positum habemus. 
sae; capsula oblonga, angusta, quinquangula, angulis parum exsertis, pilis longissimis tecta, dentibus ealycis coronata, unilocularis, subquinquespora; sporae minimae, filo affixae. - (Frutex foliis oppositis, integerrim1s, ovalibus; flores terminales super ramos, numerosi, sessiles, involucro polyphyllo cincti).

1225. CACUCIA, (CACOUCIER) CACOUCIA AUBI. JUSs. IAM. ill. tab. 359. SCHOUSBOEA WILID. (onagraria 3.). - Calyx campanulatus, limbo dilatato, patentissimo, quinquefido, caduco, corolla pentapetala; stamina saepe longissima; antherae ovales; capsula subbaccata, ovata, pentagona, fusiformis, intus pulposa, unilocularis, monospora; spora arillata. - (Elongatio sarmentosa; folia alterna, integerrima; flores alterni super spicam terminalem).

1226. MOURIRIA, (MOURIRI) AUBI. IAM. ill. tab. 36. PETALOMA sWARz, WILID. PERs. (melastomea 1.). - Culyx urceolatus, quinquedentatus, ad basin squamis quinque munitus; corollae petaia quinque inferne dilatata, calyci inserta; stamina margini calycis insidentia, magnitudine inaequalia; antherae oblongae; bacca coronata ad summum dentibus calycinis, globosa, unilocularis, tetraspora. - (Arbores Americae meridionalis ).

I227. L I MONIA (LIMONELLIER) IINN. JUSS. IAM. ill. tab. 353. (hesperidea 2.). - Calyx parvus, quinquepartitus; corolla pentapețala; bacca globosa, trilocularis, loculis monosporis. - (Arbores aut frutices : folia simplicia, ternata aut pinnata, glandulis confertis tecta, spinis axillaribus geminis aut solitariis munita ).

I228. FERONIA, (FERONIE) Roxb. tab. 191. CORREA transact. Linn. V. (hesperidea 2.). - Calyx quinquepartitus, planus; corollae petala quinque, oblonga ; filamenta basi dilatata, villosa, disco hypogynio elevato inserta; bacca corticosa, multilocularis, loculis carne spongiosa obvolutis. 
I229. COO KIA, (WAMPI) SONNER. JUSS. LAM. illustr. tab. 354. QUINARIA loureir. (hesperidea 2). Calyx minimus, inferus, quinquepartitus; corollae petala quinque patontia, aequalia; stamina libera; antherae subrotundae; ovarium loviter pedicellatum, villosum; stigma inflatum ; bacca (pomum P.) parva, ovalis, punctata, quinque - multilocularis, loculis monosporis, spora abortu obnoxia. - (Arbores; folia alterna, impari pinnata, punctis translucentibus obsita; flores in racemo paniculato terminales).

1230. HEIS T ERIA, (HEISTÈRE, Bois-perdrix) IINN. JACQ. amer. tab. 81, et pict. tab. 122. JUSS. LAM. illustr. t. 534. (hesperidea 1.). - Calyx subquinquefidus, primum viridis et minimus, deinceps coloratus et magnificatus; corollae petala quinque concava; staminum filamenta plana, alternatim breviora; antherae fere rotundae; stylus brevis; stigma subquadrifidum; drupa olivaeformis, oblonga, colorata, ad summum evasa, quinqueloba, lobis obtusis, ad dimidium calyce ampli. ficato tecta, monospora. - (Arbor liabitu Lauri; folia alterna, integerrima; flores parvi, axillares, solitarii).

123I. QUISQUAL JS, (QUISQUALIS) IINN.IAM. illustr. tab. 357. MYRODIA schreb. (daphnacea). Calyx tubulosus, longissimus, filiformis, limbo quinquefido vel quinquedentato; corolla peintapetala, tubo longissimo (squamas quinque oblonzae J.); stylus filiformis; stigma obtusum; drupa quinquangularis, mo. nospora. - (Rami cylindrici; folia opposita, inte. gerrima).

1232. MONOTROPA, (MONOTROPE, Sucepin) IINN. JUSS. LAM. illustr. tab. 362 . (ex ordine plantt. sedis incertae). - Calyx tetra-pentaphyllus, coloratus (nullus $P_{0}$ ); corollae petala quinque, hypogyna, conniventia et alterna cum calycis foliolis hisque concolora, in cylindrum coalita, et cum eo decidua, ad basin in duas appendices prolongata, interne concava, externe convexa et gibba (petala decem, quorum quinque exteriora (calycis?) basi excavato mellifera I'Ens.); stamina 
octo, decem hypogyna; antherae parvae; ovarium supoe rum: stylus cylindricus; stigma dilatatum et fure infundibuliforme: capsuia quadri - vel quinquesuica, qua. dri - quinquelosularis, quadri - quinquevalvis; polyspora: valvulae dissepimento notatae, applicato versus receptaculum centrale quatri -- quinquangulum'; sporae. numero plures, minimae, receptaculo centrali affixae.(Planta parasitica: elongatio tecta squarris latis, oblongis; flores ad elongationis summum in spica brevi).

1233. CLETHRA. (CLETHRA) GRON. LINN. JUSS. IAM. illustr tab. 369. (erica I.). - Calyx quinqueparsitus, persistens; corollue petala quinque aut corolla formata quinque petalis basi dilatatis; stamina non exserta; stigma fero trilobum; stylus apice trifidus, persistens; capsula globosa, trisulca, trilocularis, apice dehiscens in tres valvulas, calyce cincta, polyspora. - (Frutices foliis alternis, floribus dispositis in spicas axillares. aut terminales, bracteis linearibus munitis).

I234. CUELLARIA , (CUELLARIE) RUIz-PA. von. (erica - - Calyx quinquepartius; corollae pe. tala quinque aequalia; stylus trigonus; stigma trilobum; capsula trigona, trilocularis; sporae imbricața, men. branaceae. ${ }^{*}$ )

1235. PYROLA, (PYROLF) TOURN. IINN. JUse. IAM. illustr tab. 36\%. (erica 1.). - Calyx minor, quinquepartitus; corollae (quinquepartitae) petala quinque conniventia, basi dilatata; stamina non exserta; stigma capitatum, bipunctatum aut cinctum quinque crenulationibus; capsula pentagona, angulis dehiscens quinquelocularis, quinquevalvis, polyspora. - (Plantae herbaceas aut suffrutescentes; folia alterna aut fere $\nabla \in r$ i-

cil-

*) A CLETHRA LINN, cui hoc genus valde affine videtur, petalis aequalibus, filamentis compressis, antheris su: - perue bifidis, liperforatis, stylo trigono brevi, stigmate trilobo, capsula trigona depressa, sporis imbricatis, planis margiue membranaceo squamoso cinctis, receptaçulo, denique subrotundo CUELLAFIAM differri iubent als ctores systematis vegetab, forac peruvianae gravissimi. 
eillata, utplurimum radicalia : fores bracteati, terminales, spicati aut rarissime umbellati).

123\%. LE D U M, (LÉDON, LÉDE) IINN. JUSS. IAMI, ill. tab. 363. (rhodoracea 2.) - Calyx minimus, quinquedentatus vol fissus; corolla profunde quinguepartita aut pentapeiala, plana; antherae rectae oblongae; capsula basi dehiscens, acuminata, quinquelocularis, quinquevalris, polyspora; - (pedunculi bracteati; uniflori ; suffrutices : flores in corymbịs șpiciformibus aut umbelliformibus).

"1237. LEIOP HYLL UM, LANr. tab. 363. - LE: DUM LinN. Capsula apice dehiscens; folia utring̨ue glabra. (Facie a Ledis rocedit).

T23. DIQNAEA, (DIONÉE) EIIIS. IINN. VENT: jard. de Malm. t. 29. juss. LAM. tąb. 362. (ex ordine plantt. sedis incẹrtae). - Calyx pentaphyllus, persistenş; corollae petala quinque concava, antherae rotundatae; ovarium superum, rotundatum, decemsulcato. striatum, disco cinctum; stylus persistens; stigma dila. tatum, margine exserto; capsula rotundata, membrana cea, quinquangula, unilocularis gibba, polyspora; $v e$ ceptaculum centrale; embryo rectus; - (Planța herbacea; folia radicalia, duplicata, deinceps in rosulam explicata, marginibus ciliis longis rigidis fịmbriatis; fo' res super elongationem aphyllam in corymbo laxo).

1239. MURRAYA, (MURRAY) IINN. JUSS. IANR: illustr. tab 352. CHALCAS BURM IINN mant. MAR: SANA SONNER. (hesperidea 2.) - Calyx ninimus, persistens, quinquepartitus; corollae petala quinque aut sex, basi approximato connata in formam campanae, apice dilatata; stumina decem, rarius unde-vel duodecim; filamenta inaequalia, basi parumper coalita; stigma capitatum, angulosum; ovarium basi cinctum disco urceolato (nectarolyma LinN.); bacca parva, sicca, bulboso globosa, cíncta opidermito punctata, acuminata, mono-dispora; sporae cartilagineae; - (Frutex ramis alternis, folia alterna, impari pinnata, punctis translu- 


\section{CLASS. X. DECANDRIA:}

centibus tecta; flores in corymbis terminalibus; embryo sporae rectus). ${ }^{*}$ )

I140. BERGERA, (BERGERA) RUMPH. IINI. JUss. Rохв. (hesperidea 2.) - Calyx minimus, quinquepartitus; corollae petala quinque, plana, patentia; stamina alternantia breviora; stigma turbinatum, trans. verse sulcatum; bacca fere globosa, dispora; - (Arbor; folia pinnata cum impari; flores terminales, in corymbo paniculato; pedicelli bracteati).

I24I. MELASTOMA, (MELASTOME) EURM. IINN. 'Juss. IAM, illustr. tab. 360 . GROSSULARIA TOURI. TIBOUCHINA AUBL. FOTHERGILLA AUBL. (melastomea 1.) - Caly $x$ quinquefidus aut quinquedentatus, aut fere integer campanulatus; corollae petala quinque, ad summum calyci inserta; antherae longae, arcuatae; capsula baccaeformis, interne pulposa, triquinquelocularis, calyce obvoluta; sporae minutae, in pulpa nidulantes; (stamina etiam octo et tredecim; folia eleganter in plerisque nervoso-venosa; arbores mediae altitudinis, aut fruticos).

1242. TR I S T E M M A, (TRISTEMME) JUSS. MELASTOMA commers. (melastomea I.) - Calyx limbo quinquefido, prope limbum extrorsum triplici ciliarum villorumque corona membranacea cinctus; corollae petala quinque unguiculata; ovarium semiinferum; bacca ovalis, compressa, subangulata, ad summum solum. modo supera, quinquelocularis, interne pulposa, quinquangularis; - (Elongatio herbacea, tetragona; flores capitati).

I243. MICONIA, (MICONIE) RUIZ-pAv, syst. veget. flor. peruv. (melastornea r.) - Calyx quinqueden-

*) CHALCAs IINN, juss. (hesperidea 2.) - Calyx parvas persistens, quinquefidus; petala quinque, sex, recta, in ungues angustata; stigma inflatum et verrucosum; bacca oblonga, dispora; - (Frutex floribus terminalibus, paniculatis. 
dentatus; corolla pentapetala; stamina bina semper mu: nita per squamam unam bifidam ovarium tegentem (nectarolyma); antherae subulatae, rectae, plicatae, retrorsum calcare notatae; stigma simplex; capsula decemstriata, quinquelocularis, limbo calycis et squamis inrernis floris coronata; sporae numero plures, scrobiformes. (Frutices).

1244. MER I A N IA, (MÉRIANIE) swARz. WIIID. RHEXIA sWARz. (melastomea). - Calyx quinquefidus campanulatus; corollae petala quinque inserta calyci; stamina declinata; capsula quinquelocularis, polyspora.

1245. ACISANTHERA, (ACISANTHÈ RE) BRown. JUSS. RHEXIA IINN. WILID. (lythraria). Calyx ventricosus, quinquefidus; curollae petala quinque calyci affixa; antherae sagittatae, versatiles; cupsula cályce tecta et coronata, rotundata, bilocularís, polyspora; sporae insertae in quoque loculo placentao propriae; hinc placentae duae; - (Elongatio herbacea, folia opposita, flores solitarii, sxillares).

1246. AXINAEA, (AXINEE) RUIZ. PAV. JUs8. mss. (molastomea 2.) - Calyx concávus, evasus, integer, quinque-sexdentatus; corollae petala quitique aut $8 \mathrm{ex}$ dolabriformia; stamina decem vel duodecim, alterna breviora; antherae curvatae, calcaratae; ovarium quinque-sexangulum, truncatum; capsula calyce cincta, truncata, et corniculis coronata, quinque-sexlocularis; (Arbores).

1247. TIB U C H I N A, (TIBOUCHINA) AUbI. Guy. t.' 177 . Juss. (melastomea 2.) - Calyx obtectus superficie squamis parvis; limbus quinquefidus, basi cinctus quatuor aut decem glandulis magnis imbricatis et in crucem dispositis; corolla pentapetala; stamina decem; antherae basi bicornes 3 capsula quinquelocularis, quinquevalvis, calyce cincta; (Elongatio tetragona; flores solitarii axillares, aut in corymbo terminales). 
I248. MAYETA, (MAYETA) AUB̧. Guy. t. 176. juss. (melastomea 2.) - Calyx villosus, apice quinquefidus, externe quinque squamis munitus; corolla pentapetala; antherae basi bicornes, apice membranaceae; stylus brevis; bacca quinquelocularis, coronata et cincta calyce carnoso: (Elongatio tetragona, gongylosa; folia opposita, inaequali magnitudine; flores axịlares, subsessiles).

I249. TOC OC A, (TOCOCA) AUEz. Guy. t. 174. Juss. (melastomea 2.) - Calyx limbo quinquefidus, margine villosus; corollae petala quinque rotundała; stamina decem; stylus brevis; stigma poltatum; bacca coronata, trilocularis, calyce carnoso cincta, subadhaerens; (Elongatio tetragona; potiolus vesiculosus brevis; folia ovalia).

1250. JUSSIEUA, (JUSSIEUE, JUSSIÈ) IINN. JUSS. IAM. tab 280. ONAGRA PLUM. (onagra I.) Calyx superus, longus, cylindricus, limbo quatri - quinquepartita, persistens, patens; corollae petala quatuor, quinque, ovata, patentia; antherae ovales aut oblongae, versatiles; stigma capitatum, quatri vel quinquestria. tum; capsula oblonga, cylindrica aut angulosa, 'quadriquinquelocularis, angulis dehiscens, quadri-quinquevalvis, calyce coronata, polyspora; sporae numerosae minutae, receptaculo angulato centrali adnexae; dissepimenta in medio valvularum; - (Elongatio utplurimum herbacea, rarius frutescens; folia alterna; flores solitarii axillares; ab Oenothera differt limbo calycis persistente et fructum coronante).

I25I. STYRAX, (ALIBOUFIER) TOURN. IINT. Juss. LAM. illustr, tab. 369. (ebenacea I.) - Calyx urceolatus, inferus, integer aut quinquehentatus; corolla infundibuliformis, ad basin calycis inserta, tubo brovi, limbo tri-vel septempartito; stamina sex ad sedecim; filumenta basi cialita, tubo corollae inserta ; antherae rectae, oblongae; ovarium superum; stylus, stigma simplex: drupa coriacea, continens nucem monosporam, sphaericam, aut nuces duas uno latere planas altero convexas; radi- 
radicula supera; (Arbores aut frutices; flores axillares aut terminales, solitarii aut fasciculati).

\section{Flore monopetalo, aequali:}

1252. EPERU்', (ËPÉRU) AUBL, Guy. t. 142. JUSË. IAM. tab. 338. PANZERA wIILD. (leguminosa 2.) Calyx monophyllus, urceolatus, limbo magno quadripartito; corollae petaluın unum, orificio calycis insertum basi laterale convolutum, subrotundum; filamenta exserta Jongissima, basi incrassata et villoso barbata, novem fore infra coalita, decimo solitario simplici; quinque sterilia; ovarium fere pedicellatum; stylus longus; legumen longum, compressum, apice falcatum, uniloculare, elastice in duas dehiscens valvulas, quae continent tres quatuorve sporas magnas, coriaceas, intetdum uno latere emarginatas; (Arbor ramosa: folia tripinnata: flores in paniculis axillatibus et torminalibus, longis et pendulis).

1253. CODON, (CODON) ROYEN. IINN. JUSS. (ex ordine plantarum sedis incertae). - Calyx decempartitus, laciniis šubulatis; corolla monopetala, campanulata, basi tubulosa, limbo decemfido, aequali; filamersta basi squama munita, squamae conniventes: antherie incrassatae; ovarium superum ; stylus unus: stigmata duo; fructus. . . capsula bilocularis, polyspora; sporae villo8 ae, pulpa sicca circumdatae: (Elongatio herbacea; folia alterna, petiolata, glabra; flores solitarii pedunculati).

\section{ENIS I A THUS, (ENKIANTHE) IOU-} T.EIR. cochin. 339. (ex ord. plantt. sedis incertae,) Involucrum commune quinqueflorum, formatum quatuor foliolis coloratis, concavis, octo interioribus patentibus, planis; flores pedunculati ; calyx quinquefidus, laciniis parvis, coloratis; corolla monopetala, campanulata; limbus brevis, quinquelobus, stamina decern fundo calycis inserta; filamenta basi infata; ovarium superum; stylus incrassatus; bacca oblonga, pontagona, ca- 
lyco associata, quinquelocularis; sporae numero plures; - (Arbor humilis; folia conferta, oblonga, integerrima).

I255. POT ALIA , (POTALIE) AUBL. Guy. t.15r. JUSS. LAM. tab 348. NICANDRA SCHREB. (gentianeis affinis). - Calux quadripartitus, rectus, turbinatus, laciniis duabus exterioribus, duabus interioribus squamis duabus munitis ; corolla tubo brevi, limbo decempartito laciniis longis, rectis, obliquis, lateraliter imbricatis; stamina decem; antherae longas; filamenta brevia, basi connata; stigma in stylo simplici peltatum; striatum; capsula subcarnosa, trilocularis, polyspora; (Elongatio herbacea, fere lignosa, gongilosa, resinosa; folia gongilorum superiorum opposita, petiolis basi fere amplexicaulibus; flores in corymbo terminali).

1256. I N O C ARP US, (INOCARPE) FORST. IIN IV. IAxI. tab. 362. (hilospora). - Calyx bifidus; corolla tubulosa, infundibuliformis, quinquefida, laciniis lon. gis, linearibus; stigma concavum; stamina duplici serio coordinata, drupa ovalis, apice curva, maxima, nuce una reticulata monospora.

I257. CERAT TOSTEMA, (CERATOSTEMA) juSs. (erica 2.) - Calyx turbinatus, quinquefidus, la. ciniis maiusculis: corolla coriacea, tubo longo cylindrico, limbo quinquepartito; stamina perigyna; filamenta brevia; antherae longae, erectae, apice bipartitae $\nabla e l$ bifurcatae; bacca globosa, supra truncata, coronata calyce exsucco, quinquelocularis. (Frutex foliis sessilibus, floribus magnis in spica laxa et pedunculis bracteatis).

1258. ANDROMEDA, (ANDROMÉDE) IINN. juss. ram. illustr. tab. 365. ERICA tourn. (erica 1.) - Calyx inferus, minimus, quinquepartitus; corolla campanulata aut globosa ovata, quinquepartita, laciniis extrorsum flexis; stamina non exserta; capsula pentagona, quinquelocularis, quinquevalvis, valvulis dissepimento contrariis, polyspora; - (Flores quidam octandri ; 
dri, axillares aut torminales in fruticibus foliis alternis, rarissime oppositis.

1259. RHODODENDRON, (RHODODENDRON, Rosage) IINN. JUss. LAMI illustr. tab. 364, CHAMOERHODODENDROS TOURN. (rhodoracea 1.) - Calyx quinquepartitus; corclla fere campanulata aut infundibuliformis limbo patente quinquelobo, sub. obliqua; staminum filamenta declinata; antherae oblongae, rectae; capsnla quinquelocularis, quinquevalvis, polyspora; placenta centralis quinqueloba, lobis in loculos exsertis; - (Flores axillares, aut terminales, in spicis aut subcorymbis).

I 260. V A C C I I U M, (AIRELLE, myrtille.) IINN. juss. IAM. tab. 26. VITIS IDAEA et OXICOCOS Tourx (erica.) - Calyx superus integer, aut quadridentatus; corol is campanulata aut urceolata, quadri-quinquefida, laciniis reflexis ; flamenta ovario inserta; "itherae furcato bicornes, aristis duabus in dorso munitis; bacca clobosa umbilicata, quadri-quinquelocularis, oligospora: - stamina quiibusdam octo; Frutices aut suffrutices; squamar gemmarum saepo persistentes ad basin ramorum ; folia alterna, sempervirentia in quibusdam speciebus; flores pedicellati, solitarii axillares, aut approximati et terminales).

I26I. KALMIA, (KALMIE) LINN. JUSS.'IAM. ilIustr. tab. 363. (rhodoracea 1.) - Calyx quinquepartitus; corolla hypocrateriformis campanulata, patens, lim. bo recto, fere quinquefido, subtus quinquecorni, interne sulcato decem foveis, et munito externe decem tuberculis exsertis ; stamina basi corollae inserta ; flamenta recurva; antherae insinuatae ante foecundationem foveis; capsula quinquelocularis, quinquevalvis, polyspora; placenta centralis quinqueloba, lobis in loculos proeminentibus; - (Frutices foliis alternis aut oppositis: floribus dispositis in spicas aut fere in corymbos, axillares aut terminales ).

1262. URATEA, (OURATEA) AUBIET. Guy. t. 152. suss. (ex ord. plantt. sedis incertao). - Calyx pentaphyl- 
phyllus; corollae petala quinque hypogyna, roturade stamina hypogyna; antherae oblongae, approximatae in tubum transversalem adiuvante stylo; ovarium superum ; stylus unus; stigma fere quinquefidum; fructus. ...? (Arbor excelsa- lolia alterna, basi stipulacea, integerrima; fores in paniculis laxis terminalibus).

1263. EPIGAEA, (EPIGÉE) IINN.JUSS. IAM. illustr. tab. 367. (rhodoracea I.) - Calyx duplex; exterior triphyllus : interior campanulatus, quinquepartitus, persistens; corolla hypocrateriformis, inserta disco glanduloso adnato fundo calycis; tubus cylindricus, interne pilis albis rigidus; limbus pianus quinquelobus; stamina ad basin tubi insertá, inclusa; filamerita filiformia, recta ; antherae lineari oblongae, superiori filamencorum parti adnatae; stylus persistens; stigma fere urcoolatum, quinquefidum, superatum in centro quatuor vel quinque tuberculis, cylindricis; capsula fere sphaerica, quinquelocularis, quipquevalvis, polyspora; receptàculum quinquepar titum; placentá centralis quinque lateribus compressis in loculos exsertis notata: (Frutices humiles depressae, sempervirentes, pilis subulatis ubicunque partium asperae; folia alterna; flores racemosi).

1264. GAUL THERIA , (GAULTHÉRE) KALM. xINN. JUSS. IAM. illustr. tab. 367. VITIS IDAEA Tourn. (oricá I.) - Calyx duplex; exterior diphyllus; interior campanulatus, quinquefidus, nudus (extrorsum munitus duabus squamis in G. procumbente) persistens; corolla fere globosa, ọata, limbo quinquefdo, laciniis brevibas, extrorsum sevolutis : stamina inzerta bási corollae; filamenta villosa; antherae apice furcatae; ovarium depressum, circtum decem squamulis mucronatis cum filamentis staminum alternantibus (nectarolyma); capsula supera, quinquelocularis, quinquevalvis, polyspora, calyce persistente baccáeformi apice patente cincta; - (Suffrutices minimae; folia alterna aut fasciculata; flores axillares, fere solitarii aut in xacemos dispositi). 
1264. ARBUTUS, (ARBOUSIER) TOURN. IINN. - JUSS. LANה. illustr. tab. 366 . UVA URSI toURN. (erica r.) - Calyx minimus, quinquepartitus, persistens; corolla ovoidea aut globosa; limbo parvo quinquefido, laciniis extrorsum revolutis; basi pellucida; stamina non exserta; bacca supera, quinquelocularis, polyspora in ARBUTUS TOUR.N.; monospora in UVA URSI TOURN.; radicula supera; cotyledones foliaceae. - (Frutices aut suffrutices foliis alternis; flores axillares aut terminales, et fere racemosi).

1265. THIBA UDIA, (THIBAUDE) RUIZ - PAV. (erica 2.) - Calyx quinquedentatus; corolla urceolata; stamina decem; antherae fissura longitudinali dehiscontes: stylus simplex; stigma quinquangulum; bacca truncata, calyce grossificato coronata, quinquelocularis loculis folysporis; (Frutices foliis alternis aut oparsis, sempervirentibus, coriaceis; pedunculi solitarii, axillares aut terminales; flores penduli, saepe laterales; baccae subacidae, edules.

\section{Flore apetalo aut incompleto.}

1266. DAÏ S, (DAÏS) IINN. JUSS. LAMr. illustr. tab. 368. * GNIDIA Linv.? (daphnacea). - Flores aggregati in fasciculum terminalem, basi involucrati, sessiles; involucrum tetra-pentaphyllum; calyx tubulosus, elongatus, longissimus, limbo quadri-quinquefido; corolla nulla. - (PERs.: calyx nullus: corolla 4. 5. fida). - Stamina octo-decem, inserta ad medium altera super altera; ovarium inferno calyci adnatum; stylus filiformis; stigma capitatum; hacca monospora; - (Frutices; folia in quibusdam speciebus opposita.)

1267. GE T O N I A, (GETONIA) nOXB. CALYCOPTERIS гAM. ill. tab. 357.? - Calyx superus, pentaphyllus, persistens; filumenta alternatim latiora, quinque in calycis ore; spora crustata, oblonga, qृuinquestriata, calyco coronata.

$$
\text { [21] 1268. A NA- }
$$


1268. A N A I N GA, (ANAVINGUE) RHIED. JUSS. LAM. tab. 355. CASAREA JACQ. IROUCANA AUBC. ATHENAEA SCHREB. MELISTAURUM Forst. PITUMBA AUBL. (ex ordine plantarum sedis incertae). Calyx pentaphyllus vel quinquepartitus; corolla nulla; nectarolyma tetra - pentaphyllum, vel squamae octo-decem, barbato-rillosae, insertae alternatim inter stamina; stamina octo, decem, hypogyna?; ovarium superum; stigma capitatum; capsula baccata, coriacea, stylo superata, intus pulposa, unilocularis, trivalvis, valvulis sporiferis polysporis; sporae nidulantes; - (Frutices aut suffrutices foliis alternis, flores in racemis corymbosis aut aggregati super pedunculum solitarium, saepissime axillares; - hoc genus simile est Piriquetae, ofamilia cistorum).

I269) CRATERIA, (CRATERIE): CHAETO. CRATER RUrz. et parox in systemate florae Peruv. et chilensis. - Calyx quinquepartitus; corolla nulla; nectaroiyma crateriforme, setis decem hirtis coronatum; stamina breviora alternatim nectarolymatis setis inserta; stigmata tria; fructus capsula? unilocularis exsucca? *)

I 270. BUCID A, (BUCIDA, Grignon) Irra. Juss. LAмr. ill. tab. 356. (terminaliacea). - Calyx campanulatus, quinquedentatus; corolla nulla; stamina calyci lon. giora ; bacca exsucca, ovata, unilocularis, monospora, calyce coronata ; - (Folia coadunata ad apicem; flo. res in spicis axillaribus aut terminalibus).

127l. TE PII N A LIA, (BADAMIER) IINN IAM. tab. 348. 349. (terminaliacen). - Polygama; flores hermaphroditi: calyx quinquefidus, interue coloratus et vil10.

*) Anctores gravissimi pericarpinm tenervimum offenderunt, caque propter, quaenam species pericarpii esset, detcr. minare nullo pacto potuerunt; ovarium autem pericar. pinm uniloculare denotabat, vel potius cansulam diversat speciei. Caeterum ab antecedente genere hoc inpri. mis nectarolymatis forma distinctum, fortasie et frictu. 
losus, limbo patente; corolla nulla; appendiculus (nectarolyma) urceolatus, formatns e corpusculis quinque hispidis in fundo calycis (schreb. -); stamina docem; stylus unus; drupa infera, compressa, medio inflata et cincta margine tenello vel carinato; nux unilocularis, monospora ; - flores masculi : calyce, cornlla, appen. diculo, staminibus ut in hermaphrodstis. (Arbores; folia disposita in rosulas circum gongylum ramosum et spinis florum intermixta; flores in spicam dispositi ad basin hermaphroditi, ad apicem masculi.

I272. A PALATOA, (APALATOU) AUBL. CRU. DIA SChreb. TOUCHIROA lam. tab. 339. (legumirosa). - Calyx monophyllus, turbinatus, limbo quadrifido, basi munitus duabus bracteis; corolla nulla; filamenta basi dilatata; legumen (samara) totundato orbiculatum, compressum, marginatum ala membranacea $t$ undulata, subdisporum.

1273. CHUNCOA, (CHUNCHOA) JUss. PAv. pERs. GIMBERNATIA puIz-pAv. (terminaliacea). Calyx limbo quinquefido patente campanulatus, deciàus ; corolla nulla; capsula drupacea, quinquangula, angulis inaqualiter alatis, alis duabus oppositis maioribus, non coronata, monospora; - (Arbores).

1274. PAMEA, (PAMIER) AUBI. Guy. t. 359. JUS5. (terminaliacea). - Calyx trifidus; stamina. . . . ? drupa ovalis, triangula; $\varkappa u x$ monospora; - (Arbor foliis confertis in gemmis ramorum oblongorum, integrorum ).

1275. TA N I B O UCA, (TANIBOUCE) AUBL, Guy. tab. 178. Juss. (terminaliacea). - Calyx urceolatus, quinquefidus, basi bractea munitus; stamina decem; ova. rium inferum; stylus unus, stigma unum; frucius.... (Folia ovalia, integerrima).

1276. DET AR I U M, ( DETAR). Juss. cMer. (16. guminosa ro.) - Calyx quadrifidus : corolla nulla; filamentu alterna broviora 3 drupa orbiculata, mollis: rotun. 


\section{CLASS. X. DECANDRIA.}

rotundata, crassa, farinacea, continens uncem osseam compressam, monosporam, supra infraque fibris duris multis reticulatam, obtusam et glabram.

1277. C O PAÏ F ERA . (COPAIIU, Copaier) IINN. Juss. IAM. tab. 342. COPAIIVA JAcQ. (leguminosa 10.) - Calyx patens, quadripartitus, patens; corolla nulla (cal. nullus corollae petala quatuor P.); stamina distincta; antherae deflexae; legumen ovatum, bivalve, monosporum: spora arillo baccaeformi ovato pulposo obtecta: (Arbor foliis alternis, impari pinnatis; flores in racemis paniculatis axillares.

I=73 CALPIDIA, (CALPIDIE) AUBERT DE PETIT THovars. (nyctagynea *).) - Flores apotali, aggregati, in parvam umbellam dispositi, ad apicom ramorum pariculati. Calyx petaloïdeus, campanulatus, quinquepartitus, diplostemon; corolla nulla; stamina decem hy* pogyna, basi calycis inserta; stigma villosum; ovarium monosporum; fructus capsula e calyce elongato formata, prismatica, pentagona, angulis visco indutis; embryo rectus; cotyledones aequales, foliaceum tipum carnosum involventes; (Arbor humilis; elongatio ramosa, ramis maxime dissitis; folia alterna i flores terminales).

1279. GODOYA, (GODOYE) RUIZ. PAV. GODO. VIA PERs. - Caiyx triphyllus, coloratus; nectarolyma cilia quinquestriata ; antherae poris geminis dehiscentes pollen effundentes; stigma quinquangulare; capsula quinquangularis; sporae imbricatae, alatae.

128) A QUILARIA , (GARO, bois d'aigle) Juss. IAnI. tab. 356. CAv. tab 224. (ex ord. plantt. indeter. minato). - Cajx turbinato campanalatus, limbo quinquefido, persistens; corolla nulía appendiculus (nertarolyma) campanulato urcaolatus, corollae formam affectans, quinquefidus, interno staminiferus, medio calycis insertus (hinc stamina menodelpha videntur);

mare

*) Certe a nyctagineis non diffcrxs viletur nisi staminum mumero. 
margine inaequaliter decemlobatus; filamenta brevissima, inter urceolum posita; antherae oblonga $\theta$, versatiles; ovarium superum; capsula amygdaliformis, magna, bilocularis loculis monosporis, biválvis valvulis subero. sis, incrassatis, medio septiferis; sporae semiarillatae, arillo spongioso.

1281. A UGEA, (AUGEA) тHUNв, nov. gen. pars IX. - Calyx perianthium monophyllum; canalicula. tum, persistens, quinquepartitum, laciniis ovatis, obtusis, concavis, flavescentibus, erectis, lineam longis; corolla nulla; nectarolyma monophyllum, basi calycis in. sertum, ovarium cingens, decemdentatum, brevissimum; stamina: filamenta decem, dentibus nectarii inserta, capillaria, erecta, brevissima; antherae subulatae, sulcatae, erectao, flavae, calyce breviores. Pistillum: ovarium superum: stylus filiformis, erectus, flavus, brovissimus: stigma simplex, obtusum, flavum; pericarpium: capsula baccata, tereti oblonga, obtusa, glabra, decemstriata, pulposa, decemvalvis, decemlocularis, pollicaris; sporae tunica propria cinctae alba, plurimae, lenticulares, glabrao, virides. - Hinc differ. char.: calyx quinquepartitus; corolla nulla; nectarolyma decomdentatum; capsula decomlocularis, decemvalvis. - (E monadelphiis hac relatum genus). -

I282. CEODES, (CEODES) IORST. tab. 7T. JU8:. (ex ord. plantt, sedis incertae). - Calyx nullus; corolla monopetala, turbinata, limbo quinquefido; stamina decem; quinque alterna cum corollao laciniis hisque magnitudine aoqualia; quinque laciniis corollae opposita, minora; antherae rotundata $\theta$, ovarium . . . . . s stylus unus; stigma dilatatum; fructus......;- (Flores in umbellas dispositi).

1283. ADENOSTEMUM . (ADENOSTEMUM) pers. ench. p. 46\%. GOMORTEGA RUIz. PAV. (ex ord. plantt. sedis incertae). - Calyx (corolla P.) petaliformis, sex-septempartitus, foliolis basi coalitis; folioia quatuor exteriora; stamina decem triplici serio, et totidem breviora (gradatim) quot centro proxima; antherae 
oblongae, longitudinaliter filamentis affixae; filamente singula ad basin duabus glandulis podicollatis ornata; ovarium superumi: stylus brevis; stigmata duo vel tria; drupa crassa, ovalis, unilocularis; nux durissima, triquadristriata, bi-trilocularis; viuclei (sporae) compressi, in loculo quoque unus; nucis loculamenta tot, quot stigmata ; nux basiobtusa vel acuminata; nucleus unicus saepe adolescit.

1284. SAMYTA, ( SAMYDE) JACQ. IINN. JUSS. IAMr. illustr. tab. 355. (ex ord. plantt. sedis incertae),Calyx monophyllus, tubuloso-campanulatus, decemstriatus, interne coloratus; limbo inaequaliter quinquefiảo; corolia nulla; stamina decem ad duodeviginti; filamenta medio calycis inserta, in coronam connata, decem vel duodeviginti striis munitam, decem-duodeviginti dentatam dentibus antheriferis; ovarium superum; stylus et stigma numoro simplici; capsula coriacer, unilocularis, quadri-quinquevalvis, valvulis polysporis ; - (Frutices: folia alterna, stipulata; flores axillares, subsolitarii).

\section{$D \quad I \quad G \quad Y \quad N \quad I A$.}

1285. SC L ERA N T HUS, (SCLERANTHE, Gna. velle) EINN. LAM. tab. 374. ALCHEMILLA TOURN. (portulacea 1.) - Calyx monophyllus, tubulosus, ore attenuato, extremitate quinquefidus; corolla nulla; stamina calyci inserta; capsula minima, membranacea, mono-dispora, calyce tecta; - (Elongatio herbacea; folia opposita, linearia).

I286. TRIANTHE MA, (TRIANTHËME) SAUV. LINN. JUSS. LAM. illustr. tab. 375. PAPULARIA, RACOMA, GYMNOCARPOS. FORSk. ZALEIA BURM. (portulacea 2.) - Calyx quiuquefidus, 'laciniis interne coloratis, sub apice mucronatus, persisteris; corolla nulla; stamina quinque, raro decom, imo duodecim; antherae rotundatae styli unus, duo; stigmata simplicia; oycrium retusum; capsula oblonga, ad infernam partem calycos 
calyce cincta, apice truncatã, circumscisse dehiscens, bilocularis loculis disporis, sporae altera alteri superincumbentes; - (Flores axillares, capitati, sessiles; olongatio herbacoa).

1287. CHIYSOSPLENIUM, (DORINE) TOURN, LINN. JUSS. LAM. illustr, tab. 374. (saxifragea 2.) - Calyx superus, quadri-quinquefidus, ilaciniis aequalibus persistentibus intusque coloratis; corolla nul1.; stamina octo vel decem brevia; ovarium inferum, duobus stylis duobusque stigmatibus terminatum; cap. sula birostrata, unilocularis, bivalvis valvulis, polysporis; - (Flores laterales octandri, parvi, sessiles; olongatio herbacea; folia crassiuscula, simplicia, opposita aut alterna).

1288. ROYENA, (ROYENE) LINN. JUSS. rAM. illustr. tab. 370. (obenacea r.) - Calyx urceolatus, quinquedentatus vel fissus, persistens; corolla urceolata, monopetala, brevis, quiriqueloba limbo revoluto, ad basin calycis inserta ; stamina brevia; ovarium suporum, duobus stylis terminatum; capsula baccata, supera, quadrisulca, quadrilocularis, loculis morrosporis (quatuor nuces) quadrivalvis; sporae arillo cinctae; radicula supera; (Frutices; flores axillares, saepe pedunculati).

2289. HYDRANGEA, ( HYDRANGÉE) CRON. IINN. 'juss. IAM. illustr. tab. 380. (saxifragea 2.) Calyx superus, quinquedentatus ; corollae petala quinque maiora, caduca; filamenta alterna longiora; ovarium inferum; styli duo; stigmata persistentia; capsula fero hemisphaerica, bilocularis, calyce coronata, rostris (styli persistentes) duobus terminata, debiscens poro vel foraminulo centrali inter cornua stylorum s. rostra, poly. epora; sporae minimae; - (Frutex foliis oppositis; flores parpi, oppositi, in corymbum torminalem dis. positi).

1290. SAXIFRA GA, (SAXIFRAGE) TOURN, IINณ. JUss. LAMr. illustr. tab. 372. G BUUM tourn. (saxifragea x.) - Calyx quinqquefidus vel partitus, persistens; co. 
rolla pentapotala; ovarium suporum aut semiinferum: capsula rostris duobus (stylis persistentibus) torminata, unilocularis, polyspora, ad apicem dehiscens poro vel foraminulo rosiris interiecto; forma diversa, structura simili ; - (Folia alterna, rarius opposita, integra, interdum omnino omnia radicalia; flores diversimodo dispositi).

129I. TI A RELLA, (TIARELLE) IINN. JUSS. LAM. illustr. tab. 373. VENTEN jard. Malm. t. 55. MITELLA tourn. (saxifragea i) - Calyx quinquefidus vel partitus, persistens: corollae petala quinque calyci inserta, integra ; capsula oblonga, bicornis, unilocularis, bivalvis, valvnla altera multo maiori, polyspora; (Folia saepe radicalia, simplicia aut ternata).

1292. MITELLA, (MITELLE) TOURN. IINN. JUss. IAM. illustr. tab. 373. (saxifragoa I ) - Calyx quinquefidus; corollae petala quinque laciniata vel pinnatifida, calyci inserta: capsula oblonga, bicornis, unilo. cularis, bivalvis, valvulis magnitudine aequali, polyspora; - (Folia simplicia, saepe radicalia, flores in elongatione membranacea nuda).

1293. CUNONIA, (CUNONIE) IINN. JUS3. IAM. illustr. tab. 371. (saxifragois affinis). - Calyx quinquepartitus; corolla pentapotala; stumina exserta ; ovarium superum; capsula ovalis, acuminata, bilocularis, polyspora ; styli flore longiores; - (Arbores foliis oppositis, pinnatis; flores in racemis terminalibus).

1294. G YPSOPHILA, (GYPSOPHILE) IINN. JUss LAM illustr. tab. 375. LYCHNIS ToURN. (caryo. phyllea 5.) - Caly $x$ monophyllus, campanulato tubulosus, angulatus, quinquefidus laciniis' profundis, marginibus membranaceis; corollae petala qninque, ovata, vix unguiculata, subsessilia; capsula glnbosa, unilocularis, quinquevalvis, polyspora; - (Flores numero plurimi, parvi, in paniculam dispositi).

* I A T R IS med. monad. p. 1I1. calyx monophyllus, campanulatus, limbo profundo quadrifido; 
corollae petala quatuor unguicúlata, tubo filamentorum affixa; filamenta in tubum campanulatum connata, ut in rnelochia; sed in filamenta octo divisum styli cornua distantia ; fructus capsula ovalis; E. g. Gypsophylla arborea. (Clàssis octandriae civis).

2295. SA PONARIA, (SAPONAIRE, Savonnieve) IINN. JUss. LAM. illustr, tab. 376. LYCHNIS TOURN. (caryophyllea 5.) - Calyx monophyllus, tubulosus, quinquedentatus, basi nudus; corollae petala quinquo unguiculata ; capsula oblonga, unilocularis, polyspora; - (Flores axillares, saepissime potius corymbi terminales).

I296. D I A N T H US, OEILLET) IINN. JUSS. IAM. illustr. tab. 376. CARYOPHYLLUS TOURN. (caryophyllea 5) - Calyx monophyllus, tubuloso-cylindraceus, longus, coriaceus, quinquedentatus, basi squamis quatuor-octo imbricatis munitus; corollae petala quinque urguiculata, saope ad limbum dentata; styli duo, ut plurimum reflexi; capsula cylindrica, unilocularis, apice quatuor valvulis deliscens, polyspora; - (Flores terminales, solitarii aut numero plures, et tunc ag. gregati aut distincti).

121.7. ' R O K EIE K A . (ROKEIEKE) rORSK. JUSS. (portulacea 1.) - Calyx persistens, quinquefidus, laciniis margine membranaceis; corollae petala quinquo magna, marcescentia; stamina decem; ovarium superum; styli duo disiuncta; stigmata duo; capsula compressa, laciniis calycinis et potalorum persistentium cincta; - (Planta basi lignosa; rami diffusi ot articulati; foliz opposita, sessilia, integra).

\section{T R I G Y $N I A$}

1298. ARE N A R I A, ( SABLINE, Arénaire) IIN JUss. ram. illustr. tab. 378. ALSINE tourn. (caryophyllea 5.). - Calyx pentaphyllus vel quinquepartitus laciniis profundis, patens; corollae petala quinque, in- 
tegra; styli tros; capsula unilocularis, apice in valvulas quinque dehiscens, polyspora. *)

1299. S TELLARIA, (STELLAIRE) IINN: IAM. tab. 378. (caryophyllea). - Calyx quinquefidus, laciniis profundis (pentaphyllus $\mathrm{P}_{0}$ ), patens; corollae petala quinque, bipartita; capsula ovata, unilocularis, polyspora, apice valvulis (vel dentibus) sex déhiscons. (Flores axillares aut terminales).

1300. DEUTZIA, (DEUTZIE) THUNB. iap. tab. 185. LAM. tab. 380. JUSS. (ex ordine plantt. sedis incertae). - Calyx campanulatus, brevis, quinque - rarius sexfidus; corollae petala quinque, rarius sex, obtusa, indivisa; filamenta sub apice tribus rostris trifida, quinque alterne posita reliquis minora; ovarium superum, ad medium concavum; styli tres, rarius quatuor; capsula globosa, parva, perforata, triaristata stylis persistersibus, basi dehiscens, trilocularis, rarissime quadrilocularis, trivalvis, polyspora.g - (Arbor ramosa; folis opposita; flores in panicula).

330I. CUCUBALUS, (CUCUBALE, Behen ) TOURN. LINN. JUSS. LAM, illustr. tab.377. LYCHNIS tourn. (caryophyllea 5 ). - Calyx tubulosus, plerumque inflatus aut ventricosus, aut campanulatus, quinquedentatus; corollae petala quinque, unguiculata, denudata ad basin, lamina coronata ad faucem saepe bifida; capsula trilocularis, in quinque - sex valtulas apice dehiscens, polyspora. - (Flores axillares, saepius terminales, dispositi in spicas terminales ).

1302. SII,ENE, (SILÈNE, Carnillet) IINN. JUss. xAM. illustr. tab. 37\%. LYCHNIS tourn. (caryophyllea 5.). - Calyx oblongus, teres, cylincricus aut tubuloso conicus, ventricosus, quinquedentatus; corol-

*) SPERgULARIA; genus sub ARENARIA, habitu Spergulae, floribus Arenariae; elongatio trunci ad genicula stipulacea, humifusa. Cf. quae hane in rem dicta leguntur

azRsOONLO ench, pag. 505. 
lae petala quinque unguiculata unguibus rectis, longitudine calyci aequalia; lamina plana, saepo bifida, ina terne ad basin nuda aut coronata duobus appendicu. lis forma dentiura; capsula trilocularis, dehiscens apico in quinque vel sexvalvulas, polyspora. - (Pedunculi uni - multiflori, axillares aut terminales, positi interdum in dichotomia elongationis trunci ramorumque).

3303. CHERLER I A, (CHERLERIE) HALI. IIN JUSs. LAM. illustr. tab. 379. (caryophyllea 4.). - Calys quadri - quinquefidus (tetraphyllus $P_{\text {. }}$ ); corollae potala quinque, minima, omarginato bifida (nectarolyma bifidum P.); antherae alternae steriles; capsula trilocularis loculis disporis, trivalvis (polyspora SMITH). - (Planta minima; folia linearia, in rosulas disposita 3 flores 80litarii).

1304. HOR TENSIA, (HORTENSIE), COMMERS. juss. VENT. LAMT. illustr. tab. 380. HYDRANGEA вмгтн (caprifoliacea). - Flores difformes: exteriorum cymam constituentium calyx quadri aut quirquepàrtitus (pbyllus), maximus, coloratus, petalö̈deus, marcescens; corollae petala quatuor, quinque, minima, concava, subglobosa, caduca 3 stamina decem, interdum sex aut octo ; filamenta cylindrica; rudimentum ovarii abortiVum: styli duo vel tres, incrassati, conniventes; stig. mata obtusa. Flores interiores soliturii: calyx superus, quadn - quinquedentatus minutus; corollae potala quatior, quinque, calyce maiora, caduca ; stamina ut in floribus oxternis; ovurium inferum, triloculare; styli duo, tres, quatuor remoti vel disiuncti, emarginati; stigmata obtusa; fructus triloculares polyspori. - (Flores corym. bosi'; folia utrinque glabra).

305. GARIDELLA， ( GARIDELLE ) тоUлN. LINN. JUSS, LAM. illustr. tab. 379. (ranunculea 2.). Calyx parvus, pentaphyllus, petaloideus; corollae petala quinque magna, bilabiata, bifida (nectarolyma); capsulae tres oblongae, acuminatae, approximato-connexae, polysporae - (Elongatio herbacea; folia composita, laciniata; flores foro solitarii, terminales). 
1306. BAN I STERIA, ( BANISTÈRE) Houș. IINN JUss. raM. illustr. t. 381. (malpighiacea t.). Calyx parvus, tri - quadrifidus, basis laciniarum adaucta duabus glandulis; corollae petala quinque, crenulata, unguibus linearibus; staminum decem filamenta corollae breviora; styli tres; ovarium trilobum; squamae tres (una; duae abortivae), ad apicem ala longa mombranacea terminatae, monosporae. - (Arbores ot frutices plurirnum sarmentosao; foliorum petioli interdum vel ad basin vel ad apicem duabus glandulis muniti).

1307. HIRAEA, (HIRAEE) JAcQ. WILLD. FLABELLARIA cAv. (maipighiacea 1.). - Caiyx pentaphyllus absque poris melliferis; coroliae petala subrotunda, unguiculata; filamenta basi cohaerentia; samarae tres, monosporae, bialatae. - (Videtur auctoritate JUSSIEUI CUM TRIOPTERIS genere esse coniungendum).

1308. TRIOPTERIS, (TRIOPTÈRE) LINN. JUSS: IAM. tab. 382. (malpighiace 1.). - Calyx minimus, quinquefidus; corollae petala quinque, unguiculata, unguibus longis; stamina alterna breviora; stigmata tria vel sex; styli tres; samurae tres, globosae, tribus alis munitae, ala una breviori ot tenuiori, monosporae. (Frutices aut suffrutices saepe sarmentosae flores in paniculam dispositi).

1309. TETRAPTERIS, (TETRAPTÈRE) CAVAN. PERS. TRIOPTERIS wILID. (malpighiacea 1.). Calyx quinquepartitus, utrinque biglandulosus; corollae petala quinque fimbriata, unguiculata; fructus quatrialatus, alis duabus inferioribus minoribus.

I3IO. ERYTHROXYLUM, (ERYTHROXYLE) BROWN. IINN. JUSS. IAM. ill. tab. 383. (malpighiaceis affinis). - Calyx turbinatus, quinquedentatus; corollae petala quinque, non unguiculata, basi squama emarginata munita; staminum decem filamenta basi in urceolum coalita; antherae rotundatao; ovarium simplex; styli et stigmata numero terno; drupa oblonga, cylindricn, angulosa, nuce monospora; albumen nullum; embryonis 
Lobi plani, recti. - (Arbores aut frutices ramis alternis, apice compressis, interdum squamosis; folia alterna, integra; flores laterales podunculati, solitarii aut fasciculati).

13II. MALPI GHIA, (MOUREILLER, Malpighie) PLUM. IINN. JUSS. LAM. ill. tab.381. GALPHINIA cAv.? (malpighiacea 2.). - Calyx quinquefidus, laciniae quibusdam externe duabus glandulis munitae; corollue potala quinque, unguiculata unguibus linearibus; staminum filamenta aequalia, corolla breviora; styli tres; bacca drupacea, oblonga, globosa, cylindrica, continens nuces tres oblongas, angulosas, monosporas. (Arbores aut frutices interdum sarmentosao; folia quarundam specierum sive pagina interiori sive ad margines pilis rigidis centro affixis pungentibus exasperata, oxtremitate liberis; petioli saepe basi interne appendiculati et dilatati; pedunculi axillares aut terminales, uni aut mulcillori). - GALPHINIA cav. calyce infero quinquepartito, petalorum quinto superiore maiori, fructu triloculari monosporo, inprimis calyce glandulis nudo distinctum esse dicitur a MALPIGHIA. *)

\section{T $E$ E $T R R A G \quad Y \quad N I A$.}

1312. M I CROPET A LON, (MICROPETALON) PERS. SPERGULASTRUM MICHAUX. (caryophyllea?). - Calyx pentaphyllus; corollae potala minutissirna, integra, aut nulla; capsula ovata, calyco longior, guadxivalvis. - (Habitu Stellariae).

$$
\begin{array}{llllllllll}
\boldsymbol{P} & \boldsymbol{E} & N & T & A & G & Y & N & I & A
\end{array}
$$

1313. THYSANUS, (THYSANE) LOVR. conch. I. p.349. (terebintacea 4.). - Culyx profundo quinquefidus,

*) RUDOlphia MED. monad. p. nr. MLALPIGHIA IINN. Calyx perreaphyllus, foliolo singulo biglanduloso; co. rollue petald quinque ovalia, rotundata, singula diversi. mode posita et formata, unguiculata, ungue tenni; stamina ad medium in, "ubum conuatz; filamenta quis- 
dus, patens, villosus; corolla pentapetala; stamina de. cem brevia; ovaria quatuor; styli quatuor laterales; stige mata quatuor, apice parum bifida; drupae quatuor, calyce cinctae, oblongae, inflatae, apice reflexae, putamino lanoso tectae, uno tantum latere dehiscentes, monosporae: spora arillo imperfecto tecta. - (Frutex excelsa; folia pinnata; podunculi laterales uniflori).

1314. A VERRHOA, (CARAMBOLIER, Bilimbi) IINN. LAM. tab. 385 (terebintacea 5.). - Calyx par$\nabla$ us, quinquepartitus daciniis profundis; covollae petala quinque, limbo patente, unguiculata; filamenta basi coalita in nectariferum annulum, alternantia breviora, interdum omnia antherifera, interdum alterna antheri fera; ovarium quinquangulum; styli quinque, persistentes; bacca magna, carnosa, ovoïdea, pentagona, pro. funde sulcata inter angulos, pulpa acida repleta, divisa in quinque Ioculos, loculis mono- di - polysporis. CFrutices; folia alterna, impari pinnata; flores dispo. siti in racemos paniculatos insertos ad extremitatem elongationum trunci, interdum axillares, parvi, rubri; quidam abortientes).

315. CNEST S , (CNESTIS) JUss. IAM. illustr. tab. 387. (terebintacea 4 ). - Calyx externe tomentosus, quinquepartitus; corolla pentapetala (receptaculo inserta?); stamina decem, receptaculo inserta ovaria quinque pilis exasperata; capsulae quinquo (quatuor abortivae), coriaceae, breves, leguminiformes, utrinque villosae, bivalves valvulis extrorsum introrsumque pilis acutis urentibus cinctis, latero dehiscentes, monosporae. - (Frutices interdum prostratae : folia alterna, pinnata cum impari, rarius ternata; flores in spicis axillaribus ).

316. COTYLEDON, (COTYLEDON, Cotylet) ToURN, LINN, JUSS, LAM, illustr, tab. 389. UMBILI.

CUS

que breviora, quiaque longiora, recta, disinncta; ova= jium intra tubum; drupa externe carnosa, interne nucculis sporis quatror commatis repleta, monosporis sec, XLVA. SLOANE). 
CUS DECAND. (crassulea). - Calyx quinquīpartitus; corolla monopetala, tubuloso-quinquepartita vol fissa, lacin:is rectis aut extrorsum volutis; stamina ut plurinum decem, rarius quinque, super corollam inserta; ovaria quinque, unoquoque cincto ad basin squama ovali nectarifera (nectarolymata); capsulae quinque, uniloculares, interne bivalves, polysporae. - (Elongatio herbacea aut frutescens: folia opposita aut alterna, interdum pinnata; flores terminales).

13I7. SEDUM, (SEDUM, Orpin) TOURN. IINN. JUsB. IAM. illustr. tab. 390. ANACAMPSEROS TOURN. (cras= sulea). - Culyx quinquepartitus vel fissus; corolla per. tapetala; ovaria quinque, unoquoque eorum cincto al basin squamá nectarifera; capsilae quinque, uniloculares, interne bivalves, polysporae. - (Elongatio trunci lierbacea; folia alterna, plana aut cylindrica; flores in corymbis ).

13I8. PENTHORUM, (PENTHORE) IINN. JUSS. rAn. illustr. tab. 390. (crassuleis affine). - Calyx quinque-decomfidus; corollue petala quinque minima, rarissime nulla; alterna cum laciniis calycinis; antherae rotundatae; ovaria quinque, basi interne adhaorentia: fructus rotundatus, formatus capsulis quinque, basi erccto connexis, apice remotis vel liberis, ad summum omarginatis, tricuspidatis ; quinquelocularis, polysporus; sporae minimae. - Folia oblonga, non carnosa; flores in spica paniculata et terminali).

13ิ19. BERGIA, (BERGIE) LINN. suppl. THUNE: juss. (caryophyllea 4.). - Callyx patens monophyllus, caducus, quinquedentatus vel partitus; corolla pentapeıala: styli quinque broves, approximati; stigmata persistentia; capsula una, globosa, quinquelocularis (quin. quetorulosa), quinquelocularis, quinquevalvis valvulis patentibus in formam petalorum; loculis polyoporis.. (Bacca ovalis, cortice crasso, unilocularis, monospora roxв. Corom. 2. p. 22. tab. 142.). Flores verticillati ; tolia lanceolata, ad apicom doaticulata). 
1320. T A I R I A, (TAPIRIER) AUBI. I p.470. tab: 188. JÙss. LAM. illustr. tab. 386. JONCQUETIA SCHRE. willd. (terobintacea 2). - Culux (pentaphyllus?) quinquepartitus; corollae petala quinque, affixa super discum exsertum, infra ovarium collocatum: stamina insorta eodem disco hypngyno, exserto: stylus nullus; capsula unilocularis, pentacocca, quinquevalvis, ad val. vulas striato sulcata, pentaspora; sporae arillo tectae. (Arbor; folia alterna, pinnata cum impari, foliolis tri-quinquestriatis; flores in paniculis axillaros aut terminales ).

1321. SPERGULA, (SPARGOUTE) IIN. JUSS. IAM. illustr. tab. 3y2. ALSINE ToURN. (caryophyllea 4.). - Caly $x$ quinquepartitus (pentaphyllus); corollae petala quinque, integra; capsula ovata, unilocularis, quinquevalvis, polyspora; sporae emarginatae. - (Fo. lia verticillata, stipulacea aut opposita nudaque; flo. res axillares et terminales; stamina interdum quinque).

1322. CERASTIUM, (CÉRAISTE) IINN. JUSS. IAN. illustr. tab. 392. MYOSOTIS rourn. (caryophyllea 4.). - Calyx quinquepartitus (pentaphyllus): corollae petala quinque, bifida aut emarginata, passim integra; capsula globosa, magnitudine caiycis, aut foro cylindrica et calyce longior; unilocularis, apíce dentatim dehiscens, polyspora. - (Flores terminales).

1323. A GROSTEM M A, (AGROSTEVIME, Coquelourde) IINN. JUss. LYCHNIS IAM. et TOURN. (caryophyllea 5.). - Calux monophyllus, tubuiosus, angulatus. quinquedentatus ; corollue vetala quinque, unguiculata unguibus longitudine tubi calycis fauce appendiculo arguto coronata; laminae obtusae leviter emarginatae; styli quinque; capsula unilocularis, apico in quinque valvulas dehiscens, polyspora.

1324. GITHAGO, (NIELLE) DESFONT. VENT. AGROSTEMIVA IINN. LYCHNIS LAM (caryophyllea 5.). - Calyx tubulosus, angulosus, apice quingraepartitus, laciniis terminatis foliolo longo; corollae potala 
quitinque, unguiculata, ungues longitudine tubi calycis; laminae obtusae, leviter emarginatae, appendiculo nullo nndae; styii quinque; capsula unilocularis, apico in quinque valvulas dehizcens, yolyspora.

I325. LYCHNIS, (LYCHNIS, Lychnide, Lampette) TOURN. LINN. JUSS, LAM, illustr. tab. 39I. (caryophyllea 5.). - Calyx monopiryllus, tubulosus, oblongus, laevis, quinquedentatus; corollue petala quinque, unguiculata, limbo saepo bifido; capsala uni - rarius triquinquelocularis, ad apicem dehiscens in quinque valvulas, polyspora. - (Flores saepe in corymbis terminalibus, interdum in spicis paniculatis).

1326 OXALIS, (OXALIDE, SURELLE) IINN. JACQ. monegr. JUSS. LAM. tab. 39i. OXYS TOURN. MED. monad. p.i13. (geranieis affinis). - Calyx quinquepartitus (pentaphyllus), persistens: curollae hypogynae potala quinque, leviter lateraliter ad basiu unguium connexa; stumina hypogyna, inaequalia, alternantio quinque breviora; flamenta exteriora basi connata; antherae rectae, rotundatae; ovarium simplex; capsula brevis aut oblonga, pentagona, quinquelocularis, localis monopolysporis, quinquevalvis valrulis marginibus in. trantibus et atfixis placentae centrali, externe bipartitis, angulis elastico dehiscentibus; sporae subarillatae, compressa, striis transversalibus notatae, receptaculo affixae; albumen cartilagineum; embryo roctus; cos tylelones foliacare, ellipticas : vadicula supera. $\rightarrow$ (Her bae caulescentes alit scapiformes; radix interdum tuberosa (truncus superficialis): folia alterna, teruata, rarius impari pinnata, circinnation volutae ante expansionem more flicum; pedunculi terminales in elongationibus scapifornibus, axillares aut terninales in elongationibus foliaceis, et tunc unifori et ad medism partem cinatae duabus squamis, aut floribus in umbellis involu. cratis, iuvolucro brevi).

1327. ROUREA, (ROURELIE) AUBt. Guy. I. tab. 467. JUSs. ROBERGIA SCHREB, WILID. (terubintacez 1.). - Calyos quinqueparticus vel fissus, externo to- 
mentosus; corolla pentapetala ; drupa nigra, monospor ; spora putamine (testa) fragili bivalvi tecta. (Juss. , dis. crepat a CNESTIS capsula simplici, caeteris forsan ab. ortivis"). - (Frutox tortuosus, ramis sarmentosis: folia alterna, impari pinnata, ad basin duabus stipulis munita; flnres in paniculis axillaribus et terminalibus, unoquoque bracteato).

1328. BUCHANA I A, (BUCHANANIE) SPREN GEI. ap. SCHRAD. 1801. p. 234. (terebintacea). ... Calyx monophyllus ; corolla pentapetala; nectarolyma orbiculatum, decemsulcum, ovarium involvens; drupa nuce monospora, putamine bivalvi.

1329. SPONDIAS, (NIONBIN) IINN. JUSS. IAN. illustr. tab 384. MONBIN PIUM. MANGIFERA LIN suppl. CYTHEREA IAM. tab. 384. (terebintacea 2.). Caly $x$ parvus, parum campanulatus, quinquedentatus, cum corolla caducus; corollae petala quimque, patentia; stamina decem, interdum octo, inserta disco 'glanduloso, alterna breviora; styli quinque divergentes; drup.d coronata, apico notata tribus - quinque rostris, stylo. rum remanentia enatis; nucleo fibroso quinquangulari, quinqueloculari, interdum tetra et pentasporo. - (4rbores: folia pinnata cum impari, foliolis quinque vol decem seriebus posita; flores in racemis axillaribus et terminalibus; fructus flavus, edulis.

I330. POUPARTJA, ( Juss. (terebintacea 2.). - Calyx parvus, quinquefidus; corolla pentapetala; stamina decem disco crenulato inserta, hypogyna; styli quinque approximati; drupa continens nucem quinquelocularem; unus vel duo loculorum abortivi. - (Arbor insulao Bourboniensis, ubi ab incolis nominatur Bois de Poupart; folia impari pinnata, (interdum simplicia) foliola quatuor seriebus; flores in racomis axillaribus et terminalibus).

I33I. GRIELUM, (GRIEL) IIN t. tAM. tab. 398. (geraniis affine). - Calyx profunde quinquefidus ; corollae petala quiuque basi angustata; filamenta persisten- 
tia: glandulae quinque cingunt ovarium; stigma verru. cosum; pericarpia capsulae quinque monosporae. - (Fru. tex: folia aurona vel absinthii, pedunculi unifori, non bracteati).

1332. SURIANA, (SURTANE) PLUM. LINN. IUSS. LAM, illustr. tab. 389. (rosacea 5.). - Culyx profundo quinquefidus (pentaphyllıs P.); corolla pentapetala; sta. minum decem quaedam abortira; styli interiori ovarii latere inserti; capsulqe quinque, evalves, non dehiscen. tes, monosporae, hinc sporae quinque nudae. - (Fru. tex foliis simplicibus, spathulatis, exstipulaceis; pe. dunculi axillares et tormiñales multiflori; flores bra.
cteati, flavi).

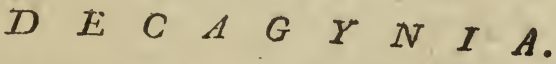

1333. NEURADA, (NEURADE) IINN. JUSS. IAM. illustr. tab. 393. (rosacea). - Calyx parvus, hemisphae. ricus, quinquefidus vel partitus; corollae petala quin. que magna, basi dilatata, inserta calycis limbo: stami. na inserta ut corolla; ovarium inferum; capsula hemisphaorica, orbiculata, infera, inferne convexs, superne plana, aculeata, munita laciniis calycinis rectis et acuminatis, decemloculeris, decaspora.")

1334. PHYTOLACCA, (PHYTOLACCA, Phyto. laque) ToUnN. trN. JUss. LAM. illustr. tab. 393. (atriplicea 1.). - Calyx petalö̈deus, quinquepartitus; co. rolla nulla (pens. calyx nullus; corollae calycinae po tala qutinque!); stamina octo, decem ; ovarium striatum; styli octo, dacem; bacca supera, orbicularis, octodecemsulcata, octo-decerulocularis, loculis monospo. ris: sporae bifidac. - (Flores in spicis, saepenumero foliis oppositi, quinque-septem-octo-decemggni; septem - octo - icosandri : elongatio frutescens; folia ad apicem mucrone recurvo terminata). *) Spora germinans capculann pexforat, annulo instar in colle
radicis, etiara in planta adulta, persiotit, 


\section{$G E N E R A \quad I N C E R T A$}

ABUTA, (ABUTE) AUBL. JUss. (menispermea). ¿ Calyx -; petala - ; stamina -; ovarium - ; baccae tres, magnae, ovales, exsuccao, parum compressae, fragiles; monosporae. - (Frutices elongatione scandente):

BA A S A, (BAGASSE) Aubl. Guy. tab. 376. JUss. (urticeis affinis). - Calyx -; stamina -; ovarium - ; bacca sphaerica, cortice granulosa obtecta, interne dura, externo pulposa, polyspora; sporae ovales. - (Arbor lactescens, ramis oppositis, stipulis caducis $;$ foliis tri. lobis ; fructu citri).

CUSSAPOA, (COUSSABOE) AUBq. Guy. tab. 36:. júss. (urticeis affinis). - Caly: -; stamina -; ova* vium - ; rèceptaculum sphaericum, onustatum sporis pulpa cinctis. - (Arbor succo flavo; folia alterna, stipulis caducis cincta, cicatricem super ramos relinquentia; flcres in racemis).

MACANEA, (MACANEE) JUSS, MIACAIIANEA AvBL. Guy. tab. 37. (gutifera 2.). - Calyx, corolla, staminc, stiłlus, stigna - ignota; bucca shagna, pyriformis, irregularis, cortice coriscoa, interne pulpasa, unilocularis; sporae quatuor aut sex, ovales, coriaceat, nidulantes in pulpa, placentis lateralibus affixae. (Frutex earmentosa; flores axillares, solitarfi). 
MAC UBEA, (MACUBEE) Juss. MACOUBEA AUḄT. Guy. tab. 378. (guttifera 2.). - Calyx, corolla, stumina et ovarium ignota; fructus, simillimus fructu citri, parum compressus, cortice tenui amictus, subasper et pun. ctatus, unilocularis, polysporus; spovae oblongae, subarcuatae, uno latere sulcato striatac, arillatae, ad parie. tes fructus fixae. - (Arbor lactescens, ramis oppositis; fa! in apposita fasciculis ductulorum rransversis; fructus in racemis).

PA MEA, (PAMIER) AUbr. Guy. tab. 359. Juss. (termirailiacea). - Calyx trifidus; stamina ...; drapa ovä lis, trigona; nux monospora. - (Arbor; folia conferta ad ramorum internodios, oblonga, integra).

PA TIMA, (PATIME) AUBr. Ǵuy. tab. 7\%. (rubiacen 9.) - Calyx quinquangulus, limbo integro; corolla -; stargina -; stigma -; bacca infera, sphaerica, coronata, quadri-sexlrcularis, polyspora; sporae pulpa cincrae. - (Elongatio herbacea; folia opposita, integer, fima; fructus in axillis foliorum)。 


\section{ADDENDA ET EMENDANDA.}

\section{$C I A S S I S P R I M A$.}

ad 1. C A N N A, (amomea). Calyx duplex: exterior - interior (corolla I.); filamenta staminum biloba, lo. bo superiori antherifero: stulus petaloïdeus, ensiformis: stigma lineare: capsula trisulca, calyco externo coronata, oligospora; sporue rotundatao, axi centrali alfixae.

ad 3. CATIMBIUM , (amomea). Calux duplex: exterior -, interior (corolla auctt.) - ; unthera linea. ris, sessilis, longitudine laciniae calycis internae; stiga ma peltatum, uni latere perforatum.

ad 4. A MOMUM, (amomea). Calyx duplex: extea rior - interior (corolla t.) tubulisus, limbo quadria partito, -; stigma obtusum: capsula coriacea, calyco externo coronata - Iociulis polysporis. - (Flores spica radicalis in gingaberis, panicula in cardamomis).

ad 7. COSTUS, (amomea). Culyx duplex: exterior tubulesus tridentatus aut -., persistens interior (corolla L.) - laciniis aequalibus, tubo inflato, super ova. rium posito; nectarolyma - labio superiori simplici. lanceolato, breviori, antherifero; antheia geminata; stigma capitatum, bilobiom capsulu calyce externo coronata - angulis dehiscess, Inculi polyspori. - (Flores maxime conferti, in spicam terminalem dispositi).

ad 8. A L P I NIA, pium. Juss. (amomea). Calux du. plex: exterior -, interior (cornlls L ) - stigma triangulum; capsula carnosa, - ; sporce super receptaculuna pulposum affixae. - (Flores in pyramida terminali). 
ad 9. MYROSMA. IrNN. suppl. Juss. (amomea). - tubus calucis interioris (corolla L.) brevissimus, limbo quinquefido, laciniis duabus magnis emarginatis, tribus munoribus longioribus; filamenta staminum inserta medio laciniae inforioris; anthera ovalis; stulus incras. satus, longitudinaliter fissus; stigma valvulaeformis; -

ad Io. MARANTA, plum, JUSs, I.AM, ill. tab. r. (amomea). Calyx duplex: exterior parvus -, interior (cornlla L.) tubulosus - ; stigma cernuum, triangulum; - nuce uniloculari -

ad II. CURCUMA, - juss. (amomea). - Calyx tubulosus, limbo quadrilobo, filumenta staminum guinque linearia, - quinto bifido, ad apicen unius fissu. rao antheritero; stigma restratum.

ad 12. Ih A E PERIA, - juss. - (amomoa). Calyx duplex: exterior -, interior (corolla L.) tenuis, - limbo magno sexpartíto - ; filimentum membranaceum, bicorne, stylum includers; anthera geminata, lateralis; stigma obtusum -. (Folia radicalia; flores subsolitarii e medio foliorum egredientes).

ad I3. THALIA, IINN. ( amomea! Calyx profundo quinquefidus; laciniae tres maiores, crispae; duae minores convolutae, -; stigma simplex; - nucem - disporam aut rarius monosporam. - (Elongatio trunci nodusa et geniculata).

ad 15. LOPEZIA, - VENT. Juss. ann. mus. no. l7. tab. 30. (onagraria 2.). - curollae petala truncata, -, rubra; - filamentum antheriferum album, basi stylum vaginans; stigma capitatum; - ; plucenta contralis. (Elongatio rrunci herbacea; flores axillares; pedunculi lougíssimi, filiformes).

ad 16. PHYLI I D RUM, - (sedis incertas). -.. (Elongatio herbacea recta, simplicissima; folia subulata, crassiuscula; flores in racemis terminalibus).

ad 1\%. VOCHISIA, - filamentum latum, - apice concavum, in cavitate autlierae duae; ovarium superum, triangulum; sty!us longus, carnosus; stigma uno latero convexum, applanatum altero; fructus trilocularis, poJysporus. - (Arbores excolsae; rami oppositi; folia opposita, integerrima).

ad 18. QUALEA, A UzL. Guy. tab. 1. Juss. - - corolla -, petalo inferiori maximo; stamen hypogynum; filamentumi oblongum; anthera oblonga; ovarium superum ; stylus unus; stigma simplox; fructus unilocularis, -; 
sporas in pulpa nidulantes. - (Arbntes foliis opposir tis, integerrimis, nervis (fascicnlis ductu? paralleitis; petioli ad basin stipulis dusbuj caducis muniti: tores ia paniculis terminalibus; an familiae Guttiferurum congener.?).

ad 20. BOFRHAAVIA, - Juss. - ; Invclucrum squamis mininis compositum, flores multos aut raris: sima unum circumdantibus, - stamina unum, duo; spora elongata, basi angulosa (interdum inter angulos muricata:- - (Flores in pedunculis solitariis, axillaribus ot ramificatis, dirisione singula basi squama ad. aucta, uni-multifora) - Jam vero menic. monado pag. 74. Srhaoc: livolucrum ccnicum, supra subpatens, coriaceum; calyx (corolla auct.) involucro insidens, tubo brevi, dein dilatatus, campanulatus, tortus; stamina in calycis fundo orta, membrana connata ambiunt ovariun: deinde intra involucrum disiuncta in filameri ta duo aut tria, quao cavitates calýcis et involucri permeant, latera non attingunt; ovarium superum intra involucrum staminumgue thbum, parvum, oblongum, terminatum stylo nno, stigmate globoso; involucrum persistens post anthesin clausum, induratum, sporam continet unam, putamine proprio tectam involucriformi, spora non replet sporangium, sed apice vacuum remit: tit. - Caeterum iam gravissimus MEDicus hoc genus ad Diandriam referri déebere recte iussit.

Ad 2t. SALMONEA, - SALIIONIA IOUR. (rhinanthacea 1.). -

ad 22. POILICHIA, - (sedis incertae). - ; (Elongatio cylindrica; folıa verticillata; fores sessiles, in capitulum connati;.

ad 23. SALICOR NIA, TounN, - Juss. - (atri plicea 4.). - stamina unum, duo; stylus bifidus; stigmata duo; - (Elongatio herbacea aut frutescenco aphylla; flores minimi, sessiles aut singulo latere ternati).

d 34. HIPPURIS, - JUss, - stamen unum, epifgsnum? filamentum breve; anthera oblonga; ovarism inerum; - spora nuda. - Elongatio trunci cylindrica, simplex; folia verticillata; folia sub aqua reversa, supra aquam assurgentia, acuta; fiores axillares).

2d 25. LA CISTE M A, (sedis incertae). - Calyx (corolla) quadridentata; ovarium globosum; stigmata tria ; - ; spora o valis.

ad 25. CORY SPER MU M, - juss. - (atriplicen 3.j. Stameil unum, rarius duo, tres, quatuor, quingue; 
styli duo. - (Floros axillares, solitarii, sessiles; supe. riores monartieri).

ad 27. CALLITRICITE, - xuss. STELLARIA $\checkmark A \mid L i$ - filamentum longum; Qvarium unum; styli ot stigmata duo. - (Folia opposita; flores axillaros; Di: cotyledo seç. GAERTN.).

ad 28. BLI T U M, (BLĖTE) - Juss. - (àtriplicea 4.) - stuii duo. - (Flores parvi, sessiles, in capitulos axillares aut terminales conferti).

ad 29. CIN N A, - juss. - C. Clores in paşicula, intordum staminibus quinque; undo coniungi cum $t$ AGROSTIS posse videtur).

ad 30. JAR A.VA, - juss. mss. - - corollae (calycis) gluma - arista torta, - pili pappum referunt; stami nis unius anthera oblonga, bifurça.

\section{$C I A S S I S S E C U N D A$.}

ad 3r. N Y T A N T ES, - Juss. - Calyx tubulosus - ; - antherae subsessiles, intra tubum; capsula dorso inflata, laterious compr., - ; spora una in fundo loculi singuli; radicula infera; (Fiarniterragoni; pecun. culi axillares et terminales, multifori; pedicelli triflo rị et bibracteati, ant quinqueỉori et quadribracteati).

ad 33. I A SMINU MI, - rourw. - juss. - ; Cias: minea 2.) - stamina intra tubura; - spora nna aborit. ens; (Folia ternata, aut subpinnata; pedunculi axilla. res et torminales, multifori).

ad 34. SYRIN GA, - I ILA C. TOURN. JUSS. IAM. - (iasminea r.) - stemina intra tubum ; interdum tria, in rarietate S. persicae IINN.; cupsuia - compressa, - lo. culi dispori; sporae - superiori parte dissepimenti, quod in acanthaceis se dividit, affiaae; (Flores oppositi in pañicula terminali).

ad 35. FORSY THIA, - (sedis incertae) siaminc duo: filamenta brevissima: ovarium superum, ovalo; stylus filiformis; - (Elongatio flexuosa; Hures sohitarii, pedunculati).

ad 36. LIGUSTRU M, - rounw. - juss. - (ias. minea 2.) - corolla tubo brevi, limbo patente, qua. drifido: - (Frutex foliis oppositis, ovalibus, integris, foribus in racemis terminalibus; interdum staminatria), 
ad 37. PHYLLIREA, - TourN. - juss. (jasmis) $2^{\prime}$ ) - aibumey carnosum; (Folia sempervirentia; flores in fasculis intra foliorum axilla).

ad 33. OLE $\Lambda$, - TourN. - Juss. (jasminea 2.) Calyx parvas, - ; corolla tubo brevi - ; drupa baccata - ante maturitatem bilocularis et dispora, matura unilocularis ot monospora: - (Folia sempervirentia, opposita, rarissime alterna; flores in paniculis axillaribus aut terminalibus).

ad 39. O I N US, - FRAXINUS TOURN. IINN. JUSS. x.Ast. ill. tab. 858. (jasminea I.) -; stamina duo hypogyna; sticma bifidun : (capsula plana, ala membranacea terminata, monospora). - (Elongatio arborescens: folia alata, impari pinnata, rarissine simplicia; flotes in panicula terminali).

ad $4 \mathrm{c}$. CHIONA N THUS, - Juss. (jasminea 2.) Calyx quadripartitus; coroila tubo brevissimo, limbo -; untherue subsessiles, tubo insertac; - ; - ( Af. boyos; folia simplicia staminasaeje tria; flores in corymbis axillaribus et termualibus).

ad 4i. FONTANESIA, - juss, mss. - (jasminea r.) - stumina basi corollae inserta; stigmata duo acuta, inlexa; capsuía subovalis, - emarginata, - ut plurimum in centro bilccularis -; - (Frutex ramis in iuventute tetragonis; foliis sempervirentibus).

ad 43. ERANTHEMUM, - (verbenacea 3)-rAM. iil. tab. 17. - corolla limbiss planus, parvus; - ana therae subscosiles, interdum corollam superantes; (Plantae herbaceae ant frutescentes; folia opposita aut altera; flores in spicis terminalibus:

ad 47. GALIPAEA, - AUBr. Guy. tab. 259. JUss. - corolu tubulosa, super discum inserta; - - ovarium supertm - ; - (Frutex folis ternatis integerrimis; flores in corymbis axillaribus et termsnalibuy).

ad 49. PAEDEROTA, - Juss. - ; (personata 2.) - corolla limbus patens, bilabiatus - ; fiunenta filifornia: stigma simplex; capsula ovalis - ; - (Herbae folits opposicis, floribus axillaribus aut in spicis ter. minalibus).

ad 50. VERONICA, - TourN. - TUss. - ( rhi. nantíacea 1.) - corollı rotata, - ; càpsitlu cordata, rarius ovalis - ; - (Plantace lierbaceae, larissime srutescentes; folia ut plurimum opposita aut verticillata; flores in spicis terminalibus aut axillaribus). 
ad st. GRATIOLA. - suss.. - DIGITALIS TOtnx. MONNIERA Brown. (personata 3.) - Calys quinquepartitus, basi duabus bracteis auctus: corolla tubúlosa, tubo rotundato, calyce longiori, striata, subbilabiata; labio superiori bilobo aut emarginato; infe. riori a equaliter trifido, interdum interne barbato; filamenta - duo fertilia; quinti rúdimentum; stigma bilobum; capsula ovö̈dea, - valvulao saepe ad apicem bifidae: dissepimentum simplex: - Elongatio trunci herbacea; folia opposita, podunculi axillares, uniflori).

Inseratur: * CHAET OCHYLUS , (CHAETO.

- CHYLE) vart. (ex ord. plante. sodis incertae). Calyx quinquefidus, subbilabiatus; covolla tubulosa; limbo quinquefido, laciniis linearibus, duabus brevissimis; filamenta staminum duo ad medium tubi inserta: ovarium superum; stigma obtusum; capsula acuminata, bilocularis, polyspora; - (Elongatio frutescens; rami alterni, cylindrici; folia alterna, integerrima; pedunculi solitarii, uniflori).

ad 53. SARMIENTA, - (sedis incertae). - caro sula ovalis, - ; - (Elongatio scanders; folia opposita, carnosa; pedunculi terminalea, filiformes, uni aut biflori).

ad 54. S A NCHEZIA, - (sedis incertae) - - ano therae villosas, -; ovarium oblongum, superum; stylus filiformis; cupsula - acuminat?, - polyspora; - (Elon. gatio trunci ramosa; folia subsessilia; bracteae linea. res).

ad 55. CYRTA N'DRA, - EORST. gen. tab. 3. Juss. (personata 3.) Calyx - inaequalis : corolla - tubo recurvato, ad facem dilatato, limbo patente, quinquelobo: - filamenta curva; stylus unus ; stigma bilamella. tum; bacca oblonga, - loculi polyspori, dissepimen. tum carnosum et utroque latero convexum,

ad 56.' S WEN IT IA, (SCHOUANKE) - ROYEN juss. (personatis affinis) - corollao fauce clausa plicis quinque glandulosis, radiatis, - limbo subaequali: stamina numero quinque; fiamenta duo magna, fertilia; tria parva, setacea, sterilia; stuius uni2s; stigma simplex ; capsula compressa, or bicularis, calyco persistente longior, - - ; receptaculum subglobosum: - (Planta herbacea; folla alterma; fores axillares; $S$, americana IXN.)

In se= 
Insercetu: A XIA, (AXIE) Lour. Juss. (nyctagynon 2.) - Involucrum triphyllum foliolis inaequalibus ot caducis, uniflorum; calyx parvus campanulatus, ad summum decemlobus; stamina tria; spara basi calycis tecta, striata, villosa; - (Elongatio trunci ramosa, nodosa et repens ; folia opposita, inaequalia; flores in racemis terminalibus).

ad 5\% GLOBIFERA, - MICRANTHEMUM MICH. fl amer. bor. 1. p. 10. tab 2. (primulacea I.) Calyx profunde quadrifidus - ; corolla tubo brevissimo, - limbo inaequaliter quadripartito, lacinia superiori maxima : - antherae rotundatae; ovarium globosum; styius brevis; stigma capitatum; capsula calyce persistente cincta, - - - ; - (Elongatio herbacea, minima, repene; folia opposita, integra; flores axillares, solitarii, altorni, minimi).

ad 58. JUSTITIA - IrNN. IUss. - '(acanthacea 2.) - corolla tubo gibbo; - - capsula basi attenuae ta - ; - (Plantae herbaceae aut suffrutescentes; folia raro verticillata, rarissime alterna; in quibusdam speciebus spinae axillares; flores solitarii aut in spicis. axillares aut terminales).

* DIA N THER A, (DIANTHÈRE) IIN⿴, JUSS. (acan* thacea 2.) - Calyx quinquepartitus; corolla tubus brevis, limbus bilabiatus, labio superiori bifido, inferio. ri tripartito, labo medio latiori ; filamenta duo, utroque duabus, antheris minico; sigma simplex; capsula compressa, loculi monospori, rarius dispori; - (Elongatio herbacea; folia opposita, linearia; flores in spicis axillaribus; D. americuna IINN. PLUK.)

ad 59. ELYTRARIA, - MrcrAUx f. amer. bar. tab. 1. (acanthacea 2.) Calyx - basi bibracteatus; corollue tabus rectus, limbus quinquefidus, subbilabiatus; stamina pillaris; - capsula -, subcylindrica, - valvulis rersus medium semiseptiferis. - (Elongatio trunci nulla : scapus radicalis longissimus; folia apico integra).

ad 6o. CA TALPA, - (bignoniea 2.). - corollae tu* bus ventricosus; - stigma bilamellatum; capsula longa, - bivalvis; dissepimentum valvulis oppositum. ad 6r. PIN GUICULA, - rourn: - Cprimulaceis affinis). Calyx - labio superiori trifido, inferiori bifido: corolla irregularis. - fauce attenuata, -; stamin duo brevissima; stylus brevis; stigma bilamellatum, an* 
Meras obtegens; capsula unilocularis, -; veceptacuium centrala, liberum. - (Eolia radicalia, nitida: scapus uniflorus; foris calcar cylindraceus, longitudine co. rollae ).

ad 62. UTRICULARIA, - Juss - LENTIBULARIA TOUPN. VAILL. (primulaceis affinis). - corolla parum tubulosa, irregularis, - labium superius rectum - staminiferum; inferius - integrum, cum tunica cordata extrorsum proëninens -; filuménta recurva; antherae connatae; stylus unus; stigma simplex; - recepsaculum cenirale !iberum. - (Plantae herbaceae, repo tantes el marcescentes, aut liberae ot in aquariam superficie navigantes; fores ut plurimum in spica super scapum aphyllum).

ad 63. SCIIZA N THUS. - (sedis inceriae). $\rightarrow$ (Elnngatio herbacea; folia alterna, pinnata; pedunculi sexflori).

ad 64. CA L C EOLARIA, - Fturit. - Jus3. (personata 2.) - corolla tubo brevi, - labio infero concavum - et refiexum; filamenta brevia; antherae recurvase; stigma simplex; cupsula conica. - (Elongatio lierbarea; folia saepissime opposita; pedunculi unimultiflor, axillaros aut in corymbis terminalibus).

a 65 . 1. AEA, - ccmarks. - - (personata 2.). filumenta staminum incrassata, atcuata; antheras conni. ventes: stigma simplex; - (Folia radicalia; scapi uniat multiflori, tenues, foliis breviores; flores in um. bellas dispositi, bractea parva muniti ad punctum in sertionis).

ad 66. T A MO N E A, - Av3z. Guy. tab. 268. jus5s -; (verbenacea 2.). - filamenta intra tubum, - : stig. ma sec. A UB⿱丷. quadrifidum; - (Elongatio herbacea; folia opposita; flores alterni in spicis axillaribus, singuli braciea lineari muniti).

ad 67. RAPUTIA, - AUBL, Gus. tab. 272, JUss. corollu hypogyna, - I stamina - duo fertilia, basi squamis duabus munita; ovarium quinquangulum, super dis. cum carnosum positum; stylus unus; stigma incrassatum, subtrilobum: - (Frutex ramis oppositis: folia opposita ternata; flores in spicis axillaribus, bracteati).

ad 69. VERBENA, - ToURN. - JUss. - (varbenacea 2.). Calux persistens - ; - stamina non exserta, - s stigma obtusum; sporce quatuor, nudae, calyce tectao. (Plantae herbaceae, rarius irutescentes: folia opposita; flores bractea una muniti, in spicam breven: et oblos: gatam dispositi). 
ad 7o. Z A PANIA, - - vent. (verbenacea 2.). Ca. lyx quadricientatus, brevis; corolla - tubo cylindrico, limbo patento - lacinis inaequalibus: stamina - inclusa; stigma angulosum; sporue - calyce coinmutato et quasi bivalvi tacto tectae - (Folia spathulata, interdum ternata, elongatio hispida; flores in spicis lon. gissimis, nudis, crassiusculis).

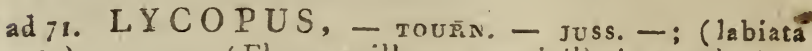

1.). - - (Flores axillares, verticiliati, sessiles).

ad 72. A E THYSTEA, - JUss. - ; (labiata 1.). - corollae - Labio bilobo, lobis patentibus; inferiori trilobo, lobo medio concavo, longiori; -. (Flores in corymbis axillaribus et terminalibus).

ad 75. ZIZIPHORA, - juss. - (labiata 1.). Calyx cylindricus, longus, villosus, - (Flores glomerasi. axillares aut terminales).

ad 76. MONARDA, - juss. -; (labiata 1.) -; corolla cylindracea, calyco longior, - labio supetiori recto, integro, attenuato - , inferiori reflexo," latiori, - lobo medio longiori - (Floresaxillares, verticillati aut in capitulis terminalibus).

ad 77. ROSMIARINUS, - Tourin. - IUSS. - (la. biata 1.). - Culux - Labio inferiori bifido; corolla tubulosa, tubo calyce longiori, labio inferiori - lacinia media maxima et concava; stamina exserta; flumentasubulata - -; (Frutex spinescens; rami recti; tolia li. nearia, attenuata, subtus albentia, marignibus revolu. tis ).

ad 79. SALVIA, - tounN. - juss. - (labiata 1.). Calyx striatus, - - labio inferiori bifido; curolla tubulosa, tubo vix dilatato, apice compresso, calyce In giori; - labio superiori convexo, - aut integro; inferiori trilobo, lobis lateralibus, appressis, lobo medio maximo, rotundato: filamerîu - articulata, et super pe. dicellum particularem corollae insertum versatilia, basi connata, per omnem longitudinem libera, apice numita unthera oblonga, unilotulari. - (Yerbae aut suffrutices; flores unus - tres ex axiliis folii aut bracteae enascen. tes, saepe in spicam dispositi; saepe duo rudimenta parva staminum sterilium sub forma glandularum pe. dicellatarum in corolla apparen ).

ad 79. CUNILA, - JUss. - (labiata 1.). Calyx de. cemstriatus, - - ; faux calycis pilis clausa sporas fovet et includit; - (Flores in corymbis aut in verticillis, axillares et terminales). 
ad 80. COLINSONIA, - juss. - (labiata r.). Ca. lux - labio inferiori bifido; corulla calyco-longiorinfundibuliformis -; (Folia rugosa, cordata; flores in panicula pyramidali).

ad 92. LINOCIERA, - swarz, - - (ex ord. plantt. sedis incertae) - - ; (Folia petiolata, opposita, integra; - Chionantho maxime affine genus; JAUNE II. pag. 340 ad Triandriac mosogynias relulit).

ad 83- ACAENA; - mutis. - - (Apud jAUME Iİ. pag. 185. Ancistrum ab Acaena disjunctum reperitur).

Inseratur: S TRIG A, (STRIGE) Lovrelr. (ex ord. plantt. sedis incertae). Calyx quadifidus; corolla quadrifida, lacinia superiori maxima, emarginata; filamenta duo brevissima; ovarium oblongum; stigma simplex; capsula uniloculari, polyspora. - (Elongatio trumci herbacea, simplex; folia sessilia; flores axillares).

ad \$5. DI A LIUM, - вUrm. juss. - stamina ad latus superius ovarii posita; anthercé oblongae, quasi didimse; ovarium superum; stylus unus; stigma sisuplex; (Genus proximum I'einmannicie secundum LAxt. - Arbor foliis alternis pimatis; flores in paniculis declinatis ).

ad s6. MORINA, - TourN. - juss. -; (dipsacoa 1.). Culux particularis duplex: exterior suporus, tubulnsus, limbo dentibus spinosis inaequalibus munitus; - interior inferus, - persistens; corolla - tubo - parum arcuato, ad summum dilatato: labium suporius bilobum; stamina exserta; stigma simplox; spora ovöidea, -: (Elongatio herbacea, folia verticillata numero quaterno, sinuato-spinosa; fores dispositi in verticillis, offormantes spicam terminalem ).

ad 8\%, CIRCAEA, - Tourn. - Juss. - (onagra. ria 2.). Calyx caducus -; capsula ovalis, - bival. vis - ; sporce ad summum loculorum affixas: - (Elon. gatio herbacea, reçta; folia opposita; flores in spicio terminalibus).

ad 88. GLOBRA, - Juss. - GRAMEN, TourN, Calyx duplex : exterior -i interior (corolla L.) aequa. lis - ; stamina duo, altero aburiente; -; C Mores in spicis terminalibus aut lateralibus); forsan ad primam classem iusta Cannan portuere vidotur? 
ad 93. A N T H OXANTHU M, - JUss. - ; (gIsIninea 1.) -; squamae duae interna ; stamina dua; -; (Clores in spica terminali).

ad 94. CRYPSIS, -; (graminea'z.)

GUNNERA, IINN. JUss. (urticeis affinis). Calyx nullus, aut superus et bidentatus; ovariam apice bidentatum; styli duo dentibus ovarii interpositi; stigmata duo: stamina epigyna; antherue subsessiles et oppositae; spora calyce crustaceo tecta unica; (Elongatio herbacea: folia redicalia; flores terminales amentiformes, amento ramoso in apice scapi).

ad 95. MN I A R UN, - IIN N. suppl. - ; Calyx rectus - -; stamina ut plurimum ad calycis medium inserta; ovarium inferum, calyco longius; stuli et stigmata duo: ovarium maturitate conmutetum in fructum calycis incrassatione -; (Plania herbaces, foliis confer tis tecta; fores terminales, per'paría dispositi).

ad 9\%. PIPER。 - BAUTr. - JUSS. - SAURURUS rourx. (urticea 3.) Spadix - Anribus - et appres:is; interdum cinctus spachis, ut plurionum nudus : singulus flos squana nna munutus; Calyx nullus, nîsi habeas externum ovarii amicum pro calyce; -; ovavium cinctum duobus antheris sessilibus: stylus - aut subnullus; stigmata - aut quatuor viliosa; - ; bacra rotundata -; (Elongatio herbacea aut trutescens, ut plurimum scandens et dichotoma, ramosa; rami nodo. si, quasi ariculati: folia in petiolis amplexicaulibus, nunc opposita et tuncaltero caduco aut abortiente: spadix foliis alternis opposittis, oppositis asillaris, plurimum solitarius et nudus, rarius fasciculatus et bracteatus).

\section{$C L A S S I S T E R T I A$.}

ad 99. VALERIANA, - TourN. JUss. - ; (dipsacea 2.). Calyx - enascens in aristam - et plits mosam; corolla - limbo aequali aut rarissime inaequas Ii; stigmata unum, rria; - ; ulbumen nullum. (Folia simplicia aut pinnata; flores plurimum dispositi in corymbos terminales).

ad 100. FEDIA, - (äipsacea 2.). - corolla régula: tis ant irregularis, - - ; stumina dno ad quinque; stigmats unum ad tria; - - ; albumen nullum.

ad 1or. MELOTHRIA. - JUss. - ; (cucurbitacea 3.). Calyx tubulosus, - fliformis super ovarium, limbo deceme 
decemfido; laciniae quinque exteriores minores, acutae; quinque interiores (corolla L.) alternae, magnae, coloratae, rotundatae, rotato patentes; stamina tria; filamenta conica, duo gerunt antheras gerninas, tertinm monandrum; stylus :cylindraceus ; stigmata tria; bacca oblonga, - - (Pejunculi uniflori; flores interdum tantum masculi).

ad 102. CROCUS, - тoUrin. - JUss. - ; (iridea 2.) Calyx - - laciniis - rectis: stigmata - convoluta: - - ('Truncus subterıaneus (radix L.) tunicatus, duplex; spatha unifora, monophylla; elongatio trunci nulla; flures subsolitarii).

ad 103. GALAXIA, - juss. - ; (iridea 1.). Calux juss. (corolla linv.) tubulosus, rectus, - limbo dilatato, sexlobo, -; - . (Truncus subterraneus (radix L.) bulbosus; folia radicalia: flores inter foliorum medium positi; elongatio subnulla).

ad 104. W A T S O N I A, - Juss. - (iridea 2.). Calux (corolla LINN.) -- tubo filiformi, longissimo; limbo subsequaliter sexpartito et parum labiato; - stylus filiformis, trifidus; stigmata tria bifida: - (Truncus sub. terraneus (radix L.), tubernsus, tunicatus, hemisphae ricus, margine dentatus; flores in elongatione simplici aut parum ram sa; spathae uniforae, bivalves, valvula exteriori maxima).

ad 105. A N T HOLIZ A, - juss. - (iridea 2.). Calyx (corolla 2 ) infundibuliformis -; - (Truncus subterraneus 'radix L.) tuberosus, tunicatus; elongatio simplex; fores in spicis; spathae uniflorae. Genus adhuc parum a genere Gladioli distinctum).

ad 106. GLADIOLUS, - TOURN. - jUss. - ; (iridea 2.). Calyx (corolla L.) - labii suporioris laciniao tres approximatae aut conniventes: inferioris laciniae tres pius minus patentes; -; stumina sub labio superiori posita; - ; sporae arillatao, aut ad margines meu-

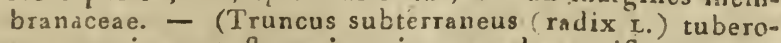
sus, turicatus; flores in spica; spatbao uniflcrae; germinatio subsimilis germinationi Ixiae; lobus sporao absolute sessilis).

ad iog. I R IS, - mourin. - juss. tAat. illustr. tab. 3r. XiPHIUM et HERIMODACTYLUS TOURN SISYRINCHIUM TOURN. (iridea 2.). - Calyx (corolla $L_{0}$ ) regularis, tubo longitudine orarii, limbo magno, sexpariito; laciniae tros alternae roctae, tres alternao reflezae, introrsum barbatae aut imberbes, basi staminiferao; - stylus brevis; , stighata - oblunga, magna,

$$
\text { [23] super }
$$


super stamina declinata, interme stria longitudinali suI. cata; - (Elongatio trunci saepe compressa: spatha uni-multiflora: truncus superficialis (radix LINN) XI. PHII тоur. bulbus simplex, SISIRINCHII toupN. bulbus duplex, bulbo uno alteri superposito, HERMODACTYLI TOUn. tubulosus; IRIDIS TOURN. carnosus, oblongus, reptans).

ad iro. IXIA, - juss - (iridea 2.). Calux 'corollar.) - ; stigma trifidum, subfiliforme; - - (Truncus subter aneus (radix LINN.), fibrosus aut tuberosotunicatus; folia plana, per margines vaginantia, aut canaliculata aut nervosa, et granineis simillima. Elongatio saepo compressa, saepe subnulla; flores terminales, aut in paniculam, aut in spicam, aut in capitulum congesti; spathae subuniflorae: sporae lobus germinatione affixus dorso priniae foliorum vaginae, et subsessilis).

ad 113. ARISTEA - AIt hort Kew 1. p.67. (iridea 2.) Calyx (corolla I.; - limbo sexpartito, lacintis profundis, aequalibus, patentibus: stamina brevia. styIus arc"ratus et assurgens: stigma - marginibus fimbriatis et tribus lateribus parum rotatis:- capsu!a oblonga, trigona - ; - . (Folia disticha; flores in capitulo; scapus angulosus).

ad̉ 21. MO $\mathrm{A} \perp \mathrm{EA},-$ juss. (iridea 2.). Calyx (co. rolla I.) - laciniis (petalis. ..) - tribus alternis refle$x i s$, interne barbatis aut imbarbibus, et basi stamini. feris; stylus brevis stigmata - potaloïdea, magna, oblonga, super stamina nutantia, interne stria longitudinali sulcata. - (Flores terminales -; spathae uni aut biflora folia ensiformia, vaginantia aut cenoliculata; truncus subterranous (radix L.' tuberosus, tunicatus).

ad I15. SISYRIN CHIUM, - juss, - : BERMU. DIANA Touns, irıcea -) - Calyx (corolla L.) tubo brevi, - - - i stigma trifidum : - (Truncus subterraneus radix I fibrosus: elongatio compressa et ramosa flores terminales aut axillares: spathae carina:as, compresste, - BER VUDIANA MEDIC monad. pag. 76. s. - Calyx ovario insidens - stuminum tubus ovario innatus, terminatus iribus antheris; fiamenta nulla: stulus intra tubum adsurgens, trifidus stignata tria patentia: copsula trigono-rotundata, - dissopimen. tum Inngitudinale sporiferum. Cf. et ADANs. fanill. dr plantes to 2. p. 60. SCHPEB. nov act. nat. curios. Vol. 3. pag. 341 .

ad i6 TIGRIDIA, - vent. REDOUTE liliac. tab. 5. -- iridea I ) Calyx (corsli,, ; tubo brevi, limbo magno plano, - - interioribus ad unguem et sub 
sub apice attenuatis -; filumenta connata in tribum longissimum:- (Elongatio herbacea, cylindracea, le: viter flexuosa; folia glabra).

FERRARIA, - (iridea 1.). Calyx (corolla L.) tubo brevi, limbi - patentes; filamenta - basi connata; -. (Planta herbacea; flores spathacei, terminales; folia radicalia, longa; ensiformia, ancipitia) - MEDIC. monad. pag. 29. - tubus filumentorum super ovarium, terninatus filis tribus brivissimis, singulo antherifero; - capsula conica, triangula, - polyspora.

ad r17. DILA T R IS, - eERG. Juss. - (irideis affnis). Calyx (corolln t.) superus, externe villosus, lim. bi lacinia sex rectne, aecuales, pẹsistentes, oblongae, canaliculatae, basi staminiferae; - flamenta sex, tria brevia sterilia, tria longa fertilia; antherae tres aequales, unica saepe non decidua; ovarium inferum; sty: lus unus; - : capsula - villosa - - trivalvis, (Folia radicalia vaginantia, elongationis sessilia; fores in corymbo terminali et villoso).

Ii seratur: HERITIERA, (HERITIERE) CIIEI. MICHAUX fl, am. bor. t. 4: (iridea 2.). - Calyx sexpartitus, laciniis petaloĩdeis, tribus linearibus, tribus alternis lanceolatis; rubens; staminum filamenta pilosa; ovarium inferum; styius triangulus, exsertus; capsula coronata limbo marcescente, trilocularis, oligospora, angulis dehiscens; sporae rotundatae, margine tenui munitae. - (Elongatio herbacea; folia iridea, acuta: Hores in panicula subcorymbiformi, fasciculati, tomentosi. (PERs, erich. pag. 54. b. cum genere Ditutris coniunxit).

TAPEINA, (TAPEINE) COHMERs, JUSS. MORAEA mugellanica wistd. (iridea 2.). - Caly2 tubulosus, lim. bo $r$ ofunde in partes sex aequales fisso ; sitgma trifidum: - (Planta herbacea minima; flores minimi, terminales, solitarii, subsessiles in medio foliorum).

ad 118. WI T SE INIA, - JUs5. - (iriuea 2.). Calyx (corolla I.) rectus, - - exterioribus villosis (Planta trunco subterraneo (radice trNv.) tuberoso: elongationo trunci angulosa, ramosa aut simplici, ad basin obtecta foliis margine raginantibus, imbricato flabelliformibus aut per paria dispositis'; spatha partialis singuli @oris brevis). 
adIg. CIPURA, - AUBL Guy. tab. 15. - (iridea 2.). Curux (curolla L.) - basi unbulosus, - ; stumina tria ubo insesta: - stulus - triangulus -. (Truncus sutrerian os ladix L.) tubernsus, tunicatus. folia quaedam radicalia, vagun ntia et ensiformia; flores terminales super el ngarionem aphyllam).

ad 1 :o. Vi \& C HENDORFIA, - Juss. - (irideis affinis). Culux (corolla L.; basi tubulusus? - laciniis tribus magnis rectis, tribus parris fatentibus -; sta. mins tra cernua, marginibus laciniarum asternantium

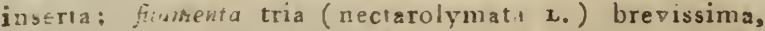
sterilia et staninubus intermixta : ovarium superum; stylur unus; stigma simplex; capsula int ra, - trivalvis -; receptacuium centralo trigonum; sporae şeltatae. - (Truncus snbterraneus (radix .) tuberosus, truncatus: folia raicalia vaginanua, elongationis sessilia; flores in panicula aut corymbo.

ad 122. XIPHIDIUM. - zOEFL. AUBL. Guy. 1. p. 33. tab. It. Juss - Ca $y x$ (corwlla L.) super 1 s - la. ciniae tres alternae interne positae: stamina tria - basi leciniarum atfixae; ovurium superum: stslus unus stigma sinplex -; capsula baccaeformis -. (Truncus subteryaneus radix $L$ : raptans, fibrosus folia alierna, subvazinantia, sterilia; flores in panicula terminali, bractea muniti!

ad 1:3 LEPTANTHUS. - meHAUx amer. bor. tab. ร. f. 1. - (arrarrllidea 2.) Culy $x$ (corolla z.) - ; stamina tria ad summum inserta, ad orficium calycis; - : uvurium unum; stulus longutudine tubi: capsula obl nga, -; sporae numero flur!mae. duplici serio dispositae. (Plantae aquaticae; folia alterna, vaginar:4 tia flores solitarii, axillares.

ad 124. HETERANTHERA, - (amaryllidea 2.). Culyx corolla c) tubo longo ad limbum atienuato, stamina tra; - - ovarium unum; stulus filif.rmmis, rectus: stigma trifidum: capsula oblonga - trivalris; spo. rae plurimae, membrana cinctae (Elongatio herbacea, trunco reptante; folia roniformia; flores tres aut quatuor in spatia singula). *)

ad 125.

*) Etsi LEPTANTHUS et HETERANTHERA tribus staminibus gandeant, tamen a genere PONTEDERIAE non esse quoad familiam seiungenda, quum ct variae Po:1lederiae species huvere rideatur tolidem stamina, arbitrantem legimuS JAUME I. 140. 
2d 125. COMMELINA, - Juss. - : (juncinea 2.) Caiyx aequalis aut uaequalis, laciniis duabus tubusvo interioribus petaloïdeis (corolla $x_{\text {. }}$ ); stamina sex, Interdum cuncta fertilia, ut plurimum tra aut quatuor sterilıa (nectarulymata L) - ; stylus uaus; stignia simplex: - ; sporue valvulis arfixae iuxta disspumento. rum insertionem. - (Elongatio trunci ferbacea, nodosa; folıa cum nodis alterua; vaginae foliomm iongae, fissae 3 rami ad basin spathacer; pedinculs axiliares aut terminales, uni aut multiflori; fl res inclusi bracteis cruatis, duplicatis, ot quisi spallaceis).

ad 126. OXYBAPHUS, - cAvAN. ic. \&. pag. I?. tab. 19. - (nyctaginea .). Invoincrum (calyx) nionophyllum, quinquelobum, unflor.m; caiyx (coroila L.) brevissimus, vix involucrum sustineus, - stumina tria, rarius quatuor; -, (Flores terminales, axillares et in thyrsis).

ad 127 MACROLORIU M, - - ATBL Guy.ıab. 7 - (leguminosa i. . - - ovarium prícellaima. egumen - obtusum: - ; spcra, rotunda!a (Aibcres foliis coniugatis; flores in panicula au in coryabis axillaribus vel termmalibus,.

ad 128. OUTE A, - AurL. Guy. tab. 9 Juss. - : (le. guminosa 1.). Culyx - bracieae magriae; corolae -, relıqua minora et aequali magnitudine; fiumerta staminum numero quatuor, unum - bruve, - rillosum - : tria opposita, longissima, fertilia; antherae versatıles: ovurium pedicellatum ; legumen - ....; (Arbor foliis pinnatis; flotes in spicis axsllaribus).

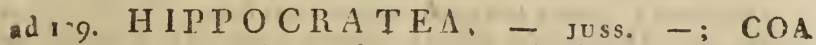
TIUN. - ; corollue petala maxima, basi dilatara, cucnllata, apice concava; stuminu tria; filamenta basi dilatata; antherce apice transversim deliscentes: ovarium simplex, ad medium disco tectum aut tubo flameninrum: stulus unus; stigma simplex; capsula -, subovales, magnae, -; sporae compressae -. (A rbor sarmentosa, ramis oponsitis: folia stipulis minimis aucta, opposita; flores in umbella dichotoma).

ad 130. TONSEL L A _ - ; stamina tria disco (s. nectarolymate) aequaliter affixa; ovurium unum (inferum?) - ; stulus unus: stigma simplex; bucca - sphas. rica, - tetraspora. (Frutex scandens ranis opposvis; folia opposita, integra: fores in pasiculis axillaribus ot terminalibus, bracieis muniti).

ad 131. LOEFLINGIA. - juss. - (caryophvllea 1.). - - ; stylus unus; stigmata tria; - (Folda oppo- 
sita, stipulis appendiculiformibus munita ; flores solit 3 rii, axillares,' sessiles).

ad 132. W I L L I C H I A, - juss. - Calyx quinque. fidus, persistens ; curolia - , limbo plano -; stamina tria inserta tribus lobis superioribus corollae; ovarium superum; stylus unus; stigma simplex; -; dissepinenta capsulae opposita; receptaculum globosum. (Elongatio irunci herbacea, reptans; folia alterna; petioli longisoimi; flores parvi, colore rosei).

C A L L ISIA, - Juss. - (juncinea 2.). Calycis laciniae tres interiores (corolla I ) petaloideae; filamenta tria, singulo duabus antheris onustato, -; capo sula - loculo tertio abortiente? (Facies Commelinae; elongatio herbacea, repenș; folia alterna, vaginantia).

ad 133. SYENA, -; MAYACA AUB土. Guy. tab. I5. JUSS. - JAUne (juncinea 2.). Calyx - laciniis tribras exterioribus acutis, tribus interioribus (corolla auct.) rotundatis, petaloïdeis; stamina tria; antherae - biloculares; stylus unus : stigma trifidum; capsula stylo acuminata - versus medium dispora. (Planta habitu calyp. tratarum (muscorum LiNN.); folia alterna, linearia; flores solitarii et axillares).

2d 134. TAPURA, - AUBL. Guy.tab. 4S. JUss. ROR. FIA schreb. (sedis incertae) -; corolla hypogyna -; labio superiori concavo, recto, inferiori breviori latiori, bilobo; stamina quinque *) epipetala, quatuor di. dynama, sub labio superiori posita, quinto longissimo ad basin labii inferioris collocati; ovarium ouperum, triangulum; stylus Iongus; stigma trilohum : fructus ...; (Genus Bignonieis affine; frutex ramosus; folia altorna, stipulis caducis munita; pedunculi axillares, solitarii, multiflori).

âd 135. CALYPSO, - aUz. peтrtir. plant. aff. austr. tab. 6. (sedis incertae). Calyx minimus-; corulla - petala inserta calyci -; discus - carnosus : - antherae ad summum adnatae filamentis ; - ; stylus brevis; zacca rotundata, acuminata, - (Frutex ramis rectis, cylindracois; folia opyosita; flores fere in umbella -).

ad 136. R U M P I A, - jrss. - (terebintacea 2.) - ; stamina tria, petalis aequalia; antherae parvao; stylus unus; stigma trigonum; - - . (Arbor; folia petiolata, alterua, simplicia, Tiliae foliis similia; flores in racemis axillaribus). ad $13 \%$.

*) Hinc ad Class. V. monogyniam Jatae II. p. 348. retulit, 
ad 137. FISS IL IA, - coMMERs. - - ; (hesperidea 1.). Calux brevis, - - ; coroila hypogyna, calyce lon. gior, apparens monopetala -, - sed divisa aut partibilis in tria petala conniventia recta, - versus apicem, tertio integro ; - filamenta petalis affixa: tria fertilia, quinque sterilia, disposita numoro duorum sterilium uno fertili intermedio in singuli petali fissi apice; ot unum fertile unumque sterile in petalo integro: stylus unus; stigma truncatum aut trigonum; $u u x-$ - angu. statim cincta fere usque ad summum calyce magnificato, longiori et capsulaeformi reddito. - (Arbor habitu Lauri, sempervirens: folia alterna, integra; flores in parvis racemis axillaribus, paucifloris, interdum subsolitarii :

ad 138. CNEORUM, - JUss. - (terebintacea 2.). Caly $x$ - persistens; corollae petala - oblonga : stamina tria brevia; antherae minutae; -; bacca parra, -; (Fruiex; folia alterna, foliis Oleae similima; pedunculi ax Ilares uni aut triflori !

ad 339. CO MOCLAD IA, - BROWN. - JACQ. JUSs: -; ( terebiutacea :.). - ; corolla - petala calyce maiora; stamina tria brevia: antherap rotundatae; stylus nullus; stigma simplex: druva - - , succulenta, ad summum trirostrata; nux eiusdem formae, - (Arbores quae incisuris factis praebent succum lacteum aut aquosum, glutinosum mediante aere nigricantem; folia impari pinnata; foliola opposita, villosa et dentata, aut integra et glabra; flores in racemis paniculatis et axillaribus, numerosi, parvi, conformantes racemos maximos: interdum stamina quatuor et calyx quadri. dentatus).

ad 140 XYRIS, - Juss. - (juncinea 1.) - laciniao calycinae tres basi staminiferae - ovarium unum; stigma trifidum:- ; - (Elongatio trunci simplex, aphylla, enodis, folia radicalia, vaginantia: flores terminales in spica angustata, oquamae imbricatae et uniflorae; habitus Schoeni).

ad 141. TR IP TERELLA, - мrсн. amer. bor. I: 9. tab. 13. - (exord. plantt. sedis incertao) - stamina tria inclusa, sub dentibus calycinis inserta; ovas vium inferum: stulus unus; stigmata - brevissima; capsula rudimento floris coronata, membranacea, - ; (Plantae minimao horbaceae, fere aphyllae).

ad 142 OLAX, - - juss. (sapoteis affinis). - Cäs lux - infundibulif rmis corolla tubulosa - nectarolymatis foliola unguiculata; stamina super tubum affixa, et cum appondaculis sive nectarolymate alternis; stulus 
unus; stigma simplex; - - - Arbor parum cognita Ceylaniae; folia alterna, integerrima; pedunculi axillares, brevissimi).

ad I43 ROTALA, - Juss. (caryophylleis affinis) -; -; stulus unus; stigmvta tria; cupsula minima, - - ; - Planta herbacea, ramis articulatis, interioribus oppositis, superioribus alternis: folia verticilla. ta linecria; flores numero quaterno axillares, sessiles, minimi).

ad 144. ORTEGIA, - IOEFL. - Juss. - (caryo. phyllea I , - - stylus unicus; stigma capitatum; - - sporae fundo capsula affixae. - (Folia oppo. sita, stipulis minimis munita; pedunculi saspe multiflori, axillares aut terminales).

ad 145. POL Y C N E M M, - Juss. - (atriplicea 2.) - : - styius bifidus; - - ; - (Elongatio herbacea diffusa; tolia linearia, mucronata ; floresminimi).

ad 147. SCHOENUS, - JUss. - GRAMEN et SCIRPUS ToUnN. (cyperacea 2.) - - spora setis aut pilis brevissimis -, saepissime non pilosa; - (Flores inferiores interdum abortiunt, et squamas vacuas relinquunt elongatio trunci cylindrica aut triquetra).

ad i48. CYPERUS, - rourn. - Juss. - (cyperacea 2.) - (Paleae inferiores quaedam vacuae: elongatio plurimarum specierum triquetra, spiculae appro. ximatae, pedunculata et in umbellas dispositae).

sub Cypero locum teneat:

IRIA, (IRIER) PICHARD. PER8. ench. pag. 65. a. CYPERUS IINN. WILID. потн. (cyperacea 2.) - Spica simplex, squamis subdistiche imbricatis, summis corgestis, infimis aristatis; stamen unum; stigmata duo ; spura mutica.

ad r5o. SC IRPUS, - rouns. - Juss. - CYPE. RUS roURN. (cyperacea 2.)

ad 152. ERIOPHORUM, -; LINAGROSTIS TOURx. (cyperacea 2.)

ad 153. MIA P N IA, - AUBL. Guy. vol. 1. pag. 47. vol 3. tab. 17. JUss. --: (cyperacea 2.) - ; stylus unus : stigmata tria. - Elongatio trunci simplex, triangularis; flores in capitulo sessili, cincto involucro maximo triphyllo). 
ad 154. LIMNETIS, - SPARTINA - SCHREE. Juss. mss (griminea Ix.) - Gluma bivalvis, uniflnra, compressa; valvula interior longa, lata, mucronata, dorso striata; caiyx bivalvis, corolla minor: stamina uria styius filiformis, staminibus longior; stigmata duo villosa; spura calyce inclusa.

ad 155. NARDUS, - JUss. - GRAMEN TOURN. (graminea เo.) - (setacea, recta; flores unilaterales).

ad 156. $R E M I R E A$, - AubL. Guy. tab. 16. Juss. - - (graminea 11.) - (Elongatio foliis penitus obtecta, flures in panicula densa).

Inseratur: TRYOCEPHALUM, (TRYOCE. PHALE) FORST. nor gen. tab. 65. Juss. (cyperacea 2.) - Paleae uniflorae; stamina tria; stigmata duo; spora rotundata; - (Elongatio triquetra; spica conferta, terminalis, involucro triphyllo munita; flores unus aut duo inferiores fominei).

ad 157. KYLLIN GA, - готтв. - juss. - (cyperacea 2.) - - ; spura - obinnga, glabra. - (Elon. gatio triangula, filiformis; flores in capitulis glome: ratis).

ad 158. L Y GEUM, - Jus8. - : GRAMEN tounN. (graminea 10.) - Glumue - valvula magna, - ; caly. ces (corollae L.) duo bivalves, valvulis basi coalitis; -; ovarium cun calycibus connatum; stigma simplex; fructus: nux bilocularis, dispora, villosissima, non de. hiscens. - (Folia fliformia, argustata, juncorum foliis simillima).

ad 159. $\mathrm{POMMEREULA,} \mathrm{-} \mathrm{IINs.} \mathrm{suppl.} \mathrm{JUss.}$ - POMNEREULlia schreb. (graminea I1.) Gluma - valvulis indequalibus -; calyx (corolla auctt.) - inaequali magnitudine, valvula exterior ma. xima, quatrifida, aristata, interior minor, integra, non aristata - : stylus unus; stigmata duo; spora glaberrima; - (Elongatio ramosa; flores omnos hermaphro. diti),

ad 16c. SACCH A RUM, (SUCRE) - Juss. ARUNDO toURN. (graminea 2.) - ; (Flores in panicula).

ad 162. FUIRENA, - juss. - ; (cyperacea 2.) - ; culycis (corollae auctt.) - squamis s. valvulis - termi. natis barba cyrrhiformi ; stigmuta duo; - - (Elongatio angulosa, striata.) 
ad 166. CHRYSITR IX, - juss. - (cyperacea 2.) - Capitulum florum compressum, imbricarim squa. mis oblong18, coriaces, formantibus fasciculum densam: - paleae multae, setaceae, - ; filanenta inearia; ovaria obl.nga obtusa - stylo filiformi; stigma simplex: JAUMe. - (Folia ensiformia, vaginantia, angest ta; scapus compressus iuxta summum se dis. iungens ad emittendum florem unum).

ad 168. COR N UCOPI AE, - juss. - : (gramirea il) - Involucrum - marginibus crenulatum aut integrum, -; -; spora tecta.

ad I'ig. PAN I CUM, - Tourn. - jUss. - ; MILIUV et GRAMEN TOURN. (graminea 2.) - corollae (calycis Juss.) - cartilaginea, persistens: - (Flores dispotiti in panicula laxa aut dersa, saepe setacei).

ad 70. DIG ITARIA, - HAIL. - ; (graminea 2.) - Calyic - valrulis inzequalibus, mucronatis, - ; (Flore unilaterales, dispositi in spicis digitatis).

ad 174. P A SPALUM, - Juss. - ; (graminea 2.)

àd r75. A R IST I D A, _ Juss. -; (graminea 2.) (Panicula ramosa, spiculis diffusis;).

Insevatur: BOBARTIA, ( BOBARTIE) IINN. JUss. IAM. ill. tab. 41. (graminea 2.) - Gluma (calyx L.) multivalvis, valvulis imbricatis : calux (corolla L.) bivalvis; - (Flores agglomerati, spicao in capitulum coalitae Cyperi in modum).

ad -6. ALOPECURUS, - Juss. - ; GRAMEN TOURN. (graminea 2.)

ad 177. POLYPOGON, - - (graminea 2.)

ad 178. PHLEUM, - JUss. - GRAMEN TOURN. (graminea 2.) - (Flores sessiles, conlerti in spicam ramosam).

ad 17\%. PHALARIS, - JUSS. - GRAMEN TOURN. (graminea 2.) - (Flores in spicis, aut in spicis paniculatis ot ramosis).

ad 180. MIL I UM, - Juss. (graminea 2.) - (Florea in panicula barbati aut nudi).

ad 8r. A GROSTIS, - Juss, - (graminea 2.) -; (Fiosculi in panicular. 
ad I86. LAGURUS, - JUss - (graminea 2.) -; (Flores in spicis).

ad 188. MÜHLENBER G IA, - juss. mss. (gra. minea 1.)

ad 189. STEGOSIA, - JUss. mss. (graminea 2.) -; -. ; valvula interiori - breviorique reliquis, spo. ra rotundata, - ;

ad 19r. PEROTIS, - SCHreb. wIIID. - (Flon: gatio ramosa; folia plana, ad internodia barbata; IAMARK cum succhari genere coninnxit, a quo vix, nisi arista corollae (calycis Juss.) differt).

àd 192. SPINIFEX, - Juss. - ; (graminea 4.) flos unus hermaphroditus, alter masculus; - ; (Flores per paria diṣositi in rachi seta terminata).

ad 195. MANISURIS, - JUss. LAM. ill. tab. 839. (graminea 4.) Monoïca; - ; - ; stigmata duo plu. mosa.

ad 196. A IRA, - IINN. JUss. - ; (graminea 5.) glu. ma - valvulis acutis, membranaceis; - ; - ; (Flores in penicula; ad unum omnes colore argentea).

ad 19\%. MELICA, - 'JUSS. - ; GRAMEN TOURN. (graminea 3.) - ; (Flores in spicis paniculatis).

ad 198. COLLADOA, - cAvAN. ic. tab. 460. (graminea 4.) - (Spicae per paria dispositae in podicello communi axillari aut terminali).

ad 199. AEG ILOPS, - jUss.-; GRAMEN TOURN. (graminea 4.)

ad 202. HOLCUS, - JUSS. - GRAMTEN TOURN, (graminea 3.) - -; Flores masculi: calyco (corolla auctt.) et pistillo destituti mixti hermaphroditis et minores; - (Flores in spicis paniculatis).

ad 205. SE HIMA, - JUSs, mss. (graminea 4.) - ; (Spica simplex; spiculae coniugatae, biflorae; flore masculi non aristati, hermaphroditi aristati).

ad 208. D AC'TYLIS, - JUSS. GRAMEN TOURN, (graminea 6.)

ad 209. UNIOLA, - JUss. - (graminea 8.) - Flo. res in panicula: -;

ad 210. B RIZA, (AMOURETTE - ) - JUss. - ; GRAMEN TOURN. (graminea 8.) - Paricula flotum diffusa. 
ad 2II. POA , - JUss. GRAMEN tourn. (graminea 8.) ad 212. FESTUCA, - JUSs. - GRAVIEN TOURN. (graminea 8.) Gluma (calyx alcctu.) bivalvie, multif rus; culyx (corolla auctt.) - valvulis oblongis, inaequalibus, acumınatis, exteriori longiori et ut plurimum apice aristata. - (Flores in panicula).

ad 214. B R OMUS, - JUss. - ; GRAMEN TOURN. (graminea 8.) -;

ad 315. AVENA, - ToUrN. - Juss. - ; GRAMEN ToURN. (gramine 8.) - calyx (corolla auctt.) - oblongus, valvulis acutis - ; - : (Flores in panicula laxa aut densa).

ad 2Iא. ARUNDO, - TOURN. - Juss. -; GRA. MEN TUURN. (graminea 8.) -; (Flores in spicis terminalibus).

ad 218. $\triangle P L U D A,-$ JUss. - ; (graminea 10.) - ; (Flores in panicula).

ad 219. PAPPOPHORUM, - JUss, mss. (graminea 4.)

ad 221. CE NCHRUS, - Juss. (graminea 4.) - ; Gluma bivalvis, biflora, flore altero hermaphrodito, al. tero masculo; - ; (Filres spicati).

ad 222. ROT TBOELLIA, - IINN. suppl. JUss. -; (graminea 4.) - Culyx - univalvis uniflorus floro hermaphrodito, aut bivalvis et bifl rus flore altero masculo; corolla - maior gluma (calyce L.), inaequalis.

ad 223. SECALE, - tourn. - juss. - (graminez 7.) - corolla (calyx Juss.) bivalvis, exteriori valvula aristata.

ad 224. TRITICU M, - TOURN. - JUSS. - : GRA. MEN TOURN. (graminea 7.) - gluma et flores rachi oppositi; culyx (corolla L.) bivalpis, aristatus aut non aristatus.

ad 225. HORDE UM, - ToURN, - JUss. - (graminea 7.) - Glumae (calycos auctt.) duáo aut tres in singulo axeos dente, bivalves, uniflorae; -

ad 226. ELY MUS, - JUSS. - ; GRAMEN TOURN. (graminea 7.) - Glumae (calyx) duae vel tres in singulo axeos dente, bivalves, (Elym. hystrix valvulae nul. lae) - aut saepo multifloris. 
ad 227. L OLIUM, - JUss. -.; ; GRAMEN TOURN. (graminea 7.) - Flores oppositi axi aut rachi.

ad 228 CYNOSURUS, - Juss - ; (graminea 7.)

ad 229 ELEUSINE, - (graminea \%)

ad 232. SESLERIA, - ARD. - - GRAMEN TOUHN ( rawi.ea 7.)

ad 233. A NDRO POGON, - JUss. -; GRAMEN TOUR.. (gramines 3.)

ad 234. A N THIS T I RIA, - Juss-

ad 235. HOLOSTEUM, - JUSs. -; ALSINE TOURN. (caryophyllea $x_{0}$ ) - Calyx quinquepartitus; - ; corollue petala - bitida -

ad 236. POLYCARPON, - juss. - ; (caryophyllea t.)

ad 237. LECHEA, - KALM. - juss. - ; (caryophilleis affinis) - ; stuminu tria, interdum quatuor, quinque : stuli nulli -; capsula - placentae tros lineares in centro capsulae positae; ; albumen carnosum:- (Plantae herbaceae aut suffrutescentes, habitu similimae Lino; folia alterna ant opposita; flores axil. lares aut dispositi in paniculas terminales.

* ad 239. E R I O C A L O N, - juss. - : (iuncinea I) Monoica : Culyx glumaceus : - ; - ; evarium unum; -; Folia graminea; elongatio trunci ut plurimum nuda, gracilis, ad summum sustunens capitulum parvum squamosurn, compositim centro foribus masculis, femineis in peripleria, in calyco communi imbricato).

ad 240. MON T I A - MICr. - Juss. - ; ALSINÖ̈. UES vAILL. (portulacea 1.) - Culyx bivalvis vel - ; -; styli tres; stigmuta tria; - (Elongatio herbacea. minima; folia opposita, fore connata; flores axillares et terminales ).

ad 241. MOLLUG O, - Juss. - ; (caryophyllea ı.) ad 243. QUERIA, IOEFL. - juss. - ; (caryophyllea i.) - ; styli tres - ( Elongatio tonuis; bracteae setaceae, rectae, ad apicem mucrone recurvo hamoso nunitae).

ad 244. KO E N I G I A, - juss. - ; (polygonea): - ; stylus nullus: - (Elongatio herbacea, nodosa . folia alterna, basi vaginantia, terminalia et quaterna; fores torminales, fasciculati).

ad 245 . 
ad 245. DONA T I A, - rorst. nov. gen. tab. 5. jussi. -; (caryophyllea $I_{0}$ ) -. (Elongatio trunci simplex, tecta foliis imbricatis, linearibus).

ad 246. PROSERPINACA,' - Juss, - ; TRI. XIS GAERTN. - ; (onagraria 1.) - ; styli nulli, stigmata tria -; - ; alhumem carnosum, - Genus antea familiae Hydrocharideum civis; plantae aquaticae; folia al. terna, inferiora pinnatifida; flores sessiles, axillares).

\section{$C L A S S I S P U A R T A$.}

Inseraţur: PHYLA, (PHYLE) tour. I. pag. $83^{\circ}$ (primulaceis affinis). - Calyx communis polyphyllus, imbricatus; proprius diphyllus, foliolis lanceolatis; co. rolla monopetala, irregularis; staminum filamenta brevia; ovarium rotundatum; stylus brevis; stigma incras. satum; sporae nudao, solitariae, receptaculo communi insertae. - (Elongatio trunci repens; folia opposita; pedunculi laterales solitarii).

ad 247. GLOBULAPIA, - тоurx. - juss. - ; (primulaceis affinis) involucrum - - homisphaericum, - ; - corolia tubulosa, - - ; ovarirm superum; stylus unus; stigma simplex; - . (Elongatio trunci herbacea, simplex, uniflora; folia radicalia saepo spathulata; Globulariae habitu proximao Dipsaceis sed dissitae et re: motae inserione staminum; difforunt etiam a primulaceis sporis nudis et florm dispositione; proteacae, corollis deficientes, nililominus aliquam similitudinem intrant cum Giobulariis, quae, novam indicare videntur familiam naturalem).

ad 248. DIPSACUS, - тourN, - Juss: - ; Involucrum (calyx communis) - multiflorum; - ; sta. mina quatuor exserta stigma simplex; spora oblonga, angulosa, - calyce proprio coronata: - (Elongatio trunci berbacea, aspora, aut aculeata; folia interdum basi connata; flores terminales, in capitulos conicos aut globosos dispositi).

ad 249. SCABIOSA, - TourN. - Juss. - ; involu. crum - foliolis uno aut multiplici ordine dispositis, acquale aut inaequale, herisphaericum ; calyx - extevior ovarium cingens, - ; ixterior sub ovario; corolla tubo longo, - ; stamina quatuor exserta; stigma emarginatum ; - ; receptaculum conico -; - (Folia sim: 
plicia aut ṇinnatifida; flores saepe terminales; species qisaedam suffrutescentes).

ad 250. K N A U TA, - Juss - ; SCABIOSA varrt. -: invoucrum - formatum foliolis rectis, simplici serie dispositis, -; calyx - inferus., porsistens; - - : corotlu - tubo oblongo, - ; stamina quatuor inclusa; stigma bifidum; spora oblonga, - (Flores terminales).

ad 251. ALLIONIA, - juss. - Calux proprius parvus, infundibuliformis, limbo inaequali, latere exteriori quatrilobus - ; spora basi calycis, cusus pars superior caduca est, tecta:- - (Flores in pedunculis solitariis et axillaribus ).

ad 5:. PERAMA. - AUBL. tab. 18. - ; (verbena-. cea 2.) - ; stigma simplex, sporae duae-quatuor minimae; (Elongatio herbacea, villosa; folia opposita, sest slia, multinervia; fores in capitulis terminalibus, pedicellati, bractea una muniti).

ad 253. PYROSTRIA, - commers. - ; - ; stigma capitutum; - drupa - parva, non coronatá, -; (Arbor. humilis folia integerrima, coriacea; petunculi axil. lares, uni autmultifori).

ad 254. MYONIMA, - commers. -; (rubiacea 8.) - Corolla - laciniis obtusis - ; antherae oblongao, exsertae; stigma incrassatum; baccu exsucca, cerasiformis, lepressa, non coronata, - nuce quadriloculari - ; sporae uno latere concavae, altero convexae; (Frutex foliis integerrmmis, nitidis; pedunculis axillaribus, termiualibus, uni-trifloris).

ad 255. PETITI A, - juss. (verbenacea 1.) Calux parvis, - ; corulla - tubo longo, - stamina brevia; stigma simplex;-; ( Frutex foliis integerrimis, glabris ; floribus in paniculis axillaribus et oppositis).

ad 256. A QUARTIA, - sUss. - ; (solanea 2.) - ; Corolia - tubo brevissimo, - ; flamenta brevia: stigma simplex; bacca pisiformis -; (Elongatio spinosa aut inermis, tomentosa; folia tomentosa; habitus Soluni).

ad 257. ROUSSEA, - ; (campanulea I) -; corolla - Laciniis acutis -; stamina corolla longiora; -; spovae multae;

ad 258. C A L L I C A R P , - JUss. - ; (verbenacea 1.) - corolla tubulosa, -; stamina exserta, magnitudine ac. quali ; stigma simplex; - ; (Frutices gossypiosao A.res axillares, subverticillati; pedunculi dichotomi, multiflori). 
ad 259. W A L L E N A, - (sedis incertae) - ; ovivium unum; stylus unus; - ; (Arbor ramis cylinulaceis folia alterna, integerrima; flores in racemis terminalibus ).

Inseratur post WALLENIAM: F O S C R E N A, (FOSCAPENE) VAND. flor. bras. (sedis incertae) Ca. $l y x$ inferus, laciniis quatuor aequalibus ; corolla monopotala, infundibuliformis, longa; antherae quatuor sub. sessiles, longae, fauci corollae iusertae; ovarium....; stylus... ; stigma ... ; drupa nuce monospora ; (Elongatio trunci arborescens, tubsrculosa, spinosa; folia integerrima, subtus villosa; flores in capitulis axillaribus).

URCEOLA, - (URCEOLE) vANDEL. flor. brasil. tab. I. f. 4. (sedis incertae) Calyx hexaphyllus: corolla infundibuliformis, tubo longo; limbo lobis quatunr patentibus, extrorsum recurvis; stamina exserta; ovarium superum; stylus anus; stigma glcbosum; capsula urceolata, bilocularis, polyspora.

ad 201. WITHERINGIA, - Juss. - (Solanea 2.) Calux persistens -; corolla - foveolis melliferis; fauco pilis clausa; limbo patente; stumina cum foreolis alterna, versus medium tubi inserta; flamenta brevia, villosa basi appendiculata; antherae - oblongae, lateralitet dehiscentes; stylus tznus; stigma capitatum; bacco - polyspora? (Truncus subterraneus (radix auctt.) fusiformis; flores axillares).

ad 262. A E G I HIL A, - Juss. - ; (verbenacea 1.) Calux parvus - persistens: corolla - limbo plano, aeCalux parvus - puali longitudine exserta; - stigmata
quali; stamina a equalio oblonga; - nucculi quatuor monospori, quibusdam
duo quab ter abortivis; (Frutices pedunculis multifloris, floribus ternatis ).

* M A N A A E, (MANABO) aubr. Guy., tab. 23. JUss. (verbenacea 1.) Calyx turbinatus, quatridentatus; corolia tubo brevi, limbo aequali, quadrifido; stamina exserta ; stigma bifidum; baccabilocularis, dispora, calyco cincta; ( Frutex ramis villosis; folia integra; flores in capitulis densis, axillares). 263. NUXI A, - COMMERS. - (sedis incertao?)
-; antherae ovales; ovarium supcrum ovale; - ; (Ar
bor foliis vertcillatis, integerrimis: flores in racemis bor foliis vertucillatis, integerrimis: flores in racemis axillaribus; - Genus a CO MMERSONIO constitutum cum Manabaea coniunctum esse voluit IUSSIEU.)

Inse. 
Inseratur: * M O S CHARIA, (MOSCHAPIE) FORsK. Juss. (sodis incertae); Calyx globosus, villosus, apico quinquedentatus, persistens; corolla membranacea, clausa ; antherae sessiles, connatae, et intra corollam positae: ororia quatuor: stylus unus; stigma simplex; sporae quatuor nudae; (Genus labiatis analogum).

ad 264. CEPHALANTHUS, - jUss. -; PLATA. NOCEPHALUS, VAIIL. (rubiacea 10.) - calux proprius angulosus -; corolla tubo gracili -; stamina brovia, parum exserta; stigma globosum; -; cupsulae circumpositae in glohulos; - loculi duo abortientes. (Frutices; pedunculi elongati, tèrminales aut axillares).

ad 265. ROUHAMON, - (apocineis affinis) stumina super tubum inserta; antherue biloculares; ovarium unum; stulus unus; stigma bifidum; - : (Frutex ramis oppositis; folia integerrima, trinervia; flores in corymbis axillaribus, oppositis; cyrrhi solitacii in foliorum axillis).

ad 268. CENT TNCÚLUS, - JUss. - ANAGAL. LIS, vaIIL. - : (Fnlia oppusita et alterna; flores solitarii, axillares; interdum stanina quingre, et quinque limbi corollae lacinine, et tunc congener Anagallidisj. ad 270. PLANTAGO, - TOURt. - Juss. - CO. RONOPUS, toURN. -; (Folia omnia radıcalia: flores in spicis densis, bractea muniti).

ad 27I, PSYLLIU M, - Touriv. - ; (Folia opposita).

ad 272. POLYPREMU M, - jUss. - ; stamina brevissima; stylus unus; stigma simplex; (Elongatio berbacea, pendula; folia verticillata; pedunculi solitarii, unis fori, in dichotomia foliorum positi).

ad 27.3. BUDLEIA, - jUss. - ; Calyx parvus - ; corolla - subcampanulata, - ; capsula - valvulis into: gris, dissepimentum simplex; (Frutices; folia verticillata; flores axillares).

ad 275. EXA C U M, - Juss. - : (géntiantea 2.) (Flores axillares aut terminales, ant dispositi in corymbum dichotomum ad punctum dichotomiae uniflorum). ad 277. COU T OU B E A, - AUBL. Guy. tab. 27. JUss. -; -; filamenta basi dilatata; antherae sagittatae; stylus unus; -; (Fleres in spicis axillaribus et terminabus ). 
ad 9-9. T'ACHIA, - AvBL. Guy. tab. 29. - stylus longu\&; stigma bilamellatum; - ; (Frutex lactescens: rami oppositi. tetragoni, sarmentosi; folia opposita; flores solitarii in axillis foliorum).

ad 279 POUTERIA, - juss. - : (ebenacea 1.) -; corolla bas: caljcis inserta - tubo vertricoso, -; capsula vilinsa, -; sporae -, amygdaliformes; (Flores axillares, saepe pedunculati ).

Inseratur: ERIPHIA, (ERIPHIE) вRowri: jam. 2\%0. Jus; (sedis incertae). - Calyx ventricosus, quinquedentatus: corolla tubulosa, fauce dilatata, limbo parv(), quinquelobo: stamina epipetala ; filamenta connventia, arcuata; antherae adglutinatae; quinti staminis rudimentum; ovarium superum; stulus unus: stigma bifidum; bacca coronata, calyce tecta, glob.ssa, utroque latere glandulosa, unilocularis, polyspora; sporae parvae, recoptaculo centrali insertae. - (Genus proximum Scrophulariis; - elongatio herbacea; flcres conforti, exillares).

ad 280. PENAEA, - juss. - ; - corolla calyce duplo longior et latior, - limbo brevi; stumina ad summums corollae inserta et cum laciniis alterna; artherae rectae; ovarium superum; stylus filiformis, aut quadrangul. - membranaceus; stigma capitatum aut - ; Frutices ad basin asporae per foilorum delapsum; supra munitae foliis cruciformiter nppositis, sessilibus, fl ralibus colcratis: flores terminales, sessiles, solitarii aut coaliti. fructus similis fructu acanthi, sed est quadrilocularis; - num ad familiam acanthearum pertinet?).

a 281. B L A ERIA, - juss. - (erica 1.). Calyx laciniis linearibus; corolla - tubo cylindraceo, - ; antherae oblongae, rectae, emarginatas, ut plurimum exsertae; - ; capsula - oligospora. (Snffrutices habitu ericarum: folia parra, verticillata; flores in capitulis terminalibus aut remotis).

ad 282. C HO MEL, I A, - Juss, - ; Calyx parrus, - corolla infundibuliformis - tubo - gracili, -; antherae superiore tubi parte insertao, lineares, subses. siles, non exsartae; -. (Frutex ramosissima et spinosissima: spinae super ramos sparsae, et axillures: folia in ram rum extremitatibus, integerrima; pedunculi solitarii et axillares). 
Inseratur: CUSSAREA, - (CUSSAREF) AUBI, Guy. tab. 38. (rubiacea 6.). - Calyx quinquedentatus; corollae tubus. brovis, limbo acuto quinquepartito; antherce oblongas, ad faucem corollae subsessiles, exsertae; stigma quadri aut quinquefidum; bacca unilocularis, parva; nux monospora; spora coriacea. - (Frutex foliis integerrimis; flores saepe terminales).

ad 283. MALANEA, - $\mathrm{ATB}^{\mathrm{T}} \mathrm{A}$. Guy tab. 41. Juss. -; (rubiacea 6.). Corolla parra, - tubo brevi -; stamina exserta ; bacca parsa, - (LAMArcir cum hoc genero genus Antirhea conIrerson coniunctrm esso roluit. Frutex sarmentosa ramis pendulis; folia revoluta; flores in spicis axillaribus ad ramorum extremitates.

ad 234. SCOLOSANTHUS, --; Calyx quadridentatus ; - antherae longitud.ne tubi ; - (Kami cylindracei; folia subsessilia, integerrima).

ad 285. PAVETTA. - rнeED. - ; corolia - limbo patente, - laciniis acutis; antherae subsessiles et exsortae; - (Irutex folis integerrimis; florss in cọ. rymbis terminalibus::

ad 286. IXORA, - jiss: -; corulla - laciniis obtusis -; antherue subsessiles et exsertae; -. (Frutices; flores in corymbis terminalibus).

ad 287. PETESIA, Joss. - (Frutices; pedunculi axillares, uni aut muluflori),

ad 289. CA TESBEA, - Juss. - (rubiacer 5.) corolla magna, -; - ; antherue oblongae, exsirtao -; (Frutex spinosissima; folia parva, integra; flires solitarii, axillares).

ad 289. FR OEL I C H I A, -; ( Arbor parum elata; rami tetragoni, glabri ; folia opposita, integerrima, flores in panicula terminali).

Inseratur: POLYOSUS, (POLYOSE) rour. (sedis incertae) - Calyx inferus, quadridentatus. corolla monopetala, tubo brevi, limbo quadrilnbo; stamina nuatuor, brevia, in tubo affixa; antherae oblongae, vvavium unum; stylus unus stigma magnum, emarginatum; bacca calycinalis, unilncularis, dispora; - (Arbor excelsa, folia bipinnata; flures in racemis asillaribus et brevibus ).

ad 291. ER NODE A, - ; stigma truncatum: - ; (Frutex ramis tetragonis; folia opposita, sessilia, integerrima ). 
ad 292. SIDERODENDRUM, - Calyx profunde quadrifidus; - : filamenta staminum brevissima; stulus longitudine corollae; -; (Flores in pedunculis axiliaribus ).

ad 293 FER NELIA, - ; Calux - laciniis subulatis? corolla - tubo brevi : - bacca pisiformis, parum carnosa -; (Frutex; folia opposita ; flores subsolita. rii, axillares).

ad 296. M I T C HEL L A, - Juss. -; Calycesadríatao per internam superficiem, -; corolla - tubo cylindrico -; stamina vix exserta; -; (Elongatio herbacea, articulata ;).

Inseratur: PA TABEA, (PATABEE) AUEE, Guy. t. 43 Juss. IAx. tab. 65. (rubiacea 10.) Flores capitati, distincti interpositis bracteis; capituli florum squamati; calyx quadridentatus; corolla tubulosa, quadrifida; antherae subsessiles; fructus .... (Frutex ramis dissi. tis : florum capituli solitarii et subsolitarii).

FA PA MEA, (FARAMEE) AUnL. t. 40. f. r. JUSs, (ru= biaceis affinis); Calyx turbinatus, quadridentatis; cc, rolla tubulosa, limbo quadrifido; stumina non exserta; ovarium inferum; stylus unus; stigma bilobum; fructus bilocularis; (Frutex foliis oppositis, integerrimis; pe: dunculi terminales, multiflori).

ad 29\%. EVEA, - AUBL. Guy. tab. 43. JUSs. - ; - ahtherafoblongae, subsessiles, non exsertae; --; (Frutex ramis tetragonis: folia ovalia, integerrima; flores in capitulis axillaribus, solitarii).

ad̉ 298. HED Y O T IS, - Juss. - (Herbae aut suffrutices; flores terminales aut axillares).

ad 292. O LDENLA NDIA, - PLU.M. - (rubiacea 3.) - ; (Elongatio herbacea, interdum sublignosa; pedunculi terminales aut axillares, uni aut multiflori; folia basi connata membrana ciliata).

ad 301. N A C I B A EA, - Juss. - ; corolla ad faucem tubi attenuata, -; capsula -.. valvulis navicularibus, marginibus intrantibus et membranaceis; quasi capsulao duae; - (Pedunculi solitarii, axillares, multiflori).

ad 302. CARPHALEA, - ; Calyx turbinatus, - laciniis oblongis, - persistentibus; corolla - tubo longo, filiformi, ventricoso, - laciniae acutae, angustatae; an- 
therae oblongae, subsessiles, fauce tubi insertae; stigma acutum? - ; (Frutex elongatione cylindrica; folia in fascrculis oppositis et verticillatis; flores oppositi in corymbis terminalibus).

ad 304. SANGUISORBA, - juss. - PIMPINEL. - LA, Tourn. - ; ovaria, styli, stigmatu, simplicia duo;--

ad 305. HOUSTON I A, - anon. - Juss. - ; ru. biacea 2.) -; stamina minima; ovarium superum; - ; (Flores terminales).

ad 306. A N THOSPERMUM, -; (Frutex; folia verticillata, minima; flores axillares).

ad 307. IR UBIA, - tourn. Juss. - ; - ; sporae glabrae; (Plantae nitidao aut muricatae; flores in corymbis axillaribus ot terminalibus).

ad 308. G ALIIU M, - TOURN. JUss. - ; APAPINE, TOURN. - - ; sporae glabrae in Gallium TOURN., hispidae in Aparine tounN, non coronatae; (Elonga. tio trunci herbacea, glabra aut aspera; flores termina. les aut axillares, saepe dispositi in corymbis paniculatis).

ad 309. ASPERULA, - Juss. -; GALIUM, APARINE, CRUCIATA TOURN. - ; corolla - interdun trifida, et stamina tria; -; (Fores terminales; aut axillares ).

ad 311. SHERARDIA, - - APARINE, TOURN, - ; (Flores axillares aut terminales).

ad 312. SPERMACOCE, - ; (Flores axillares; in. terdum verticillati aut in capitulis terminalibus).

ad 3I3. II NOXIA, - yuss. - ; Calyx - quadridentatus; corolla tubulosa, - limbo quadridentato; -; (Flores alterni, dispositi in spicam terminalem).

ad 3I4 D IO D I A, - juss. - ; - corolla tubulosa. - ; (Elongatio trunci prostrata; flores sessiles).

ad 315. CR UCIA NEL L A, - Juss. - RUBEOLA, TOURN. - ; corolla tubulosa, - -; rarius quinquefida et stamina quinque.

ad 316. SIPHONAN THUS, - Juss. - ; ovarimm quadrifidum; stylus unus; stigmu simplex; - (Elongatio trunci herbacea, simplex; folia ternata, sessilia; flores in racemis terminalibus).

ad 31\%. EPIMEDIUM , - rounN. - Jess. - ca. byx - patens; - foliola duo basi bractea munita; co- 
rollae petala - patentia, similia calyci eiusque foliolia opposita : - stulus lateralis ; stigma simplex; -. (Elongatio herbacea, inxta truncum (radicem auctt) squamis cincta: folia bi aut triternata).

ad 318. P TELEA, - jues. - ; calyx parrus, -; ovarium simplex; stulus brevis; -; sporae oblongae. (Frutices; folia alterna, ternata, rarius impari pinnata, et glandulis subcutaneis translucentibus obsita ; fores fere in corymbo axillari et terminali; inter. dum petala quingue et quinque stamina, stigmata tria et loculi tres; interdum etiam dioici).

ad 3,9. PTELIDIUM, - (Frutex foliis oppositis integerrimis).

ad 32I. SKI M M I A, - - ; calyx - persistons: co. rolla - minima; - stylus unus, stigma. .; bacca pisiformis, - (Frutex foliis alternis, floribus in panicula terminali).

Inseratur: ALLASIA . (ALLASIE) Lotr. (sedis incertae). - Calyx monophyllus, limbi laciniis quinque villosis; corollae petala quatuor parva, concava; filamenta crassiuscula; antherce biloculares ; ovarium unum; stylus unus; stigma acutum ; bacca carnosa, mag. na, oblonga, unilocularis, in pulpa continens sporams unam. - (Arbor oxcelsa; folia digitata, integerrima; pedunculi multıflori).

ad 322. AZIMA, - joss. - Calyx ventricosus, cam. panulatus, - ; corollae petala - linearia, altorna cum laciniis calycis, apice parum reflexa: stamina recoptaculo inserta, alterna cum petalis ; filamenta recta, incrassata; antherae oblongae, cernuae; ovarium subquadrangulum; stylus brevis, stigma acutum; cupsula subcarnosa, pisiformis, unilocularis - ; sporae orbiculares, leviter compressae, alteía abortiva. - (Genus analogiam cum Tw STRYCHNOS habere videtur; frutex sempervirens; rami rigidi, spinosi; folia opposita, coriacea, integerrima, spinose mucronata, ad basin spinis-quatuor verticillatis munita; flores solitarii axillares, sessiles, parvi).

ad 324. HA TOGIA, - SCHREBERA - JAUME; (rhamnea 2.) - Calyx parvus, - quinquepartstus? Corolla infundibuliformis, quinquefida? stamina quinque fauci corollae inserta; filamenta introrsum squama 
ciliata munita; ovarium bilnbum; styli duo; stigmatd duo: fructus bilocularis, disporus. - (Frotex foliis alternis; pedunculi numerosi, mụltiflori et axillares).

Inseratur: DORA T IU M,' (DORATIE) solAND. juss. mss. (rbamnea 2.) - Calyx profunde quadrifidus; corollae petala quation calyce longiora; stamina quatuor petalis breviora; ovarium superum; stylus unus; stigma apice quadrifidum aut apice quinquepartitum; drupa globosa, nuce ossea, aeque globosa, quadri aut quinqueloculari, tetra aut pentaspora.

M A Y PEA, ( IAYEPEE) AtBL. Guy. tab. $22 \%$ Juss. (rhamiea 3.) - Calyx quašrifidus, patens; corollae petala quatuor inter lacinias calycinas inserta, basi concava, versus summum in longum filamentum attenilata; stamina quatuor; antherae sessiles et positae intr petalorum foveas: stylus nullus: stigma incrassatum concavum; dripa olivaeformis, nuce monospora, lignosa.

ad 325. CUR T ISIA, _- - ; (Frutex; folia opposi. ta; flores in panicula terminali).

Insevitur: CONTRARENIA , (CONTRARE. N(E) vaND A. brasil. tab. 3. f. 20. (sedis incertae). Calux tubu!osus, bifidus; corolla parva, ligulàta, apico trifida; siamina inciusa; ovarium unum: stylus tenuis, intus curvatus; stigma globosum; capsula bilncularis, associata calyco et corolla exsiccaia, polyspora (VANo Detre hanc plantain ad Didynamiam amandavit).

ad 326. FAG A R A, - juss, - ; (terobinthaceis affi. nis) - capsula una (rni:ss duae-quinque) - unilocularis, - ; spora - nitida. - (Frutices aut suffrutices, spinosao aut inermes: folia alterna, simplicia aut ter. nata, saepissime impari innata", glandulis subcutaneis translucontibus obtecta, flores fasciculati aut in racemis axillaribus?

ad 33r. TRA PA, - juss. - : TRIBULOÏDES TOURN.

ad 332. CISSUS, - juss. - ; - (Folia uni-biternata -). 
ad 333. VOTOMITA, - (Frutex ramis tetrogonis: folia opposita integerrima; pedunculi axillares tri au\& quadriflori).

ad 352. PERSOONIA, - VENT. jard. malm. tab. 82. - corolla (calyx sec. Juss.) - laciniis rectis, apice aucuatim revolutis, -; filamenta linearıa, compressa; antherae Inngitudinaliter dehiscentes; ovarium in pedun: culo brevi, basi glandulis exsertis cincto; stylus cylindricus; stigma truncatum -

Iuxta 570. inseratur:

C A T O N IA, (CATONIE) BROWN. JUSs. (sedis incertae). - Calyx superus, quadrifidus: corolla nulla; stamina quatuor; ovarium inferun, globosum; stylus unus; stigma simplex; bacca carnosa, coronata; spo:at quaiuor, una aut duabus saepe abortientibus. - (Frutex foliis oppositis, margine parallele venosis).

Inseratur: LA GEN ULA, (LAGENULE) IoUR, Juss. mss. (cucurbitaccis affinis). - Calyx quadrifidus; stamina quatuor; ovarium cinctum et disco carnoso tectum, quadrilobum; sillus incrassatus; stigma simplex; bacca parva, ampullaeformis, bilocularis loculo singulo disporo. - (Frutex elongatione scandente; folia pedata).

OCT ARILLUM, (OCTARILLE) LOUR. conch. 113. (eleagni). - Calyx urceolatus limbo quadripartito; staminum quatuor filamenta brevissima; ovarium oblongum: stylus staminibus longior; stigma incrassatum; bacca ovali oblonga, monospora; spora arillata. (Frutex foliis alternis, iutegerrimis; pedunculi uniflori, solitarii, axillares).

\section{$C L A S S I S P U I N T A$.}

In seratur: GONUS, (GONE) Lovr conch.pag. 809: (terebintacea 3.) - Calyx profunde quadrifidus, post florescentiam caducus; corolla tetrapetala; stamina breo 
vissima; ovarium tetragonum; stigmata quatuor sessilia; drupue quatuor, singula monospora. - (Frutex: folia impari pinnata; flores in spicis subterminalibus).

ad 394. NYCTAGO, - IAI,APA MEDICUs monad. pag. 83. - filamentu super ovarium connata, tubo coriaceo sporam ambiesite; e margine tubí surgunt filàmenta quinque recta, chordaram in modum extensa, ubi corollae tubum attingunt eo adglutinantur, deinceps libera; ovarium intra tubum staminum, supra pyriformo. styius longus, exsertus, stigma capiszto globosum, glandulis pedicellatio obtectum; influrescentiae tempore giobulus snpera parte vacusus, nisi filamentis extensis permeantibus; foeoundatione peracra, ovarium grossificatur, cortlae tubo deciduo globulus indurescit, supra clauditur, nuciformis evadit, sporis repletar. Conspici antem omsi temporis momento adhuc valent quae restant flamentorum tubi mombranulae scariosae inter nucem sporasque - Propier filainentnrum singularem fabricam ad monadiplpias medicus Ialappam retulit rid 1 l. pag. 84 . et ibid. de invo. lucro et calyce recte dijudicatus est.

ad 399. PLU M BAGO, - Miedicus monad. pag. 86 . - covolla - infra parum rentricosa, tubo aequali supra dilatato -- ; stumina intra ventricosam cornllae parte: connata in tuburn crassiusculum: filumeata quinque; antherae violaceae: - stulus longitudine flamentorum; stigma - lacıniis linearibus radiantibus; capsula oblonga, quinquangula, acuminata, clausa, lateraliter angulos valvulis quinque dehiscens, apice coniunctis remanestibus spora ut capsula oblonga, propria testa sua vestita: stulus capsulum peretrat, receptaculum efformans centrale fundo calycis innatum, sporis maturis filum longitudinaliter decurrentem effin. gens.

ad 429. A N A G A L I S , - MEDrcus monad. pag. 89. - corolla campanulato rotata - Linbis rodundatis stamina basi arachnoideo - c nnata; e tubo staminum filumenta quinque; capsula rotundata, - ; receptaculum capitatum, sporiferum; sporaetrigonae.

an 430. LYSIM A CHIA, - MEDICUs monadelph. pag. 89. - corolla basi tubo brevi cumuata petala quinque patentia, filamenta e tubo connato recta qunnque; receptuculum capitatum, sporifernm sporae trigonae; capsula semper apico quinquevalvis, valvulis sponto se. parabilibus. 
ad 539. TOURNEFORTIA, - VERRUCARTA MEDrc. monadelph. pag. I03. - corolla - lacisiae subulatae; stamina - supra filo coriaceo coniuncta; ová vium rotundatum ; -.. fructus drupa - nuces quadrivalves, coriaceae, -

ad 542. SOLANUM, - AMATULA MEDIC. monad. pag. 106. calyx - laciniae ovali acuminatas; - fila. menta basi connata, membranacea elongatione apicesubquinquefida ; -

ad 692. B A L S A MIN A, - MEDIC. p. 7 O. - stylus conicus; stigmata quinque radiata. - 


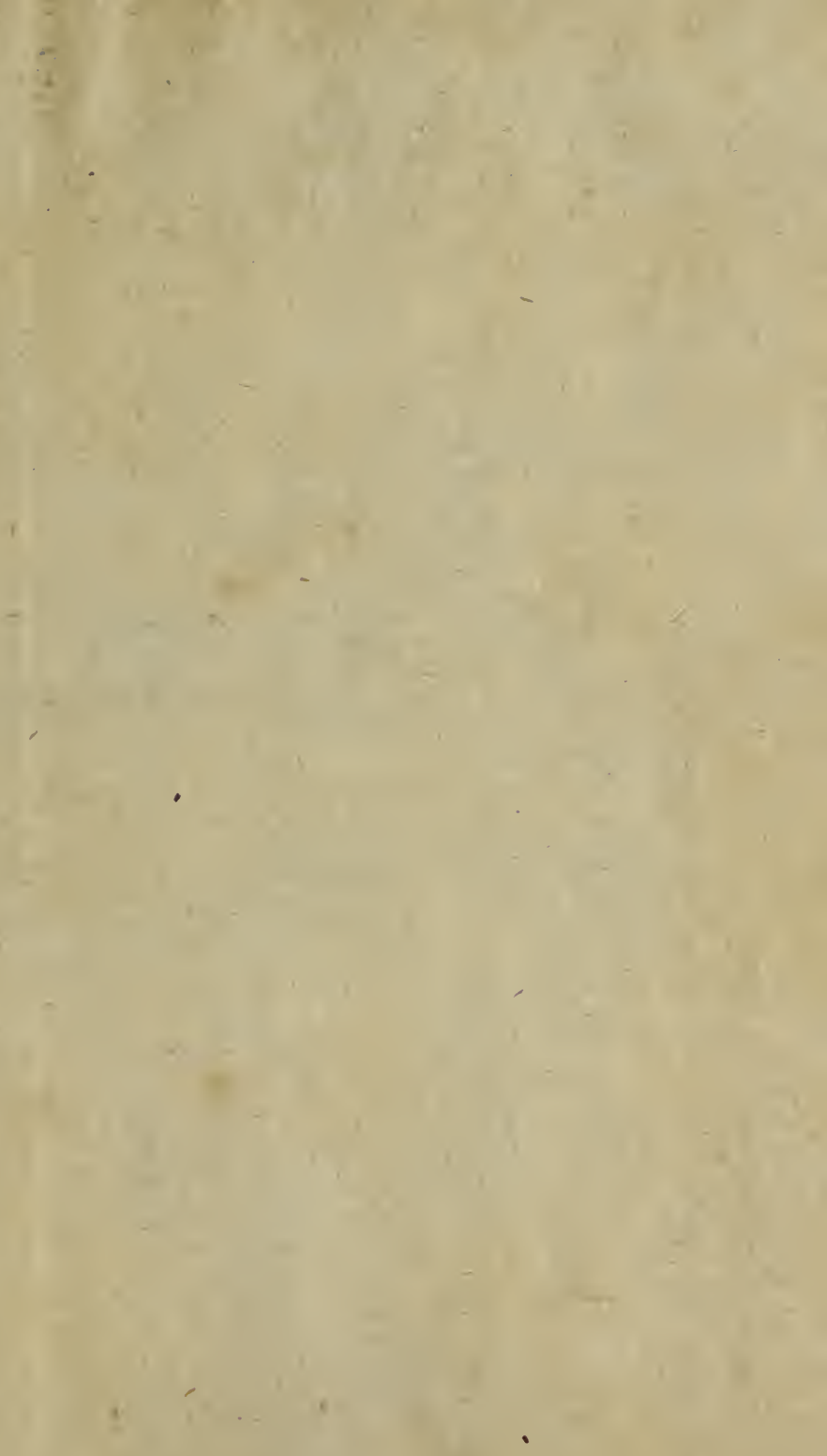





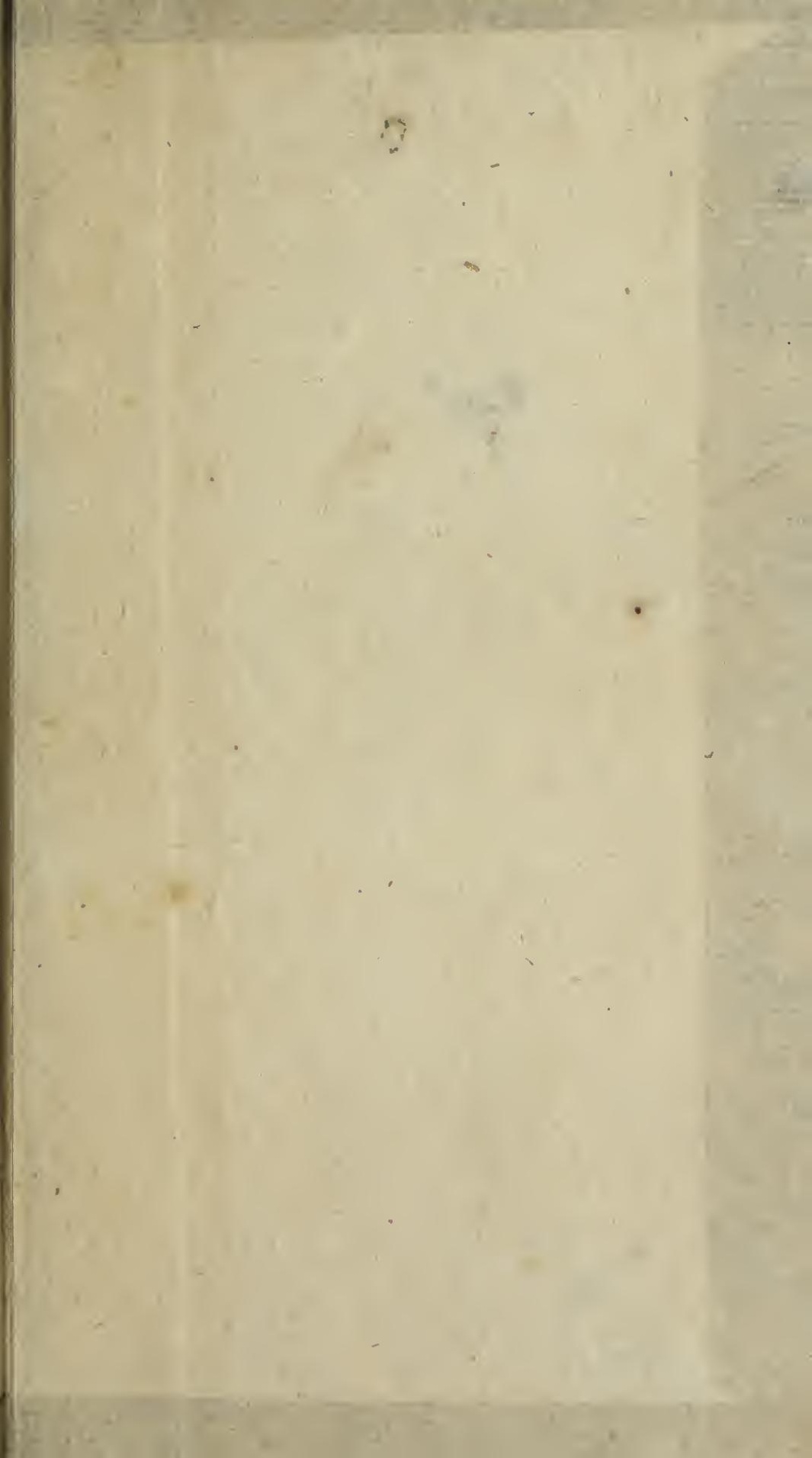




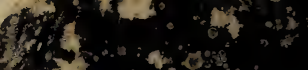 \\ $4 x^{x}$}

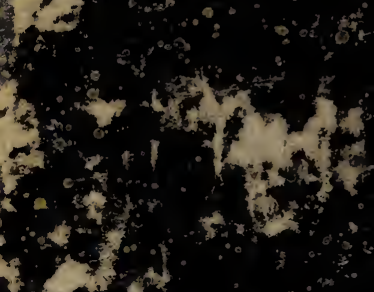
21 $21+$

- 3

8

है?

s.

$x$

$\therefore \operatorname{los}^{+}$

$-30: x$

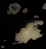

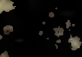

$\therefore 4$

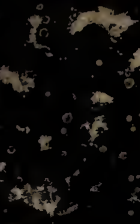

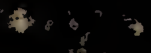

$\therefore$

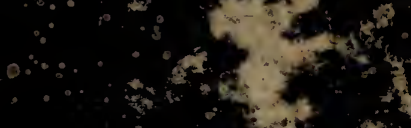

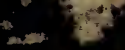

$\tan ^{2}+x^{2}+x^{2}$

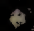

$\because 0,4$

and

$-4$ $x^{2}+x^{2}$

1

28

$y^{2} x^{2}+x^{2}+2$

4x)

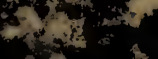

$-x^{2}+3$

\section{$\%$}

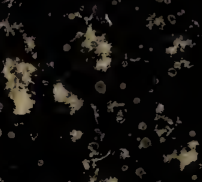

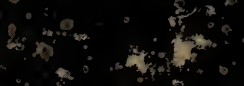

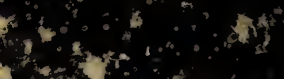

600 a

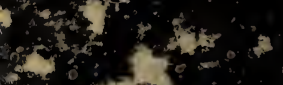

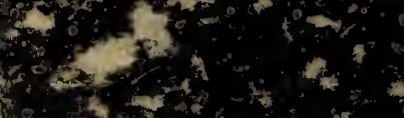

s.

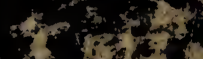
the

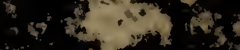

$\therefore$ a 